Nicholas H. Schaffner, Neubrückstrasse 95, 3012 Bern

\title{
Wege ins Erwachsenenleben
}

Integrationsprozesse an einer Schule in der Nordwestschweiz

\author{
Dissertation \\ zur Erlangung des philosophischen Doktorgrades \\ an der Philosophischen Fakultät \\ der Georg-August-Universität Göttingen
}

vorgelegt von

Nicholas H. Schaffner

aus Sydney, Australien

Göttingen 2012

Diese Arbeit entstand im Rahmen des nationalen Forschungsprogramms „Integration und Ausschluss“ (NFP 51) des Schweizerischen Nationalfonds (2003-2006) 


\author{
Versicherung \\ gemäss $§ 11$ der Promotionsordnung \\ der Philosophischen Fakultät \\ der Georg-August-Universität Göttingen
}

Hiermit versichere ich an Eides statt, dass ich die eingereichte Dissertation "Wege ins Erwachsenenleben" selbständig und ohne unerlaubte Hilfe verfasst habe. Anderer als der von mir angegebenen Hilfsmittel und Schriften habe ich mich nicht bedient. Alle wörtlich oder sinngemäss den Schriften anderer Autorinnen oder Autoren entnommenen Stellen habe ich kenntlich gemacht. Die Abhandlung ist noch nicht veröffentlicht worden und noch nicht Gegenstand eines Promotionsverfahrens gewesen.

N. Schaffner 


\section{Inhaltsverzeichnis}

Vorwort

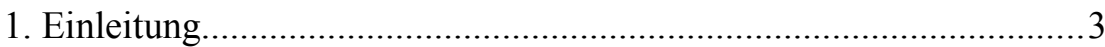

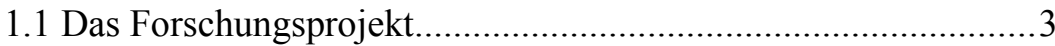

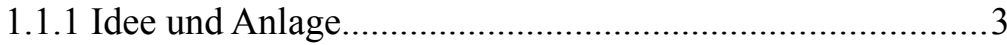

1.1.2 Tatsächlicher Verlauf........................................................

1.1.3 Meine Position im Feld ....................................................12

1.2 Die Erforschung von Schulen in der Europäischen Ethnologie. 13

1.3 Zum Aufbau dieser Arbeit.......................................................29

Teil I: Methoden und Feldzugang...........................................................31

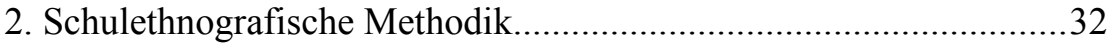

2.1 Ethnografische Perspektiven auf Schulen..................................35

2.1.1 Schulethnografie als Organisationskulturforschung...........37

2.1.2 Spezifik der schulethnografischen Praxis............................40

2.1.3 Implementierung der Methodik..........................................48

2.1.4 Validität, Reliabilität, Triangulierung...................................50

2.1.5 Rollen, Beobachtungsfokus, Langfristigkeit.......................51

2.1.6 Gatekeepers, Setting, Aufzeichnungsgattungen..................53

2.1.7 Strategie der Anwesenheit...............................................56

2.1.8 Spezifik der Forschung mit Jugendlichen..........................60

2.2 Strukturierte und phänomenografische Interviews.....................61

2.3 Quellenproduktion: Aufsätze, Objekte, Schriftliches.................68

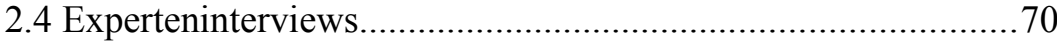

2.5 Integration des Forschers in das Feld.........................................

2.6 Methoden der Auswertung und Verschriftlichung.......................86

2.6.1 Verschriftlichung mit Blick auf die Lesenden.....................87

2.6.2 Elektronische Datenauswertung durch ein Wiki................91

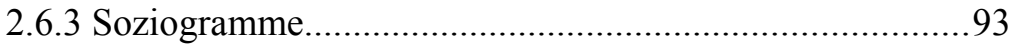

2.6.4 Überlegungen zum Aufbau des Textes...............................95 
3. Arbeitsweisen und die Situation im Forschungsfeld.......................95

3.1 Die Schulklasse 2D als kulturelle Gruppe..............................96

3.2 Die historische Situation der WBS..........................................99

3.3 Vergleich der Integrationsbemühungen Schweden/Schweiz....106

3.4 Verlängerung der Schule in den Brückenangeboten................112

Teil II: Jugend in der sozialwissenschaftlichen Forschung...............115

4. Historische Bedingungen von Jugend........................................116

4.1 Der jugendliche Gesellschaftsvertrag wird neu verhandelt......116

4.2 Die erste Generation des neuen Jahrtausends.........................125

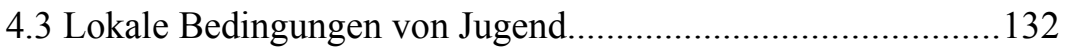

5. Begriffe, Konzepte und theoretische Felder...............................135

5.1 Integration und Ausschluss................................................. 136

5.2 Jugend und Gesellschaft................................................. 158

5.3 Jugendkultur und Kultur der Jugend.................................165

5.4 Jugend und Moratorium.................................................. 171

5.5 Übergangsriten und Statuspassagen..................................... 178

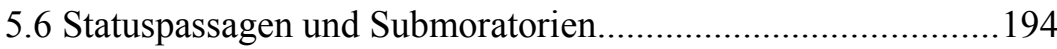

5.7 Normalität und Integration ..............................................208

Tabelle: Verlauf der Integration in die Erwachsenenwelt.................209

Teil III: Die Fallstudie Klasse 2D: Schule, Berufsübergang, Freizeit. 213 6. Zerfallserscheinungen am Ende der Schulzeit..............................214

6.1 Gruppierungsprinzipien...................................................216

6.2 Das letzte Klassenzimmer..............................................221

6.3 Gruppenbildung bei den jungen Männern............................2224

6.3.1 Die Einheimischen..................................................225

6.3.2 Hip-Hopper (Basketball)...........................................226

6.3.3 Zwischen Skater und „halb irgendetwas“......................233

6.4 Junge Frauen und ihre Dyaden............................................235

6.4.1 Sonia, Janine (und Loredana)....................................237

6.4.2 Monika, Rosa (und Fiona)........................................257

6.5 Der Einzelgänger: Stefan..................................................259 


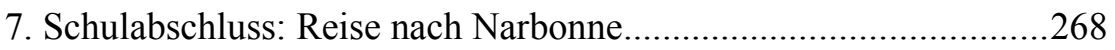

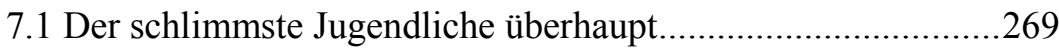

7.2 Rituelles Ende der Schulzeit auf dem Zeltplatz........................278

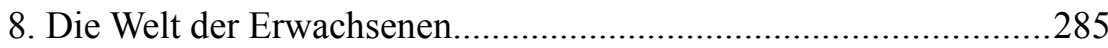

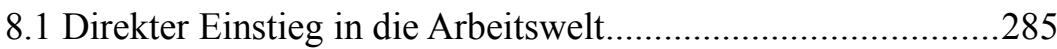

8.1.1 Berufliche Integration einer Lehrtochter..........................286

8.1.2 Integration eines Lehrlings Vorlehre A.............................289

8.1.3 Weitere Beispiele aus der 2D.........................................294

8.2 Noch keine Perspektive im Brückenangebot.............................304

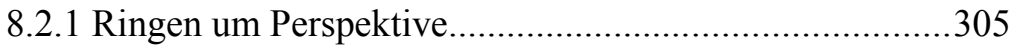

8.2.1.1 Widerstände, Hemmungen, Zögern..........................307

8.2.1.2 „Sinnvoll“ ist die „,anständige“ Lehre.......................310

8.2.2 Gute und schlechte Berufe................................................. 316

8.2.3 Werte und Zukunftsvorstellungen.....................................321

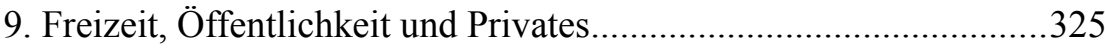

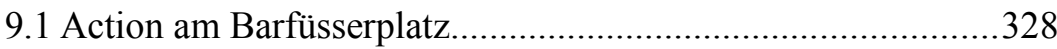

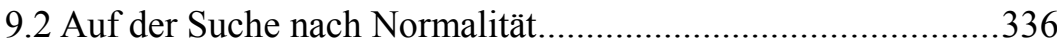

9.3 Die Wohnung: Spielplatz der Normalität.........................................

9.3.1 Geschlecht, Körper, Sexualität.........................................351

9.3.2 Treue und Beziehungen....................................................353

9.3.3 Gender: Sexualität und Männlichkeit.................................356

9.3.4 Konsumkultur: Hanf und Alkohol....................................359

9.3.5 Real Time: Das Leben in Echtzeit...................................372

9.3.6 Verhältnis zu den Autoritäten...........................................373

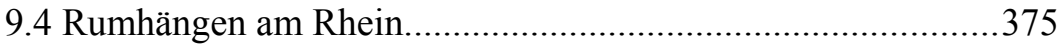

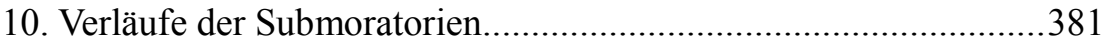

11. Schlussbemerkungen und Ausblick...............................................390

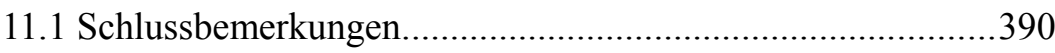

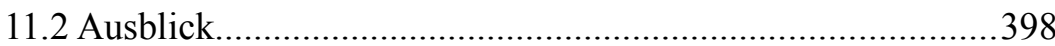

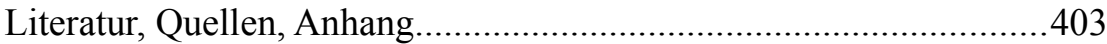

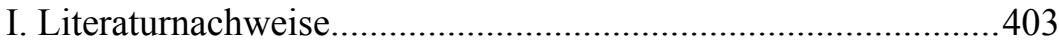

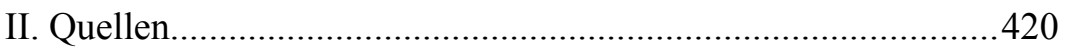


II.1 Dokumente amtlicher Stellen.......................................420

II.2 Originaldokumente...................................................421

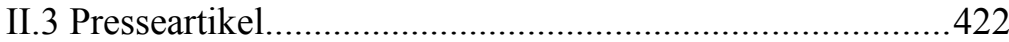

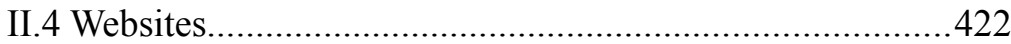

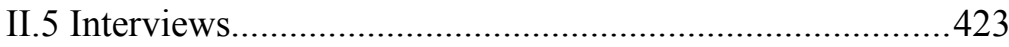

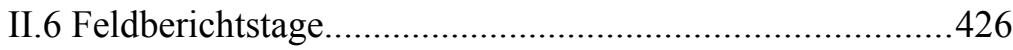

III. Anhang.................................................................... 426

III.1 Leitfaden strukturiertes Interview vom Juni $2004 \ldots \ldots \ldots . . .426$

III.2 Fragen für das unstrukturierte Interview, Herbst 2005....429

III.3 Liste von Denkeinheiten................................................429 


\section{Vorwort}

Diese Arbeit führt ins Basel des ersten Jahrzehnts des 21. Jahrhunderts. Da besonders die rechtsrheinischen Quartiere des schweizerischen Kantons Basel-Stadt überschaubar sind, wurden sämtliche Namen und Ortsbezeichnungen im Text bewusst vage gehalten, geändert oder mit ähnlichen Bezeichnungen anonymisiert. Die Namen sind mit einem Zufallsverfahren aus dem Telefonbuch ausgewählt worden. Allerdings wurde bei der Auswahl darauf geachtet, den kulturellen Hintergrund der Akteure bei der Namensgebung durchscheinen zu lassen.

Die Arbeit hätte ich nicht ganz ohne fremde Hilfe zu Stande gebracht. Ganz besonderen Dank gebührt zuerst den Schülerinnen und Schülern der in dieser Arbeit 2D genannten Klasse und dem Lehrpersonal an der Weiterbildungsschule Basel-Stadt (WBS). Danken möchte ich auch den Klassenlehrern und -lehrerinnen aller zweiten WBS-Klassen im untersuchten Schulhaus im Jahre 2003/2004, insbesondere der Lehrerin, die in diesem Buch Isabelle Gisler heisst. Sie hat mich geleitet und beraten. Auch im Brückenangebot Basis, einer weiterführenden Schule, wurde ich im Herbst 2005 von Paula Lang sehr hilfsbereit empfangen. Die Person hinter dem Pseudonym hat ein ganz grosses Dankeschön verdient, wie auch die Mädchen und Jungen, die mich in ihrer Klasse aufnahmen.

Zur Abfassung mancher Texte, die Grundlage dieser Studie wurden und die hier zum Teil eingearbeitet sind, konnte ich auf die Hilfe verschiedener Mentorinnen zählen. Johanna Rolshoven hat immer den richtigen Input zur richtigen Zeit geleistet, Gisela Unterweger hat mir mit ihrem grossen Wissen über Schulen und Schulethnografie viele fruchtbare Perspektiven aufgezeigt. Michaela Heid stand immer für Diskussionen zur Verfügung. Auch konnte ich im Gespräch mit Christi- 
ne Burckhardt-Seebass mein Wissen über Jugendliche in Basel vertiefen. Die schwedischen Texte habe ich selber übersetzt. Zudem möchte ich ganz herzlich Regina Bendix danken. Ohne ihre kritische Auseinandersetzung mit dem Stoff dieses Buches hätte dieser Text nie den jetztigen Stand erreicht.

Ein ganz grosser Dank gebührt schliesslich Janine und Loredana. Ohne die Mädchen hinter diesen Namen wäre die Arbeit gar nicht durchführbar gewesen. Sie haben mich aufgenommen, wie ich es nie erwartet hätte. Es war unglaublich bereichernd, diesen Mädchen beim Erwachsenwerden zusehen zu dürfen. Dafür möchte ich mich nochmals bei ihnen bedanken. 


\section{Einleitung}

\subsection{Das Forschungsprojekt}

Integration wurde in den 1990er Jahren zu einem vieldiskutierten Konzept in der Schweiz, da sich im Zuge der sogenannten Globalisierung und der damit verbundenen Migrationsbewegungen ein Unbehagen bemerkbar machte; es wurde diskutiert, ob denn alle diese fremdkulturellen Menschen nicht die Gastgesellschaften destabilisieren könnten. In diesem Zusammenhang wurde das Thema akut, wie denn genau Integrationsprozesse ablaufen. Unter anderem zur Beantwortung solcher Fragen hat die Schweizerische Eidgenossenschaft das Nationale Forschungsprogramm 51 (NFP 51) aufgelegt.

\subsubsection{Idee und Anlage}

Diese Arbeit entstand im Rahmen des NFP 51-Teils "Integration und Ausschluss". Das Projekt hiess' "Integrations- und Ausschlussprozesse bei Jugendlichen in Ausbildungsgruppen". Es war darauf angelegt, zwei Abschlussklassen in Bern und Basel am Ende ihrer obligatorischen Schulzeit zu vergleichen. Die dabei gewonnenen Erkenntnisse sollten

1 In dieser Arbeit wird die in der Schweiz übliche Rechtsschreibung verwendet, insbesondere ohne $\beta$. Des weiteren soll die Sprache möglichst geschlechterneutral sein. Dieser Anspruch tritt jedoch in den Hintergrund, etwa wenn ich meine männliche Erfahrung als Feldforscher reflektiere oder Dinge beschreibe, die in rein männlicher Umgebung statt fanden. Interviewpassagen, welche die erhobenen Daten illustrieren, suchen einen Kompromiss zwischen der blumigen, kreativen Dialektsprache der untersuchten Jugendlichen und der hochdeutschen Standardsprache, um die Sprachstimmung vor Ort wiederzugeben. 
die empirische Ausgangslage bilden, um Integration in ihrer alltagsbezogenen Dimension zu verstehen. Grundlegende Idee der Forschungsanlage war die These, dass Interaktionsmuster beschrieben werden konnten, in denen Bedeutungen ausgehandelt würden. Dieses Aushandeln führe zu einer bestimmten Konstruktion von Normalität, die als zentrale Motivation zur Integration oder Ausgrenzung verstanden werden sollte. Unser Ziel war es, Empfehlungen zu Handen staatlicher Akteure auszuarbeiten, um die Steuerbarkeit von Integrationsprozessen zu verbessern.

Die empirischen Daten wurden mit verschiedenen Methoden erhoben. Teilnehmende Beobachtung, Gruppengespräche, strukturierte und unstrukturierte Einzelinterviews sowie Analysen sozialer Netzwerke erlaubten es, die ethnografischen Befunde mit dem gesellschaftlichen Kontext und theoretischen Hintergrund zu verknüpfen. Das Projekt begriff die Jugendlichen als aktive Kulturproduzenten, die Normvorstellungen aushandeln und Zugehörigkeiten in einem fragilen Prozess herstellen und ausformen. Deshalb wurde die emische Sichtweise, die Sicht der Akteure, zur wichtigsten Forschungsperspektive.

Ausgangspunkt des Nationalfondsprojektes war eine Forschungsarbeit von Gisela Unterweger aus dem Jahre 2002, deren Erkenntnisse sie im Buch "Klasse und Kultur"2 präsentiert hat. Unterweger hatte eine Schulklasse der Oberstufe im Kanton Zürich mit Schülerinnen und Schülern aus zwölf verschiedenen Nationen teilnehmend beobachtet und gezeigt, wie sich kulturelle Identität in einem multikulturellen Umfeld gestaltet. Diese ist primär gelebte Alltagspraxis und entwickelt sich in Prozessen des Aushandelns, Vergleichens und Bewertens. Die Frage war, wie die Jugendlichen damit umgehen, dass sie verschiedene soziale und kulturelle Hintergründe haben. Die Arbeit zeigte, wie sich Ju-

2 Unterweger, Gisela: Klasse und Kultur. Verhandelte Identitäten in der Schule, Zürich 2002 (Zürcher Beiträge zur Alltagskultur, Bd. 12). 
gendliche im Austausch mit anderen Jugendlichen, beeinflusst von Medien und gesellschaftlichen Diskursen, kulturelle Symbole und Systeme aneignen, um sich in ihrem sozialen Umfeld zu behaupten und zu einer akzeptablen Position zu gelangen. Dabei lag das Augenmerk auf den komplexen Verhandlungen rund um eine sich bildende Identität.

Am Ende ihrer Untersuchung hatte Unterweger gefordert, eine Langzeitstudie mit dem Fokus auf Identitätsprozessen von Einzelpersonen durchzuführen. Nur so könne aufgezeigt werden, wie vielfältig, komplex und dynamisch sich kulturelle Identität in einer von Migration beeinflussten Gesellschaft für die Jugendlichen gestaltet. Diese Langzeitstudie konnte von 2003 bis 2006 dank der Unterstützung des Nationalfonds durchgeführt werden. Zu den Ergebnissen dieser NFP 51-Studie "Integrations- und Ausschlussprozesse bei Jugendlichen in Ausbildungsgruppen" sei auf den 2006 erschienenen Schlussbericht ${ }^{3}$ verwiesen. In ihm wird auch ausführlich auf die Differenzen zwischen den Untersuchungsorten Bern und Basel eingegangen.

Die vorliegende Arbeit geht nicht auf die Unterschiede zwischen zwei verschiedenen Schulorten ein, sondern beschränkt sich auf den Schauplatz Basel. Auf der Grundlage der in Basel erhobenen Daten soll versucht werden, folgende Fragen zu beantworten: Wie gehen einzelne Jugendliche als kulturelle und soziale Akteure in einem für ihre gesellschaftliche Integration wichtigen Lebensabschnitt mit Fragen der Integration und Ausgrenzung um? Wie stellt sich das Ringen um Normalität für die Gruppe und für den Einzelnen dar? Neben diesen spezifischen Fragen ging es auch um die Gesamtschau. Was bedeutet Erwachsenwerden in den nördlichen Stadtquartieren von Basel zu Beginn des 21. Jahrhunderts?

3 Rolshoven, Johanna: Integrations- und Ausschlussprozesse von Jugendlichen in Ausbildungsgruppen. (Schlussbericht Projekt Nr. 4051-40-69227 im Rahmen des NFP 51 "Integration und Ausschluss"), 2006. 
Um diese Fragen zu beantworten, mussten Grundannahmen und Hypothesen festgelegt werden, die theoretische Leitplanken für das Forschungsvorhaben darstellten. Ein wichtiges Forschungsfeld der Europäischen Ethnologie sind Gruppen und deren Kulturen. Das gemeinsame Merkmal der hier untersuchten Gruppe war ein äusseres, willkürliches: Die Zugehörigkeit zu einer Schulklasse der Weiterbildungsschule des Kantons Basel-Stadt (WBS). Die WBS bildet in der sekundären Schulstufe die nichtgymnasiale Laufbahn an, welche die Schülerinnen und Schüler nicht für die tertiäre Bildungsstufe vorbereitet, sondern für eine Berufslehre oder Fachausbildung. Dieser Fokus ist in der Jugendforschung aussergewöhnlich. Die WBS ausgerechnet dieses Schulhauses wurde gewählt, weil das Rektorat der WBS-Schulen diesen Ort als Hotspot bezeichnet hatte, der von besonders viel Integrationsproblemen betroffenen sei. Diese Wahl konnte nicht umgestossen werden, obwohl sich darin ein Widerspruch manifestiert: Wir wollten Jugendliche als normale Akteure in einer bestimmten biografischen Situation auffassen, dazu wäre die Wahl eines Hotspot eher unlogisch gewesen.

Innerhalb der Klasse, nahm unsere Forschungsgruppe an, gab es verschiedene Integrationstiefen, die mit ethnischen Fragen, Geschlechterfragen und der sozialen Stellung einer Person zu tun hatten. Die Integrationsverhältnisse sollten mit theoretischen Anleihen aus der Jugendforschung, der Identitätsforschung und der Statusforschung interpretiert werden. Für unser Vorgehen hatten wir ein Bild der Jugendlichen im Kopf. Sie waren für uns nicht passiv Erleidende, sondern aktiv Handelnde, die für ihre Situation rationale Entscheidungen treffen. Die Idee war, das schulische Abschlussjahr und den darauffolgenden beruflichen Einstieg von rund einem Dutzend Schülerinnen und Schüler über längere Zeit aus der Nähe zu beobachten. Im Verlauf dieser Zeit wurden diese Leute erwachsen, und es war für den Feldforscher sehr faszinierend, dies mitzuerleben. 
Dieser Vorgang eröffnete auch einen Blick auf die Reproduktion der Gesellschaft. Er wirft ein Licht darauf, wie die Stellung eines Erwachsenen in unserer Gesellschaft entsteht. Sie ist vielfältig mit den unterschiedlichsten Bereichen der Gesellschaft verzahnt, mit dem Arbeitsleben, mit dem sozialen Leben, mit der Abfolge der Generationen.

Gleichzeitig entsteht eine soziale Position, eine Verbindung mit verschiedenen Gesellschaftsebenen, mit der sozialen Hierarchie. Während dieser Zeit versuchen die Jugendlichen, die Kontrolle über diesen Prozess aufrechtzuerhalten und so eigene Träume und Wünsche in die Erwachsenenwelt hinüberzuretten. Das führt zu Reibereien mit den Forderungen und Beschränkungen, die ihnen von der Gesellschaft auferlegt werden.

Der Übergang ins Erwerbsleben ist nicht nur für das Leben der Jugendlichen sehr wichtig, sondern auch für die Gesellschaft als Ganzes. "Der Prozess des Überganges von der Schule zur Arbeit ist ein Prozess, der kontinuierlich die Gesellschaft wiederherstellt, indem neue Kohorten junger Arbeitskräfte bereit gestellt werden, die Positionen auf dem Arbeitsmarkt einnehmen", wie Harvey Krahn über kanadische jugendliche Schulabgänger schrieb. ${ }^{4}$

Das Ziel war, ein differenziertes Bild von Basler Jugendlichen an der Schwelle zum einundzwanzigsten Jahrhundert und an der Schwelle zum Erwachsenenleben zu zeichnen. Dadurch sollte diese oft zwischen Problemfall und Vorbild, zwischen Ablehnung und Vereinnahmung stehende gesellschaftliche Gruppe in ihren Handlungsweisen, Gefühlslagen und Motivationen besser verstanden werden. Und zwar durch die Erklärungen und Deutungen, die die Akteure selbst als wichtig erachteten.

4 Harvey Krahn: The School to Work Transition in Canada: New Risks and Uncertainties, in: Heinz, Walter R. (Hg.): The Life Course and Social Change: Comparative Perspectives, Weinheim 1991, 43-69, 66. 


\subsubsection{Tatsächlicher Verlauf}

Leider liess sich dieses Vorhaben nicht so wie geplant durchhalten, soviel kann vorweg genommen werden. Eine Schwierigkeit in diesem Forschungsunternehmen war die zu jenem Zeitpunkt schlechte Situation auf dem Lehrstellenmarkt. Die Forschungsanlage ging davon aus, dass die meisten Jugendlichen nach der WBS eine Lehrstelle antreten würden, wo der Einstieg in die Berufswelt beobachtet werden könnte. Doch eine klassische Lehre machten von 19 Schülerinnen und Schülern gerade drei, die anderen gingen in weiterführende Schulen oder nahmen eines der verschiedenen sogenannten Brückenangebote des Kantons Basel-Stadt in Anspruch, die um die Jahrtausendwende aufgrund der Schwierigkeiten der Jugendlichen, eine Anstellung zu finden, aufgebaut worden waren.

Ein zweites wichtiges Ziel, die Feinmechanik der Integrations- und Ausschlussprozesse anhand von Bedeutungskonstruktionen und Aushandeln von Normalität sichtbar werden zu lassen, liess sich ebenfalls nicht erreichen. Zum einen gab es eine methodische Schwierigkeit: die Jugendlichen liessen sich nie so nah beobachten, dass solche Prozesse erfasst werden konnten. Die Jugendlichen waren bestrebt, gewisse Vorgänge vor Abgesandten der Autoritäten wie einem Forscher verborgen zu halten. Zum anderen liess sich die These nicht bestätigen, dass Bedeutungskonstruktionen und Differenzen über Normalitätsauffassungen über Integration oder Ausschluss entscheiden. Vielmehr spielten andere Faktoren eine Rolle, wie weiter unten gezeigt werden wird, etwa ästhetische Fragen und zeitlich und örtlich pragmatisch umgesetzte Integrationsbedürfnisse.

Diese Schwierigkeiten waren für das Projekt aus ethnologischer Sicht kein Problem, da Europäische Ethnologie eine phänomenologische Wissenschaft ist, die versucht, die Lebenswelt des Menschen un- 
mittelbar durch ganzheitliche Interpretation alltäglicher Situtationen zu verstehen. ${ }^{5} \mathrm{Zu}$ diesem Zweck nimmt der Forscher die Perspektive der untersuchten Personen ein und versucht, die Welt durch ihre Augen zu sehen. Die Untersuchten unterrichten die Forschenden darin, wie sie denken und handeln. Die Forschenden untersuchen einen Prozess in einer so wenig wie möglich veränderten Umwelt, leben und befinden sich im besten Fall eine längere Zeit im Feld. Dort leben die Gewährspersonen und äussern sich, was wiederum dokumentiert wird. Die Ethnografen analysieren die Informationen kontinuierlich und vertiefen die Analyse zum Schluss in einem beschreibenden und narrativen Text. ${ }^{6}$ Eine ethnologische Forschung gleicht deshalb eher einer Reise, bei der die Forschenden an der Hand genommen werden und ein Stück des Weges mitgehen dürfen. Wohin es geht, bestimmen die Untersuchten. Wie auf jeder Reise verändern sich die Reisegefährten, Forscher und Beforschte, im Laufe der Reise.

Während dieser Reise stellt der Ethnologe selbst das Untersuchungsinstrument dar. Sein durch die Auseinandersetzung mit der ethnologischen Theorie und Praxis geschultes Bewusstsein bestimmt, welche Phänomene für die Auswertung erfasst werden. Er muss versuchen, möglichst wie die Anderen zu sehen und zu fühlen und gleichzeitig sich selber treu zu bleiben. Der Spagat zwischen diesem systematisch-reflektierten und alltäglich-unreflektierten Wissen ist für die Beobachter besonders anstrengend und bedarf der Unterstützung durch eine Forschergruppe sowie der Supervision. ${ }^{7}$

5 Muri, Gabriela: Pause! Zeitordnung und Auszeiten aus allltagskultureller Sicht, Frankfurt a. M., Campus, 2004, 16.

6 Kullberg, Birgitta: Etnografie i klassrummet, Lund, Studentlitteratur, 1996, 32-33.

7 Zum Thema Haltung während der Feldforschung u.a. Hauser-Schäublin, Brigitta: Teilnehmende Beobachtung, in: Beer, B.: Methoden und Techniken der Feldforschung, Berlin 2003, 33-54. 
Die Jugendlichen waren, wie bereits erwähnt, mit einer schlechten Arbeitsmarktsituation konfrontiert. Dies führte dazu, dass sich vor allem diejenigen mit schlechterer Vernetzung in den Brückenangeboten wiederfanden. Die Schule für Brückenangebote (SBA) verlängert die Übergangszeit von der Schule ins Berufsleben um mindestens ein Jahr. Der Zweck dieser speziellen schulischen Angebote besteht darin, zunächst einmal die Jugendlichen über ihre Möglichkeiten zu informieren und möglicherweise vorhandene Defizite in der Persönlichkeit und im Wissen der jungen Menschen auszugleichen, um deren Chancen auf einen geglückten Übergang ins Berufsleben zu erhöhen.

Nach Ablauf einer ersten Beobachtungsphase am Ende der für die Jugendlichen obligatorischen Schule stellte sich die Frage, wie mit dieser unvorhergesehenen Situation umgegangen werden sollte. Da nur eine Minderheit eine Lehre begann, war es nicht möglich, den Berufseinstieg einer grösseren Gruppe von Jugendlichen zu vergleichen und so zu Erkenntnissen über die Berufs- und damit Gesellschaftsintegration zu gelangen. Die Forschungsgruppe beschloss daher, dass der grössten Gruppe der Jugendlichen zu folgen sei. Die Beobachtungen wurden in eine Schulklasse des Brückenangebots "Basis" verlegt, in dem drei der ehemaligen Schülerinnen und Schüler untergekommen waren. Gleichzeitig gab es dauernde, leider erfolglose Bemühungen, Kontakt mit den anderen Schülerinnen und Schülern zu halten, die in anderen Brückenangeboten der Stadt untergekommen waren.

Glücklicherweise erwies sich eine der drei Schülerinnen und Schüler in der "Basis", Janine, mir gegenüber als sehr offen. Vielleicht war sie froh, in der fremden Umgebung ein bekanntes Gesicht zu sehen. Sie hatte mich in den letzten Monaten der regulären Schulzeit etwas mehr akzeptiert als andere und hat mir einige Bereiche ihres Privatlebens eröffnet. Ich konnte sie und ihre Freundinnen Loredana und Martina in der Folgezeit weiter in ihrer Freizeit treffen und den Alltag dieser jungen Frauen aus nächster Nähe miterleben, ihre Freunde und Freundin- 
nen kennenlernen, ihre Probleme mit der Schule und dem Berufseinstieg, ihre Liebesgeschichten miterleben, ihre Auseinandersetzungen mit ihren Eltern, die ersten Erfahrungen mit Alkohol und Drogen. Ein glücklicher Zufall war hierfür entscheidend. Loredanas Mutter hatte im Winter 2004/2005 eine Wohnung aufgegeben, deren Miete bis Ende März 2005 noch bezahlt war. Die Jugendlichen verbrachten dort viel Zeit, und ich war willkommen. Ein grosser Teil der Erkenntnisse, die in dieser Arbeit präsentiert werden, basiert auf Material aus dieser Zeit.

Nicht nur beim Vorgehen im Feld glich dieses Forschungsprojekt einem Schiff auf rauher See, auch bei den theoretischen Grundlagen gab es einen völligen Kurswechsel. Ausgehend von den Fragen, welche das Forschungsdesign vorgab, diejenigen nach Integration, Ausschluss und den damit verbundenen Identitätsprozessen und Normalitätskonzepten, wurde bei der Untersuchung der Gruppen in den Schulklassen immer deutlicher, dass individuelle Reifungsprozesse und ihr Stand grundlegend für die zu untersuchende Grundfrage nach der Integration in das Berufsleben waren. Diesem Umstand wurden die zunächst unterstellten theoretischen Ansätze nicht gerecht. Deshalb wurde im Verlauf dieser Arbeit das theoretische Konzept der Submoratorien entwickelt, das zunächst eine untergeordnete Rolle spielte, aber im Verlauf der empirischen Forschung immer wichtiger wurde.

$\mathrm{Zu}$ Beginn der Feldforschung wurde allerdings das Hauptaugenmerk auf Integrations- und Ausschlussprozesse gelegt. Entsprechend wurden zunächst Gruppen in der Klasse beschrieben. Erst allmählich wurde der Zusammenhang der Gruppendynamiken mit den Verläufen der Submoratorien deutlich, die Analysekraft des Konzepts erkannt. Deshalb wird das Konzept der Submoratorien im Verlauf der empirischen Datenerhebung immer wichtiger und kulminiert am Schluss in einer Übersicht zum Stand der Submoratorien bei einzelnen Schülerinnen und Schülern, bei denen die Quellenlage dies erlaubte. Die Datenlage zur Beurteilung der Submoratorien ist unter anderem deshalb lückenhaft, weil dieses 
Konzept erst im Verlauf der Forschung entwickelt wurde. Der Fokus der Feldforschung verschob sich also von der Gruppe zum Individuum und den Bedingungen, welche seine Gruppenanbindung mitbestimmten. Es wäre wünschenswert, wenn zukünftige Forschungen zum Thema Erwachsenwerden das Konzept der Submoratorien einbeziehen, überprüfen und verfeinern würden.

\subsubsection{Meine Position im Feld}

Für mich überraschend war die Tatsache, dass ich als Mann Mitte vierzig anscheinend relativ problemlos ein Stück weit in ein lose Gruppe von Mädchen integriert wurde, die zu jungen Frauen heranwuchsen. Weshalb dies so war, darüber kann ich als Nichtpsychologe nur spekulieren. Jedenfalls scheine ich gegenüber Mädchen dieser Altersgruppe aus Gründen, die wohl in meiner Persönlichkeit zu suchen sind, einen einfachen Zugang zu haben. Dies zeigt sich auch in meiner Arbeit als Kulturanimator und Veranstalter eines Kochkurses für Jugendliche im 2011, bei der ich neben einer Jugendlichengruppe eine Gruppe von sechs muslimischen albanischstämmigen Mädchen in der Vorpubertät betreue. Bei diesen Kursen treffen meine pädagogischen Interessen und die Interessen der Mädchen aufeinander.

Wir haben viel Freude zusammen. Immer mehr Mädchen und manchmal auch ein kleiner Bruder schliessen sich der Gruppe an. Die oben genannte Interessenlage zeigt sich beim Einkauf. Während ich beispielsweise rigoros die bekannten zuckerlastigen Produkte internationaler Getränkehersteller aus dem Einkaufswagen mit dem Hinweis auf unsere Doktrin der gesunden Ernährung entferne, setzen die Mädchen meist eine Rivella-Flasche oder einen Liter Eistee trotz meinen 
Protesten durch. ${ }^{8}$ Auch hat sich trotz meiner gegenteiligen Bemühungen das Ritual etabliert, dass die Mädchen nach dem Dessert den Anlass mit dem Hinweis auf die vorgerückte Stunde verlassen und den Abwasch den männlichen Jugendlichen überlassen.

\subsection{Die Erforschung von Schulen in der Europäischen Ethnologie}

Während die Forschungsliteratur zu Methode, Begriffen und theoretischen Grundlagen im Teil I dieser Arbeit vorgestellt wird, soll hier als Abschluss der Einleitung ein Blick auf die bisher zum Thema Schule geleistete Forschung in der Europäischen Ethnologie geworfen werden. Wenn die hier vorgestellten Ansätze vereinzelt und marginal erscheinen, möge dies als Ansporn für nachkommende Forscher und Forscherinnen verstanden werden, sich der Bildungsinstitutionen und deren wachsenden Bedeutung für das Leben in der Spätmoderne anzunehmen.

Leider gibt es recht wenig Material über die Gruppe der jugendlichen Schülerinnen und Schüler. Die volkskundliche Forschung erfasste selten die Jugendlichen in ihren Klassenverbänden. Es gibt viele Forschungen über Kinder- oder Jugendkultur sowie über Schülerinnen und Schüler und die Schule im Allgemeinen. Doch über Jugendliche in einer Schulklasse und ihre Rolle als aktive kulturelle Akteure findet sich erst in den letzten Jahrzehnten Material.

Diese Situation erstaunt, denn schon früh geriet die Institution Schule in das Blickfeld der Volkskundler. Vor allem in der zweiten Hälfte des 19. Jahrhunderts und im frühen 20. Jahrhundert waren die Schulen ein wichtiges Mittel im Aufbau eines Nationalbewusstseins, das auf bestimmten Annahmen über die Volkskultur des betreffenden Landes be-

8 Rivella: Aus Molke hergestellte Süssbrause. 
ruhte. Die Nationalstaaten Europas bedienten sich volkskundlichen Wissens, um über die zentral organisierte Institution Schule die Kultur ihres Gebietes zu homogenisieren und dem so neu geschaffenen Staatsvolk das richtige Wissen über die nationale Kultur zukommen zu lassen.

Aus diesem Grunde wurden die Lehrer in ihrem Werdegang volkskundlich ausgebildet und mit den entsprechenden Unterrichtsmitteln versehen. Zum Beispiel in Schweden: So schrieb etwa der Gründer des Nordiska Museet und des Skansen in Stockholm, Arthur Hazelius, in den 1940er Jahren als Hauptmitarbeiter am „Lesebuch für die Volksschule" mit. ${ }^{9}$ Es wurde ein wichtiges Unterrichtsmittel in allen schwedischen Schulen und sollte den volkskulturellen Kanon Schwedens vermitteln.

Für die Schweiz hat Hans Trümpy die Unterrichtsmittel seit der Helvetik in der Schulbuchsammlung im Pestalozzianum untersucht und festgestellt, dass seit früher Zeit sehr gerne volkskundliche Themen als Unterrichtsstoff aufgegriffen wurden, obwohl Pestalozzi selber dazu geraten habe, von Mord- und Räubergeschichten im Unterricht Abstand zu nehmen. ${ }^{10}$ John Meier gab 1928 ein praxisorientiertes Büchlein mit dem Titel „Lehrproben zur deutschen Volkskunde“"11 heraus, in dem Lehrer „,an Hand ausgewählter Beispiele“ ihren Fachgenossen ,zeigen, wie sie sich volkskundliche Themata mit Nutzen im Unterricht der Volksschule behandelt denken“. In diesem Buch weist er auf das im vorigen Jahr erschienene Buch „Deutsche Volkskunde“ hin, das theoretisch ausgerichtet war und den Untertitel trug: ,Insbesondere zum Ge-

9 Svensson, Sigfrid: Folkkulturkunskap som undervisningsämne i seminarierna, Rig 1941, Separatdruck, 75-78, 75-76.

10 Trümpy, Hans: Schule, Volksbrauch und Volksglaube, Separatum aus der Basellandschaftlichen Schulnachrichten, 1964, 72-78, 77-78.

11 Meier, John (Hg.): Lehrproben zur deutschen Volkskunde, Berlin und Leipzig, 1928. 
brauch der Volksschullehrer“. Leider musste die Lehrprobe „Märchen“ wegen Erkrankung des Verfassers ausfallen, bedauert Meier. ${ }^{12}$ Das Vorwort endet mit einem Dank an die Lehrer, welche die Lehrproben verfasst haben. „(...) wir (danken) herzlich für den Dienst, den sie dadurch unsrer gemeinsamen Sache geleistet haben." Die Sache der Volkskundler und der Lehrer wurde also von Meier als eine Gemeinsame angesehen. ${ }^{13}$

In Stockholm empfahl das Leitungsgremium des Nordiska Museet zudem, dass die Lehrer in den geisteswissenschaftlichen Fächern des Lehrerseminars akademisch ausgebildete ,Volkslebens“-Forscher ${ }^{14}$ sein sollten, um den Einfluss des fremden und oft allzu theoretischen Lehrstoffes zurückzudrängen. ${ }^{15}$ Auch Hans Trümpy fordert in seiner oben genannten Untersuchung aus den 1960er Jahren, dass an den Lehrerseminarien Volkskunde, am besten mit Heimatkunde verbunden, gelehrt werden solle. ${ }^{16}$

In Schweden sind die Auswirkungen dieser Forderung noch heute zu spüren. Als ich nach meinem Umzug im Jahr 1999 in dieses Land einen Sprachkurs für Migranten besuchte, waren volkskulturelle Themen im Unterricht häufig. So lasen wir etwa eine Sage, welche das Sprichwort „Koka soppa på en spik“ erklärt. Der Titel heisst wörtlich „Mit einem Nagel Suppe kochen“ und wird in der Bedeutung ,,aus dem Nichts auf Kosten der anderen etwas herstellen“" verwendet. ${ }^{17}$ Die Sage wurde mit

12 Ebd., 3.

13 Ebd., 4.

14 Folkslivsforskning: Damalige schwedische Bezeichnung für Volkskunde.

15 Svensson, Folkkulturkunskap som undervisningsämne i seminarierna, 1941, 75-76.

16 Trümpy, Volksbrauch und Volksglaube, 1964, 78.

17 Ein Landstreicher kommt zu einer Suppe, indem er einer alten Bauernfrau verspricht, aus einem Nagel eine Suppe zu kochen. Die Frau wird neugierig, als der Landstreicher in einem Topf einen Nagel im Wasser kocht. Das werde eine leckere Suppe, verspricht der Landstreicher, aber mit ein paar Rüben würde sie noch besser. Als die Bauersfrau diese dazugibt, fordert 
einem Abschnitt eingeleitet, der ein paar fachgeschichtliche Themen vermittelte, wie etwa das Verhältnis von Schriftlichkeit und mündlicher Überlieferung.

Volkssagen sind Sagen, welche alle Menschen in allen Zeiten einander erzählt haben. Im 19. Jahrhundert begann man in Europa, Volksdichtung, -gesang und -sagen zu sammeln. Sagenbücher wurden schnell populär und in viele verschiedene Sprachen übersetzt. Die folgende Sage ist Schwedisch und wurde am Ende des 19. Jahrhunderts niedergeschrieben. ${ }^{18}$

So wurden die Migranten im Sprachunterricht an die Inhalte der schwedischen Volkskultur herangeführt. Doch ist das rein Schwedische durchbrochen. Auch die Sage „Råttfångaren från Hameln“ wurde im Unterricht benutzt. „Der Rattenfänger von Hameln“ ist Teil der deutschen, somit europäischen Überlieferung und ist deshalb im Zeitalter der EU-Mitgliedschaft Schwedens und der Bedeutungsminderung des Nationalstaats auch als Unterrichtsmittel statthaft.

Mit der volkskundlichen Ausbildung der Lehrer wurden die Schulen in Europa zum Arbeitsplatz vieler mehr oder weniger professioneller Volkskundler. Klassisch ist der Dorfschullehrer als Gewährsmann, häufig waren bedeutende Volkskundler des 20. Jahrhunderts im Vorleben Schullehrer. Als Beispiele aus der Gegend von Basel können Hans Trümpy, der Gymnasiallehrer war, oder Eduard Strübin ${ }^{19}$, der an der

der Landstreicher weitere Zutaten mit dem Hinweis, die Suppe würde noch weiter verbessert. Zum Schluss hat er eine vollständige Gemüsesuppe vor sich, der Nagel war nur eine Art Katalysator.

18 Folkuniversitetet, Stockholm: Ausgeteilte Fotokopien im Unterricht in den Sprachintegrationskursen III - V, 1999.

19 Eduard Strübin hat unter anderem bedeutende Werke über das Volksleben im Kanton Basel-Landschaft verfasst. 
Schule in Gelterkinden (BL) arbeitete, genannt werden. Max Lüthi, ein zürcher Erzählforscher, legte 1935 die Gymnasiallehrerprüfung ab. ${ }^{20}$

Volkskundliche Forschung wurde oft von Lehrern getragen. Sie kamen nach der Ausbildung als Ortsfremde in ein Gebiet und mussten die lokalen Überlieferungen eines Ortes studieren, schon um sich zu integrieren. Ihnen ist ein Grossteil des volkskundlichen Wissens über die historische Kultur der Schweiz zu verdanken. ${ }^{21}$ Für sie war es naheliegend, dass sie auch aus Interesse an der Heimatkunde Ethnografie an der Schule betrieben. Allerdings erscheinen die Schülerinnen und Schüler in erster Linie nicht als eigene kulturelle Gruppe, sondern als Lieferanten von Informationen über das Volksleben, als Gewährsleute neben Pfarrern, Honoratioren und alten Leuten.

In der Schweizerischen Lehrerzeitung aus dem Jahre 1917 empfiehlt Georg Küffer ${ }^{22}$, den Schülern volkskundliche Stoffe als spannende Unterrichtsthemen zu präsentieren und sie als Gewährsleute zu nutzen. Unter dem Titel „Volkskundliches im Deutschunterricht“ schlägt er beispielsweise vor, ...

... statt die hellen Kinderaugen unermüdlich in die Druckerschwärze bohren zu lassen, schlagen wir das grosse Buch des Lebens auf und lehren wir (den Schülern und Schülerinnen), die Buchstaben kennen zu lernen, die dort drin verzeichnet sind! (...) (Wir sollten) auch im Deutschunterricht (daran denken,) das Nächstliegende zu (erwerben, bevor) wir die übrige Welt erobern. ${ }^{23}$

20 Schenda, Rudolf: Lüthi, Max, in: Enzyklopädie des Märchens 8, 1996, 1307-1313, 1307.

21 Trümpy,Volksbrauch und Volksglaube, 1964, 78.

22 Küffer, Georg: Volkskundliches im Deutschunterricht, in: Schweizerische Lehrerzeitung 22, 1917, 199-201.

23 Ebd., 199. 
Den passenden Stoff für den Deutschunterricht sollen die Kinder selber sammeln, aber ...

... nicht planlos! Der Lehrer gibt die Richtung an, und mir scheint, dass in den Sagen unseres Landes der prächtigste Stoff zu unserer Verfügung meist brach daliegt. (...) Eine einzige Klasse von zweiunddreissig Schülern (es waren zwölfjährige Buben) brachte mir in sehr kurzer Zeit schier ein halbes hundert Sagen zusammen, zudem Mitteilungen von über zwei Hundert Aberglauben, Gebräuchen und dergleichen. Dies in einer Industriestadt. Alles aus dem Volksmunde gesammelt, nichts aus

Büchern. $^{24}$

Das Forschungsinteresse an den Schulen und mit den Schülerinnen und Schülern war also in erster Linie historisch, wie es für die Volkskunde bis in die 1980er Jahre üblich war. Dies war Ausdruck eines Gedankens der Volkskunde in der zweiten Hälfte des 19. Jahrhunderts, dass man möglichst viel von der untergehenden ,alten“ Zeit zu retten habe und dass theoretische Überlegungen weniger wichtig waren. ${ }^{25}$

Strübin hat mir im persönlichen Gespräch anvertraut, dass er oft die Schulkinder Aufsätze zu volkskundlichen Themen schreiben liess, etwa über Bräuche, die in der Gegend durchgeführt wurden. Diese Informationen verwendete er in seinen Werken. Oft richtete sich sein Interesse dabei auf die für ihn wichtige Frage der sogenannten Biografie von

\section{Ebd., 199.}

25 Vgl. z.B. Ludwig-Uhland-Institut für Empirische Kulturwissenschaft: Von der Volkskunde zur empirischen Kulturwissenschaft, mit Beiträgen von Hermann Bausinger, Utz Jeggle, Gottfried Korff, Martin Scharfe, u.a., Tübingen 1974 . 
Bräuchen, also auf die Frage, ob ein bestimmter Brauch in einer bestimmten Gegend noch „lebe“, also durchgeführt wurde. Auch erfasste er neu entstehende kulturelle Erscheinungen, sozusagen die „Geburt“ eines Brauchs.

Als Beispiel möge der Brauch des „Räbeliechtli-Umzuges“ dienen. Dies ist ein Brauch, dessen älteste Erwähnungen im Aargau und in der Ostschweiz anzutreffen sind. Vor allem im Kanton Zürich ist er schon Mitte des 20. Jahrhunderts weit verbreitet gewesen. Der Brauch wurde mit der Hochkonjunktur der 1960er Jahre im Kanton Basel-Landschaft heimisch, als viele Kindergärtnerinnen- und Lehrerinnenstellen mit Bewerberinnen aus der Ostschweiz besetzt wurden. Der Umzug wurde seither in vielen Gemeinden von Kindergärten und Schulklassen durchgeführt. Der Brauch wurde nicht von Männern eingeführt. Die Elemente des Brauchs, Rüben, Lieder usw. stammten aus der Ostschweiz, was als störend empfunden wurde, man solle doch die hiesigen Bräuche fördern, mahnten die Kritiker. In diesem Beispiel hat das Kindergartenseminar in Liestal, welches für die Aus- und Weiterbildung der Lehrerinnen zuständig ist, eine bedeutende Rolle in der Ausbreitung des Brauchs eingenommen. ${ }^{26}$

Es lässt sich beobachten, dass in Strübins Beschreibungen die Schülerinnen und Schüler einen ziemlich unwichtigen Platz einnehmen. Sie erscheinen nicht als Akteure, die eigene kulturelle Ausdrucksformen entwickeln, sondern als passive Träger eines von aussen aufgepfropften Brauchs, entsprechend dem damaligen Menschenbild der Volkskunde.

Strübin weist zudem darauf hin, dass man mit volkskundlichen Befunden den Unterricht interessanter gestalten könne:

26 Strübin, Eduard: Jahresbrauch im Zeitenlauf, Kulturbilder aus der Landschaft Basel, Liestal, Verlag des Kantons Basel-Landschaft, 1991, 402406. 
Die Kinder schätzen es, wenn man zur Auflockerung des Unterrichts ,nebenbei“ für Herz und Gemüt etwa ein paar Knacknüsse aus der Fülle volkstümlichen Rätselgutes darbietet. Die kindertümliche Poesie sollte namentlich den Mädchen als den zukünftigen Müttern weitergegeben werden. Schnellsprechsprüchlein leisten als Sprechübungen gute Dienste. ${ }^{27}$

Im weiteren empfiehlt Strübin pädagogische Kniffe für den Unterricht. An Ostern mit Eiern zählen zu üben, an Weihnachten mit Kerzen, im Deutschunterricht Märchen und Sagen verwenden, das sind nur einige davon. Danach bespricht er die Rolle der Schule als Trägerin des Brauchlebens, etwa eines Einläutens am 6. Dezember, dem Nikolaustag. ${ }^{28}$

Schülerinnen und Schüler kamen dagegen dort als eigenständige kulturelle Akteure vor, wo man glaubte, dass sie Träger gesunkenen Kulturgutes seien. William Wells Newell, Begründer des ,Journals of American Folklore", legte 1883 ein Werk über die Spiele und Lieder von Kindern in New York vor ${ }^{29}$. Dort argumentiert er, dass diese kulturellen Erscheinungen gesunkenes Kulturgut europäischer Hochkulturen seien. Die Kinderreigen von heute zeigten uns diejenigen Tänze, welche einst den Königshof und das Alte England erfreuten, bevor sich das Neue darüber legte, glaubte Newell.

Die Idee des sinkenden Kulturguts sollte in den 1920er Jahren mit Hans Naumann und John Meier wieder an Bedeutung gewinnen. Aber Newell war auch Evolutionist. Er vertrat die Ansicht, dass die groben

27 Strübin, Eduard: Baselbieter Volksleben, Sitte und Brauch im Wandel der Gegenwart, 2. Auflage, Basel 1967, 276

28 Ebd., 276-277.

29 Newell, William Wells: Games and Songs of American Children, New York, Harper and Brothers, 1883. 
und seiner Meinung nach bruchstückhaften kulturellen Erscheinungen, die er bei Kindern beobachten konnte, Zeugnisse einer Zeit seien, die als Jugendzeit in der Geschichte des menschlichen Verstandes anzusehen sei. ${ }^{30}$

Falls sich herausstellen sollte, dass der menschliche Geist langsam erwachsen geworden ist, wird man auf diese Vergangenheit mit liebevollem und doch unvernünftigen Interesse zurückblicken wie ein erwachsener Mann, der sich seiner Jugendzeit erinnert. ${ }^{31}$

Newell sah also die Kinderspiele als Produkt einer historischen Epoche an, deren man sich später so erinnern würde wie der Jugend eines Mannes. Aufschlussreich wäre zu wissen, weshalb Männer wie Newell liebevoll, doch unvernünftig auf ihre Jugendzeit zurückblicken und weshalb sich die Metapher Jugend für die auf den Strassen New Yorks gemachten Beobachtungen anbot.

Doch Newell war kein Lehrer, und er beschränkte sich nicht auf die Kinderkultur innerhalb der Schule. Kommt die Schule als volkskundliches Thema vor, wird sie oft als Dorfinstitution verstanden, die mitten im Dorfleben der damaligen Zeit stand und deshalb volkskundlich attraktiv war. Ein Beispiel dieser Art von Ethnografie ist etwa Max Wirsings Broschüre „Oberfränkisches Schulleben in älterer Zeit“, die als „Heimatbeilage“ des Amtlichen Schulanzeigers des Regierungsbezirks Oberfranken erschien. ${ }^{32}$

30 Bendix, Regina: Amerikanische Folkloristik, Berlin, Reimer, 1995, 38-39.

31 Zitiert ebd., 39.

32 Wirsing, Max: Oberfränkisches Schulleben in älterer Zeit am Beispiel des früheren Schulortes Pilgramsreuth, Ldkr. Hof, Heimatbeilage zum Amtlichen Schulanzeiger des Regierungsbezirks Oberfranken 138, Bayreuth 
In diesem Typus volkskundlicher Untersuchung wird über die Geschichte einer Dorfschule seit den Anfängen meist im 16. Jahrhundert berichtet, die verschiedenen Dorfschullehrer und ihr Wirken dargestellt und Anekdoten erzählt. Die Schülerinnen und Schüler kommen in diesen auf schriftlichen Quellen beruhenden Schilderungen eher am Rande vor. Jugendliche waren noch seltener anzutreffen, da die Dorfschulen meist nur die Primarschule abdeckten, welche mit 13 Jahren endete. ${ }^{33}$ Danach mussten die Jugendlichen arbeiten gehen, häufig auf dem elterlichen Hof.

Der Europäischen Ethnologie ging es im Forschungsfeld Schule meist um Kinder zwischen sieben und dreizehn Jahren, also nicht um Jugendliche. Jugendliche tauchen in anderen volkskundlichen Diskussionen auf, etwa über die Knabenschaften. Dies ist nicht erstaunlich, denn für die Bevölkerung, die nicht der Oberschicht angehörte, endete die Schule normalerweise mit dreizehn, danach machte man eine Lehre. Für städtische Jugendliche am Gymnasium ging die Schule jedoch schon vor der Zeit nach dem Zweiten Weltkrieg bis in die jugendlichen Lebensjahre hinein.

In diesem Bereich gibt es schulische Überlieferungen, die genuin jugendlich sind und in Einzelfällen auch beschrieben wurden: Die Bräuche zum Abschluss der Matur oder des Abiturs. Der pensionierte Lehrer Henri Mercier zum Beispiel schrieb zwar 1934 über die Genfer Maturitätsfeiern, blieb aber bei dem oben beschriebenen Muster der Institutionengeschichte einer Dorfschule. ${ }^{34}$

1987.

33 Ein Bespiel aus unserer Gegend: Baumann, Josef: Von Schulhäusern, Schulmeistern und der Schule in Oberwil, Liestal 1986, Separatum aus Baselbieter Heimatblätter 1986.

34 Mercier, Henri: Les Promotions du Collège de Genève de 1798 jusqu'a nos jours, Genève, Villard \& Rabot, 1934. 
Bereits 1964 haben Christine Burckhardt-Seebass und Elsbeth Liebl für die Jahre 1950 bis 1963 die Maturvergnügungen der Basler Schülerinnen beschrieben, was ein willkommenes Stück frühe Mädchenforschung darstellt. Mädchen spielten in der volkskundlichen Forschung keine Rolle, man fasste sie nicht als eigenständige kulturelle Agenten auf. Paul Hugger berichtet von den ,fast nicht existenten Wesen in der volkskundlichen Zeit des vorindustriellen Zeitalters" und fügt hinzu:
Die Rituale im früheren Leben der Mädchen (sind) kaum fassbar, (es) verwischen sich deren Konturen. Offensicht- lich ist die weibliche Jugend weitgehend auf die Knaben ausgerichtet, ihr Leben war von ihnen mitbestimmt wie ein Schattenbild. Man kann von einer eigentlichen Vormund- schaft sprechen. ${ }^{35}$

Burckhardt-Seebass und Liebl beziehen sich auf eine Arbeit von Johanna von der Mühll aus dem Jahre $1944^{36}$ und vermuten, dass die Volkskunde den kulturellen Formen von Schülerinnen und Schülern deshalb nur selten Beachtung geschenkt hat, weil den Volkskundlern wohl das Verbindliche und Grundständige an der Schülerkultur zweifelhaft erschien. ${ }^{37}$ Gemeint ist damit die Idee, dass Volkskunde sich mit dem Volk abzugeben habe und in der Oberschicht keine interessanten Beobachtungen von allgemeingültiger Bedeutung zu machen seien.

Diese Ansicht ist heute überholt. Seit rund zwei Jahrzehnten verstehen sich Volkskundler immer mehr als Europäische Ethnologen, die

35 Hugger, P.: Pubertätsriten - einst und jetzt - aus der Sicht des Volkskundlers, in: Kosinski, Günter (Hg): Pubertätsriten, Bern Stuttgart Toronto, Hans Huber, 1991, 25-39.

36 Von der Mühll, Johanna: Basler Sitten, Basel 1944.

37 Burckhardt-Sebass, Christine und Elsbeth Liebl: Maturvergnügungen der Basler Schülerinnen, Basel 1964. 
heutige Alltagserscheinungen untersuchen. Seit den 1970er Jahren bezeichnet man die europäischen Disziplinen der Volkskunde, Folklore oder Art et Traditions Populaires mit diesem Begriff. Er hat sich im deutschsprachigen Raum als Fach- oder Institutsname im Zuge eines Modernisierungs- und Entnationalisierungsprozesses der Volkskunde eingebürgert. ${ }^{38}$ Doch es gibt auch andere Namen, deshalb wird Volkskunde auch als Vielnamenfach bezeichnet. ${ }^{39}$ In diesem Zusammenhang wird europäisch als kulturelle und nicht geografische Kategorie verstanden. Europäische Ethnologie ist also in dieser Auffassung die Wissenschaft der europäischen Kulturen in aller Welt und nicht der Kulturen in Europa.

Nachdem auch Gruppen forschungsrelevant geworden waren, die nicht dem traditionellen Volk der Volkskundler angehörten, richtete sich der Blick der Forscher und vor allem der immer zahlreicheren Forscherinnen immer mehr auf die Schülerinnen und Schüler als eigenständige kulturelle Akteure. Auch die Gruppe der Mädchen wird immer mehr beforscht, meist von Frauen. Angela McRobbie ${ }^{40}$ und Helena Wulff ${ }^{41}$ sind hier zu nennen. Sie haben private Bereiche der Mädchen untersucht. Aber die Forscherinnen kritisieren immer noch die weitgehende Un-

38 Rolshoven, Johanna: Europäische Ethnologie - Diagnose und Prognose einer kultur- und sozialwissenschaftlichen „Volkskunde“, in: Österreichische Zeitschrift für Geschichtswissenschaft, Wien 2004, 73-87, 75ff.

39 Z. B. in Göttsch, Silke und Albrecht Lehmann (Hg.): Methoden der Volkskunde, Positionen, Quellen, Arbeitsweisen der Europäischen Ethnologie, 2. Auflage, Berlin, Reimer, 2007. Ein Oral History Zugang zu diesem Thema: Ein Aufklärer des Alltags. Hermann Bausinger im Gespräch mit Wolfgang Kaschuba, Gudrun M. König, Dieter Langwiesche, Bernhard Tschofen, Wien, Köln, Weimar, Böhlau, 2006, 88ff.

40 Etwa McRobbie, Angela und Jenny Garber: Girls and Subcultures, in: Hall, Stuart und Tony Jefferson: Resistance through rituals, youth subcultures in post-war Britain, London, Routledge, (1975) 2002, 177-188.

41 Etwa Wulff, Helena: Bästa vänner, Förtrogna flickor på upptäcksfärd i livet, in: Kulturella perspektiv, Svensk etnologisk tidskrift 3-4, 1992, 23-31. 
sichtbarkeit der Mädchen in der Jugendforschung. Sie glauben, dass die meist männlichen Forscher nicht adäquat auf die Prozesse der Mädchenpubertät eingehen können und zeigen auf, wie Mädchen eigene, wenig sichtbare, verborgene Formen der Erwachsenwerdung pflegen. Die vorliegende Arbeit beweist jedoch, dass Forscher durchaus zum Thema Mädchen beitragen können.

Ein Beispiel zur Analyse der Schülerkultur ist die Schrift der Musikethnologin Brigitte Bachmann-Geiser über den Lärmbrauch des Zürcher Schulsylvesters ${ }^{42}$, der in die Zeit vor 1800 zurückreicht. Auch hier klagt Bachmann-Geiser darüber, dass dieser beliebte und alte Brauch mit über hunderttausend Beteiligten und seinen traditionellen Lärminstrumenten selten erwähnt wird, weil er eine Angelegenheit der Kinder unter Ausschluss der Erwachsenen sei, wie sie vermutet. ${ }^{43}$ Ihre Studie ist auch deshalb eine Ausnahme, weil sie alle Schülerinnen und Schüler umfasst, die am Schulsylvester mitmachen. Das sind alle Jahrgänge der obligatorischen Schulausbildung, neben Kindern also auch Jugendliche. Eine weitere Arbeit ist die Untersuchung von Eberhard von $1976^{44}$.

1993 legte Werner Mezger eine Arbeit zu den Bräuchen von Abiturienten und Abiturientinnen vor. ${ }^{45}$ Auch er stellt das Fehlen von Arbeiten über die Schülerkultur fest und führt dies in nicht ganz nachvollziehbarer Weise auf eine instinktive Abneigung der Mitglieder unserer Gesellschaft gegenüber dem komplexen System Schule zurück, das einem das

42 Bachmann-Geiser, Brigitte: Der Zürcher Schulsylvester, Zürich, Kommissionsverlag Hug \& Co., 1984.

43 Ebd., 3.

44 Eberhard, H.: Schulbräuche: Eine volkskundliche Untersuchung der traditionellen Elemente an den Volksschulen der Stadt Graz, Graz 1976, unpubliziert.

45 Mezger, Werner: Die Bräuche der Abiturienten: Vom Kartengruss zum Supergag, ein Beitrag zur Schülervolkskunde, Konstanz, Universitätsverlag Konstanz GmbH, 1993. 
ganze Leben lang in irgendeiner Form begleite. Deshalb habe sich die Volkskunde nur sehr zögerlich mit diesem Forschungsfeld auseinandergesetzt, obwohl bereits Mitte der 1960er Jahre Leopold Schmidt die Schülervolkskunde als wichtiges Desiderat angesehen habe. Ingeborg Weber-Kellermann habe dann gemäss Mezger zwei Jahrzehnte später immer noch feststellen müssen, dass das schulische Leben von der Volkskunde ganz vernachlässigt wurde. Dabei bilde die Schule einen wesentlichen Bestandteil unserer Kultur, deren spezifische Erscheinungsformen eigentlich schon längst einer intensiveren Betrachtung hätten unterzogen werden sollen. Nur punktuell seien Untersuchungen gemacht worden, etwa zur Schülersprache, zu unerlaubten Kommunikationsformen, zur Schülerkleidung oder zur Prügelstrafe. Eigentliche Schwerpunkte der volkskundlichen Forschung bildeten Bräuche, die sich im Umfeld der Schule abspielten, und in dieser Themengruppe die Übergangsrituale wie Schuleintrittsbräuche oder Schulabschlussrituale. ${ }^{46}$ Dieser blinde Fleck in der volkskundlichen Wahrnehmung führe dazu, dass wir über die Kulturformen der unterschiedlichsten sozialen Schichten, selbst über die der Randgruppen, relativ genau Bescheid wissen, während ein umfassender Überblick über die Schülerkultur nach wie vor aussteht, meint Mezger. ${ }^{47}$

Tatsächlich wurde die Forschungsperspektive auf die Jugend als aktive Kulturproduzentin erst gefördert durch den Aufstieg der Jugendkulturen nach dem Zweiten Weltkrieg. Durch die Entstehung einer Massenkonsumgesellschaft und den Nachkriegsbabyboom entstanden eigenständige ästhetische jugendliche Ausdrucksformen, die spätestens

46 Etwa Kipar, Mechthild: Penne ade! Festaktivitäten zum Abitur zwischen Tradition und Suche nach neuen Formen, Münster, Waxmann, 1994 oder der Film von Frebel, Lisa und Sabine Helms: Wunderbare Jahre? Magisterfilm, Institut für Kulturanthropologie/Europäische Ethnologie, Göttingen, 2009.

47 Mezger, Die Bräuche der Abiturienten, 1993, 8-11. 
seit 1968 auch das Interesse der Volkskundler und Europäischen Ethnologen erregten. Doch ging es bei diesen Forschungen zunächst um soziologische Subkulturforschung, die Erforschung von Peer-Groups, die Gender- und Armutsforschung. Die Schule spielte dabei nur eine untergeordnete Rolle, damit hat Mezger recht.

Eine Ausnahme bildet die Studie von Paul Willis ,Learning to Labour “48 , die der Frage nachgeht, welche kulturellen Mechanismen dafür sorgen, dass männliche englische Jugendliche, die Kinder von Fabrikarbeitern sind, in der Schule der 1960er Jahre derart schlecht abschneiden. Deswegen stehe ihnen nur der Weg der Fabrikarbeit offen, was ihren sozialen Aufstieg verhindere. In seiner Arbeit beschreibt Willis eindrücklich die Kultur der von ihm untersuchten männlichen Jugendlichen und zeigt auf, dass die Schule als obrigkeitliche Institution den Werten, welchen Arbeiterjugendliche in ihrer Mannwerdung nachleben wollten, nicht entsprechen konnte. Deshalb wurden Bildungsbemühungen der Schule mit passivem Widerstand unterwandert ${ }^{49}$, im Bewusstsein, dass sich in der Zeit der permanenten Hochkonjunktur der 1960er Jahre ein Job allemal finde.

Im Sinne eines Ausblicks sei zur Zeit der Redaktion dieser Arbeit auch auf die seit November 2007 laufende und von der Deutschen Forschungsgemeinschaft (DFG) und Evangelischen Kirche (EKD) geförderten Forschungsprojekts „Schulkultur“ der Georg-August-Universität Göttingen verwiesen. In Aufsatz „Fenster in die Schulkultur: Organisation und Gestaltung ritueller Übergänge ${ }^{\text {“ } 50}$ stellen Regina Bendix et. al. erste Ergebnisse vor. Ausgehend von einer Beschreibung der Einschu-

48 Willis, Paul E.: Learning to Labour. How working class kids get working class jobs, 7. Auflage, Aldershot, Gower, 1983.

49 Ebd., 89-106.

50 Bendix, R., M. Kraul, C. Kessler, M. Nietert und K. Koch: Fenster in die Schulkultur: Organisation und Gestaltung ritueller Übergänge, Zs. f. Vk I/106, Münster, u.a., Waxmann, 2010, 1-22. 
lung an einem niedersächsischen Gymnasium als in komplexer Weise ritualisiertes Ereignis erklären die Autorinnen diese mit der „für Wohlstandsgesellschaften typischen Verdichtung von festlichen Markierungen im Lebens- und Jahreslauf ${ }^{\text {‘f51 }}$. Diese Arbeit hier befasst sich jedoch mit Jugendstatusübergängen am Ende der Schulzeit und versucht, die Rolle von Statusfragen in Bezug auf die Erwachsenwerdung zu verstehen.

Für die Schweiz hat Gisela Unterweger, wie bereits erwähnt, sich in die Schule begeben und Jugendliche untersucht. Sie tat dies nicht primär aus Interesse an der Schule. Ausgehend von Fragen der kulturellen Identität stellte Unterweger fest, dass es , in einer wahren Flut an Literatur, die sich ausschliesslich auf die durch Migration verursachten Probleme und Konflikte konzentriert", keine Studien gab, welche die Entwicklung von Identiät bei Migrantinnen und Migranten erforschte sowie bei Menschen aus Gastgesellschaften, die von Migration beeinflusst sind. Deshalb wählte sie eine Schule als Untersuchungsfeld, in dem beide gleichzeitig interagieren und sich dabei beobachten liessen.

So kam ich schliesslich auf Umwegen zu der Idee, eine „multikulturelle“ Klasse zu beobachten. Vor allem Jugendliche interessieren mich, denn im Alter zwischen ungefähr 14 und 20 Jahren sind Fragen der Identifikation besonders virulent. ${ }^{52}$

Zum Schluss ihrer Untersuchung forderte Unterweger, dass eine längerfristige Beobachtung durchzuführen sei, während der einzelne Personen in ihren Integrationsverläufen begleitet würden. Nur so könne man die vielen Facetten erfassen, die Identitäten aufweisen und aufzeigen, wie vielfältig, komplex und dynamisch sich kulturelle Identität in

51 Ebd., 2.

52 Unterweger, Klasse und Kultur, 2002, 8. 
einer von Migration beeinflussten Gesellschaft gestaltet. ${ }^{53}$ Dieser $\mathrm{Zu}-$ gang ist neu. So können lohnende Einsichten in die Jugendkultur und das Erwachsenwerden entstehen, indem die Integrationsverläufe der einzelnen Schülerinnen und Schüler einer Klasse aufgezeigt werden. Im Folgenden wird versucht, diese Forderung zu erfüllen. Doch zunächst werden die verwendeten Methoden diskutiert, das Forschungsfeld vorgestellt und theoretische Konzepte und Begriffe erläutert, welche die gewonnenen Erkenntnisse erklären sollen.

\subsection{Zum Aufbau dieser Arbeit}

Diese Arbeit schildert und reflektiert in einem ersten Teil die für diese Studie genutzte Methodik und umreisst den Forschungsort und das Forschungsfeld. Es wird die Spezifik der schulethnografischen Praxis erläutert, deren Implementierung und Legitimation, deren menschliche und materielle Dimension. Hier werden erste Aspekte zur Verbindung von schulischem Übergang ins Berufsleben und der damit verbundenen Integrationsbemühungen geschildert. Als Vergleichsfolie sowohl für die methodischen Überlegungen wie auch Fragen der Integration baue ich auch auf eigene Erfahrungen ebenso wie Forschungsliteratur aus Schweden auf, die mir dazu verhalfen, das eigentlich vertraute heimische Feld aus der Perspektive eines "Remigranten" - ich hatte mehr als vier Jahre in Schweden gelebt - zu betrachten.

Ein zweiter Teil befasst sich ausführlich mit der Forschungsliteratur verschiedener Fachgebiete und deren Begrifflichkeiten, die es für diese Studie zu vereinen galt. Dies war von vorneherein Absicht, denn im jugendlichen Erwachsenwerden wirken körperliche, kulturelle, soziale

53 Ebd., 204. 
und gruppenspezifische Faktoren zusammen. Dies betrifft die Jugend als Lebensphase in ihrer historischen Entwicklung unter lokalen Bedingungen, und in ihren verschiedenen, oft ideologisch überhöhten Nuancen sowie unter ökonomischen und gesellschaftspolitischen Ansprüchen. Darauf aufbauend werden für die Feldforschungsparameter massgeblichen Begriffe erörtert. Während Termini wie Integration und Ausschluss sowie Jugend und Gesellschaft einen wesentlichen Rahmen dieser Arbeit gebildet haben, gaben meine Forschungserfahrungen den Anlass dazu, mich mit den Begriffen Moratorium und Submoratorium in ihrer Relevanz auf die Übergangsphase, welche die begleiteten Jugendlichen durchliefen, vertieft auseinander zu setzen. Es wurde auch anhand der gewonnenen Daten eine Typologisierung und Systematisierung erarbeitet.

Im Dritten Teil liegt der Fokus auf der Schulklasse 2D. Die erhobenen Daten werden in Kapiteln, welche die Statuspassagen der Jugendlichen festhalten, analysiert. Sie dokumentieren im Rahmen eines chronologischen Ablaufs von der letzten Schulbank bis an die Arbeitsplätze in den Lehrbetrieben die unterschiedlichen Wege der Jugendlichen in die Arbeitswelt. Hier wird das Ineinandergreifen von Geschlechtsreifung und -stilisierung, ökonomischen Dimensionen, verschiedenen Ebenen der Beziehungsentwicklungen in Schule, Arbeitsplatz und Freizeit vielschichtig sichtbar. 


\section{Teil I}

Methoden und Feldzugang 


\section{Schulethnografische Methodik}

Im Folgenden wird erläutert, welche methodischen Vorgehensweisen sich für Schulethnografie am besten eignen. $\mathrm{Zu}$ diesen gehören neben der teilnehmenden Beobachtung strukturierte und unstrukturierte Interviews mit Akteuren und verschiedenen Experten, Tiefeninterviews, spontane Gespräche, das Sammeln von Gegenständen und Dokumenten. Die Erhebungsmethoden werden in Bezug auf ihre jeweilige Reichweite in diesem spezifischen Forschungsfeld evaluiert.

Bei der Auswahl der Forschungsmethodik für das spezifische Forschungsfeld Schule wurde grundsätzlich ein Vorgehen gesucht, das im Sinne einer „lebensweltlichen Ethnografie“, wie sie von Anne Honer ${ }^{54}$ gefordert wird, dazu beiträgt, die von der sozialen Praxis konstruierte Wirklichkeitssicht zu rekonstruieren. So soll die emische Sichtweise, die Innensicht, erhoben werden, also die Sichtweise der Beforschten. Dazu werden verschiedene ethnografische Methoden herangezogen, die sich gegenseitig kontrollieren. Die Perspektive der lebensweltlichen Ethnografie fordert den Forschenden auch als Person: Die Kerntätigkeit der Feldforschung als klassische Forschungsmethode der Europäischen Ethnologie, die teilnehmende Beobachtung, wird zur beobachtenden Teilnahme, weil der Forscher nicht „als ob“ mitmacht, sondern wirklich mit-tut. Es ist also für den Forscher notwendig, sich ein Stück weit auch existenziell einzulassen auf die Ereignisse im Feld. Nur so, argumentiert Honer, könne man die Schwächen der klassischen Methoden zur Erschliessung sozial konstruierter Wirklichkeit überwinden. Die ziehen meist die Zuverlässigkeit der erhobenen Daten ihrer Gültigkeit vor, was oft die einzelnen Menschen auf der Strecke lasse. Die Sachverhalte sol-

54 Anne Honer: Einige Probleme lebensweltlicher Ethnografie. Zur Methodologie und Methodik einer interpretativen Sozialforschung, in: Zeitschrift für Soziologie 4, 1989, Jahrgang 18, 297-312. 
len nicht in erster Linie erklärt werden, sondern der Forscher soll unter Reflexion des vorgängigen eigenen alltäglichen Verstehens ,settings“ beschreiben, um Alltags-Erklärungen und Alltags-Handeln verstehen zu können. Die lebensweltliche Ethnografie möchte verstehend beschreiben und gleichzeitig kleine soziale Lebens-Welten durch Beschreibung verstehen. ${ }^{55}$ In Honers Forderungen wird also die Balance zwischen Teilnahme und Beobachtung in Richtung Teilnahme verschoben, was Auswirkungen auf die Datenerhebung und Interpretation hat. Auch die Resultate dieser Arbeit sind geprägt durch eine grosse Nähe zu den Beforschten.

Eine weitere methodische Grundausrichtung besteht im Konzept der ,interkulturellen Forschung“ in der Arbeit mit Jugendlichen. Obwohl die hier beforschten Jugendlichen der Kultur des Forschenden angehören, hat ihre Gruppenhaftigkeit eine deutlich unterschiedliche Ausprägung, sowohl was den generationellen Unterschied zum Forscher, wie auch die vielfältige Herkunft der jugendlichen betrifft. Diese Subkulturalität ist eine Legitimation dafür, interethnische und interkulturelle Forschungsansätze einzubringen.

Die Aufgabe interethnischer und interkultureller Forschung ist es, die Codes, die der Interaktion kulturell unterschiedlicher Gruppen zugrunde liegen, zu erschliessen und im Kontext des Forschungsfeldes nachvollziehbar zu machen. Sie fragt nach Bildern und Symbolen, in denen sich Differenz ausdrückt, sowie nach Formen interkultureller Kommunikation, die ethnisch-kulturelle Barrieren im Alltagsleben und -handeln überbrücken und überschreiten lassen. $^{56}$

55 Ebd., 297ff.

56 Eisch, Katharina: Interethnik und interkulturelle Forschung, Methodische Zugangsweisen der Europäischen Ethnologie, in: Göttsch, Silke und Al- 
So formuliert Katharina Eisch den Ansatz interkultureller und interethnischer Forschung. Die Methodik muss dabei prozessual und kontextbezogen den Gegebenheiten des Feldes folgen und damit der Tatsache gerecht werden, dass kulturelle Bedeutung jeweils situativ und dialogisch ausgehandelt wird. Die Akteure agieren im Schnittpunkt zwischen individueller und kollektiver Identität und verfolgen aus der Mikroperspektive in Lebensläufen und im Alltagshandeln den Reflex gesellschaftlicher Prozesse. Dabei wird dem Raum, eine wichtige analytische Kategorie, wenn es um Jugendliche geht, eine wichtige Bedeutung beigemessen:

Kulturelle Selbstverortung ist immer auch räumlich fundiert, selbst vor den fliessenden Horizonten globalisierter Lebenswelten. Im Kontext von Migration und neuer Mobilität versuchen Menschen, sich relativ zu ihrer räumlichen Umgebung zu definieren und Landschaften und Städten ihre Spuren und Gedächtnisorte einzuschreiben; das Fehlen räumlicher Identifikationspunkte wird als Fremdheit empfunden. ${ }^{57}$

Gleichzeitig sind die hier untersuchten Jugendlichen selbst Teil unterschiedlichster Immigrantengruppen. Sie konstruieren unter sich be-

brecht Lehmann (Hg.): Methoden der Volkskunde. Positionen, Quellen, Arbeitsweisen der Europäischen Ethnologie, 2. Auflage, Berlin, Reimer, 2007, 141-169, 148-149.

57 Ebd., 148ff. 
reits ein Feld komplexer Interethnik, die sich innerhalb der Institution Schule und im Klassenverband auch nur in manchen Situationen zu einer kollektiven Identität, der Schüleridentität, zusammenfügt.

\subsection{Ethnografische Perspektiven auf Schulen}

Zunächst aber soll gezeigt werden, warum es sich für eine empirisch arbeitende Kulturwissenschaft lohnt, in der Schule Ethnografie zu betreiben. Als wichtigster Grund dafür kann angeführt werden, dass die Schule aus der Sicht der Gesellschaft eine symbolische Arena darstellt. So interpretiert unter anderem die Sozialanthropologin Ann Runfors ${ }^{58}$ deren Rolle in ihrer Studie über schwedische Schulen in von Segregation gekennzeichneten Vororten von Stockholm. Für Runfors ist die Schule eine Institution, die sowohl Gesellschaftsvisionen wie -veränderungen spiegelt als auch gesellschaftliche Bedingungen formt. An ihr werden verschiedene Konzepte deutlich, etwa was unter Ausbildung verstanden wird oder was aus Kindern werden soll und welchen Weg in die Zukunft eine Gesellschaft gehen will. Zudem ist die Schule eine Projektionsfläche gesellschaftlicher Ängste und Hoffnungen sowie ein Feld der Auseinandersetzung. ${ }^{59}$

In dieser Studie wird die Schule als Ort gesehen, der den institutionellen Rahmen vorgibt, innerhalb dessen Jugendliche permanent gezwungen sind, in Klassenverbänden ihre Identität auszuhandeln. Dabei ringen die Verhandelnden immer wieder um Bedeutungen, welche bestimmten Komponenten ihrer Identität beigemessen wird. In den Schul-

58 Runfors, Ann: Mångfald, motsägelser och marginalisieringar. En studie av hur invandrarskap formas i skolan, Stockholm, Prisma, 2003.

59 Ebd., 67. Für den Zusammenhang von Schule als Arena und community action siehe auch Bunar, Nihad und Magnus Dahlstedt: Segregationen, 'community action' och skolan som arena, Umeå, Norrköping, Växjö, PfMI, 2000. 
gruppen präsentieren die Akteure ständig Identitätsentwürfe, die auf mehreren sozialen Kategorien und Gruppierungen beruhen. Diese können etwa ihre Identität als erotisches Wesen sein, ihre Zugehörigkeit zu einer nationalen oder ethnischen Gruppe, oder ihre Zugehörigkeit zu einer Szene. So formt sich die Identität immer wieder in manchmal überraschenden Bedeutungssprüngen neu. Dieser Prozess ist eng mit der Frage nach Integration und Ausschluss verzahnt, da die Gruppen sich nach bestimmten Zugehörigkeiten ausrichten und bilden.

Ein Beispiel mag diesen Zusammenhang illustrieren. Ein junger Mann besass eine dunkle, aber nicht schwarze Haut. Damit war er in der Gruppe der Schwarzhäutigen automatisch akzeptiert. Doch er selbst fand, dass er nicht wirklich schwarz war, deshalb mied er angeblich jene Gruppe und wies ihr Aufnahmeangebot ab. Dieser Junge gab also einem Gruppenmerkmal eine andere Bedeutung als diejenigen Gruppenmitglieder, welche sich bereits vorher über die Bedeutung der schwarzen Hautfarbe als gemeinsames Merkmal und wichtigsten Integrationsfaktor geeinigt hatten. ${ }^{60}$

Ein weiterer Grund für die Wichtigkeit des Forschungsfelds Schule ist deren Bedeutung für das Leben jedes Einzelnen. In der biografischen Rückschau fallen einem sofort Begebenheiten aus dem Schulleben ein. Aus oben genannten Gründen wird jeder und jede durch die Schule enorm geprägt; gute und schlechte Schulerlebnisse haben einen grossen Einfluss auf die Einstellungen und Ansichten Einzelner über die Gesellschaft. Nicht zuletzt nimmt man auch sozial sehr viel mit, die ältesten und stabilsten Freundschaften stammen oft aus dieser Zeit: In der Schule schliesst man Freundschaften fürs Leben. Weshalb dies so ist, soll unter anderem in dieser Studie aufgezeigt werden.

60 Feldbericht 2004, Seite 72, im folgenden mit FB04, 72 abgekürzt. 


\subsubsection{Schulethnografie als Organisationskulturforschung}

In Bezug auf die Methodik kann eine Schule auch als bürokratisch organisierte Institution aufgefasst werden. Für die Erforschung von Organisationskultur hat Irene Götz ${ }^{61}$ methodische Vorgehensweisen aufgearbeitet. Hierbei fragt die Europäische Ethnologie nach gruppengebundenen Werten und Normen, Einstellungen und Haltungen, die auf der Auseinandersetzung der Menschen mit der internen und externen betrieblichen Welt entstehen, welche die Handlungen und Wahrnehmungen der Organisationsangehörigen prägen, in symbolischer Form existieren und als Traditionen weitergegeben werden. Die betriebliche Lebenswelt dieser Subkultur konstituiert sich aus dem selbstverständlichen und traditionalen Wissen, Handeln und Erleben der interagierenden Subjekte. Deren Alltagspraxis wird vom Alltagsdenken beherrscht, das aus der Innenperspektive der Lebenswelt als gesichert und bewährt erlebt wird. Das wiederum beruht auf jenem Wissen, welches das Verhalten in der Alltagswelt reguliert und welches die Alltagswelt als sinnhaft und daher begreifbar erscheinen lässt. Das sind die zentralen Dimensionen von Organisationskultur. Sie lässt sich nur über die subjektiven Interpretations- und Handlungsweisen verstehen und nur im historischen, soziokulturellen Kontext, in dem diese subjektiven Interpretations- und Handlungsweisen verständlich werden. Im Mittelpunkt der Untersuchung von Organisationskultur steht daher der einzelne Beschäftigte oder Mitarbeiter des Betriebs, also die Situation des Individuums in der Struktur. ${ }^{62}$

61 Götz, Irene: Empirische Erhebungen in Industriebetrieben und bürokratischen Organisationen, in: Göttsch, Silke und Albrecht Lehmann (Hg.): Methoden der Volkskunde. Positionen, Quellen, Arbeitsweisen der Europäischen Ethnologie, 2. Auflage, Berlin, Reimer 2007, 249-270.

62 Ebd., 251-252. 
Schule lässt sich als Organisation im Sinne eines Betriebs auffassen, der subkulturelle Charakter ist jedoch spezifisch durch die Tätigkeit des Lehrens und Lernens geprägt. In der vorliegenden Arbeit ist der Blick noch weiter eingegrenzt, nämlich auf Integrations- und Ausschlussprozesse innerhalb und zwischen Gruppen von Jugendlichen einer Schulklasse, also auf Dynamiken von Gruppenkulturen innerhalb einer Subkultur. Trotzdem sind methodische Überlegungen der Organisationsethnologie für die Feldforschung in der Schule hilfreich. Götz schlägt folgende Indikatoren für deren Erforschung vor:

1. Die Selbstdarstellung der Organisation nach aussen

2. Die Gesamtanlage, die Architektur, die räumliche Gliederung der Untereinheiten, die Zimmerverteilung, das Mobiliar, die Kleidung als Statusindikator, das Auftreten der Einzelnen, Manifestationen besonderen Stils

3. Die Leitung: wie sind die offiziellen Grundsätze, Werte und Verhaltensvorschriften und wie werden sie im Alltag rezipiert und aktualisiert?

4. Dokumentation der inoffiziellen, verdeckten Organisationskultur, also der lebensweltlichen Aspekte im Arbeitsalltag, z.B. Xeroxlore, Witze, Graffiti

5. Informelle Betriebskultur

6. Erzählungen, die bereits Gruppenbesitz geworden sind

7. Individuelle Geschichten: Beziehungen, Werte und Leitbilder

8. Funktion des Erzählens im Betrieb: Persönliche Identität des Einzelnen in der Gruppe, Bedürfnis nach Solidaritätserlebnissen, Konflikte am Arbeitsplatz, Nischen, Freiräume

9. Institutionalisierte Zusammenkünfte, rituelle Inszenierungen, Anlässe mit hohem Symbolwert zeigen die Diskrepanz zwischen offiziellen symbolischen Botschaften und Wirklichkeit

10. Objektive Faktoren, die von oben vogegeben sind 
11. Ausserhalb liegende Informationsquellen wie Verbände, Behörden, Gewerkschaften

12. Verschiedene Informationen aus verschiedenen Hierarchiestufen über Vorstellungen, Arbeits- und Führungsstile, Geschlechterrollen, subjektive Konzepte wie Zeit oder Leistung ${ }^{63}$

Götz betont die Notwendigkeit, offene ethnografische Ansätze zu wählen, damit Fragestellungen, Methoden und Quellen den Feldgegebenheiten angepasst werden können. Die Hypothesen über ein Feld sind im Prozess des empirischen Arbeitens induktiv zu entwickeln. Anhand eines gesellschaftlichen Problems sei eine Ausgangsfragestellung zu entwickeln und durch Vergleiche zu überprüfen. Folgende Vergleiche sind möglich:

1. Eigene Werte der Betriebsrealität versus im Feld vorgefundene Werte

2. Offizielle gegen inoffizielle Organisationskultur

3. Einstellungen und Verhalten zwischen oben und unten

4. Zwischen verschiedenen regionalen Niederlassungen

5. Zwischen Gegensätzen wie Männer - Frauen, Anspruch - Wirklichkeit, früher - heute, u.a

Diese Vergleiche bedingen eine gewisse Distanz zum Feld. „Indem die Forschenden einer fremden Kultur zunächst aus der Distanz der Aussenstehenden begegnen und sie mit der eigenen, vertrauten Kultur vergleichen, wird für sie die neue Kultur - aus der Differenz zur eigenen - verstehbar." Dies setzt voraus, dass die Organisation eine eigene Subkultur innerhalb der grösseren nationalen und regionalen Kultur ist. Durch die Teilnahme am Betriebsalltag werden die Forschenden zuneh-

63 Ebd., 255-258. 
mend mit den Werten und Vorstellungen der Betriebsangehörigen vertraut und können deshalb mit der Zeit auch verschiedene emische Sichtweisen einnehmen. Dabei sollten sie im Betrieb mitarbeiten und die teilnehmende Beobachtung grundsätzlich als gleichwertige Kommunikation konzipieren. Auch sollen die Daten diskursiv ausgewertet werden, also unter Einbezug der Beforschten. ${ }^{64}$

\subsubsection{Spezifik der schulethnografischen Praxis}

Diese methodischen Überlegungen flossen in die Gestaltung der Feldarbeit als Grundlage dieser Arbeit mit ein. Jedoch war das Erkenntnisziel nicht die Beschreibung der Kultur einer Organisation oder einer Unternehmenskultur, sondern Integrations- und Ausschlussprozesse unter den Bedingungen dieser Organisationskultur, wie sie zwischen sowie innerhalb von Jugendlichengruppen in Ausbildung vorkommen. Kulturelle Spezifika von Jugend schränken das oben dargestellte Methodikprogramm ein. Jugendliche schaffen sich andere kulturelle Praxen wie Erwachsene, sind also nicht eine Subkultur innerhalb einer regionalen Kultur, sondern eine kulturelle Gruppe per se. Die Gruppen innerhalb der Schulklassen sind keine Subkulturen, sondern auch eigene, wenn auch lose und temporär gestaltete kulturelle Gruppen, mit eigenen Stilen, Sprachen, Darstellungsweisen, Werten.

Die Widerständigkeit, mit der Jugendliche den Forschungswünschen eines Erwachsenen entgegentreten sowie deren in diesem Fallbeispiel noch geringe formale Bildung, erschweren methodische Ansätze wie etwa die von Götz vorgeschlagene diskursive Datenauswertung oder gleichwertige Kommunikation in der teilnehmenden Beobachtung. Passend ist jedoch der Indikatorenkatalog und die Forderung nach einem

64 Ebd., 259-263. 
dynamischen Forschungsansatz, der der Dynamik jugendlicher Kulturen gerecht wird.

Neben den Überlegungen zur Organisationsethnologie stützt sich diese Studie allgemein auf das klassische Methodeninstrumentarium der Europäischen Ethnologie, wie sie etwa von Brigitta Schmidt-Lauber ${ }^{65}$ beschrieben werden. Ihre Arbeiten haben in für die Methodik der Europäischen Ethnologie anleitende Qualität erhalten. Für diese Arbeit zentral wurden zudem methodische Überlegungen, wie sie im schwedischen Handbuch für Schulethnografie von Brigitta Kullberg ${ }^{66}$ spezifisch für die Forschungstätigkeit in Schulen und mit Kindern und Jugendlichen entwickelt worden sind. Diese Ansätze wurden arbeitet, um den Pädagogen Möglichkeiten aufzeigen, Informationen über ihre Schüler zu sammeln, um den Alltag in der Schule verbessern zu helfen. Sie stützen sich auf Methoden aus der Ethnologie und der qualitativen Sozialforschung allgemein und stellen eine methodische Verfeinerung dar, wie ich sie in der deutschsprachigen Europäischen Ethnologie nicht angetroffen habe. Aus diesen Gründen stütze ich mich in dieser Arbeit stark auf diese Überlegungen.

Grund für den hohen Entwicklungsgrad schwedischer Schulethnografie kann in der komplexen Integrationsproblematik dieses nordischen Landes vermutet werden. In einigen Regionen ist Schweden stark von Segregationsproblemen geprägt. Die seit den 1970er Jahren eingewanderte ausländische Bevölkerung konzentriert sich auf die Satellitenstädte von Stockholm, Göteborg und Malmö, wo eigentliche ausländische Kleinstädte entstanden sind. ${ }^{67}$ An den Schulen in diesen Gemein-

65 Schmidt-Lauber, Brigitta: Feldforschung. Kulturanalyse durch teilnehmende Beobachtung, in: Göttsch, Silke \& Albrecht Lehmann (Hg.): Methoden der Volkskunde. Positionen, Quellen, Arbeitsweisen der Europäischen Ethnonogie, 2. Auflage, Berlin, Reimer, 2007, 219-248.

66 Kullberg, Birgitta: Etnografi i klassrummet, Lund, Studentlitteratur, 1996.

67 Schweden legte zwischen 1960 und 1980 ein staatliches Bauprogramm auf, das zum Ziel hatte, eine Million neue Wohnungen zu schaffen. Dazu 
den gibt es Ausländeranteile von bis zu 90\%. Diese Situation hat eine rege ethnologische Forschungstätigkeit nach sich gezogen und in deren Folge vertiefte Überlegungen zur Methodik. Kullbergs Buch wird seit Jahren für solche Forschungen herangezogen.

Dazu gibt es Vorläuferbewegungen. Seit den 1950er Jahren gibt es im angelsächsischen Sprachraum, vor allem in den USA, aber auch in England und Australien, die ,teacher-researcher"-Bewegung. Ziel dieser Bewegung war es, die Rolle der Lehrer als Reflektierende über das Lehren und Lernen zu stärken, weil sie die einzigen seien, welche die Vorgänge im Klassenzimmer aus eigener Erfahrung beurteilen könnten. Ein Problem der Pädagogik sei es, dass viele pädagogische Experten gar nie vor einer Klasse gestanden haben, was dazu führe, dass das Unterrichten einer der wenigen Berufe sei, in dem das Wissen über Arbeit bei Leuten liege, die sie nicht ausführten. Durch eine ethnologische $\mathrm{Zu}$ gangsweise, beispielsweise durch teilnehmende Beobachtung in der Schulklasse könne das Lehrpersonal dagegen eine wichtige Rolle einnehmen in der Theoriebildung darüber, welche pädagogische Konzepte funktionierten und welche nicht. Es habe sich gezeigt, dass Lehrerinnen und Lehrer, welche die Position eines Ethnografen einnähmen, wesentlich effizienter arbeiteten, was auch den Schülerinnen und Schülern einen Gewinn brachte. ${ }^{68}$

errichtete man ausserhalb der grossen Städte grosse Überbauungen in industrieller Bauweise, eigentliche zehnstöckige Kleinstädte, die von bis zu 50’000 Leuten bewohnt werden konnten. Zunächst zogen dort vom Lande zugezogene Schweden ein, die jedoch bald von - wie die Schweden sagen - Arbeitskrafteinwanderern aus anderen europäischen Ländern und dann von Menschen aus arabischen Staaten und Afrika verdrängt wurden. Dennoch sind die Integrationsprobleme nicht so akut wie in ähnlichen Wohngebieten anderer Länder, etwa den Banlieus in Frankreich.

68 Myers, M.: The teacher-researcher. Urbana, Illinois, ERIC Clearinghouse on Reading and Communication Skills and the National Council of Teachers of English, 1985, 2-5. 
Auch die deutschsprachigen Erziehungswissenschaften interessieren sich immer mehr für qualitative Zugänge zu ihrem Forschungsfeld. Weil die vorliegende Arbeit fachlich im Bereich der Europäischen Ethnologie angelegt ist, kann auf diesen Forschungszweig nur verwiesen werden, obwohl sich gerade in den letzten beiden Jahrzehnten verschiedene fruchtbare Kooperationen ergeben haben. Wesentliche Impulse kamen vom breiten Werk von Christoph Wulf, der durch seine internationale Tätigkeit im Bereich "Anthropologie und Erziehung" nicht nur in der deutschsprachigen Forschung richtungsweisende Ideen eingebracht hat. ${ }^{69}$ Der ethnografischen Forschung kulturanthropologischer Prägung besonders nah sind die Arbeiten von Forscherinnen und Forschern wie Helga Kelle ${ }^{70}$ oder Georg Breidenstein ${ }^{71}$. Auch die qualitativen Projekte wie etwa diejenigen von Sabine $\operatorname{Reh}^{72}$ zeigen wichtige methodische Schnittstellen zu ethnografischen Ansätzen auf. In der Deutschen Ge-

69 Aus dem enorm breiten Werk von Wulf seien zwei Werke erwähnt: Wulf, Christoph (Hg.): Einführung in die Anthropologie der Erziehung, Weinheim und Basel, Beltz Verlag, 2001 und Wulf, Chistoph (mit Birgit Althans, Kathrin Audehm, Gerald Blaschke, Nino Ferrin, Ingrid Kellermann, Ruprecht Mattig, Sebastian Schinkel): Die Geste in Erziehung, Bildung und Sozialisation: Ethnografische Feldstudien, Wiesbaden, VS Verlag, 2011.

70 Etwa Kelle, Helga: Ethnografische Methodologie und Probleme der Triangulation, am Beispiel der Peer Culture Forschung bei Kindern, in: Zeitschrift für Soziologie der Erziehung und Sozialisation (ZSE) 21 (2001) 2, 192-208 sowie Kelle, Helga: Mädchen und Jungen in Aktion. Ethnografische Ansätze in der schulischen peer culture Forschung, in: Horstkemper, Marianne und Margret Kraul (Hg.): Koedukation - Erbe und Chancen, Weinheim, Deutscher Studien Verlag, 1999, 157-183.

71 Z.B. Breidenstein, Georg: Teilnahme am Unterricht, Ethnografische Studien zum Schülerjob, Wiesbaden, VS Verlag für Sozialwissenschaften, 2006 und Breidenstein, Georg und Helga Kelle: Geschlechteralltag in der Schulklasse, Ethnografische Studien zur Gleichaltrigenkultur, Weinheim und München, Juventa, 1998.

72 Reh, Sabine, Kerstin Rabenstein und Carla Schelle: Unterricht als Interaktion, Bad Heilbrunn, Klinkhardt Verlag, 2010. 
sellschaft für Erziehungswissenschaft hat sich die Kommission für Quantitative Bildungs- und Biografieforschung gebildet, die bereits mehrere Kongresse ausgerichtet hat. Dieser Forschungszweig arbeitet zunehmend qualitativ-ethnografisch in Schule und Unterricht und mit den Themen Kindheit und Jugend. ${ }^{73}$

Doch wenden wir uns zunächst dem klassischen methodischen Instrumentarium der Europäischen Ethnologie und den ihm zugrunde liegenden Grundsätzen zu. Brigitta Schmidt-Lauber bezeichnet Feldforschung als zentrales methodisches Verfahren der Europäischen Ethnologie, dessen Spezifik ein perspektivenreicher, meist multimethodischer Zugang ist, der auf der aktiven, beobachtenden Teilnahme am alltäglichen Leben der Beforschten basiert. Dies dient dem Ziel des sinnverstehenden Miterlebens und Nachvollziehens von Wirklichkeitszusammenhängen. ${ }^{74}$ Dabei können unterschiedliche qualitative und quantitative Methoden wie diverse Interview- und Beobachtungsverfahren, Netzwerkanalysen oder ähnliches zum Einsatz kommen. Kennzeichnende Schlüsselmethode ist jedoch die teilnehmende Beobachtung, mit der die Feldforschung häufig auch gleich gesetzt wird. Gemeint ist damit die unmittelbare Partizipation des Forschenden am alltäglichen sozialen Leben im jeweiligen Untersuchungsfeld und ein empathiegeleitetes, nachvollziehbares Verstehen bei gleichzeitig gewahrter analytischer Distanz. Dabei soll eine sinnverstehende Deutung sozialen Handelns erlangt werden, das in einen überindividuellen kulturellen Sinnzusammenhang eingeordnet wird. ${ }^{75}$ Synonym zur Feldforschung wird in verschiedenen Fächern auch der Begriff Ethnografie verwendet, im Gegensatz zu theoretischen Überlegungen, die als Ethnologie bezeichnet wer-

73 Für weiterführende Informationen siehe http://www.dgfe.de/sektionen/sektion-2-allgemeine-erziehungswissenschaft/kommission-qualitative-bildungs-und-biographieforschung.html (11. Februar 2012).

74 Schmidt-Lauber, Feldforschung, 2007, 219.

75 Ebd., 220-221. 
den. In der Ethnologie selbst wird der Begriff Ethnografie als methodische Verfahren zur Datengewinnung gebraucht, also als Oberbegriff für verschiedene Verfahren der Feldforschung. ${ }^{76}$

Schmidt-Lauber geht mit bemerkenswerten Überlegungen auf Konzeption und Planung von Feldforschung ein, auf die Formulierung einer Fragestellung und eines Erkenntnisziels, auf Untersuchungsfelder, auf zeitliche Abläufe und formale Fragen. ${ }^{77}$ Da diese Aspekte in der vorliegenden Forschung durch die Auftraggeber bereits festgelegt waren und eine Diskussion irrelevant gewesen wäre, soll an dieser Stelle nicht näher auf die Fachdiskussion um diese Fragen eingegangen werden. $\mathrm{Zu}$ diesem Themenbereich sei auf die Schrift von Schmidt-Lauber verwiesen.

Schmidt-Lauber ist der Meinung, dass teilnehmende Beobachtung ein dialogisches Verfahren ist, bei dem die Person der Forschenden in besonderem Mass selbst zum Forschungsinstrument wird. ${ }^{78}$ Im Hintergrund dieser Überlegungen stehen verschiedene Fachdiskussionen, wie sie von Schmidt-Lauber summiert werden. ${ }^{79}$ Hier wurde etwa die Frage nach der Balance zwischen Distanz und Nähe, also der Ausgleich von Teilnahme und Beobachtung im Forschungsfeld zentral, ebenso wie die in der sogenannten „Krise der Ethnografischen Repräsentation“ aufgeworfenen Zweifel an der Glaubwürdigkeit ethnografischer Darstellungen. In den 1960er Jahren nährten Restudies, Wiederholungen von Forschungen derselben Einheit zu unterschiedlichen Zeitpunkten, die Skepsis gegenüber ethnologischen Berichten, die offensichtlich von Faktoren wie Geschlecht, Status, Anwesenheitsdauer oder Höflichkeitsregeln beeinflusst waren. ${ }^{80}$ Störungen und Irritationen im Feld sollten

76 Ebd., 221. Für die disziplinäre und fachgeschichtliche Einordnung vgl. ebd., 221-227.

77 Ebd., 227-230.

78 Ebd., 230.

79 Vgl. Ebd., 244-248.

80 Ebd., 231-233. 
nicht vermieden und ausgeblendet werden, sondern als Informationsquellen über Interessenkonflikte, soziale Strukturen, Verhaltensnormen oder politische Kontexte genutzt werden. Das Diktum von der Angst des Forschers vor dem Feld, das Rolf Lindner 1981 geprägt hat, fasst diese Problematiken in eine griffige Formel. ${ }^{81}$

Die Erkenntnis, dass die Feldforschung eine bistimmte Konstellation antrifft und dass ihre Erkenntnisse an die Person und Herkunftskultur des Forschenden gebunden ist, hat dazu geführt, dass das methodische und analytische Repertoire der Europäischen Ethnologie in den 1980er Jahren erweitert wurde. Zudem wurden Forschende in Teams zusammengefasst oder hielten sich mehrmals im Feld auf. Supervision wurde institutionalisiert, um Gegenbertragungen ${ }^{82}$ und Abwehrstrategien zu erkennen und als Quelle zu nutzen. ${ }^{83}$

Kullberg bricht diese Erkenntnisse auf die Ebene der Feldforschung in Schulklassen hinunter, betont den Aspekt der rollenden oder dynamischen Forschung und entwickelt diese Ansätze mit Erkenntnissen aus der qualitativen Sozialforschung weiter. Zentral ist hier die Idee des "grounded theorizing" von Anselm Glaser und Barney Strauss ${ }^{84}$. Diese

81 Lindner, Rolf: Die Angst des Forschers vor dem Feld. Überlegungen zur teilnehmenden Beobachtung als Interaktionsprozess, in: Zeitschrift für Volkskunde 77, 1981, 51-66.

82 Gegenübertragungen finden in jeder sozialen Begegnung statt, der Begriff stammt aber aus dem Vokabular der Beziehung zwischen Patienten und Therapeuten in der Psychoanalyse. Schildert der Patient dem Therapeuten seine Phantasien, kann dieser mit Abscheu oder Zuneigung reagieren, was man als Übertragung bezeichnet. Richtet nun der Therapeut aufgrund dieser Äusserungen Vorurteile, Gefühle, Wünsche oder Erwartungen an den Patienten, nennt man das eine Gegenübertragung. Für die Ethnologie hat als erster Georges Devereux methodische Konsequenzen aus diesen Phänomenen gezogen (Devereux, Georges: Angst und Methode in den Verhaltenswissenschaften, Frankfurt am Main, Berlin, Wien: Ullstein 1976).

83 Schmidt-Lauber, Feldforschung, 2007, 234.

84 Glaser, B. und A. Strauss: The Discovery of Grounded Theory, Chicago 1967. 
führten die Vorstellung ein, dass Informationsbeschaffung, fortlaufende Informationsanalyse, Theorieentwicklung und die strategische Ausrichtung des Forschungskonzeptes dynamisch verwobene Aspekte der Forschungstätigkeit sind.

Ein weiteres Konzept der Grounded Theory ist das Bracketing, eine Bezeichung für den Versuch, sich immer wieder neue Ausblicke auf das Forschungsobjekt zu verschaffen. Dies kann durch die aktive Mithilfe der Untersuchten geschehen, indem man ihnen zum Beispiel die Interpretation gewisser Vorgänge überlässt. Methodisch soll der multiperspektivische Zugang durch Triangulierung geschaffen werden. Dies ist eine Umsetzung des an sich einfachen Grundsatzes, dass man etwas von unterschiedlichen Seiten betrachten soll. Auf die Situation der Feldforschung übertragen bedeutet dies, dass man durch das Kombinieren verschiedener Untersuchungsmethoden und das Kontrastieren derer Resultate die Nachteile und blinden Flecke einzelner Erhebungsmethoden ausgleicht. So sind wesentlich zuverlässigere Aussagen zu erhalten. ${ }^{85}$ An dieser Stelle führt Kullberg Norman K. Denzin ein, einen Forscher, der sich stark mit methodologischen Problemen der qualitativen Sozialforschung befasst hat und schlägt vier verschiedene Typen von Triangulierung vor: Forschertriangulierung, die Idee, dass mehrere Leute das gleiche untersuchen sollen, Datentriangulierung, den Vorschlag, verschiedene Quellen und Situationen zur Informationsbeschaffung über das gleiche Thema herbeizuziehen, Theorietriangulierung, verschiedene theoretische Perspektiven einzunehmen und schliesslich die Methodentriangulierung, die Forderung, gänzlich verschiedene Methoden auf ein Feld anzuwenden oder mindestens eine Methode zu variieren. ${ }^{86}$

85 Webb, P.: Unobstructive Measures. Nonreactive Research in the Social Sciences, Chicago, Rand McNally \& Co., 1966.

86 Denzin, Norman K.: Sociological Methods. A Sourcebook, Chicago, Aldine, 1970. 
Die Qualität der Resultate und deren Validität kann zudem gesteigert werden, wenn möglichst viele verschiedene Methoden angewendet werden.$^{87}$ Ethnografie ist multimethodisch: Die Anzahl der Methoden ist im Prinzip unbegrenzt, was die Forschenden herausfordert, auf die Forschungssituation spezifische Methoden zu entwickeln. In Anlehnung an die ethnologische Begriffsauffassung ist für Kullberg Teilnehmende Beobachtung ein Oberbegriff für alle möglichen ethnografischen Techniken. Kullberg fordert in konsequenter Umsetzung der Idee, dass der Forscher ein Instrument zur Erforschung einer sozialen Situation ist, also eine Art sozialer Resonanzkörper, alle Sinne zu benutzen: Nase, Gehör, den Tastsinn, den Geschmackssinn, u.a. ${ }^{88}$

\subsubsection{Implementierung der Methodik}

Diese Forderungen mussten zunächst im Feld umgesetzt werden, was eine gewisse Implementierungszeit erforderte. Neben den bewährten Zugängen geb es viel auszuprobieren. Es erforderte beispielsweise, dass ich versuchte, das Essen und Trinken der Jugendlichen zu besorgen, um das Gefühl dafür zu schärfen, in welchen Geschmacks- und Geruchswelten sie leben. Regina Bendix führt diesen Gedanken konsequent weiter: Alle wahrgenommenen Stimmungen, Emotionen und Eindrücke sind Möglichkeiten, Erkenntnisse zu gewinnen. Sie fordert eine Art intensive mentale Aufmerksamkeit im Feld. Auch diskutiert Bendix die methodischen und theoretischen Voraussetzungen dieses Vorgehens und lässt die wahrnehmungstheoretischen Aspekte nicht ausser acht, etwa den Einfluss von rauschhaften Zuständen ${ }^{89}$ Neben den Sinnesein-

87 Ganzer Abschnitt Kullberg, Etnografi i klassrummet, 1996, $117 \mathrm{ff}$.

88 Ebd., 66-67.

89 Bendix, Regina: Was über das Auge hinausgeht: Zur Rolle der Sinne in der ethnografischen Forschung, SAVk 102 (2006), 71-84. 
drücken sollten also auch die von ihnen ausgelösten Emotionen beachtet werden.

Über die von mir erlebten Sinneseindrücke und Emotionen führte ich detailliert Buch, um nachher die Gründe für meine Gefühle wie Ärger oder Freude zu analysieren. Diese Analysen rühren sehr tief an individuelle und kulturelle Identitätsfragen. Auch auf diese bereitet Brigitta Kullbergs Schrift vor. Sie ist ganz besonders hilfreich, weil sie die methodischen Prinzipien für die feldforschende Praxis umsetzt. Ihre praktischen Überlegungen erwiesen sich für meine Studie besonders dann als hilfreich, wenn ich während meines Feldaufenthalts auf Schwierigkeiten traf. Diese Schlüsselstellen wurden für diese Arbeit besonders wichtig.

Doch schon zu Beginn meines Feldaufenthalts machte ich mir aufgrund von Kullbergs Überlegungen, die stark auf der Grounded Theory aufbauten, Gedanken über die verschiedenen Rollen von Schülern, über Möglichkeiten, meine Erhebungen zu triangulieren und über die Produktion von Theorie während der Aufzeichnung von Informationen.

Beispielsweise ist es, wie Kullmann zeigt, völlig normal, dass sich die Ausgangsfragen während der Studie verändern, denn die Theorien, welche zu neuem Wissen führen sollen, werden während der Studie geschaffen. "One is free to discover what the problem is rather than to be obliged to pursue a inquiry into a predominant problem." zitiert Kullberg den Schulethnografen Walcott. ${ }^{90}$ Bei meiner Arbeit im Schulzimmer hatte ich immer ein Logbuch dabei, in das ich mit kurzen Sätzen oder stichwortartig meine Beobachtungen eingetragen habe. Später habe ich diese Notizen ausformuliert und in Tagebuchform überführt. So entstanden zu jedem Feldbeobachtungstag ein- bis mehrseitige Berichte, die ich chronologisch ablegte. Diese versuchte ich nach theoreti-

90 Walcott, H.: Criteria for an Ethnografic Approach to Research in Schools. Human Organisation 34 (2), 1975, 111-127, nach Kullberg, Ethnografi i klassrummet, 1996, 36. 
schen Überlegungen durchzukämmen und laufend zu analysieren, um die nächsten Beobachtungstage vorzubereiten.

Dieses Vorgehen entspricht der konsequenten Anwendung von Grounded Theory. Der Feldforscher soll während seinen Studien theoretische Gedanken laufend zu Papier bringen. Das wird als theoretischer Output bezeichnet und hat seinen Grund in dem, was während der Studie geschieht und was sich in der kontinuierlichen Analyse zeigt. Der theoretische Output kann zu einer veränderten Problemstellung führen. ${ }^{91}$ Tatsächlich flossen bei meiner Feldforschung auch auf den banalsten Feldtagebuchblättern fast unbewusst immer wieder kleine Überlegungen ein, die als Abstraktionen oder Interpretationen der Beobachtungen zu werten waren und deren theoretischer Gehalt die Ausrichtung und den Fokus der Untersuchung beeinflussen sollten.

\subsubsection{Validität, Reliabilität, Triangulierung}

Trotz dieses Vorgehens dürfen die Forscher nicht die Validität, das Mass der Übereinstimmung zwischen Untersuchungsthema und dem tatsächlich Untersuchten, und die Reliabilität, die Nichtzufälligkeit der Resultate, aus den Augen verlieren. Obwohl beide Begriffe aus der quantitativen Forschung stammen, wo es Messinstrumente und Gemessenes gibt, haben sie auch für die qualitative Forschung Bedeutung. Europäische Ethnologen arbeiten hauptsächlich qualitativ und sind selbst das Forschungsinstrument. Sie messen ihre Daten nicht, sondern interpretieren sie. In diesem Licht kann die Validität mit den oben beschriebenen Triangulierungen gesteigert und sichergestellt werden. Eine weitere Art, die Validität zu steigern, besteht im Einbezug der Erforschten in die Interpretation des gesammelten Materials.

\footnotetext{
91 Ebd., 49ff.
} 
Die Reliabilität kann mit Kriterien wie der im Feld verbrachten Zeit oder der Vielfalt und Menge der erhobenen Daten gut eingeschätzt werden. ${ }^{92}$ Meine Untersuchung lief über mehr als zwei Jahre. Sie produzierte mehrere hundert Feldbeobachtungstagebuchseiten und sonstige Quellen. Obwohl das Mass der Reliabilität in diesem Fall nicht als Zahl angegeben werden kann, darf davon ausgegangen werden, dass die Reliabilität genügend hoch ist, obwohl gewisse, weiter unten noch dargestellte Schwierigkeiten die Datenerhebung teilweise behinderten.

\subsubsection{Rollen, Beobachtungsfokus, Langfristigkeit}

Feldforschung in der Schule unterliegt besonderen Bedingungen beim Dokumentieren, im Aushandeln von Rollen und im Festlegen von Beobachtungsthemen. Diese Arbeit in einem Schulzimmer erfordert Engagement, aktives Zuhören, intensive Beobachtung und ständiges Analysieren, was auch körperlich anstrengend sein kann.

$\mathrm{Zu}$ Beginn der Arbeit werden als erstes die Rollen festgelegt. Die Rolle des Forschenden und die damit verbundene spezifische Kommunikation ist zunächst für anderen Beteiligten unklar. Nimmt man am Unterricht teil, ist aber kein Schüler, ist man dann nicht ein Lehrer? Wenn er kein Lehrer ist, was ist er dann? Die Rolle des Forschers, seine Position muss aktiv und bewusst ausagiert werden. Auch die Schülerinnen haben wechselnde Rollen: etwa als Auszubildende und als Gewährspersonen. Auch diese Sachverhalte müssen berücksichtigt sein, weil sie über eine Interpretation entscheiden können.

Schwierig ist es auch, im Schulalltag zu wissen, was man beobachten soll. Eine Schulstunde kann derart langweilig sein, dass man gegen den Schlaf ankämpfen muss. Kullberg empfiehlt, mit einem möglichst breiten Fokus anzufangen. Durch das ständige Notieren und laufende 92 Ebd., 54 und $117 \mathrm{ff}$. 
Analysieren findet der Ethnologe zu den wichtigen Ereignissen und Situationen. Im übrigen ist Beobachten anstrengend. Mehr als drei Stunden intensives Observieren am Stück ist wegen Ermüdung unrealistisch.

Ein weiteres kniffliges Problem ist, was der Feldforschende auswählt für seine Notizen. Die Frage ist sehr wichtig, weil sie die Generierung der zu interpretierenden Daten entscheidend beeinflusst. Diese haben wiederum einen grossen Einfluss auf die Auswertung und damit die Forschungsresultate. ${ }^{93}$ Schmidt-Lauber spricht dieses Problem in ihrer Methodenschrift nicht an. Sie meint bloss, dass ,eine möglichst ausführliche Beschreibung der Beobachtungen (...) erforderlich ist ${ }^{‘ 94}$, doch ganz offensichtlich kann man nicht alles notieren. Es muss also eine Auswahl getroffen werden.

Grundsätzlich gibt es, meint Kullberg, zwei Strategien: The critical incident strategy: Abweichende, spezielle Situationen sollen abgewartet werden, beispielsweise wenn Schüler besonders starke Reaktionen zeigen. Eine anderer Zugang besteht darin, ,periodisch“ zu arbeiten: Beispielsweise alle fünf Minuten Notizen zu machen, auch wenn scheinbar nichts passiert. Das Umfassende und damit die Objektivität der Beobachtung kann auch gesteigert werden, indem eine Liste der Perspektiven erstellt wird. Etwa Räume, Akteure, Aktivitäten, Objekte, Handlungen, Geschehnisse, Zeitrahmen, Ziele, Gefühle können dafür in Frage kommen. ${ }^{95}$

Auch diese Empfehlungen erwiesen sich als sehr brauchbar. Die periodischen Notizen zum Beispiel waren eine ausgezeichnete Hilfe, Dinge zu beobachten, die einem gar nie aufgefallen wären. Gerade das alltäglich Unscheinbare war ja ein spezieller Fokus der Untersuchung. Auch zu notieren, unter welchen Umständen bestimmte Gefühle auftra-

93 Ebd., $73 \mathrm{ff}$.

94 Schmidt-Lauber, Feldforschung, 2007, 234.

95 Kullberg, Etnografi i klassrummet, 1996, 74-77. 
ten, etwa Peinlichkeit oder Widerwillen, waren eine grosse Hilfe zur Objektivierung.

Auch zum Thema Interview mit Schülern stellt Kullberg viele Überlegungen an, die zu grundlegenden Zugangsweisen führen. Die wichtigsten Grundsätze sind, dass man zwar zu den Gewährspersonen hingehen, aber sie auf neutralem Grund treffen solle. Man solle das Gespräch auf Tonband aufnehmen und für die Fragen Feldbeobachtungen beiziehen. Dieser Hinweis deckt sich mit der Empfehlung, Feldbeobachtungen von den Jugendlichen selbst interpretieren zu lassen. ${ }^{96}$ Dies liess sich schlecht umsetzten, denn ich machte die Erfahrung, dass die Jugendlichen erst nach der Schulzeit bereit waren, wirklich aussagekräftige Informationen über ihre Klassenkollegen zu geben, und versuchten, sie während der Schulzeit durch unterschiedliche Strategien zu schützen, um nicht sich selber nicht zu exponieren.

\subsubsection{Gatekeepers, Setting, Aufzeichnungsgattungen}

Besondere Aufmerksamkeit und Rücksicht verdienen die sogenannten "Gatekeepers", also Akteure, welche Schlüsselressourcen vor Ort kontrollieren und den Zugang zum Feld ermöglichen. Diese Gatekeepers müssen dazu gebracht werden, einen durch das Tor (Gate) aufs "Feld" zu

lassen. ${ }^{97}$ In meinem Fall waren dies die Schulbehörden, die Lehrer und die Eltern, die ich um Erlaubnis bitten musste, meiner Arbeit nachgehen zu können. Diese Erlaubnis wurde mir in allen Fällen ausser einem erteilt. Ein türkischer Vater hat meine Ausführungen am Telefon sprachlich nicht verstanden und die Erlaubnis, seinen Sohn befragen zu dürfen, verweigert.

96 Ebd., 79ff.

97 Ebd., 92-93. 
Ist der Forscher im Feld etabliert, in diesem Fall in einem Schulzimmer, empfiehlt Kullberg ihm, sich als erstes ein Setting aufzubauen:

Einen Ort zu schaffen, wo Forscher und Erforschte unter günstigen Vorzeichen zusammentreffen können. Oft wird einem temporär oder permanent ein Raum zur Verfügung gestellt. Dieser kann gestaltet werden. Dies einerseits physisch durch das Aufhängen von Bildern, Einrichten eines Briefkastens, Auflegen von Tage- und Gesprächsbüchern oder das Platzieren von wichtigen Gegenständen im Raum. Oder psychisch durch Strukturieren des Beobachtungstages, durch Abmachungen, wann Gespräche, Beobachtung und Pausen stattfinden sollen. ${ }^{98}$ Auch diese ausgesprochen wichtige Erhebungshilfe habe ich beherzigt: Ich sorgte dafür, dass ich in einem unbenutzten Schulzimmer meine Interviews führen konnte. Ich legte das Tonband auf einem runden Tisch bereit, um eine unhierarchische Sitzordnung zu haben, sorgte für frische Getränke, lüftete das Zimmer, legte Dokumente wie Fragelisten oder Broschüren zur Schulorganisation oder Lehrstellenwesen aus und bezog die Umgebung immer wieder ins Gespräch mit ein, indem ich etwa nach Musikstars auf den aufgehängten Postern fragte.

Am Ende meines Feldaufenthaltes von September 2003 bis Sommer 2005 war ich im Besitz folgender Quellen:

64 Protokolle über Beobachtungstage in und ausserhalb der Schule

Bericht über 5 Tage Klassenfahrt in Südfrankreich

4 Protokolle Gruppengespräche

8 Interviews aus dem Frühjahr 2005

18 Interviews mit Schülerinnen und Schülern der WBS März bis Juni 2004

4 Experteninterviews

98 Ebd., $97 \mathrm{ff}$. 
15 Aufsätze von Schülerinnen und Schülern zum Thema: Ein Tag in meinem Leben: der 4. Mai 2024

Verschiedene Dokumente der Schule

Gegenstände: Spickzettel, Wurfzettel, Schreibzeug, Plakate aus dem Klassenzimmer, CD's

Medienprodukte: Presseausschnitte, Ausdrucke Websites, Jugendmagazine, Hefte, u.a.

Chat- und SMS-Protokolle

Diese Quellen sind folgendermassen entstanden: Jeden Tag schrieb ich meine Beobachtungen in ein Feldnotizbuch. Das war ein Heft im Format A5/6, in das ich meine Aufzeichnungen logbuchartig eintrug. Um wirklich sicher zu sein, dass die Notizen nicht unbemerkt von Dritten gelesen werden konnten, schrieb ich möglichst unleserlich oder auf Schwedisch. Ich war ja zur Geheimhaltung verpflichtet, denn eine Bedingung der Auftraggeber und eine Hilfe für die Motivation zum Mitmachen war, dass die Beforschten anonym blieben und die Ergebnisse nicht zuzuordnen waren.

So bald als möglich setzte ich das stichwortartig Aufgeschriebene um: Ich formulierte die Sätze aus und ergänzte Informationen aus dem Gedächtnis. Dies ergab mehrseitige Tagesberichte. Ein Spezialfall waren die Beschreibungen, die ich auf der Schulabschlussreise nach Südfrankreich aufgeschrieben habe. Diese habe ich in meinem Zelt bereits in einem Heft ausformuliert und zuhause nur noch abgetippt. Weiter unten, im Abschnitt über die Auswertung des Materials, wird über die Art berichtet, wie diese Unterlagen für die Analyse erschlossen wurde. 


\subsubsection{Strategie der Anwesenheit}

Vor Ort entwickelte ich eine Art Strategie der Anwesenheit, um ergiebige Situationen beobachten zu können. Für mich waren das Orte, an denen es verstärkte Interaktion gab. Es hat sich etwa kaum gelohnt, in die regulären Schulstunden zu gehen, da hier eine stark hierarchische Situation herrschte. Eine Schulklasse beispielsweise, die sich über mathematische Rechenaufgaben beugt, interagiert kaum. Ich versuchte deshalb, in Situationen anwesend zu sein, von denen ich vermutete, dass sie für mich ergiebiges Material produzieren würden. Dies waren meistens unhierarchische Situationen, Pausen, Gruppenarbeiten, Konzerte, Vorführungen im Hauptgebäude der Schule, in denen die Schüler sich ohne Aufsicht trafen. Beispielsweise hielt ich mich vor der Schulstunde vor der Zimmertüre auf wie die Schülerinnen und Schüler, die in dieser Zeit stark interagierten, wohl weil sie nachher Schweigen und Zuhören mussten; ich begab mich auf dem Pausenhof in den Raucher ecken, obwohl ich kaum rauche; ich nahm den vollgepfropften Achtuhrbus, obwohl ich mit dem Fahrrad weniger lang gebraucht hätte. Ich machte mich auch erreichbar indem ich mich für das Internetprogramm MSN Messenger anmeldete mit einem erkennbaren Namen, um für Chats erreichbar zu sein. In diese Kategorie fällt auch das Austauschen von Mobiltelefonnummern, um per SMS kommunizieren zu können. Dieser Kanal erwies sich in der Folge als besonders wichtig.

Doch stellte sich für mich immer die Frage, ob es besonders sinnvoll ist, dorthin zu gehen, wo ich das Gefühl hatte, am meisten zu sehen. Dies waren oft Randstunden. In diesem Zusammenhang taucht die Frage auf, ob es nicht problematisch ist, sich auf solche Situationen zu konzentrieren, wo sozusagen der Schulalltag gegenüber einer Brückenoder Endzeit wie das Mittagessen oder die letzte Wochenstunde zurücktritt. Oder ist gerade dort das meiste zu beobachten? 
Als Feldforscher wollte ich meine Ressourcen möglichst effizient einsetzen, also in möglichst kurzer Zeit möglichst viel erfahren. Die wichtigste Ressource ist meine Anwesenheit. Dabei verhielt ich mich so, dass ich möglichst wenig wahrgenommen wurde, versuchte sozusagen mit der Umgebung zu veschmelzen, nahm, wie ein Schauspieler eine andere, unauffällige Persönlichkeit an. Dieser Wandel ist anstrengend, da er mit einer verstärkten Selbstkontrolle einhergeht.

Um die Ressource Anwesenheit möglichst effizient einzusetzen, habe ich immer, als ich das Gefühl hatte, vor einer Situation zu stehen, die besonders reich an Interpretierbarem war, ähnlich wie der Hahnenkampf, der von Clifford Geertz ${ }^{99}$ beschrieben worden ist, geistig ein Fenster aufgemacht mit dem Titel: der Soundso-Vorfall. Das Setting wie die Problemstellung dieser Arbeit haben mit denen von Geertz keine Gemeinsamkeit. Auch die von Geertz angenommene Grammatik von Kultur als verwobenen Handlungsabläufen kann jedenfalls in dieser Statik nicht direkt übernommen werden. Doch die Vorgehensweise, bestimmte Augenblicke oder Handlungssequenzen sozusagen in Zeitlupe vertieft zu interpretieren, erwies sich als nützliches Vorgehen.

Ein Beispiel soll diesen Sachverhalt verdeutlichen. Am Morgen des 5.12.2004 um 9.20h: Prüfung im Fach INU (Integrierter Naturkunde-Unterricht: Wissenschaftsfach mit Physik, Chemie und Biologie). Sämtliche elektronischen Geräte wie Mobiltelefone oder CD-Player sind im Unterricht - ob Prüfung oder nicht - generell verboten. Die Schülerinnen sitzen allein an je einem Tisch. Janine Maggiorini ${ }^{100}$ packt unter dem Tisch einen CD-Spieler aus und hört Musik. Frau König, die Lehrerin, schlendert zu ihr und konfisziert das Gerät. Kurz darauf ver-

99 Situationen, in denen viel zusammenkommt. Siehe Geertz, Clifford: Dichte Beschreibung, Beiträge zum Verstehen kultureller Systeme, 4. Auflage, Frankfurt a. Main, Suhrkamp, 1995.

100 Die Schülerinnen und Schüler werden in den Fussnoten mit Vor- und Nachnamen und Interviewstelle zitiert, im Text nach einer Weile ohne Nachnamen angeführt. In der Schule galt die Sie-Anrede und Vornamen. 
sucht Jürg Beller das gleiche. Auch sein Player wird sofort eingezogen. Nach der Stunde verteilt König Strafaufgaben an die Sünder und händigt die Geräte wieder aus. Die Schüler ziehen mit ärgerlichen Gesichtern ab. Ich frage mich und richte die Frage auch an die Lehrerin: Wie kann man nur so blöd sein, in einer Prüfung einen CD-Spieler auszupacken? Ist das eine Art Ritual? Frau König weiss es auch nicht. Als ich die Frage an Janine richte, sagt sie: "Beim Lehrer X. darf man das eben." Der Fehler der beiden Ertappten war also, dass sie die Strenge der Regelumsetzung bei Frau König zu niedrig eingeschätzt hatten.

Der Vorfall erlaubt fruchtbare Ausblicke auf Werte, Persönlichkeiten und Umgang mit Regeln. Er war jedoch im Sinne von Geertz nicht „dicht“, obwohl ich das zunächst glaubte, weil der Bezug zu übergeordneten, weiteren kulturellen Werten fehlte. Er warf jedoch die Frage auf, wie die Schülerrolle je nach Lehrer in verschiedenen Ausprägungen zu interpretieren ist. Dies wiederum stellt ein Training dar, um in Hierarchien zu funktionieren.

Bemerkenswert war auch, dass Schülerinnen und Lehrer begriffen, dass dem Ethnologen mit "dichten" Situtationen am meisten gedient war. Sie versuchten deshalb, mir zu Situationen zu verhelfen, die aus ihrer Sicht für mich informativ sein könnten oder wiesen auf diese Situationen hin. Frau Gisler verliess häufig kurz den Raum, um etwas zu holen oder zu kopieren, und man weiss ja: "Ist die Katze aus dem Haus, tanzen die Mäuse", wie ich erwiderte, als sie mich darauf ansprach, ob ihre kurzen Abwesenheiten mir nützen würden. Daniel Spreng, ein Schüler, empfahl mir, als ich ihn bitte, seine Bemerkung über den angeblich abnormalen Geisteszustand zweier Kameradinnen zu erläutern, in den Hauswirtschaftsunterricht zu kommen, "dann würde ich schon sehen", wie er andeutet. Oder Lehrer Schladerer meint, als ich mich über die Unruhe in seine Stunde wundere: "Für Sie muss das ja sehr interessant sein." 
Ich hatte immer ein sehr mulmiges Gefühl in diesen Situationen. Ich hatte den Verdacht, man wollte mir sozusagen etwas "bieten". Ich hätte dies durch Abwesenheit unterlaufen können, ging aber trotzdem hin; wenn man mich auf etwas hinweist, kann ich es nicht auslassen, da ich sonst nicht erfahre, weshalb man mich auf etwas hingewiesen hat und weshalb diese Person dies als wichtig erachtet. Meine Anwesenheit veränderte also das Setting insofern, als dass die Akteure meine Aufgabe mit bedachten. Dieses Problem liess sich kaum vermeiden, denn die Essenz der Teilnehmenden Beobachtung ist ja das Anwesendsein, das Dort- und Dabeisein, das Vor-Ort-Sein.

Diesen Sachverhalt diskutierte ich mit Margrit Ritter, der Sozialarbeiterin am Schulhaus, während einer Pause, in der ich sie bat, mir Gesellschaft zu leisten. Sie verwendet auch die Teilnehmende Beobachtung als Arbeitsmittel und weist darauf hin, dass die Schüler wissen, dass die Sozialarbeiterin nur in bestimmten Klassen Einsitz nimmt.

Frau Ritter denkt sich, die Schüler denken: "Sind wir besonders schlimm?" Ich entgegne, dass ich mehrmals über meine Tätigkeit aufgeklärt habe und es klar ist, dass die Klasse aus reinem Zufall ausgesucht wurde. Dann bleibt die Frage: "Sind wir besonders interessant?" und daraus resultiert ein bestimmtes Verhalten, z.B. dass man sich besonders produziert. ${ }^{101}$

Diese Verhaltensänderung lässt sich nicht vermeiden. Dennoch dürften die negativen Effekte auf das Datenmaterial relativ gering ausfallen. Die Auseinandersetzung mit den Methoden der Quellenerhebung führte dazu, dass dieses Problem von Anfang an einkalkuliert wurde. Ausserdem wirkten die Methodentriangulierung und die langfristige Anlage

$101 \mathrm{FB} 04,50$. 
des Projekts dem verzerrenden Effekt entgegen. Diese Schwierigkeit konnte ich also einkalkulieren. Von anderen methodischen Problemen blieb ich aber nicht verschont.

\subsubsection{Spezifik der Forschung mit Jugendlichen}

Ein ganz schwieriges Problem hat beinahe dazu geführt, dass ich das Forschungsprojekt nicht hätte durchführen können: Jugendliche möchten sich ganz einfach nicht erforschen lassen. Weder Schmidt-Lauber noch Kullberg berücksichtigen diese Prämisse, sondern nehmen offensichtlich an, dass die Beforschten kooperativ sind. Dies war in dieser Forschungsarbeit über lange Zeit überhaupt nicht der Fall. Wo sich Erhebungen machen liessen, blieben sie oft unüberprüfbar und lückenhaft.

Diese Nebenbedingung hat starke Auswirkungen auf die Wahl der Forschungsmethoden. Das Phänomen ist aus der Literatur bekannt: Der Zürcher Volkskundler Paul Hugger beispielsweise schreibt, dass die "bewährten volkskundlichen Erhebungsmethoden, wie Interviews ${ }^{102}$ oder teilnehmende Beobachtung, [bei Jugendlichen] nur bedingt und unter erschwerten Umständen zum Zuge kommen. (...) Denn (...) der Jugendliche ist als Interviewpartner schwierig anzugehen und an sich problematisch." 103 Mit der Problematik "an sich" meint Hugger vermutlich die Tendenz "des" Jugendlichen, grundsätzlich Informationen vor

102 „Ein Interview ist ein planmässiges wissenschaftliches Vorgehen, bei dem (...) Gesprächspartner durch Erzählstimuli oder gezielte Fragen zu verbalen Äusserungen veranlasst werden“". (Schmidt-Lauber, Brigitta: Das qualitative Interview oder: Die Kunst des Reden-Lassens, in: Göttsch, Silke und Albrecht Lehmann (Hg.): Methoden der Volkskunde. Positionen, Quellen, Arbeitsweisen der Europäischen Ethnologie, 2. Auflage, Berlin, Reimer, 2007, 169-188, 174).

103 Hugger, Pubertätsriten - einst und jetzt - aus der Sicht des Volkskundlers, 1991, 28-29. 
den Erwachsenen verborgen halten zu wollen. Die Jugendlichen entwickelten sogar eine grosse Kreativität im Vertuschen, Verschleiern und Auflaufen lassen. Dieser Sachverhalt unterminiert beispielsweise die bewährte Methode, die Interviewpartner reden zu lassen.

\subsection{Strukturierte und phänomenografische Interviews}

Schmidt-Lauber geht in ihrer Abhandlung über Interviewtechniken in der Europäischen Ethnologie anscheinend von der Voraussetzung aus, dass ein Gespräch überhaupt zustande kommt. Sie imaginiert eine hierarchische Interviewsituation, in welcher der Interviewer Macht über den Interviewten hat, etwa eine Kooperationsanweisung eines Vorgesetzten, oder ein Statusgefälle zwischen Akademiker und Interviewten. Sie empfiehlt für eine solche Situation zurecht, „das starre Frage-Antwort-Schemata im Gespräch durchbrochen und der Spielraum der Fragenden sowie v.a. der Interviewten“ gross werden zu lassen, damit „eine entspannte Erzählsituation“ entsteht, „die es dem Gesprächspartner ermöglicht, seine Erfahrungen und Vorstellungen in einer ihm angemessenen und vergleichsweise gewohnten Form zur Sprache zu bringen. ${ }^{“ 104}$ Mit dem Interviewten soll also eine ihm angemessen erscheinende Form der Erzählung gefunden werden, um die hierarchische Situation zu durchbrechen. Dies soll der zentralen Forderung qualitativer Erhebungen gerecht werden, eine Dialogizität zu erreichen. ${ }^{105}$

Diese Form des offenen oder narrativen Interviews ist in der Europäischen Ethnologie vor allem gebräuchlich im Zusammenhang mit lebensgeschichtlichen Fragestellungen und wird auch als biografisches Interview charakterisiert. Die andere sehr gebräuchliche Form ist das

104 Schmidt-Lauber, Das qualitative Interview oder: Die Kunst des Reden-Lassens, 2007, 175.

105 Ebd., 172. 
leitfadenorientierte Interview, das dann zur Geltung kommt, wenn ein bestimmter, eingegrenzter Katalog von Themen und Fragen abgearbeitet werden soll und/oder ein komplexes Thema vorab bekannt ist. ${ }^{106}$ Diese Form wurde in der vorliegenden Arbeit zu Beginn als Haupterhebungsmethode eingesetzt, weil sie als am ergiebigsten eingeschätzt wurde. Grundlage dafür ist ein Gesprächsleitfaden, in dem Fragen- und Themenvorgaben enthalten sind. Er kann sehr ausführlich oder knapp gehalten werden, bringt aber auf jeden Fall eine stärkere Strukturierung und Vereinheitlichung der Gespräche mit sich, was die Vergleichbarkeit gewährleistet. Dabei ist ein starres Fragen und Antworten zu vermeiden; vielmehr sollen die Fragen situationsangemessen ins Gespräch einfliessen. ${ }^{107}$ Ziel ist es, dass die Balance gewahrt wird zwischen Nähe, Aufbau einer ungezwungenen Gesprächsatmosphäre und vertrauensvoller Beziehung sowie gleichzeitig gewahrter Distanz durch das Erkenntnisinteresse und die analytische Durchdringung des Gesagten. ${ }^{108}$

Kullberg zieht eine Interviewtechnik vor, die der ethnografischen Arbeit mit Kindern und Jugendlichen besser angepasst ist. Sie bezieht sich dabei auf die Technik des phänomenografischen Interviews, das zum Ziel hat zu ergründen, wie die Interviewten ihr Verhalten organisieren. Diese Methode wurde seit den 1980er Jahren in der Bildungsforschung entwickelt und von Marianne Hansson Scherman auf Asthmaund Allergiepatienten angewandt, um zu erfahren, welche Ansichten einer rationalen Durchdringung des medizinischen Problems entgegen stehen, was die Heilung erschweren kann. ${ }^{109}$ Patienten stehen wie Ju-

106 Ebd., 175. Für eine eingehende Diskussion der Terminologie und Varianten der Interviewformen siehe ebd., 174-175.

107 Eine ausführlichere Diskussion der verschiedenen Teilaspekte von Interviewführung findet sich ebd., 178-180.

108 Ebd., 180.

109 Hansson Scherman, Marianne.: Att vägra vara sjuk. Göteborg, Acta Universitatus Gothoburgensis, 1994, zitiert nach Kullberg, Etnografi i klassrummet, 1996, 89-90. 
gendliche in einer Situation, wo beunruhigende körperliche Prozesse ablaufen, die Gefühle wie Scham auslösen können, und haben deshalb kein Interesse an Forschern, die mit Interviews ihr Innenleben erforschen wollen.

Mit dieser Methode versuchen Interviewer, die Gedankenwelt des Interviewten zu erfassen, was ein intensives Zuhören erfordert, aber auch provozierende und vertiefende Fragen notwendig macht. Der Interviewer sucht dabei nach einem nahezu zufälligen Anwesendsein. Das oberflächliche Floskelniveau des Gesprächs soll jedoch durchbrochen werden, um die Umweltwahrnehmung des Interviewten zu ergründen. Dabei ist der Forscher ähnlich wie in der teilnehmenden Beobachtung das Instrument des Interviews und hat sich gegenüber den Interviewten informell aber distanziert zu verhalten. Die Interviewten werden ein ganz bestimmtes Verhalten einnehmen. Hieraus kann unter anderem bestimmt werden, was der Forscher aus Sicht der Erforschten im Verlauf des Forschungsprozesses erfahren und wissen darf und was nicht. Diese Veränderungen im Umgang sind wichtige Informationen. Ein solches Vorgehen, betont Kullberg, ist anspruchsvoll und fordert viel Übung und Vorbereitung. Im Hinblick auf das Setting des Interviews fordert Kullberg die Wichtigkeit einer neutralen Umgebung, wo beide Personen allein anwesend sind, aber das Milieu das gleiche wie das des Forschungsfeldes ist. Falls nicht anders möglich, soll der Interviewer die zu interviewende Person aufsuchen. ${ }^{110}$

Dies ist für die Arbeit mit Jugendlichen angepasster als der Vorschlag von Schmidt-Lauber, die fordert, das Interview bei den Beforschten zu Hause durchzuführen, um die Einrichtung der Wohnung als Information zu erschliessen. ${ }^{111}$ Diese Information wäre an sich interessant, doch wohnen Jugendliche oft noch bei der Familie, was zu

110 Kullberg, Etnografi i klassrummet, 1996, 88-91.

111 Schmidt-Lauber, Das qualitative Interview oder: Die Kunst des Reden-Lassens, 2007, 178. 
Ablenkungen oder Komplikationen im Interview führen kann. Die Bedingungen der Arbeit mit Jugendlichen macht es auch notwendig, nach jedem Interview zu analysieren, wie die Interviewsituation verbessert werden kann. ${ }^{112}$ Deshalb wurden im Verlaufe der Arbeit solche Interviewformen und informelle Gespräche immer wichtiger.

Um die geforderte Dialogizität zu erreichen, also eine unhierarchische Produktion von Interviewtexten, in welchen sich die Interviewten selber erkennen können, mussten die Interviews für diese Arbeit lange vorbereitet werden. Für mich fiel zwar das Problem weitgehend weg, wie ich meine Interviewpartner auswählen sollte, da sie durch die administrative Einheit Schulklasse gegeben waren ${ }^{113}$, doch fühlte ich mich zu Beginn sehr fremd in der Schulklasse und die Schülerinnen und Schüler ignorierten mich weitgehend. Ich war entmutigt, doch ich spielte auf Zeit; ich wusste, dass ich vergleichsweise lange anwesend sein würde und übte mich in Geduld. Ab und zu gab ich auch einen Input, um die Schülerinnen und Schüler herauszufordern, mich zur Kenntnis zu nehmen, Informationen zu teilen. Zunächst veranstaltete ich Gruppengespräche, in der Meinung, dass die Jugendlichen in der Gruppe viel eher bereit sein würden, zu reden und dass ich sie näher kennenlernen würde. Das erste erwies sich als falsch, das zweite als richtig. Auch hat die Klassenlehrerin, Isabelle Gisler, dazu geraten, die Gespräche während der Schulstunden abzuhalten; wenn ich sie während der Freizeit der Schüler/innen abhalten würde, würde ich die jungen Leute verärgern, glaubte sie. Die Jugendlichen würden dann erst recht nichts preis geben. Für Gespräche eigneten sich die beiden Stunden am Mittwochnachmittag am besten, Laufbahnberatung und Deutsch, empfahl mir Frau Gisler.

112 Kullberg, Etnografi i klassrummet, 1996, 89-91.

$113 \mathrm{Zu}$ diesem Thema vergleiche Schmidt-Lauber, Das qualitative Interview oder: Die Kunst des Reden-Lassens, 2007, 173-174. 
Drei bis vier Monate vor dem Ende der regulären Schulzeit begann ich mit strukturierten Einzelinterviews ${ }^{114}$. Auf der Grundlage eines Fragesystems von Gisela Unterweger ${ }^{115}$ entwarf ich eine Liste von Fragen, die zunächst erzählerische ${ }^{116}$, aber auch aufzählende Antworten ${ }^{117}$ provozieren sollten. Daneben wollte ich auch Fakten eruieren ${ }^{118}$ und stellte Nonsense-Fragen ${ }^{119}$, die verblüffen und auflockern sollten. Diese Mischung sollte weniger ermüden und zum Nachdenken anregen. ${ }^{120}$

Dennoch traf ich auf einige Schwierigkeiten bei meinen Gesprächen. Ich spürte eine diffuse Unwilligkeit, deshalb wählte ich für meine $\mathrm{Ge}$ spräche einen sanften Einstieg: Ich erklärte zunächst, dass alles gänzlich anonym sein werde und die Interviewprotokolle mein Büro niemals verlassen würden. Dann begann ich das eigentliche Interview mit den Fragen zur Person, fragte dann nach der Familie und arbeitete mich erst zum Schluss zu den für die Fragestellung wirklich wesentlichen Fragen vor.

Dennoch gaben einige Interviewte ihren Widerstand nie ganz auf und erschwerten mir mit verschiedenen Strategien die Arbeit. Widerwillig antworten war eine; eine andere bestand darin, mich zu reizen. Eine bestimmte Schülerin konnte das ganz gut. Sie war überhaupt schwer zugänglich, ich konnte sie nur einmal interviewen. Es wurde ein unerfreuliches Interview, weil ich ihr immer die sprichwörtlichen Würmer aus der Nase ziehen musste und sie mit Banalitäten oder mit platten Stammtischsprüchen auf meine Fragen antwortete.

114 Vgl. zu diesem Thema ebd., 177.

115 Da unser Projekt auf der Arbeit von Gisela Unterweger aufbaute, erschien es mir als sinnvoll, thematisch ähnlich vorzugehen. Unterweger, Klasse und Kultur, 2002, 214-217.

116 Z.B.: Beschreibe einen typischen Mittwochabend.

117 Z.B.: Nenne deine vier besten Schulkollegen (Schulfreunde).

118 Z.B.: Wer bestimmt, wie ein Klassenzimmer eingerichtet wird?

119 Z.B.:Weshalb sitzen alle Mädchen am Fenster?

$120 \mathrm{Vgl}$. Fragebogen im Anhang. 
Diese Aussagen haben mich etwas aus der Fassung gebracht, da die Schülerin mich so lange hat auflaufen lassen und dann plötzlich auf meine erwachsene Ebene wechselte und dort proletenhafte Dummheiten von sich gab. Darauf hin geriet ich ins Schlingern und beging einige Interviewfehler, schob etwa auf unbeantwortete Fragen weiter Fragen nach, anstatt zu warten. Es war aufschlussreich zu sehen, wie diese junge Frau zwar dem Befehl, an einem Interview zu erscheinen, nachkam, mich dann aber mit wertlosen Informationen fütterte. So hat sie mein Ansinnen, Einblick in ihr Leben und Denken zu gewinnen, erfolgreich unterwandert. Diese Taktik war anscheinend generell erfolgreich: Frau Gisler hat mir später gesagt, dass sie über diese Schülerin praktisch nichts wisse.

Zuviel Information kann auch Probleme bereiten und den Interviewer von den wirklich wichtigen Fragen ablenken: In einer grossen Pause redet ein Schüler über Minigolf. Er berichtet über Bälle, Balltypen, Bahnen, Materialien, Strategien, usw. Er ist recht gut, zu jener Zeit Platz 22 der Schweizer Minigolf-Bestenliste. Meine Gegenstrategie war hier, das Gespräch auf dieser Sachebene zu belassen, also dieses typische Sportfachgespräch weiterzuführen, um dann von dort auf Persönliches zu sprechen zu kommen.

Eine andere Art von Eloquenz stellte ich bei einem Schüler fest, die ich als Therapiegewandtheit bezeichnen würde. Er ist während seines Lebens immer wieder mit den Autoritäten in Konflikte geraten und stand am Rande des Schulausschlusses. An ihm fiel auf, dass er sehr gewandt über sich selbst reden konnte. Sein erster Satz war: "Ich bin Stefan Staub und wohne in der Aussenwohngruppe des Waisenhauses." Die Aussenwohngruppe des Waisenhauses ist eine betreute Wohnmöglichkeit für Jugendliche, die mit dem Gesetz in Konflikt geraten sind und kein Zuhause haben. Er war straffällig geworden, konnte aber alle Schwierigkeiten auf irgendwelche Zufälle und Umstände zurückführen 
und redete dann von sich wie von einem Fremden. Man hatte den Eindruck, dass er alle Antworten schon mal gegeben hatte.

Jedenfalls merkte ich sehr bald, dass die Schüler grundsätzlich wenig bereit waren, viel über ihr Leben im Gespräch preiszugeben. Daher war es für mich schwierig, überhaupt aussagekräftige Daten zu erheben. Auch hatte mich die Lehrerin auf eine gewisse Forschungsmüdigkeit der Schülerinnen und Schüler hingewiesen, die anscheinend schon oft befragt worden waren und Fragebogen ausfüllen mussten, was sie inzwischen angeblich verabscheuten. Ich empfand es als etwas frustrierend, dass manche Gespräche wie ich den Eindruck hatte, nie aus der Aufwärmphase herauskamen. Ich tröstete mich dann damit, dass weitere Interviews folgen würden, in denen ich mehr erfahren würde. Dies war zum Teil tatsächlich auch der Fall. Dennoch blieb dieses Problem die grösste methodische Schwierigkeit in diesem Forschungsvorhaben.

Schwierig war es auch, Unterschiede in der Bildung zu überbrücken. Beispielsweise war das Vorhaben, Bedeutungskonstruktionen abzufragen, die als Normalität angesehen wurden und welche zu Integration oder Ausgrenzung führten, nicht einfach zu erklären. Dieses Konzept konnte ich 15jährigen Jugendlichen schwer erläutern und musste es auf ihre Bildungsebene übersetzen. Auch gab es keine auskunftsfreudigen Gewährsleute wie bei anderen Untersuchungen über soziale Gruppen, die mit Stolz über Interna berichten würden.

Um so grösser war meine Überraschung, als ich die Jugendlichen im neuen Schuljahr 2004 an verschiedenen Orten wieder traf: Die meisten freuten sich, mich wieder zu sehen, was bei mir ebenfalls Freude auslöste, nachdem ich zuvor oft Ablehnung verspürt hatte. Aus dieser Zeit stammen die tiefsten Einblicke, die ich in das Leben der untersuchten Gruppe nehmen konnte - nach der eigentlich dafür vorgesehenen Forschungszeit an der Schule. Offensichtlich war es erst möglich, über die Klasse 2D offen zu sprechen, als sie nicht mehr existierte. 
Die Interviews ab dem Herbst $2005^{121}$ fanden in einem informellen Rahmen statt, den sich die Betreffenden aussuchen konnten. Wieder waren es strukturierte Gespräche, wobei der Leitfaden auf die Spezifiken der betreffenden Person angewendet wurde. Das Gespräch begann ich mit der Erkundigung, wie es der betreffenden Person heute gehe, ob sie sich Sorgen um ihre Zukunft mache, was ihre Pläne seien. Dann gingen wir uns bekannte Leute aus der Klasse und ausserhalb durch, ein Gesprächsmodus, der aufforderte, über die Personen zu klatschen, und damit auch brisante Informationen preiszugeben. Dabei flocht ich Fragen ein, die ich mir anhand meiner Unterlagen im voraus notierte hatte, wo es im Sachverhalte ging, die ich mit der betreffenden Person abklären wollte, etwa, was in einer bestimmten Situation abgelaufen war oder was geschah, nachdem ich eine bestimmte Situation verlassen hatte. Da die Jugendlichen nach dem Auseinandergehen der Klasse 2D kaum mehr Kontakt zueinander hielten, waren sie eher bereit, "aus dem Nähkästchen" zu plaudern und ich erhielt Aufklärung über manch eine mir unklar gebliebene Begebenheit aus dem Vorjahr.

\subsection{Quellenproduktion: Aufsätze, Objekte, Schriftliches}

Eine weitere Art, Informationen zu bekommen, war, die Schüler einen Aufsatz schreiben zu lassen. Ich liess zum Thema "Ein Tag in meinem Leben: Der 4. Mai 2024" schreiben. Meine Idee war, "key scenarios" beschreiben zu lassen. Der Begriff geht auf Sherry Ortner ${ }^{122} \mathrm{zu}-$ rück, die damit ein Bild des Erfolgs oder des guten Lebens bezeichnet. Jede Kultur hat eine Vorstellung davon, wie dieser Erfolg definiert wird, und welche Mittel am Besten sind, dieses Ideal zu erreichen.

$121 \mathrm{Vgl}$. Anhang II, 8.

122 Sherry Ortner: On key symbols, American Anthropologist 75(5), 1973, 1338-1346. 
"Schlüsselszenarien" ist ihre Bezeichnung für diese Kategorie von Symbolen. Diese stellen Handlungsstrategien und klare und eindeutige Handlungsarten dar, die je nach Kultur vorgeschlagen werden für das Erreichen eines glücklichen Lebenswandels, also eine Mittel-Ziel-Relation. Eine sehr bekannte Handlungsanweisung ist "Vom-Tellerwäscherzum-Millionär", die für die US-amerikanische Kultur massgebend ist. Jede Kultur besitzt solche Schlüsselszenarien. Sie zeigen sowohl passende Ziele auf und schlagen passende Massnahmen vor, diese zu erreichen. Mit anderen Worten: Szenarien sind Schlüsselstrategien einer Kultur. ${ }^{123}$

Diese methodische Überlegung erwies sich als richtig, doch gab es Schwierigkeiten in der praktischen Umsetzung. Nachdem ich mein Anliegen in der Deutschstunde erklärt hatte, brach allgemeine Ratlosigkeit aus. Eine Schülerin meldete sich daraufhin und fragte, wie lange der Aufsatz werden solle. Als ich etwas verunsichert erwiderte, dass er mindestens eine Seite lang sein solle, gerne aber länger, waren alle beruhigt und begannen zu schreiben. Doch hatte ich nicht damit gerechnet, dass die Schülerinnen und Schüler die Anweisung, eine Seite zu schreiben, so wörtlich nehmen würden, dass fast alle ihren Text in der unteren rechten Ecke des Blattes abbrechen lassen würden.

Weiter Informationen residieren in den von mir gesammelten Gegenständen. Von den jungen Menschen selbst erhielt ich nicht sehr viel, was mich angesichts knapper Taschengeldbudgets nicht wunderte. Drei Mal wurden mir Süssigkeiten angeboten, die ich nahm und deren Geschmack mich sehr verblüffte. Zusammen mit den Befunden über die Ernährung der Jugendlichen gab mir das einen Einblick über die jugendliche Esskultur und Geschmackswelt, die stark von derjenigen der Erwachsenen differiert. Beim Ausräumen des Klassenzimmers in der letzten Woche des Schuljahres gelangte ich in den Besitz dreier Plakate. Zwei stellen ein Arrangement von Fotos aus den Ski- und Sommerla123 Ebd., 1338ff. 
gern des Jahres 2003 mit den zweiten WBS-Klassen dar, eines ist ein Themenplakat über Eisbären und weitere zwei stellen je drei Fussballer des FCB (Fussball Club Basel) dar. Eine aussagekräftige Quelle ist in einem A4-Blatt zu sehen, das anscheinend zwischen einer Schülerin und einer Schulkameradin in der 2D hin und her geworfen wurde. Das Blatt ist übersät von Schmähungen, die einer dritten Schülerin galten. Dieses Blatt war überhaupt das einzige schriftliche Dokument eines Ausschlussprozesses.

\subsection{Experteninterviews}

Neben diesen Interviews mit den Schülerinnen und Schülern führte ich auch drei Experteninterviews zum Thema Integrations- und Ausschlussprozesse durch. Das erste führte ich mit Sandra Müller, einer Handballtrainerin, über Integration in Mannschaften und über die Rolle des Sports für die Integration von Jugendlichen. Ich sprach auch mit Beatrice Ledergerber, die zu jener Zeit ein als erfolgreich beurteiltes Mentoring-Projekt leitete. In diesem wurde Jugendlichen eine Mentorin oder einen Mentor zugeteilt, die helfen sollten, eine Lehrstelle zu finden. Mir ging es um die in der Integrationsbegrifflichkeit enthaltenen Idee, dass Integration durch Helfer vorangetrieben werden kann. attraktiv ist die Tatsache, dass in den letzten Jahren sehr viele Sozialprojekte entstanden sind, eine regelrechte Integrationsindustrie, die professionelle Helferinnen zur Integration von Jugendlichen einsetzen. Zuletzt interviewte ich Stefan Blülle, Leiter der Jugendstaatsanwaltschaft BaselStadt zum Thema Jugendliche, Delinquenz und Integration.

Für diese Forschungsarbeit mussten die Methoden, welche die Europäische Ethnologie bereitstellt, für die Arbeit an Schulen und mit Kindern und Jugendlichen modifiziert werden. Gründe dafür sind die grosse kulturelle Andersartigkeit der Jugendlichen und die damit verbunde- 
nen kommunikativen Einschränkungen und Bedingungen. So gesehen lässt sich die Forschungsarbeit mit der Erfoschung fremder Kulturen vergleichen und nicht mit der Arbeit in der eigenen Kultur, die ein Stück weit kennzeichnend ist für Methodik und Interpretation innerhalb der Europäischen Ethnologie. Auffällig war auch die lange Zeitdauer, die es brauchte, Zutritt zum Feld zu erhalten und eine tragfähige Arbeitsbeziehung mit den Jugendlichen aufzubauen.

\subsection{Integration des Forschers in das Feld}

Von Anfang an war mir klar, dass das Erlangen von aussagekräftigen Informationen nur möglich war, wenn es mir gelang, von den Jugendlichen ein Stück weit akzeptiert zu werden. Diese Akzeptanz war eine Grundbedingung der ,lebensweltlichen Ethnologie“, der emischen Perspektive, die ich anstrebte. Diese war aber nur durch eine Annäherung an die Jugendlichen, einen Integrationsprozess ins Feld, zu erreichen. Dieser Prozess war also entscheidend für den Erfolg meiner Arbeit, und dessen Ausgang war überhaupt nicht vorauszusagen.

Am 12. September 2003 trat ich meinen ersten Feldforschungstag an, nicht ohne Nervosität. Die Schule ist eine Arena, in der die Eltern auf den Zuschauerrängen sitzen, wo es Mitspieler, Helden, Versager, Schiedsrichter, Trainer und Beobachter gibt. Ich würde also genau so beobachtet werden wie ich beobachten würde. Was hatte ich ausserdem nicht alles in den Medien über schlimme Jugendliche gelesen. Gewappnet mit den Überlegungen von Rolf Lindner über verschiedene Rollenerwartungen, setzte ich mich ins Klassenzimmer. ${ }^{124}$ Frau Gisler erklärte im Deutschunterricht, was in dieser Stunde für die Schule gemacht werden musste und dann kam sie auf mich zu sprechen: "Das ist

124 Lindner, Die Angst des Forschers vor dem Feld, 51-66. 
Herr Schaffner, der macht eine Forschung über euch", erklärte sie, was Stirnrunzeln auslöste. Ich erhielt zum zweiten Mal, nachdem ich mich im Sommer vorgestellt hatte, Gelegenheit, mein Projekt und meine Pläne zu erläutern, doch scheinen diese Ausführungen mehr Fragen aufgeworfen als Antworten geliefert zu haben. Dies glaubte ich anhand der ratlosen Gesichter festzustellen. Ein Phänomen, das für Europäische Ethnologen nicht unbekannt ist, scheinen doch Menschen, die das erste Mal mit einem solchen Forscher zu tun bekommen, Schwierigkeiten zu haben, zu verstehen, was der eigentlich will. Dies wusste ich und war mir deshalb bewusst, dass ich meine Rolle sehr bald ausagieren musste, um zu demonstrieren, wer ich bin.

Ich bin ganz offensichtlich kein Lehrer, aber was dann? Ich dringe in den Alltag einer Schulklasse ein, und die betroffenen Menschen - Schülerinnen und Schüler, Lehrer, Schulhauspersonal - müssen mit mir eine gewisse Zeit lang auskommen. Zunächst wissen sie nicht, mit wem sie es zu tun haben. Die Rolle des Ethnologen ist speziell, und die Beforschten müssen in einem Prozess die Regeln und Formen dieser Beziehung erlernen, da sie meistens das erste Mal mit so jemandem konfrontiert werden.

Dieses Eindringen in einen fremden Alltag und diese besondere Sozialbeziehung erfordern eine bestimmte Haltung des Feldforschers. Betritt er das Forschungsfeld, ist er mit einem Problem konfrontiert: Er interessiert sich für Leute, aber nicht in erster Linie um der Leute willen, sondern, um etwas über die Kultur dieser Leute zu erfahren. Deshalb ist er bestrebt, eine stabile Beziehung zu den Beforschten aufzubauen. Für die Jugendlichen wirkt das befremdend, löst Misstrauen aus. Was will dieser Erwachsene wirklich von mir? lautete die Frage, die oft nicht ausgesprochen wurde. Dieser Zugang löst zwischenmenschliche Prozesse aus, die eine Eigendynamik entwickeln, die für die Forschungsergebnisse nicht ignoriert werden dürfen. 
Ich sass meistens im hinteren Teil des Schulzimmers, und ab und zu äugte jemand zu mir, um abzuschätzen, ob ich eine Bedrohung sei. Vermutlich wurde ich wie die Sozialarbeiterin als ein irgendwie Prüfender oder Überwachender eingeordnet. Die damit verbundenen Bedenken zerstreute ich vermutlich, als die Lehrerin einmal die Klasse verliess. Ein Schüler probierte daraufhin einige obszöne Gesten gegen einen anderen aus, und ich habe nicht reagiert, auch nichts notiert. Damit war meine Ungefährlichkeit bewiesen und es war unklar, welche Informationen ich sammelte.

Aber war ich gefährlich für die Lehrer? Als ich mit nein antwortete, als ein Schüler mich fragte, ob ich die Macht hätte, die Lehrer zu feuern, war auch das klargestellt. ${ }^{125}$ Zudem achtete ich darauf, in Konfliktsituationen, in denen ich in der Klasse als Schiedsrichter angerufen wurde, da ich aus Sicht der Akteure irgendwie Protokoll führte, neutral zu bleiben und mich auf meine Schweigepflicht zu berufen. Die Schweigepflicht war eine entscheidende Voraussetzung, mit der Zeit das Vertrauen einzelner Schülerinnen und Schüler zu gewinnen. Auch die Jugendlichen beobachteten meine Arbeit und wussten, dass ich mit der Zeit alle Beteiligten bis hinauf in die Schulhierarchie interviewen würde. Dieses Wissen entfachte einige Neugier und ich musste aufpassen, welche Teile ich davon bekannt gab, um weitere Forschungsmöglichkeiten zu erschliessen. z.B. durch Bezugnahme auf Bemerkungen von Interviewten, und welche Teile ich für mich behielt. Auch im Sozialleben der Jugendlichen ausserhalb der Schule wurde manchmal ein Bezug zu Aussagen hergestellt, die ich über Themen oder Leute gemacht habe, oft ohne die Konsequenzen meiner Aussagen sogleich zu erfassen.

Es entstand mit der Zeit das, was Sven Sauter in seiner Abhandlung über die Mentorenrolle ${ }^{126}$ des Forschers als Arbeitsbündnis bezeichnet. In einer Untersuchung über adoleszente Ablösungsprozesse von Ju125 Ebd., 49. 
gendlichen aus Immigrantenfamilien reflektierte Sauter seine Rolle als Forscher. ${ }^{127}$ Das Arbeitsbündnis charakterisierte er als eine der grössten Schwierigkeiten empirischen Forschens und bezeichnete es als "stabiles Setting ... das tragfähig genug ist, um den Forschungsprozess über einen verlässlichen Zeitraum aufrecht zu erhalten" und forderte zu Recht, dass die Reflexion über das Arbeitsbündnis für die Wissensproduktion fruchtbar gemacht werde.

Man kann Arbeitsbündnisse danach ordnen, wie breit und allgemein sie gelten. Das konkrete Arbeitsbündnis lässt (sich) auf allen diesen Ebenen gleichzeitig beschreiben. In der Analyse des Arbeitsbündnisses werden sie wie in einer Spirale auf- und abwärts durchlaufen. Kulturelle, institutionelle, situative, interpersonelle und idiosynkratische Aspekte der Arbeitsbündnisse konstituieren die Bandbreite möglicher herstellbarer Beziehungen und die daraus resultierenden Ergebnisse. Genaue Analyse dieser Aspekte geben einerseits Aufschluss über hegemoniale Wissensbestände und Verhaltensregeln (...) und verlangen andererseits einen selbstreflexiven Umgang mit subjektiven Empfindlichkeiten und ihrem Einfluss auf die Interaktion zwischen Befragten und Forscher/inn/en. ${ }^{128}$

126 Mentor war in der griechischen Mythologie die Göttin Athene, die sich in einen väterlichen Freund und Ratgeber von Telemachos verwandelte, während dessen Vater Odysseus unterwegs war (Schwab, Gustav: Sagen des klassischen Altertums, Wien Heidelberg, Überreuther, 1974, 418 ff). Sauter, Sven: Der Forscher als Mentor, in: Ethnopsychoanalyse 6. Forschen, erzählen und reflektieren, Frankfurt a. M., 2001, 70-93.

127 Sauter, Sven: Wir sind Frankfurter Türken. Adoleszente Ablösungsprozesse in der deutschen Einwanderungsgesellschaft. Frankfurt a. Main 2000. 128 Zitiert nach ebd., 73. 
In seiner eigenen Feldforschung war Sauter gezwungen, wegen der Schwierigkeit, mit den Untersuchten Kontakt zu halten, ihnen etwas anzubieten: Mithilfe bei den Hausaufgaben, Berufsberatung, Suche nach einem Praktikumsplatz oder nach grösseren Räumen für die Gruppe. ${ }^{129}$

Auch ich fühlte mich unbewusst veranlasst, irgendetwas zurückzugeben, für das Privileg, Informationen sammeln zu dürfen. Mir war klar, dass eine asymetrische Beziehung bestand, bei der ich als Bittsteller auftrat und nur Erfolg haben würde, wenn mir Einblicke gewährt würden. Zunächst machte ich Geschenke: Süssgetränke, Süssigkeiten, etwas zu Essen, Absenz von einer Schulstunde. So konnte ich mit der Zeit doch gewisse Beziehungen aufbauen, auf denen die Informationsbeschaffung fusste. Ich war gespannt, wie sich die Beziehungen weiterentwickeln würden, denn ich war mir bewusst, dass ich von den Jugendlichen nicht als einer der Ihren angesehen und daher immer von vielen Situationen und Informationen ausgeschlossen bleiben würde. Dennoch erwiesen die Beziehungen sich als erstaunlich stabil und über längere Zeiträume haltbar.

Nach einiger Zeit war auch für die Schülerinnen und Schüler klar, dass dieser Mann mittleren Alters tatsächlich nicht einfach nur für eine Schulstunde anwesend gewesen war, sondern dass er seine Ankündigung wahr machen würde, dass er die Klasse über längere Zeit beobachten würde. In der Folge wurde ich verschiedenen "Tests" ausgesetzt, die meine Lust, anwesend zu sein, auf die Probe stellen sollten. Ich versuchte deshalb, geduldig dem Image entgegenzuarbeiten, das ein Mittvierziger gegenüber Jugendlichen haben musste. Ich vermied jede Symbolik und Insignien der Macht, etwa in meiner Kleidung, meinem Habitus, meiner Sprache und Ausrüstung. Ich zog mich etwa so an, wie ich mich im Arbeitsbereich anziehen würde, bequeme, lockere Kleidung, bemühte mich aber um einen eigenen "Style", der stark von meiner Bekleidung im vorherigen Wohnort Schweden bestimmt war und sich von 129 Ebd., 76-77. 
der Kleidung hierzulande unterschied. Mit dieser wollte ich vermeiden, dass man mich mit Schweizerischen Stilkriterien in eine Hierarchie einordnen konnte. Ich mied schulische Autoritätspersonen. Ich hielt mich an Orten auf, wo sich die Jugendlichen aufhielten. Ich unterwarf mich den üblichen Beschwichtigungsritualen, falls ich einmal zu spät kam. Ich versuchte, nicht belehrend oder altklug zu wirken und hielt deshalb meist den Mund. Es war mir bewusst, dass das Machtgefälle Erwachsener-Jugendlicher nicht einfach verschwindet, und dass jugendliche Abwehr das grösste Hindernis meines Unterfangens darstellen würde.

Den ersten Test erlebte ich bereits in der ersten Schulstunde: Einige Zeit nach meinen Erläuterungen über das Forschungsprojekt war die Stunde vorbei. Alle Jugendlichen drängen aus dem Zimmer, wobei ich, mit den Strömungsverhältnissen bei der Türe unvertraut, ins Abseits gedrängt wurde. Ich musste mich von diesem Moment an entscheiden, ob ich vor der Klasse oder nach der Klasse in ein Zimmer gehen wollte. Dieses Gedrängte der Raumnutzung von Jugend in Gruppen war mir zu dieser Zeit noch unvertraut und ich erfuhr gerade körperlich den ersten Ausgrenzungsprozess.

Gleichentags ging ich nach einer Pause, die ich mit Frau Gisler im Lehrerzimmer verbracht hatte, mit ihr zügig los zu einem Nebengebäude, wo Hauswirtschaftsunterricht stattfinden sollte. Plötzlich klatschte es, und Frau Gisler wies zwei Schüler zurecht, die aus dem dritten Stock schauten. Erst jetzt bemerkte ich drei grosse Spuckeflecken auf dem Boden. Gisler schimpfte und eilte die Treppen hoch zum Schulzimmer, aus dem die beiden Jungen gespuckt hatten, um ihnen Arrest zu erteilen. Ich war froh, nicht getroffen worden zu sein und machte mir Gedanken über die Risiken meines Vorhabens.

Bei einer weiteren Prüfung versage ich: Im Hauswirtschaftsunterricht wurden zwei Kochgruppen gebildet, eine Mädchen- und eine Jungengruppe. Aufgabe: Eine Spaghettisauce kochen. Beim Essen wurde es peinlich: Eine Schülerin hatte die Mädchen-Spaghetti-Sauce mit 
Aromat ${ }^{130}$ zugewürzt. Alle fanden sie eklig. Eine andere Schülerin fragte mich, ob ich sie gut finde. Alle schauten mich an und ich log: "Sehr gut!" Im selben Moment war es mir peinlich; ich war ganz offensichtlich der Situation nicht gewachsen. Die erste Schülerin hatte mich etwas überrumpelt, da sie in einer früheren Hauswirtschaftsstunde als einzige Töpfe und Teller vor dem Essen laufend abgewaschen hat. Sie hat offensichtlich Ahnung, wie eine Küche effizient geführt wird. Deshalb interpretierte ich ihre Aromatüberdosis als Zumutung oder als Test. Die Jungen dagegen hatten eine deutlich bessere Sauce gekocht.

Oder ein weiterer Test, verbunden mit einem Ausschlussversuch: Ich frage in einer Pause ein Mädchen nach ihrem Namen. Es tritt nahe an mich heran, bringt ihr Gesicht in $10 \mathrm{~cm}$ Abstand zu meines und schnauzt mir zu: "Lore, von Loredana, heschs tscheggt?!" 131 "Ja," nicke ich und behalte den Namen von da an tatsächlich im Gedächtnis - obwohl ich sehr schlecht mit Namen bin. ${ }^{132}$

Diese Eingangstests oder kleinen Prüfungen hielten die Schülerinnen und Schüler auch in der 10B des Brückenangebots Basis für mich bereit. Dort setzte ich meine Studien nach der WBS fort. Da ich von den drei mir bekannten Jugendlichen in der Art begrüsst wurde, wie sich gut bekannte Jugendliche untereinander begrüssen, war die Integration in die Schulklasse für mich einfacher, weil mit dem Gruss gezeigt wurde, dass man mich wohlwollend behandeln sollte und ich keine Gefahr darstellte. Dennoch prüften mich die anderen, mit mir noch unvertrauten Schülerinnen und Schüler. Ein Beispiel vom 5. Januar 2005: Ich komme als letzter in die Klasse, aber noch rechtzeitig. Es ist kein Stuhl mehr frei. Ich nehme mir den Hocker vom Computertisch-

130 Schweizer Streuwürze.

131 FB 04, 34. Tscheggen: merken, schnallen, von to check (engl.). Die Schülerinnen und Schüler werden im Hauptteil näher vorgestellt.

132 Alle Beispiele aus FB04, 20. 
chen und setze mich ans Ende der Ersten Reihe. Das Mädchen neben mir sagt laut und deutlich: "Scheisse!"133

Die Sache mit der Spucke ging nicht mich in erster Linie an, dennoch zeigte es mir, dass alle Erwachsenen auf dem Schulhof sozusagen gefährlich leben und immer wieder Opfer jugendlichen Übermuts werden konnten. Die spuckenden Schüler mussten dann in den Arrest. Die beiden anderen Vorkommnisse interpretierte ich als Versuche, mich an einen Platz zu verweisen oder Hürden für mich aufzurichten. Da ist einer, der will was wissen, aber wir Schülerinnen und Schüler sind nicht von vorneherein bereit, ihm Informationen zu geben. Wer ist das überhaupt? Ist er gefährlich oder harmlos? Die Macht eines Lehrers besitzt er anscheinend nicht, und dann liegt es nahe, ihn wie einen neu hinzugekommenen Schüler zu behandeln und ihn Prüfungen auf seinem Integrationsweg bestehen zu lassen. Immerhin lernte ich so den Integrationsverlauf von neuen Schülern kennen. Die Integration des Forschers und möglicherweise auch neuer Schüler geschieht in vier Stufen.

In ihrer Untersuchung über Strassenjugendliche in einer niedrigschwelligen Anlaufstelle im Hauptbahnhof einer norddeutschen Grosstadt unterscheidet Ute Marie Metje vier Phasen der Integration in die Feldsituation. ${ }^{134}$ Die erste Phase ist die des ersten Kontakts, der Unsicherheit nach der Ankunft. ${ }^{135}$ Diese Phase dauerte für mich rund einen Monat: Ankommen, sich einrichten, sich mit dem Setting vertraut machen, sich auf dem Gelände zurechtfinden, wichtige Personen kennenlernen. Aus der Sicht der Schülerinnen war klargeworden, dass ich keine Bedrohung war und auch keinen Einfluss auf den Unterricht nehmen würde. Ich war auch nicht ein unkontrollierbarer Informationsverbrei-

133 FB05, 1.

134 Metje, Ute Marie: "Hier haben ja alle Löffel Löcher" Forschung im eigenen Land: Eine ethnologische Untersuchung über Strassenkids, in: Ethnopsychoanalyse 6 . Forschen, erzählen und reflektieren, Frankfurt a. Main 2001, 42-69.

135 Ebd., $55 \mathrm{ff}$. 
ter, dem man mit Misstrauen begegnen würde, das hatte ich mehrfach bewiesen, indem ich mich auf meine Schweigepflicht berufen hatte.

Metje nennt als zweite Phase die Ablehnung. ${ }^{136}$ Dazu passt in meinem Fall das Angeschnauztwerden. Aber es gab auch andere Umgänge mit mir: Ignorieren und Tolerieren. Bis zum 4. April 2004 wurde ich von den meisten Jugendlichen der Klasse ignoriert. An jenem Tag verteilte eine Schülerin, Rasiah Sunteralingam, in der Deutschstunde Frau Gisler hatte das Zimmer verlassen - Daims, eine Süssigkeit. Bei mir zögert sie. Auf Fürsprache des Schülers Akdis Saliba hin bekam ich dann doch eins. Danach revanchiert sich Akdis an der Klasse mit Pfefferminz-Pastillen, von denen ich auch zwei kriege. ${ }^{137}$ Damit wurde ich das erste Mal in einen Warenkreislauf integriert. Am 3. Mai wurde ich erstmals bei einem Gespräch toleriert. Fiona und Sonia, zwei Schülerinnen, reden die längste Zeit über die Erlebnisse des Wochenendes und über Handball und lassen sich von mir, der gerade gegenüber sitzt, nicht stören. Ich denke, sie signalisieren damit, dass ich als Passivhörer akzeptiert bin. Aus Dankbarkeit tue ich so, als ob nichts sei, und signalisiere damit Frau König, dass die beiden nichts Unstatthaftes während des Unterrichts machen. ${ }^{138}$ Ich war wirklich froh, dass ich toleriert wurde. Es wurde mir in diesem Moment klar, dass damit die Durchführbarkeit meines Auftrages in Reichweite kam.

Als dritte Phase bezeichnet Metje die Phase des gegenseitig SichEinlassens ${ }^{139}$. Hier kam mir zwei Umstände zu Hilfe: Ich hatte sehr bald einmal beschlossen, meine Untersuchung auf die Parallelklassen der 2D auszuweiten, da ich den Eindruck hatte, die Klasse würde sich in den Pause den ähnlichen ethnischen oder Style-Gruppen anderer Klassen zuwenden, etwa, dass sich die türkischstämmigen Jugendlichen eher

136 Ebd., $57 \mathrm{ff}$.

137 FB04, 59.

138 Ebd., 57.

139 Metje, "Hier haben ja alle Löffel Löcher", 2001, 59ff. 
mit den türkischstämmigen Jugendlichen anderer Klassen in der Pause abgaben als mit ihren eigenen Klassenkameraden. Aus diesem Grunde sass ich auch einmal in der 2C. Ich bemerkte, dass Sonia Plüss und Janine Maggiorini von Loredana Di Costanzo aus dieser Klasse während eines Vortrags unterstützt wurden, und beschloss, weil sie sich nach meinem Auftrag erkundigt hatte, was sonst keine Schülerin und kein Schüler gemacht hatte, auch mit ihr ein Interview zu führen. Während diesem sprach ich sie auf eine Begebenheit an, die mir zu Ohren gekommen war, nämlich, dass Loredana und Janine einen Termin bei der Jugendstaatsanwaltschaft wahrnehmen mussten. Was ist denn da passiert, fragte ich. Sie vertraute mir an, dass sie mit Cannabis in der Tasche von der Polizei erwischt worden waren. Ich gab daraufhin meiner Überzeugung Ausdruck, dass der Staat in Sachen Cannabis etwas überreagiere und dass ich auch schon mit Cannabis Bekanntschaft gemacht hatte. Die Reaktion war verblüffend: Loredana fiel sozusagen aus allen Wolken, da sie sich nicht vorstellen konnte, dass ein Erwachsener schon "Dope" geraucht habe. Ob ich sie mal besuchen kommen würde? Überrascht und erfreut, von einer Jugendlichen eingeladen zu werden, stimmte ich zu. Am 19. Juni, kurz vor der Schulabschlussfahrt nach Südfrankreich und nur zehn Tage vor Abschluss der WBS und damit der obligatorischen Schulzeit, wurde ich an eine Stelle am nördlichen Rheinufer in der Nähe von Kleinhüningen bestellt. Da es sich bei den folgenden Begebenheiten um einen Wendepunkt in dieser Forschung handelt, zitiere ich aus dem Feldtagebuch:

Als ich mit dem Fahrrad am vereinbarten Treffpunkt ankomme, ist es mir peinlich, in aller Öffentlichkeit von zwei kichernden Mädchen empfangen zu werden. Ausserdem mache ich mir ziemlich Sorgen über die Legalität meines Unterfangens. Was ist, wenn die Polizei mich in Begleitung zweier minderjähriger Mädchen erwischt, die illegale 
Substanzen auf sich tragen? Ich darf gar nicht daran denken.

Ich grüsse freundlich und wir wenden uns Richtung Rhein, es ist mir leicht peinlich, nicht so recht zu wissen, was reden, versuche aber, mich zu entspannen; Loredana humpelt dahin mit ihrem Gipsfuss (sie hatte die Bänder des linken Fusses gezerrt) und Janine packt einen riesigen Joint aus. Sie macht Anstalten, ihn auf der Strasse anzuzünden, aber Loredana weist sie zurecht: "Du Scheisskind, jetzt mach das Ding weg!" Janine weigert sich und steckt sich den Riesenkonus in den Mund. Ich sage beunruhigt: "Wart doch mal ab, bis wir sitzen! Keinen Stress!" Janine ist nicht beeindruckt, zündet den Joint an und stösst riesige Rauchwolken aus. Wir gehen weiter, Janine schwenkt das qualmende Ding wie eine Keule, auch als uns eine Gruppe junger ausländischer Mütter mit ihren Kindern entgegenkommt. Ich versuche so zu tun, als sei ich nicht da und schiebe unschuldig mein Fahrrad. Janine geht voraus dem Rhein entlang und steigt eine Treppe das Rheinbord hinunter zum Wasser. Am Fusse der Treppe hält sich eine Gruppe ausländischer Jugendlicher auf und schwatzen. Sie verstummen angesichts unseres Anblicks. Wir verschwinden in eine stillgelegte Unterführung einer Schiffsanlegestelle neben der Treppe.

Etwas zurückversetzt in der stillgelegten Unterführung steht ein Sofa und ein Couchtisch, ein Papierkorb demonstriert Ordnungsanspruch. Hinter dem Sofa da und dort Müll. Ich packe eine Flasche mit Mineralwasser aus, die ich mitgebracht hatte, Janine Chips und Orangensaft, eine Flasche für jeden. Die Stimmung ist gespannt, dennoch lehne ich mich zurück und versuche zu entspannen. Es will 
kein so rechtes Gespräch aufkommen. Loredana sagt ständig Sie zu mir, was mich nervös macht. Ich schlage vor, dass wir uns duzen. Loredana: "Der Obi"140. Wir schütteln die Hände. Janine schweigt und isst Chips. Ab und zu versucht sie, das Sofa mit dem Feuerzeug anzuzünden, was Loredana unterbindet.

Die Unterhaltung: Zuerst eine Unsicherheit mit dem Sie und $\mathrm{Du}$, dann eine peinliche Stille. Worüber soll man sich mit Jugendlichen unterhalten, die bekifft sind? Ich warte ab. Nichts passiert. Der Rhein fliesst. Loredana lächelt die ganze Zeit und Janine sitzt nur da und fährt damit fort, irgendwelche Sachen mit der Flamme ihres Feuerzeuges zu bearbeiten. Loredana erzählt einen abstrakten Witz. Loredana fragt, ob ich auch einen Witz kenne. Ich verneine und bedaure, dass ich Witze nie behalten kann. Sie erzählt weiter: "Zwei Hochhäuser sitzen im Keller und stricken Milch." Sie lacht, das Bild sei fantastisch, findet sie. Irgendwie beginnt Janine über die Schule zu reden. Sie möchte nicht zum Brunch gehen morgen früh. Ich erschrecke; den Brunch Freitagmorgen hatte ich vergessen, das Beobachten in der Schule hatte mich ziemlich angestrengt, und ich war froh, dass die Ferien bald anfingen. Jetzt noch dieser letzte Tag. Ich frage, was das war, was ich einkaufen hätte gehen sollen, für den Frühstückstisch. Ich habe es vergessen. Janine: Gipfeli ${ }^{141}$. Das stimmt.

140 Mein Spitzname bei den Jugendlichen. Sie wissen nicht, woher der Name kommt, er soll aber von Französisch Observateur abgeleitet sein. Manchmal wurde auch Obi Wan Kanobi, eine väterliche Figur aus dem Film Star Wars (USA 1977) genannt.

141 Hörnchen, Croissants. 
Loredana fragt, welches Sternzeichen ich bin. Ich sage: Jungfrau. Sie lächelt und will die Hand schütteln. Da Janine so schweigsam ist, frage ich sie: Und was bist du? Sie sagt: Widder. Das hätte ich mir denken können, ertappe ich einen Gedanken. Das passt dazu, wie sie den Lehrer Boder in der Mathelektion attackiert hatte, denke ich. Der Rhein fliesst weiter. Man sieht nur einen Ausschnitt des Flusses durch den Tunnel der Unterführung: einmal gleitet ein Schiff vorbei, einmal startet eine Ente. Die Sonne senkt sich. Gegen $21 \mathrm{~h}$ sagt Janine, dass sie langsam gehen muss, sie werde um $9 \mathrm{~h}$ erwartet. Ich erkundige mich, was die beiden daheim erwartet. Sie stöhnen und winken ab. Offensichtlich diversen Ärger. Sie wirken bedrückt. Wir packen zusammen und verlassen den Ort. Die Jugendlichen am Eingang sind verschwunden. Loredana leert brav ihren Aschenbecher in den Papierkorb und nimmt ihn mit. Das Sofa und der Couchtisch bleiben leer zurück und wir steigen die Treppe hoch. Sie gibt mir das Orangensaftfläschchen und meint, das das mir gehöre. Ich nehme es verwundert über den Ordnungs- und Eigentumssinn, es ist praktisch leer, ich hätte es stehen lassen und damit zur Vermüllung beigetragen. Oben verabschiede ich mich und nehme das Velo, während die beiden zur Tramhaltestelle streben. ${ }^{142}$

Obwohl ich mich unbehaglich gefühlt hatte, hatte ich den Test bestanden, was sich bald zeigte. Weitere Türen gingen auf: Die Jugendlichen wurden zugänglich, als sich am nächsten Tag verbreitete, dass ich am Rhein dabei gewesen war. Janine erzählte es überall herum. Die

142 FB04, 74-75. 
Mädchen der Klasse steckten die Köpfe zusammen, tuschelten und schauten zu mir hinüber. Das Getuschel war mir peinlich, ich schwitzte. Ausserdem machte ich mir aus rechtlichen Gründen Sorgen. Ich empfand es als Mutprobe, die man bestehen muss, um in einer Gruppe aufgenommen zu werden, eine Initiation. Etwas Illegales tun. Ich habe meinen Gruppennamen erfahren. Die Situation in der Unterführung war die erste mit Jugendlichen, bei der Hierarchien keine Rolle spielten.

Dieser Abend markierte ein davor und danach. Vorher liefen Jugendliche, die mit mir sprachen, Gefahr, ausgeschlossen zu werden. Beispielsweise unterhielt sich Günter Robert mit mir einmal in einer Pause und wir waren überrascht, als er von einigen seiner Klassenkameraden als "Schleimer" angemacht wurde. Sprach ich mit mehreren, gab es immer solche, die die Gruppe verliessen. ${ }^{143}$ Von jetzt an war das nicht mehr so, von jetzt an bekam ich aussagekräftige Informationen. Ich mag mich täuschen, aber etwas Komplizenhaftes schwang nachher im Umgang mit den Jugendlichen immer mit, ein gemeinsames Geheimnis. Es war der Wendepunkt meiner Feldforschungstätigkeit, fast ein Jahr nach deren Beginn.

Wir waren in der vierten Phase der Integration ins Feld nach der These von Metje angekommen: Der Phase des prekären Vertrauens. ${ }^{144}$ Es bleibt ein Vertrauensbonus, der verspielt werden kann und auf Widerruf gewährt wird. Martina, eine Freundin von Janine und Loredana, vermutete beispielsweise immer wieder, und zog mich damit auf, dass ich ein versteckter Polizist oder ein Spion sei. Sie hat sogar im Scherz damit gedroht, dass ihr Vater daheim eine Waffensammlung habe und wenn ich nicht brav wäre, dass er dann was an mir ausprobieren würde. ${ }^{145}$

143 Ebd., 57.

144 Metje, "Hier haben ja alle Löffel Löcher", 2001, $63 \mathrm{ff}$. 145 FB05, 5. 
Ich war für Loredana zunächst genauso Mitglied eines fremden Stammes, wie sie das für mich war. ${ }^{146}$ Metje weist darauf hin, dass im Forschungsprozess die kulturelle Identität an Bedeutung verlieren kann gegenüber Kategorien wie Bildung, dem Geschlecht, dem Status, der sexuellen Orientierung oder der Rasse. "Das heisst, die Wirklichkeit, die sich in der Begegnung zeigt, kann stärker von der Individualität der ForscherInnen determiniert sein als von deren kultureller Identität. Und schliesslich ist die Qualität der Forschungsbeziehung abhängig von den zwei Menschen (oder mehr), die in diesem Prozess aufeinander treffen. ${ }^{147}$ Möglicherweise konstituiert das gemeinsame bestehen einer illegalen Situation eine Klammer ähnlich wie oben genannte Kategorien, welche die Identität der Einzelnen über Verschiedenheiten wie etwa der Altersgruppe heraushebt und eine gemeinsame Basis der Kommunikation schafft.

Diese Basis war ziemlich tragfähig. Überrascht war ich, dass ich nach Abschluss der Schule eingeladen wurde, in "die Wohnung" zu kommen, wo sich die Mädchen in ihrer Freizeit trafen. Ich habe mich gefragt, weshalb ich in fast selbstverständlicher Weise hinzugeladen wurde. Es stellte sich heraus, dass ich einer der wenigen erwachsenen Männer war, den Loredana auf einer kollegialen Basis kannte. Diese Tatsache könnte die Erklärung dafür sein, dass ich derart problemlos Zugang zu diesem intimen Bereich erhalten habe. Da Loredana nicht weiss, was ein Mann ist, bin ich auch nicht vorbelastet. Ich bin für sie genauso ein fremdes Wesen und damit ethnologisch interessant, wie sie das für mich ist. Es ist ein gegenseitiges Testen und Tasten: beide wollen Informationen: Der Ethnologe über die Jugendlichen und die Jugendlichen über die Erwachsenen. Im Übrigen finde ich die Tatsache, dass Jugendliche erwachsen werden, ohne einen Mann als Ansprechpartner zu haben, bedenklich. So bleibt ein grosser Teil menschlicher

146 Ebd.

147 Metje, "Hier haben ja alle Löffel Löcher", 2001, 65. 
Erfahrung für die Jugendlichen nicht zugänglich. Dieses Manko äusserte sich auch in den Fragen an mich.

\subsection{Methoden der Auswertung und Verschriftlichung}

Nach Abschluss der Feldforschungsphase besass ich sehr viel Material, das thematisch nicht gegliedert, sondern chronologisch aufgebaut war. Das Problem stellte sich, wie diese Materialien für die Auswertung erschlossen werden sollten. Laut Schmidt-Lauber kann die Annäherung an die Analyse des Materials auf verschiedene Weise erfolgen. „Neben dem wiederholten Lesen des meist umfangreichen Tagebuchs, aus dem sich Leitthemen für die Analyse der Lesarten der kulturellen Realität aufdrängen, kann dies die systematische Verschlagwortung aller Daten oder die visualisierende Clusterbildung sein oder auch das Herausgreifen eines Einzelfalls, der detailliert dargestellt und gedeutet werden soll." "148

Diese Anweisung stellt einen Ansatz zur Bewältigung eines wichtigen Meilensteins im Verlauf eines Forschungsprojekts dar. An dieser Stelle findet der entscheidende Übergang vom Dateninput zur Repräsentation der Ergebnisse statt, also zum wissenschaftlichen Output. Für die Forschenden ist dieser Moment mit grossem psychischen Druck verbunden. Sie stehen am Ende einer je nach Forschungsplan jahrelangen Feldforschung und müssen die vielleicht tausende von Eindrücken, Notizen, Tonbandaufzeichnungen, gesammelten Gegenständen und anderen, oft heterogenen Materialien in einen theoretisch fundierten und wissenschaftlich relevanten Text umgiessen. Oft hängt die weitere Forscherkarriere davon ab, wie kompetent dieser Moment bewältigt wird.

In anderen Disziplinen, der Journalistik zum Beispiel, wird diesem Moment grosse Beachtung geschenkt, denn hier geht es ums Ganze: Es 148 Schmidt-Lauber, Feldforschung, 2007, 235. 
werden die wichtigsten und entscheidendsten Beschlüsse für die Darstellung der Forschungsergebnisse im gewählten Medium getroffen. Das ist eine grosse Geistesleistung, welche die höchsten Anforderungen an die Kompetenz und Erfahrung der Forschenden stellt, und die den weiteren Verlauf der Repräsentation entscheidend prägt. Es lohnt sich, grosse Sorgfalt auf die Entscheidungen in diesem Moment zu legen. Anderseits darf nicht zu lange gezögert werden, denn zieht sich die Niederlegung der Ergebnisse zu lange heraus, verliert die Analyse an Qualität, weil die Eindrücke nicht mehr frisch sind. Die Überforderung mit diesen Bedingungen kann beim Ethnografen zu negativen Gefühlen wie Niedergeschlagenheit, Lähmung und Verzweiflung führen, was das ganze Forschungsprojekt gefährden kann.

\subsubsection{Verschriftlichung mit Blick auf die Lesenden}

Schmidt-Lauber erwähnt die Methode, das Material mehrfach zu lesen und sich aufdrängende Leitthemen für die Analyse zu notieren, alle Daten systematisch zu Verschlagworten oder in der Clusterbildung zu visualisieren. Man könne auch einen Einzelfall herauslesen. ${ }^{149}$ Sie meint wahrscheinlich mit ihrer Anweisung, das Material mehrmals zu lesen, die aus Journalistenschulen und Schreibkursen bekannte Methode, sich mit Eindrücken, Fakten, Informationen und Stimmungen sozusagen wie ein Schwamm vollzusaugen, um diese im Kopf halb unbewusst, halb bewusst zu gliedern, strukturieren, in eine gewünschte Abfolge zu bringen und dann auf Papier festzuhalten. So arbeiten unter anderem Schriftsteller. Das weist darauf hin, dass das Schreiben einer Abhandlung eine künstlerische, schriftstellerische Leistung ist. Es gibt dazu inhaltliche und formale Arbeitshilfen, die in Journalistenschulen und in Ausbildungen für Schriftstellende gelehrt werden.

149 Schmidt-Lauber, Feldforschung, 2007: 235. 
Gegen die Niedergeschlagenheit kann angegangen werden. Beispielsweise ist es in einer solchen Situation hilfreich, auf formale psychologische Methoden wie Supervision oder Coaching zurückzugreifen oder auf informelle, wie Gespräche mit Kollegen oder in der Forschungsgruppe. Kullberg betont, wie wichtig in diesem Moment die Unterstützung der Kollegen sein kann. Auch lohnt es sich, nicht bis zu diesem Moment mit der Niederschrift von Ergebnissen zu warten, sondern schon seit Anfang der Forschung laufend zu versuchen, Kapitelentwürfe zu schreiben. ${ }^{150}$ Damit ist das Eis schon ein Stück weit gebrochen, der entscheidende Einstieg möglicherweise schon geschafft.

Die Verschriftlichung der Forschungsergebnisse wurde in der Europäischen Ethnologie nicht zum methodischen Thema, sondern zum Stoff für Literaturkritik der Monografien. Beispielsweise hatte die „Writing Culture“-Diskussion in den 1980er Jahren, die auf James Clifford und George Marcus ${ }^{151}$ zurück geht, die theoretische Reflexion der Produktion von Texten über Kulturen zum Inhalt, etwa die Frage, mit welchen stilistischen Mitteln der Ethnografie die Aura von Authentizität und Richtigkeit verliehen wird. Die beschriebenen Kulturen werden von Autoren konstruiert. Dieses Verfahren bezeichnet man als Othering. Ebenfalls auf die ethnologische Textproduktion wirkten sich verschiedene erkenntnistheoretische und interdisziplinäre Wendepunkte aus: Der sogenannte "linguistic turn" nahm Anstösse aus der Linguistik auf, in denen es um die welterschliessende Bedeutung von Sprache ging, der sogenannte "interpretative turn" machte die Standortgebundenheit jeglicher Aussage zum Thema. Der "narrative turn" schliesslich sensibilisierte für die Reflexion der narrativen Strukturen und rhetorischen Stile wissenschaftlicher Beschreibung.

150 Kullberg, Etnografi i klassrummet, 1996, $111 \mathrm{ff}$.

151 Clifford, James \& George E. Marcus: Wrtiting Culture: The Poetics and Politics of Ethnografy, Berkeley 1986. 
Ein weiterer einflussreicher Darstellungsstil war auch die sogenannte Dichte Beschreibung ${ }^{152}$. Dieser Begriff beschreibt eine Methode, eine Theorie und eine Textualisierungsstrategie zugleich, die ein theoretisches Verständnis von Kultur als Text impliziert. ${ }^{153}$

Infolge dieser Auseinandersetzungen gab es eine wachsende Sensibilisierung für Fragen der Machtausübung, die nach neuen Schreibformen verlangte. Nach der Dekonstruktion ethnografischer Texte als subjektive, hegemoniale Narrationen wurde es zunehmend möglich, die Subjektivität des Forschers zu betonen. Dies führte zu einer Phase des ethnografischen Realismus, in der die Monografie ein neutrales, objektives Abbild der beschiebenen Kultur zu bieten vorgab, ein Darstellungsduktus, der auch als Bekenntnisliteratur bezeichnet wird.

Als Folge dieser Diskussionen erhielten die Ethnografien eine Diskurslastigkeit, die sich in einer Vielzahl von Meta-Anthropologien, von Analysen von Analysen, ausdrückte, welche die empirische Forschung und das Verfassen ethnografischer Texte in den Hintergrund treten liess. Auch dies stiess immer mehr auf Kritik, etwa von Pierre Bourdieu. ${ }^{154}$ Immerhin haben alle diese Debatten auch zu einer qualitativen Verbesserung von Ethnografien geführt. Kritische Reflexion der Bedingungen der Feldforschung und des Schreibens sind heute eine Selbstverständlichkeit. Auch darf der Forscher im Text erscheinen, allerdings nur dort, wo es für die Ergebnisse von Bedeutung ist.

In letzter Zeit spielen Forschungen eine Rolle, welche die Machtrelation berücksichtigen. "Research up" nennt man Untersuchungen von Machteliten und "studying down" Forschungen, welche sozial Schwache zum Thema haben. Die räumliche, soziale oder institutionelle Abgrenzbarkeit von Kulturen wurde wichtig. Deren Geschlossenheit wur-

152 Vgl. Geertz, Dichte Beschreibung, 2003.

153 Schmidt-Lauber, Feldforschung, 2007, 238-239, in Anlehnung an Geertz,

Dichte Beschreibung, 1995.

154 Pierre Bourdieu zitiert nach ebd., 240. 
de im Zuge der Globalisierung in Frage gestellt, die Grenzbereiche rückten ins Zentrum des Interesses. Diese Entwicklung führte zu Forschungsansätzen wie der "multi-sited ethnography", bei der der Forscher dem Gegenstand oder den Untersuchten an verschiedene Orte hin folgt und dort mehrere Sippvisiten zubringt. Mit dem Verlassen ortsfixierter Kulturkonzepte und der Hinwendung zu einer multilokalen, mobilen Ethnografie verbindet sich das Anliegen, die Relationalität, Kontextualität und Prozesshaftigkeit von Kulturen anschaulich zu dokumentieren. ${ }^{155}$

Merkwürdig an diesen Debatten scheint das völlige Fehlen der Interessen der Leser von Ethnografien zu sein. Wenn man schon feststellt, dass Ethnografien Literatur sind und die Resultate von Feldforschung und Teilnehmender Beobachtung in plastischer und lebendiger Weise eine Annährung an den gelebten Alltag der Beforschten darstellen, fragt man sich, weshalb nicht Formen und Methoden literarischer Darstellungen rezipiert werden, etwa von Jane Kramer ${ }^{156}$ oder Hans Magnus Enzensberger ${ }^{157}$. Seit der Jahrtausendwende ist der perspektivenreiche und lebensnahe Einblick in kulturelle und soziale Wirklichkeiten ein hochgefragtes Konzept, das einem Verlangen nach sinnlicher Erfahrung und einer Ästhetik des Unmittelbaren entgegenkommt. ${ }^{158}$

Aber zurück zur Methodik der Auswertung: Auch hier hilft Kullberg mit konkreten Anweisungen weiter. Sie drängt darauf, die gewonnenen Informationen laufend zu kodieren. Dabei werden ihre Quelltexte in Beschreibungen, Überlegungen, Theorien, und andere Textsorten aufgeteilt und markiert, z.B. mit farbigen Filzstiften. Dabei werden folgende Fragen an die Daten gelegt: Was passiert eigentlich? Was sind die

155 Ebd., 240-243.

156 Z.B. Kramer, Jane: Sonderbare Europäer. Gesichter und Geschichten,

Frankfurt a. M., Eichborn, 1993.

157 Z.B. Enzensberger, Hans Magnus: Ach, Europa!: Wahrnehmungen aus 7

Ländern, Frankfurt a. M., Suhrkamp, 1987.

158 Schmidt-Lauber, Feldforschung, 2007, 243. 
grundlegenden sozialen und psychologischen Probleme, mit denen die Akteure konfrontiert werden? Was sind die grundlegenden sozialen und strukturellen Prozesse, welche die Probleme sichtbar werden lassen? Was erklärt die grundlegenden Prozesse und Probleme?

In dieser Endanalyse geht es darum, die ethnografischen Fakten umzuorganisieren und nach Hauptkomponenten zu suchen. Viele Forscher empfehlen, ein System von Denkeinheiten zu schaffen, die aus der Schlussanalyse herauswachsen. Diese Hauptkomponenten variieren je nach Forschung. Stichworte könnten sein: Inhalte, Episoden, Sitten, Gepflogenheiten, Treffen, Rollen, Beziehungen, Gruppen, Organisationen, Ansiedlungen, soziale Welten, Lebensstile. ${ }^{159}$ Strauss schlägt folgende Kategorien vor: Bedingungen, Zusammenspiele, Konsequenzen. ${ }^{160}$

\subsubsection{Elektronische Datenauswertung durch ein Wiki}

Im Falle meiner Forschungsarbeit war klar, dass diese Komponenten aus dem Material selbst entstehen und auf einander Bezug nehmen sollten. Deshalb entschloss ich mich, die Informationen elektronisch auszuwerten und ein sogenanntes Wiki einzurichten. Ein Wiki ${ }^{161}$ ist ein System, mit dem Websites sehr einfach online ediert werden können. Das System führt auch Buch über die angebrachten Änderungen, so dass es einfach wieder in einen früheren Zustand überführt werden kann. Fol-

159 Kullberg, Etnografi i klassrummet, 1996, 124-125.

160 Strauss, A.L.: Qualitative Analysis for Social Scientists, Cambridge, Cambridge University Press, 1987, 27.

161 Die Gründungslegende besagt, dass Ward Cunningham 1995 dieses System erstmals für das Internet programmiert und nach der Wiki Wiki Fahrspur der Schnellbusse auf dem Honolulu International Airport benannt hat. Wiki wiki heisst auf Hawaiianisch schnell oder sich beeilen. (Wiki - Wikipedia, http://de.wikipedia.org/wiki/Wiki\#Vorl.C3.A4ufer, 4.3.2009). 
gendes Prinzip leitet die Informationseingabe: Begriffe, die einen Grossbuchstaben enthalten, wie zum Beispiel BuchHülle, werden durch die Software zu Links gemacht. Zudem wird eine Seite erzeugt mit dem Titel BuchHülle und der Aufforderung, diesen Begriff zu definieren. Auf diese Seite kann von anderen Seiten her verwiesen werden. So entsteht ein Geflecht von Bedeutungen, das durch Begriffe und ihre Links repräsentiert wird. Das bekannteste Wiki ist wikipedia.org, eine freie Enzyklopädie, wo zum Zeitpunkt des Endes meiner Feldforschung über 900'000 englischsprachige und 300'000 deutschsprachige Webdokumente durch die Benutzer selbst unterhalten werden.

Jeder Beobachtungstag im Feld ergab mehrere Seiten Notizen in kleinen, A5/6-formatigen Heftchen, die von einem Datum eingeleitet wurden. So bald als es mir möglich war, übertrug ich diese Kritzeleien ins Reine und ergänzte sie aus dem Gedächtnis, was einen fortlaufenden Text ergab, den ich mit der Jahreszahl, beispielsweise 2004 und der Seitenzahl erschloss. Gegen Ende meines Forschungsauftrags, als es auf das Niederschreiben zu ging, sah ich sämtliche Texte, die ich produziert hatte, nach Inhalten durch. Dies lief konkret so ab: Sämtliche Textstellen kategorisierte ich nach einem Hauptgedanken. Wenn ein Schüler mir beispielsweise erzählte, dass er eine Schnupperlehre bei einem Orthopäden gemacht hatte und es ihm nicht gefallen hätte, trug ich diese Aussage unter dem Titel "Beobachtungen über die Bewertung einzelner Berufe" ein, mit dem mit einem Link zum Begriff Schnupperlehre und der Textstelle. Nach der Auswertung sämtlichen Materials ergab dieses Vorgehen eine Liste mit rund hundertzwanzig Begriffen, die Gegenstände, Verhaltensweisen, Gefühle, Sozialbeziehungen und anderes beeichneten. ${ }^{162}$ Diese versuchte ich, in Obertitel zu fassen und so thematisch zu gliedern. Der Obertitel Delinquenz, Devianz, Antisoziales Verhalten beispielsweise enthielt die Begriffe Antisoziales Verhalten, Gesetzes-

162 Siehe Anhang, Liste von Denkeinheiten gemäss Kullberg, Etnografi i klassrummet, 1996, 124ff. 
bruch, Lügen, Randale und Stehlen. Auf diese Weise gelang es, die oben beschriebenen Denkeinheiten aus dem Material selbst herauszuarbeiten. Diese thematischen Perspektiven waren über die vom Wiki erzeugten Links miteinander verknüpft. Damit wurde es möglich, übergeordnete Kategorien wie Prozesse, Verbindungen, Beziehungen oder Konflikte zu erkennen. Diese zum Teil längeren Abschnitte waren durch einen Verweis auf den Feldtagebuchjahrgang und die Seite des reingeschriebenen Textes, der aus den Notitzen entstanden war, an das ursprüngliche Material zurückgebunden. Das gleiche Verfahren wurde mit den Interviews angewandt. Nach dem Transkript der Tonbandaufnahmen entstand ein Text, dessen als wichtig erachtete Stellen im Wiki erfasst und mit relevanten Passagen anderer Provenienz über Links verknüpft wurde.

\subsubsection{Soziogramme}

Auch benutzte ich grafische Methoden. Erwin Herrmann stellte für den Lehrergebrauch eine Broschüre mit diesen soziometrischen Methoden ${ }^{163}$ zusammen, die auch ich als Anregung für diese Forschung hier verwendet habe. Sie erlauben gewisse Einblicke in das innere Gefüge von sozialen Systemen, etwa von Schülergruppen:

Wo Menschen in Gruppen leben und arbeiten, treten Gruppeneffekte auf, die innerhalb des Gesamtverbandes das Eigenleben der Gruppe, den Gruppenprozess, darstellen; auch die Schulklasse ist also ein Gruppenphänomen mit oft ausgeprägten Strukturwandlungen etwa innerhalb eines

163 Herrmann, Erwin: Methoden und Möglichkeiten der Soziografie in der Schule (anhand oberfränkischen Materials), Heimatbeilage zum Amtlichen Schulanzeiger des Regierungsbezirks Oberfranken, Bayreuth 1985. 
Schuljahres. (...) Ab etwa dem 12./14. Lebensjahr (...) gibt es komplexe Verbindungen innerhalb der Klasse, allerdings auch dezidierte Ablehnungen vonseiten der Gruppe wie auch vonseiten manchen Individuums. Eine Gruppe (also auch eine Klasse) entwickelt prozesshafte Tendenzen, kennt Anziehung und Abstossung, Initiative und Selbstbehauptung wie auch Zurückhaltung und Submissivität, also Unterwerfungsverhalten (...). Der einzelne ist also sozusagen ein Körper im sozialen Kraftfeld. ${ }^{164}$

Die Begrifflichkeit weist darauf hin: Der Mensch in der Gruppe wird sozusagen wie ein Stück Eisen in einem Magnetfeld angesehen, auf das bestimmte Kräfte wirken. Deshalb stellt die Soziometrie Gruppen mit Signaturen wie Pfeil, Stern, Dreieck und Kreis dar, zwischen denen - ähnlich wie in der Physik Kraftlinien - Antipathie, Sympathie, Machtgefälle, Sitzordnungen, und andere Beziehungen mit verschieden gekennzeichneten Linien oder Vektoren eingetragen werden.

So weit bin ich nicht gegangen. Regelmässig habe ich die Sitzordnungen in der Klasse aufgezeichnet, um nachher darüber nachzudenken, wer mit wem wie verbunden ist, doch stiessen diese Überlegungen bald an Grenzen, denn die Sitzordung in den Schulklassen wurden auch von verschiedenen gruppenexternen Faktoren beeinflusst und waren deshalb für das Verstehen der inneren Gruppendynamik nur ein Stück weit hilfreich. Weit sinnvoller waren Soziogramme für das Verstehen der verschiedenen Beziehungen innerhalb der klasseninternen Schülergruppen. Im Interview fragte ich nach den vier besten Freunden in der Klasse und nach denen vier, mit der eine Person am wenigsten zu tun hat. So gelang es mir, zusammen mit den Feldbeobachtungen, Aussagen über die Gruppendynamiken zu gewinnen.

164 Ebd., 3-4. 


\subsection{4 Überlegungen zum Aufbau des Textes}

Mit diesen von Kullberg und anderen vorgeschlagenen Methoden hatte ich also meine Feldergebnisse in eine Form gebracht, die es erlaubte, sie in einem Text niederzulegen, welcher wissenschaftlichen Traditionen und Gepflogenheiten entsprach. Nach der Abwägung verschiedenster Forderungen aus der Forschungsgeschichte der Europäischen Ethnologie, wie sie Schmidt-Lauber beschreibt, entschloss ich mich, diesen Text grundsätzlich chronologisch aufzubauen und nicht primär thematisch. Verschiedene Gründe veranlassten mich dazu.

In dieser Arbeit sind Wege in das Erwachsenleben das Thema. Die chronologische Reihenfolge des Materials ist eine Information, welche mit dem Erwachsenwerdensprozess der untersuchten Jugendlichen korrelliert und somit das Begleitende meiner Forschungsweise betont. Die Chronologie ist zudem ein Spiegelbild meiner Fortschritte als Feldforscher im Eintauchen in das Forschungssetting. Auf dieser Reise in das Erwachsenenleben wurden aber auch Fragen aufgeworfen, die für die Jugendlichen wichtig waren. Deshalb wird der Text durch thematische Einschübe und Vertiefungen unterbrochen, etwa zur Frage der Normalität oder zur Interpretation besonderer Vorkommnisse. Diese Fragen und Vorkommnisse deuten auf Themen hin, welche für die Jugendlichen genau in diesem Alterszeitpunkt virulent wurden.

\section{Arbeitsweisen und die Situation im Forschungsfeld}

Äusserlich ist das Schulhaus eine typische Bildungsmaschine der 70er Jahre: Betonplattenbauweise, grosse Glasfronten, mehrere tausend Schülerinnen und Schüler, die täglich verschiedene Oberstufenschulen besuchen. Der Grund, dass ich dort gearbeitet habe und nicht an einer 
den anderen sechs Standorte der WBS, liegt darin, dass das Integrationsproblem hier von der Rektorin als besonders brennend beschrieben wurde. Dass meine Wahl auf die Klasse 2D fiel, lag daran, dass ich den Eindruck hatte, dass die Lehrerin der 2D am auskunftsfreudigsten im Vergleich aller Klassenlehrer war, die in Frage kamen. Diesen Sachverhalt schätzte ich für meine Arbeit als sehr wichtig ein. Die Wahl der zu erforschenden Gruppe ergab sich aus den äusseren Bedingungen der Forschungsanlage.

\subsection{Die Schulklasse 2D als kulturelle Gruppe}

Zunächst hatte ich mit dem Gedanken gespielt, mehrere Klassen zu beobachten, um das Umfeld besser abzudecken, aber das erwies sich als zu aufwendig. Ich brauchte relativ lange, um mich einzuleben. Später revidierte ich meine Entscheidung teilweise, da die Klasse vielfältige Beziehungen zu den Nachbarklassen und den klassenfremden Schülern des gleichen Zuges unterhielt. Diese Beziehungen beruhten vor allem auf ethnischen und stylemässigen Affinitäten.

Das war im Frühjahr 2003, und es hat sich dann ergeben, als sich die Feldforschungsphase im November 2003 intensivierte, dass ich meistens am Dienstag, Mittwoch und Freitagmorgen anwesend war. $\mathrm{Zu}$ nächst habe ich bloss die Stunden von Frau Gisler besucht, bis ich das Gefühl hatte, Fuss gefasst zu haben, nicht ganz fremd zu wirken, was etwa einen Monat dauerte. Dann weitete ich meine Beobachtungen über den ganzen Stundenplan aus. Dabei achtete ich darauf, alle Stunden einmal besucht zu haben und bei allen Lehrern in der Stunde gesessen zu haben.

Ausgangspunkt war also die Klasse 2D. Doch dies bezeichnet zunächst eine verwaltungstechnische Einheit und hat nicht per se gemeinsame kulturelle Merkmale. Auch die Kultur einer verwaltungstechni- 
schen Einheit kann man untersuchen, wie die eines Staates. Ein Staat stellt ja zunächst einmal eine politisch-administrative Einheit dar, die Grundlage einer Kultur werden kann. Die Schulklasse spielt eine ähnliche Rolle. Ihre zu entstehenden Gemeinsamkeiten stellen bei der Zusammenstellung der Klassen ein derart wichtiges Kriterium dar, dass sie immer wieder in der politischen Diskussion über die Ausgestaltung der Schule ins Feld geführt werden. ${ }^{165}$ Die Arbeit hier beschreibt die dynamische Entwicklung dieser Gemeinsamkeiten, versucht, über die lebensweltlichen Praxen ${ }^{166}$ der Schülerinnen und Schüler innerhalb und ausserhalb der Institution Schule die Merkmale der Schulklasse als kulturelle Gruppe und als Ansammlung von kulturellen Gruppen zu verstehen.

Der Grund dafür war, dass ich mich auf schwedische Vorbilder berief. Seit den 60er Jahren, berichtet Kullberg, betrachten die schwedischen Forscher Gruppen im Klassenzimmer als Kulturen. Diese Gruppen - die Klasse selbst oder Gruppen innerhalb dieser - können als Träger unterschiedlicher Kultursysteme aufgefasst werden. Die Aufgabe des Ethnografen sei es, zu untersuchen, wie gut integriert diese Kulturen sind. Die Schule ist eine kulturelle Institution und Ausbildung ist ein Kulturprozess. Schulen sind "cultural sites", ein Begriff, der von Paolo Freire und anderen eingeführt wurde. Als solche erzeugen sie und verkörpern Unterstützung für bestimmte kulturelle Praxen, wie Arten zu Sprechen, Legitimierung bestimmter Formen von Wissen, Bevorzugung gewisser Geschichten und Autoritätsmuster, Bestätigung bestimmter Arten, die Welt zu erfahren und zu sehen. Schulen geben oft den Anschein, eine gemeinsame Kultur zu transportieren, aber tatsächlich legitimieren sie zumeist, was als dominante Kultur bezeichnet wird. Die

165 Zur Geschichte der politischen Auseinandersetzungen über die Schule im Kanton Basel-Stadt vergleiche den nächstfolgenden Abschnitt.

166 Bourdieu, Pierre: Die feinen Unterschiede: Kritik der gesellschaftlichen Urteilskraft, Frankfurt a. M., Suhrkamp, 2010. 
Vermittlung und Implementierung dieser Werte wird auch als "heimlicher Lehrplan" bezeichnet. ${ }^{167}$

Die Konzentration auf die Schulklasse hat gleichzeitig Vor- und Nachteile. Die Schülerinnen und Schüler finden sich bei Antritt der Schule in einer Verwaltungseinheit wieder und müssen sich innerhalb von dieser eine Identität und soziale Position aushandeln. Dieser Prozess hat wiederum Auswirkungen auf den Klassengeist, der bestimmt, wie die Klasse von aussen wahrgenommen wird. Die Konzentration auf die Einheit Schulklasse kann aber auch einschränkend wirken. Die Klasse 2D war zum Beispiel sehr heterogen und unterhielt auch starke Bindungen zu anderen Klassen und zu aussenstehenden Personen, weshalb ich bald meine Beobachtungen auf Jugendliche ausserhalb ausweitete. Dieser Schritt war eine entscheidende Weichenstellung für mein Projekt. Mit ihm verabschiedete ich mich auch vom Konzept der Schulklasse als Gemeinschaft, in die sich der oder die Einzelne zu integrieren habe und seine Identität so lange anpasse, bis dies gelungen sei. Dies bedeutete auch den Abschied von einer normativen Vorstellung von Normalität, der sich die Jugendlichen unterwerfen, um sich zu integrieren. Offensichtlich gab es auch die Optionen Parallelklasse oder Einzelgängertum, um im Verband zu funktionieren und sich eingebunden zu fühlen.

Die Frage, ob die Klasse eine eigene kulturelle Klammer darstelle und so eine eigene Gruppe konstituiere, habe ich an die Schülerinnen und Schüler weitergegeben. Ich fragte, ob die Klasse einen Klassengeist besitze. Die Schüler fassten die Frage aber nicht kulturell auf, sondern sozial; für sie zielte sie auf den Zusammenhalt in der Klasse, auf die Art und Tiefe ihrer Integration und nicht auf irgendwelche gemeinsamen kulturelle Züge, die ihre Gruppe konstituierten und die aus ihrer Sicht kaum vorhanden waren. Deshalb waren die Schüler gezwungen, sich darüber klar zu werden, was für sie unter Integration zu verstehen sei

167 Kullberg, Etnografi i klassrummet, 1996, 102-103. 
und diesen Zustand für ihre Schulklasse in Worte zu fassen. So kam ich unbeabsichtigt zu einem Integrationsbegriff.

Die 2D bestand aus sieben Mädchen und zwölf Jungen, die im Juni 2004 das Ende der obligatorischen Schulzeit erreichten. Die Schule hiess WBS (Weiterbildungsschule) Basel-Stadt. Sie ist nach ihrer eigenen Darstellung eine Schule, "in der Jugendliche das 8. und 9. Schuljahr der Volksschule absolvieren und ihre obligatorische Schulzeit mit einem nach Leistung differenzierten Abschlusszertifikat beenden können. Die WBS ist eine Schule zum Wohlfühlen, eine lebendige Schule, in der neben leistungsorientiertem Unterricht nach Stundentafel auch Freiräume bestehen für Lager, Projekte und verschiedene Unternehmungen". ${ }^{168}$ Ausser der WBS gibt es im Kanton Basel-Stadt auf der Mittelstufe das Gymnasium. 2004 besuchten insgesamt 1699 Schüler die WBS an ihren sechs Standorten mit 80 Klassen. Das von mir untersuchte Schulhaus war das mit zwölf ersten und vierzehn zweiten WBSKlassen mit 187 Schülern der grösste der sechs Standorte. Die Klassengrössen bewegten sich zwischen fünfzehn und zwanzig Leute, wobei der Durchschnitt neunzehn betrug, davon pro Klasse neun bis sechzehn "fremdsprachige" Schüler aus 49 Nationen. Die drei grössten Gruppen waren die Jugendlichen aus der Türkei (208), aus Italien (176) und aus den Ländern zwischen Griechenland und Österreich.

\subsection{Die historische Situation der WBS}

Die WBS passt sich ständig den veränderten politischen Rahmenbedingungen an, die geprägt werden von der Auseinandersetzung über das Vorgehen bei der Integration von ausländischen Schülern. Da hier unterschiedliche Integrationsbegriffe im Hintergrund eine Rolle spielen,

168 Porträt WBS (Weiterbildungsschule Startseite, http://wbs-basel.ch, 4.3.2009). 
soll diese Geschichte kurz skizziert werden. "Seit gut 20 Jahren prägt die Frage um das richtige Schulsystem und die richtige Bildung die Politik des Kantons Basel-Stadt. Die Diskussion um die Basler Schulreform - also das Ringen um die richtige Gliederung der Sekundarstufe I verlief sehr kontrovers. Der Konsens über die Schulpolitik war nicht selten schmal und labil", schreibt das Erziehungsdepartement. ${ }^{169}$ Diese Situation führte zu dauernden Reformen, die je nach politischem Standpunkt mehr oder weniger geglückt ausfielen. Die grossen Differenzen entstehen aus einem Dilemma: Das Vorantreiben von Integration und Ausbildung hat im Schulalltag die Konsequenz, dass die richtige Mischung der Klassen in den Blickpunkt rückt, denn das wichtigste Mittel zur Integration von kulturellen Minderheiten ist die Mischung mit Einheimischen. ${ }^{170}$ Mischt man aber zuviele fremdkulturelle Kinder mit Einheimischen, leidet die Aufnahme von Schulstoff, da viele Energien für die Integration aufgewendet werden. Die Einheimischen lernen zwar wichtige weiche Kompetenzen wie den Umgang mit Gleichaltrigen aus anderen Kulturen, dafür aber weniger harte Fakten, die an Prüfungen zu guten Noten und damit verbesserten Aufstiegschancen im Bildungssystem führen würden. Das Erziehungsdepartement bezeichnet zwar diese "kulturellen Kenntnisse" als "hervorragende Bildungschancen"171, doch bleibt die Frage, ob die Eltern und damit die Stimmbürgerinnen und Wähler, die sich um die Laufbahnchancen ihrer Kinder sorgen, von den Vorteilen dieser Chancen wirklich überzeugt sind. Jedenfalls scheint es so, dass die Linke eher die Integration fördern will, während die politi-

169 Erziehungsdepartement, Ressort Schulen: Medienmitteilung "Leitbild für die Schulen des Kantons Basel-Stadt”, 1.12.2005 (Kanton Basel-Stadt, Medienmitteilungen, Leitbild für die Schulen des Kantons Basel-Stadt, http://www.bs.ch/mm/ed/2005/12/ed-20051201-001.html, 4.3.2009). 170 Runfors, Mångfald, motsägelser och marginalisieringar, 2002, $181 \mathrm{ff}$. 171 Erziehungsdepartement 2005. 
sche Rechte sich sorgt, dass die heterogenen Klassen niemandem wirklich gerecht werden können. ${ }^{172}$

Dieses Problem ist stark akzentuiert im sozial und kulturell vielfältigen Stadtkanton Basel. Besonders betroffen ist das untersuchte Schulhaus, das auf praktisch freiem Feld zwischen den reichen Vorortgemeinden Riehen und Bettingen steht. Während Riehen und Bettingen vorwiegend eine grossbürgerliche Sozialstruktur und zum Zeitpunkt der Erhebung einen Ausländeranteil von 18\% aufweisen, ist Basel-Nord durch einen niedrigen Sozialstatus und einen Anteil der ausländischen Wohnbevölkerung von $44 \%$ gekennzeichnet. ${ }^{173}$ Beide Teile des Kantons gehören zum Einzugsgebiet derSchule.

Im Jahre 1998 führte man eine Nachbesserung der Schulreform durch, die einige Jahre vorher überhaupt die Weiterbildungsschule gebracht hatte. Anstelle von homogenen Klassen wurden zwei Leistungszüge eingeführt, welche die schulische Ausrichtung von der Integration weg hin zu mehr Leistung verschob. Doch anscheinend reichte auch diese Nachbesserung nicht.

Die WBS ist eine heterogene Schule. Sie nimmt alle diejenigen Schülerinnen und Schüler auf, die nicht das Gymnasium besuchen. Da schneller und langsamer Lernende, hoch und gering motivierte Schülerinnen und Schüler zusammenkommen, steht diese Schule hinsichtlich der Integration verschiedenster Anforderungen wie z.B. auf dem Feld des Unterrichtsmanagements, des sozialen Lernens, der Lehrkräftezusammenarbeit und der Schulorganisation um nur einige zu nennen - vor enormen konzeptionellen und pragmatischen Herausforderungen. Konzeptionelle

172 Basler Zeitung, 30.4.2003, 18.

173 Für 2004 aus den Statistiken der rechtsrheinischen Quartiere errechnet (Basel-Stadt Statistik Startseite, http://www.statistik-bs.ch, 4.3.2009). 
Überlegungen wie z.B. die Verkleinerung der Klassengrösse oder die Aufteilung der Schülerschaft in noch differenziertere Leistungsniveaus sind nicht selten auch von der Vorstellung eines Abbaus von Heterogenität motiviert. Dem steht die insgesamt erstaunliche und erfreuliche soziale Integrationsfunktion dieser Schule gegenüber, die vor allem das Ergebnis täglichen Bemühens vieler Lehrkräfte darstellt. ${ }^{174}$

Es wurden also immer noch zu viele Energien für die Integration aufgewendet. Deshalb scheint auch diese Lösung nicht befriedigt zu haben: Im Juni 2000 wurde aufgrund des Zwischenberichts einer externen Evaluation das sogenannte Aktionsprogramm I "zur raschen Verbesserung der WBS" gestartet, das einige Sofortmassnahmen brachte, wie Förderung der leistungsstarken Schülerinnen und Schüler, weniger Verlust von Lernzeit und bessere Koordination beim Schulwechsel und Übertritt in die Lehre. ${ }^{175}$

Vielleicht wollte man auch politischen Vorstössen der erstarkten populistischen Rechte zuvorkommen, die in anderen Kantonen zur Lösung dieses Konflikts getrennte Klassen für Schweizer Kinder und für solche mit fremdsprachigem Hintergrund forderte. ${ }^{176}$

Meine Feldforschungstätigkeit fiel also in eine Zeit ständigen Umbaus. Als ich dort war, wurde der Unterricht in den Hauptfächern Deutsch, Französisch und Mathematik für eine Schulklasse in zwei Leistungsniveaus ( $\mathrm{G}$ für Grundniveau und $\mathrm{E}$ für erweitertes Niveau) geführt. In den Nebenfächern fand der Unterricht im Klassenverband statt.

174 Weiterbildungsschule Basel: Bericht der Arbeitsgruppe “Allgemeine Integrationsarbeit" zur Vorbereitung der Strukturänderung, 2003, 2.

175 Weiterbildungsschule Basel-Stadt: Jahresbericht 2000/2001, 3-4.

176 Für eine vertiefte Auseinandersetzung mit diesem Thema siehe Ochsner, Peter, Urs Kenny und Priska Sieber: Vom Störfall zum Normalfall. Kulturelle Vielfalt in der Schule, Chur und Zürich 2000, Rüegger. 
Daneben gab es vielfältige Förderangebote und Wahlfächer, für Schülerinnen in akuten Krisensituationen die Kriseninterventionsstelle (KIS), wohin Schüler aus dem regulären Unterricht zeitweise ausgegliedert werden konnten.

Doch bereits im Schuljahr 2004/2005 wurde alles anders, weil sich die Regierung Sorgen machte. Auf rechtsrheinischem Gebiet waren nur $28 \%$ der Schülerinnen und Schüler ins Gymnasium übergetreten, im Gegensatz zum Kantonsdurchschnitt von 40\%. Es lief eine Versuchsphase mit zu Klassenverbänden gefassten, getrennten Leistungszügen an, die Entscheidungsgrundlagen für eine grössere Reform im Jahre 2008 liefern sollte. ${ }^{177}$ Dabei wurde der Grund-Leistungszug in A-Zug umbenannt, das A steht für Allgemein, weil der G-Zug und damit seine Teilnehmenden während seiner kurzen Lebensdauer bereits als "Ghetto"-Zug stigmatisiert wurde. Bei diesem Versuch wurde die Schulklasse auf Kosten der Leistungszüge aufgewertet. Die Entmischung wurde also forciert, indem die Leistungszüge sich zu Schulklassen entwickelten.

Der Klassenverband wird das tragende Element integrativer Schulung an der neuen WBS. Wünschenswert wäre sogar, wenn er vom fünften bis zum neunten Schuljahr stabil bleiben könnte, damit die Jugendlichen auf ihrem Weg in die Verunsicherung der Pubertät auf die Sicherheiten aus der Kindheit zurückgreifen können. ${ }^{178}$

Aus ethnologischer Sicht bemerkenswert ist auch das Selbstbild der WBS, das im Leitbild 2003 formuliert worden ist. ${ }^{179}$ Unter dem Titel

177 Basler Zeitung, 30.4.2003, 18.

178 Weiterbildungsschule Basel: Bericht der Arbeitsgruppe “Allgemeine Integrationsarbeit", 6 . 
"Lernen ein Leben lang" wird anerkannt, dass diese Schule einen liminalen Raum darstellt: "Im Zentrum steht der Übergang". ${ }^{180}$

Die WBS schliesst die obligatorische Volksschule ab und bildet die Schwelle zu den Berufslehren und den weiterbildenden Schulen. Sie bereitet die Jugendlichen auf die veränderten Anforderungen der nachobligatorischen Ausbildung vor und hilft ihnen, realistische Lebens- und Berufsziele zu entwickeln.

Das Ziel ist Integration: Die WBS setzt sich dafür ein, dass alle ihre Schülerinnen und Schüler einen würdigen Platz in der Arbeitswelt und in der Gesellschaft finden können. ${ }^{181}$

Die WBS hat für sich auch einen Integrationsbegriff definiert:

Integration (ist nicht) allein (...) Integration von ausländischen Jugendlichen in die schweizerische Gesellschaft, obwohl dieser Aspekt speziell berücksichtigt werden muss. Es geht generell darum, dass sowohl jeder jugendliche Mensch sich in die Gesellschaft, in die er hineinwächst, eingliedern soll, als auch darum, dass die Gesellschaft jeden jugendlichen Menschen aufzunehmen bereit ist. (...) Sich einbringen und eingelassen werden ist nur möglich, wenn man vertiefte Kenntnisse voneinander hat. Und dies ist wiederum nur möglich, wenn Jugendliche beides kön-

179 Weiterbildungsschule Basel: Bericht der Arbeitsgruppe "Leitbild" zur Vorbereitung der Strukturänderung, 2003.

180 Ebd., 6.

181 Ebd., 6. 
nen: sowohl in ihrer eigenen kleinen Welt zu Hause sein als auch zur grösseren, sie umgebenden Welt sich zugehörig fühlen. ${ }^{182}$

Integration ist also für die Schule mit dem Erwerb von Kenntnissen verbunden, die es möglich machen sollen, sowohl in der kleinen wie auch in der grossen Welt zu Hause sein zu können. Auf der Ebene des alltäglichen Unterrichts bedeutet das, dass die Lehrpersonen in beiden Zügen unterrichten sollen, damit sie Kenntnis und Erfahrung in beiden Bereichen sammeln können. Auch für die Jugendlichen sei das wichtig.

In den Pflichtwahlfächern ist es möglich, A- und E-Schüler/innen gemeinsam zu unterrichten, was wiederum dem Prinzip vom kleinen Eigenen zum grösseren Anderen entspricht. Ein Schüler, eine Schülerin ist eben nicht nur AZügler/in oder E-Zügler/in, sonden auch WBSler/in. Dieses erweiterte Bewusstsein in den Jugendlichen zu verankern, wird Aufgabe der unterrichtenden Lehrpersonen sein. Für sie ist die Durchmischung in den Pflichtwahlfächern eine grosse Herausforderung. ${ }^{183}$

Das Finden des richtigen Mischungsverhältnisses war in der WBS Basel-Stadt im Jahre 2005 noch im vollen Gang. Die Leistungszüge waren zwar nominell zu eigenen Klassen geworden, dennoch mochte man das Mischen nicht aufgeben, weil für die Schule das wichtigste Ziel die Integration ist. Auf organisatorischer Ebene verband man deshalb die Schulklassen zu sogenannten Stammgruppen, um flexibel den

182 Weiterbildungsschule Basel: Bericht der Arbeitsgruppe “Allgemeine Integrationsarbeit" zur Vorbereitung der Strukturänderung, 2003, 4. 183 Ebd., 5. 
Durchmischungsgrad zu regeln und so eine stufenweise Eingliederung zu erreichen.

Die Grösse dieser Gruppe kann je nach Schulstandort unterschiedlich sein, aber mindestens je eine Klasse aus dem A-Zug und eine Klasse aus dem E-Zug bilden ein Tandem. Damit ist ein Grundprinzip, auf dem Integration aufbaut, gewährleistet: sowohl unter sich als auch mit Fremden zu sein, also die stufenweise Vergrösserung des integrativen gesellschaftlichen Bewegungsraumes. ${ }^{184}$

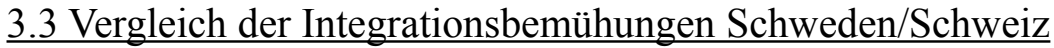

Zur weiteren Erhellung der Integrationsbegrifflichkeiten und verschiedenen Zugehensweisen zu Integrationsfragen in Schulen soll abschliessend ein grenzüberschreitender Vergleich herangezogen werden. Diese Studie arbeitet sehr stark mit schwedischer Literatur, vor allem zum Thema Integration im Schulwesen, was auf einen Zufall zurückzuführen ist: Damals wohnte ich in Stockholm. Es könnte allerdings auch mit Literatur aus Österreich gearbeitet werden, denn dort wurde Anfang der 1990er Jahre das sogenannte „Interkulturelle Lernen“ eingeführt. ${ }^{185}$

184 Ebd., 6.

185 Für einen Überblick vgl. Fillitz, Thomas (Hg.): Interkulturelles Lernen. Zwischen institutionellem Rahmen, schulischer Praxis und gesellschaftlichem Kommunikationsprinzip. In: Bildungsforschung 18, Insbruck, Wien Bozen, Studien Verlag, 2003 oder für eine praxisbezogene Sicht Binder, Susanne: Schule und Migration, Einblicke in die Praxis des interkulturellen Lernens, in: SWS-Rundschau, Schwerpunkt ,ZuwandererInnen-Integration mit Formen interkulturellen Lernens in Wien“, 4/42, Wien 2002, 422-440. 
Die Schulen nicht nur in der Schweiz und in Schweden sind mit ähnlichen Problemen konfrontiert: Da wie dort müssen fremdethnische Schülerinnen und Schüler in Klassenverbände integriert werden und sie müssen nach der Schule eine Perspektive in den Arbeitsmarkt aufweisen. Deshalb war es aufschlussreich, zu sehen, wie die jeweilige lokale Kultur die Auffassung von Integration, deren Ziele und Methoden beeinflusste. Diese Differenzen liessen Rückschlüsse über das Phänomen der Integration zu. Da wie dort war das richtige Mischverhältnis von Fremden und Einheimischen ein grosses Thema. Das Prinzip des Mischens von ausländischen und einheimischen Jugendlichen zur Förderung der Integration ist aus ethnologischer Sicht offensichtlich erklärbar. Migranten treten von einer Kultur in eine andere über; auch dieser Übergang wird wie jeder Übergang in drei Phasen vollzogen, die rituell markiert sind. ${ }^{186}$

Die schwedische Schulethnologin Ann Runfors bezeichnete diese drei Schritte in ihrer Schrift über Probleme mit Migranten in schwedischen Schulen ${ }^{187}$ als Abtrennen, Formen und Einfügen. Dieses Prozedere arbeitet mit Bedeutungszuschreibungen und markiert die Grenze zwischen den Kulturen - konstruiert also Menschen der Kategorie Migrantinnen - und bietet gleichzeitig ein Mittel, diese Grenze zu überwinden: Ausschliessen, um zu integrieren. Im Folgenden möchte ich den schwedischen Zugang zur Integrationsproblematik an Schulen vorstellen, bevor ich die Gründe erläutere, weshalb ich am WBS-Schulhaus und gerade in der 2D und nachher im Brückenangebot Basis arbeitete.

186 Van Gennep, Arnold: Übergangsriten (Les rites de passage),

Frankfurt/New York, Campus, 1986. Dieses zentrale theoretische Konzept wird im Kapitel 5.5 Begriffe, Konzepte und theoretische Felder ausführlich behandelt.

187 Runfors, Mångfald, motsägelser och marginalisieringar, 2003, $98 \mathrm{ff}$. 
Das schwedische Lehrpersonal hatte die Ambition, die Kinder von Immigranten in einem dreiteiligen Prozess in die schwedische Kultur einzugliedern. Dies war die Übersetzung der Forderung von Regierungsdirektiven ${ }^{188}$ in die Praxis. Diese Direktiven sahen kulturelle Vielfalt als etwas an, das es zu respektieren und erhalten galt und als Ressource, mit der man leben lernen musste. Die konkrete Umsetzung überliess man dem Lehrpersonal. Dies ist nicht als Vernachlässigung aufzufassen: Der Schwedische Staat kann bereits seit dem Spätmittelalter als Nationalstaat angesehen werden, dessen Beamtenschaft aufgrund der teilweise enormen Distanzen zum Hof weitgehend selbständig arbeiten musste. Im 17. Jahrhundert gab es Kolonien im heutigen Nordschweden, Finnland, Russland, im Baltikum, Deutschland und in den heutigen USA. Die Zentrale hat angesichts dieser geografischen Begebenheiten eher richtlinienartige Weisungen erteilt, nicht im Detail regiert und auf die Kreativität der lokalen Vertreter der Krone vertraut.

Dieser Zugang findet sich auch in den Formulierungen zur Integrationspolitik. Die Lehrerinnen und Lehrer sahen jedoch die wegen der kulturellen Differenzen anders gelagerten Erfahrungen der eingewanderten Schülerinnen und Schüler trotz des Integrationsanspruchs im Schulalltag selten als etwas Positives an. Sie konzentrierten ihre Anstrengungen hauptsächlich auf Erfahrungen und Fähigkeiten, welche diesen Schülern fehlten und auf solche, die sie erwerben mussten, um kulturell kompetent nach schwedischen Vorstellungen zu werden. Diese Vorstellung war stark von den persönlichen Erfahrungen des Personals geprägt. Was als kulturelle Ressource angesehen werden konnte, war das, was als akzeptabel innerhalb sozial dominanter Werte galt. Das Kulturelle konnte aber auch als negativ angesehen werden, wenn es mit diesen dominanten Werten in Konflikt geriet. Dann musste es verändert oder bekämpft werden. Möglicherweise wurden soziale oder ökonomische Probleme umdefiniert oder reduziert auf kulturell geprägte Befun1881994 erlassen. 
de wie Modernitätsrückstand, kulturelle Differenz und soziales Kompetenzdefizit. In diesem Feld ist Sorgfalt angebracht: Wolfgang Kaschuba warnt davor, Kritik an der Gesellschaft in Kritik an der Kultur umzuinterpretieren. Dies könne eine fragwürdige politische Strategie darstellen. ${ }^{189}$

Im Alltag wurde Kultur als das interpretiert, was vom Normalen, Gewöhnlichen oder Wünschenswerten abwich. Dies konnte eine positive Bedeutung annehmen, akzeptiert oder gar erwünscht sein. Oft waren dies individuell erworbene Fähigkeiten im expressiven Bereich, etwa Esskultur, Musik, Tanz, Brauch oder Ritual. Als negativ angesehen wurden jedoch oft von der kulturellen Gemeinschaft aufgezwungene, kollektive Kulturzüge, die schwierig, mühsam oder unerwünscht wirkten. Ein Beispiel dafür waren etwa Beziehungen, welche die Schule und die Gesellschaft als unakzeptabel ansahen und die Vorrang vor dem Individuellen hatten. Von solchen unerwünschten kulturellen Erscheinungen mussten die Schülerinnen und Schüler sozusagen getrennt werden.

Dann sahen die Lehrer ihre Rolle darin, in einem nächsten Schritt die Schüler zu formen. Eine Schlüsselrolle nahm dabei der Sprachunterricht ein. Es wurde angenommen, dass wenn die Schüler sogenannt gutes Schwedisch sprächen, sie am besten integriert seien. ${ }^{190}$ Diese Annahme erwies sich als falsch, da Sprache subtile kulturelle Differenzen transportieren kann, die im Nachhinein praktisch nicht mehr erlernbar sind. Daneben wurden Werte einer urbanen Mittelklassekultur vermittelt. Legitimiert wurde das Formen der Schüler durch pädagogische Normen und Ansichten über Gleichheit und Autonomie. Das Abtrennen

189 Kaschuba, Wolfgang: Kulturalismus: Kultur statt Gesellschaft? in: Geschichte und Gesellschaft 21, 1995, Heft 1, 83.

190 Für den Zusammenhang von Sprache, Alltag und Integration vergleiche auch Jacobsson, Anna: En plats i klassen, en plats i världen, en studie om eleverna i en klass i norra Botkyrka, Botkyrka, Mångkulturellt Centrum, 1995. 
und Angleichen war Voraussetzung für den nächsten Schritt, das Mischen mit "kulturreinen" Schweden.

In der Schulpraxis wurde Integration hauptsächlich durch Mischen von Immigranten- und Schwedenkindern in liminalen Situtationen wie auf Reisen oder bei Feiern angestrebt. Menschen in liminalen Situationen zeichnen sich durch eine intensive Kameradschaft aus, was integrierend auf eine Gruppe wirkt. Die Mischung war auch ein Symbol dafür, dass das Ziel der Partizipation erreicht worden war und sollte die Gleichheit vertiefen. Das Gleichmachen war mit Ähnlichkeit als Ideal verbunden. So gesehen waren kulturelle Differenzen etwas Problematisches, vor allem wenn sie zum Beispiel als ungerecht angesehen wurden. Dann mussten sie zum Verschwinden gebracht werden.

Das Lehrpersonal versuchte also, Migrantenkinder in einer Schulklasse als eine imaginäre, hierarchiearme Gemeinschaft zu integrieren, mit der schwedischen Sprache als wichtigstem Zusammenhaltsfaktor. Ziel war Gleichheit im Sinn einer kulturellen Uniformität, so wie es den wesentlichsten Werten der egalitären schwedischen Gesellschaft entsprach. Die Klasse war also zu einer Gruppe geworden, welche die Lehrervision eines zukunftgerichteten, modernen Schwedens im Kleinen darstellte, und kompatibel mit der Gesamtgesellschaft sein sollte. ${ }^{191}$ Das stellt einen Zugang dar, der die schwedische Auffassung von Integration als einen Gesellschaftszustand, bei dem ein starker Zusammenhalt vorhanden ist, reflektiert.

In Schweden begreift man kulturelle Vielfalt zwar offiziell als Ressource, mit der man aber merkwürdigerweise zu leben lernen hat, wie wenn diese Ressource eine Belästigung wäre. Wie das konkret umzusetzen ist, überlässt man den Lehrern, also einer aus historischen Gründen unabhängigen kulturhomogenen Beamtenschaft, im Vertrauen auf deren stark normative schwedischen Werte. Es liegt auf der Hand, dass

191 Runfors, Mångfald, motsägelser och marginalisieringar, 2003, 197 und 241-248. 
dieser Zugang dort an Grenzen stösst, wo es zuwenig Einheimische gibt, mit denen man Einwanderer mischen könnte, etwa in den von Segregation gekennzeichneten Vorstädten der grossen Zentren.

In der Schweiz gelten andere Werte. Hierzulande stellen kulturelle Besonderheiten grundsätzlich kein Problem dar, weil die Schweiz sich als eine Willensnation verschiedener Kulturgruppen ${ }^{192}$ sieht und kein Zentralstaat ist, der sich über eine homogene Kultur und Sprache definiert. Alle Kantone traten einst der Eidgenossenschaft durch einen Willensakt bei, meist aus Gründen der inneren und äusseren Sicherheit. Sie hatten zum Teil gemischte Kulturen, wie etwa Bern, Freiburg oder Graubünden, und behielten im überwiegenden Masse ihre Verwaltung bei, wurden also nicht durch eine entfernte Zentralverwaltung regiert. Diese Faktoren spielen in der Integrationspolitik eine Rolle: Sie wird von den Kantonen umgesetzt, wobei der Bund die Vorgaben macht.

In der baselstädtischen Ausprägung der schweizerischen Integrationspolitik begreift man kulturelle Eigenheiten nicht als Mangel. Zwar berücksichtigt man speziell die Integration von ausländischen Jugendlichen in die schweizerische Gesellschaft, betont aber, dass es darum geht, Individuen einzugliedern und die Gesellschaft an ihre Pflicht zu erinnern, jeden jungen Menschen aufzunehmen, egal welche kulturellen Züge dieser Mensch mitbringt. Das Personal gehört auch nicht einer homogenen Kultur an und erhält deshalb einen konkreten Auftrag: Es muss das Verankern dieses Bewusstseins noch lernen und hat zu diesem Zweck mit gemischtem Unterricht Erfahrungen zu sammeln.

192 Etwa Bundesverfassung der Schweizerischen Eidgenossenschaft vom 18.4.1999: Präambel. 


\section{$\underline{3.4 \text { Verlängerung der Schule in den Brückenangeboten }}$}

Für die Klasse 2D endete die obligatorische Schulzeit mit einer Reise nach Südfrankreich Ende Juni 2004, an der ich teilnahm. Am Morgen nach der Nacht ihrer Rückkehr erhielten die Schülerinnen und Schüler ihr Schlusszeugnis, danach waren Ferien. Am 8. August 2004 begann also das nachschulische Leben für die ehemaligen 2D-ler. Zwei traten in die Fachmittelschule FMS (früher: DMS, Diplommittelschule) ein, zwei besuchten neu die Gewerbeschule und zwei die InformatikMittelschule. Einer begann eine Lehre als Motorradmechaniker, einer eine Vorlehre als Maler, drei wollten nicht mehr beforscht werden und acht besuchten sogenannte Brückenangebote.

Die Brückenangebote bestehen aus Kursen, die ab 2001 im Zuge der Lehrstellenkrise vom Kanton Basel-Stadt eingerichtet wurden. Sie sollen in einem intensiven 10. Schuljahr punktuelle Bildungsdefizite ausbessern, um die Schülerinnen und Schüler auf den Lehrstellenmarkt vorzubereiten. Es gab zehn verschiedene Brückenangebote, zwei mit allgemeiner Richtung, drei eher für Migrantinnen mit Deutschdefiziten und fünf mit Schwerpunkt auf praktischen Berufserfahrungen. ${ }^{193}$ Sie verlängern, wie der Name antönt, die Übergangszeit von der Schule ins Berufsleben um ein Jahr. Diese verlängerte Übergangsphase ist eine Zeit, in der die Bildungslücken der vom nicht geglückten Übergang betroffenen Menschen sozusagen ausgebessert werden sollen, bis sie fähig sind, den Übergang ins Erwachsenenleben zu schaffen und sich ökonomisch zu integrieren.

Da acht der von mir begleiteten Jugendlichen in verschiedene Brückenangebote überwechselten, beschloss ich nach Konsultation der Kolleginnen, dieser grössten Gruppe in ihr Zwischenjahr zu folgen. Drei Schülerinnen und Schüler, Janine Maggiorini, Akdis Saliba und

193 Vgl. Brückenangebote Basel-Stadt sba-basel.ch (Schule für Brückenangebote Basel: Start, http://www.sba-basel.ch, 4.3.2009). 
Stefan Staub, wechselten in das Brückenangebot Basis über, in die neue zusammengestellte Klasse 10B. Sie bestand aus 17 jungen Menschen, die alle aus der WBS hergekommen waren. Der Lehrplan war sehr stark darauf ausgerichtet, den jungen Menschen Fächer zu vermitteln, in denen sie Schwächen hatten, wie etwa Mathematik und Französisch. Daneben gab es einen sehr starken Praxisbezug: häufig waren Leute abwesend zu Praktika, es wurden Standortbestimmungen durchgeführt, und Bewerbungstheorie wurde gross geschrieben. Dort hielt ich mich vom August 2004 bis zum Juni 2005 auf und konnte beobachten, wie sich die jungen Menschen, die ich bereits kannte, in diesen neuen Zusammenhang integrierten und sich gleichzeitig weiter stark darum bemühten, sich eine Perspektive über dieses erzwungene 10. Schuljahr hinaus zu verschaffen. 


\section{Teil II}

Jugend in der sozialwissenschaftlichen Forschung 


\section{Historische Bedingungen von Jugend}

Nachdem ich im vorhergehenden Abschnitt ausgehend von methodischen Überlegungen beschrieben habe, wie sich die Einbettung des Forschers in das Feld der Schule gestaltete und welche praktischen und theoretischen Überlegungen den Forschungsverlauf beeinflussten, möchte ich in diesem Abschnitt auf die historischen Bedingungen des Jungseins nach der Jahrtausendwende in den Industrieländern und und danach konkret und lokal eingehen. Diese Ausführungen dienen dazu, die historischen Bedingungen der Lebenswelt der hier im Fokus stehenden Jugendlichen darzustellen. Verschiedene unten beschriebene Faktoren beeinflussen das Erwachsenwerden in den untersuchten Stadtteilen und die Einstellung der Jugendlichen zur ökonomischen Selbständigwerdung erheblich und haben so einen grossen Einfluss auf die Integration der Jugendlichen. Zunächst sollen die globalen Entwicklungen skizziert werden.

\subsection{Der jugendliche Gesellschaftsvertrag wird neu verhandelt}

An der Schwelle zum neuen Jahrtausend sind ganz grundlegend neue ökonomische Bedingungen des Erwachsenwerdens festzustellen. Für die schwedische Jugendforscherin Britta Jonsson liegen die Gründe darin, dass die westeuropäischen Gesellschaften eine tiefgehende Strukturveränderung durchlaufen. ${ }^{194}$ Auf theoretischer Ebene sind diese Veränderungen unterschiedlich beurteilt worden: Von Forschern wie Lyo-

194 Jonsson, Britta: Unga vuxna, deras livsprojekt och tankar om framtida yrke, in: Hagström (red.): Ungdomar i övergångsåldern - handlingsutrymme och rationalitet på väg in i arbetslivet, Lund, Studentlitteratur, 1999, 58-89. 
tard und Baudrillard als historische Epochenwende ähnlich wie der Übergang vom Mittelalter zur Neuzeit, die - historisch nach der "Moderne", als "Postmoderne" bezeichnet wird. ${ }^{195}$ In dieser postmodernen Ära seien die klassischen Gesellschaftstheorien nicht mehr anwendbar, traditionelle Muster, welche das Leben der Individuen mehr oder weniger voraussehbar machten, und Schlüsselbegriffe wie Klasse oder Geschlecht seien nicht mehr erklärungskräftig.

Andere Theoretiker sind etwas vorsichtiger und glauben, es handle sich um eine Weiterentwicklung der modernen Ära. Giddens spricht beispielsweise von Hochmoderne oder Spätmoderne ${ }^{196}$ und Lash verwendet den Begriff reflexive Modernität, wo das Individuum erhöhte Verantwortung für sein Leben übernehmen muss, was mehr persönliches Überlegen, Strategie und Nachdenken erfordert. Vielleicht gab es nach dem Ende der Sowjetunion 1991 ein Bedürfnis, eine Epochengrenze zu konstruieren; Furlong und Cartmel ${ }^{197}$ etwa geben sich solchen Theorien gegenüber skeptisch und betonen die Kontinuität im Modernisierungsprozess, indem sie den Begriff Spätmoderne verwenden. Die zu beobachtende Schwächung traditioneller Bindungen, mehr Anonymität in den Beziehungen und versteckte Ausbeutung im fortgeschrittenen Kapitalismus seien Erscheinungen, die bereits im Kommunistischen Manifest von 1848 kritisiert werden, und somit nichts Neues. ${ }^{198}$

195 Vgl. Lyotard, J.-F.: The Postmodern Condition. A Report on Knowledge, Minneapolis, University of Minneapolis Press, 1984 und Baudrillard, J.: Selected Writings, Oxford, Oxford University Press, 1988.

196 Giddens, Anthony, The Consequences of Modernity, Oxford, Polity, 1990 sowie Modernity and Self Identity, Self and Society in the Late Modern Age, Oxford, Polity, 1991.

197 Furlong, A. und Cartmel, F.: Young People and Social Change. Individualization and Risk in Late Modernity. Buckingham, Open University Press, 1997.

198 Nach Jonsson, Unga vuxna, deras livsprojekt och tankar om framtida yrke, 1999, 60. 
Jedenfalls lässt sich vor allem nach 1980 eine Erscheinung festmachen: die sich weiter ausdehnende Individualisierung. Der Fokus schiebt sich von sozialen und wirtschaftlichen Strukturen zu Prozessen, von Status zu Funktion, von Rollen zu Initiativen, von Sozialisationsprozessen zu Individualisierungsprozessen, von politischen Anstrengungen, die auf gemeinsame soziale Fortschritte zielen, zum Bestreben, das individuelle Lebensprojekt voranzubringen.

In der modernen Ära wurde es als normal angesehen, dass das Leben von Individuen in Abschnitte mit dazwischenliegenden Übergangszeiten aufgeteilt war: Kindheit - Erwachsenenleben - Alter, oder, wenn man die Arenen betrachtet: Ausbildung - Arbeitsleben - Pension. Jugend wurde dabei als die einzige Übergangszeit von Kindheit zum Erwachsenenleben charakterisiert, in der die gesellschaftliche Aufgabe darin bestand, in die Ausbildung zu investieren, um den Nachwuchs an notwendiger Arbeitskraft und damit den Fortbestand der Gesellschaft zu garantieren. ${ }^{199}$ Auf der Ebene des Lebenslaufs waren dies Altersphasen und nicht Phasen, die getrennt waren von prinzipiell vom Lebensalter unabhängigen biografischen Ereignissen.

Die meisten Jugendlichen wurden in Jahrgangsgruppen in das Arbeitsleben integriert. Es war normal, nach dem Schulabgang ins Erwerbsleben einzutreten, was für die meisten den definitiven Übergang von der Jugend- und Ausbildungsphase in ein produktives Arbeits- und Erwachsenenleben bedeutete. Das Altersintervall dieser Übergangsphase war begrenzt. Nur eine Minderheit jeden Jahrgangs verlängerte die Jugend- oder Ausbildungsphase mit einem Studium.

Die Veränderung der Biografien beruhen auf einer tiefgreifenden Umstrukturierung der Wirtschaft. In den beiden Jahrzehnten nach 1980 kam es wegen der flächendeckenden Einführung des Computers, der

199 Wallace, C., und Kovacheva, S.: Youth in Society. The construction and Deconstruction of Youth in East and West Europe, Basingstoke, MacMillan Press Ltd.,1998. 
fortschreitenden Automation in der Industrie, der Einführung des Internets und einer Vielzahl von Phänomenen, die man unter dem Begriff Globalisierung ${ }^{200}$ zusammenfasst, zu enormen Produktivitätssteigerungen. Der Arbeitsmarkt wurde komplexer und die Berufsbilder und Lebensläufe differenzierten sich immer mehr aus. Dieser Prozess nahm an Fahrt zu, als der Kommunismus zusammenbrach oder reformiert wurde, weil diese Ökonomien in die Weltwirtschaft integriert wurden. Plötzlich standen hunderte von Millionen Arbeitnehmerinnen und Arbeitnehmer in Osteuropa und Asien in Konkurrenz zu den westlichen Arbeitskräften. Diese Entwicklung hatte erhebliche Auswirkungen auf den "Jugendlichen Gesellschaftsvertrag". Gesellschaftsvertrag deshalb, weil die Rechte der Individuen gegenüber dem Staat, aber auch die Ansprüche des Staats gegenüber Individuen in unserer Gesellschaft durch einen rousseauschen contrat social geregelt sind.

Hier haben sich die Gewichte verschoben. Traditionell funktionierte die Schule als wichtigste Vorbereitung für den Eintritt ins Arbeitsleben. Bis zum Ende der Systemkonkurrenz von Kapitalismus und Kommunismus galt folgendes Angebot: Wer sich den Regeln der Schule unterwirft, erhält nach der Schule eine Stelle. Dabei korrelierte die Schuldauer mit der Qualität der Tätigkeit, die nachher aufgenommen werden konnte.

Da Stabilität, Ruhe und Ordnung für die Produktion sehr wichtig waren, betonte man diese Werte auch in der Schule. Die Schüler unterwarfen sich den Regeln und zeigten Respekt gegenüber Autoritäten. Sie wurden nicht für eigene Ideen oder Lösungen belohnt, sondern für Disziplin und Kooperation, und so für die hierarchisch organisierte Ar-

200 Weltweite wirtschaftliche Verflechtung, Migration und andere Phänomene. Ein wichtiger Aspekt ist die Ausweitung des tertiären Wirtschaftssektors, des Dienstleistungssektors, zulasten des primären und sekundären. Die sekundäre Wirtschaftssektoren wanderten in sogenannte Schwellenländer wie China und Indien ab. 
beitswelt vorbereitet. Dieses Konzept spiegelte sich auch in der Einrichtung der Schulzimmer: Die Ausgestaltung mit Bänken gab den Lehrern die Möglichkeit zur Beaufsichtigung nach denselben rationalen Prinzipien wie in der Fabrikhalle. Wie diese Hierarchien reproduzierende Konstellation auf die Jugendkultur und die Integration der Jugendlichen prägend wirkte, hat beispielsweise Paul Willis in seiner Studie über englische Jugendliche der Arbeiterklasse gezeigt. ${ }^{201}$

Doch ist es zum Zeitpunkt dieser Studie für viele Jugendliche Realität, dass eine lange Ausbildung nicht automatisch zum wohl entlohnten Job mit Status führt. Nach dem Ende der Systemkonkurrenz und der Vertiefung der Globalisierung war dieser Gesellschaftsvertrag im Westen nicht mehr haltbar. Plötzlich gab es keine Versprechen mehr für ihre Zukunft. Deshalb erleben viele Schülerinnen und Schuler die Ausbildung als reine Verschwendung ihrer Zeit. Sie können auch nicht erwarten, eine lebenslange Anstellung zu erhalten wie ihre Eltern. Sie können sich nicht ein für alle mal ausbilden, sondern es kommt zu "lebenslanger" Ausbildung. Das gesellschaftliche Angebot an die Jugendlichen hat sich also drastisch verschlechtert.

Tiefgreifende Veränderungen haben die westliche kapitalistische Wirtschaftsweise umgestaltet. In der stetig sich ausweitenden und verändernden Warenproduktion haben sich die Arbeitsbedingungen grundlegend geändert. Internationalisierung, technische und elektronische Innovationen haben die Produktion in Richtung Flexibilität und Beweglichkeit umstrukturiert. Um schnellere Wechsel der Produkte und ihrer Herstellungsart zu erreichen, bieten sich drei Vorgehensweisen an. Erstens kann Flexibilität durch neue Technologien, Logistik- und Arbeitsmethoden erhöht werden, zweitens erlauben es flexible Organisationen, arbeitsintensive Herstellungsschritte in Länder mit niedrigeren Lohnkosten $^{202} \mathrm{zu}$ exportieren und drittens kann die Finanzierung der Produk-

201 Willis, Learning to Labour, 1983. 
tionsmittel dank elektronischer Mittel innert Sekunden sichergestellt oder auch verweigert werden.

Unqualifizierte Arbeiten, auf welche Einsteigerinnen und weniger gut ausgebildete Arbeitnehmer angewiesen waren, fallen in Mitteleuropa zum grossen Teil weg. Die grossen, hierarchischen Belegschaften sind ausgetauscht worden gegen kleine, wissensintensive Gruppen. ${ }^{203}$ Arbeiten wie etwa Werbung oder die Betreuung der IT-Infrastruktur, die früher innerhalb einer Firma ausgeführt wurden, werden heute an Beratungsfirmen, Unterlieferanten und Zeitangestellte ausgelagert. So haben die Arbeitgeber immer stärkere Kontrolle über den Arbeitsprozess, gleichzeitig haben immer weniger Arbeitende sichere Anstellungsbedingungen.

Doch die sichere Anstellung war wohl historisch immer die Ausnahme. Davon profitieren konnten die Arbeitnehmer, die nach dem Zweiten Weltkrieg in den Arbeitsprozess eintraten. Vor allem nach 1950 begann ein globaler Aufschwung, der zunächst alle entwickelten Industrieländer betraf und nach 1960 vor allem in den westlichen kapitalistischen Ländern weiter anhielt. Diese Zeit bezeichnet der britische Historiker Eric Hobsbawm als die Goldenen Jahre. ${ }^{204}$ Doch dieser Auf-

202 Beispielsweise die Auslagerung von Softwareentwicklung nach Indien. Outsourcing: Verschieben von firmeninternen Aktivitäten zu einer Firma ausserhalb, welche die Aufgabe kostengünstiger erledigen kann. Seit den frühen Neunzigerjahren haben grosse Firmen wachsende Teile ihrer Geschäfte ausgelagert mit der Begründung, dass beide Firmen davon profitieren: die outsourcende Firma konzentriert sich auf Aktivitäten, wo sie am meisten Wert erzeugen kann, die entgegennehmende Firma kann ihre Effizienz durch Skalenerträge und Spezialisierung steigern und somit billiger liefern. In der Praxis stösst Outsourcing bald an Grenzen der Beherrschbarkeit, vor allem für komplexe Aktivitäten. (Siehe auch Outsourcing Wikipedia, http://de.wikipedia.org/wiki/Outsourcing, 22.2.2012).

203 Stichworte: Kravattensilos, Hollywoodisierung, Outsourcing.

204 Hobsbawm, Eric: Age of Extremes. The Short Twentieth Century 19141991, London, Abacus, 1994, 257ff. 
schwung betraf nicht die Dritte Welt, die erst mit der Globalisierung so starke Fortschritte machte, dass sie die westlichen Länder konkurrenzierte und in Schwierigkeiten brachte.

Für die einzelnen Firmen und die Menschen, die in ihnen angestellt sind, ergaben sich Perspektivenveränderungen in allen Bereichen. In der Produktion etwa verschob sich der Fokus von der Standardisierung hin zur Kundenanpassung und zum Servicedenken. Für die Firma bedeutete dies Dezentralisierung anstatt Zentralisierung, qualitativ hochstehende Kleinserien anstatt Massenproduktion. Es gab allerdings auch Beispiele sehr grosser Firmen, die erfolgreich wurden, vor allem wenn sie auf Lifestyle-Produkte setzten, wie IKEA, Nokia, H\&M, Apple. Doch allen Firmen war gemeinsam, dass die früher ausgeprägten Hierarchien flachen Organisation mit wenigen Hierarchiestufen Platz machten. Die Menschen hatten sich auch zu ändern: Einfache Aufgaben verschwanden, qualifizierte Arbeit nahm zu, die Arbeitszeiten wandelten sich von "acht bis fünf" zu "wann auch immer", Gruppenarbeit ersetzte immer mehr die Einzelarbeit, die Einzelne wurden sozusagen vom austauschbaren Zahnrad zu Individuen mit einmaliger Kompetenz, die aber gleichzeitig eine hohe Flexibilität aufweisen mussten.

Lebenslanges Lernen wurde gleichzeitig ein Schlüsselbegriff dieser spätmodernen Gesellschaften und wird stark diskutiert. Der Begriff drückt einen permanenten Prozess persönlicher Entwicklung und darüber hinaus eine institutionelle Rahmung zur Ermunterung dieser Entwicklung aus. Aber lebenslanges Lernen beinhaltet auch eine Vielzahl von Fertigkeiten, das Vermögen, Kenntnisse aufzunehmen, diese zu hinterfragen, kritisch zu beleuchten und weiterzuentwickeln. Das wiederum bedeutet, dass Lernen einen Eigenwert erhält, also an sich wichtig ist, und nicht nur einen Nutzwert beinhaltet wie das Aufnehmen von Wissen.

Um sich in der postmodernen Gesellschaft orientieren zu können, brauchen die Jugendlichen das Vermögen, neue Bedeutung in ständigen 
neuen Zusammenhängen herstellen zu können und Mehrdeutigkeiten wie etwa widersprüchliche Forderungen wie Spezialisierung und gleichzeitige Flexibilität zu tolerieren. Es sind die Fähigkeiten und Haltungen gegenüber dem lebenslangen Lernen, deren Grundlagen in der Schule gelegt werden können, aber das verlangt auch eine Anpassung der rationellen standardisierten Jahrgangsorganisation und des normativen Denkens. $^{205}$

Diese Umstrukturierung ist ziemlich weit fortgeschritten. Beispielsweise macht man in Schweden bereits Versuche zur Neuausrichtung. Der letzte nationale Lehrplan 1994 weitet die Möglichkeiten der Schüler aus, flexibel eine individuelle Wahl zu treffen bezüglich der Schule und des Ausbildungsprogramms. Diese Reform spiegelt die Forderungen des Zeitgeists, der von Individualisierung, neuen Massenmedien und der schnell wachsenden Informationstechnologie geprägt ist.

Ende der neunziger Jahre ist es so, dass die Jugendlichen länger in der Schule behalten werden, da sie als Ungelernte nicht im Arbeitsleben benötigt wurden. ${ }^{206}$ Durch die Schulreformen der Sechziger und Siebziger Jahre in Deutschland und anderen europäischen Ländern verlängerten sich die Ausbildungszeiten und die Anzahl Jugendlicher, welche eine verlängerte Ausbildungsphase in Anspruch nahmen.

Es wurde immer weniger üblich, einen definitiven Übergang von der Ausbildung zur Arbeitsphase zu finden. Die Forscher stimmen überein, dass der Arbeitsmarkt und damit die Ausbildungswege immer differenzierter und komplexer werden. Damit werden sie auch schwerer vorausplanbar, strategisches Verhalten ist nahezu unmöglich geworden. Während längerer Lebensphasen pendeln die Individuen zwischen Ausbil-

205 Jonsson, Unga vuxna, deras livsprojekt och tankar om framtida yrke, 1999, $60 \mathrm{ff}$.

206 Vgl. Andersson, B.-E.: "Does School Stimulate Young People's Development?", in: Johnsson, B. (Hg.): Studies on Youth and Schooling in Sweden. Stockholm, HLS Förlag, 1995: In Schweden würden 34\% der Oberstufenschüler eine Arbeit annehmen, wenn sie könnten. 
dung, Arbeit, Arbeitslosigkeit und Arbeitsmarktmassnahmen hin und her; Auslandsaufenthalte und Perioden mit einer ganz anderen Beschäftigung können längere Abschnitte in Anspruch nehmen.

Weil dieses Hin- und Herschwanken zwischen verschiedenen Rollen nicht mehr begrenzt ist auf eine Zeit nach der Schule, sondern einen immer grösseren Teil des Lebens immer grösserer Bevölkerungskreise charakterisiert, kann festgestellt werden, dass die Jugend- und damit die Übergangsphase sozusagen auf das ganze Leben ausgedehnt wird. Die Erwachsenen übernehmen kulturelle Züge der Jugendlichen, um flexibel zu bleiben. Zeugin und Gros stellen in ihrer Studie über die Jugendkultur in der Schweiz aus dem Jahre 1991 fest, dass die Jugendkultur sich nicht mehr von der Erwachsenenkultur abgrenzt. "Die Erwachsenen gehen formal auf die Jugendlichen zu", schreiben sie, "und berauben sie um traditionelle jugendliche Verhaltensweisen". ${ }^{207}$ Die Erwachsenen müssen jugendlich bleiben. Lebensbahnen sind erratisch geworden. Jederzeit kann jemand gezwungen sein, sein Leben von Grund auf neu zu beginnen, eine neue Ausbildung anzufangen, einst ein Zeichen von Jugend. Der stagnierende Arbeitsmarkt in Mitteleuropa und fehlende Mittel sorgen dafür, dass es Junge immer schwerer finden, mit einer Familie und einem Job in Ruhe zu leben.

Aus diesen Gründen sollte man die Jugendzeit nicht mehr Übergangszeit nennen. Der Charakter des Übergangs kann das ganze Leben hindurch anhalten. Das bedeutet, dass sich die kulturellen Grenzen zwischen Jungen und Erwachsenen auflösen, dass die Funktion der Jugend als Vorbereitung auf das Leben immer unwichtiger wird. Das hat teilweise erwünschte Effekte auf die Freiheit der Individuen und deren Identitätsentwicklung. Sogar die Lebensstile sind immer weniger altersgebunden. Doch dies ist die Sicht der Erwachsenen, der bestimmenden Altersgruppe. Dreht man die Perspektive um, könnte man sogar argu-

207 Zeugin, Peter und Dominique Gros: Jugendkultur in der Schweiz, Kurzfassungen der Projekte des NFP 21, Basel 1991, 4-5. 
mentieren, dass das Erwachsenenleben wie es in der Moderne bekannt war, immer mehr am Verschwinden ist und dass die Menschen in den westlichen Kulturen immer länger kulturell jugendlich bleiben, bis sie in Rente gehen. Auch scheint es ein Problem zu sein, die Altersgruppe der Rentnerinnen und Rentner aufrecht zu erhalten, glaubt man den Prognosen der Demografen.

Heutige Jugendliche zeichnen sich im Gegensatz zur Jugend bis etwa 1980 durch ausgebreitete Individualisierung und Autonomie aus. Das hat grössere Bedeutung für deren Haltungen, Werte, Lebensprojekte und Verhalten wie etwa der Familienhintergrund und andere institutionalisierte Sozialisationsmilieus. Jugendliche sehen subjektiv weitgehende Wahlmöglichkeiten. Sie fassen ihre Biografie zwar als flexibles Projekt auf, dennoch hat die Tradition über Begriffe wie Klasse oder Geschlecht immer noch Einfluss darauf, wie Junge ihr Leben formen. ${ }^{208}$

\subsection{Die erste Generation des neuen Jahrtausends}

Im Folgenden möchte ich auf die Erkenntnisse verschiedener Studien eingehen, die in den letzten Jahren in Deutschland durchgeführt wurden und uns ein sehr genaues Bild der Werte und Einstellungen von Jugendlichen nach der Jahrtausendwende liefern. In diesem Abschnitt stütze ich mich hauptsächlich auf Material von Jürgen Zinnecker, einer der bedeutendsten Jugendforscher im deutschsprachigen Raum.

Die verschiedenen Jugendstudien, die seit den Fünfziger Jahren von der Firma Shell in Deutschland gefördert wurden, lassen Zinnecker ein ziemlich genaues Bild der jungen Generation des neuen Jahrhunderts zeichnen, die auch für die Verhältnisse in der Region Basel von Bedeu-

208 Jonsson, Unga vuxna, deras livsprojekt och tankar om framtida yrke, 1999, 77. 
tung sein dürften. Er stützt sich dabei auch auf Material von Jugendstudien aus dem Jahre $2001^{209}$ und $2002^{210}$ und stellt fest, dass die alten $\mathrm{Zu}$ schreibungen und Erfahrungen des vergangenen Jahrhunderts nicht mehr stimmen. Gegenwärtig formiere sich in Deutschland eine junge Generation, deren Mentalität anders sei, ja es zeige sich sogar ein radikaler Wandel von Jugend, meint er. ${ }^{211}$

Die Mentalität der Jugend hat sich in Richtung bürgerlicher Mitte verschoben. Noch in den achtziger Jahren waren Systemkritik und utopische Gegenentwürfe zur bestehenden Ordnung wichtig, heute ist die Loyalität zum System grösser. Jenseits ausgearbeiteter ideologischer Systeme werden pragmatische Lösungen für die grossen gesellschaftlichen und persönlichen Probleme gesucht. Die Mehrheit hält die globalen Probleme der Welt wie Krieg, Armut, Umweltzerstörung und Arbeitslosigkeit für nicht lösbar, doch für machbar hält sie einiges. Sie blicken daher optimistisch in eine ungewisse Zukunft der Spätmoderne und suchen nach privaten Lösungen für ihren eigenen Lebensweg.

Alte Werte, wie sie Jürgen Zinnecker nennt, Leistung, gute Bildungsabschlüsse, Karriere oder Treue, stehen bei den Jugendlichen hoch im Kurs. Das Bedürfnis nach Sicherheit ist ausserordentlich stark entwickelt. Auch haben sich die sogenannten postmaterialistischen oder nach Zinnecker „Neue Werte“, also Selbstverwirklichung und Verzicht auf Konsum, ganz und gar nicht durchgesetzt. Die jungen Leute sind konsumfreudig und an materiellen Genüssen orientiert und kombinieren

209 Eine mehrstündige schriftliche Befragung von rund 8000 10-18jährigen Menschen, Herbst 2001.

210 Jugendstudie der Deutschen Shell, Frühjahr 2002. Es wurden rund 2500 Jugendliche zwischen 12 und 25 Jahren in mündlichen standardisierten Interviews befragt.

211 Zinnecker, Jürgen: Alles ist möglich und nichts ist gewiss. Deutschlands erste Jugendgeneration im 21. Jahrhundert, in: Coolhunters. Jugendkulturen zwischen Medien und Markt, Frankfurt a. Main 2005, Suhrkamp, 175176. 
diese Werte mit einer hohen Wertschätzung der Werte der Kreativität und Toleranz. Beide Seiten zusammen bilden eine zentrale Orientierungsdimension.

Innerhalb der untersuchten deutschen Jugend lassen sich vier Gruppen mit unterschiedlichen Wertegewichtungen bilden. In der Jugendstudie 2002 werden diese mit den einprägsamen Etiketten pragmatische Idealisten, selbstbewusste Macher, robuste Materialisten und zögerliche Unauffällige belegt. Jede dieser Wertegruppen repräsentiert etwa ein Viertel der deutschen Jugendgeneration zu Beginn des Jahrhunderts. Als pragmatische Idealisten gelten bildungsprivilegierte Jugendliche, die aus bildungsprivilegierten Elternhäusern stammen und etwas häufiger weiblich sind. Sie sind systemloyal, betonen die idealistische Seite des Lebens, sind jedoch alles andere als postmaterialistisch ausgerichtet. Sie repräsentieren die beschriebene Synthese von Ordnung, Sicherheit und kreativem Engagement in besonderer Weise.

Die nach Zinnecker "selbstbewussten Macher" bilden als Pendant die Leistungselite unter den Jüngeren. Sie bringen den Trend der heutigen Jugendgeneration, sich aktiv und erfolgreich der aktuellen gesellschaftlichen Agenda zu stellen, besonders klar zum Ausdruck.

Dagegen sind "robuste Materialisten" und "Unauffällige" eher die Repräsentanten der Verliererseite der Spätmoderne. Sie kommen mit den Leistungsanforderungen in der Schule und Ausbildung weniger zurecht und sind teilweise arbeits- bzw. erwerbslos. Beide Gruppen blicken eher skeptisch in die Zukunft und äussern eine diffuse Unzufriedenheit mit dem gesellschaftlichen System. ${ }^{212}$

Die hier relevante Jugendgeneration wuchs in den Neunziger Jahren des vorigen Jahrhunderts auf. Diese Jahre waren durch Versuche geprägt, die seit den Siebziger Jahren dauerhaft gelockerten und liberalisierten Standards und Umgangsweisen wieder zu restaurieren. In den Schulen wurde das Personal ermuntert, neben dem Unterricht wieder 212 Ebd. 176-179. 
pädagogische Aufgaben wahrzunehmen und Ratgeber wiesen Eltern darauf hin, dass den Kindern "Grenzen" zu setzen seien. Doch die Erfolge dieser Bestrebungen waren eher bescheiden. Überforderte Eltern und Lehrpersonen, deren Machtfülle immer mehr rechtlichen Einschränkungen und Normen unterworfen wurden, während die Rechte der Kinder und Jugendlichen auf Schutz und Partizipation durchgesetzt wurden, wirkten der Restauration entgegen. ${ }^{213}$

In den neunziger Jahren nahm die Bedeutung einer ideologischen Ausrichtung ab. Die ideologische Konkurrenz zum Kapitalismus und deren Gesellschaftsform war moralisch diskreditiert und ökonomisch zusammengebrochen. Demzufolge hatte die radikale Rechte gewisse Erfolge, während die linksorientierten radikalen Utopien praktisch nicht mehr zu vernehmen waren. Auf der Ebene der Jugendkultur mussten sich die jungen Menschen mit Skinheads und Rechtsradikalismus auseinandersetzen, während Punks, Hausbesetzer oder radikale Friedensbewegte in Vergessenheit gerieten. Dennoch erlebte auch diese Jugendgeneration Debatten um die gefährdete Zukunft des Planeten. ${ }^{214}$

Die neunziger Jahre waren auch geprägt vom dualen System im Leitmedium Fernsehen. Neben den öffentlich-rechtlichen Sendern entstand eine Reihe von kommerziellen Fernsehstationen. Gleichzeitig kamen Mobiltelefonie und $\mathrm{SMS}^{215}$, Internet und Spielkonsolen ${ }^{216}$ auf. Ka-

213 Ebd. 181-182.

214 Ebd. 182.

215 Short Message Service (SMS) ist ein Telekommunikationsdienst, der für die Mobiltelefonie entwickelt wurde. (Short Message Service - Wikipedia, http://de.wikipedia.org/wiki/Short_Message_Service, 4.3.2009) Über die Weihnachtsfeiertage 2005 wurden in der Schweiz rund 55 Mio. SMS verschickt. (Tages-Anzeiger Online, http://www.tagesanzeiger.ch, 26.12.2005).

216 Zur Grössenordnung: An den Internet-Games Weltmeisterschaften in Singapur im November 2005 nahmen 700 junge Männer teil, darunter ein Mädchen, um sich in Sportarten wie Counter Strike, Warhammer und StarCraft Brood War zu messen. Sie waren in Qualifikationsspielen in 67 Län- 
belfernsehen, Zappen, Reality-Fernsehen, Doku-Soaps wie etwa Big Brother, Daily Soaps wie GZSZ (Gute Zeiten, Schlechte Zeiten), Millionen-Quizshows, Shopping-Fernsehen, E-Mail, Chats, E-Commerce sind Schlagworte, welche in den Neunziger Jahren wichtig wurden. Diese Jugendgeneration hat eine völlig andere Mediensozialisation erlebt wie ihre Eltern, die noch mit dem Leitmedium Print und dem öffentlich-rechtlichen Fernsehen aufgewachsen war. ${ }^{217}$

Das letzte Jahrzehnt des Zwanzigsten Jahrhunderts und die ersten Jahre des Einundzwanzigsten waren auch Jahre der beginnenden Krise des westeuropäischen Wohlfahrtsstaates. Internet-Aktienboom und -crash, die Einführung des Euro, die Folgen der Wiedervereinigung Deutschlands, der Niedergang der Industrie zugunsten der Dienstleistungsbranche prägten diese Zeit. Für die Jugendlichen wurden die Ausbildungswege unübersichtlicher, der Einstieg ins Berufsleben ungewisser und unplanbarer, die Phasen der Arbeitslosigkeit drohten nun auch der jüngeren Generation. Auch waren die wachsenden Kosten des Sozialstaates mit einer immer älter werdenden Bevölkerung zu tragen. ${ }^{218}$

Zinnecker ortet in dieser Unsicherheit die Gründe für die Tatsache, dass die von ihm als alt bezeichneten Werte wie Ordnung und Sicherheit bei der ersten Jugendgeneration des Jahrhunderts so hoch im Kurs stehen. Jugendliche benötigen zur Planung und Steuerung ihres Lebenslaufes und ihrer Zukunft gewisse verlässliche Ordnungen und Orientierungen, denen sie vertrauen können. Sie haben auch verstanden, dass sie selbst zu deren Aufbau und Erhaltung beitragen können und müssen.

dern aus 1,25 Mio. Kandidaten ausgewählt worden. Man schätzt die Zahl von Internet-Gamern weltweit auf 300 Millionen, die fast alle unter 35 Jahre alt sind. Deren Zahl wird sich gemäss Schätzungen der Industrie innert 10 Jahren verdoppeln. Im Jahr 2002 hat dieses Medium die Filmindustrie als populärstes Unterhaltungsmedium abgelöst. (The Economist, 26.11.05, 70).

217 Zinnecker, Alles ist möglich und nichts ist gewiss, 2005, 182-183. 218 Ebd. 183. 
Viele Stichworte der aktuellen Pädagogik weisen darauf hin: Selbstbildung, Selbsterziehung, Selbstorganisation, Selbstmanagement und ähnliche Begriffe stehen in der Erziehungsdebatte hoch im Kurs.

Die legendäre sogenannte 68er-Generation war die Letzte, welche sich innerhalb der westlich-christlichen Kultur dazu berufen fühlte, die Welt zu befreien. Sie wollte bestehende Ordnungen umstürzen. Doch die gemäss Zinnecker "entfesselte Moderne" besorgt das Bilder- und Denkmalstürmen, das Niederlegen traditioneller Ordnungen mittlerweile viel radikaler und effektiver. Die Jugendlichen werden durch die vorzeitige Auflösung und Transformation der Ordnungen bedroht. In welche Ordnung sollen sie sich integrieren? Erwachsene fühlen sich immer weniger dazu imstande, der jungen Generation sichere und stabile Bedingungen für das Aufwachsen und die Zukunft zu garantieren. Oft scheint durch, dass die Älteren selber Angst haben, nicht genau wissen, wie Probleme zu lösen sind. Sündenböcke für die Unfähigkeit der erwachsenen Generation, eine verlässliche Ordnung des Lebens zu bewahren, sind die Politiker und deren Institutionen. Durch ihre Unfähigkeit, Konsens unter Erwachsenen herzustellen, werden sie selbst zu einem Faktor der Instabilität und Unordnung. Die Jungen wollen deshalb die bedrohten Ordnungen stützen, und das gelingt am bestem im familiären oder privaten Nahbereich.

Auch wird laut Zinnecker das Bildungssystem immer mehr zur zentralen Lebenswelt der heutigen Jugendgeneration. Bildungstitel zu erlangen ist etwas Erstrebenswertes. Es wird keine Unordnung gestiftet, indem etwa verunsichernde Sinnfragen gestellt werden, sondern die Jugendlichen richten sich optimal in der bestehenden Ordnung ein. Gegen die Schule wird nicht rebelliert, sondern sie wird im besten Fall zu einem Treffpunkt, einem Kommunikationsraum mit Gleichaltrigen, wobei das System des Unterrichts den jugendlichen Tagesablauf optimal reguliert. ${ }^{219}$

219 Ebd. 186-189. 
Nach Zinnecker hat die jüngste Jugendgeneration noch eine zweites, wichtiges Merkmal. Sie ist eine Generation der Gelegenheitsjäger. Die Spätmoderne entfesselt nicht nur bestehende Ordnungen, sie eröffnet auch neue Möglichkeiten. Auf diese wartet die jüngste Generation. Es kommt darauf an, das Optimum auszunützen, zur rechten Zeit am rechten Ort zu sein und zuzugreifen. Ihr Optimismus beruht darauf, dass sie sicher sind, dass sich solche neuartigen Gelegenheitsräume für sie auftun werden. Sie sind sich auch sicher, dass sie besser als die vorangegangenen, mittlerweile älter gewordenen Generationen für die künftige Jagd gerüstet sind. Sich entspannen, hier und dort zu experimentieren, alles einmal anzufassen, auszuprobieren und wieder fallen zu lassen, das erscheint ihnen als beste Vorbereitung für die Gelegenheitsjagd. Sich langfristig und einseitig in jungen Jahren festzulegen, birgt die Gefahr in sich, zu unbeweglich, unflexibel zu sein. Viele von ihnen häufen deshalb mit einer gewissen Langmut Bildungstitel auf Bildungstitel an, Gelegenheitsjob auf Gelegenheitsjob - nützliche wie sinnlose. Man kann eben nie wissen. Gemäss der Philosophie der Spätmoderne: Alles ist möglich, doch nichts ist gewiss. ${ }^{220}$

Der oben beschriebene gesellschaftliche Wandel hat auf der Ebene der populären Kultur einige Auswirkungen. Seit der beschleunigten Globalisierung mit dem Ende des Kalten Krieges sind für die Jugendlichen andere kulturelle Bedingungen eingetreten. Beispielsweise sind besonders computerkundige, modisch bewusste Jugendliche genügend wohlhabend geworden, um agressiv beworben zu werden. Sie stehen daher unter einem grossen Druck, sich möglichst früh in die erwachsene Konsumkultur zu integrieren, was zu Problemen bei der ökonomischen Integration führen kann.

Ausserdem lässt sich beobachten, dass ähnlich wie in der Zeit vor der Jahrhundertwende zum 20. Jahrhundert, die als erste Globalisierung bezeichnet werden kann, ein starker Kulturwandel stattfindet. Wir Eu220 Ebd. 190. 
ropäischen Ethnologen sollten deshalb hellhörig werden. Schon damals galt die Parole „Retten, was zu retten ist“, um angeblich bedrohte kulturelle Erscheinungen zu erhalten oder zu sammeln. Dieses Gefühl des kulturellen Verlusts lässt sich heute beim Wechsel zum digitalen, globalisierten Zeitalter wieder ausmachen.

Dieser Niedergang ist jedoch kein Problem: Wahrscheinlich steht uns eine Zeit der „Invention of Tradition“ ${ }^{\text {‘221 }}$ ins Haus, wie vor dem Ersten Weltkrieg, als die Erste Globalisierung die Welt erfasste. Das sind abwechslungsreiche Zeiten für Ethnologen, Kulturwissenschaftlerinnen und Populärkulturforscher.

\section{$\underline{4.3 \text { Lokale Bedingungen von Jugend }}$}

Oben beschriebene Tendenzen lassen sich auch bei den untersuchten Jugendlichen feststellen. Probleme an zwei Schnittstellen standen vor allem der ökonomischen Integration der Jugendlichen im Wege: Der Übergang von der Schule in die Lehre und der Übergang von der Lehre an einen regulären Arbeitsplatz. Für diese Untersuchung, die in den Jahren 2003 und 2004 ihre Daten erhob, ist die Diskussion über den seit einigen Jahren sich immer mehr verstärkenden Lehrstellenmangel vor allem bedeutend, da die hier beschriebenen Jugendlichen Lehrstellen antreten sollten.

Mehrere Firmen der Industrie, die eine grössere Zahl von Lehrlingen ausgebildet haben, haben ihre Tätigkeit aus unterschiedlichen Gründen eingestellt. Die Gewerbeschule beider Basel etwa bildet heute nur noch halb so viele Elektroniker-Lehrlinge aus wie vor fünf Jahren. Es gibt für Basel-Stadt nur noch eine Klasse, und die soll mit derjenigen des Halb-

221 Hobsbawm, Eric: Mass-Producing Traditions: Europe, 1870-1914, in: Hobsbawm, Eric und Terence Ranger (Hg.): The Invention of Tradition, Canto Auflage (8. Auflage), Cambridge 1992, 263-309. 
kantons Basel-Landschaft zusammengelegt werden. Der Lehrstellenmangel und damit die Möglichkeiten zum Berufseinstieg ist in der Zwischenzeit so stark, dass sich die Kantonsregierung prioritär dieser Problematik angenommen hat und sie drängt die lokale Wirtschaft dazu, mindestens 700 zusätzliche Lehrstellen anzubieten.

Verstärkt wird diese Problematik durch die Trägheit des Ausbildungssystems. Früher traten Jugendliche eine Lehrstelle an, das heisst, sie lernten beim Arbeiten und gingen gleichzeitig zur Berufsschule. Durch den relativ raschen Wandel der Berufswelt gehen aber immer mehr Berufe, die in dieser Weise erlernt wurden, ab. Angeboten werden Lehrstellen unter anderem in Berufen wie Gerber, Hafner, Sattler und Schuhmacher oder solche, die in grösseren Zahlen nur noch durch staatliche Regulierung geschützt sind wie Kaminfeger oder Landwirt. ${ }^{222}$ Doch Regulierungen stehen generell unter Druck. Leider scheint es so, dass neue Berufsbilder eher zögerlich geschaffen oder bestehende durch eine Aufwertung zu höheren Ausbildungsgängen, die von den neu geschaffenen Fachhochschulen angeboten werden, dem Lehrstellenmarkt entzogen werden. Für die hier untersuchten jungen Leute, die auf eine Lehrstelle angewiesen waren, war die Entwicklung ungünstig.

Die Regierung reagierte auf die Probleme, indem eine neue Ausbildungsmöglichkeit geschaffen wurde. Die sogenannte Attestausbildung ist eine zweijährige Grundausbildung, welche die Lücke zwischen Schule und Lehre schliessen soll. Doch diese "greife noch nicht genügend", wie Reto Baumgartner, Leiter der Berufsbildung beim Gewerbeverband Basel-Stadt, im Frühjahr 2005 bedauerte. ${ }^{223}$

222 Online-Liste der freien Lehrstellen der Ämter für Berufsbildung und Berufsberatung beider Basel (Lehrstellennachweis beider Basel, http://www.lenabb.ch, Januar 2006).

223 Reto Baumgartner, Leiter Berufsbildung beim Gewerbeverband BaselStadt, Basler Zeitung 28.5.2005, Beilage Stellefant, 1. 
Auf der anderen Seite klagt die lokale Wirtschaft, die schulischen Leistungen der Bewerber für Lehrstellen seien zu schlecht. ${ }^{224}$ Im Zuge der oben beschriebenen Globalisierung steigen die Ansprüche der Wirtschaft an die Arbeitnehmer, die zukünftigen Lehrlinge sind hier keine Ausnahme. Anscheinend soll die Lehrfrau oder der Lehrling nicht mehr von der Pike auf ausgebildet werden, es wird neuerdings erwartet, dass die Bewerbenden gewisse Fähigkeiten bereits mitbringen. Die Folge ist, dass es keinen Platz mehr für Leute mit schwächeren Schulleistungen oder anderen Handicaps gibt. ${ }^{225}$

Als diese Studie durchgeführt wurde, bestand also die Situation, dass viele Jugendliche aus der WBS 2D keine Aussicht hatten, sich ökonomisch zu integrieren. Damit geriet auch ein Teil ihrer Integration in die Erwachsenenwelt in Verzug. Erschwert wurde dieser Sachverhalt durch eine noch nicht erfolgte Normalisierung der Verlängerung dieser Übergangsphase. Erst im Laufe der Jahre nach 2003 entstanden viele unterschiedliche Angebote im Sozialbereich, die mit verschiedenen Mitteln diesen Übergang erleichtern helfen sollen. Wie die Jugendlichen auf diese Situation reagierten und wie ihre Erwachsenwerdung davon beeinflusst wurde, ist Thema des Hauptteils dieser Arbeit. Doch zunächst sollen einige Begriffe und theoretische Konzepte erklärt werden, welche die Interpretation der gemachten Befunde ermöglichen sollen.

224 Basler Zeitung, 25.11.2004, 20.

225 Interview mit Niklaus Gruntz, Leiter des Amtes für Berufsbildung und Berufsberatung Baselland, in: Basler Zeitung, Beilage Stellefant, 25.9.2004, 1. 


\section{Begriffe, Konzepte und theoretische Felder}

Im Zentrum dieser Arbeit steht die Suche von Jugendlichen nach einem Übergang in die Erwachsenenwelt, insbesondere in die ökonomische Welt, unter den Bedingungen, welche die Gruppendynamik und die Lebenswelt der Jugendlichen vorgibt. Zum Verständnis dieses Prozesses greift die Studie auf verschiedene Zugehensweisen zurück, die innerhalb der sozial- und kulturwissenschaftlichen Jugendforschung und Ethnologie entwickelt worden sind. Das waren insbesondere die Integrations- und Ausschlussforschung sowie die Moratoriumskonzepte und Forschungen im Bereich der Statuspassagen. Im Folgenden sollen einige wichtige theoretische Konzepte aus diesen Forschungsfeldern dargestellt werden. Sie stellen verschiedene Angebote unterschiedlichen Disziplinen dar, wie Jugend aufgefasst werden kann.

Zunächst wird die wissenschaftliche Debatte um die Begriffe Integration und Ausschluss aufgegriffen, danach werden die Begriffe Jugend und Jugendkultur aus der Sicht verschiedener Disziplinen evaluiert. Diese Analysen bilden die Grundlage für das wichtigste theoretische Konzept der Arbeit, die der Submoratorien. Danach folgt ein Abschnitt über den Schlüsselbegriff der Normalität und seine Bedeutung für den Integrationsverlauf im Leben von Jugendlichen. Es kann gezeigt werden, dass mit diesem theoretischen Instrumentarium eine Vielzahl von Phänomenen, welche Jugendliche auszeichnen, erklärt werden können. Diese Überlegungen kommen sodann im Hauptteil dieser Arbeit in der Interpretation des empirischen Materials zur Anwendung. 


\section{$\underline{5.1 \text { Integration und Ausschluss }}$}

Integration und Ausschluss sind Begriffe, zu denen um die Wende zum 21. Jahrhundert in den Sozial- und Kulturwissenschaften viele Studien verfasst werden. Gemäss dem Soziologen Max Bergmann haben diese "traditionellen (sozialwissenschaftlichen) Studien" alle den Nachteil, dass sie die Bedeutung von Strukturen und Institutionen überbewerten und sich zu stark auf "exkludierte" Gruppen konzentrieren. ${ }^{226}$ Ausserdem sei man weggekommen von den Begriffen Integration oder Ausschluss, weil der angesprochene Sachverhalt nicht in polaren Begriffen gefasst werden könne. Die aktuelle Begriffsdiskussion dreht sich, so Bergmann, um den Begriff Exklusion, doch würden die beispielsweise im Jahre 2004 publizierten über 300 sozialwissenschaftlichen Arbeiten, welche das Wort im Titel führen, erstaunlich wenig Anhaltspunkte dafür liefern, wie dieser Begriff eigentlich definiert ist. Auch deckt der Begriff Exklusion eine grosse Bandbreite von Bedeutungen ab. In diesem Fall spielten, argumentiert Bergmann, forschungspolitische Strategien eine Rolle: Regierungen geben viel Geld für die Untersuchung von Exklusion aus, da möchte niemand an der Festlegung oder Eineingung des Begriffs arbeiten, aus Angst, von zukünftigen Aufträgen ausgeschlossen zu werden.

Doch Exklusion ist negativ konnotiert. Oft ist Exklusion an die Mitgliedschaft in einer bestimmten Gruppe gebunden, und beschreibt die

226 Bergmann, Max: Vortrag zu den Zwischenergebnissen des Moduls 6, NFP51, "Wer ist drinnen und wer ist draussen? Dynamiken der soziokulturellen und politischen Integration und des Ausschlusses in der Schweiz", gehalten an der Hochschule für Pädagogik und Soziale Arbeit, Basel 2005. Die Überlegungen dieses Vortrages wurden weiterentwickelt und eingearbeitet in den Begriff Motility, der verschiedene Ebenen und Formen von Mobilität beschreibt (Kaufmann, Vincent, Manfred Max Bergman and Dominique Joye, Motility: Mobility as Capital, in: International Journal of Urban and Regional Research, Volume 28.4, 745-756, 749). 
spezifischen Schwierigkeiten der jeweiligen Gruppe mit der Mehrheitskultur, ein Beispiel dafür sind die alltäglichen Diskriminierungen beispielsweise der Pakistani in London. Bergmann selbst favorisiert die Vorstellung, dass es "tangible or intangible commonly desired goods" gebe, die von den Exkludierten nicht erlangt werden können. Diese konkreten oder geistigen, in einer Kultur normalerweise zu erlangenden Güter können sozialer Natur sein, aber auch aus dem ökonomischen, politischen oder kulturellen Bereich stammen. Die Definition ist deshalb so bestechend, weil mit "goods" alle möglichen Güter gemeint sein können. Politisch kann damit das Wahlrecht gemeint sein, kulturell etwa die Ansicht, dass ein Museumsbesuch etwas Bereicherndes ist, ökonomisch ein gewisser Lebensstandard und sozial beispielsweise der Umgang mit Freunden anstatt ein Leben in Einsamkeit.

Die Charakterisierung dieser Güter als "tangible" oder "intangible", also als greifbar, berührbar aber auch immateriell, weist darauf hin, dass diese Güter einen Kapital-Charakter haben und es eine Aktion oder Bemühung braucht, um sie zu erlangen. Für die Einzelnen tun sich nach Bergmann sogenannte "action windows" auf, also zeitlich begrenzte Gelegenheiten, die zur Erlangung dieser Güter genutzt werden können. Dieser Sachverhalt führt zu der Frage, unter welchen Prämissen jemand angesichts einer solchen Gelegenheit passiv bleibt oder aktiv wird. Es geht auch darum, wie sich Individuen in Institutionen einpassen und weshalb es Aufsteigerinnen gibt, die trotz widriger Umstände Erfolg haben, und soziale Absteiger, die trotz guten Bedingungen scheitern.

Die sozialwissenschaftliche Begrifflichkeit um Integration und Ausschluss hat sich also laut Bergmann in Richtung der Frage entwickelt, welche institutionellen und individuellen Faktoren Ausschluss beeinflussen. Diese negativ gefärbte Sichtweise von Exklusionseffekten kann jedoch für eine kulturwissenschaftliche Definition nicht genügen, da implizit behauptet wird, Exklusion führe zum sozialen Abstieg. Kultur 
wird in diesem Konzept leider kaum thematisiert, obwohl sie genau institutionelle und individuelle Faktoren erleuchten könnte. ${ }^{227}$

Die Schwierigkeiten um den Begriff Integration beklagte auch Gunnar Olofsson von der Schwedischen Universität Växjö: "Wir glauben zu wissen, worüber wir sprechen, wenn wir die Worte Integration und Integrationspolitik, Integrationsamt und Integrationskommitee brauchen: über tiefe Gräben, vor allem wenn die ethnische Dimension mitgemeint ist." ${ }^{228}$ Die schwedische Diskussion entwickelte sich vor allem um das Begriffspaar Integration und Segregation, wobei Segregation als unerwünschter Gegensatz zur Integration angesehen wurde. Deshalb ist die Tiefe der gesellschaftlichen Gräben in dieser Diskussion ein wichtiger Aspekt.

Dies stehe im Kontrast zur Begrifflichkeit anderer politischer Einheiten, meinte Olofsson: Auch die EU beispielsweise verwendet die Begriffe Integration und Exklusion. Aus ihrer Sicht beschreibt Integration Gesellschaften, die von einer starken Kohärenz geprägt sind. Die Politik habe in dieser Sichtweise die Aufgabe, einen stabilen Rahmen zu bilden, um das soziale Leben zu ermöglichen. Historisch wurzelt diese Begrifflichkeit in Gesellschaftsvorstellungen der sozialistischen, sozialdemokratischen und katholischen Bewegungen und in der EU-Gemeinschaftstradition der politischen Integration.

Lehrreich ist in dieser Sichtweise der Zusammenhang zwischen Gruppenintegration, staatlichem Zusammenhalt und dem laufenden Integrationsprozess der Europäischen Union. Diese politische Vorstellung deckt sich mit der auf das lateinische Verb integrare zurückzuführenden

227 Bergmann, Max: Vortrag zu den Zwischenergebnissen des Moduls 6, 2005.

228 Olofsson, Gunnar: Om begreppen segregation och integration, in: Olofsson, Gunnar et al.: Integration och segregation - i teori och praktik, Växjö 2001, 10-12, 10. 
Bedeutung: Etwas, das zersplittert, geteilt oder zerbrochen war wie Europa, wieder ganz machen.

Zudem stellte Olofsson fest, dass der Begriff Integration sehr breit verwendet wird. Diese ist das Resultat einer Analyse von über $900 \mathrm{zwi}-$ schen 1994 und 2001 erschienenen sozialwissenschaftlichen Artikeln, in denen der Begriff vorkommt. Ein weiteres Resultat war, dass der Begriffsinhalt viel genauer wird, wenn in der Definition der oft mitgedachte Gegensatz mit berücksichtigt wird. Dieser Gegensatz kann für die Definition entscheidend sein. Folgende Beispiele führte er an:

Integration und Inkusion gegen Marginalisierung und Exklusion betreffen vor allem den Arbeitsmarkt.

Integration kontra Konflikt oder Streit ist gemeint, wenn es um Gesellschaften mit starken Konflikten geht. Die Konfliktparteien können miteinander verbunden sein, etwa wenn einzelne Gesellschaftsklassen integriert sind, aber die Gesellschaft als ganzes durch Konflikte auf Gesellschafts- oder Gruppenniveau geprägt ist.

Integration im Gegensatz zu Auseinanderfallen, das Fehlen gemeinsamer Normen innerhalb einer Gruppe.

Integration kann als Gegensatz zu Aussenvorsein ${ }^{229}$ angesehen werden, was ein psychologisches Phänomen bezeichnet.

229 Utanförskap: Wörtlich Aussenvorsein. Diese Erscheinung bezeichnet in Schweden unter anderem das Gefühl, nicht akzeptiert zu sein, beschreibt also ein gesellschaftlich unerwünschter individualpsychologischer $\mathrm{Zu}$ stand. 
Das Gegensatzpaar Integration - Segregation meint Integration in Bezug auf die Qualität der Wohnsituation und das soziale Leben (Ghettobildung)

Auffällig bei diesen Beispielen sind laut Olofsson die unterschiedlichen Gesellschaftniveaus, die durch die Begriffe beschrieben werden. Akteure in Bezug auf den Arbeitsmarkt können Individuen sein, Gruppen bei gesellschaftlichen Konflikten oder der Staat, etwa im 19. Jahrhundert, als die Bevölkerung in den Nationalstaat integriert werden musste. Über dieses Phänomen und seine Bedingungen dachten unter anderem Theoretiker wie Adam Smith, Karl Marx und Emile Durkheim nach.

Ausserdem wies Olofsson auf den wichtigen Unterschied zwischen Systemintegration und Sozialintegration hin, Begriffe, die David Lockwood in die Diskussion einbrachte ${ }^{230}$ : Systemintegration beschreibt, wie Teile einer Gesellschaft zusammenpassen, ob es in dieser Hinsicht Harmonie oder Konflikte gibt. Dies geht auf Karl Marx' Diskussion über die Spannung zwischen den Produktivkräften und Produktionsverhältnissen zurück. Es kann aber auch die Spannung zwischen den Ansprüchen der Familie, des Arbeitslebens und der Schule gemeint sein, der das in postmoderner Fragmentierung lebende Individuum ausgesetzt ist. Sozialintegration ist dagegen das Verhältnis zwischen Akteuren, zwischen Menschen innerhalb von Gruppen oder Organisationen. Lockwood meint, dass die beiden Integrationsarten miteinander verknüpft sind. Es sei eine analytische Perspektive, zu untersuchen, wie Systemintegration zur Sozialintegration wird, ob Spannungen und Konflikte

230 Lockwood, David: Soziale Integration und Systemintegration. In: Zapf, Wolfgang (Hrsg.): Theorien des sozialen Wandels, Köln und Berlin, Kiepenheuer \& Witsch, 1970, 124-137. 
bestehen, wie solche Prozesse innerhalb von Organisationen wie etwa der Schule ablaufen. ${ }^{231}$

Auch im Rahmen des engeren kulturwissenschaftlichen Rahmens gibt es viele verschiedene Färbungen der Begriffe von Integration und Ausschluss. Im Forschungsprogramm "Integration und Ausschluss" des Schweizerischen Nationalfonds vertraten verschiedene Forschungsgruppen jeweils unterschiedliche Begriffsfärbungen. An einer Konferenz im April 2005 wurden die verschiedenen Gruppen aufgefordert, den für ihre spezifische Forschung angepassten Begriff zu erklären. Es zeigte sich, dass von verschiedenen Begriffen und Theorien ausgegangen wurde und die Begriffe Integration und Ausschluss auf unterschiedlichen Ebenen ansetzten: Während beim Projekt, das sich mit Bildquellen befasste ${ }^{232}$, die Verknüpfung von In- und Exklusion mit Unsichtbarkeit und Sichtbarkeit im Vordergrund stand, beschäftigte sich das Projekt Raum ${ }^{233}$ mit offiziellen Integrationsbegriffen. Für das Projekt Ak$\operatorname{ten}^{234}$ waren hingegen das Konzept der Stigmatisierung und die damit verbundenen Effekte wie Kriminalisierung, Pathologisierung und Diskriminierung wichtig. Das Projekt Jugend, auf dessen Forschungsergebnissen diese Arbeit beruht, ging vom kulturwissenschaftlichen Konzept der kulturellen Identität aus. Ein Projekt, das sich mit Stigmatisierung befasste ${ }^{235}$, widmete sich eher dem Aspekt personaler Identität. Trotz

231 Olofsson, Om begreppen segregation och integration, 2001, 11-12.

232 Forschungsgruppe Sabine Maasen und Cornelia Renggli: Integration und Ausschluss durch Bilder des Anderen.

233 Das Forschungsmodul öffentliche Räume umfasste vier Projekte, u. a. Integration und Ausschluss im Kontext von Raumstruktur, Raumwahrnehmung und Raumnutzung von Rosmarie Anzenberger und Sabine Eggmann.

234 Roger Sablonier und Thomas Meier: Aktenführung und Stigmatisierung. Institutionelle Ausschlussprozesse am Beispiel der Aktion „Kinder der Landstrasse" 1926 - 1973.

235 U.a. Thomas Huonker: Unterwegs zwischen Verfolgung und Anerkennung. Formen und Sichtweisen in der Integration und Ausgrenzung von 
dieser ganz verschiedenen Interpretationen des Begriffs Integration liessen sich einige Schnittmengen feststellen, welche zentrale Begrifflichkeiten enthielten.

Wichtig waren allen Forschungsgruppen die Kategorien der Identität und Normalität und damit die ganz grundlegende Frage nach Zugehörigkeit. Des Weiteren wurde ersichtlich, dass alle Projekte von prozesshaften Vorstellungen, von einem Aushandeln von Integration und Ausschluss ausgehen. Schliesslich teilen die Projekte die Auffassung, dass es keine klare Konzeption des Innen im Gegensatz zum Aussen gibt. Viel bemerkenswerter als diese Pole ist das Dazwischen, weshalb auch andere Begriffe und die damit transportierten Theorien, etwa jene der Ambivalenz und der Differenz, für die interessierenden Fragen als erkenntnisreich betrachtet wurden. ${ }^{236}$

Auffällig war in der Debatte, dass die Begrifflichkeit wenig fassbar war. Schon die Frage, ob Integration ein Prozess oder ein Zustand sei, war nicht zu beantworten. Der Begriff ist für beide Bedeutungen gebräuchlich. Zwei Faktoren sind für diese Entwicklung verantwortlich: Erstens ist der Begriff Integration, wie bereits Olofsson zeigte, in den letzten Jahren im Gebrauch sehr alltäglich geworden. Damit erweitert sich das Bedeutungsfeld, was dazu führt, dass er in ganz unterschiedlichen Diskursen aufgegriffen wird. Zweitens wird der Begriff Integration oft in Kombination mit anderen gebraucht: Die Begriffe Integration und Ausgrenzung oder verwandte Begriffe wie Exklusion und Inklusion werden oft in einem Atemzug genannt, was die Diskussion weiter kompliziert.

Diese Sachverhalte spiegeln sich auch in den administrativen Unterlagen des Schweizerischen Nationalfonds. Beispielsweise verwendet

Jenischen, Sinti und Roma in der Schweiz vor 1800 bis heute.

236 Eggmann, Sabine und Cornelia Renggli: Schlussbericht zum NF-

P51-Workshop "Begriffe und Theorien zu Integration und Ausschluss",

Basel 2005, 3. 
der Fonds im "Final scientific report" durchgehend das Begriffspaar "Integration und Ausschluss", einmal auch "Integrations- und Ausschlussprozesse". ${ }^{237}$ Dies spiegelt ein Oszillieren zwischen den Auffassungen, dass der Begriff einen Zustand und einen Prozess bezeichnen kann, wobei die zu jener Zeit geltende staatspolitische Auffassung favorisiert wird, dass Integration ein gesellschaftlicher Zustand sei, bei dem soziale Konflikte eine untergeordnete Rolle spielen. Kommt noch eine Übersetzung dazu, wird es noch verwirrender: Auf Englisch heisst das Programm "Social Integration and Social Exclusion", obwohl es auch um kulturelle Prozesse geht, auf Französisch "Intégration et exclusion".

Diese Komplexität der Begriffe war zu erwarten. Es gibt eben nicht nur Integration oder Ausgrenzung, sondern auch viele Schattierungen dazwischen, die vom Kontext abhängig sind. Die Kulturwissenschaften sollten sich gegen falsche Dichotomien und gegen den einengenden "Charme der Eindeutigkeit" wehren, um sich die Möglichkeit zu erhalten, für bestimmte Forschungssituationen angemessene Varianten herzuleiten. Eine wichtige Aufgabe meines Forschungseinsatzes war aufgrund dieser Überlegungen, herauszufinden, was als Integration in meinem Forschungsfeld bezeichnet wurde und daraus eine eigene, für mein Forschungsvorhaben passende Begrifflichkeit zu formen.

Deshalb sollen an dieser Stelle die Akteure und Beforschten dieser Studie zu Wort kommen. Es ist zwar nicht üblich, im theoretischen Teil einer Arbeit empirische Resultate zu präsentieren, doch soll der Anspruch dieser Arbeit eingelöst werden, die Betroffenen zu Wort kommen zu lassen. Sie haben auch zu theoretischen Fragen einiges beizutragen und dürfen durchaus helfen, an der Entwicklung von Theorie mitzuarbeiten, welche dazu verwendet wird, sie selbst zu untersuchen. Da sie in ihrem Alltag mit der Problematik der gesellschaftlichen Forde-

237 Schweizerischer Nationalfonds, Division Targeted Research, National Research Programmes (NRP): NRP 51 "Social Integration and Social Exclusion", Final scientific report. 
rung nach Integration und ihrer alltäglichen Umsetzung konfrontiert waren und zur Bewältigung dieses Alltags unbewusst theoretische Konzepte entwickeln mussten, haben sie auch Allgemeingültiges gedanklich entwickelt. Über die Übertragbarkeit und Reichweite dieser Begrifflichkeit wäre zwar noch zu diskutieren, doch aufgrund der Äusserungen schält sich überraschenderweise ein ziemlich eindeutiger und brauchbarer Begriff von Integration, jedenfalls für dieses Projekt, heraus.

Der Scherz der Lehrerin Paula Lang ("Integration ist wie Mayonnaise: man rührt zwei Teile zusammen, und manchmal gelingt sie, manchmal nicht"238) zeigt zwei Dinge auf: Es geht ums Mischen, und daraus soll etwas Neues, Drittes entstehen. Das Resultat wird von Faktoren kontrolliert, die aufgrund von Komplexität, Unkenntnis oder fehlender Steuerbarkeit nicht beeinflusst werden können.

Ein weiterer Integrationsbegriff, der für mein Forschungsfeld relevant war, wurde von den lokalen Autoritäten gebraucht, weil er die Arbeit der Experten determinierte. Von der Regierung des Kantons BaselStadt wurde Integration im Jahre 2004 wie folgt definiert: "Integration ist ein gegenseitiger Prozess, der von Einheimischen wie von Zugezogenen eine aktive Mitgestaltung erfordert." Die Regierung betont also das Prozesshafte und lädt zu einer aktiven Mitgestaltung von Integration ein. "Fördern und Fordern" ist dabei die Haltung der Integrationspolitik, mit dem sozialpolitischen Ziel der "Vermeidung sozialen Abstiegs" ${ }^{239}$. Darin steckt also wiederum die häufig von der Politik ge-

\section{FB05, 2.}

239 Alle drei Zitate aus: Kantonale Integrationsstelle Basel-Stadt "Integration Basel": Integration Basel-Stadt, Basel 2004, 11-12. Der Basler Regierungsrat hat 1999 von der Ethnologin Rebekka Ehret das Integrationsleitbild und Handlungskonzept für Basel-Stadt erarbeiten lassen. Es wurde 2004 vom Interdepartementalen Netzwerk Integration (INI) überarbeitet und den aktuellen Bedürfnissen angepasst, was zum Aktionsplan 20042007 führte. 
brauchte, nicht zwingende Konsequenz des Integrationsbegriffs, dass mangelnde Integration zu sozialem Abstieg führe.

Für die Klassenlehrerin Isabelle Gisler beispielsweise kam der Integrationsbegriff im Zusammenhang mit meiner Frage nach dem Integrationsgrad des Schülers Jürg Beller zur Sprache.

Nicholas: Jürg. Hat auch wenig Integration in der Klasse gehabt, hab ich gefunden (...) er sass immer (allein) in der Mitte da ...

Gisler: Die Frage ist eben in dem Fall, was ist Integration? (...) Ist Integration etwas aktives, wo zwei Parteien was aktiv tun müssen, um jemanden zu integrieren, oder ist Integration schon dann geleistet, wenn zwei Gruppen, die absolut (unterschiedlich sind) sich in einer Gruppe vereinen und einen Weg gemeinsam gehen können, ohne dass es irgendwelche Konflikte gibt (...) ist das auch schon? Weil dann ist bei ihm eine sehr starke Integration. Gewesen. (...) Er ist nämlich Kleinklassenschüler ${ }^{240}$ gewesen, und dann in die WBS gekommen, und (...) er ist - ich drücks mal krass aus - wie der Behinderte der Klasse. (Das) war allen klar, da gab es nix, es hat aber niemand ihn damit konfrontiert, im Gegenteil, er wurde aufgenommen, (...) von allen, aufgenommen in diese Gruppe, die geistig und sozial durchaus begriffen haben, was es für diesen Menschen nun bedeutet, in dieser Klasse zu sitzen. Und sie haben ihn nie ausgelacht, wenn er nun das Dümmste von sich gegeben hat, im Vergleich zu den Anderen. Wenn ein anderer (...) wirklich dumme Sachen losgelassen hat, ist (er) bei jedem

240 Schüler, die im normalen Unterricht Schwierigkeiten haben, werden in kleineren Klassen unterrichtet, damit die Lehrpersonen sie intensiver betreuen können. 
Buchstaben schon ausgelacht worden. Ein Jürg nie. Es hat einmal jemand in den ersten zwei Wochen WBS aus der Parallelklasse zu ihm irgendwie gesagt, du bist dumm oder irgendwie so was. Dann sind vier Leute vor Jürg gestanden und haben sich den gepackt und gesagt, wenn du noch ein einziges Mal, dann ... schlagen wir dir in die Fresse. (...) Der Jürg hat gar nicht richtig begriffen, was da passiert ... für mich war das dann pure Integration in wenigen Stunden. Aber sie haben jetzt natürlich auch nie ihre Freizeit miteinander gestaltet. Oder ... aber das wäre jetzt auch viel zu viel verlangt. Aber sie haben ihn aufgenommen, so liebevoll, wie ich es von Pubertierenden eigentlich nicht erwartet hätte. ${ }^{241}$

Integration bedeutete also gemäss der Lehrerin für die Schulklasse 2D der WBS im Jahre 2003, dass verschiedene Gruppen in der Klasse einen Weg gemeinsam gehen können, ohne dass es Konflikte gibt. Integration erfolgte also nur bis zu einem gewissen, mit Begrifflichkeit der Intensität zu bezeichnenden Grad, und zwar so weit, dass eine Art Arbeitsfrieden hergestellt war. Integration bedingte auch eine Kompetenz, nämlich Sozialkompetenz, zu begreifen, was im anderen, vielleicht weniger begabten vorgeht, und den Willen, ihm eine massgeschneiderte Lösung anzubieten, welche die Stärke dieses Willens und den Ausgangspunkt des Problems reflektiert: Nach aussen wird Jürg als Mitglied der Gruppe verteidigt, aber innerhalb der Klasse wird er nicht in

241 Gisler, Isabelle, 20. Des weiteren mit dem Geschlechtsnamen bezeichnet. (...) sind Auslassungen und (ein Wort) ist eine Ergänzung um des Sinns oder der Grammatik willen. Interviews werden mit Nachnamen, Vornamen, Gesprächsnummer zitiert, falls es mehrere gab, und Seitenzahl des Transkripts. Eine vollständige Liste der Interviews ist im Anhang zu finden. 
einer der vorhandenen Gruppen einbezogen, man verbrachte seine Freizeit nicht mit ihm.

Um dieses Phänomen zu verstehen, bietet es sich an, gemäss Lockwood Integrationsphänomene auf sozialer und individueller Ebene zu unterscheiden. Während Sozialintegration Vorgänge auf der Ebene des Individuums erfasst, beschreibt Systemintegration die Beziehungen von Teilen eines sozialen Systems zueinander. ${ }^{242}$ Im Fall von Jürg war also die Systemintegration gelungen, die Sozialintegration nicht. Dennoch war Gisler beeindruckt von der Empathie und Geschwindigkeit dieses Integrationsprozesses.

Ein anderer Fall illustriert verhinderte Integration oder einen aktiven Ausschlussprozess. Die Schülerin Monika Bauer erzählte während eines informellen Gesprächs von der Basler Fasnacht. Sie ist Tambourin bei einer bekannten Clique ${ }^{243}$, und schwärmte von der Fasnacht 2005, obwohl das Wetter sehr kalt gewesen sei. Bei ihrer Clique gebe es recht viele Tambourinnen. Nicht so wie bei anderen Cliquen. Das finde sie schön. In der WBS durchlebte sie eine schwere Zeit, da sie als einzige Baslerin in eine Klasse kam, in der ethnische Gangs das Klassengeschehen bestimmten (die "Chicks" und die "Typen", wie sie sagt). Die sagten, als sie kam: "Ah, die Schweizerin kommt!". Sie hat dann nach einigen Monaten in die 2D wechseln können und fühlte sich erst dann einigermassen wohl. Zu den alten Klassenkameraden der 2D hat sie keinen Kontakt mehr, ausser zu Akdis Saliba, den sie manchmal noch im Tram sieht. ${ }^{244}$

Eine Person kann also auf einer Ebene völlig integriert sein und gleichzeitig auf einer anderen Ebene ausgeschlossen werden. Kennt man die Bedeutung der Trommlerinnen in den Cliquen für die lokale Kultur, muss man diese junge Frau als hochgradig integriert bezeich-

242 Lockwood, Soziale Integration und Systemintegration, 1970, 124-137. 243 Die Trommler und Pfeifer der Basler Fasnacht treten in Cliquen auf. 244 Bauer, Monika, 1. 
nen. Gleichzeitig wurde sie auf der Ebene der Schulklasse so stark gemobbt, dass sie die Klasse wechseln musste. Die Sozialintegration in der städtischen Gesellschaft war hier also kein Problem, während diejenige in der Klasse - und damit auch die Systemintegration - misslang.

Diese Beispiele sollen auch die Kompetenz illustrieren, welche die Akteure auch auf theoretischer Ebene entwickelten, um mit den sich stellenden Zugehörigkeitsfragen umzugehen. Auf den Integrationsbegriff der Schülerinnen und Schüler bin ich gestossen, als ich der Frage nachging, ob die Klasse einen Klassengeist besitze. Diese Frage stellte ich, um herauszufinden, ob die Schülerinnen und Schüler der 2D ihre Klasse als Gruppe wahrnahmen. Sie fassten die Frage nach dem Symbol ihrer Gruppenhalftigkeit aber nicht als eine nach kulturellen Faktoren in der Praxis auf, wie ich intendiert hatte, sondern als eine Frage nach den Bedingungen ihres inneren Zusammenhalts, nach Integration in der Klasse und nach theoretischen Begriffen, welche das beschreiben. Sie machten sich also, entdeckte ich, aufgrund ihrer Alltagserfahrung Gedanken darüber, was Integration sei, und äusserten sich dazu bei verschiedenen Gelegenheiten. Thomas Holdener meinte zur Frage, ob es einen Klassengeist in der 2D gebe:

Thomas Oppliger: Ich nehme an schon. Ich würde es sehr stark vermuten. Auch wenn es bei uns nicht so aussieht. Nicholas: Also, das ist jetzt eine merkwürdige Antwort. Ich nehme an ... also, du hast ihn persönlich zwar noch nie gesehen, aber ...

T: Doch! Schon, aber es ist so, er kommt eben raus, er kommt ein Stück weit raus bei Sport- und Spielsachen, in der Art, (wir) schauen für einen, das wir das schaffen, wir sind schon ein bisschen eine ehrgeizige Klasse in dem Sinn ... ${ }^{245}$

245 Oppliger, Thomas, 11. 
Für Thomas ist Integration also ein Faktor, der in kompetitiven Situationen aufkommt und nicht an kulturelle Bedingungen geknüpft ist, sondern an den Leistungswillen. Jürg bringt den Zusammenhang Klassengeist und Integrationsbegriff ins Spiel, was darauf hinweist, dass der Klassengeist das Ergebnis eines Integrationsprozesses darstellt und begrifflich gefasst werden kann.

Jürg: Also, wo ich vor allem den Geist gespürt habe, war im E-Niveau, dort auf jeden Fall. Im G-Niveau ist es zwar auch schön, aber sie machen einfach zuviel Scheiss ${ }^{246}$. Und das nervt einfach irgendeinmal.

Nicholas: Also, für dich ist der Klassengeist so eine Art Atmosphäre, in der man gut arbeiten kann?

$\mathrm{J}$ : Atmosphäre, wo man gut arbeiten kann. ${ }^{247}$

Hier zeigt Jürg das aus dem fehlenden Klassengeist resultierende fehlende Arbeitsklima. Andere Schülerinnen und Schüler meinten, diesen Gruppengeist gäbe es gar nicht oder nur zeitweise. Der Vorfall mit Jürg, als einige junge Männer aus der Klasse ihn nach einer Beleidigung verteidigen wollten, wies in diese Richtung. Ob Claude den Klassengeist spürt?

Claude. Ich glaube nicht. Also, überhaupt nicht, im Gegensatz zu ...

Nicholas: ... zu früher ...

C: ... zur früheren Klasse.

246 „Blödsinn machen“, „Scheiss“ oder „Scheissdreck“ machen: Tätigkeiten, welche Integration verhindern, den sozialen Frieden stören, einem die $\mathrm{Zu}$ kunft ruinieren.

247 Beller, Jürg, 8 . 
$\mathrm{N}$ : Also, du meinst, vergleich $\mathrm{OS}^{248} \mathrm{zu}$ WBS, dann.

C: Ja. Eigentlich gar keinen. Joh, im Sport (...) manchmal

ein bisschen, aber sonst

nicht. $^{249}$

Günter Robert glaubte, dass die inneren Spannungen der Klasse den Klassengeist praktisch verhinderten, im Gegensatz zu den Erfahrungen, der er anscheinend in Deutschland gemacht hatte.

Günter: (Den Klassengeist) hats nicht wirklich.

Nicholas: Nicht wirklich?

G: Das war in meiner anderen Klasse anders.

(...)

G: Die Klasse geht mit sich selber auch nicht gut um und

so. Schon manchmal, aber ...

(...)

$\mathrm{N}$ : Habe ich auch den Eindruck.

G: Die ganze Zeit sind sie am dissen ${ }^{250}$ und so ... ${ }^{251}$

248 Orientierungsstufe, die Schulstufe vor der WBS.

249 Beottcher, Claude, 5.

250 Ein wichtiger Ausschlussprozess: Dissen. Dieser Ausdruck wurde popularisiert von MC's und entstammt dem afroamerikanischen Strassenslang. "To diss (oder dis)" ist ein Verb und bedeutet jemanden runtermachen, eine Verkürzung von "to disrespect someone", jemanden nicht respektieren, ja sogar aktiv herabwürdigen. MC: Master of Ceremonies: Im HipHop-Bereich der Gastgeber einer Bühnenshow oder Aufführung. Er präsentiert üblicherweise die Künstler, hält das Publikum bei Laune und die Show am Laufen. Siehe http://www.de.wikipedia.org/wiki/MC_(Musik), 13.6.2006.

251 Robert, Günter, 2, 6 . 
Lehrerin Isabelle Gisler wies darauf hin, dass die Klasse vor allem dann einen starken Klassengeist aufwies, wenn es darum ging, nach aussen Geschlossenheit zu zeigen, wie etwa am Sportstag oder als Jürg verteidigt werden musste. Sonst falle sie ziemlich auseinander, gehe nicht gut mit sich selber um, indem man sich nicht genügend respektiere. Die Gemeinsamkeit der Klasse war also so etwas wie eine Ressource, die dann aktiviert wird, wenn dies wegen eines äusseren Anlasses angebracht erschien, etwa in einer kompetitiven Situation.

Thomas Häberlin, der in seiner Freizeit Gruppenleiter bei den Pfadfindern war, und deshalb wohl eine gewisse Sozialkompetenz aufwies, betonte in seinen Ausführungen das Prinzip der Assimilation:

Thomas Häberlin: Integration? Das Wort selber ... oder ... zum Beispiel bei Ausländern, dass wenn die in die Schweiz kommen, dass sie sich integrieren ... so ...

Nicholas: Und was ist das für dich ... ?

T: Einen Fremdkörper einpassen ... also irgendwo hineinpassen ...

$\mathrm{N}$ : Äh ... gibt es die gelungene Integration. Oder die missglückte ... gibt es das?

T: Ich weiss jetzt kein Beispiel ...

N: Pfadi, zum Beispiel. Du bekommst jetzt ein neues Kind ...

T: Genau, das ist eine gute Idee ... da haben wir jetzt einen, der heisst Pavel. Der ist nett zu allen und so, aber der macht Scheissdreck ... wie nichts. Wir probieren, dass er sich hält ... er macht sich jetzt einigermassen gut ... aber er macht Scheissdreck ...

$\mathrm{N}$ : Ja, ja, das ist der Grund, mit den Leuten ... die kommen aus irgendwelchen Gründen irgendwo dazu, und du musst mit denen funktionieren. 
T: Er ist so ein bisschen - was willst du eigentlich von mir. So in dieser Stimmung. (...) Macht nicht, was wir ihm sagen.

N: Also, z.b. im Lager: He - kannst du abwaschen, und er macht es nicht.

$\mathrm{T}$ : Ja, genau.

$\mathrm{N}$ : Integration wäre dann, wenn er es machen würde.

T: Also wäre es ... so ja. ${ }^{252}$

Für Thomas Häberlin war Integration erstens das Einpassen von etwas vorher unbekanntem, auch einem räumlich verstandenen Ding ("Fremdkörper") in eine bereits vorhandene, historisch gewachsene Struktur und bildete die Voraussetzung, in einer bestimmten Situation zusammenarbeiten zu können. Der Schüler Thomas Oppliger verstand folgendes unter Integration:

Also, wenn man so Leute (...), Aussenstehende, integriert,
dass die zur Gruppe finden, ausser natürlich ... wenn er gar
nicht will oder immer Blödsinn macht ... dass man einfach
jedem eine Chance gibt und nicht von vorneherein ... und
wenn er dann die Chance nicht nutzt, ist er selber schuld. ${ }^{253}$

Beiden Aussagen gemeinsam war die Erwähnung von Blödsinn machen oder "Scheissdreck" machen. Öffnet sich die Gruppe für jemanden oder leistet gar einen Aufwand, diese Person einzubeziehen ("Wir probieren, dass er sich hält.") damit diese Person sich auch bemühen kann (“... zur Gruppe findet ...”), kann das Individuum Integration durch asoziales Verhalten (Tobias H.: "Er ist so - was willst du von mir") verhindern.

252 Häberlin, Thomas, 11.

253 Oppliger, Thomas, 6. 
Integration braucht auch, meinte der Schüler Jürg Beller, der einige Jahre im spanischsprachigen Ausland gelebt hatte, jemanden, der sich für einen einsetzt.

Jürg: Ja, wirklich, was ist (Integration)? Viele nehmen Integration als, dass man sich einsetzt. Also, ich bin eine Person, und es hat acht Schüler, und ich setze mich für die acht Schüler ein. Für etwas. Ich würde es in die Richtung nehmen. Also, im Spanischen wird das Wort extrem benutzt.

Nicholas: Ah, sicher?

$\mathrm{J}$ : Ja, io me vol integrar cor ailio ... und so weiter ...

$\mathrm{N}$ : Was heisst das übersetzt?

J: Also, ich werde so einmischen ... Eher in Richtung einmischen, es geht schon ums einsetzen, darum geht es, also, das ist eigentlich die beste Übersetzung, du setzt dich irgendwie ein bei den Leuten. Ein Wort wie Hilfsbereitschaft. ${ }^{254}$

Die Idee der Person, die einem bei der Integration hilft, kam auch im Interview mit Loredana Di Costanzo zur Sprache.

Loredana: Wenn ein neuer Schüler kommt, dass man den nicht draussen lässt, sondern ... (spielt vor:) tschau, los, wie heisst $\mathrm{Du}$, woher kommst $\mathrm{Du}$, was machst $\mathrm{Du}$ hier ... das sind die und die Leute, komm, wir machen etwas, so. Also, meiner Meinung ... also, ich mach das. (Hochdeutsch:) An der Hand nehmen.

254 Beller, Jürg, 8-9. 
Nicholas: Also, man braucht einen Freund, der einem so ein bisschen führt. ${ }^{255}$

Kommt jemand neu hinzu, hat die Person zunächst die von Thomas Oppliger beschriebene, ablehnende "was willst du von mir"-Haltung und wehrt Annäherung möglicherweise ab. Hier ist ein Freund nötig, welcher diese Haltung überwinden hilft und einen einführt.

Doch Integration hat auch eine ästhetische Komponente. Der erste Schritt zur Integration sei eine äussere Anpassung, die auch vorgespielt sein kann. Günter Robert, ein Schüler, der kürzlich von Deutschland in die Schweiz gezogen war, erklärte den Sachverhalt so:

Günter: Sich integrieren, sich irgendwo rein integrieren, ja, sich einfügen, reinkommen, oder so. in ner Gruppe, sich ... wie ein Chamäleon. Wenn es grün ist, ist es grün, und wenn es was Gelbes ist, ist es gelb. ${ }^{256}$

Loredana sprach auch über bereits gefestigte Gruppen und die Schwierigkeit, in eine solche hineinzukommen. Sie meint, dass der Eintritt in eine Gruppe im Prinzip kein Problem sei, aber es komme auf den Style an, in welche Gruppe hinein man sich integriert, also auf eine Vorselektion, die auf ästhetischen Präferenzen beruht. ${ }^{257}$

Evaluiert man die in obigen Beispielen aufscheinenden Bedeutungsfelder, verwendeten Termini und Metaphern zur Integration, fällt auf, dass eine teleologische Komponente die Definition von Integration erheblich mitbestimmt. Je nach dem, welcher Zweck oder welches Ziel mit Integration gemeint ist und welche Programmatik unterliegt, ergeben sich andere Definitionen. Für die Politik beispielsweise ist wichtig,

255 Di Costanzo, Loredana, 6-7.

256 Robert, Günter, 2, 7.

257 Di Costanzo, Loredana, 2-3. 
dass keine soziale Unrast entsteht, und deshalb definiert sie Integration als Abwesenheit von sozialem Abstieg, also durch ein Ausschlusskriterium. Sie wertet Integration positiv und Ausschluss negativ.

Für die Sozialwissenschaften stehen forschungspolitische Anliegen im Vordergrund. Die Wissenschaften haben das Interesse, die Behauptung aufrecht zu erhalten, dass sie in der Lage sind, der Politik Grundlagen zur Verfügung zu stellen, welche die Mechanik von sozialem Aufstieg und Abstieg in komplexen Gesellschaften beschreiben oder systembedingte Ursachen von Ausschluss aufzeigen können. ${ }^{258}$ Das ist für die Politik attraktiv, dafür werden Forschungsgelder zur Verfügung gestellt. Deshalb wird in der Forschung Integration bevorzugt als etwas Statisches dargestellt, das durch Abwesenheit von Exklusion gekennzeichnet ist. Diese führe angeblich zu sozialem Abstieg, wobei kulturelle Komponenten von Exklusion, also grosse und wichtige Bereiche, ausgeklammert werden.

Die Nachteile dieser Zugangsweise sind evident. Forschungspolitische Interessen sollten für die Definition von wissenschaftlichen Begriffen keine Rolle spielen. Die Kulturwissenschaften sollten zudem Exklusionsprozesse nicht grundsätzlich als negativ ansehen, sondern deren Bedeutung für eine Gruppenkultur untersuchen. Wir sollten auch versuchen, zu Positivkriterien zu gelangen und nicht einen Sachverhalt durch Abwesenheit von etwas zu definieren. Eine kulturell erwünschte Exklusion kann beispielsweise das Bestreben einer gesellschaftlichen Schicht sein, sich durch eine bestimmte kulturelle Praxis zu unterscheiden. Ob dieses Abgrenzen für die Gesellschaft als Ganzes erwünscht

258 Das Programm NFP51, welche diese Studie hier ermöglichte, war nach dem Skandal um die "Kinder der Landstrasse" aufgelegt worden. Jahrzehntelang wurden Kinder von Fahrenden ihren Eltern weggenommen und in Pflegefamilien gegeben. Begründet wurden diese Massnahmen mit oftmals tendenziösen Einträgen in die Dossiers der Betroffenen, ein Phänomen, das auf die Rolle der Administration in Ausschlussprozessen ein Licht warf. 
ist, stellt eine andere, durchaus legitime Frage dar, die jedoch nichts mit der Definition von Integration zu tun hat.

Zur Illustration dieses Sachverhalts ein Beispiel: Glaser und Strauss berichten, dass Menschen, die einen Aufstieg in die obersten sozialen Schichten erlebt haben, starke Integrationsprobleme haben können. Dies erklärt, dass es viele Institutionen gibt, die den Aufsteigern beibringen, sich richtig zu kleiden, essen, sprechen oder zu gehen. Hochglanzbeilagen von Zeitungen zeigen beispielsweise, wie das Geld richtig ausgegeben wird oder wie und wo man seine Zeit verbringen soll. Die Aufsteiger werden feststellen, dass diejenigen, die ihre Position geerbt oder vor ihnen erreicht haben, sich zum Teil weigern, die neureichen Zuzüger zu akzeptieren. Oft entstehen dann Style-Cliquen jener Personen, denen es trotz den nötigen materiellen Voraussetzungen nicht gelungen ist, sich in die höchsten Schichten zu integrieren. ${ }^{259}$ Diese Integrationsprobleme mögen wir als Luxusprobleme abtun; sie spielen jedoch für das Verstehen von politischen Vorgängen, etwa Parteiungen zwischen ,altem“ und „neuem“ Geldadel einer Gesellschaft möglicherweise eine bedeutende Rolle.

Neben der aus der wissenschaftlichen Literatur bekannten Definitionen des Integrations- und Ausschlussbegriffes gibt es also weitere Begrifflichkeiten, die durch den jeweiligen Kontext geprägt werden, beispielsweise den Integrationsbegriff des hier untersuchten Forschungsfeldes. Aus diesen Erkenntnissen ergeben sich unterschiedliche Forderungen.

Zunächst muss das je nach Kontext hinter dem Integrationsbegriff liegende Programm aufgezeigt werden. Die Handhabung des Begriffs wird erheblich vereinfacht, wird ein Positivkriterium oder eine teleologische Komponente gesucht. Sie kann auch von vorne herein festgelegt werden. Man kann sie auch völlig weglassen: Dann sollte Integration und Ausschluss nicht negativ oder positiv bewertet werden. Zudem ver259 Glaser und Strauss, Status Passage, 1971, 169. 
folgt Integration immer das Ziel, eine Arbeitsatmosphäre herzustellen, ein funktionierendes Ganzes, die Aufnahme in eine bestimmte Gruppe zu einem thematisch abgegrenzten Zweck oder für eine bestimmte Zeitdauer. Aus der Perspektive der Systemintegration, etwa im Fall der Gesamtgesellschaft, heisst dieses Arbeitsklima sozialer Frieden, bei einer Schulklasse beispielsweise, die personelle Grundlage und Klammer für diese Studie, könnte man diese Stimmung Klassengeist nennen. Für eine erfolgreiche Integration kann ein Ausschlussprozess, wie etwa dass ein Schüler aus einer Gruppe herausgenommen und vom Lehrer an einen anderen Tisch versetzt wird, weil er stört, durchaus positiv sein, weil damit die Arbeitsatmosphäre der ganzen Gruppe positiv beeinflusst wird. Für die Schüler herrschte dann Integration, wenn eine gute Arbeitsatmosphäre hergestellt war, die es erlaubte, ein Ziel zu erreichen.

In der Praxis kann Integration durch Anpassung erleichtert werden. Hilfreich sind auch Personen, die einen sozusagen in eine Kultur einführen, oder die eine Einladung aussprechen, sich einer Gruppe beizugesellen. Diese Einladung sollte von der Gruppe ausgehen, aber die Person, welche die Einladung erhält, sollte sich um ihre Integration bemühen und nicht ablehnend reagieren.

Gleichzeitig wird eine konkrete, beschreibbare Zugehörigkeit von Individuen zu einem übergeordneten Ganzen hergestellt, welches die Perspektive der Systemintegration darstellt. Ausserdem wird der zu integrierenden Person Raum zur Verfügung gestellt, eine Person wird in einen Zusammenhang eingepasst. Wird Integration nicht erreicht, weil jemand sich nicht adäquat verhält, also ein befriedigendes Arbeitsklima nicht hergestellt werden kann, resultiert möglicherweise daraus Ausschluss, der unter bestimmten Umständen zum ökonomischen oder sozialen Abstieg führen kann. 


\section{$\underline{5.2 \text { Jugend und Gesellschaft }}$}

Die gesellschaftliche Gruppe der Jugendlichen charakterisiert sich dadurch, als dass sie nicht fremd ist, aber dennoch eigene kulturelle Züge, eine eigene Kultur oder Kulturen besitzt. Es existieren eigene Regeln, etwa für Treffen; die Topografie der Stadt wird anders genutzt. Auch bewegen sich Jugendliche in der Öffentlichkeit anders, meist viel dynamischer, als Erwachsene. Hoch- und runterrennen, hin- und herhüpfen, den Wagen der Strassenbahn wechseln sind Dinge, welche Erwachsene kaum machen. Auch die Ernährung ist anders. Die Mädchen und Jungen stillten den Hunger mit Nutella-Schnitten, esslöffelweise Ovomaltinepulver ${ }^{260}$, Süssigkeiten, Eis-Tee und anderen Produkten mit hohem Nährwert, während viele Erwachsene sich bemühen, überflüssige Kalorien möglichst zu meiden. Jugendliche sind also anders, doch die Frage nach dem Wesen dieser Andersartigkeit wird je nach Standpunkt verschieden beantwortet.

In der Literatur werden Jugendliche häufig als defizitäre oder unvollständige Erwachsene aufgefasst, was ein gewisses Unverständnis gegenüber Jugendlichen reflektiert. Werner $\mathrm{Heiz}^{261}$ definierte in einer Studie über Schweizer Jugendliche der 1980er Jahre, die stark von den Problemen der sozialpsychologischen Jugendberatung ausging, den sozialen Alterstypus der Jugendlichen anhand von vier Kriterien. Diese sind für die Kulturwissenschaften kaum fruchtbar, dennoch sind sie bemerkenswert, da sie mit den weiter unten zu besprechenden Submoratorien erklärbar sind. Heiz' Überlegungen gehen auf die Charakterisierung von Friedhelm Neidhardt ${ }^{262}$ zurück: Erstens sind Jugendliche geschlechtsreif, aber nicht verheiratet; zweitens sind sie ausserhalb der

260 Nutella: Nussschokoladeartiger Brotaufstrich, Ovomaltine: energiereiches Getränk.

261 Heiz, Werner: Die “Unerreichbaren”, Probleme der Jugendberatung, Diss., Zürich 1983. 
obligatorischen Schule, aber noch in der Lehre oder im Studium, d.h. ohne Position in einer beruflichen Hierarchie; drittens sind sie rechtlich nur teilweise mündig und viertens politisch rechtlos. ${ }^{263}$ Heiz deutete auf wesentliche kulturelle Unterschiede hin, die zu den Erwachsenen bestehen.

Sprache: Die Sprache der Jugendlichen und der Erwachsenen ist verschieden.

Gruppen: Die Phase der Jugendzeit entsteht (...) als Übergangszeit, während sich die Jugendlichen in altershomogenen Gruppen zusammenschliessen.

Reife: Jugendliche sind geschlechtlich reif, von den Erwachsenen wird ihnen die soziale Reife aber nicht anerkannt.

Warten: Jugendzeit ist Wartezeit. Jugendliche sind meistens noch in Ausbildung und darum finanziell und emotional von den Eltern abhängig.

Beziehungen: Die Beziehungen zwischen den Erwachsenen und Kindern sind meistens klar und konkret, während die zwischen Jugendlichen und Kindern und Jugendlichen und Erwachsenen unklar sind. Die Rollenerwartungen sind damit auch unklar und diffus.

Kinderrechte: Folgende Rechte der Kinder dürfen die Jugendlichen nicht mehr beanspruchen: übermütig herumtollen; selbstvergessen spielen; das Recht auf Geborgenheit. Erwachsenenrechte: Folgende Rechte der Erwachsenen dürfen die Jugendlichen noch nicht beanspruchen: das Recht auf sexuelle Unabhängigkeit (Heirat); das Recht auf

262 Neidhardt, Friedhelm: Die junge Generation: Jugend und Gesellschaft in der Bundesrepublik, Opladen 1967.

263 Heiz, Die "Unerreichbaren”, 1983, 10. 
eine berufliche Position; das Recht auf Prestige; das Recht auf politische Partizipation; das Recht auf eine eigene Wohnung. ${ }^{264}$

In dieser Liste kommt deutlich heraus, dass Jugendliche eine kulturelle Gruppe sind, die sich aufgrund biologischer Entwicklungen auch kulturell von den Kindern und Erwachsenen unterscheidet. Die Biologie konstituiert also eine Gruppe, die durch defizitäre Rollenvorstellungen aus Sicht der Erwachsenen geprägt ist. Ausserdem reflektiert die Liste die Probleme der Behörden in den 1980er Jahren, als sich in manchen Schweizer Städten Jugendunruhen ereigneten: Sozial unreif, emotionale und finanzielle Abhängigkeit, fehlende Kinder- und Erwachsenenrechte, lauteten die Diagnosen der überforderten Obrigkeiten, die dem Phänomen teilweise hilflos gegenüberstanden.

Obige Liste zeigt, dass der Status der Jugend gesellschaftlich erzeugt wird. Hier spielen sogenannte Gatekeeping-Experten wie Heiz eine entscheidende Rolle. Ihre Autorität beruht auf Imaging: Ein Bild über eine Person wird durch verschiedene Prüfmethoden erzeugt. Lehrerinnen machen Prüfungen, Schulpsychologen und Sozialarbeiter Abklärungen, Ärztinnen Diagnosen. Diese Methoden sollen Aussagen über ein Individuum in einer strukturierten Art erzeugen und werden in aufwendigen Verfahren validiert und justiert. Mit diesen angeblich objektiven Methoden, in die die Öffentlichkeit ein hohes Vertrauen hat, wird die Autorität der Gatekeeping-Experten erhöht und gesichert. Das Übertragen des privaten, individuellen Wissens auf eine öffentliche, allen zugängliche Ebene ist eine Form der Machtausübung. ${ }^{265}$ Für diese Studie hier wichtig ist die Tatsache, dass dieses Fachwissen den Gatekee-

264 Ebd., 11.

265 Stone, Deborah A.: Gatekeeping Experts and the Control of Status Passages, in: Heinz, Walter R. (Hg.): The Life Course and Social Change: Comparative Perspectives, Weinheim 1991, 203-220, 205 und 214. 
pers die Macht verschafft, die Statuspassagen im Leben der Menschen im Namen der Gesellschaft zu kontrollieren, etwa den Zugang zu Unterstützungsangeboten, welche den Zugang zum Arbeitsmarkt erleichtern können. ${ }^{266}$ Es wird beispielsweise evaluiert, wie weit erwachsen und damit arbeitsmarktfähig ein Jugendlicher ist. Damit wird auch diagnostiziert, wie er oder sie arbeitsmarktfähig gemacht werden kann. Wie dies im einzelnen geschieht, wird weiter unten im Detail ausgeführt.

Neben dem Bild des defizitären Erwachsenen gibt es auch die Idee, dass die Jugend eine Vorreiterrolle in der Gesellschaft spielt. Für diese Idee gibt es bereits im 18. Jahrhundert Belege, doch Breitenwirkung erhielt sie erst mit Walt Whitman, einem US-amerikanischen Schriftsteller, der 1855 „Leaves of Grass“ herausbrachte. ${ }^{267}$ Diese hymnische Verherrlichung der freien Natur, der offenen Strasse und der Harmonie der Welt lässt sich als Kritik der neuen Institutionen des 19. Jahrhunderts lesen und drückte die neue Lebensstimmung der zu einem Massenphänomen gewordenen bürgerlichen Grossstadtjugend aus. Keine festen Gefüge, keine Regeln, keine Verwalter des eigenen Lebens, zuletzt auch keine abstrakte Beweisführung, sondern nur das Leben in Gemeinschaft mit Gleichgesinnten ist das Programm für die Jugend, die Whitman als "birds of passage" bezeichnet. Sie sind die Pioniere der Gesellschaft, ungeduldig, voll von "action", stolz und erfüllt von Freundschaft, frisch und stark, unbekannte Wege erkundend. 1907 hat Johannes Schlaf den zweiten Gesang aus dem Buch XXVII, "birds of passage" mit "Wandervögel" übersetzt, eine Bezeichnung, die als Name für eine der ersten jugendkulturellen Bewegungen in Deutschland aufgenommen wurde und die Whitmans Programm enthielt. ${ }^{268}$

266 Ebd., 208.

267 Aktuelle Übersetzung: Whitman, Walt: Grasblätter. Übersetzt von Jürgen Brocan, München, C. Hanser, 2009. 
Nahe verwandt mit dem Glauben an die Jugend als Pioniere der Gesellschaft ist der Gedanke, dass die Jugend ein Motor gesellschaftlichen Wandels sei. Dieser Gedanke geht auf Eduard Spranger zurück, der 1924 schrieb: "Die erwachsene Gesellschaft ist durch und durch geregelt (...), Der Jugendliche aber widerstrebt solchen von aussen kommenden Regelungen; er will Ungebundenheit und Bewegungsfreiheit (...). ${ }^{269}$ Deshalb würden die Jugendlichen sozusagen automatisch die Gesellschaft verändern.

Häufig trifft man auch den Gedanken an, dass die Jugend ein Anzeiger für meist niedergehende gesellschaftliche „Zustände“ sei. Dieses in allen Zeiten anzutreffende „o tempora, o mores “ ${ }^{\text {‘270 }}$ hat beispielsweise der Reformator Melanchton im 16. Jahrhundert sehr hübsch auf das Höchstmögliche zugespitzt: "Der grenzenlose Mutwillen der Jugend ist ein Zeichen, dass der Weltuntergang nahe bevorsteht". ${ }^{271}$

Möglicherweise stimmt die Annahme, dass die Jugend eine Vorreiterrolle spiele, für einige Gesellschaften. Doch ist nicht einzusehen, weshalb die Jugendlichen in einer spätmodernen Gesellschaft besser als Anzeiger für gesellschaftliche Entwicklungen anzusehen sind als etwa Migranten oder Computerexperten. Die historischen Aussagen über die Jugend beschreiben häufiger damalige Probleme der Erwachsenengesellschaft als wirklich vorhandene Eigenschaften von Jugendlichen.

268 Oelkers, J.: Jugendbewegung als Erziehungsform: Historische Anmerkungen, in: Klosinski, Günther (Hg.): Pubertätsriten, Bern, Stuttgart, Toronto, Hans Huber, 1991, 65.

269 Spranger, Eduard: Psychologie des Jugendalters, 28. Auflage, Heidelberg 1966, 132.

270 Cicero, Marcus Tullius, Erste Rede gegen Catilina, Abschnitt 2. Cicero verwendet den Ausruf für den moralischen Niedergang der Oberschicht Roms und nicht für die Jugend.

271 Zitiert in Klosinski, G.: Pubertätsriten - Äquivalente und Defizite in unserer Gesellschaft. Einführene Anmerkungen des Jugendpsychiaters, in: Kosinski, Günter (Hg.): Pubertätsriten, Bern Stuttgart, Toronto, Hans Huber, 1991, 11. 
Jugendlich sein ist zudem keine einheitliche Kategorie: Dreizehnjährige sind völlig anders als Siebzehnjährige. Oft werden auch Menschen im öffentlichen Diskurs als Jugendliche bezeichnet, die gar keine sind, wie etwa Achtzehnjährige, die auch juristisch als erwachsen anzusehen sind, oder grossgewachsene Kinder um zehn. Lokale Jugendliche zur Zeit dieser Studie sind nicht problematischer als andere Bevölkerungsgruppen, und auch nicht pathologisch. Dieser Satz ist zentral für die vorliegende Studie, die Jugendliche als eine gesellschaftliche Gruppe versteht, die durch von Experten festgelegte kulturelle und soziale Besonderheiten gekennzeichnet ist.

An dieser Stelle soll nicht den mannigfaltigen Bedeutungen von Begriffen wie Pubertät, Teenagealter, Prä-, Früh- , Hoch- und Spätadoleszenz, Jugendzeit, Jugend, Jugendlichkeit, u.a. nachgegangen werden, die in verschiedenen Literaturen auftauchen. Für diese Studie prägend ist der Begriff "kurze Adoleszenz" von Howard Parker. ${ }^{272}$ Dieser pragmatische Begriff enthält ein Arbeitsprinzip, das sich als praktisch für die von Parker in einem benachteiligten Gebiet der Innerstadt von Liverpool untersuchten Jugendlichen erwiesen hat. In diesem Quartier, genannt Roundhouse, hat er eine Studie über männliche Jugendliche durchgeführt. Die Definition orientierte sich am Selbstverständnis von Adoleszenz, wie dies von den Bewohnern von Roundhouse, ein aus Plattenbauten bestehendes Wohngebiet, wie man in Deutschland sagen würde, geteilt wurde.

Parker gab die Lebensgeschichte von dreissig jungen Männern aus diesem Quartier wieder, den sogenannten Boys, und diskutierte sie im Hinblick auf die Bedeutung ihrer kurzen und beschleunigten Adoleszenz. "Kurze Adoleszenz" umfasste in diesem Kontext die Periode zwi-

272 Parker, Howard: Aus Jungen werden Männer. Kurze Adoleszenz in einem innerstädtischen Wohnbezirk, in: Clarke, John, u.a., Jugendkultur als Widerstand. Milieus, Riutale Provokationen. Honneth, Axel, Rolf Lindner und Rainer Paris (Hg.), Frankfurt a. M., Syndikat, 1979, 181-216. 
schen dem Abgang von der Schule im alter von fünfzehn Jahren (Jahr 1, beginnend im April), bis zum Ende von Jahr 4, zu welchem Zeitpunkt den Jungen zumeist alle wichtigen lokalen Definitionen von Männlichkeit zugeschrieben wurden oder von ihnen selbst erworben wurden. ${ }^{273}$

Roundhouse war zu jener Zeit ein Slum, typisch für soziologische Untersuchungen von Jugendlichen, und es geht um Probleme. Manche Aspekte des Verhaltens der Boys - Schimpfworte, "Halsstarrigkeit", "Faulheit" und Gleichgültigkeit gegenüber der Arbeit bis hin zur Verletzung von Gesetzen über Jugendschutz, Alkohol, Verkehr, Drogen und Eigentum - warfen die für die damalige Forschung wichtige Frage auf, ob die Armen "achtbar" oder "verächtlich" seien, also ob Armut durch strukturelle oder pathologische Gegebenheiten bedingt ist.

Diese Frage hat Parker überwunden. Jeder, polemisierte er, der ein Jugendheim geleitet, einen Bandenkampf beobachtet, eine Schlägerei vor dem Fussballstadium gesehen hat, wähnt sich berechtigt, eine eigene Abhandlung über die sogenannte Jugend von heute vorzulegen. Alle, die sich mit Jugendforschung befassen, sind in Gefahr, die Jugend als Problemfall zu betrachten, vor allem, wenn über Arbeiterjugendliche geforscht wird. Er schrieb: "Die neuere Hit-and-Run-Forschung, was immer ihre Ziele sein mögen, beleuchtet, ohne es zu beabsichtigen, letzten Endes doch den sensationellen Aspekt auf Kosten einer ausgeglichenen Untersuchung, die sowohl den sozialen Kontext wie die breitere soziale Struktur erhellt, in der die Jugend agiert". ${ }^{274}$

Diese Einsicht hat Parkers Blick auf kulturelle Prozesse freigelegt. Obiges Verhalten, so argumentierte er, steht in enger Beziehung zur kurzen Adoleszenz, und hängt damit zusammen, dass die Jungen in einem innerstädtischen Nachbarschaftsbezirk aufwuchsen, wo bestimmte Vermittlungsprozesse wirksam waren. Tatsächlich legten Szenen, wie sie in der Umgebung von Roundhouse zu beobachten waren, den

273 Ebd., 183-184.

274 Ebd., 182. 
Schluss nahe, dass die Gruppe der Jugendlichen wohl traditionelle Praktiken übernahmen, hinter denen, wie er annahm, traditionelle jugendspezifische Werte standen. ${ }^{275}$ Obwohl die Studie von Parker ausschliesslich männliche Jugendliche berücksichtigte, vermutlich weil weibliche Jugendliche in jener Zeit kaum in der Öffentlichkeit sichtbar waren, wurde diese Erkenntnis für meine Studie wichtig. Sie schlägt vor, dass die Jungen eine eigene kulturelle Gruppe bilden, welche die Altersgruppe der 15-19jährigen umfasst. Diese Gruppe ist von einem hohen Umsatz an Mitgliedern gekennzeichnet, was die Vermittlungsformen und Traditionen prägt. Parker schloss dies aus der Tatsache, dass die Boys selber feststellten, dass "die jüngeren Burschen die älteren kopieren" und "genauso hart sein wollen wie die älteren Kumpel". ${ }^{276}$

Diese Studie möchte eine Begrifflichkeit anwenden, welche einen möglichst unvoreingenommenen ethnologischen Zugang ermöglicht. Jugendliche sind den Erwachsenen fremd, dürfen aber als Beforschte wie andere Gruppen auch unseren Respekt und unsere Unvoreingenommenheit in Anspruch nehmen. Ein "studying down" kann zwar praktisch nicht ausgeschlossen werden, da die Forschenden Erwachsene sind, sollte aber möglichst vermieden werden.

\section{$\underline{5.3 \text { Jugendkultur und Kultur der Jugend }}$}

Oben beschriebene kulturellen Phänomene werden in dieser Studie unter dem Begriff Jugendkultur im Sinne der Kultur von Jugendlichen eingeordnet. Doch soll dieser nicht mit dem Begriff Jugendkultur verwechselt werden, der gemäss Dieter Baacke ${ }^{277}$ bereits historisch besetzt

275 Ebd., 186.

276 Ebd., 187.

277 Dieter Baacke: Jugend und Jugendkulturen, 3. Auflage, Weinheim und München, Juventa, 1999, $141 \mathrm{ff}$. 
ist. Der Begriff war zur Zeit seiner Entstehung um 1900 ein Kampfbegriff, der gegen die herrschende pädagogische und gesellschaftliche Praxis gerichtet war und die bürgerlichen Traditionen, die bürgerliche Unterhaltungskultur und ihre geistige Welt erneuern sollte. ${ }^{278}$

$\mathrm{Zu}$ Beginn des 21. Jahrhunderts ist die Kultur der Jugend viel mehr als damals von ausserschulischen populärkulturellen als von bürgerlich kulturellen schulischen Einflüssen geprägt. Kultur wird auch nicht mehr nur als Bestand von Traditionen und geistigen Bildungsgütern aufgefasst, sondern als ein Lebensraum, der durch Konsum, Mode und soziale Praxen definiert wird. Der Begriff Jugendkultur besitzt also eine pädagogische Tradition, das Bildungssystem bleibt ein Bezugspunkt, obwohl die Kultur der Jugendlichen sich von der Schule weg bewegt, freizeitbezogener geworden ist.

Ende des 20. Jahrhunderts stellte Jugendkultur im Westen eine eigenständige Tradition dar, in der Innovationen und neue Konzeptionen von Individualität zu finden waren. ${ }^{279}$ Jugendkultur beinhaltete Versuche zu Alternativen, zu Gegenöffentlichkeiten. Es ist auch eine Pluralisierung der Selbst-Konzepte und Praktiken festzustellen: Jugendkultu-

278 Der Begriff stammt von Gustav Wyneken (1875-1964), ein vor dem Ersten Weltkrieg bekannter Pädagoge und Schriftsteller. Er nahm an der deutschen Landschulheimbewegung teil und gründete 1906 zusammen mit Paul Geheeb die Freie Schulgemeinde Wickersdorf. Wyneken stellt den Begriff Jugendkultur gegen die von ihm so genannte Alterskultur der bürgerlich wilhelminischen Lebensweise. In der von Wyneken propagierten freien Schulgemeinde sollen die angeblichen Mängel der "alten" Schule und der Familie überwunden werden. (Baacke, Jugend und Jugendkulturen, 1999, 141-142) Äuffällig ist die Gleichzeitigkeit und thematische Nähe mit der künstlerischen Richtung des Jugendstils. Ab Januar 1896 erschien in München eine Kunstzeitschrift "Jugend", die jegliche Form der Spiessigkeit ablehnte, also die bürgerliche Unterhaltungskultur erneuern sollte und später berühmte Künstler mit ihren Werken vorstellte. Die Arbeiten dieser Künstler wurden allmählich als Stil der Zeitschrift Jugend, als Jugendstil bekannt.

279 Baacke, Jugend und Jugendkulturen, 1999, 143-145. 
ren lösen die Jugendkultur ab. Deren Internationalisierung und Konstituierung geschieht über die Massenmedien, die auch einer Pluralisierung unterworfen sind. Insgesamt kann im Begriff eine Schwächung der Bezeichnung eines Lebensalters und die Stärkung der Bedeutung eines Lebensgefühls beobachtet werden, das prinzipiell allen Altersgruppen zugänglich ist. Am Anfang des 21. Jahrhunderts spricht man in der Jugendforschung von Jugendkultur oder Jugendkulturen. Der Begriff Subkultur ${ }^{280}$ dagegen, der in den Sechziger und Siebziger Jahren des 20. Jahrhunderts die Diskussion stark dominierte, wird heute kaum mehr benutzt. ${ }^{281}$

Die Ausführungen von Heiz weisen darauf hin, dass Jugend eine kulturelle Konstruktion der Erwachsenen ist. Sie verändert sich historisch und folgt der Entwicklung der westeuropäischen Gesamtgesellschaft hin zur Konsumgesellschaft. Der Begriff Jugendkultur kommt mit seiner Betonung der geistigen und materiellen Güter merkwürdig altertümlich daher, vergleicht man ihn mit der Entwicklung des Kulturbegriffs im Allgemeinen. Eine weitere Folge der Pluralisierung war eine Schwächung des traditionellen Bereichs, wo Jugend statt fand. Der Jugendforscher Jürgen Zinnecker ${ }^{282}$ beschrieb, wie die Autorität über die Konstruktion von Jugend sich verschob.

Nach der Mitte des 20. Jh. treten altgediente Konstrukteure von Jugend ab, während die Macht der kulturellen Definitionen an Newcomer-Institutionen übergeht. Ins zweite Glied werden die Arbeitsorganisationen, die grossen Kir-

280 Für eine ausführliche Diskussion dieses Begriffes verweise ich auf ebd., 125-141.

281 Ebd., 146-147.

282 Zinnecker, Jürgen: Metamorphosen im Zeitraffer: Jungsein in der zweiten Hälfte des 20. Jahrhunderts, in: Geschichte der Jugend, Band II. Von der Aufklärung bis zur Gegenwart, Levi, Giovanni und Jean-Claude Schmitt (Hg.), Frankfurt a. M., S. Fischer, 1997, 460-505. 
chen, das Militär, die lokale Nachbarschaft, der traditionelle, erwachsenengeleitete Jugendverband gezwungen. ${ }^{283}$

Zinnecker wies darauf hin, dass diese Entwicklung für die Jugendlichen eine Aufwertung bedeutete: Als Gewinner gingen die Massenmedien, die Freizeit- und Konsumgüterindustrie, das Bildungs- und Wissenschaftssystem hervor sowie die Jugendlichen selbst. Der Modus der Konstruktion von Jugend veränderte sich dabei von einer autoritativ "verordneten" Zuschreibung durch Institutionen, die direkte Verfügungsgewalt über Jugend hatten, in Richtung eines marktgenerierten Wettbewerbs um die zugkräftigste, marktgerechteste Definition des Jungseins. ${ }^{284}$ Jugendliche wurden also von Befehlsempfängern zu Marktteilnehmern. Es entwickelte sich eine Allianz zwischen den Jugendlichen und einem Teil des sich nach dem Zweiten Weltkrieg ausweitenden Dienstleistungssektors, der Kulturindustrie, die auch die Wünsche der Jugendlichen nach Abgrenzung und Emanzipation von den Erwachsenen bediente. Diese Kulturindustrie, insbesondere deren Medien- und Konsumgüterindustrie, trat dabei nicht als direkte Kontrolleure von Jugendlichen auf, sondern als indirekt operierende Agenten, die sich zudem als Stellvertreter und Erfüllungsgehilfen jugendlicher Wunschproduktion gerierten. ${ }^{285}$ Bemerkenswert ist das Paradox, dass die Jugendlichen als Kunden so mündig, also erwachsen geworden sind, dass ihre Bedürfnisse bedient werden, sie also ökonomisch integriert werden, trotz des Widerstandes traditioneller Kontrolleure von Jugend, und dass gleichzeitig Jugendkultur konstituiert wird, also Abgrenzung entsteht. Nach Zinnecker entwickelte sich eine Komplizenschaft zwischen der Medien- und Konsumgüterindustrie und den Jugendlichen:

283 Ebd., 476.

284 Ebd., 477.

285 Ebd., 478. 
Jugendliche werden Ko-Konstrukteure: Jugendliche sind nicht bloss Objekte und Opfer der Methaphern, Bilder und Mythen, die mächtige Institutionen von Jugend produzieren und durchsetzen, das waren sie nie. Besonders seit den fünfziger Jahren ist diese Rolle jedoch weiter fortgeschritten (...). Die mediale "Wirklichkeit" von Jugend beruht auf Bündnissen, um nicht zu sagen Komplizenschaften, zwischen diesen Konstrukteuren und der jüngeren Generation. Kulturindustrie und Werbebranche näherns sich Jugendlichen im Bestreben, für diese Zielgruppe attraktiv zu sein, und Jugendliche greifen dieses auf, um ihre Wünsche und Vorlieben auf dem Markt zur Geltung zu bringen. ${ }^{286}$

Dieses Bild der Jugendlichen als Konsumenten der Medien- und Kulturgüterindustrie verschleierte effizient die Wahrnehmung von Jugend, weil eines der wichtigsten Bedürfnisse von Jugendlichen mit professionellen Strukturen bedient wurde, nämlich, dass die Erwachsenen sie nicht verstehen sollen.

Das erste Mal, als diese Komplizenschaft in Erscheinung trat, waren gemäss Zinnecker die sogenannten Halbstarkenkrawalle Mitte der fünfziger Jahre. Sie bildeten das Urmuster für später folgende wechselseitig nützliche Komplizenschaften. Die Krawalle entzündeten sich an den Anfängen einer populären Kultur für das junge Zielpublikum. Ein Auslöser war der aus den USA importierte Rock'n'Roll. ${ }^{287}$ Die Musik eignete sich als Transporteur eines neuartigen jugendlichen Le-

\section{Ebd., 479-480.}

287 Der “King” des Rock'n'Roll, Elvis Presley, schaffte es als Erster, Millionen von Platten zu verkaufen. Von diesem Zeitpunkt an war die Musikindustrie zum Big Business geworden, das von grossen Konzernen dominiert wurde und entsprechend professionell organisiert war. 
bensgefühls und war verbunden mit kulturell homologen Objekten, Kleidungsmoden, Haarstilen und einer entsprechenden Bewegungskultur (Motorrad, Tänze, lässiger Körperhabitus). Die Medienkampagnen, die diese Welle jugendlicher Populärkultur begleiteten, erzeugten Aufmerksamkeit, die die Jugendlichen sogleich für eigene, begleitende Auftritte nutzten. ${ }^{288}$

Das Muster des Rock'n'Roll sollte sich in der Zukunft mit Hardrock, Pop, Punk, New Wave und weiteren populären Stilrichtungen wiederholen. Rocker, Mods, Teddyboys, Skinheads, Punks, Hooligans und Technos/Raver hiessen die Akteure, deren

ritueller, manieristischer Widerstand gegen die Normalität des durchschnittlichen Aufwachsens ohne die wirksame Verstärkung und Verbreitung durch Medien-, Musik- und Modeproduzenten kaum denkbar wäre. Die soziale Identität, die diese Gruppen exponierter Jugendlicher durch solche Ko-Konstruktionen gewinnen, ist jedoch mehrdeutig. Sie schwankt grundsätzlich zwischen einer positiven $\mathrm{Zu}$ schreibung, der Rolle des Innovators, und einer negativen, der Rolle des öffentlichen Bösewichts, vor allem, wenn Gewalt im Spiel ist. Dies kann ins soziale Abseits führen, wenn sich öffentliche Panik mit strafrechtlichen Sanktionen kombinieren. ${ }^{289}$

Doch seit den 80er Jahren hat sich das Gewicht in den von Zinnecker beschriebenen Arbeitsbündnissen der jeweils aktuellen Jugendgenerationen mit den Produzenten des Dienstleistungssektors immer mehr in Richtung Jugendliche verschoben. Grund hierfür ist ein immer weiterer Ausbau des Dienstleistungssektors bei gleichzeitiger Abnahme der

288 Zinnecker, Metamorphosen im Zeitraffer, 1997, 481.

289 Ebd. 481-482. 
Anzahl Jugendlicher infolge des Geburtenrückganges in westlichen Ländern ab 1963. Fernsehkanäle, Radiosender, Zeitungen vervielfältigten sich, das Internet mit seinen ahierarchischen und dezentralen Kommunikationskanälen entwickelte sich. Jugendliche wurden mit Stilangeboten überschwemmt. Es entstand ein von Kunden, also den Jugendlichen, bestimmter Markt: Man musste nicht mehr unbedingt die neueste Rolling-Stone-Platte haben, um statusmässig als Jugendlicher mithalten zu können, da es auch andere Identifikationsangebote gab.

Die obigen Ausführungen zeigen, dass sich die kulturelle Konstruktion von Jugend zwar im Laufe der Geschichte der Entwicklung der Massenkonsumgesellschaft verändert, doch bleibt das Besondere und Singuläre der Jugendzeit im Lebenslauf eines Menschen. Diese Zeit ist in der Biografie durch spezifische Faktoren gekennzeichnet, die mit der Konstruktion des kulturellen Status des Erwachsenseins einhergehen. Dieser Prozess kann mit verschiedenen theoretischen Mitteln gefasst werden.

\subsection{Jugend und Moratorium}

Seit den Siebziger Jahren hat Jürgen Zinnecker in seinen Arbeiten über das Konzept Jugend immer wieder die "theoretische Leitfigur" 290 des Jugendmoratoriums eingebracht. Der Begriff Moratorium ist ursprünglich juristisch und bezeichnet einen Aufschub von Fälligkeit, eine Stillhaltephase. Es wird der Erfüllungstermin einer Verpflichtung in die Zukunft verschoben. Im soziologischen Zusammenhang ist der Moratoriumsbegriff viel weiter gefasst, Jürgen Zinnecker sprach in seinem Artikel über die Jugendzeit als Moratorium von einem Regen-

290 Zinnecker, Jürgen: Jugend als Moratorium, in: Reinders, Heinz und Elke Wild (Hg.): Jugendzeit - Time Out? Zur Ausgestaltung des Jugendalters als Moratorium, Opladen, Leske + Budrich, 2003, 37-64, 37. 
schirmbegriff, der den Freiraum bezeichnet, den die Gesellschaft der Jugend gewährt. Dieser äussert sich beispielsweise in einem separaten Jugendstrafrecht mit eigenen Behörden und Regeln was die Tatsache reflektiert, dass die Gesellschaft die Heranwachsenden als reduziert verantwortlich ansieht. Weil der Begriff Moratorium für diese Studie von zentraler Bedeutung ist, möchte ich die Vielfalt und Geschichte dieses Begriffes nach Zinnecker darstellen. ${ }^{291}$ Danach werde ich eigene Gedanken entwickeln, die auf diesen Erkenntnissen aufbauen und sie für diese Studie anwendbar machen.

Jede Diskussion über Jugend als Moratorium muss unweigerlich mit Rousseau beginnen, dem Begründer des europäischen Jugendbegriffs. Jean-Jacques Rousseau stellte 1762 einen Modelljugendlichen vor: Emile. ${ }^{292}$ Anhand dieses Modelljugendlichen zeigte Rousseau, wie das für ihn richtige Aufwachsen und die für ihn richtige pädagogische Begleitung und Steuerung des jugendlichen Aufwachsens künftig aussehen sollte. Die Elemente dieser Auffassung von Jugend ist für die Geschichte von Jugend in Europa hoch bedeutsam und bis heute stark prägend. Deshalb wird dieses Jugendmodell kurz rekapituliert.

Die Jugend von Emile wird mit der Krise der Pubertät als einer zweiten Geburt des Menschen eröffnet, einer Art Urknall, den Rousseau in der Metapher eines biologischen Gewitters beschreibt: "So wie das Grollen des Meeres das nahende Unwetter anzeigt, kündigt sich diese stürmische Revolution durch das erste Aufbegehren der entstehenden Leidenschaft an. ${ }^{\circ 293}$ Erst jetzt beginnt die Erziehung im engeren Sinn. Die Einführung in die moralische Welt und die Förderung von Leistung und Kompetenz beim Heranwachsenden sollen die Kindheit nicht belasten, diese sollte im Gegensatz dazu stressfrei verlaufen.

$291 \mathrm{Im}$ Folgenden beziehe ich mich auf ebd.

292 Die Reclamausgabe: Rousseau, J.-J.: Emile oder Über die Erziehung,

1762, herausgegeben von M. Rang, Stuttgart, Reclam, 1968.

293 Ebd., 439. 
Emile wächst in einer ländlichen Idylle auf, fernab der verderblichen zivilisatorischen Grossstadteinflüsse. Dieses Motiv der pädagogischen Provinz soll ein optimiertes Umfeld für die Entwicklung und Erziehung bereitstellen. Weil Emile allen Einflüssen ferngehalten wird, die Rousseau für unnatürlich und schädlich hält, lebt er in einer Gegenwelt, einer pädagogischen Enklave. Aus dieser kommt laut Rousseau die Erneuerung von Kultur und Gesellschaft. Emile ist als Rebell und Reformer prädestiniert. Die pädagogische Enklave ist ausschliesslich dem männlichen Geschlecht vorbehalten. Nur bei diesem sieht Rousseau einen radikalen Bruch mit der Kindheit. Nur durch die getrennte Erziehung kann der junge Mann die notwendige Askese und Sublimierung seiner Antriebe erlernen.

In „Emile“ wurden die wesentlichen Komponenten genannt, die wir bis heute kennen: Jugend als Krise, die besonderer pädagogischen Betreuung bedarf, Jugend als Teil einer Gegenwelt, der pädagogischen Provinz, Jugend als Erneuerung der Gesellschaft, Jugend als Domäne männlicher Askese und Sublimierung. In der Folge verstand sich Jugendforschung vorwiegend als Sozialgeschichte der Jugend. Bis mindestens zur 68er-Generation gilt die Denkfigur von Jugend als Zeit des Sturm und Drangs und das Konzept des Jugendlichen als Rebell und Reformer.

Dieses Bild war nicht negativ unterlegt. Erst Erik H. Erikson ${ }^{294}$ hat in den 1950er Jahren den Begriff des Moratoriums als Lebenskrise und Identitätsstatus genauer spezifiziert als psychosoziales Moratorium, und leider auch pathologisch gefärbt, in die Fachdiskussion eingeführt.

Ein Moratorium ist eine Aufschubsperiode, die jemandem zugebilligt wird, der noch nicht bereit ist, eine Verpflichtung zu übernehmen, oder die jemandem aufgezwungen

294 Erikson, Erik H.: Jugend und Krise. Die Psychodynamik im sozialen Wandel, Stuttgart, Klett-Cotta, 1974. 
wird, der sich selbst Zeit zubilligen sollte. Unter einem psychosozialen Moratorium verstehen wir also einen Aufschub erwachsener Verpflichtungen oder Bindungen und doch handelt es sich nicht nur um einen Aufschub. Es ist eine Periode, die durch selektives Gewährenlassen seitens der Gesellschaft und durch provokative Verspieltheit seitens der Jugend gekennzeichnet ist. (...) Meistens fallen diese Moratorien mit Lehrzeiten und Abenteuern zusammen, die mit den Wertskalen der jeweiligen Gesellschaft übereinstimmen. Das Moratorium kann eine Zeit zum Pferdestehlen oder oder der Suche nach einer Vision sein, eine Zeit der Wanderschaft oder der Arbeit "draussen im Westen" oder "drüben am anderen Ende der Welt", eine Zeit der "verlorenen Jugend" oder des akademischen Lebens, eine Zeit der Selbstaufopferung oder dummer Streiche - und heute ist es oft eine Zeit für Patiententum oder Kriminalität. ${ }^{295}$

Das jugendliche Moratorium ist gemäss Erikson durch die Gleichzeitigkeit von gesellschaftlichem Gewähren und jugendlichem Fordern gekennzeichnet. Dabei kann davon ausgegangen werden, dass die Gestalt des Moratoriums historischen und kulturellen Variationen unterliegt. In westeuropäischen Gesellschaften beispielsweise kann ein sukzessiver Wechsel vom Übergangs- zum Bildungsmoratorium beobachtet werden: Dabei bezeichnet Übergangsmoratorium einen relativ klar abgegrenzten Übergang von der Kindheit zum Erwachsensein, der im Kern durch eine relativ kurze Jugendphase gekennzeichnet ist. Die Heranwachsenden haben dabei die Rolle von Amateuren, die von der älteren Generation lernen. Die Wichtigkeit dieser Phase fällt hier vergleichsweise gering aus. Demgegenüber ist das Bildungsmoratorium 295 Ebd., $161 \mathrm{ff}$ 
aufgrund der Ausdehnung der in Bildungseinrichtungen verbrachten Lebenszeit ein verlängerter Lebensabschnitt mit relativ hoher Wichtigkeit. Jugend lernt nicht nur von Erwachsenen, sondern etabliert mit Peers eigene Lebensstile und Kompetenzen. Die Jungen können sozusagen als Profis gelten, die z.T. einen Wissensvorsprung gegenüber der älteren Generation besitzen.

Seit dem Zweiten Weltkrieg wurde das historische Modell des arbeitenden Jugendlichen, der innerhalb des Büros, der Lehrwerkstätte, der Fabrik erwachsen wird, immer mehr durch das Muster der Jugendlichen ersetzt, die innerhalb von Bildungsinstitutionen erwachsen werden. Die Jugendlichen müssen sich viel mehr mit Institutionen, Normen, Leistungszumutungen und Laufbahnen des Bildungssystems auseinandersetzen. ${ }^{296}$ Das Moratorium kann durchaus auch durch äussere Umstände, etwa ökonomische Faktoren, aufgezwungen werden. ${ }^{297}$ Dies kann zu einer unfreiwilligen Verlängerung der Jugendzeit führen.

In den 70er Jahren des letzten Jahrhunderts, als das Thema der sozial ungleichen Bildungschancen in der empirischen Bildungsforschung diskutiert wurde, kam immer mehr Kritik an Rousseaus Jugendkonzeption auf. Diese Kritik stellte die Ausformung des jugendlichen Moratoriums als rein männliche Domäne und als Lebensabschnitt der Askese und Sublimierung in Frage. ${ }^{298}$

In Zinneckers Promotionsschrift über die Sozialgeschichte der Mädchenbildung ${ }^{299}$ bestand ein zentraler Leitgedanke in der Idee, dass das

296 Zinnecker, Jugend als Moratorium, 2003, 52-53.

297 Schumann, Michael und Burkhard Fuhs (Fotos): Übergangsverläufe: Ende der Jugendzeit? in: Jugendwerk der Deutschen Shell (Hg.): Jugend '92. Lebenslagen, Orientierungen und Entwicklungsperspektiven im vereinigten Deutschland. Band 1: Gesamtdarstellungen und biografische Portraits, Opladen, Leske + Budrich, 1992, 173-183, 183.

298 Zinnecker, Jugend als Moratorium, 2003, 38-41.

299 Zinnecker, Jürgen: Sozialgeschichte der Mädchenbildung. Zur Kritik der Schulerziehung von Mädchen im bürgerlichen Patriarchalismus, Wein- 
Jugendmoratorium ein Privileg im Lebenslauf darstelle, das Jungen häufiger und intensiver als Mädchen gewährt werde. Entscheidend sei die Möglichkeit, qualitativ hochwertige Bildungslaufbahnen in Anspruch zu nehmen. Mädchen werde im Modell des sogenannten sozialen Wartestandes die Herausbildung eines individuellen Ich-Ideals und Wertekanons verwehrt; für jedes anspruchsvolle Jugendmoratorium eines meist bürgerlichen Jungen sei dieser das selbstverständliche Hochziel.

Auch die These der Askese und Sublimierung widerspricht der Mentalität der sechziger und siebziger Jahre des 20. Jahrhunderts. Diese Zeit ist als Epoche der "Entsublimierung" bekannt, ein Begriff, der die Veränderung alltäglicher Umgangsformen zwischen den Geschlechtern wie erotisch-sexuelle Normen oder die gesellschaftliche Regelung von heterosexuellen und homosexuellen Paarbeziehungen beschrieb. Jugendliche nahmen jetzt erotisch-sexuelle Beziehungen nicht erst nach dem schulischen Moratorium auf, sondern bereits während ihrer frühen Schülerjahre. Erste sexuelle Erfahrungen verlagerten sich vom Ende auf den Beginn der Jugendphase und wurden zunehmend gesellschaftlich toleriert. Das Bildungswesen wurde in dieser Zeit in ein koedukatives System überführt.

Auch das Moratorium der Studenten änderte sich. Der in den Nachkriegsjahrzehnten dominierende Typus des Studierenden, der enthaltsam und als Single lebt und lernt, wurde seit Mitte der 1960er Jahre zunehmend seltener. Das studentische Moratorium formte sich in einen sozialen Raum um, wo feste Paarbeziehungen in z.T. eheähnlichen Formen, meist unverheiratet und mit relativ spät geborenen Kindern dominierten. Auch arbeiteten die Studierenden oft neben dem Studium. Die von Rousseau und seinen Nachfolgern vertretenen Vorstellungen von

heim, Juventa, 1973. 
einem Jugendmoratorium als separate, monogeschlechtliche Bildungszone war endgültig abgelöst. ${ }^{300}$

Die ethnopsychoanalytische Zugangsweise zeigt die Konsequenzen dieser Erkenntnisse für die Einzelnen auf. Mario Erdheim ${ }^{301}$ verknüpfte die gesellschaftliche und die individuelle Ebene mit seiner These, dass die Akzeleration des Kulturwandels mit einer verlängerten Adoleszenz zusammenhängt. Der beschleunigte Kulturwandel bedingt also eine entschleunigte Adoleszenz und umgekehrt. Dies sei so, da der Triebdurchbruch in der Pubertät die vorher von der Familie gebildeten psychischen Strukturen lockert und damit die Voraussetzungen für eine nicht mehr auf den familiären Rahmen bezogene Neustrukturierung der Persönlichkeit schafft. Die Adoleszenz stellt die psychischen Bedingungen für den Wandel bereit. Es ist nun die Dehnbarkeit der Adoleszenz, welche die Akzeleration des Kulturwandels gestattet. Je schneller sich die Kultur verändert, desto länger wird nach Erdheim die Adoleszenz ihrer Angehörigen und umgekehrt. Die verlängerte Adoleszenz stellt die psychischen Energien frei, die den Wandel beschleunigen. ${ }^{302}$ Diese Beobachtung wies darauf hin, dass eine Entgrenzung der Adoleszenz in unserer Gesellschaft zu beobachten ist, die mit dem beschleunigten Kulturwandel in der Zeit der Globalisierung nach dem Ende des Kalten Krieges akzentuiert worden ist.

300 Zinnecker, Jugend als Moratorium, 2003, 43-44.

301 Erdheim, Mario: Zur Entritualisierung der Adoleszenz bei beschleunigtem Kulturwandel, in: Kosinski, Günther (Hg.): Pubertätsriten, Bern Stuttgart Toronto, Hans Huber, 1991, 79-88, 83. 302 Ebd., 79. 


\section{5 Übergangsriten und Statuspassagen}

Die genuin europäisch-ethnologische Theorie der Übergangsrituale lässt sich sehr gut mit den Überlegungen zu den Moratorien verbinden. Die Theorie der rites de passage ist zudem anhand der Phänomene des Erwachsenwerdens entwickelt worden und ist schon allein aus diesem Grunde für diese Studie unverzichtbar. Die Theorie wurde von Arnold van Gennep formuliert, und seit ihrer Publikation 1909 ist der Begriff des Übergangsrituals fast zu einem kulturwissenschaftlichen Gemeinplatz geworden und auch ausserhalb der Wissenschaft bekannt.

Die Rituale, welche Übergänge zwischen Zeiten, Räumen, verschiedenen sozialen Stellungen und anderen Beziehungen regulieren, lassen sich in drei Unterrituale aufteilen: ein Abtrennungsritual, ein Schwellenritual - nach dem lateinischen Wort für Schwelle ein liminales Ritual - und ein Aufnahmeritual. Van Gennep hat festgestellt, dass die liminale Phase und Menschen, die in ihr stehen, bestimmte Eigenschaften aufweisen. ${ }^{303}$

Diese Eigenschaften beschäftigten den amerikanischen Sozialanthropologen Victor Turner ${ }^{304}$, der anhand seiner Feldforschungen in Zentralafrika feststellen konnte, dass die Liminalphase relativ unstrukturiert, undefiniert und vieldeutig ist, im Gegensatz zur üblichen hierarchischen Organisation der Gesellschaft, wo alles und alle ihren festgelegten Platz haben. Turner benutzte für diese Eigenschaft den Begriff Communitas oder soziale Antistruktur. In einer Communitassituation entsteht eine direkte Kommunikation zwischen den Beteiligten, die ihren sozialen Status ablegen und damit gleichwertig werden. Gesellschaftliche Übergänge geschehen in solchen Situationen, die zu Scharnierpunkten von Übergängen von Sozialstatus zu Sozialstatus werden.

303 Van Gennep, Übergangsriten (Les rites de passage), 1986, 182.

304 Turner, Victor: Das Ritual, Struktur und Anti-Struktur, Frankfurt/NewYork, Campus, 1989. 
Die Menschen in liminalen Situationen weisen bestimmte Eigenschaften auf. Sie rutschen durch das Netz der Klassifikation, welches normalerweise die Positionen im kulturellen Raum festlegt. Sie stehen "betwixt and between"305, fallen also sozusagen zwischen Stuhl und Bank. Sie sind oft Adepten, die sich passiv oder demütig verhalten müssen, erleiden Spott und Schmerzen, werden gestraft, haben einen unklaren Status, keine Insignien. In dieser Situation wird spielerisch mit Symbolen umgegangen. Untereinander entwickeln die Liminalen eine intensive Kameradschaft und Egalitarismus.

Turner beschrieb also zwei grundsätzlich verschiedene Modelle menschlicher Bindungen, die sich im Leben abwechseln. Das erste ist die Stellung in der Gesellschaft, die als strukturiertes, differenziertes und hierarchisches System gesellschaftlicher Positionen erscheint, wo ein oben und unten existiert. Das zweite ist die soziale Antistruktur, die in liminalen Phasen vorherrscht. Hier ist die Gesellschaft unstrukturiert, eine Gruppe gleicher Einzelner, die sich unter die Autorität rituell älterer begeben. Die Inhaber der höheren Position anerkennen damit ein allgemeingültiges gesellschaftliches Konzept, denn in liminalen Phasen, die sie in ihre Position gebracht haben, haben sie erfahren, dass ein oben nicht ohne ein unten sein kann. Am Ende der Kindheit etwa ist die Stellung des Individuums in der Gesellschaft nicht mehr haltbar. Eine Statuspassage drängt sich auf, die Individuen in dieser Situtation begeben sich unter die Autorität rituell älterer, um in die statusmässig nächsthöhere gesellschaftliche Gruppe, die der Erwachsenen, integriert zu werden.

Das soziale Leben, das gilt für Individuen wie auch für Gruppen, ist nach Turner ein dialektischer Prozess zwischen oben und unten, Struktur und Antistruktur, Homogenität und Differenziertheit, Gleichheit und Ungleichheit. Jede Gesellschaft setzt sich aus Menschen zusammen, die ihre eigenen Entwicklungsgänge haben. Während dieser Entwicklung 305 Ebd., 95. 
überschreiten die Menschen immer wieder Statusschwellen, das heisst, während ihres ganzen Lebens sind sie Struktur und Antistruktur ausgesetzt. ${ }^{306}$

Von diesen Überlegungen ging wiederum Terence S. Turner aus. Er stellte fest, dass die Ausführungen von Victor Turner davon ausgehen, dass die Phänomene der liminalen Phase einem abstrakteren Niveau angehören als diejenigen der normalen Sozialstruktur. Bezieht man zudem die regulierende Funktion der Übergangsrituale in die Überlegungen ein, liegt der Schluss nahe, dass hier ein kybernetisches System vorliegt, das die Gesellschaft stabil hält, obwohl immer neue Generationen nachstossen und anderskulturelle Menschen, die von aussen kommen, integriert werden müssen.

So gesehen wird die liminale Phase eines Übergangsrituals zu einer "transformational operation", wie Terence Turner schrieb, zu einer Übergangsoperation, wobei Operation im Sinne einer mathematischen Operation verstanden wird. Ein bestimmter Satz von gesellschaftlichen Eigenschaften, die einem Akteur vor einem Übergang anhaften, wird mit einem anderen Satz von gesellschaftlichen Eigenschaften, die dem zukünftigen Sozialstatus entsprechen, nach einem höheren formalen Prinzip so verknüpft, dass als Resultat ein neuer Satz von gesellschaftlichen Eigenschaften entsteht.

Auf die in dieser Studie untersuchte Gruppe angewendet, bedeuten diese Ausführungen Folgendes: Wenn beispielsweise ein Jugendlicher, dessen Satz von Eigenschaften mit jugendlich, also elternabhängig, sexuell inaktiv, unmündig, etc. bezeichnet werden kann, erwachsen wird, werden diese Eigenschaften in Frage gestellt, was die Sozialstruktur dieser Person gefährdet und damit die sie tragende Gesellschaft destabilisiert. Dies bedeutet, dass ein Übergang zu einem anderen Status nötig wird, der mit Erwachsensein bezeichnet wird und auch einen bestimmten Satz von Eigenschaften wie Elternunabhängigkeit, sexuelle Aktivi306 Ebd., $96 f f$. 
tät, Mündigkeit, etc. beinhaltet und wieder stabil ist. Nun werden in einem Übergangsritual, etwa in einer Konfirmation, beide Sätze sozialer Eigenschaften in einem Communitasrahmen aufgehoben und so der Übergang ermöglicht. Trotzdem behält der Akteur gewissen anderen Menschen gegenüber den alten, abgelegten Satz sozialer Eigenschaften manchmal bei, beispielsweise gegenüber seinen Eltern oder Geschwistern.

Demzufolge bedeutet die Integration der beiden Sätze sozialer Eigenschaften innerhalb des Beziehungsfelds des Akteurs eine Erhöhung des gesellschaftsstrukturellen Niveaus: Der Akteur steigt gesellschaftlich auf. Das Beziehungsfeld besteht also, formal gesehen, aus drei Komponenten: erstens aus den verschiedenen Sets sozialer Beziehungen, die im Gegensatz zueinander definiert werden können, zweitens aus den Übergangsoperationen, welche die Übergänge zwischen den verschiedenen Beziehungsarten regeln und drittens aus dem gesellschaftlich vorgeschriebenen Muster der verschiedenen Übergänge, welches durch ein formales Prinzip vorgeschrieben wird. ${ }^{307}$

Diese Überlegungen beschreiben das Muster eines gesellschaftlichen Aufstieges. Leute, die einen hohen gesellschaftlichen Status geniessen, haben im Laufe ihrer Karriere einen cursus honorum durchlaufen, der kulturspezifisch vorgeschrieben ist. Sie haben im Laufe dieses Aufstiegs häufig Übergänge durchlaufen, die rituell markiert sind. Auch haben sie im Laufe dieses Aufstieges eine Vielzahl von Sets sozialer Beziehungen integriert, die ihnen erlauben, mit anderen hochstehenden Persönlichkeiten zu interagieren, die je nach gesellschaftlichem Aktionsfeld anders gestaltete Aufstiegsmuster hinter sich haben. Die Aufstiegsmuster im Sport oder in der Wirtschaft etwa sind anders als dieje-

307 Turner, Terence S.: Transformation, Hierarchy and Transcendence: A Reformulation of Van Gennep's Model of the Structure of Rites de Passage, in: Moore, Sally F. und Barbara G. Myerhoff: Secular Ritual, Assen/Amsterdam, Van Gorcum, 1977, 53-56. 
nigen in der Politik. Dieses System hält die Gesellschaft stabil, indem der Zugang zu Statusstufen reguliert wird.

Die kulturell definierten Muster der Übergänge beschreiben die Statusdynamik des Erwachsenenlebens und regulieren Integrations- und Ausschlussprozesse. In dieser Studie soll beobachtet werden, wie Jugendliche, die erstmalig mit diesen Prozessen kontrontiert werden, mit diesen Umgehen und wie sie entstehende Probleme meistern lernen.

Doch ist Jugend zuallererst mit tiefgreifenden körperlichen Veränderungen verknüpft. Die regelmässige Blutung definiert die junge Frau, junge Männer bekommen Stimmbruch und Bartwuchs. Als Kulturwissenschaftler interessieren wir uns jedoch eher für die Prozesse der Ablösung und Selbstfindung, welche wichtige kulturelle Veränderungen nach sich ziehen. Ethnopsychoanalytische Ansätze können diese Ebenen verknüpfen. Sie sind sehr hilfreich für das Verständnis der Prozesse des Erwachsenwerdens. Mario Erdheim beispielsweise sah die stammesgesellschaftlichen Übergangsriten, die in der Pubertät durchgeführt werden, als Anpassungsprozesse an, die nach Paul Parin und Goldy Parin-Matthèy die Identifikation mit der Rolle als Mann oder Frau und mit der Zugehörigkeit zu einer bestimmten Kultur zu tun haben. Diese haben auch einen dreiteiligen Verlauf, wobei die erste Phase die Trennung von den Eltern und die zweite den eigentlichen Übergang darstellt, der nach Van Gennep von Ambivalenzen gekennzeichnet ist. Sie ist, stellte der Jugend-psychiater Günter Klosinski ${ }^{308}$ fest, in unserer Gesellschaft im Gegensatz zu den Stammesgesellschaften stark verlängert.

In nichtindustriellen Gesellschaften wird der Übergang gelegentlich durch die Absonderung der Adepten in eine Hütte oder Höhle markiert und durch spezialisierte Erwachsene unterstützt. Hier erarbeitet Mensch

308 Klosinski, Günter: Pubertätsriten - Äquivalente und Defizite in unserer Gesellschaft. Einführende Anmerkungen des Jugendpsychiaters, in: Kosinski, Günter (Hg): Pubertätsriten, Bern Stuttgart Toronto, Hans Huber, 1991, 11-20, 12. 
seine Identität, gleichzeitig wird die Frage nach dem Menschensein in der jeweiligen Kultur durch Erzählungen über die Geschichte der Gruppe, ihrer Gebräuche und Riten beantwortet. Hier sind Mentoren wichtig, die nicht aus der Gleichaltrigengruppe stammen und diese Informationen vermitteln können. ${ }^{309}$

Die Jugendlichen dieser Studie unterhielten eine Art Hütte: Eine früher als Praxis gebrauchte Einzimmerwohnung war durch Geschäftsaufgabe frei geworden, aber noch nicht weiter vermietet. Hier trafen die Jugendlichen sich, spielten, schliefen, assen, verhandelten Normalitätskonzepte und aktuelle Fragen um das Erwachsenwerden. Manchmal wurde der Forscher mit einbezogen, was ich dazu nutzte, den gesellschaftlichen Diskurs über bestimmte Fragen zur Diskussion zu stellen. So konnte ich die Stelle als rituell Älterer einnehmen und eine Hierarchiebildung möglichst vermeiden. Über die Rolle der Wohnung als Äquivalent der Höhle oder Hütte wird im Abschnitt über ,die Wohnung" mehr berichtet.

Victor Turner und die Ethnopsychoanalytiker entwickelten ihre Theorien vor dem Hintergrund kleiner Stammesgesellschaften. In jenen Gesellschaften, die Claude Lévi-Strauss wegen ihrer Neutralisierung des Kulturwandels die Kalten nannte ${ }^{310}$, wird die Adoleszenz stark ritualisiert. Diese starke Ritualisierung ist sinnvoll, weil die Gesellschaft streng strukturiert ist: Die Rollen der Erwachsenen sind weitgehend definiert und die Ambivalenz der Adoleszenz gilt als unrein und gefährlich. Die Rituale beherrschen das Unreine, tradieren die kulturelle Identität und festigen die Gruppe. Psychologisch gesehen dienen die Rituale der Angstreduktion, der emotionalen Stabilisierung und damit der Krisenbewältigung. ${ }^{311}$

309 Ebd., 15-17.

310 Lévi-Strauss, Claude: Rasse und Geschichte. In: Lévi-Strauss, C.: Strukturale Anthropologie II, Frankfurt a.M. 1975, 365-407. 
In postmodernen, dynamischen Gesellschaften sinkt zwar die Bedrohung der Gesellschaftsstruktur durch nachstossende Generationen, die integriert werden müssen, doch werden die Integrationsverläufe komplexer. Es ist zu beobachten, dass sich das Jugendmoratorium in eine Vielzahl kleinerer rituell markierter Übergänge aufgliedert, welche Kinder und Jugendliche zu bestehen haben. Riten des Überganges bei Einund Ausschulung, religiöse Zeremonien wie Erstkommunion, rechtliche Übergänge wie Führerscheinerwerb und solche der Raumnutzung wie die verschiedenen Stadien der Motorisierung, Stufen des Auszuges aus dem Elternhaus, Riten und Abfolgen der Teilhabe an Paarbeziehungen, vom ersten date über die verpflichtende Beziehung bis zur Heirat gilt es zu durchlaufen. Eine solche Vervielfachung von Initiationsriten, die ihren eigenen Logiken folgen, entspricht der Struktur ausdifferenzierter Lebensläufe in modernen Gesellschaften. Aus der Dezentrierung und zeitlichen Separierung von Initiationsriten ergibt sich auch eine andere Forschungsperspektive: Jugend lässt sich als Kette kleinerer und grösserer normativer Lebenslaufereignisse verstehen und untersuchen. ${ }^{312}$

Dabei soll jedoch in der Interpretation restriktiv vorgegangen werden, wie Gerrit Herlyn ${ }^{313}$ angesichts der verschiedensten Sinn- und Bedeutungszuschreibungen von Ritualen argumentiert hat. Qualitativ sehr unterschiedliche soziale Handlungen werden mit obigen Theorien erklärt. Bisweilen hinterlasse der unterschiedliche Umgang mit der klassischen Ritualliteratur den Eindruck, dass zu viele soziale Handlungen unter den Ritualbegriff gefasst wurden. Neben der Forderung, nicht an der attraktiven Oberfläche der Rituale zu bleiben, um gesellschaftliche Problemstellungen zu erkennen, findet Herlyn die Forderung berechtigt,

311 Erdheim, Zur Entritualisierung der Adoleszenz bei beschleunigtem Kulturwandel, 1991, 83.

312 Zinnecker, Jugend als Moratorium, 2003, 48-49.

313 Herlyn, Gerrit: Ritual und Übergangsritual in komplexen Gesellschaften, Sinn und Bedeutungszuschreibungen zu Begriff und Theorie, Hamburg, Lit Verlag, 2002. 
rituelles Handeln von nicht-rituellem Handeln abzugrenzen. Hochzeiten und Beerdigungen als Rituale zu fassen, fällt noch leicht, der interpretative Anteil bei der Beschreibung von Taxi-Fahrten als Übergangsritual ist doch als (wohl zu, Anmerkung ns) hoch einzuschätzen. ${ }^{314}$ Um dieser Kritik gerecht zu werden, wurde in dieser Studie versucht, die beobachteten Phänomene nur bis zu einem gewissen, aus quellenkritischer Sicht als vernünftig erachteten Grad interpretativ auszuschöpfen.

Das Problem der sehr unterschiedlichen Handlungen, die als Ritual aufgefasst werden können, wurde jedoch für die Lebenslaufereignisse angegangen. Glaser und Strauss haben in ihrem Werk „Status Passage“ ${ }^{315}$ ein Klassifikationssystem vorgelegt, das erlaubt, solche normativen Lebenslaufereignisse zu typologisieren. Der Übergang kann unvermeidbar sein wie das Erwachsenwerden oder vermeidbar wie etwa die Heirat. Er kann reversibel sein wie eine Beförderung oder Degradierung oder wiederholbar, wie die Wahl in ein Amt. Das Umfeld kann eine Rolle spielen. Er wird kollektiv im Jahrgangsverbund, allein oder aggregiert in einer wie auch immer definierten Gruppe erlebt. Er kann bewusst oder unbewusst erfolgen sowie freiwillig oder unfreiwillig, wie etwa nach einer Verurteilung vor Gericht, wenn jemand in den Häftlingsstatus gerät. Ein weiteres Kriterium ist der Grad der Kontrolle, welche verschiedene Agenten ausüben, eingeschlossen jene Person, die den Übergang erlebt. Eine Mutter kann etwa ihren Sohn davon abhalten, die Fahrprüfung zu machen, was den Jungen in den Status eines Führerscheinbesitzers bringen würde. Der Übergang muss unter Umständen durch einen legitimierenden Agenten abgesegnet werden, wie etwa, wenn bei einem Todesfall der Arzt einen Totenschein ausstellt. Erst dann erhält jemand den Status "tot". Ein weiteres Kriterium ist die Klarheit des Überganges. ${ }^{316}$ Es fällt auf, dass diese Typologisierung kei-

314 Ebd., 73-75.

315 Glaser und Strauss, Status Passage, 1971.

316 Ebd. 4-6. 
nem übergeordneten Konzept folgt, sondern einfach die Passagen durch bestimmte arbiträre Eigenschaften gruppiert. Deshalb wird weiter unten ein Klassifikationssystem vorgeschlagen, welches die Typologisierung der Statuspassagen in einen Zusammenhang mit dem Jugendmoratorium stellt. Damit wird auch das von Gerrit Herlyn angesprochene Problem entschärft.

Statuspassagen haben, stellen Glaser und Strauss weiter fest, eine bestimmte zeitliche Abfolge, die sie die sentimentale Ordnung der Statuspassage nennen. Damit meinen sie, dass diejenigen, welche mit der Statuspassage zu tun haben, eine ziemlich genaue Vorstellung eines Art Fahrplans haben, welche Zeremonien, Rituale, Verzögerungen, Beschleunigungen, Schrittfolgen wann genau zu erwarten sind. Zeitliche Änderungen wie unplanmässige Verzögerungen, Rückgängigmachungen oder schlechtes Timing enttäuschen diese temporalen Erwartungen und werden von allen Beteiligten als beunruhigend bis desaströs empfunden. ${ }^{317}$ Deshalb gibt es Verhandlungen zwischen den Leuten in der Passage, den Agenten, die den Übergang regeln und den offiziellen Legitimatoren, welche die Hoheit über den Zeitplan haben. Dabei werden sogenannte Zeitblöcke ${ }^{318}$ ausgeschieden, die den Zeitplan puffern helfen, um den Übergang kontrolliert ablaufen zu lassen. Gleichzeitig werden Notfallsysteme aufgebaut, welche die Folgen von schlechtem Timing auffangen sollen. ${ }^{319}$

Glaser und Strauss zeigen, dass ein Statusübergang einen Verlauf hat, der sich aus Richtung und Timing der Passage zusammensetzt. Im Original verwenden die Autoren den Begriff "shape", also Form oder Gestalt. Über diese Form müssen sich die Personen im Übergang und die Agenten, die den Übergang regeln, einigen. Das ergibt ein Gleich-

317 Ebd. 43.

$318 \mathrm{Im}$ Original: temporal cushions: Zeitkissen.

319 Ebd. 45. 
gewicht von Kontrolle über den Prozess. ${ }^{320}$ Dieser kann sich schnell destabilisieren, seine Form verlieren, beispielsweise wenn die Zeichen der Passage nicht richtig interpretiert werden, deshalb Probleme entstehen und eine Möglichkeit zur Korrektur nicht ergriffen wird. Meistens sorgen aber kleine Fehler, Beschleunigungen, Verzögerungen oder Rückschritte dafür, dass die Passage ausser Form gerät. In diesem Fall entsteht Abweichung ${ }^{321}$ und eine Debatte über die Möglichkeiten, die Form wieder herzustellen. Dies ist etwas anderes als zu verneinen, dass eine Passage überhaupt stattfindet, was auch vorkommen kann. Im Fall der Abweichung gibt es Experten, wie Ärzte, Sozialarbeiter, Anwälte, die dafür sorgen, dass die Passage ihre Form wieder findet. ${ }^{322}$

Im Fall unserer Jugendlichen waren es die sozialen Institutionen des Kantons Basel-Stadt, welche die Form des Übergangs in das Berufsleben aufrecht zu erhalten versuchten. Die in dieser Studie unterstuchten Jugendlichen hatten im untersuchten Zeitraum vor allem mit dem Lehrstellenmangel zu ringen, was ihren Übergang in die Berufswelt behinderte. Dieses Problem war in der Stadt Basel im Vergleich zu anderen Kantonen besonders akut. ${ }^{323}$ Um diesen Mangel zu bekämpfen, hatten die offiziellen Legitimatoren aus dem pädagogischen Bereich Notfallsysteme aufgebaut. Einige davon wurden unter dem Begriff Brückenangebote zusammengefasst und boten, wie der Name sagt, ein Zusatzjahr in der Schule mit vielen Integrationsmöglichkeiten. Zudem hat sich seit 2002 im Kanton Basel-Stadt ein Zweig des Sozialbereichs ausgeweitet, der sich mit der Übergangssituation der Jugendlichen in das Berufsleben befasst. Aufschlussreich aus ethnologischer Sicht ist die Tatsache, dass der Sozialsektor, seine Entscheidungsträger und die Regierung er-

320 Ebd. 57-58.

321 Original: mild deviance.

322 Ebd. 73-76.

323 Im 2005 waren über 40\% der Schulabgänger ohne Anschlusslösung. 
kannten, dass die Statuspassagen ganzer Jahrgänge aus der Form zu geraten drohten und ein Ausbaubedarf im Sozialbereich bestand.

Eine Typologie dieser Notfallsysteme ist informativ, weil sie zeigen, wo alles Übergänge aus der Form geraten können. Schaut man sich die Liste der in diesem Bereich tätigen Institutionen an, lassen sie sich grob in zwei Bereiche gliedern. Der erste Bereich ist derjenige des sekundären Arbeitsmarktes. Werkstätten, Ämter wie die Stadtgärtnerei und soziale Einrichtungen bieten schätzungsweise rund eintausend in irgend einer Form geschützte Arbeitsplätze an, in denen die Jugendlichen eine Zeit lang Berufserfahrung sammeln können. Berufserfahrung ist also eine wichtige Voraussetzung für einen stabilen Übergang und soll überhaupt möglich gemacht werden.

Der zweite Bereich umfasst alle Tätigkeiten, die den Jugendlichen im Kanton Basel-Stadt Begleitung anbieten und so die Form des Übergangs wieder herstellen sollen. Sie weisen darauf hin, welche Faktoren die Statuspassage aus der Form geraten lassen können und zeigen Wege aus diesen Blockaden auf. Die Faktoren und das Ziel des Unterstützungsangebots sind in Klammer zur Institution gesetzt.

Organisation: Ziel/Tätigkeit (Faktor Statuspassage, Zweck_ in Bezug auf Statuspassage)

Sozialhilfe Basel-Stadt: Begleitung von 18-25jährigen, die die Sozialhilfe aufsuchen (Armut, sehr niedrige soziale Stellung verhindert Statuspassage, Start der

Statuspassage)

Sozialhilfe Basel-Stadt: Integration von

Fürsorgebezüger/innen in den ersten Arbeitsmarkt (Neustart des Übergangs nach Fehlstart)

Fachstelle Migration und Integration: Jugendliche institutionenübergreifend begleiten (Migrationshintergrund, d.h. doppelte Integrationsforderung, Fehlen der ersten Stufen 
des cursus honorum, Fehlen von kulturellem Kapital, damit sehr niedriger Status. Früherkennung von Blockaden, Form des Übergangs erhalten)

Lehrplatz für Migrantinnen: Patin begleitet Jugendliche/n nach der obligatorischen Schulzeit (Fehlendes soziales Kapital bereitstellen, Form des Übergangs erhalten) Netzwerk Lernberatung AGS: Jugendliche in der Lehre, Vermittlung von Stütz- und Förderangeboten, Hilfe bei Teilleistungsschwächen und allgemeinen Problemen (Motivationsmangel und Angst bekämpfen, Statuspassage unterstützen)

Projekt "last minute": Finden einer Lehrstelle, Beratung bei einzelnen Schritten wie Bewerbungen schreiben, etc. (Unter Zeitdruck fehlende formale Kenntnisse ersetzen, damit Start der Statuspassage ermöglicht wird) berufslehre.ch/brückenangebote: Informationsangebot über Brückenangebote (Fehlende Kenntnisse über Formreparatur- und Unterstützungsangebote bereitstellen) Brückenangebote: Verbesserung der Grundkenntnisse (Fehlen fachlicher Kenntnisse, Chancen der Bewältigung der Statuspassage soll erhöht werden)

Verein Neustart: Schwerstintegrierbaren Jugendlichen erste Lösungsansätze aufzeigen (Neustart des Übergangs nach Scheitern von Statuspassagen)

Kiebitz: Beratung für Stellenlose durch Standortbestimmung (Blockade, Neustart des Übergangs bei Identitätsproblemen)

Help: Psychologische Unterstützung am Arbeitsplatz (Motivation, Übergang durchzuziehen wird angeboten) 
Kontaktstelle für Arbeitslose: Begleitung und Unterstützung im Umgang mit den Behörden (Organisation der Formreparatur bei verschiedenen Experten)

Schwarzer Peter: Jugendliche und junge Erwachsene begleiten, die auf der Strasse leben (Statuspassage defekt wegen extrem niedrigen sozialen Status, Formreparatur wird angeboten)

Basler Freizeitaktion: Stärken von Handlungskompetenzen von Jugendlichen (Statuspassage nicht gefährdet, Stützung durch Kompetenzstärkung)

Basler Freizeitaktion Jugendberatung: Psychosoziale Unterstützung, damit das Leben selbständig gemeistert werden kann (Statuspassage unsicher, Formreparatur durch Hilfe bei den grundlegenden Kulturtechniken) lotse: Hilfe für fremdsprachige Jugendliche bei der Lehrstellensuche (Migrationshintergrund) InTeam: Jugendlichen den Einstieg in die Berufswelt erleichtern (Hilfe beim Start der Statuspassage) JobFactory Basel: Erhöhung der Vermittlungschancen durch Training "on the job" (Formstabilisierung durch Kompetenzvermittlung)

Interkulturelles Foyer: Tagestruktur bereitstellen, Motivation und Fähigkeiten fördern (Formstabilisierung durch Unterstützung bei grundlegenden Kulturtechniken und verbessern der Motivation, die Statuspassage durchzustehen $)^{324}$

324 Abteilung Jugend, Familie und Prävention des Justizdepartementes BaselStadt: Die Situation sozial schwacher Jugendlicher im Übergang zwischen Schule und Beruf, Übersicht über Programme, Akteure, Schnittstellen in Basel-Stadt (und Basel-Land), 24. Oktober 2003. 
Diese Unterstützungsangebote für Jugendliche mit Formdefekten in der Statuspassage lassen sich wie folgt typologisieren: Zunächst gibt es Angebote, die versuchen, die Statuspassage zu starten oder neu zu starten. Dann gibt es Angebote, welche während der Statuspassage wirken: Die Motivation, die Statuspassage durchzustehen, wird erhöht. Es werden auch Kompetenzen verstärkt, die zur Bewältigung der Statuspassage nötig sind oder kulturelles Kapital wie Beziehungen, Behördenkontakte oder kulturelles Wissen zur Unterstützung der Passage bereitgestellt. Drittens werden Formreparaturen für blockierte Übergänge angeboten, indem der Blockadefaktor bearbeitet wird, etwa mangelnde Kulturtechniken oder fehlende Motivation. Dabei erfolgt die Arbeitsteilung nach Zielgruppen.

Es lässt sich beobachten, dass einige Jugendliche recht lange bei diesen Angeboten verbleiben und es schwer haben, in den regulären Arbeitsmarkt zu wechseln. ${ }^{325}$ Bei ihnen wird der Statuspassage ein relativ langer Zeitraum eingeräumt, da viele Programme über die reine Jugendzeit hinausreichen und Leute umfassen können, die bis 25 Jahre alt sind. Es gibt auch Programme, die nicht zwischen Jugendlichen und Erwachsenen unterscheiden, was darauf hinweist, dass diese Problem kaum mehr exklusiv Jugendliche betreffen.

Neben diesen Angeboten gibt es ein eigentliches Mentoringprogramm, das im Kanton Basel-Stadt von der Europäischen Ethnologin Beatrice Ledergerber geleitet wurde und das den Jugendlichen eine Begleitperson mit hohem Sozialstatus zur Verfügung stellt. Mentoring ist nicht institutionengebunden, sondern wird von Privatpersonen getragen, die Jugendliche bei ihrer Berufswahl begleiten. Hier wird also den Jugendlichen eine Expertenperson mit kulturellem Kapital zur Seite gestellt, die den ganzen Statusübergang coachen soll, damit Formverluste möglichst schnell erkannt und bewältigt werden können. Entstanden ist

325 Mündliche Klage einer Betreuerin anlässlich einer Tagung über Probleme bei der Lehrstellensuche, März 2003. 
das Programm als Reaktion auf die Feststellung, dass junge Migrantinnen die grösste gesellschaftliche Gruppe darstellen, die ohne Abschluss bleiben. Bei dieser Gruppe ist die Gefahr also am Grössten, dass die Statuspassage aus der Form gerät, denn hier kombiniert sich niedriger sozialer Status, fehlendes kulturelles Kapital, die jugendliche Statuspassage und die Migrationsstatuspassage. Vermutlich spielt auch der von Zinnecker in den frühen 70er Jahren auch für westliche Mädchen beschriebene soziale Wartestand eine Rolle, welche Frauen in gewissen Kulturen einnehmen müssen.

Dies ist wahrscheinlich vor allem für Töchter aus sogenannten traditional-sklerotisierten Migrationsfamilien der Fall, die auf die moderne urbanisierte Gesellschaft des Gastlandes mit Rückzug und Abschottung reagieren. Nach einer Migration gibt es, wie der Kinder- und Jugendpsychologe Andrea Lanfranchi festgestellt hat, zwei Zugehensweisen zur Kultur des Gastlandes: Die traditional-vorwärtsgewandte Familie, die trotz psychosozialen Belastungen und Diskriminierungserfahrungen in der Lage ist, ihr Schicksal in die eigenen Hände zu nehmen und autonomiebezogene Kriterien von Lebenswirklichkeit zu entwickeln, und die sogenannte traditional-sklerotisierte Familie, die auf die moderne urbanisierte Gesellschaft des Gastlandes mit Rückzug und Abschottung reagiert. Die Vorwärtsorientierung, die sich durch Selbstbestimmung und Handlungsfähigkeit auszeichnet, ist die wichtigste Ressource der Jugendlichen in der Übergangssituation der Migration. ${ }^{326}$

Als Grund für die schlechte Ausbildungssituation wird angenommen, dass junge Migrantinnen von allen Bevölkerungsgruppen die grössten Schwierigkeiten haben, Beratung und Hilfe zur Integration in die Berufswelt aus öffentlich zugänglichen Quellen in Anspruch zu nehmen. So ist 1997 eine Initiative entstanden, die dafür sorgt, dass eine erwachsene Frau, die "Motivationsdame", eine junge Frau zu Be-

326 Ledergerber Beatrice: Damit mangelnde Integration nicht zur Desintegration führt, SuchtMagazin 1, Basel 2005, 22-24, 24. 
ratungsstellen begleitet. Sie soll mit ihrem Vorbildcharakter auch dazu motivieren, die angebotenen Ausbildungsgänge durchzustehen, auch wenn "lebensweltliche Fragen dazwischen kommen" 327 . Die Form wird wieder hergestellt, indem die Mentorinnen den jungen Frauen kulturelles Kapital wie Wissen und Kontakte im Umgang mit Arbeitgebern und Behörden zur Verfügung stellen. Obige Liste zeigt auch, dass es für alle erdenklichen Zielgruppen und Problemstellungen Expertinnen und Experten gibt, die beigezogen werden können, um die stillstehende Passage wieder in den zeitlichen Verlauf zu zwingen. Manchmal klappt dies trotzdem nicht und den Jugendlichen gelingt es nicht, den Übergang weiter voranzubringen.

Obwohl diese Überbrückungsangebote angesichts des Lehrstellenmangels zu Beginn des 21. Jahrhunderts in Basel ausgebaut oder bereitgestellt wurden, gibt es eine lange Geschichte solcher Zeitpuffer: Die Lehr- und Wanderjahre. Der europäische Ethnologe Ueli Gyr hat dies am Beispiel des Welschlandjahres für junge Deutschschweizerinnen gezeigt. ${ }^{328}$ Bildungsaufenthalte im Ausland oder in anderen Landesteilen als Page, Student oder kaufmännischer Praktikant waren seit dem 15. Jahrhundert für die Oberschicht bekannt. Erst in der Industrialisierung kam das Bürgertum in den Genuss dieser Bildungsmöglichkeiten. Seit dem 19. Jahrhundert wurden sie als Zwischenjahr zwischen Schulabschluss und Lehrantritt eingeschoben, die jungen Welschlandfahrerinnen waren dann zwischen 16 und 17 Jahre alt. Für die niederen sozialen Schichten wurde 1880 der Aufenthaltstypus des Volontariats für Dienstboten und Haushaltshilfen geschaffen. ${ }^{329}$

327 Ebd., 23 und Ledergerber, Beatrice, 3

328 Gyr, Ueli: Welschlandaufenthalte als Übergangs- und Kontaktmuster, in: Hugger, Paul (Hg.): Handbuch der Schweizerischen Volkskultur, Leben zwischen Tradition und Moderne, Ein Panorama des schweizerischen Alltags, Band 1, Zürich 1992, 119-128.

329 Ebd., 119-122. 


\section{$\underline{5.6 \text { Statuspassagen und Submoratorien }}$}

Das Moratorium bei Jugendlichen ist eine krisenhafte, schwerfällige, peinliche und unsichere Zeit, wo die Identitäten in den Bereichen Körper, Geschlecht, Geschlechterbeziehungen, Beruf und Ausbildung, Verhältnis zur Familie und zu Autoritäten aufgrund äusserer, unter anderem körperlicher Entwicklungen unklar, diffus und vieldeutig werden. Eine Statuspassage drängt sich auf. Wie diese zu verstehen ist, wurde im vorhergehenden Abschnitt gezeigt.

Gleichzeitig ist das Jugendmoratorium mit neu entstandenen Freiräumen verbunden. In dieser Zeit werden den Jugendlichen Freiräume zur Verfügung gestellt, indem die Erfüllung von Erwachsenenfunktionen aufgeschoben wird. Jugendliche schaffen oder besetzen auch selbst Freiräume, wenn die Erwachsenen das zulassen. In diesen Freiräumen arbeiten die Jugendlichen an ihren Statuspassagen. Für die seit den späten 1990er Jahren Aufgewachsenen ist das Internet ein neuer Raum, der hierzu intensiv genutzt wird. Die hier untersuchten Jugendlichen nutzten diese Möglichkeit intensiv. Im Internet lassen sich Identitäten einfach konstruieren, kombinieren und vorspiegeln, d.h. im Unklaren lassen oder ausprobieren. Welche Konsequenzen der Aufstieg der neuen Technologien für die Jugend hat, ist noch nicht abzuschätzen und wäre ein Gegenstand weiterer Forschung.

Deutlich festzustellen ist ausserdem eine Art Staffelung von Übergängen in verschiedenen parallelen Entwicklungsfeldern. In diesen Feldern wirken Submoratorien, die es den Jugendlichen erlauben, ihre Energien auf die Bewältigung von Übergängen in einzelnen Bereichen zu konzentrieren und andere Bereiche zeitweise zu vernachlässigen oder die Entwicklung ganz einzustellen. Diese Staffelung erleichtert die 
Bewältigung von Übergängen, die sowohl psychisch wie körperlich anstrengend $\operatorname{sind}^{330}$.

Diese Belastung eines Überganges kann derart gross werden, dass sie krisenartige Formen annehmen kann. In diesem Fall, stellten Glaser und Strauss fest, tendieren diese Krisen dazu, wie sie formulieren, das ganze Leben zu überfluten ${ }^{331}$, so dass alle anderen Statuspassagen stagnieren. Dies ist der Fall etwa bei Migrantentöchtern, die wegen lebensweltlicher Fragen oder kultureller Eigenheiten in ihrem von der Gesellschaft gewünschten Integrationsverlauf stecken bleiben. Ein anderer Krisenzustand ist der sogenannte Notfall. Dieser stellt einen $\mathrm{Zu}$ sammenbruch dar, dessen Totalität es nötig macht, für diesen Fall vorzusorgen. Hier sind alternative Handlungsweisen vorgesehen, was die Krisenhaftigkeit mindert. ${ }^{332}$

Angeregt wurde die hier vertretene Idee der Submoratorien von Zinneckers Artikel über die, wie er sagt, "natural history" des Moratoriumsbegriffs. Dort blieb er konsequent im Singular. Nur einmal, bei der Erörterung einer Methode der Jugendstudie 92 der Deutschen Shell, wechselte er zum Plural: Die Forscher liessen die Jugendlichen schätzen, in welchem Alter sie gewisse Lebenslaufereignisse erlebten. So konnten Zeitreihen über die lebensgeschichtliche Verlagerung einzelner Übergänge oder Übergangscluster im Verlauf der letzten Jahrzehnte gebildet werden. ${ }^{333}$

330 Dies mag die energiereiche Ernährung der Jugendlichen erklären. Im Hauswirtschaftsunterricht wurde die Frage gestellt, ob jemand bereits eine Diät gemacht habe. Thomas Oppliger stellte fest, dass er mehr Hunger habe, seit dem er in der Pubertät ist, und Nanny sprach von ihrer "negativen Diät", die sie gemacht habe, um zuzunehmen. Leute, die den Übergang bereits bewältigt haben, versuchen eher abzunehmen. Ein Beispiel dafür wäre Fiona. (FB04, 20).

331 Glaser und Strauss, Status Passage, 1971, 144-146.

332 Ebd. 144-146.

333 Zinnecker, Jugend als Moratorium, 2003, 49-50. 
Die erfragten Übergänge - je nach Survey werden zwischen 10 und 30 Übergänge abgefragt - lassen eine Clusterung zu, beispielsweise nach Lebensbereichen oder nach Kulminierung in bestimmten Altersabschnitten. Für eine Empirie des Jugendmoratoriums ist dabei die Typenbildung wichtig, um anhand signifikanter Konstellationen von Statusübergängen Jugendliche nach langen und kurzen Moratoriumszeiten oder nach früh bzw. spät einsetzenden und auslaufenden Moratorien zu differenzieren. ${ }^{334}$

Zinnecker wies selbst darauf hin, dass es Ungleichzeitigkeiten und separate Lebensbereiche gibt, führte aber diesen Gedanken nicht aus. In seinem Artikel über Statuspassagen zusammen mit Imbke Behnken ${ }^{335}$ wurde die Idee konkreter. Statuspassagen wurden zunächst als Häufung von Übergängen verstanden, die etwa zwischen Kindheit und Jugend oder zwischen Jugend und Erwachsenenalter stattfinden.

Wer zum ersten Mal Alkohol trinkt, zum erstenmal küsst oder ein Freischwimmerzeugnis erlangt, der vollzieht eine - vielleicht nur kleine - Statuspassage. Er bewegt sich vom Status des unerfahrenen Trinkers, Küssenden oder Schwimmenden zu einem, der zur Gemeinde der Erfahrenen und Eingeweihten gehört - wenn auch nur im Status des Anfängers. ${ }^{336}$

\section{Ebd. 50.}

335 Behnken, Imbke und Jürgen Zinnecker: Lebenslaufereignisse, Statuspassagen und biografische Muster in Kindheit und Jugend, in: Jugendwerk der Deutschen Shell (Hg.): Jugend '92. Lebenslagen, Orientierungen und Entwicklungsperspektiven im vereinigten Deutschland. Band 2: Im Spiegel der Wissenschaften, Opladen, Leske \& Budrich, 1992, 127-143. 336 Ebd. 128. 
Normalbiografien lassen sich gut entlang dreier zentraler Lebensbereiche anordnen. Das sind Stationen des Hineinwachsens in Ausbildung und Beruf, zweitens der Weg in intime Paarbeziehungen und in die Familiengründung und drittens das lebensgeschichtliche Feld einer Partizipation am Markt- und Konsumgeschehen bzw. an der politischgesellschaftlichen Öffentlichkeit. ${ }^{337}$

Diese Beobachtungen sind korrekt, obwohl dabei das Konzept der Statuspassagen etwas strapaziert wird, da sie teilweise nicht oder kaum rituell markiert werden. Ritualisierungen sollten restriktiv interpretiert werden, wie Gerrit Herlyn ${ }^{338}$ ausführte. Auch sollte für heutige Phänomene das Konzept der Normalbiografie hinterfragt werden. Wie auch immer: Meine Feldbeobachten suggerieren, dass sich die Lebensbereiche aus verschiedenen Gründen sinnvoller gruppieren lassen. Bereits 1991 gehen die Autoren davon aus, dass die "Statuspassagen, die von der Kindheit zum Erwachsenenstatus führen, in Ostdeutschland schneller durchlaufen werden (... als ...) in Westdeutschland. Es dauert länger, bis aus Kindern Jugendliche und aus Jugendlichen junge Erwachsene werden. ${ }^{" 339}$ Auch eine Familiengründung ist beispielsweise ein Cluster von Statuspassagen und hat wichtige Übergänge in rechtlicher, sozialer und ökonomischer Hinsicht zu Folge. Ausserdem ist die Partizipation am Markt- und Konsumgeschehen eher mit dem Bereich Ausbildung und Beruf verknüpft als mit dem Bereich politisch-gesellschaftliche Öffentlichkeit, der eher mit sozialem Umgang und Hierarchien verbunden ist.

\section{Ebd. 129.}

338 Herlyn, Ritual und Übergangsritual in komplexen Gesellschaften, 2002, 73-75.

339 Behnken und Zinnecker, Lebenslaufereignisse, Statuspassagen und biografische Muster in Kindheit und Jugend, 1992, 131. 
In dieser Arbeit soll für die Bereiche Körper, Geschlecht, Geschlechterbeziehungen und Ausbildung, Beruf, Konsum sowie den Umgang mit der Gesellschaftsstruktur der Begriff Submoratorien eingeführt werden. Sie werden Geschlechtssubmoratorium, ökonomisches Submoratorium und soziales Submoratorium genannt. Die Vorsilbe weist darauf hin, dass Submoratorien verschiedene Aspekte des jugendlichen Moratoriums darstellen und miteinander verzahnt sind.

Das Geschlechtssubmoratorium behandelt alle Fragen rund um die körperliche Veränderung in der Pubertät, der geschlechtlichen Identität und dem erotischen Umgang mit Partnern. Das ökonomische Submoratorium betrifft einerseits den beruflichen Werdegang, welcher ein Aspekt der Einnahmenseite des ökonomischen Handelns darstellt, aber auch den Bereich des mündigen Umgangs mit Geld, etwa das Erlernen und den Umgang mit der Konsumkultur. Dies stellt sozusagen die Ausgabenseite des ökonomischen Handelns dar ${ }^{340}$.

Das soziale Submoratorium gliedert sich auch in zwei Bereiche: Einmal den Umgang mit vertikalen Sozialbeziehungen, mit Autoritäten, wie Eltern, Lehrmeistern, Lehrern, staatlichen Beamten, aber auch transzendenten Autoritäten wie Gott oder unpersönlichen wie der Tradition. In diesem Bereich zeigen die oben angeführten Überlegungen zu den Übergangsritualen von Terence S. Turner ihr analytisches Potential. Ein zweiter Bereich betrifft horizontale Sozialbeziehungen: Das Schaffen eines Freundeskreises beispielsweise, das sich Bewegen in Menschengruppen, der adäquate Umgang mit Bekannten, Kollegen oder Nachbarn. Hier wirken die von Victor Turner formulierten Überlegungen zur

340 Als ich Devi Mahic in meinem Büro interviewte, erlaubte ich mir, einen Kaffee zu kochen. Devi, der vorher hochgradig erwachsen über seine beruflichen Pläne gesprochen hatte, rief plötzlich aus: "Weshalb die Erwachsenen ständig Kaffee trinken müssen, habe ich nie begriffen!" (Mahic, Devi, 2, 2) Erwachsene Konsumkultur war für ihn also noch nicht verständlich, obwohl er so tat, als sei seine Zukunft sicher. 
egalitären und gemeinschaftsstiftenden Communitassituation erkenntnisleitend.

Das gemeinsame Durchleben der Übergänge eines Submoratoriums befestigt die Beziehung zwischen den Akteuren, wirkt also integrativ. Im gemeinsamen Durchleiden der liminalen Situation entwickelt sich eine intensive Kameradschaft und ein Egalitärismus. Menschen, die gleichzeitig am gleichen Submoratorium arbeiten, kommen sich deshalb näher; umgekehrt entfremden sich beispielsweise zwei Freundinnen, wenn die eine ihre Entwicklung in einem benachbarten Submoratorium als die Freundin weiterbringt. Zu einem bestimmten Zeitpunkt wird sie beispielsweise auf die andere hinabschauen und sie als kindisch empfinden.

Der hier differenzierte Jugendmoratoriumsbegriff verbessert die analytische Schärfe, indem die Thesen der Entstandardisierung ${ }^{341}$ und Entsynchronisierung ${ }^{342}$ berücksichtigt werden. Die These der Entstandardisierung besagt kurz gefasst, dass die einst selbstverständlich erschienenen Grenzen zwischen Kindheit und Adoleszenz sowie zwischen den verschiedenen Phasen der Spätadoleszenz und dem Erwachsenenalter fliessend geworden sind. Die These der Entsynchronisierung verweist darauf, dass Entwicklungen in den verschiedenen Bereichen nicht mehr synchron mit anderen verlaufen. Beispielsweise dauert das Erreichen der ökonomischen Selbständigkeit länger, während die Aufnahme sexueller Aktivitäten früher erfolgt. ${ }^{343}$ Die Jugendstudie der Deutschen Shell $1992^{344}$ stellt in ihrer Begriffsdefinition Jugend fest: "Jungsein umfasst mittlerweile die Altersgruppen der Dreizehn- bis Dreissigjährigen, also

341 Nach Olk T.: Jugend und Gesellschaft, in: Heitmeyer, W. (Hg.): Interdisziplinäre Jugendforschung, Weinheim, Juventa, 1986.

342 Siehe Von Trotha, T.: Zur Entstehung der Jugend. Kölner Zeitschrift für Soziologie und Sozialpsychologie 34, 1982, 254-277.

343 Fend, H.: Frühentwicklung in der Adoleszenz und ihre Risiken, in: Kosinski, Günter (Hg.): Pubertätsriten, Bern Stuttgart Toronto, Hans Huber, 1991, 102-128, 103. 
rund eineinhalb Jahrzehnte." ${ }^{345}$ Diese Dehnung der Altersspanne zeigt die Probleme des Jugendbegriffs auf. Der Submoratoriumsbegriff lässt die Definition von Jugend unabhängig von Alterszahlen werden und erfasst Phänomene, die sich bis weit ins Erwachsenenalter hinein erstrecken; Bindestrich-Begriffe wie Post-Adoleszenz oder Nach-Jugendphase sind damit hinfällig.

Diese Begriffe sind nämlich - jedenfalls für Phänomene des beginnenden 21. Jahrhunderts - wenig praktikabel. Auch Eva Breitenbach stellte dies am Ende ihrer Studie über junge Mädchen fest, dass sich während der Studie "die Vorstellung einer inhaltlich eindeutig definierbaren Phase der Adoleszenz zugunsten einer Beschreibung von adoleszenten Praktiken und Orientierungen und zugunsten der Vorstellung einer Inszenierung von Adoleszenz auflockert." ${ }^{\text {346 }}$ Die Schärfung der Beobachtung liegt demnach auf Praxen, die verschiedenen Submoratorien zugeordnet werden können.

Das Ende dieser Submoratoriumsphasen ist dann erreicht, wenn um einen umgangssprachlichen Ausdruck zu gebrauchen - der "Knopf aufgemacht" wird. Umgangssprachlich ist damit ein Moment gemeint, in dem jemand einen mentalen Durchbruch erlebt, wie etwa folgende Feldbeobachtung zeigt:

344 Jugendwerk der Deutschen Shell (Hg.): Jugend '92. Lebenslagen, Orientierungen und Entwicklungsperspektiven im vereinigten Deutschland, Opladen, Leske und Budrich, 1992.

345 Fischer, Arthur und Jürgen Zinnecker: Einleitendes zur Jugendstudie 1992, in: Jugend '92. Lebenslagen, Orientierungen und Entwicklungsperspektiven im vereinigten Deutschland. Band 1: Gesamtdarstellungen und biografische Portraits, Opladen, Leske und Budrich, 1992, 9-22, 12.

346 Breitenbach, Eva: Mädchenfreundschaften in der Adoleszenz. Eine fallrekonstruktive Untersuchung von Gleichaltrigengruppen, Opladen, Leske + Budrich, 2000, 328. 
Ich frage die Lehrerin Paula Lang, was das Knopfaufmachen bedeutet und ob das ein feststellbarer Moment ist. "Ja," antwortet sie, "es gibt den Moment. Plötzlich geht jemandem auf: Lesen ist toll! Französisch ist super! Und vor allem: es geht um mich!"347

Ein Mensch entdeckt also, dass eine Anstrengung in einem Feld bereichert. Schlagartig erlangt eine Person die Einsicht, dass es um einen selber geht. Es ist auch keine Hilfe mehr von aussen nötig, um ein Verhalten an den Tag zu legen, das für die Erwachsenen verständlich ist und das allgemein als vernünftig bezeichnen wird. Die Ambivalenz in den genannten Submoratorien geht also in eine eigene Identität mit klaren Perspektiven über, die Strukturen der Erwachsenenrolle bilden sich aus.

Im alltäglichen Umgang mit den jungen Menschen erweisen die Erwachsenen den unterschiedlichen Submoratorium unbewusst Reverenz, indem sie zwischen der formalen Anrede Sie und dem jugendlichen Du schwanken. Ich habe mich beim Beobachten immer wieder dabei ertappt, dass ich in den Notizen für die gleiche Person die Bezeichnung junge Frau oder Mädchen nutzte, je nach dem, in welchem Submoratorium ich mich gerade thematisch bewegte. ${ }^{348}$ Für männliche Jugendliche war die entsprechende Bezeichnung im Sprachgebrauch der Jugendlichen selbst „Typ“ im Gegensatz zum „Mann“.

Mit dem Gewinn klarer Perspektiven ist nicht gemeint, dass die lebensweltliche Situation klar ist. Perspektive heisst in diesem Zusammenhang, dass jemand weiss, dass er beispielsweise Automechaniker werden will, er braucht aber noch keine Lehrstelle in diesem Bereich gefunden zu haben.

347 FB05, 42.

348 Ebd., 35. 
Die Zeit des ökonomischen Submoratoriums steht im Zentrum der hier vorgelegten Untersuchung, da sie die eigentliche Integrationsphase ins Berufsleben darstellt. Sie kann lange dauern: Behnken und Zinnecker haben festgestellt, dass für deutsche Jugendliche im Jahre 1991 "rund vier Jahre" benötigt wurden, "um von der ersten Gewissheit, was man beruflich einmal werden will, bis zur vollen Berufstätigkeit zu gelangen, in der man genug Geld verdient, um für sich selber sorgen zu können." ${ }^{349}$ Dieser Zeitraum dürfte inzwischen für manche weit länger geworden sein.

Britta Jonsson wies darauf hin, dass die jungen Erwachsenen nach dem Niedergang des jugendlichen Gesellschaftsvertrages im Übergang zum Arbeitsleben immer weniger von den Strukturen der Gesellschaft kontrolliert oder geleitet werden. Sie haben die Aufgabe, selber zu wählen. Gleichzeitig haben Sie es schwer, realistisch zu beurteilen, was eine bestimmte Berufswahl für die Zukunft bedeutet, weil sich die Gesellschaftstruktur aufgrund ökonomischer Prozesse schnell verändert. Sie sind mehr oder weniger gezwungen, sich sozusagen als Navigatoren in unbekannten Gewässern zu verhalten. Um diese Situation mit den vielen Möglichkeiten, zu scheitern, zu bewältigen, müssen sie nachdenklich sein, eigene Beurteilungen vornehmen, von sich selbst in ihrer Wahl ausgehen und ein gutes Timing aufweisen. Im empirischen Teil dieser Arbeit wird gezeigt, wie sich im Lichte dieser Ausführungen ein erheblicher Teil des jugendlichen Verhaltens erklären lässt.

Roberts $^{350}$ zeigte, dass es vor diesem Hintergrund für Forscher schwerer wird, auf einfache Weise die Übergänge der Jugendlichen zum Arbeitsleben zu kategorisieren. Es wird immer notwendiger, von

349 Behnken und Zinnecker, Lebenslaufereignisse, Statuspassagen und biografische Muster in Kindheit und Jugend, 1992, 132-133.

350 Roberts, Ken: Structure and agency: the new youth research agenda. In: Bynner, J., Chisholm, L., \& Furlong, A.: Youth, Citizenship and Social Change in a European Context. Aldershot, Ashgate 1997, nach Johnsson, Unga vuxna, deras livsprojekt och tankar om framtida yrke, 1999, 58. 
der Akteursperspektive auszugehen und auf die eigene Rationalität und Werte der jungen Individuen zu hören. Der Begriff Lebensprojekt kam in diesem Text schon mehrmals vor. Theoretisch fängt dieser Begriff die Akteursperspektive und das individuelle Verhalten gegenüber der Zukunft ein, das aktualisiert wurde durch die Theorien über den Individualisierungsprozess in spätmodernen Gesellschaften. Das Leben und die Zukunft wird als eigenes Projekt angesehen, eben als individuelles Lebensprojekt. Gleichzeitig verfällt auch die Vorstellung davon, was als normaler Übergang ins Erwerbsleben zu gelten hat. Der Übergang von Ausbildung zur Arbeit ist viel zu komplex geworden, um ihn in traditionellen Modellen, was als normal oder abweichend verstanden werden kann, zu fassen. ${ }^{351} \mathrm{Er}$ schwebt für die hier untersuchten Jugendlichen häufig auch im dauerhaften Provisorium des Bildungswesens, der prekären Arbeitsverhältnisse oder der Arbeitslosigkeit.

In der Psychologie gibt es das Konzept der Entwicklungsaufgaben. Die Idee der gestaffelten Submoratorien wie auch Behnken und Zinneckers Konzept der Statuspassagen auf dem Weg zum Erwachsensein sind sehr nahe an diesem Konzept, also an der Idee, dass es Aufgaben zu lösen gilt auf dem Weg zum Erwachsensein, um Selbständigkeit zu erreichen. Dies stellen auch Behnken und Zinnecker fest. ${ }^{352}$ Doch Entwicklungsaufgaben sind nicht Submoratorien. Die ersteren bezeichnen psychologische Marksteine, deren Nichterreichen als Problem gesehen wird, während Submoratorien kulturelle Merkmale aufweisen, die nicht gewertet werden. Der Entwicklungspsychologe Hans Fend hat

\section{Ebd., $58 \mathrm{ff}$.}

352 Das Konzept der Entwicklungsaufgaben wurde von R. Havighurst vorgeschlagen (Havighurst, R.J.: Developmental Tasks and Education, New York 1972) und spielt in der Sozialisationsforschung und Entwicklungspsychologie eine Rolle (Behnken und Zinnecker, Lebenslaufereignisse, Statuspassagen und biografische Muster in Kindheit und Jugend, 1992, 141 und ebenda 1992, Fussnote 2). 
untenstehende Entwicklungsaufgaben definiert. ${ }^{353}$ Unten sind die Entwicklungsaufgaben von Fend im Alter von 12 bis 16 Jahren nach den hier postulierten Submoratoriumsbereichen geordnet.

Ökonomisches Submoratorium (Beruf, Erwerbsarbeit, erwach sene Konsumkultur)

Grössere Anschaffungen selbst bestimmen und aussuchen; Anfangen, sich über die eigene Zukunft Gedanken machen; Zum ersten Mal auf eigene Faust eine Urlaubsreise machen;

Das erste eigene Auto fahren;

Die erste Berufsausbildung abschliessen;

Aus dem Elternhaus ausziehen;

Genug Geld verdienen, um für sich selbst sorgen zu können;

Geschlechtssubmoratorium (Körper, Paarbeziehung)

Zum ersten Mal verliebt sein;

Selbst sexuelle Erfahrungen (mit dem anderen und/oder gleichen Geschlecht) machen;

Soziales Submoratorium (Umgang mit Hierarchien und

Mitmenschen)

Weggehen und Heimkommen, wann man will;

Selbst bestimmen, wie man aussehen will;

Über gute Umgangsformen verfügen;

Von den meisten Leuten mit Sie angesprochen werden;

Gegenüber Vorgesetzten seinen eigenen Standpunkt vertreten können; ${ }^{354}$

353 Fend, Frühentwicklung in der Adoleszenz und ihre Risiken, 1991, 102108.

354 Nach ebd., 103-104. 
Das Konzept der Entwicklungsaufgaben dient in der Psychologie als Diagnosemittel, um festzustellen, wo die Passage aus der Form geriet und in welchem Bereich Jugendliche in ihrer Entwicklung sozusagen versagt haben, indem zu frühe oder verspätete Entwicklungsbereiche ausgemacht werden. Fend schrieb von Früh- und Spätentwicklern, wobei mit Frühentwicklern Jugendliche gemeint waren, die angeblich risikohafte Verhaltensweisen an den Tag legen, die sich "lebenshungrig am schönen Leben unserer Konsumwelt orientieren”, mit Spätentwicklern solche, die nach Sicherheit streben und "noch intensiver in den Normalentwurf von Leistung und Anstrengung eingebunden, langfristig planend und normativ kontrolliert" sind. ${ }^{355}$ Beide Jugendbilder sind seltsam negativ. Diese Studie hier geht nicht vom Bild der Problem- oder defizitären Jugendlichen aus, die karrieristisch zu spät oder moralisch verwerflich verfrüht erwachsen werden, sondern von Menschen, die in einer gegebenen Situation Strategien entwickeln müssen. Sie werden nach kulturwissenschaftlichen Gesichtspunkten und möglichst unvoreingenommen geschildert.

In den Bereich des sozialen Submoratoriums gehört auch die Identitätsentwicklung. Diese ist verzahnt mit dem ökonomischen Submoratorium, da in den spätmodernen Gesellschaften die Arbeitswelt zum wichtigen identitätsstiftenden Bereich geworden ist, vor allem für männliche Akteure. Dieses Phänomen wurde in den 1980er und 1990er Jahren hitzig diskutiert. ${ }^{356}$ Doch die Erscheinung der Unsicherheit ist eher die historische Regel als die Ausnahme und deshalb nichts neues: Schon Karl Marx klagte über die Zerstörungskraft des Kapitalismus, der jede Tradition und Bindung zum verschwinden bringe. Die gesellschaftliche Forderung, sich aus vorgefertigten Fragmenten und Versatzstücken eine eigene Biografie und eine eigene Identität zu konstruieren,

\section{Ebd., 117.}

356 Eickelpasch, Rolf und Claudia Rademacher: Identität, Bielefeld, transcript, 2004, 5. 
stellt für den Einzelnen ein anstrengendes, störungsanfälliges, riskantes Unternehmen dar, stellten Eickelpasch und Rademacher in ihrem Werk über die Identität am Ende des 20. Jahrhunderts fest. ${ }^{357}$

Gemeinsam ist den Identitätskonzepten die Annahme, dass Sinngebung und Identitätsbildung in der zersplitterten Sozialwelt zu einer privaten Angelegenheit jedes Einzelnen geworden ist. Verschiedene Begriffe wie Bastelmentalität ${ }^{358}$, bei der der Mensch sozusagen zwischen verschiedenen am Markt angebotenen Stilpaketen wählen könne, der "flexible Mensch" von Richard Sennett ${ }^{359}$ und die postmodernen Lebensstrategien wie etwa Touristen, Spieler oder Vagabunden von Zygmunt Bauman ${ }^{360}$ stellen meiner Meinung nach viel zu stark die pessimistische Seite der postmodernen Wirtschaftsentwicklung in den Vordergrund. Identität wird als prekär und zerrissen aufgefasst, als schwierig zu bewerkstelligen, da keine kulturell vorgegebenen Muster mehr existierten. ${ }^{361}$ Beizustimmen ist hingegen der Feststellung, dass Identität nicht mehr in der Adoleszenz erworben und dann durch einen festen Kern definiert ist, sondern immerwährende Arbeit erfordert, die im Prinzip niemals beendet ist.

Auch Heiner Keupp lehnte die kulturpessimistische Rhetorik ab und weist darauf hin, dass die postmoderne oder Patchwork-Identität ${ }^{362}$, ein kreativer Prozess der Selbstorganisation, die eine gelungene, oft überra-

357 Ebd., 6.

358 Gross, Peter: Bastelmentalität: ein 'postmoderner' Schwebezustand?, in:

Thomas Schmid (Hg.): Das pfeifende Schwein, Berlin, Wagenbach, 1985, 63-84.

359 Sennett, Richard: Der flexible Mensch. Die Kultur des neuen Kapitalismus, Berlin, Berlin Verlag, 1998.

360 Bauman, Zygmunt: Flaneure, Spieler und Touristen. Essays zu postmodernen Lebensformen, Hamburg, Hamburger Edition, 1997.

361 Eickelpasch und Rademacher, Identität, 2004, 14 und 18.

362 Keupp, Heiner: Auf der Suche nach der verlorenen Identität, in: Keupp, Heiner und Helga Bilden (Hg.): Verunsicherungen, Göttingen, Hogrefe, 1989, 47-69. 
schende Verknüpfung von Lebensmustern, Stilelementen und Sinnangeboten darstelle, einen ästhetisch-kreativen Prozess von Selbstorganisation sei. Wir haben es nicht mit Zerfall zu tun, meint Keupp, sondern mit einem Zugewinn an kreativen Lebensmöglichkeiten. ${ }^{363}$

Wie auch immer: Karrieren werden in der Postmoderne anscheinend unsicherer und das Leben wechselhafter. Die zentrale Stellung der Ökonomie führt dazu, dass die Erwerbsarbeit hierzulande zum alles überragenden Identitätsfaktor geworden ist. Sie ist das Hauptmedium der Vergesellschaftung und Identitätsbildung, vor allem deshalb, weil sie dem gesamten Lebenslauf ihren zeitlichen Rhythmus aufprägt. ${ }^{364}$ Für junge Erwachsene ist die Erwerbsarbeit als Sinn- und Identitätsanker ohne Alternative. Ein Arbeitsplatz gilt nach wie vor als Eintrittskarte in unsere Gesellschaft. Deshalb ist Jugendarbeitslosigkeit und die damit verbundenen Ausschlussprozesse das gravierendste Problem der Gesellschaft und wird zurecht als die grösste Belastung und Beeinträchtigung der gesellschaftlichen und persönlichen Zukunft empfunden. ${ }^{365}$ Aus diesen Gründen ist das ökonomische Submoratorium auch politisch wichtig. Leider, muss festgestellt werden, ist in manchen westeuropäischen Ländern keine Verbesserung auf diesem Gebiet erreicht worden. Es muss von der Politik verlangt werden, dass sie Rezepte für die Lösung dieser Probleme einbringt und so bald als möglich umsetzt.

363 Eickelpasch und Rademacher, Identität, 2004, 28.

364 Ebd., 30-31.

365 Ebd., 36. 


\section{$\underline{5.7 \text { Normalität und Integration }}$}

Die Unsicherheit der Identitätsentwicklung in der Adoleszenz wirft auch die Frage auf, was eigentlich normal ist. Diese Frage ist aus zwei Gründen für diese Studie wichtig: Zum Ersten ging die Forschungsanlage davon aus, dass Integrations- und Ausschlussprozesse davon bestimmt wurden, welche Bedeutungskonstruktionen von Normalität in einer Gruppe vorhanden waren. Zweitens konnte im Feld festgestellt werden, dass Normalität immer wieder in verschiedenen Formen als Thema bei den Jugendlichen auftauchte. Möglicherweise ist das Einpendeln von Verhaltensweisen auf von der Gesellschaft akzeptierte Bereiche auch eine Form, Submoratorien zu bearbeiten und weiter zu entwickeln. Doch dieser Frage konnte in der vorliegenden Studie nicht weiter nach gegangen werden, da sich das Konzept der Submoratorien erst aus dem empirischen Material ergab.

Aus diesen Gründen soll der Begriff Normalität an dieser Stelle diskutiert werden. Zu diesem Thema hat Jürgen Link eine Studie vorgelegt, die weit in die Geschichte dieses Begriffs zurückgeht. ${ }^{366}$ Darin zeigte er, dass der Begriff Normalität aus dem statistischen Begriff der Normalverteilung herausgewachsen ist. Mittels Statistik wird das Normale konstituiert. Daraus erwächst ein gesellschaftlicher Diskurs, der sich vor allem in der zweiten Hälfte des zwanzigsten Jahrhunderts auf allen gesellschaftlichen Ebenen etabliert hat.

Normalität zeige sich in wissenschaftlichen wie in alltagspraktischen Diskursen. Radio, Presse und Fernsehen bringen immer wieder Meldungen, die anhand von Statistiken Entwicklungen aufzeigen, die die Grenzen des Normalen zu sprengen drohen und daher angeblich problematisch sind. Immer wieder kommt zum Beispiel die Frage auf, ob Frauen, die in den Medien auftreten, nicht zu dünn sind, ob sie nor366 Link, Jürgen: Versuch über den Normalismus: Wie Normalität produziert wird, 2. Auflage, Opladen, Westdeutscher Verlag, 1999. 
mal aussehen oder nicht. Diese Ansicht unterliegt Schwankungen und kulturellen Bedingungen.

Doch Normalität ist etwas anderes als Normativität, die Werte, Normen und Paradigmata vorschreibt, denen man nachzuleben habe. Den Diskurs über Normalität nennt Link Normalismus. Dieser unterteilt sich in Protonormalismus und flexiblen Normalismus. Protonormalismus geht in Richtung von Normativität und bemüht sich sozusagen um die Einengung des Normalitätsfeldes, des Bereichs dessen, was als normal gilt, während der flexible Normalismus die Normalitätsgrenzen im Alltag so pragmatisch handhabt, dass eine Vielzahl von Phänomenen darin Platz haben. Ein Beispiel: Der Protonormalismus behauptet, dass etwa Homosexualität abnorm ist. Der flexible Normalismus misst dagegen zuerst ein Phänomen und stellt dabei beispielsweise fest, dass der homosexuelle Anteil der Bevölkerung zwischen fünf und zehn Prozent der Bevölkerung schwankt. Dieser Anteil ist daher normal. ${ }^{367}$

\section{Tabelle: Verlauf der Integration in die Erwachsenenwelt}

Nächste Seiten: Submoratorien: Die einzelnen Submoratorien sind unabhängig, beeinflussen einander jedoch. Die Bearbeitung eines Übergangs in den einzelnen Submoratorien ist mit innerem und äusserem Aufwand verbunden. Deshalb werden sie unterschiedlich schnell und zeitlich gestaffelt durchlaufen. Durch extreme äussere Ereignisse kann die Entwicklung stillstehen.

In der Tabelle wurde versucht, die Vielzahl der in verschiedenen Publikationen (Behnken und Zinnecker, Lebenslaufereignisse, Statuspassagen und biografische Muster in Kindheit und Jugend, 1992, Glaser und Strauss, Status Passage, 1971, Fend, Frühentwicklung in der Adoleszenz und ihre Risiken, 1991) beschriebenen Statuspassagen nach kulturellen Kriterien neu zu ordnen.

367 Ebd., 92. 


\begin{tabular}{|c|c|c|}
\hline & $\begin{array}{l}\text { Kindheit } \\
\text { Kindliches Verhalten. Ende } \\
\text { der Kindheit durch biologi- } \\
\text { sche Prozesse. Eine Sta- } \\
\text { tuspassage drängt sich auf. }\end{array}$ & $\begin{array}{l}\text { Jugend } \\
\text { Moratorium, "Jugendlicher } \\
\text { Leichtsinn". Keine Per- } \\
\text { spektiven, Kulturtechniken } \\
\text { teilbeherrscht, Freiraum, } \\
\text { unklare Situation, Ablösen } \\
\text { von der Kindheit }\end{array}$ \\
\hline $\begin{array}{l}\text { Geschlechtssubmorato- } \\
\text { rium }\end{array}$ & $\begin{array}{l}\text { Kindliche Erotik, kindli- } \\
\text { cher Körper }\end{array}$ & $\begin{array}{l}\text { Pubertät setzt ein, Körper- } \\
\text { veränderungen problema- } \\
\text { tisch, Interesse an Erotik, } \\
\text { Suchen einer Beziehung } \\
\text { ("Dates"), erotische Orien- } \\
\text { tierung unklar. }\end{array}$ \\
\hline $\begin{array}{l}\text { Ökonomisches Submora- } \\
\text { torium (Einnahmen) }\end{array}$ & $\begin{array}{l}\text { Taschengeld, wirtschaftli- } \\
\text { che Elternabhängigkeit }\end{array}$ & $\begin{array}{l}\text { Erste Jobs, Praktika, } \\
\text { Schnuppern, zeitlicher } \\
\text { Lohn, Elternabhängigkeit } \\
\text { geht zurück }\end{array}$ \\
\hline $\begin{array}{l}\text { Ökonomisches Submora- } \\
\text { torium (Ausgaben) }\end{array}$ & $\begin{array}{l}\text { Fahrrad, lokaler Horizont, } \\
\text { erste Erfahrungen mit der } \\
\text { Konsumkultur, Eltern ma- } \\
\text { chen grössere Anschaffun- } \\
\text { gen }\end{array}$ & $\begin{array}{l}\text { Konsumkultur für Alltags- } \\
\text { bedarf beherrscht, erste Er- } \\
\text { fahrungen mit Genussmit- } \\
\text { teln, Schokogetränke, Mo- } \\
\text { ped, regionaler Horizont }\end{array}$ \\
\hline $\begin{array}{l}\text { Sozialsubmoratorium } \\
\text { (vertikale Beziehungen) }\end{array}$ & $\begin{array}{l}\text { Rechtliche Elternverant- } \\
\text { wortung, ausserhalb von } \\
\text { Hierarchien, Du, Aussehen } \\
\text { von Eltern dominiert }\end{array}$ & $\begin{array}{l}\text { Jugendstrafrecht, reduzierte } \\
\text { Elternverantwortung, "ju- } \\
\text { gendlicher Leichtsinn", Sie } \\
\text { und Vorname, Aussehen } \\
\text { weitgehend selbst bestimmt }\end{array}$ \\
\hline $\begin{array}{l}\text { Sozialsubmoratorium } \\
\text { (horizontale Beziehungen) }\end{array}$ & Freunde gleichaltrig & $\begin{array}{l}\text { Ausprobieren verschiede- } \\
\text { ner Gruppen, Styles und } \\
\text { Freunde, Erwerb von Iden- } \\
\text { titätskompetenz }\end{array}$ \\
\hline
\end{tabular}




\begin{tabular}{|c|c|c|}
\hline $\begin{array}{l}\text { Jugendwendepunkt } \\
\text { "Knopf geht auf", liminale } \\
\text { Situation, Wendepunkt von } \\
\text { der Kindheit zum Erwach- } \\
\text { senenleben, es wird er- } \\
\text { kannt, dass es um einen } \\
\text { selbst geht }\end{array}$ & $\begin{array}{l}\text { Jugend } \\
\text { Moratorium geht zu Ende, } \\
\text { Perspektive bildet sich, kul- } \\
\text { turelle Strukturen des Er- } \\
\text { wachsenseins bilden sich } \\
\text { aus, Kulturtechniken be- } \\
\text { herrscht, Situation klärt } \\
\text { sich }\end{array}$ & $\begin{array}{l}\text { Erwachsen sein } \\
\text { Vollwertiges Mitglied einer } \\
\text { Kultur, erwachsenes Ver- } \\
\text { halten, anzustrebender Zu- } \\
\text { stand, Einzelkriterien wer- } \\
\text { den häufig nicht erreicht }\end{array}$ \\
\hline $\begin{array}{l}\text { Erste erotische Kontakte, } \\
\text { erste Beziehung }\end{array}$ & $\begin{array}{l}\text { Körperveränderung akzep- } \\
\text { tiert, erotische Kontakte } \\
\text { normal, erotische Orientie- } \\
\text { rung klärt sich, kurze Be- } \\
\text { ziehungen }\end{array}$ & $\begin{array}{l}\text { Feste Beziehungen, Heirat, } \\
\text { Familie, Kinder, Single }\end{array}$ \\
\hline $\begin{array}{l}\text { Erkenntnis, dass man selbst } \\
\text { für seinen Unterhalt auf- } \\
\text { kommen muss, Stolz auf ei- } \\
\text { gene Leistungen, Berufseh- } \\
\text { re }\end{array}$ & $\begin{array}{l}\text { Berufswunsch klar, Lehr- } \\
\text { stelle, reduzierter Lohn, El- } \\
\text { ternabhängigkeit stark re- } \\
\text { duziert }\end{array}$ & $\begin{array}{l}\text { Berufserfahrung, Dauerstel- } \\
\text { le, voller Lohn, Elternunab- } \\
\text { hängigkeit, Karriere }\end{array}$ \\
\hline $\begin{array}{l}\text { Erste Erfahrungen mit grös- } \\
\text { seren Anschaffungen, Lek- } \\
\text { tionen durch gelegentlich } \\
\text { exzessiven Genussmittel- } \\
\text { konsum, erste Reise ohne } \\
\text { Eltern }\end{array}$ & $\begin{array}{l}\text { Genussmittelkonsum be- } \\
\text { herrscht, Roller, überregio- } \\
\text { naler Horizont, erste grös- } \\
\text { sere Anschaffungen selber } \\
\text { machen }\end{array}$ & $\begin{array}{l}\text { Genussmittelkonsum im } \\
\text { kulturell akzeptierten Rah- } \\
\text { men, Auto, internationaler } \\
\text { Horizont, grössere An- } \\
\text { schaffungen selber machen }\end{array}$ \\
\hline $\begin{array}{l}\text { Jugendstrafrecht, Durchlau- } \\
\text { fen kleinerer Statuspassa- } \\
\text { gen wie etwa der Führer- } \\
\text { scheinerwerb, reduzierte } \\
\text { Elternverantwortung }\end{array}$ & $\begin{array}{l}\text { Mündigkeit, Lernen des } \\
\text { Umgangs mit Hierarchien, } \\
\text { kaum mehr Elternverant- } \\
\text { wortung, Aussehen weitge- } \\
\text { hend selbstbestimmt, Aus- } \\
\text { zug aus dem Elternhaus, } \\
\text { Steuerpflicht }\end{array}$ & $\begin{array}{l}\text { Volljährigkeit, angemesse- } \\
\text { ner Umgang mit Hierarchi- } \\
\text { en, Erwachsenenstrafrecht, } \\
\text { Elten gleichwertig, Sie und } \\
\text { Nachname, Aussehen } \\
\text { selbstbestimmt, Beginn des } \\
\text { Aufstiegs durch die Hierar- } \\
\text { chie, Steuerpflicht }\end{array}$ \\
\hline $\begin{array}{l}\text { Gemeinsames Durchleiden } \\
\text { der Strapazen des Über- } \\
\text { gangs festigt Freundschaf- } \\
\text { ten, Finden der Identität }\end{array}$ & $\begin{array}{l}\text { Feste Freundschaften ("für's } \\
\text { Leben"), neue Freunde, ei- } \\
\text { gener Style, Identitätskern } \\
\text { klar }\end{array}$ & $\begin{array}{l}\text { Alte Freunde, neue Freund- } \\
\text { schaften seltener, Umgang } \\
\text { gemäss Höflichkeitsregeln, } \\
\text { Identität als kreativer Pro- } \\
\text { zess, soziale Vernetzung }\end{array}$ \\
\hline
\end{tabular}




\section{Teil III}

Die Fallstudie Klasse 2D

Schule, Berufsübergang, Freizeit 


\section{Zerfallserscheinungen am Ende der Schulzeit}

Von dieser Stelle an präsentiert diese Studie den grössten Teil des empirischen Materials. Thema ist von jetzt an die Gruppendynamik in der Schulklasse 2D, die Schülerinnen und Schüler, ihre Freundschaften, Gruppen und Konflikte am Schluss der obligatorischen Schulzeit. Das Ende der Schulzeit wird mit der Abschlussreise an einen Zeltplatz in der Nähe von Narbonne markiert, danach geht die Klasse auseinander. Die Schülerinnen und Schüler machen erste Erfahrungen auf dem Weg in das Erwachsenenleben.

Zum Einstieg in diesen Abschnitt wird über die Prinzipien reflektiert, welche die Gruppenbildung in der Klasse bestimmten. Danach wird das physische und soziale Setting geschildert, welches zu Beginn der Studie den Rahmen des beobachteten Geschehens bildete. Dazu beschreibe ich das Klassenzimmer, seine Einrichtung und Dekoration im Zusammenhang mit den Gruppen in der Klasse und soweit bekannt, ihre Geschichte, Kultur und mögliche Konflikte zwischen ihnen. Die Entwicklung von sozialen Kompetenzen in diesem Bereicht gehört in das horizontale Sozialsubmoratorium.

Die nun folgende Beschreibung des Forschungssettings und der in der Schulklasse befindlichen Gruppen ist eine Zugehensweise, welche aufgrund des Forschungsdesigns gewählt wurde. Es ist in Ethnografien traditionell und sinnvoll, in der Empirie mit der Beschreibung der Umgebung und der Akteure anzufangen. Dabei wird deutlich, dass das im theoretischen Teil beschriebene zentrale Konzept der Submoratorien in diesem Abschnitt zunächst eine untergeordnete Rolle spielt, um dann im Verlauf der empirischen Forschung immer wichtiger zu werden. Die Forschungsanlage war darauf angelegt, Integrations- und Ausschlussprozesse zu beobachten. Dies wurde zu Beginn der Feldforschung auch gemacht, deshalb sind die Gruppen in der Klasse beschrieben. Erst all- 
mählich wurde der Zusammenhang der Gruppendynamiken mit den Verläufen der Submoratorien deutlich, die Analysekraft des Konzepts erkannt. Deshalb wird das Konzept der Submoratorien im Verlauf der empirischen Datenerhebung immer wichtiger und kulminiert am Schluss in einer Übersicht zum Stand der Submoratorien bei einzelnen Schülerinnen und Schülern, bei denen die Quellenlage dies erlaubte. Der Fokus wandelte sich also von der Gruppe zum Individuum und den Bedingungen, welche seine Gruppenanbindung mitbestimmten.

Das letzte obligatorische Schuljahr ist eine Zeit, in der sich die in der Schule entstandenen Gruppenstrukturen aufzulösen beginnen. Vor allem gegen Ende der Schulzeit beschleunigte sich dieser Prozess. Vermutlich beruht diese Beobachtung auf den Wegfall des Drucks, ein funktionierendes Arbeitsverhältnis im Klassenverband aufrecht zu erhalten und wurde durch die generell sehr dynamische Beziehungsstruktur Jugendlicher gefördert.

Um einige dieser Prozesse im Detail anzusehen, wird im Folgenden die Dynamik beobachtet, mit welcher sich die Gruppen in der Klasse verändern. Dabei muss unterschieden werden zwischen Mädchengruppen und Jungengruppen, denn sie organisieren sich nach unterschiedlich gewichteten Kriterien. Bei beiden spielt das Kriterium Style als Vorselektionsprinzip eine Rolle. Bei den Mädchengruppen handelt es sich häufig um Dyaden. Als Beispiel für die Umgruppierung, welche gegen Ende des Schuljahres einsetzt, wird beschrieben, wie Janine ihre beste Freundin wechselt: Sonia wird fallen gelassen und Loredana tritt an ihre Stelle. Bei den jungen Männern bilden sich die Gruppen auch nach Style-Kriterien, die aber von anderen kulturellen Zügen überlagert werden können. Lalander und Johansson ${ }^{368}$ warnen jedoch davor, junge Mädchen bloss in Dyaden zu sehen und junge Männer in Gruppen. Dies

368 Lalander, Philip und Thomas Johansson: Ungdomsgrupper i teori och praktik, 2. Auflage, Lund, Studentlitteratur, 2002. 
ist ein undifferenziertes und grobes Bild, welches der These widerspricht, dass Geschlecht eine formbare und konstruierte Sache sei. ${ }^{369}$

Um den Anspruch dieser Arbeit einzulösen zu helfen, dass primär die Jugendlichen zu Wort kommen sollen, werden Interviewpassagen und Beschreibungen aus dem Feldtagebuch absichtlich zum Teil etwas ausführlicher als nötig gelassen. Dies dient dazu, die Sprech- und Denkweise der Schülerinnen und Schüler sichtbar werden zu lassen und ein möglichst farbiges und lebensnahes Bild entstehen zu lassen. Die Sprache der jungen Menschen war in ihrer Ausdruckskraft und Treffsicherheit oft beeindruckend.

\subsection{Gruppierungsprinzipien}

Die Geschlechter werden im Folgenden gesondert besprochen, weil sich das Geschlecht als wichtigstes Gruppierungsprinzip erwies. Die einzigen, die beim anderen Geschlecht Kontakte hatten, waren Fiona, die wegen ihrer Sportlichkeit eher "in die männliche Komponente hinüberreichte" und Claude, der "in die weibliche hinüberreichte", wie sich Lehrerin Gisler ausdrückte. ${ }^{370}$ Ansonsten war die Welt innerhalb der Schule streng getrennt zwischen Mädchen und Jungen. Die Geschlechter hätten auch als eigene Gruppen aufgefasst werden können, weil sie intern völlig anders organisiert waren. Bei den Mädchen gibt es weniger Gruppierungen als Dyaden ${ }^{371}$. Auf die Frage, wer denn ihre vier besten Freunde seien, breitete sich bei den Mädchen oft Ratlosigkeit aus. Mit dieser von mir ausgehenden Kategorie konnten sie nichts

\section{Ebd. 129.}

370 Gisler, Isabelle, 18.

371 Eine Dyade ist eine Gruppe von zwei Leuten, die kleinste kulturerzeugende Gruppe. Vgl. Oring, Elliott: Dyadic Traditions. Journal of Folklore Research 21: 19-28. 
anfangen. Darauf hin weisen Bemerkungen wie "es sind schon viel mehr wie vier" ${ }^{372}$ oder "eigentlich sind alle meine Freunde" ${ }^{373}$. Alle können wohl nicht beste Freunde sein, sondern nur eine. Fiona und Nanny wirkten wie eine Gruppe, wobei beide auch hin und wieder wechselten, wie auch Monika und Rosa sowie Janine und Sonia. Rasiah scheint nirgends aktiv dabei gewesen zu sein, dennoch war sie zu beliebt, um ausgegrenzt zu erscheinen; sie schien irgendwo immer dabei zu sein. ${ }^{374}$

Ein weiteres, allerdings nicht so strenges Gruppierungsprinzip war die Zugehörigkeit zu einem Leistungszug. Bei den Jungen waren Thomas Häberlin., Klaus, Thomas Oppliger, Claude und Devi im E-Zug und bei den Mädchen gehörten Sonia, Nanny, Rosa und Monika dazu. Dieses Kriterium deckt sich ein Stück weit mit der ethnischen Kategorie Schweizer und mit einem räumlichen Kriterium: Das Schulgebäude steht auf der Grenze der Stadt Basel zur Landgemeinde Riehen ${ }^{375}$. Diese Grenze markiert auch eine soziale Schwelle zwischen der sogar für schweizer Verhältnisse sehr reichen Landgemeinde mit niedrigem Ausländeranteil Riehen und den einkommensschwachen nördlichen Quartieren der Stadt Basel mit hohem Ausländeranteil bis zum Badischen Bahnhof. Nördlich des Bahnhofs gibt es schweizerische mittelständische Quartiere, die in reiche Wohnzonen der Landgemeinden übergehen. Die meisten ethnisch schweizerischen Jugendlichen stammen aus den Landgemeinden oder den Quartieren nördlich des Bahnhofs und die nichtschweizerischen aus den nördlichen Innerstadtquartieren. Die eth-

372 Van Eijk, Nanny, 3.

373 Maggiorini, Janine, 1, 2.

374 Gisler, Isabelle, 16.

375 Eigentlich gibt es keine Einwohnergemeinde Basel-Stadt. Es gibt nur den Kanton Basel-Stadt und die sogenannten Landgemeinden Riehen und Bettingen. Das Kantonsparlament ist deshalb auch das Gemeindeparlament der Stadt, in dem aber auch Abgeordnete der kleinen Landgemeinden sitzen. 
nische Zugehörigkeit trat als Gruppierungsprinzip also in Gestalt von sozialen und räumlichen Kriterien besonders bei den jungen Männern auf und war augenfällig. Deshalb wird in der Besprechung der Gruppen bei den Jungen dieses Kriterium als primäre Einteilung übernommen.

In gewissen Unterrichtsstunden wird in Gruppen gearbeitet, etwa in der Hauswirtschaft oder in den Miniprojektwochen. Auf die Frage, wie sich die Gruppen bilden, antwortet Gisler: "Die Gruppen bilden sich von selbst." ${ }^{376}$ Arbeitsgruppen bilden sich also entlang den Linien von Freundschaftsgruppen. In eine andere Gruppe versetzt werden, oder auch bloss aus seiner Gruppe genommen zu werden, gilt deshalb als milde Strafe. Im Schulalltag kann dies so ablaufen:

Halil stört und muss neben Jürg sitzen. Absichtlich missversteht er die Aufforderung und setzt sich neben Stefan. Der Lehrer Müller insistiert. Halil ist es sichtlich unangenehm, er verhandelt, während Jürg nachsichtig und freundlich lächelt. Halil setzt sich schliesslich zu ihm, aber pünktlich aufs Läuten zischt er ab. ${ }^{377}$

Dieser Ausschlussprozess war also eine Strafe. Es gibt aber auch Integrationsprozesse, die ich als Beispiel für die Herstellung eines typischen Arbeitsverhältnisses bezeichnen würde. Die folgende Beobachtung stammt aus dem Haushaltsunterricht; es soll in zwei Gruppen gekocht werden.

Ein Problem taucht auf: es gibt zwei Gruppen: Rasiah, Monika, Rosa und Thomas Häberlin, Claude, Akdis, Da-

376 FB04, 34.

377 Ebd., 24. 
niel. Wohin soll Stefan? Rasiah schlägt vor: er soll halt zu uns kommen. ${ }^{378}$

Aufschlussreich ist das im Schweizerdeutschen häufig und vielschichtig verwendete Füllwort "halt": Die Mädchen schicken sich darein, einen jungen Mann zeitweise aufzunehmen, da sie einsehen, dass es nicht eine Dreier- und Fünfergruppe geben kann. Im Hauswirtschaftsunterricht gibt es bestimmte Aufgaben für jedes Mitglied einer Vierergruppe. Also sind zwei Vierergruppen für diese einige Stunden dauernde Situation angebracht und das normalerweise strenge Zuteilungsprinzip Geschlecht wird dieser Forderung untergeordnet.

Das Aussehen als Merkmal von Gruppenzugehörigkeit in der Jugendkultur ist detailliert diskutiert worden im Zusammenhang mit der Subkulturdiskussion. Die Äusserlichkeiten und ihre Unterschiede sind auch die auffälligsten Merkmale der Jugendkultur. In seinem Werk „Subculture - The Meaning of Style ${ }^{\text {“379 }}$ bespricht Dick Hebdige die Erscheinungsbilder und Rituale subkultureller Gruppen vor dem Hintergrund semiotischer und marxistischer Theorie sowie devianzsoziologischer Ansätze. Im Jahr 1979, als sein Buch erstmals in England, wo er seine Studien durchführte, erschien, gab es stark auffällige Styles. Die Punks oder Teddys gaben Anlass zu heftigen gesellschaftlichen Debatten. Dies ist in westlichen Demokratien nicht mehr der Fall. Durch die Entwicklung in den letzten zwanzig Jahren des 20. Jahrhunderts im Medienbereich zersplitterten die Ausdrucksformen der Jugendszenen immer mehr, eine letzte mediengerecht auffällige und emotional berührende Subkultur sind zum Zeitpunkt dieser Untersuchung wohl die Gothics (Grufties), von denen Janine und Sonia gewisse Elemente übernahmen.

378 Ebd., 27.

379 Hebdige, Dick: Subculture. The Meaning of Style, 14. Auflage, London und New York, Routledge, 1979. 
Die Vervielfachung und damit auch Abflachung der Wirkkraft von Jugendsubkulturen kann beispielsweise für Deutschland auf jugendszenen.de ${ }^{380}$ abgerufen werden. Dort werden im Januar 2006 folgende Subkulturen aufgelistet: Antifa, Blackmetal, Comic, Deathmetal, Drogen, Gothic, Graffiti, Hardcore, Hiphop, Junghexen, LAN-Gaming, Punk, Rollenspieler, Skateboarding (Skater), Sportklettern, Techno, Warez.

In der Tat konnte man die Schülerinnen und Schüler der 2D nach ihrer Kleidung typologisieren. Auffällig war, dass die männlichen schweizerischen Jugendlichen weniger stylebetont angezogen waren, also für mich normal und unauffällig. Zu dieser Gruppe gehörte Daniel, Jürg, Thomas Häberlin, Tobias und Klaus. Diese Wahrnehmung bestätigte sich, als ich in einem Gruppengespräch anmerkte, "ihr wart ja die Normalen". Die Anwesenden lachten alle, und als ich meinen Fehltritt korrigierte und umformulierte auf: „die nicht so einen Style ausgelebt haben“, wurde dies mit Zustimmung bedacht.

Es kann aber auch sein, dass diese Jugendlichen einfach noch keinen eigenen Style entwickelt haben. Sonia berichtet im März 2005 aus der $\mathrm{FMS}^{381}$, dass es dort ziemlich viele "Strebertypen" gebe, "die noch die Kleider von Mami anhaben", oder Migros ${ }^{382}$-Kleider, welche die Mutter eingekauft habe. Diese Leute seien für sie nicht interessant. ${ }^{383}$ Sonia wertet also den wenig ausgeprägten Style als nicht erwachsen und damit unattraktiv. Dieser Sachverhalt lässt sich mit der Feststellung erklären, dass die schweizerischen Jungen die Submoratorien eher langsamer durchlaufen als die ausländischen.

380 Sehr empfehlenswertes Internetportal für Szenenforschung, basierend auf Material von Ronald Hitzler, Universität Dortmund und anderen Quellen. http://www.jugendszenen.com/, 15.1.2006.

381 FMS: Fachmittelschule, die Nachfolgerin der DMS, der Diplommittelschule.

382 Migros: ein Grossverteiler, wo preiswert eingekauft werden kann.

383 Plüss, Sonia, 1, 2. 


\section{$\underline{6.2 \text { Das letzte Klassenzimmer }}$}

Das Klassenzimmer, in dem die 2D ihre letzten Monate verbrachte, war rund zehn auf zehn Meter gross und vier Meter hoch. Trat man durch die Tür, sah man vor sich eine grosse Fensterfront, die ab Hüfthöhe begann und zur Decke reichte. Sie war von zwei Säulen in drei grosse Einzelfenster geteilt und gab den Blick auf eine Böschung frei, auf welcher Büsche und Deckpflanzen wuchsen. An der Säule rechts war ein Plakat der Band No Angels ${ }^{384}$ an gebracht.

Wandte man den Blick nach rechts, sah man die Wandtafel. Davor, zur Fensterwand geschoben, stand der Schreibtisch von Isabelle Gisler, der Klassenlehrerin, daneben befand sich ein Hellraumprojektor. Hinter dem Schreibtisch war auf Kopfhöhe leicht nach vorne gekippt, eine weisse Projektionsfläche für den Projektor angebracht, rechts neben der Wandtafel hing eine Küchenuhr an der Wand. Wiederum rechts daneben, im Eck des Zimmers, befand sich ein Wandschrank, in dem Schulmaterial untergebracht war. Daneben befand sich die Tür; dann folgte eine Pinwand von der Türe bis zur Rückwand des Klassenzimmers. Über der Pinwand waren drei Plakate befestigt: eine Basketball-Szene aus den USA, ein Plakat von Kobe Bryant, einem berühmten NBABasketballer ${ }^{385}$, und ein weiteres Basketball-Plakat, Allstar Power, das fünf bekannte Basketballer unter dem Korb in ein Handgemenge verwickelt zeigte.

Meist ging ich von der Tür kommend nach links und setzte mich in die hinterste Bank an der Innenwand. Die Schulbänke waren in drei

384 Deutsche sogenannte Girl-Group. Sie wurde 2000 in der Sendung "Popstars" gewählt und war bis 2003 aktiv http://de.wikipedia.org/wiki/No_Angels, 29.2.2012.

385 NBA: National Basketball Association, die US-Amerikanische Liga der Profi-Basketballvereine. Kobe Bryant war nach der Jahrtausendwende einer der zehn besten Basketballer der USA. Vgl. Kobe Bryant - Wikipedia, http://de.wikipedia.org/wiki/Kobe_Bryant, 28.2.2009. 
Reihen und Zeilen mit gleichförmigen Abständen angeordnet, eine zehnte Bank war als Reservebank an die Fensterbank gestellt worden und lag nun leicht hinter meinem Platz links. Hinter meinem Rücken befand sich das zwei Meter breite Bibliotheks-Bücherregal mit Atlanten und nicht mehr benutzten Plakaten von Miniprojekten ${ }^{386}$, daneben ein Kleiderständer auf Rollen. Zwischen diesem und einem Schrank, der neben der hintersten Reservebank im Eck zur Fensterwand stand und auch Schulmaterial enthielt, hing ein Plakat vom Sommerlager 03, das ein Arrangement von Fotos von Schülerinnen und Schülern der ersten WBS-Klassen zeigte. Darüber hing ein Plakat, dass in schwarzweiss drei Spieler des FC Basel in seiner Aufstiegsphase nach 2000 abbildete, Hakan Yakin, Christian Giménez und Hervé Tum in nonverbaler Kommunikation mit dem Publikum. Über dem Schrank hing ein Plakat eines Hip-Hoppers der Bling-Bling-Fraktion ${ }^{387}$ in Trainingskleidung, Nelly ${ }^{388}$, der ein diamantenbesetztes Nummer-Eins-Zeichen zur Kamera streckt. Daneben befand sich noch ein Plakat desselben Künstlers mit Kelly Rowland von Destiny's Child ${ }^{389}$, die er wie ein Besitzstück im Arm hält.

386 In einem Miniprojekt arbeitet eine Zweier- oder Dreierschülergruppe ein Thema auf. Es dauert eine Woche und endet mit einem Vortrag, einem Plakat und einer Mappe, die abgegeben werden muss.

387 Bling-Bling ist Hip-Hop-Slang und verweist auf teuren, massivgoldenen und diamantenübersäten Schmuck und darüberhinaus auf einen Lebensstil, der durch das Ausgeben von absurd viel Geld gekennzeichnet ist. Das Wort Bling-Bling stammt aus Jamaika und bezeichnet das Geräusch, wenn in Trickfilmen Licht auf einen Diamanten fällt und Reflexe dargestellt werden. Vgl. Bling Bling - Wikipedia, http://de.wikipedia.org/wiki/BlingBling, 28.5.2006.

388 Hip-Hop-Musiker.

389 Amerikanische R\&B-Gruppe. Beyoncé Kowles, Kelly Rowland und Michelle Williams, die wichtigsten Mitglieder, verkauften zwischen 1990 und 2005 über 40 Millionen CDs und verfolgen seither Solokarieren. Vgl. Destiny's Child - Wikipedia, http://de.wikipedia.org/wiki/Destiny's_Child, 15.6.2006. 
Nach der Einrichtung zu urteilen, war das Klassenzimmer gleichzeitig Sozial- und Arbeitsbereich: alle Geräte und Möbel in diesem nüchternen, grosszügigen Raum dienten dem Lehren oder Lernen, grosse Fenster liessen viel Licht hinein und boten einen Ausblick auf einen lebhaften, grünen, aber ablenkungsfreien Bereich. Kontrapunkte boten die Plakate an den Wänden, die auf wichtige Idole der verschiedenen Styles verwiesen: Hip-Hopper und Basketballer für die Hip-Hop-Fans an der Innenwand und eine Girl-Group für die sogenannten Chicks, ein wichtiger Style für die Mädchen, an der Fensterwand. Die Mädchen sassen unter dem Plakat. An der Rückwand gegenüber der Wandtafel befanden sich Plakate der allgemein akzeptierten oder kombinierten Idole: Fussballer des lokalen Vereins und eine Girl-Group-Hip-HopKombination, Nelly und Kelly.

In der Bank vor mir sassen Günter und Halil, die Bank davor war leer. In der Bank links neben mir arbeiteten Klaus und Daniel, in der Bank vor ihnen sass Jürg alleine, davor Akdis, auch alleine. An der Fensterfront sassen die Mädchen: in der Bank, die direkt an das Lehrerinnenpult anschloss, Nanny und Fiona, dahinter Janine und Sonia, dahinter Monika und Rosa. In der Reservebank hinter ihnen hatten Claude und Thomas Oppliger Überblick über das Geschehen. Prominent abwesend war Stefan.

Dieses Sitzarrangement war erstaunlich stabil, bedenkt man, dass in einigen Hauptfächern die Schülerinnen mit Schülern aus anderen Klassen Unterricht hatten und häufige Fragen wie "Sitzt du neben mich?" oder "Wo sitzt du?" darauf hinwiesen, dass die Platzvergabe diskutiert werden konnte. Zwei Ordnungsprinzipien kreuzten sich mehr oder weniger: das Prinzip der Freundschaft, das die Schüler gruppierte, gegen das Prinzip des geringsten Störpotentials, welches die Lehrerinnen anwandten. In dieser Momentaufnahme, die im Deutsch des G-Niveaus stattfand, sind nicht alle Schülerinnen und Schüler anwesend. 
Selbst innerhalb dieser relativ kleinen Schulklasse formten sich, oft überlappend, Gruppen, die allerdings fast nie die Geschlechtergrenze überschritten. Diese Gruppen hatten bei den Schülern keine grosse Integrationskraft, da sie den Charakter von ad-hoc-Arrangements besassen, die den Aufenthalt in der Schule so angenehm wie möglich zu machen hatten. Bei den Schülerinnen gab es praktisch nur Dyaden, die sich jedoch sehr dynamsch im Verlauf des Beobachtungszeitraums um- und neugruppierten.

\subsection{Gruppenbildung bei den jungen Männern}

Die Kriterien der Gruppenbildung bei den jungen Männern werden in Folgenden herausgearbeitet und Integrations- und Ausschlussprozesse zwischen und innerhalb dieser Gruppen skizziert. Im nächsten Kapitel, das die Schulreise nach Südfrankreich zum Thema hat, wird deutlich, dass alle diese Gruppen eine Tendenz hatten, sich aufzulösen, weil die Gruppierungen ihren Zweck erfüllt hatten und immer weniger benötigt wurden.

Bezüglich Gruppenbildung bei den jungen Männern spielte die ethnische Zugehörigkeit im Hintergrund immer eine Rolle. Devi, Akdis und Halil waren eine Gruppe, die wegen ihrer ethnischen Besonderheit sofort ins Auge fiel, wobei Devi auch oft mit dem aus Berlin stammenden Günter und dem Schweizer Thomas Oppliger zusammen war. Während Devi, Akdis und Halil ihren Migrationshintergrund teilten - Devis Familie wanderte in den 1980er Jahren aus dem Kosovo ein, Akdis' aus dem christlichen Sudan und Halils aus der Türkei - hatten alle fünf ein gemeinsames Interesse an Basketball, was sie zusätzlich locker miteinander verband. Aber jeder wies eine Besonderheit auf: Die Hip-HopKultur spielte für alle ausser dem Schweizer Thomas Oppliger eine wichtige Rolle, dessen Kontaktperson zur Gruppe Günter war und der 
eher am Basketball interessiert war. Halil war dagegen nicht sportlich und deshalb nicht integrierbar in den Basketballdiskurs. Günter, der erst kürzlich aus Deutschland eingewandert war, scheint Akdis gegenüber eher kühl eingestellt gewesen zu sein. An dieser Gruppe fällt auf, dass Devi mit seinen Balkan-Wurzeln sozusagen als Scharnier zwischen den zwei jungen Männern mit muslimischer Kultur und den beiden Schülern mit schweizerisch-deutschen Kulturausrichtung fungierte. Die beiden Extreme in diesem kulturellen Kontinuum, Akdis und Günter, scheinen einander nicht besonders nahe gestanden zu haben. Diese Gruppe war am wenigsten locker und wird deshalb hier an erster Stelle aufgeführt, obwohl man auch die Gruppengrenze zwischen Akdis/Halil und Thomas Oppliger/Günter hätte ziehen können - mit Devi als Wanderer zwischen den Gruppen. Doch das Interesse an Basketball konnte diese inneren Differenzen überbrücken.

\subsubsection{Die Einheimischen}

Eine eher lockerere Gruppe hat die ethnische Kategorie Schweizer als Integrationsprinzip. Sonst lassen sich kaum Gemeinsamkeiten ausmachen. Thomas Häberlin und Klaus Ganz, die ein Interesse an Informatik teilen und eine entsprechende Lehrstelle suchen, sind hier als Ausnahme zu nennen. Daniel ist eher ungebunden, neigt einerseits zu Devi, Akdis, Günter und Thomas Oppliger, nennt aber auch Thomas Häberlin und Klaus als seine engsten Freunde. Tobias scheint allein zu sein innerhalb dieser Klasse und verkehrt mit Leuten ausserhalb, wie auch Jürg, der bloss Klaus als Kollegen nennt. Im Gegensatz zu Tobias ist Jürg aber in der Klasse kein Aussenseiter. Claude neigt auch keiner der vorhandenen Gruppen zu, behauptet von sich, dass "er nicht gross 
mit Leuten zu tun hat" ${ }^{\prime 390}$, nennt aber die beiden Thomas seine Freunde. ${ }^{391}$ Stefan ist auch eher ein Einzelgänger. Er ist zu diesem Zeitpunkt sechzehn, also ein Jahr älter als die anderen und wirkt etwas fremd in dieser Klasse. Er hat eher Verbindungen zu Schülern in anderen Klassen, sagt er.

\subsubsection{Hip-Hopper (Basketball)}

Eine andere Gruppe, Devi, Thomas Oppliger, Akdis, Halil und Günter waren als Hip-Hopper auszumachen, wobei Akdis sich als halber Skater bezeichnet hat. Diese Gruppe mehrheitlich ausländischer Jungs deckte sich mit den Basketball-Fans, mit Ausnahme von Halil, weil er zu unsportlich war. Als türkischer Junge durfte er keinen extremen Style haben. Er stellt sich loyal hinter diese Entscheidung seiner Eltern, begründet dies aber mit einer Geschmackspräferenz.

H: Meine Eltern würden mir ... sind dagegen, dass ich ... würden also schon etwas sagen, wenn ich würde so ... skatermässig oder als HipHopper mit richtig breiten Hosen ... wo die Knie unten sind ... würden sie schon dagegen sein ... ich habe das selber auch nicht gern. ${ }^{392}$

Loredana meint, dass der Style der türkischen Jungs trotzdem hervorsticht. Sie trügen meist sehr teure, enge, "mega-gestylte" Kleider. ${ }^{393}$

390 Boettcher, Claude, $1,1$.

391 Diese Abschnitte beruhen auf verschiedenen Interviewaussagen und zwei Soziogrammen, die auf den Interviewfragen beruhen, welche sich nach den "vier engsten Freunden" und "die vier, die du am wenigsten magst" erkundigten.

392 Demir, Halil, 1, 5.

393 Di Costanzo, Loredana, Gesprächsnotiz. 
Mit "mega-gestylt" ist wohl ein gewisser Manierismus gemeint, der damit kulturell abgrenzend wirkt: Einmal war ich überrascht, als Halil mit durchsichtigen Sportschuhen in die Schule kam. Ich fand die auf die Spitze getriebene Ästhetik ziemlich abgedreht.

Der einzige Schweizer in der Hip-Hopper-Gruppe war Thomas Oppliger, der ein glühender Basketball-Fan war und deshalb mitmachte. Günter kam im Sommer 2003 aus dem grossstädisch geprägten Berlin in die Schweiz und trug diesen Style am ausgeprägtesten. Breite Hosen, gerne auch Trainingsanzugshosen, lange T-Shirts, Baseball-Kappen mit Basketball-Symbolen und Schuhe bestimmter Marken stellen das Äussere dar.

Hip-Hip entstand als afroamerikanische und Latino-Subkultur in New York City der 1970er Jahre und zeichnet sich durch eine bestimmte Sprache, Musik, einen bestimmten Theaterstil und spezielle Bekleidung aus. Die vier Hauptaspekte dieser Subkultur, MC-ing (rapping), DJ-ing, das Sprayen von Graffiti und Breakdancing, sind seit den 1990er Jahren weltweit anzutreffen. Die Musik unterteilt sich in Dutzende von Unterstilen, unter anderem den Gangsta-Rap, deren Protagonisten sich als Verbrecher stylen. Dieser durch Homophobie, Misogynie, Rassismus und Materialismus gekennzeichnete Stil ist der kommerziell erfolgreichste Zweig der Rap-Musik und wird durch Musiker wie Tupac Shakur, Snoop Doggy Dogg, Dr. Dre und 50 Cent vertreten. ${ }^{394}$ Die Jugendlichen in der 2D sind während des Siegeszugs des Hip-Hop aufgewachsen, der in der Schweiz zwischen 1997 und 1999 kulminierte und in Basel schon Anfang der 1990er Jahre von Musikgruppen wie P27 aufgegriffen wurde. P27 produzierten als erste in der Schweiz Mundart-Rap. Die Schülerinnen und Schüler haben Helden der HipHop-Musik wie Nelly und 50 Cent im Klassenzimmer als Plakate aufgehängt, was auf eine gewisse Verehrung hinweist.

394 Gangsta-Rap - Wikipedia, http://de.wikipedia.org/wiki/Gangsta-Rap, 30.7.2012 
Ausserdem wurde ein Ausschlussprozess dieser Kultur importiert, der einem gewissen Basler Kulturzug, der Neigung zum "Anzünden" 395 und zur verbalen Kritik in Versform wie etwa Schnitzelbänken entgegenkommt. Er wurde von Leuten mit afrikanischem Hintergrund kultiviert: Das Dissen. Diese Form der Beleidigung stammt aus dem Umfeld der Hip-Hop-Kultur und der Rap-Musik. Diese steht in einer langen Reihe afroamerikanischer Gattungen gereimter Erzählformen. In den Urspungsländern der Afroamerikaner sind Musikalität, Rhythmus und Rhetorik wichtige Kommunikationselemente. In der Neuen Welt lebten sie insbesondere im religiösen Bereich auch in der Sklavenzeit fort, war doch die Kirche eine der wenigen Orte, wo Sklaven Kraft schöpfen und Zusammenhalt erleben konnten. Die afroamerikanischen Prediger mit ihrem halb gesungen Vortrag, der von der aktiven Teilnahme der Kirchgänger getragen wird, waren für den Rap wichtige Vorgänger. ${ }^{396} \mathrm{Be}-$ kannt aus den afroamerikanischen Quartieren amerikanischer Städte sind auch rhetorische Kämpfe wie "dozens", relativ kurze, rhythmische Spontangedichte, in denen traditionelle Figuren auftreten, Trickster wie "monkey" oder "Stagalee", und die tabuisierte Themen wie Sex oder Kriminalität aufgreifen und die Schlauheit des Tricksters feiern. Vorgetragen werden diese Gedichte oft in Duellen, "playing the dozens", mit dem Zweck, einen Gegner durch eigene bessere Poesie und grössere Beleidigung zu übertreffen. ${ }^{397}$ Diese verbalen Formen des Wettbewerbs, die Konkurrenz und Kräftemessen ermöglichen, sind auch in anderen Milieus und Gruppen zu finden, doch ist anzunehmen, dass die massenmediale Verbreitung dieser Formen auch für die Jugendlichen der untersuchten Klasse eine Rolle spielten. Das Dissen war für Günter einer der wichtigsten Gründe, weshalb er sich an der Schule nicht so wohl fühlte.

395 Jemanden leicht angriffig ansprechen.

396 Bendix, Amerikanische Folkloristik, 1995, 117-118.

397 Ebd., 118. 
$\mathrm{N}$ : In der Schule und so ... ist der Ton irgendwie rauher als in Berlin?

Günter: Ja, das Dissen und so.

$\mathrm{N}$ : Ja, Dissen.

G: Eigentlich geht es den ganzen Tag so. Also in Berlin wars sozusagen nicht so, sondern, wenn was war, dann wars härter. Aber hier ist die ganze Zeit dieses Gedisse, diese ... höhö ... (Anmachgeräusch) ... weisste so ... Hundefaces (?) die ganze ... komm du (?) ... Scheisse .. weisst du was ich meine?

N: Jaa-aa. Also, runtermachen, aber Prügel irgendwie nicht. In Berlin Ruhe, aber dann wird richtig geprügelt. G: Weisst du - ja klar - vielleicht mal ... aus Spass ... so und so ... aber hier zieht sich das wirklich über den ganzen Tag in der Schule. Also, mich stört das nicht grossartig, aber irgendwie nervt das dann schon mal, wenn die ganze Zeit so ne Scheisse kommt.

$\mathrm{N}$ : Und wie reagierst du dann? Also, auch zurückdissen?

G: Jaja, klar, irgendwie so, aber ... ich denke, scheiss drauf. Ist ja nie ernst gemeint, aber trotzdem ... ist schon komisch irgendwie, seit ich hier bin, ist mir das wieder so aufgefallen ... ganz komisch eigentlich.

$\mathrm{N}$ : Also, so im halb-ernsthaften Bereich. Also da nimmt man zum Beispiel eine Schwäche oder so ... was weiss ich ... ich erfind jetzt irgendwas ... du stinkst aus dem Mund ... oder so was ... und dann wird auf diese Kerbe gehackt, den ganzen Tag. Muss man sich das so vorstellen? G: Nee, es kommt die ganze Zeit irgendwas irgendwie, dann ... dann ... läufst du so vorbei ... (und jemand sagt) höhö, Rattenface, oder so ... und (ich zurück) fick dich doch oder so. Dann ist so was anderes ... oder so ... naja, 
du Penner oder so ... einfach so ne kleinen dummen

Dumpfheiten... das ist ziemlich komisch, weil das ja andauernd passiert, und in Berlin ... weiss auch nicht ... nie so ne Scheisse gelaufen ist.

$\mathrm{N}$ : ... ist das wirklich annervend gemeint, oder ist das ... irgendwie ... wie soll ich sagen ...

G: Naja, bei vielen nicht ... oft nicht ... manchmal schon, kommt drauf an ... ich finds wirklich komisch, weil in Berlin, wenn irgendwas ist, wurde dann geschlagen, oder was weiss ich, und in der Klassengemeinschaft sowieso nie und nie irgendwie so ein Scheiss.

(...)

G: (...) es wird irgendwie zum Alltag, einfach. Es wird normal einfach, und das ist irgendwie total dumm.

$\mathrm{N}$ : Ist es denn auch Unhöflichkeit, so ne Art ...

G: Nee, die tun blöd, einfach. ${ }^{398}$

Akdis mit seinem afrikanischen Kulturhintergrund sah die Sache wohl eher von der sportlichen Seite und verstand Dissen eher als verbindend:

A: Hier macht man sich die ganze Zeit gegenseitig runter, wenn wir zu zweit in der Stadt sind, macht man das schon auch, ... aber hier macht man das richtig.

$\mathrm{N}$ : Wieso ist das wohl so?

A: Weil es viel Leute sind. Da muss man zeigen, dass man der Bessere ist.

$\mathrm{N}$ : Also wegen der Konkurrenz.

398 Robert, Günter, 2, 7. 
A: Ja, genau. Auch alle Lehrer meinen, ich streite mich immer mit Devi, aber wir machen nur so (spielen es nur). Eigentlich sind wir Kollegen. ${ }^{399}$

Als verbal überlegen und witzig zu gelten, war für Akdis besonders wichtig. Im Gruppengespräch frage ich ihn, ob es für ihn besonders schwierig war, eine Schnupperlehrstelle zu finden. Er antwortet:
A. Nein, ich habe einfach keine gefunden.
Daniel. Wieso?
A. Weil ich dein Kollege bin (Weil ich Dich kenne).
(Alle lachen)
A. (Fühlt sich geschmeichelt): Wer kann besser runterma- chen? Ich oder er?
D. Der hat keine Chance (Ich bin Akdis überlegen).
A. (zweifelnd): Jojo! ${ }^{400}$ (Wohl eher nicht)

Akdis war im Zwiegespräch eher schüchtern und zurückhaltend und nicht wie in der Klasse der Spassmacher und Performer. Auch Gisler bestätigt, dass er ohne die Umgebung Schule, die ihm ein Publikum bot, nicht so extrovertiert war:
Also, wenn der Vater neben ihm sass, oder der Walter ${ }^{401}$ hier Nachhilfe gab, dann war er (sehr schüchtern). Ich bin dann da gesessen, zehn Minuten, und hab dann gesagt, Ak- dis, wenn du jetzt nicht mal den Mund aufmachst, und mir

\footnotetext{
399 Saliba, Akdis 1, 4.

400 Gruppengespräch 1, 4.

401 Der Partner von Gisler, Walter, gab manchmal Nachhilfeunterricht und half auch sonst an der Schule aus.
} 
das Gefühl gibst, du bist noch halbwegs normal, ich erkenn dich ja nicht mehr wieder. ${ }^{402}$

Leider behinderte dieses Verhalten von Akdis auch seine Integration, etwa bei den Mädchen. Nanny macht deutlich, dass Akdis nicht gerade ihr engster Freund gewesen ist.

Na: Jo, also ich verstehe mich schon mit ihm, aber ...

$\mathrm{N}$ : Er ist nicht gerade dein engster Kollege.

Na: Nein, ich erzähle ihm nie etwas.

$\mathrm{N}$ : Jaja. Hast kein Vertrauensverhältnis zu ihm.

$\mathrm{Na}$ : Er ist eher einer, der mich fertigmacht und ich ihn fertigmache ... so Spassfertigmachen.

$\mathrm{N}$ : Jaaa, nicht gerade innige Freunde und so ...

Na: Nein. ${ }^{403}$

Spassfertigmachen, Dissen oder rhetorisches Kräftemessen kann also sowohl verbindend wie trennend wirken. Dass Günter besonders empfindlich auf Dissen reagierte, lag vielleicht daran, dass er besonders davon betroffen war.

N (eine Liste in der Hand): Ich habe (...) gefragt, ob (die Jungs der 2D) nicht ein Pseudonym wählen wollen, es ist ja alles anonym und so ...

Monika (schaut auf mein Blatt): Wie heisst (Günter)? Berlin ....?

N: Berlin Shaker.

(Gekicher)

Nanny (spöttisch): Ja, sicher!

402 Gisler, Isabelle, 19.

403 Van Eijk, Nanny, 1, 3. 
Monika: Da kann er ja gleich Berliner hinschreiben ... (Gekicher) $^{404}$

Dies ist eine Anspielung auf Günters Körperfülle, da ein Berliner (Pfannkuchen) ein in Öl ausgebackener Süssteigklumpen ist. Gekicher ist laut McRobbie und Garber ein Zeichen dafür, dass Mädchen sich aus einer männerdominierten Situation zurückziehen und ihre Gruppenoder Cliquenhaftigkeit betonen. ${ }^{405}$ Hier handelt es sich also um einen Ausschlussprozess gegen mich und Günter und um einen Integrationsprozess innerhalb der Mädchengruppe.

\subsubsection{Zwischen Skater und „halb irgendetwas“}

Der wichtigste Style neben dem Hip-Hop war der Skateboarderoder Skater-Style. Skater tragen normalerweise sehr weite, stabile und funktionale Hosen, weite, meist einfarbige Oberbekleidung mit Aufdruck und Schuhe ganz bestimmter Marken. Skater markieren im öffentlichen Raum mit "Halfpipes", Rampen und Relings ihre Anwesenheit, bevorzugen bestimmte Musik und investieren viel Zeit in die Beherrschung ihres Rollbretts. Einmal konnte ich beobachten, wie ein Dutzend Skater die Freie Strasse, eine wichtige Einkaufsmeile Basels, in Formation hinunterfuhren und in dieser für Fahrzeuge gesperrten Fussgängerzone einiges Aufsehen erregten. Die Winterversion des Skaters ist der "Snöber", der Snowboarder. Die meisten Jugendlichen der Klasse adaptierten in irgend einer Form Skater-Elemente. Ein echter Skater war dagegen Claude, der einzige, bei dem ich ein Skateboard gesehen habe, der sich aber "normal" kleidete.

404 Gruppengespräch 3, 1.

405 McRobbie und Garber, Girls and Subcultures, (1975) 2002, 210. 
Loredana begann 2003 punkartig mit Ketten, Nieten und Stacheln, verlor aber diesen Style im Laufe der Zeit immer mehr für skaterartige Kleidung und Selbergestricktes. Sie selbst bezeichnet sich im Sommer 2005 als Freestyler, also als jemanden, der einen eigenen Style hat. Sie hatte sich in der Parallelklasse der 2D, der 2C, unwohl gefühlt, ein wichtiger Grund dafür war, dass sie sich stylemässig nirgends anschliessen konnte.

Janine begann als Chick. Das ist ein Style, der sich durch enge Hosen bestimmter Marken wie Miss Sixty sowie enge Tops auszeichnet. Die bevorzugten Farben sind Pink und Rosa. Diese Elemente betonen das Mädchenhafte der Schülerinnen. Bei ausländischen Mädchen wird dieser Style nach Vorbildern aus der Hip-Hop-Kultur ins leicht "nuttige" gezogen. Gisler beschrieb den Stil, als wir in Südfrankreich spät abends beim Bus auf die Schülerinnen und Schüler warteten, die vom Ausgang zurückkamen. Im Dunkeln waren nur Silhouetten auszumachen, und ich fragte die neben mir stehende Klassenlehrerin, ob das unsere Leute seien. "Das sind WBS-lerinnen," lautete die trotz Dunkelheit bestimmte Antwort, und als ich erstaunt nachfragte, woran sie das erkenne, meinte sie: "an den billigen hochhackigen Riemchenschuhen und den Riesenkreolen an den Ohren." Sie deutete mit beiden Zeigefingern einen Ring zwischen Ohrläppchen und Schultern an.

Die oben aufgelisteten Styles von Jugendszenen.de betreffen Deutschland. In der Schweiz stellt sich die Situation anders dar. Dies hat verschiedene Gründe. In der kulturell stärker fragmentierten und eher vertikal als horizontal integrierten ${ }^{406}$ Schweiz reicht es, mit Ausnahme der grösseren Städte, insbesondere Zürich, rein zahlenmässig nicht, Szenen mit einer gewissen Eigendynamik zu bilden. Die Gruppe der 15-19 Jährigen ist in Basel in der Zahlenreihe seit 1920 (13772)

406 Kulturelle Merkmale der politischen Einheiten, die alle Gruppen betreffen, wie etwa die kantonale Kultur, sind stärker als kulturelle Merkmale, die über alle politischen Einheiten hinweg eine Gruppe betreffen. 
vom Höchststand um 1960 (14547) auf fast die Hälfte (8634) geschmolzen, was 4,6\% der Bevölkerung entspricht. Es werden deshalb nur die wichtigsten Styles aufgegriffen. Die Jugendlichen gehören auch verschiedenen Szenen an oder nehmen bloss gewisse Elemente eines Styles an, um in anderen Zusammenhängen nicht ausgeschlossen zu werden. Eine ergänzende Interpretation wäre, dass Styles eher in einem späteren Alter wichtiger werden, mit 16 und 17, dann etwa, wenn die Jugendlichen auch Konzerte bis spät in die Nacht besuchen können, und nicht schon um neun oder zehn zuhause sein müssen. Stefan bestätigt diese Vermutung und meint, dass die "extremen" Gruppierungen "dann eigentlich überall so aufgeteilt" sind. ${ }^{407}$

Jedenfalls ist auffällig, dass die meisten Jugendlichen sich als halb-irgendetwas bezeichnen oder einen eigenen Style gefunden haben. Loredana bezeichnet in ihrem Bekanntenkreis Janine als Halb-Chick Halb-Skater, Martina habe einen eigenen Style mit Skater-Elementen. die Jungen neigen eher zum Hip-Hop. Rafael sei halb Hip-Hop, weil er eine Vorliebe für weite Kleider hat. Peter sei halb Skater, Pascal habe seinen eigenen Style, einfach weite Hosen. Melanie sei eher Öko.

Dass im Bereich Kleidung Ambivalenz zu Perspektive übergeht, könnte darauf hinweisen, dass Styles in den Bereich des sozialen Submoratoriums gehören, wo eigenständige kulturelle Ausdrucksformen gruppenbasierte ablösen und Erwachsensein demonstrieren.

\subsection{Junge Frauen und ihre Dyaden}

Bei den Mädchen schienen Zweiergruppen die wichtigste Art von Gruppen zu sein. Zwei dieser Dyaden, Sonia und Janine sowie Monika und Fiona werden im Folgenden beschrieben. Mädchendyaden, das Zwiegespann bester Freundinnen, die gemeinsam die ersten Schritte ins 407 Staub, Stefan, 1. 
Leben machen, hat Helena Wulff erforscht. Sie weist darauf hin, dass nahe Freundschaft eine freiwillige und erworbene Beziehung ist. Es gibt jedoch Begrenzungen, wie Freundschaften in welcher Klasse und in welchem Geschlecht ausgeformt werden. Nahe Freundschaft sei eine informelle Sozialbeziehung, die nicht zu Ausschlussprozessen führe. Mädchen ohne beste Freundin werden nicht ausgeschlossen oder stigmatisiert. Die Wahl einer besten Freundin werde zu einem gewissen Mass von der Ethnizität bestimmt. ${ }^{408}$ Die Beziehung zur besten Freundin kann auch einseitig sein, d. h. ein Mädchen kann behaupten, sie sei die beste Freundin einer anderen, obwohl jene ein anderes Mädchen als beste Freundin angibt.

Beste Freundinnen haben bestimmte Charakterzüge: Sie gleichen sich so stark wie möglich, sie teilen alle Geheimnisse miteinander und verbringen mehr Zeit miteinander als mit anderen Mädchen. Die beste Freundin kann auch problemlos in die Familie eines Mädchens aufgenommen werden und dort sogar zeitweilig wohnen. Beste Freundinnen sind meist gleich alt oder nicht mehr als ein Jahr auseinander, die Freundschaft dauert von wenigen Wochen bis zu sechs Jahren, Mittelwert ist ein Jahr. Trotz des gleichen Aussehens gibt es etwas Komplementäres an der Beziehung zweier besten Freundinnen. Die Eine ist oft etwas grösser als die Andere und eher die treibende Kraft beim Erforschen der Welt. Sie bändelt für die andere mit Jungs an und verabredet sich, geht voran in die Disco, nimmt als erste Drogen. Doch das mehr nach aussen gewandte Mädchen erkennt sich wieder in der Zurückhaltung des anderen.

Beste Freundschaften, wie alle Sozialbeziehungen, werden durch Reziprozität weiterentwickelt. Dazu eignet sich die bequeme Abgeschiedenheit des Schlafzimmers. Es stellt eine private Sphäre dar, in der Musik gehört und mit Make Up und Frisuren experimentiert werden kann oder die Mädchen können auch verschiedene Kleider ausprobie408 Wulff, Bästa vänner, 1992, 23. 
ren. Dabei werden die Körperhaltungen, Gesten und Sprechweisen der älteren, in geschlechtlichen Dingen erfahreneren Schwestern durchprobiert, während die Mädchen heimlich Zigaretten oder Cannabis rauchen. Beste Freundinnen teilen Geheimnisse, reden lange über Jungs, die nicht erreichbar sind, teilen sich über Probleme mit und geben sich Trost. Beste Freundinnen können lange Abende, ganze Wochenenden und Schulferien zusammen verbringen. ${ }^{409}$

Doch auch die Mädchendyaden waren sehr dynamisch und lösten sich gegen Ende der Schulzeit zugunsten einer Orientierung ausserhalb der Klasse auf: Sonia und Janine trennten sich wegen Loredana; Monika und Fiona lösten sich voneinander, wozu Fiona beigetragen hatte.

\subsubsection{Sonia, Janine (und Loredana)}

Eine auffällige Gruppe bildeten für ungefähr ein Jahr Sonia und Janine. Sie waren sehr eng zusammen und experimentierten mit Styles, bis Janine Sonia für Loredana aufgab. Die Geschichte dieser Mädchendyade soll kurz rekonstruiert werden. Die Geschichte dieser Dyade begann im Frühjahr 2003, Janine war bereits in der WBS, als Sonia nach einem halben Jahr Gymnasium neu in die Klasse kam. Janine berichtet:

Im ersten halben Jahr ist (Sonia) zu uns gekommen, vom Gymnasium, und da hatten wir keinen Kontakt, und dann fanden wir, am Rhein ist es so schön, und drei Mädchen von der Klasse, ich, Rosa und Sonia, haben abgemacht, und dann hat Rosa gerade gehen müssen, und wir beide sind noch lange geblieben, haben geschwatzt und so, und

409 Ebd., 23-28. 
haben gefunden, ist eigentlich noch cool, und haben dann immer wieder abgemacht. ${ }^{410}$

Janine benutzte die Bekanntschaft mit Sonia, um ihren Style zu wechseln. Vorher war sie eher ein „Chick“, und wertete dies im Mai 2005 als eine Phase, die jede einmal durchmacht, was darauf hinweist, dass die Jugendlichen selber die Styles auch als Spiel auffassen und in jugendlicher Leichtigkeit mit ihnen experimentieren. Sie mokierte sich sogar über sich selbst. ${ }^{411}$ Sonia beschreibt den Anfang ihrer Freundschaft weniger idyllisch:

In der WBS habe sich Janine an sie gehängt, J. sei megaunsicher gewesen und habe den Style von ihr kopieren wollen. J. habe immer gleich alles nachgemacht, wo sie ihre Hosen her habe und so. ${ }^{412}$

Jedenfalls machte Janine dann die “Ökogrufti”-Phase ${ }^{413}$ durch mit schwarzen Kleidern und Haaren, die durch gestrickte Schals, geringelte Stulpen und schwarzlackierten Fingernägeln ergänzt wurden. Diesen Style gab es im vom ökologischen Bewusstsein her wohl grüner gelagerten Gymnasium und er wurde durch Sonia in die WBS importiert. Der Stylewechsel war derart krass, dass noch ein Jahr später Halil darüber berichtet:

H: (...) Das ist mit den Kollegen (so) ... wenn die Kollegin etwas macht, dann will sie das auch machen ... die hat auch einen ganz anderen Style gehabt, früher.

410 Maggiorini, Janine 2, 2.

411 FB05, 38.

412 Plüss, Sonia, 2, 1.

413 Gisler prägte diesen Begriff (Gisler, Isabelle, 23). 
N: Die hat ihren Style gewechselt.

H: Die war wie Rosa, Monika und so war sie .. als Sonia in die Klasse kam, wurde sie so ... Wie heisst das ... sag mal ... der Stil ...

N: Jaja, Satanistinnen.

H: Satanistinnen, genau. ${ }^{414}$

Aus meiner teilnehmenden Beobachtung erschien mir merkwürdig, dass sich mehrere Erwachsene über das fehlende Selbstbewusstsein von Janine äusserten. Für mich wirkte sie nicht so, sondern in Gesellschaft ihre Klassenkameraden eher entschlossen. Auch bei Sonia sei das angeblich fehlende Selbstbewusstsein ein Problem, meinte die Lehrerin:

Deswegen waren auch meines Erachtens ihre Probleme, ihre pubertierenden, von mir als Laie beurteilt, eher auf die Familienverhältnisse oder auf die schwierige Situation zuhause zurückzuführen, mit der Mutter. Nicht auf die Leistung, sondern sie hat das einfach irgendwie nicht gebacken gekriegt, sondern die drei älteren Geschwister, die eben gut, Abitur, super und gefestigt, die Mutter hat jetzt wieder eine Ausbildung gemacht, weil sie sind weg, und die Sonia ist irgendwie zurückgeblieben, so mit 14, und ihr blieb nichts anderes übrig als sich schwarz anzuziehen und von der Rheinbrücke zu springen. ${ }^{415}$

In diesem Abschnitt kommt sehr gut die Idee der unterschiedlich schnell durchlaufenen ökonomischen Submoratorien zum Ausdruck. Drei Mädchen werden verglichen: die älteren sind "gut", also auf dem rechten Weg und "gefestigt", also haben Strukturen ausgebildet, wäh-

414 Demir, Halil, $1,7$.

415 Gisler, Isabelle, 22. 
rend die jüngste einen sozialen Abstieg vom Gymnasium in die WBS hinter sich hat. Dorthin bringt sie den Style des "Ökogrufties" mit, der starke Reaktionen auslöst und aufgrund des Stillstands im Sozialsubmoratorium wegen des Rückschlages im ökonomischen Submoratorium stark zelebriert wird. Dass Janine diesen Style übernimmt, zeigt die Mechanik des sinkenden Kulturgutes auf.

Das Springen von der Rheinbrücke muss völlig anders interpretiert werden. Die Anspielung bezieht sich auf einen Vorfall, der einige Wochen vorher passiert war: Janine und Sonia sind von der Mittleren Rheinbrücke gesprungen, wurden dabei gesehen und mussten deswegen vor den Rektor. Dies ist an sich nichts Ungewöhnliches. Viele Jugendliche springen im Sommer von der Rheinbrücke, eine klassische Mutprobe und verbotene Tat. Mein Onkel, der in Basel-Nord aufgewachsen ist, erzählt dies schon für die 1940er Jahre:

W: Wir sind unmittelbar unten an der Oetlingerstrasse ${ }^{416}$ haben wir unsere Kickhösli ${ }^{417}$ hingelegt und sind den Rhein hochgelaufen, immer am Bord ${ }^{418}$ entlang, und wo es kein Bord gehabt hat, musstest du durchrennen, weil dich kein Polizist hat sehen dürfen und dann bist du durch und bis zur Wettsteinbrücke und noch weiter, und da war auch kein Bord, (und wenn jemand intervenierte) in den Rhein geflüchtet. Dann über den Rhein, und wenn du das Gefühl gehabt hast, dass sie dich weiter verfolgen, bist du gerade wieder zurück (lacht) ... Den Zickzack gemacht. Und (man

416 Die Oetlingerstrasse verläuft im rechten Winkel zum Rhein und endet dort. 417 Fussballshorts.

418 Das Rheinufer wird durch eine liegende Mauer, die im 45-Grad-Winkel zur Uferstrasse aufsteigt, gebildet. Einen halben Meter über der Wasseroberfläche verläuft ein Treidel- oder Barmenweg, der erst gesehen werden kann, wenn man sich direkt am Bord befindet. 
durfte nicht) von den Brücken springen ... wir klebten dann an den Pfeilern ...

$\mathrm{N}$ : ... man kann ja so dran (hoch) ...

W: ... jaja, wir sind dann hoch, die Johanniterbrücke zum Beispiel hatte ja so Rillen dran ... und wenn du hoch gekommen bist, ist gut, und sonst bist du einfach reingefallen, weil du irgendwann einmal abgerutscht bist. Und dann alles nochmals von vorne. (...) Und zwischendurch wollten (meine Brüder) dann auch noch von der (Mittleren Rhein)Brücke runterspringen. (...) die haben dann schon ... sehr oft abends, im Sommer, von der Mittleren Brücke, dann hast du gewartet, bis es genug Leute gehabt hat, und dann (...) sind sie gesprungen. Und wenn die Schmier ${ }^{419}$ gekommen ist, haben sie natürlich ab(-rennen) müssen. $\mathrm{N}$ : Die sind dann gekommen wegen dem? $\mathrm{W}$ : Ja, das sind sie, aber die haben dich nie erwischt. Hingegen, während der Kriegszeit, sind wir mal unter der Brücke durch, und dann mussten wir vor die Jugendstaatsanwaltschaft.

$\mathrm{N}$ : Also von oben springen und dann unten durch ...

W: Nein, nur unten durch schwimmen. Es war verboten, dort unten durch zu schwimmen, weil es eine Militärische Sperrzone gewesen ist. ${ }^{420}$ Und die haben uns dann ins Gewissen geredet ... Wir könnten erschossen werden, das sei militärisch bewacht. ${ }^{421}$

419 (rheinisch:) Polizei.

420 Die Brücke war mit Sprengstoff geladen und hätte bei einem deutschen Angriff zerstört werden sollen.

421 Schaffner, Werner, 8. 
Schliesslich wurde Sonia von ihrer Mutter rausgehauen, die Isabelle Gisler telefonisch versicherte, dass ihre Tochter nicht von der Rheinbrücke springt, das könne gar nicht sein, dafür lege sie ihre Hand ins Feuer. ${ }^{422}$

Paul Hugger weist darauf hin, dass Bandenbildung und Mutproben typische kindliche Verhaltensweisen sind und nicht der Erwachsenenwelt angehören, wobei Hugger ganz offensichtlich Jugendliche auch als Kinder versteht.

Kinder, besonders in den unteren Primarschulklassen, schliessen sich gerne zu Banden zusammen, die zum fruchtbaren Nährboden von Ritualen werden, welche die Bande gegen aussen abgrenzen: Sie entwickeln dabei Geheimsprachen, verständigen sich über Symbole, die nur dem Eingeweihten bekannt sind, legen über die vertraute Umgebung eine zweite, nur den Mitgliedern zugängliche Topografie. ${ }^{423}$

Mutspiele aller Art gehören zum Ritualbestand Heranwachsender. ${ }^{424}$ Hugger führt als "klassisches Beispiel" das Durchschreiten einer langen, dunklen Röhre an. Sein Beispiel stammt übrigens aus Kleinhüningen, das auch in Basel-Nord liegt und sozial gleich gelagert ist. Einige Schüler der Klasse stammten aus Kleinhüningen.

Im Kleinhünigen der Jahrhundertwende gehörte das "Tunneli" zu den Mutspielen der Grösseren. Oben an der Wiese befand sich eine Röhre von mehreren hundert Metern Län-

422 Gisler, Isabelle, 21.

423 Hugger, Pubertätsriten - einst und jetzt - aus der Sicht des Volkskundlers, 1991, 29. 424 Ebd., 30. 
ge, die in der Mitte einen Knick aufwies, so dass es darin stockdunkel war. Der Waghalsige, der die Höhle durchwanderte, bediente sich eines Stocks, den er waagrecht hielt, um so den Verlauf der Wanderung auszumachen. (...) Spielte sich einer auf, dann stellte man ihm die Frage: Bisch du scho durs Tunneli? Gang du zerst dert duure! Wer es gewagt hatte, gehörte zum harten Kern.

Dieses Prestige musste durch ein entsprechendes Ritual erworben werden, wichtig war aber, dass es Zeugen gab. Ein solches Ritual wurde also angekündigt. (...) Dann stellten sich die Zeugen, die Neugierigen, die Neidischen und die Bewunderer ein. Auch die hämisch Triumphierenden, wenn er, ohne bis zum Ende gegangen zu sein, zurückkehrte. $^{425}$

Das Beispiel vereinigt in besonders schöner Weise die Idee der Höhle, Hütte oder des Engnisses, der Gang durch die Dunkelheit in der Erde, der für eine Initiation durchschritten werden muss, sowie den Action-Aspekt der Mutprobe und den Aspekt, dass das Beispiel aus der örtlich gleichen Kultur stammt.

Hugger weist zudem darauf hin, dass auch Mädchen Mutrituale durchführen, er führt ein zürcherisches Beispiel auch mit Wasser an.

In den Badeanstalten am See oder Fluss z.B. gehört es zu den noch heute prickelnden Wagnissen, unter dem Floss oder dem Holzraster durchzuschwimmen, auf dem sich die Leute lagern. In Zürich unterlässt es offenbar kaum ein Mädchen, bevor es dabei ins Wasser geht, die magische

425 Ebd., 30. 
Formel zu sprechen: "Scho (Schere) - Schtai (Stein) Papier!" ${ }^{\prime 26}$

Diese Formel scheint eine Tradition zu sein unter den Mädchen der Zürcher Primarschulen. Im Sommer 2005 besuchte ich Freunde in Zürich und wir gingen bei der Landiwiese am See baden. Als ich mich auf dem rund 20 Meter vom Ufer entfernten Holzfloss sonnte, sprang ein etwa zehn Jahre altes Mädchen neben mir ins Wasser und rief vorher zu meiner Verblüffung diese Formel aus.

Das Schere-Stein-Papier spielt dabei auf eine Zuteilungsritual hin, das ähnlich wie Münzenwerfen, eine Gewinnerin und einen Verlierer produziert, was als ein Hinweis auf die existenzielle oder Action-Komponente des Mutrituals gewertet werden kann. Hugger nennt als weitere bekannte Mutrituale das Stehlen im Warenhaus und das Sprayen. ${ }^{427}$

Jedenfalls scheint der neue Stil von Janine und Sonia einigen Leuten auf die Nerven gefallen zu sein, was die Gruppenhaftigkeit dieser Mädchendyade verstärkte. Akdis berichtet:
A: Weil mit Janine haben alle Stress gehabt in der (...)
Klasse. Also alle Buben und so. Ich war der einzige, der angefangen hat zu reden, und so. ${ }^{428}$

Janine bestätigt im ersten Interview, dass Akdis der einzige Junge gewesen war, mit dem sie in der 2D überhaupt einmal gesprochen hatte. ${ }^{429}$ Akdis erzählt weiter:

426 Ebd., 31.

427 Ebd., 30-31.

428 Saliba, Akdis, $1,4$.

429 Maggiorini, Janine 1, 2. 
N: Was heisst Stress ... kein Kontakt?

A: Günter hat sie gehasst, sie hat ihn gehasst, genau gleich mit Devi und Halil, Thomas Oppliger, Thomas Häberlin, Tobias, alle ausser Claude. Alle.

$\mathrm{N}$ : Wieso denn das? Ist das eine Mann-Frau-Frage?

A: Nein. Beim Devi schon, beim Halil auch. Ja. Allgemein

Mädchen sind komisch miteinander. Aber Günter hat sie einfach gehasst. ${ }^{430}$

Die Mädchen bilden zum Teil einen derart undurchdringlichen Block, dass sie für die jungen Männer völlig uninterpretierbar werden. Am schlimmsten, erinnert sich die Klassenlehrerin Gisler, war Janine in ihrer "Küblböck"-Phase. Wir unterhalten uns über sie, und kommen überein, dass sie ein Mädchen ist, das sich für gar nichts interessiert.

NS: Janine war ja ein Mädchen, die hat sich ja für überhaupt nix interessiert, irgendwie ...

IG: Doch, Daniel Küblböck. ${ }^{431}$

NS: Nein! Echt?

IG: Das war die schlimmste Phase mit ihr. Da schrie sie immer DANIEL! und schmiss sich über den Tisch und

430 Saliba, Akdis, 2, 4.

431 Am 6.10.2002 kam Küblböck in der Casting-Show "Deutschland sucht den Superstar" unter die ersten 30, da der gelernte Kinderpfleger ohne jegliche Gesangsausbildung durch seine eigenwillige Interpretationen und unkonventionelles Auftreten auffiel. Im März 2003 erschien seine erste Single "You drive me crazy", die in den deutschen Charts gleich auf Platz 1 stieg. Sein Image wurde in hohem Masse manipuliert. Im Verlauf des Jahres 2003 liess man die Stimmung kippen: öffentliche Äusserungen über Küblböck wurden negativ. Im Januar 2004 wurde er in einer Fernsehshow "Die 100 nervigsten Deutschen" auf Platz 1 gewählt. Vgl. http://de.wikipedia.org/wiki/Daniel_K\%C3\%BCblb\%C3\%B6ck, 2.3.2009. 
schmierte sich voll mit Daniel, Daniel, Daniel, Daniel, Daniel, Daniel ... (lacht)

NS: Das ist doch jetzt so ein Kunstprodukt pur ... Plastik ... irgendwie ... naja ... da fehlt mir das Verständnis ...

IG: Ja. S'ist, wies ist. Aber dann war er wohl gottseidank out, und dann war er aber richtig out. Also war er dann auch total Scheisse. ${ }^{432}$

Wegen ihrer Grufti-Phase, die allen auf die Nerven ging, wurden Janine und Sonia von den Klassenkolleginnen und -kollegen beschimpft, erinnert sich Gisler. ${ }^{433}$

Der Konflikt wurde so wichtig, dass er vor der Klasse verhandelt wurde. In der Klassenstunde vom 22.1.04 beschuldigt Janine Daniel, er mache sie "immer fertig". Daniel: "Ich fühle mich durch sie immer gestört" und Fiona habe an die Tafel geschrieben: Daniel ist schwul. In diesem Moment gibt es anzugliches Gejohle und Pfiffe. Sonia: "Wenn wir Musik machen, sagt Daniel: Scheissskaters. Das nervt. Er soll uns in Ruhe lassen." ${ }^{434}$ Ein Auszug aus dem Gruppengespräch zwischen mir $(\mathrm{N})$, Daniel, Akdis, Jürg, Devi und Klaus in der Stunde darauf, als ich ergründen wollte, was vor sich ging:

$\mathrm{N}$ : Du bist ja vorher ziemlich angegriffen worden von der Janine.

Daniel. Mmmh.

$\mathrm{N}$ : Ist das normal?

D: Ja, Sie, ... die zwei Mädchen sind einfach, ich sage es jetzt so, psychisch gestört. Ohne Scheiss.

Akdis: Stimmt schon.

432 Gisler, Isabelle, 21.

433 Gisler, Isabelle, 33.

434 FB 04, 29. 
$\mathrm{N}$ : Du redest von der Fiona und ...

D: Nein, ich rede von der Sonia und der Janine. ...

(...)

A: Also: Fiona, Janine und Sonia. Haben ihm etwas gemacht. Er hat zurück schlagen wollen. Sie haben aufgehört.

Devi (überrascht): Was träumst du, Akdis?!

A: Sie haben mir etwas gemacht, ich habe geschlagen, sie haben aufgehört. Sie haben dem Günter etwas gemacht, er hat geschlagen, sie haben aufgehört. Sie wissen, dass (Daniel) nicht schlägt. Deshalb nehmen sie ihm Sachen weg. Jürg: Ich gebe ihnen einen Tip: kommen sie in den INU-Unterricht ${ }^{435}$, da werden sie wissen, was die drei sind. Devi: Nein, sicher nicht, Akdis.

A: Aber ...

Daniel: Ich habe auch geredet mit der Frau Gisler und versprochen, ich machs nicht mehr.

$\mathrm{N}$ : Ich erinnere mich noch.

Daniel. Ich habe mal im Hauswirtschaft megalange geredet mit ihr. Seit dem ist gut.

(Zustimmendes Stimmengewirr)

$\mathrm{N}$ : Wie äussert sich das?

Daniel: Jo, die tun einfach dumm, und dann wenn sie das tun, dann gibts halt etwas zurück. Und wenn dann halt irgend .... eine Hand ausrutscht, dann haben sie geschissen.

A: Jo, so quasi.

$\mathrm{N}$ : Sind sie dumm?

Daniel: Ich weiss nicht, ob sie dumm sind, sie sind einfach psychisch gestört.

A: Sag doch einfach, sie sind Goa.

435 Wissenschaftsfach der WBS. 
Daniel: Jo, Goa!

A: Jo, sie hören etwas ähnliches. Wissen Sie was das ist, Goa?

$\mathrm{N}$ : Naja, hmm, so Musik ...

A: Die tanzen halt so (macht vor: streckt die Arme zombieartig vor und rückt mit den Schultern). ${ }^{436}$

$\mathrm{N}$ : Total elektronisch? Was hörst denn du für Musik?

A: Hip-Hop Heeey!

N: Gibt's da Namen und so ...

A: Tupac. Das macht sie heiss ...

$\mathrm{N}$ : Er hier hat gemotzt wegen Skater-Musik.

Daniel: Jo, Skater! Nicht wegen der Musik. Sie sind Skater.

A: Jo, ich bin halber.

Daniel. Jo, das hat mit Skater nichts zu tun.

$\mathrm{N}$ : Was ist Skatermusik denn?

Devi: Das ist so ...

Jürg: Krokus ...

Daniel: Nein, das ist so ... Scheissdreck.

$\mathrm{N}$ : So eine Art Hardrock?

436 Sehr schnelle, elektronische Musik mit orientalischen Einflüssen, die zu den Trance-Stilen gehört und in der zweiten Hälfte der 1990er Jahre aufgekommen ist. Drogengebrauch gehört in der Techno-Kultur zum Must und verstärkt das Musikerlebnis, schafft ein Gefühl des Einsseins mit der Umwelt, also Flow. Für mehr Information und eine Musikprobe: http://en.wikipedia.org/wiki/Goa_music, 25.4.2006. Flow: Ein Glücksgefühl, das folgende Komponenten hat: Involvierung, Fokussierung, Konzentration, Extase, innere Klarheit, das Gefühl, dass eine Aufgabe machbar ist, Sorglosigkeit, Zeitlosigkeit, Motivation aus sich selbst. Alles gelingt und läuft reibungslos. Flow entsteht etwa beim Sport, wenn ein Skifahrer perfekt fährt und Bestzeit macht. Flow entsteht oft in liminalen Situationen. Vgl. Csikszentmihalyi, Mihaly: Flow: Das Geheimnis des Glücks, 6. Auflage, Stuttgart, Klett Cotta, 1998 
Daniel: Jo ...

$\mathrm{N}$ : Eine Musik für junge Mädchen?

Daniel: Die wo Skater sind, sind Mädchen.

Akdis: Sicher nicht.

Devi: Es gibt soviel Buben wie Mädchen, die Skater sind. Akdis: So ein Scheiss. ${ }^{437}$

Was hier zum Ausdruck kommt, ist einerseits eine Spannung zwischen Janine, Sonia sowie einigen Jungen aus der Hip-Hop-Gruppe der Klasse, die sie als "gestört" empfinden, wobei ein kultureller Unterschied eine Rolle spielt. Die beiden Mädchen stellen sich mit ihrer engen inneren Bindung also bewusst ausserhalb der Normalität dar, was von den im Verständnis überforderten jungen Männern als Goa bezeichnet wird. Die Spannungen manifestieren sich als Kulturstreit zwischen Musik- und Kleidungsstilen, über deren Inhalte man sich auch noch zuerst einigen muss. Das Goa-Schulterzucken könnte auf den Grufti-Tanzstil als Zombies hinweisen.

Doch es war für Aussenstehende schwierig, wegen der von Wulff beschriebenen Ähnlichkeit der Beteiligten an einer Mädchendyade zu erkennen, wie die Strukturen innerhalb ausgesehen haben. Etwas reserviert notierte ich zu jener Zeit über Janine und Sonia als Mädchendyade:

Janine scheint die Leaderin zu sein, was erstaunt, denn sie ist deutlich aus Faulheit die Schwächste der Klasse, während Sonia aus dem Gymnasium gekommen ist. Janine ist eine Art Styleguide. Sie putzt sich ständig, läuft aber gekonnt zerrissen herum. Sonia ist noch ziemlich verlegen, experimentiert mit dem Make-up-Style. Beide kauen ihre Fingernägel. Sonia hat Janine auf den Arm geschrieben.

437 Gruppengespräch 1, 6. 
Zusammen besitzen sie ihre Aliens und eine Anti-Schule-Haltung (vermuten wohl, sie werden von Aliens entführt in eine bessere Welt). ${ }^{438}$

Und, möchte ich hinzufügen, sie haben zusammen gekifft, was ich zu jenem Zeitpunkt aber noch nicht wusste. Sonia hat anscheinend Janine mit dem Cannabis bekanntgemacht, glaubt der Moralist Akdis, der mit seinen Behauptungen und Befürchtungen jedem Erwachsenen das Wasser reichen könnte:

Akdis: Sie ist ja hochgekommen in Deutsch, (vom) G-Niveau zu E-Niveau, dann ist (zu diesem Zeitpunkt) die Sonia gekommen. Alles kaputt gemacht. Geraucht, gekifft alles mögliche, getrunken ... dann ist sie vom E-Niveau runtergefallen. (...) Ich denke, Janine hat keine Zukunft. Die wird in der Gosse enden. Wenn die so weiter macht, wird die hundert prozent(ig) in der Gosse enden. ${ }^{439}$

Als Schlüsselereignis für die Beziehung zwischen Janine, Sonia und Loredana kann kurz vor Weihnachten 2003 ein von Janine und Sonia gehaltener Vortrag über UFOs angesehen werden. Zur Unterstützung tauchte auch Loredana auf. Im Feldtagebuch steht:

Es ist der letzte Schultag vor Weihnachten. (...) Die nächsten (Vortragenden) sind Janine und Sonia, bei ihnen geht es um Aliens. Hinein kommt auch eine gewisse Loredana, eine Punkerin, die mit Nieten, Ketten und Stacheln übersät ist. Sie hilft beim Aufbau der Installationen, beim Abspielen der UFO-Musik, stellt die UFO-Paraphernalia auf,

438 Ebd., 21.

439 Saliba, Akdis, $1,7$. 
spricht aber nicht selber, sondern setzt sich auf die Fensterbank. Die Mädchen starten dramatisch: das Licht wird ausgemacht, dann kommt die UFO-Musik, dann Lichteffekte, dann wird das Deckenlicht eingeschaltet und die beiden berichten über UFOs. Obwohl mir der Anfang mit seiner Dramatik gut gefallen hat, bin ich vom eigentlichen Vortrag wenig begeistert: Eine Ansammlung von UFO-Gemeinplätzen. Die Lehrer haben nachher eine heisse Diskussion, wie sie den Vortrag bewerten sollen. Auch ich werde angefragt. Ich schlage vor, den Inhalt als Glaubenssache anzusehen und nur die Form zu bewerten. ${ }^{440}$

Über ihr Verhältnis zu jenem Zeitpunkt berichtet Loredana im Interview:

$\mathrm{N}$ : Du bist näher bekannt mit Janine und Sonia?

L: Also, nicht näher. Wir haben uns einfach durch die WBS kennengelernt und jetzt sind wir einfach volle Kollegen.

N: Du hast ja auch, als sie ihren Vortrag gehalten haben über UFOs und so, hast du ihnen geholfen.

L: Ja, wir verstehen uns einfach gut. ${ }^{441}$

Loredana hat sich an Janine und Sonia gewandt, weil die Gruppen in der Parallelklasse, in der sie war, sie nicht angesprochen haben. Das deckt sich auch mit der Beobachtung von Claude, dass die kulturellen Gruppen wie Stylegruppen, Schweizer oder bestimmte Ausländergruppen sich über Klassengrenzen hinweg zusammentun.

440 FB04, 4.

441 Di Costanzo, Loredana, 1. 
N: In der (Parallelklasse) war ich nur einmal, während der Projektwoche. Und die ist - wie soll ich sagen - vom Niveau her tiefer. Deutlich.

L: Ja, joooh. Es ist megaschrecklich: es ist so aufgeteilt: die vier Türkenmädchen, dann die K., ist allein, dann C. K. und $\mathrm{C}$. sind zusammen, und ich bin allein, was soll ich mit denen? Dann ist M, S., M. und R. ist eine Gruppe, wo alle so abgestoned sind, L. ist auch noch oft allein, und der H. ist auch noch bei den anderen und und R. und E. sind einfach so Mitläufer. Aber ich weiss nicht - pffff - mit der V. ... und dem P. kann ichs, aber mit den Mädchen weniger. Bei denen bin ich selten. Ich bin immer mit Janine. ${ }^{442}$

Das Auftauchen von Loredana scheint auch der Keim des Zerwürfnisses zwischen Sonia und Janine gewesen zu sein. Alle drei Mädchen entwickelten sich stark weiter, was die Auflösung der Gruppenstrukturen verstärkte: Janine und Loredana arbeiteten gemeinsam an ihrem Sozialsubmoratorium weiter und teilten den kulturellen Hintergrund einer starken Verbundenheit mit Italien. Loredanas Vater ist Sizilianer und Janines Vater italienischer Secondo. Sonia büffelte derweil für die Aufnahmeprüfung für die DMS (Diplommittelschule), arbeitete also am Berufssubmoratorium. Dass sie als eine der wenigen in der WBS die Prüfung bestand, beeindruckte viele Lehrer und erfüllte sie selbst mit Stolz, da der Prüfungsstoff zum Teil gar nicht im Lehrplan der WBS enthalten war. Sonia berichtet:

$\mathrm{N}$ : (Es) würde mich eigentlich interessieren, weshalb ihr euch nicht mehr sieht ... hattet ihr irgendwie Lämpen ${ }^{443}$ ?

442 Di Costanzo, Loredana, 2-3.

443 Krach, Streit. 
S: Ich weiss nicht ... wir haben einfach keinen Kontakt mehr, sie ist in einer anderen Schule, ich bin in der DMS ... weiss nicht ... sie ist schon noch mit der Loredana zusammen.

$\mathrm{N}$ : Loredana und Janine die sind ja oft zusammen, noch. $\mathrm{S}$ : Eben, und ich komme nicht gut aus mit der Loredana, und darum habe ich gesagt ...

$\mathrm{N}$ : Wow! Was ist denn da passiert?

S: Joh ... sie hat mich oft versetzt gehabt wegen der Loredana und dann habe ich gesagt, das scheisst mich an.

$\mathrm{N}$ : Irgend so ein Eifersuchtszeugs zwischen ihr und dir? S: Nein, ich habe auch gesagt, wir können schon zu dritt Sachen machen, aber wenn ich dann mit ihr allein abgemacht habe, dann ist immer Loredana noch gekommen oder sie hat gesagt, sie komme nach und dann ist Loredana noch gekommen und ... das hat mich ein bisschen angeschissen.

Heute habe sie keinen Kontakt mehr mit Leuten aus der WBS, ausser mit den beiden Marias. Heute habe Loredana die Rolle als Zugpferd bekommen, behauptet Sonia. Jetzt mache Janine einfach ihr alles nach. Vor allem das Kiffen, in das sei sie von Loredana eingeweiht worden. Die sei immer mit Hanf gekommen. Loredana habe in der ersten WBS noch nicht gekifft, das sei erst im zweiten Jahr der Fall gewesen. Sie habe Janine kaum mehr allein treffen können, immer sei Loredana mitgekommen, und dann sei bloss noch rumgehangen und gekifft worden. ${ }^{44}$

Herumhängen ist eine klassische Jugendlichen-Tätigkeit, welche Sonia jetzt, im März 2005, nicht mehr mitmacht. Merkwürdig ist auch die Verachtung des Kiffens, in das sie Janine - glaubt man Akdis - einge444 Plüss, Sonia, 2, 1. 
weiht habe. Überhaupt wirkt sie erstaunlich erwachsen. Bekleidet ist sie mit einer geschmackvollen Kombination aus schwarz und rot, wobei grosse rote Blüten dominieren, hat also einen eigenen Style, und möchte positiv auf den Interviewer wirken.

Während der Zeit, als Sonia lernte, ist also das Verhältnis von Janine mit Loredana enger geworden, so dass sie im Frühjahr 04 schon abgemacht haben, sich auch nach der WBS weiterhin zu sehen.

Ich weiss, dass ich mit Janine nach der WBS noch will zusammenbleiben. Weil wir haben so viel Shit hinter uns ... aaah (ein Geräusch des das-können-sie-sich-gar-nicht-vorstellen). ${ }^{445}$

Was beide so zusammengeschweisst hat, erzählt Janine ein Jahr drauf, im Juni 2005. Sie bedauert offensichtlich das Auseinanderwachsen, das zur "Funkstille" führte und hat anscheinend ein schlechtes Gewissen gegenüber Sonia.

J: Sonia? Wieder mal was gehört von ihr?

$\mathrm{N}$ : Nein.

J: Hej, ruf die mal an.

$\mathrm{N}$ : Ist da irgendwie schlechte ... ist da noch was offen zwischen euch, irgendwie?

$\mathrm{J}$ : Nein, es ist gar nichts offen, aber wir haben einfach keinen Kontakt mehr. Vor einem Tag auf den anderen war einfach Funkstille.

$\mathrm{N}$ : So wie ich das verstanden hab, wegen dir, oder?

J: Jo, quasi, weil ich dann (verlegenes Lachen) mehr dann mit der Loredana abgemacht habe. Weil ich ehrlich gesagt auch lieber mit der Loredana zusammen gewesen bin.

445 Di Costanzo, Loredana, 3. 
N: Das ist ein interessanter Punkt. Hast du irgendwie gefunden, dass Sonia ich weiss auch nicht ...

$\mathrm{J}$ (fällt mir ins Wort): Ja, schau, die Sonia ist auch voll cool und so, aber sie hat einfach eine fette Arroganz. Und zwar fett. Sie ist einfach hier oben (hält eine Hand über Kopfhöhe) und wir sind hier unten (die Hand fällt auf Kniehöhe). Wir sind Durchschnittsmenschen, weisst du, und sie ist hier (Hand wieder oben).

(...)

J: Die (Sonia) hat Loredana noch nie ausstehen können. Mich hat (Sonia) voll mies behandelt so. Wir waren beste Kolleginnen, ein Jahr lang oder so. Und so gegen Ende hat das ein bisschen so angefangen und so, ja sie ist die Höhere von uns beiden, so. Schlauer und so. Das hat mich einfach aufgeregt. Und dann ist sie mal ein Wochenende weggegangen - das war genau vor einem Jahr, vor zwei Wochen vor einem Jahr - ging sie mal ein Wochenende weg. Und dann hatte ich halt das Wochenende nichts zu tun und so ... und dann habe ich mit der Loredana abgemacht, das Wochenende. Und dann wurden wir von den Bullen gefickt (lacht). Das war am 31. Mai.

$\mathrm{N}$ : Und das war die Geschichte mit dem Falschgeld.

J: Am 31. Mai war es das Falschgeld und am 7. Juni war es nochmals das Gras, halt.

$\mathrm{N}$ : Scheisse.

J: Und die Sache hat uns einfach zusammengeschweisst und so.

$\mathrm{N}$ : Klar.

J: Und wir haben megaviel abgemacht und so, megaviele Juga $^{446}$-Termine gehabt, zusammen und so, Aussagen und 446 Jugendstaatsanwaltschaft. 
bla bla. Ja, und dann ist sie halt gerade einmal ... das muss gerade vor den Sommerferien gewesen sein .. ja, Anfangs Sommerferien ...

N: Dann hast du eh Sommerferien, dann siehst du dich eh nicht.

J: Ja, eben, das habe ich dann auch gedacht ... und dann bin ich halt wieder einmal ... ja, genau, ich hätte irgend ihr anrufen sollen ... früher .. ich habe ihr dann schon noch angerufen, aber es war irgendwie schon 3 (Uhr Nachmittags) und so. Und ich habe gedacht, die pennt noch und so, oder ... und dann hat sie mich einfach so angerufen, joh, wo bist du jetzt und so, hast du mit der Loredana abgemacht und bla, und dann wurde sie sauhässig ...

$\mathrm{N}:$... ah, die Konkurrenz ...

$\mathrm{J}$ : ... joh, sags nicht, die hat die Loredana schon immer irgendwie gehasst ... und so. Und dann ...

$\mathrm{N}$ : Ja, wieso denn? Ja, das ist ... (weisst Du), Spiel, irgendwo ... oder nicht?

$\mathrm{J}$ : Keine Ahnung, weiss nicht. sie hat immer gesagt, so, Loredana ist so jööh ... braucht megaviel Selbstmitleid und so, bla, bla. Weist du, sie ist schon ein bisschen anders, und so, Loredana, und so, sensibel und so, eigentlich ... (...)

$\mathrm{N}:$ Ja.

J: Und Sonia wollte das dann nicht verstehen und wääääh. Hat sie angerufen, und so, und dann gab es Streit am Telefon, und dann haben wir uns nie mehr gemeldet, seit einem Jahr. ${ }^{47}$

447 Maggiorini, Janine, 2, 7. 
Anscheinend tut es Janine leid, dass die Freundschaft mit Sonia so geendet hat. Ein Mal sitzen wir in der Wohnung zusammen und plaudern. Janine fragt mich, wie meine Arbeit voran gehe. Ich darf zwar nicht darüber reden, möchte aber noch etwas über das Verhältnis von Janine und Sonia wissen und erwähne deshalb, dass ich Sonia interviewt habe. Janine: "Sonia? Echt?" Sie ist ganz aufgeregt. Ich erzähle dann, dass Sonia es befremdend gefunden hat, dass Janine so abgesprungen sei. Martina kommentiert: "Ganz genau, Jani, da hättest du mich auch fast verloren! Die Sonia hat es dann nicht ausgehalten, als Du zur Loredana übergelaufen bist." ${ }^{448}$ Der Streit zwischen Janine und Sonia wegen Janines Verrat hat im Bekanntenkreis Kopfschütteln ausgelöst, auch Fiona, die den beiden nahestand, aber wegen ihrer sportlichen Ausrichtung und Widerwillen gegen Hanf nicht völlig integriert wurde, bestätigte, dass Janine mit Sonia Krach gekriegt habe. ${ }^{449}$

\subsubsection{Monika, Rosa (und Fiona)}

Eine weitere Mädchendyade bildete für eine kurze Zeit Monika und Rosa, wobei sich hier auch Fiona anschloss, wie sie manchmal auch bei Sonia und Janine mitmachte. Im Januar 2004 notiere ich, dass ich die Dyade als eng und undurchdringlich empfinde. ${ }^{450}$ Beim Antritt der Klassenfahrt im Juni 2004 nach Narbonne kommt es zum Bruch zwischen den beiden, als der reguläre Schulbetrieb abgeschlossen ist, wie Monika berichtet:

Rosa ist zwar von (Monikas) Mutter noch zum Treffpunkt am Bus nach Lörrach gefahren worden, aber von da an

\footnotetext{
448 FB05, 47.

449 De La Vega, Fiona, $1,1$.

$450 \mathrm{FB} 04,21$.
} 
habe Rosa sich nicht mehr um Monika gekümmert. Monika habe dann halt für sich selbst geschaut. Rosa sei dann sehr schnippisch und merkwürdig gewesen und habe sich nur noch um Fiona bemüht. Sie fände das stossend, wenn man sich um die Leute kümmert und dann das. Einmal sei sie (auf dem Zeltplatz in Südfrankreich, N.S.) in der Dusche gewesen und habe die Zähne geputzt. Dann habe sie Rosa auf das komische Verhalten angesprochen. Die habe bloss zickig getan und mit Fiona Portugiesisch gesprochen. Seit dem ist nix mehr mit Monika. ${ }^{451}$

Auch hier spielt die ethnische Nähe zwischen Rosa und Fiona eine Rolle. Beide sprechen portugiesisch. Dennoch war Fiona nirgends in der Klasse wirklich in einer Gruppe. Fiona hatte aber ganz starke Beziehungen zu Mädchen aus anderen Klassen, insbesondere zu Julia, die „Schönste der Schule“, die immer von jungen Männern umgeben war. Aber auch Rosa war begehrt, wie folgende Begebenheit zeigt:

Michael, der ein Problem mit Akne hat, macht sich in der Pause an Rosa heran. Rosa nervt sich: "Isch's geil?" Michael: "Jo". Rosa: "Ich wills gar nicht wissen." ${ }^{452}$

In dieser Beobachtung unterstellt Rosa Michael, dass er sich an sie heranmacht, weil er nicht nur sie, sondern auch das Heranmachen an sich geil findet. Michael gibt das sofort unumwunden zu. Weil aber Publikum anwesend ist, rechnet Michael damit, dass seine Schlagfertigkeit den Gesichtsverlust der Abweisung kompensiert. Rosa weist darauf hin, dass sie nicht interessiert ist, zu wissen, was Michael geil findet und damit auch, dass sie ihn nicht geil findet.

451 Bauer, Monika, 3.

452 FB04, 34 . 
Obwohl Rosa von jungen Männern umschwärmt wurde, war sie nicht interessiert, weil sie in der Weiterentwicklung des sozialen Submoratorium steckte. Im Gegensatz zu ihr waren Julia und Fiona im Geschlechtssubmoratorium deutlich weiter als der Durchschnitt der jungen Mädchen der WBS, wie auch Monika, die dank klaren Perspektiven in der Familie und im Beruf (sie hatte bereits eine Lehrstelle auf sicher) sich dem Geschlechtlichen widmen konnte.

IG: Genau. Monika war auch gut behütet, gute Familienstruktur, ruhig und zurückhaltend.

NS: Konnte aber auch schon flirten und so, schon auch an der Grenze zur Frau. Und hatte keine Schwierigkeiten, mir da mit irgendwelchen Anzüglichkeiten ...

IG: (lacht laut)

NS: ... im Gegensatz zu - weiss nicht, jetzt irgendwie Rosa oder so. Da war das völlig tabu.

IG: Ja, stimmt, die hat sich ja auch entwickelt, in den zwei Jahren, die Monika. Am Anfang, war die ja erst in der Parallelklasse, da wurde sie gemobbt, ganz komisch (und dann hat man sie zu mir versetzt) (...) und da ist sie dann auch sofort aufgeblüht und keinerlei Probleme. ${ }^{453}$

Gisler glaubt, dass Monika und Fiona Leute waren, die sich zwischen den Gruppen bewegten.

\section{$\underline{6.5 \text { Der Einzelgänger: Stefan }}$}

Einen Spezialfall stellte Stefan dar, der deutlich älter als seine Klassenkameraden wirkte, obwohl er gleich alt war. Er gehörte keiner Grup453 Gisler, Isabelle, 12. 
pe an. Durch Suchtprobleme der Eltern in der Kindheit vorbelastet, war er von seinem Verhalten und seinen Strukturen her "völlig haltlos"454, was als fehlende Kompetenzen in grundlegenden kulturellen Techniken zu übersetzen ist. Gisler erzählt, dass sie "ihn extrem in Ruhe gelassen" hat, weil sie "um seine Vorgeschichte wusste, und er hat auch ein bisschen Fuss gefasst, Vertrauen gefasst zu mir, hat es aber trotzdem nie geschafft, mir ein einziges mal anzurufen, wenn er mal eine Abszenz hatte, um sich zu entschuldigen." 455

Stefan lebte auch nicht zuhause, sondern in einer Institution für Jugendliche, wo ein strenges Regime ihm diese alltäglichen Strukturen beibringen sollte. Er musste immer mit einem Ausweis unterwegs sein, da er polizeilich ausgeschrieben war und sich stets ausweisen können musste. Ins Ausland durfte er nicht reisen. Gegen diese Disziplinierungsversuche wehrte er sich stark, er schien nicht zu verstehen, dass man ihm jeweils dann ein Stückchen von seiner Freiheit wegnahm, wenn er eine Regel des Hauses gebrochen hatte. Gisler klagte, dass

...du ihm tausend mal erklären (konntest), dass er das ja selber zu verantworten hat, also wenn er besoffen irgendwo einen Hydranten randaliert und er von der Polizei ins Heim gebracht wird. Was sollen die denn machen, als ihm Hausarrest (zu) geben? Und das hat er dann nicht verstanden, ist dann abgehauen. (...) Also selbst Verantwortung (für seine Abszenzen zu übernehmen) hat er nie geschafft, mir mal einen Zettel ins Fach zu legen, also diese Strukturen kannte er gar nicht. ${ }^{456}$

\footnotetext{
454 Ebd., 15.

455 Ebd., 15.

456 Ebd., 16.
} 
Er war auch schon vor zwei Jahren an der Schule, hatte aber soviele Abszenzen, dass das Jugendamt eingeschaltet wurde. Das nahm ihn von der Schule. Jetzt wollte er freiwillig noch einen Versuch machen, durch die Schule zu kommen. Von sich selbst glaubt er, dass er stigmatisiert sei und deshalb in der Schule Schwierigkeiten habe.

Ich bin halt ein Mensch, der sehr offen ist, was meine Vergangenheit betrifft, dass ich Scheisse gemacht habe, zum Beispiel, ich habe eine Anzeige wegen Einbruchdiebstahls, ja solche Sachen, dann machen die (Lehrer) sich ein eigenes Bild von dir, die schauen mich mit anderen Augen an als sonst, oder ... und ich bin mir so vorgekommen, als ich einen Stempel im Gesicht hätte. ${ }^{457}$

Bemerkenswert an Stefan ist seine Eloquenz. Im Interview hatte ich den Eindruck, einen Interview-Profi zu befragen, der schon viele Gespräche hinter sich hatte. Er nennt das "sehr offen" sein und berichtet über seine Vergangenheit, wie wenn er von einem Fremden erzählen würde. Kein anderer Jugendlicher war derart freigiebig mit Informationen, normalerweise musste ich die sprichwörtlichen Würmer zur Nase heraus ziehen. Bei Stefan nicht. Ich nahm an, dass das seine Strategie war im Umgang mit Autoritäten, die über sein Leben bestimmten und die er immer wieder auflaufen liess. An Stefan konnten die Grenzen der Integrationskraft staatlicher Institutionen wie die Schule aufgezeigt werden: Beispielsweise

... überschüttete man ihn mit Massnahmen, (hat ihn) gepuffert, unterstützt, (mit ihm) Gespräche geführt, (und so) hat man ihn in diese Klasse hineingleiten lassen und versucht, ihn da irgendwie aufzunehmen. Es war also wirklich wie 457 Staub, Stefan, 3. 
ein Boxkampf, du hattest so Puffer aussenrum so dick, (...) (er) musste dann so Auflagen erfüllen, hatte immer so Probephasen, bis Weihnachten durfte er (etwa) keine Abszenzen haben, da hatte er aber schon zehn, aber dann haben wir gesagt, weil es nur 10 waren im Gegensatz zu dem, dass er früher 200 hatte, ist das ja schon ein Fortschritt, und dann wurden wieder Standortgespräche geführt, mit dem Jugendamt Gespräche geführt, mit dem Schulhausleiter, mit dem Rektor, mit den Eltern und überhaupt. (...) (Tatsächlich) hat er sich in seinem Rahmen sogar entwickelt in den zwei Jahren. ${ }^{458}$

Trotz grossem Einsatz von Integrationshilfen ist es also nur beschränkt gelungen, diesen jungen Mann soweit aufzubauen, dass er in die regulären Strukturen integrierbar gewesen wäre, etwa eine Chance auf eine Lehrstelle gehabt hätte.

Trotzdem (hat er) im ersten Schuljahr 45 Abszenzen gehabt, eigentlich hätte er längst fliegen müssen. (...) Du kriegst keine Lehrstelle, das ist klar, mit dieser Art und Weise des unstrukturellen Umgehens mit deinem Leben, der hat sich (...) bis nachts um drei besoffen und kam morgens nicht aus dem Bett, ihm war schlecht, wen wunderts? $?^{459}$

Es gibt also anscheinend gewisse Strukturen im Untergrund, mit denen Leute wie Stefan umgehen können. Um ein Jahr vorzugreifen: Im Frühjahr 2005 verschlechterte sich die Situtation, weil Stefans Mutter schwer krank geworden war. Stefans Noten wurden schlechter und er 458 Gisler, Isabelle, 15. 459 Ebd., 15 
kam kaum noch in die Schule, wie ich in den Feldberichten notiert habe. Stefans Mutter weigerte sich zwar, ihren Schutz für Stefan aufzugeben, zu kooperieren und Stefan zu regelmässigem Schulbesuch anzuhalten, liess sich aber von der Basis-Lehrerin Paula Lang dazu überreden, Stefan davon zu überzeugen, in einen Informationsabend betreffend weiterführende Schulen zu kommen, das sei für ihn wichtig. Er sei dann tatsächlich gekommen, habe aber sehr schlecht ausgesehen. ${ }^{460}$

Stefan hat jedenfalls das Brückenangebot Basis nicht durchgehalten. Im Mai 2005 wurde er offiziell von der Schule verwiesen ${ }^{461}$ Seine Klassenkameradin Janine erzählt:

J: In dem ganzen Jahr, ist er wirklich, ohne Scheiss, ungefähr ein Viertel Jahr in die Schule gekommen. (...) Die Franzilehrerin (Französischlehrerin) hat ihn ein Mal gesehen. Ein Mal! Und die neue Englischlehrerin kennt ihn gar nicht. (...) Und dann haben sie gesagt - wir alle haben schon angefangen auszurufen ${ }^{462}$ und so - was passiert mit dem und so - hat er schon lange mal, etwa vor einem Monat, die Androhung gekriegt, dass er rausfliegt und so ... und ja ...

N: Du bist ja seine Gotte ${ }^{463}$ gewesen und so ...

$\mathrm{J}$ : Gewesen! Haha, juhu, jetzt muss ich nichts mehr machen ...

460 FB05, 3.

461 Ebd., 52.

462 Schimpfen, motzen.

463 Jede Schülerin oder jeder Schüler ist Pate (CH-Dt.: Götti) eines anderen Schülers oder Schülerin. Die Patin (CH-Dt. Gotte) ist ein einem relativ unübersichtlichen System wie der WBS oder Basis nötig, damit die Leute mit den Informationen à jour sind, etwa mitgeteilt kriegen, welche Hausaufgaben sie machen sollen. 
$\mathrm{N}$ : Ich erinnere mich noch, wie du seiner Mutter hinten nach telefoniert hast, und sie hat gefunden, ja, nein, der ist am Praktikum und so ...dabei war er eben voll abgetaucht (lacht).

J: Da war er gerade am Dealen oder weiss auch nicht wo ... N: Jaja!

Janines weitere Erzählung weist darauf hin, dass eine Untergrundkarriere für jemanden, der die offizielle Sozialisation nicht durchlaufen hat, durchaus eine attraktive Alternative zu einem "normalen" Lebenslauf sein kann.

J: Hej - Scheisse! Joh, der wird echt mal Gängstä, Mann.

N: Der wird echt Gangster, ja, genau.

(beide lachen)

N: Der hat auch die Verbindungen und so.

J: Der hat Connections, Mann. ${ }^{464}$

(...)

$\mathrm{N}$ : Der ist im Untergrund (...)

$\mathrm{J}:$... der stürzt voll ab im Fall. Vorher hat er sich noch in der $\mathrm{Bea}^{465}$ gemeldet und so und er hat sich voll dort abgemeldet und so ... der hat hun-dert-zwan-zig-Abs-zen-zen! $\mathrm{N}$ : Was ist Bea?

\section{J: HUN-DERT-ZWAN-ZIG!!}

$\mathrm{N}$ : Rund um die Uhr, irgendwie.

J: Jaaa!! Das ist jeden Tag! Und das sind im Fall nicht alle, er hätte noch viel mehr. Ich weiss nicht - der war ja ein Viertel Jahr in der Schule, mehr nicht! Hach - der ist jetzt arbeitslos. Selber schuld.

464 Maggiorini, Janine, 2, 3.

465 unverständlich. 
(...)

J: Finde ich voll hart, hey. Ja, nu, muss ich nicht mehr hin und her rennen.

$\mathrm{N}$ : Aber eben, der wird auch nie mehr gesehen von irgendjemandem. Der ist einfach irgendwie weg vom Fenster, oder.

J: Jooh!!

$\mathrm{N}$ : Der ist schon hier, aber nicht irgendwie dort, wo die anderen sind. ${ }^{466}$

Von diesem Zeitpunkt an war Stefan abgetaucht. Niemand im Bekanntenkreis der Jugendlichen hat je wieder etwas von ihm erfahren, da er sich in anderen Umgebungen bewegte wie die anderen Jugendlichen.

In diesem Abschnitt wurde gezeigt, wie sich die Gruppen in der Klasse 2D zusammensetzen und welche Dynamiken die innere Zusammensetzung bestimmten. Es zeigte sich auch, dass diese Gruppen eher locker geflochten waren und die Tendenz hatten, sich aufzulösen, weil es gegen das Ende des Schuljahres ging und der Zweck der Gruppe, das Arbeiten in der Schule zu erleichtern, mehr und mehr wegfiel. Während bei der Besprechung der Gruppendynamiken der jungen Männer ethnische, soziale oder andere Kriterien die Gruppendynamiken bestimmten, wurde bei den jungen Frauen bald deutlich, dass individuelle Entwicklungsdiskrepanzen ein Stück weit über ihre Anbindung an Dyaden bestimmten. Hier spielen die Beobachtungen von Helena Wulff eine Rolle, dass Mädchendyaden ausgehend von einem gewissen Mass an Ethnizität etwas Komplementäres haben und durch Reziprozität weiterentwickelt werden. ${ }^{467}$ Style-Kriterien müssen auch erwähnt werden.

Beim Wechsel der Dyade Sonia und Janine zu Loredana und Janine kommt zum ersten Mal während der Feldforschung die Idee der unter-

466 Maggiorini, Janine, 2, 5.

467 Wulff, Bästa vänner, 1992, $23 \mathrm{ff}$. 
schiedlich schnell durchlaufenen Submoratorien zum Ausdruck, indem beschrieben wird, wie die beiden Schwestern von Sonia im Leben besser gefestigt sind wie Sonia selbst. Diese haben also im ökonomischen Submoratorium Perspektiven gebildet, Sonia aber nicht. Diese arbeitet jedoch an ihren horizontalen sozialen und ökonomischen Submoratorien, mit dem Effekt, dass ihre Dyade mit Janine zunächst stark integriert wird. Das unentwegte Lernen läuft jedoch an Janine vorbei, die sich dann an Loredana wendet, welche sich wegen der fehlenden Anbindungsmöglichkeiten in ihrer Klasse zu Sonia und Janine begeben hat. Mit ihnen teilt Loredana kulturelle Züge. Als Sonia schliesslich den Sprung in die nächsthöhere Schule schafft, Janine aber nicht, integriert sich die Dyade Janine und Loredana, Sonia wird bedeutungslos.

Bei Rosa Di Lorenzo lag der Fall anders: Das geschlechtliche Submoratorium und die damit verbundenen Interaktionen lehnte sie ab, weil sie ihre wenigen neben dem Privatleben verbliebenen Energien in das einnahmenseitige ökonomische Submoratorium investierte. Julia und Fiona dagegen hatten im geschlechtlichen Submoratorium sowie im ökonomischen Submoratorium bereits Perspektiven gebildet und waren deshalb nicht auf Gruppenanbindung angewiesen. Der Einzelgänger Stefan hatte vor allem im horizontalen sozialen Submoratorium Probleme. Wegen seiner Vergangenheit hatte er Mühe, sich in die Gesellschaftsordnung einzupassen. Doch diese Beobachtungen wurden ex post in das theoretische Gebäude des Konzepts der Submoratorien eingefügt. Zum Zeitpunkt der Beobachtung waren noch vorwiegend Integrations- und Ausschlussprozesse im Fokus.

$\mathrm{Zu}$ diesem Zeitpunkt neigte sich das Schuljahr und damit die obligatorische Schulzeit dem Ende zu. Dieses wurde mit einer Schulabschlussreise der Klasse nach Südfrankreich rituell markiert. Die Reise kann als Scharnierzeit zwischen der Schule und dem nachschulischen Leben angesehen werden. Sie lag immer noch innerhalb der Schulzeit und wurde rituell deutlich und mehrfach markiert, informeller Höhe- 
punkt davon war die Abschlussparty in Südfrankreich und offiziell die Übergabe der Zeugnisse am Tag nach der Heimkunft nach Basel. Dann war die Schule aus. Nachher gehen die Wege der Schülerinnen und Schüler der 2D auseinander, nur noch Akdis, Janine und - eher selten Stefan treffe ich im Brückenangebot Basis regelmässig wieder. Dieses schulische Angebot wird im Folgenden im Hinblick auf die Funktion zur Förderung der Fortschritte im ökonomischen Submoratorium analysiert. Loredana ist von der WBS an die Fachmittelschule gekommen und Martina blieb, wie schon vorher, am Gymnasium. Grundlage dieser Analysen waren die Erlebnisse, die ich in der Freizeit dieser Mädchen in der Wohnung und an Orten im öffentlichen Raum protokollieren konnte. Diese reichhaltigen Feldaufzeichnungen zeigten vertiefte Einblicke in verschiedene Bereiche ihres Erwachsenwerdensprozesses. 


\section{Schulabschluss: Reise nach Narbonne}

Höhepunkt, Ende und Abschluss der obligatorischen Schulzeit bildete die Schulreise an einen Zeltplatz bei Narbonne zusammen mit Schülerinnen und Schülern eines weiteren WBS-Standortes. Diese Reise war eine an Bedeutungen sehr dichte Zeit und sie setzte rituell den Schlusspunkt der obligatorischen Schulzeit. Das Kapitel teilt sich in zwei Teile: Eine Beobachtung eines bestimmten Schülers, Ali, welcher laut Auskunft der Lehrer anscheinend der schlimmste Jugendliche in der WBS überhaupt war. Welche Vorkommnisse und Themen trugen dazu bei, dass dieser Schüler als derjenige Jugendliche angesehen wurde, welcher die negativen Seiten des jugendlich Seins derart lebhaft verkörperte? In der sozial verdichteten Situation der Busreise von Basel nach Südfrankreich wurden diese Seiten besonders deutlich. Der zweite Teil des Kapitels bildet die Beschreibung und Analyse der Abschlussparty am letzten Abend des eine Woche dauerenden Aufenthalts auf dem Zeltplatz.

18.6.2005: Ich war etwas aufgeregt; es soll nach Narbonne gehen, die ganze Nacht, in einem Bus voll Jugendlicher der unseres WBS Standorts und eines zweiten aus dem anderen Kantonsteil. Die Fahrt wurde ziemlich anstrengend, weil die Lehrerinnen und Lehrer vom zweiten Standort ihre Schüler ignorierten und machen liessen. Binnen einer Stunde war ein grosser Lärm und viel Gerufe und Gestreite den ganzen Weg runter bis nach Bern. Es lief ein Europameisterschafts-Match, Italien - Kroatien, was den Lärmpegel zusätzlich hochtrieb. Obwohl der Lärmpegel bereits sehr hoch war, übertönte die Stimme eines Schülers mühelos alles andere. ${ }^{468}$

468 FB04, 76. 


\section{$\underline{7.1 \text { Der schlimmste Jugendliche überhaupt }}$}

Dieser Schüler - nennen wir ihn Ali - war auffällig unangenehm. Die Lehrer bezeichneten ihn als den schlimmsten Jugendlichen überhaupt. ${ }^{469}$ Anscheinend vertrat er die unangenehmen Seiten des Jugendlichseins mit grosser Ausdruckskraft, was mein Interesse weckte. Die Einträge im Feldtagebuch sind zum Teil sehr abwertend und werden bewusst so wiedergegeben, weil sie auch meine Anspannung und meinen Widerwillen ausdrücken:

Der Schüler hatte eine unangenehm laute Stimme, die er oft und gern anwandte und die mühelos das ganze Geplapper des Busses übertönte, und eine perfekte WBS-Sprache ohne Artikel. ${ }^{470}$ Ausserdem demonstrierte er die ganze Zeit eine Agression, die abstiess, Typ gelackter Latino ${ }^{471}$. Zum Beispiel bedrängte er einen schmächtigen Jungen an einer Autobahnraststätte, hielt ihm die Gurgel im Würgegriff, während seine Begleiterin, ein Mädchen im "nuttigen" Chick-Style, ihn abzuhalten versuchte. B-Movie-mässig. Er hatte ein WBS-Mädchen im Schlepptau, auf der er ständig herumrutschte (oder sie auf ihm), demonstrativ vor aller Augen. Die Demo-Version von Sex. ${ }^{472}$

469 Ebd., 85.

470 Die Umgangssprache der ausländischstämmigen Schülerinnen und Schüler an der WBS besteht aus einem Dialekt, der Schweizerdeutsch mit einem südosteuropäischen Akzent kombiniert. Oft werden die Artikel weggelassen, was der Sprache eine vulgär und primitiv wirkende Reduziertheit verleiht.

471 Kann auch als eigener Style aufgefasst werden, der von den Mädchen "Schleimsack" genannt wurde: Gel in den Haaren, hautenges Shirt (manchmal ohne Ärmel), Trainingshosen.

472 FB04, 76. 
Erving Goffman beschreibt in "Interaktionsrituale" das Konzept der Action, des "wo was los ist" "473 Sie ist immer dort zu finden, wo jemand bewusst folgenreiche Risiken eingeht, die als vermeidbar verstanden werden. Es gibt verschiedene Ebenen, auf denen Action vorkommen kann: Sportlicher Wettbewerb, wie oben beschrieben Kampf, Sex und wie folgt, Geschwindigkeit.

Der Lehrer des zweiten Standorts kümmerte sich nicht die Bohne um den Aufruhr, was meine braven 2D-ler leiden liess. Die wurden ruhiger und stiller. Vorne, wo die anderen Schüler sassen, wurde es lauter und lauter bis $22 \mathrm{~h}$, dann stopfte ich Stöpsel in die Ohren. Laute Musik lief; Fiona ging hinunter zu Müller und bat, dass er anordnen möge, den CD-Player leiser zu stellen. Nichts geschah. Später kam Tobias und bat nochmals darum, ohne Erfolg. Auch Müller hatte sich inzwischen an einer Raststätte Ohrenstöpsel besorgt.

Um 23.30h hatte der Busfahrer, der über seine Passagiere nur den vernichtenden Kommentar "Jugendliche!!!" abgab, die schlechte Idee, einen DVD mit dem Film "The Fast $\&$ the Furious" auf dem Bord-TV zu zeigen. Der Film lässt sich am einfachsten als Autoporno charakterisieren. Meist rotlakierte, aufgemotzte und tiefergelegte Kleinwagen übernehmen dabei die Rolle der Darsteller. Die Handlung ist vernachlässigbar - sie dreht sich um illegale Autorennen - und dient als Kulisse für frisierte Autos. Von diesem Moment an übertönten jaulende Motoren und quiet-

473 Goffman, Erving: Interaktionsrituale. über Verhalten in direkter Kommunikation, Frankfurt a. Main, Suhrkamp Taschenbuch Wissenschaft,1991, 2. Auflage, 164ff. 
schende Reifen das Gejohle (...), während die Schüler vom Leonhart "Geil! Tschöss!" und ähnliche Grunzlaute der Anerkennung von "geilen" Autos und Garniermädchen äusserten. ${ }^{474}$

Der Film war um $0.30 \mathrm{~h}$ aus, der Fahrer löschte das Licht. Trotz allgemeiner Erschöpfung ging der Lärm bis $3 \mathrm{~h}$ weiter. Ich konnte zwischen 3 und 6 Uhr etwas dösen. Um 6 Uhr Rast in der Nähe von Narbonne, der erste Kaffee aus dem Automaten, Croissant aus der Fabrik, einige Schritte an der Meeresluft, Erleichterung. ${ }^{475}$

Autorennen sind deshalb als Action zu bezeichnen, da der Fahrer dadurch gewinnt, dass er häufiger die Geschwindigkeitsgrenze erreicht, die ihn die Kontrolle über sein Auto verlieren lassen könnte, als die anderen Rennfahrer. ${ }^{476}$ Obwohl Action eher zum "Kult der Maskulinität" der westlichen Kultur gehört, die besonders die lateinische Gesellschaft kennzeichnet, gibt es auch weibliche Formen von Action: die sexuelle oder Hofier-Action. Männer können Frauen als Objekte definieren, mit denen man eine mögliche sexuelle Beziehung anknüpft. Dazu gibt es in unserer Gesellschaft bestimmte Orte und Zeiten, unter anderem in der liminalen Zone des Urlaubs, des Ferienhotels, oder in unserem Falle, der Reise im Autobus. ${ }^{477}$

Der ärgerliche Ton des Feldberichts könnte auch daher rühren, dass der Beobachter diese Demonstration von Männlichkeit als nicht wirklich männlich ansieht und deshalb die ganze Show unglaubwürdig wirkt, was sie grotesk und ärgerlich macht. Zudem ärgert die öffentliche Zurschaustellung von Hofier-Action, was laut Goffman bei anderen

$474 \mathrm{FB} 04,76$.

475 Ebd., 77.

476 Goffman, Interaktionsrituale, 1991, 223.

477 Ebd., 229 und Fussnote 83. 
Männern zu Missvergnügen führen kann. ${ }^{478}$ Dies kann sogar die Forschungsperspektive beeinflussen: Angela McRobbie und Jenny Garber kritisieren etwa zurecht Paul Willis abschätzige Kommentare über Mädchen in Motorrad-Subkulturen, können diesen Sachverhalt aber nicht wirklich einordnen. Sie führen ihn auf die angeblich natürliche Verbindung zwischen männlichem Forscher und seinen Beforschten zurück. ${ }^{479}$ Wahrscheinlich war dieses Gefühl eher ein Goffmannsches Missvergnügen.

Des weiteren könnte der Ärger daher rühren, dass hier zwei Jugendliche etwas aufführen, wovon sie offensichtlich keine Ahnung haben und das für Erwachsene provozierend wirkt, weil sie in kulturell nicht anerkannter Weise mit Erotik umgehen, die für Erwachsene in den privaten Raum gehört. Wie auch immer: Auch andere ärgern sich über Ali.

Am Abend trinken Gisler, Müller, Sandmann und ich Roséwein und schwatzen noch ein bisschen über diesen und jenen, über besondere Vorkommnisse. Ganz besonders derjenige, den ich in meinen Notizen Ali nenne, gibt viel Gesprächsstoff. Dass er ganz besonders unangenehm reinkommt, scheint nicht nur mir so zu gehen. Zum Beispiel die Szene vor dem Bus, als wir auf die Abfahrt für einen Tagesausflug nach Carcassonne warteten. Vier Lehrer und ich stehen vor dem Materialkontainer beim Bushalteplatz herum, reden und warten, bis alle an Bord sind. Ali stellt sich hin, den Arm besitzergreifend über der Schulter seiner Partnerin, in seiner viel zu lauten, unangenehmen Stimme: "Malli, wo isch Bus?!" Alle Lehrer, die beim Halteplatz stehen, Malli, der sich anscheinend mit einem Spitznamen anreden lässt, eine Leh-

478 Ebd., 229.

479 McRobbie und Garber, Girls and Subcultures, (1975) 2002, 209-222. 
rerin, Sandmann, Müller und ich machen plötzlich ein Gesicht, als hätten wir beim Essen auf ein Steinchen gebissen. Der Auftritt ist auf mehreren Ebenen nervig: die Haltung des Mackers aus dem B-Movie, die Leere des Blicks des nuttigen Chicks, die Lautheit der Stimme, die fehlenden Artikel des WBS-Pidgins und die Tatsache, dass der Bus fünfzehn Meter weiter steht, dort, wo er eigentlich immer steht, und einen trockenen Strauch um zwei Meter überragt.

Wir fragen uns alle, ist das einfach Dummheit? Wenn nicht - es kann ja nicht sein, dass jemand diesen Bus NICHT sieht - was ist es dann? Will er sein Mädchen beeindrucken? Die Lehrer ärgern? Niemand weiss die Antwort. ${ }^{480}$

Die Fassungslosigkeit der Erwachsenen vor der Jugend ist eigentlich ganz klassisch. Immer wieder trifft man in der Literatur Stossseufzer von Erwachsenen, welche die Jugendlichen nicht verstehen, wobei in unserem Fall noch eine ethnische Komponente mitspielt. Wir erinnern uns noch an die provokanten Parolen der Jugendbewegung der 1980er Jahre: "Weg mit den Alpen, freie Sicht aufs Mittelmeer!" oder "Macht den Staat zu Gurkensalat!", welche die Politiker damals in Ratlosigkeit gegenüber den Forderungen der Jungen stürzten und sie sehr schlecht aussehen liessen, weil ihre Problemlösungsfähigkeit fundamental angezweifelt wurde. Erikson spricht von "provokanter Verspieltheit" ${ }^{481}$

Die Eigenschaft der Jugendlichen, in den Erwachsenen Unverständnis auszulösen, ist genau eine wichtige Vorbedingung des Erwachsenwerdens. Dieses Unverständnis geht aber irgendwann einmal in Verstehen über, die Forderungen werden wirklichkeitsnaher und integrierbarer, die Jungen erwachsen. Doch diese Bedingung des Erwachsenwer-

480 FB04, 85-86.

481 Erikson, Jugend und Krise, 1974, 161. 
dens wird im Fall von Ali noch von einem anderen Phänomen überlagert.

Gunnel Olsson beschreibt auch eine solche Situation der Ratlosigkeit in einer schwedischen Primarschule. ${ }^{482}$ Dort gab es ein Mädchen, die alle Schülerinnen und Schüler mit ihrer Art zur Verzweiflung trieb. Karin, wie Olsson sie nennt, konnte nicht auf andere hören, was zum Zusammenbruch der Aktivität von Arbeitsgruppen führte, weil die Schüler sich nicht verstanden fühlten. Auf ihrem Sitzplatz hing sie bloss mit dem halben Körper, und nach der Arbeit mit Papier wischte sie alle ihre Abfälle hinüber auf die Plätze der anderen, sodass diese ärgerlich alles zurückschoben. Mussten die Schülerinnen etwas für sich lesen, so las sie es laut vor, dass die Kameraden sie darum bitten mussten, leise zu sein, weil sie störte. Das verstand Karin wiederum nicht und setzte ihre laute Lektüre fort, was die anderen als Provokation auffassten. Karin merkte gar nicht, dass sie aneckte.

Angesichts der verfahrenen Situation kam es zu Gesprächen. Im Gruppengespräch mit der Lehrerin gestanden die Schüler sofort, Karin schlecht zu behandeln. Sie versuchten zu beschreiben, was geschah: Karin lache an den falschen Stellen. Sie befehle nur herum. Sie prahle immer so. Sie lüge ganz offensichtlich. Sie sage immer, dass sie in der Prüfung alles richtig habe, doch darf man das Blatt nie sehen. Sie sei so gut, dass sie die Schule problemlos bestehen werde. Kaum glauben könne man, was sie über ihr Pferd erzähle und ihre Kusinen.

Karin war traurig, was alle betroffen machte. Die Schülerinnen und Schüler waren bereit, ihr Verhalten zu ändern, doch es gab etwas anderes, das einer Verbesserung der Situation im Wege stand: Die Klassenkameraden verstanden Karin nicht, begriffen ihre Worte und Handlungen nicht und konnten ganz generell nicht mit ihr kommunizieren. Den-

482 Olsson, Gunnel: Mellan Rum. En studie i fysisk och mental utveckling av kommunikation med utgångspunkt i en mellanstadieklass, Stockholm, Brutus Östlings Bokförlag Symposion, 2003. 
noch versuchten sie, ihr zu helfen. Ein Mädchen nahm den Platz von Jan ein, der so lange neben Karin gelitten hatte. Karin erhielt plötzlich Zettel zugeschickt, die Schülerinnen versuchten sie zu integrieren, Karin versuchte sich anzunähern. Doch sie blieb eine Fremde in der Klasse und litt weiter. Niemand konnte verstehen, was ablief.

Bis zu der Nacht im Klassenlager, in der ein Nachtorientierungslauf anberaumt war. Gruppen bildeten sich und Karin wurde nicht gewählt. Sie brach in Tränen aus, worauf eine Mädchengruppe sich erbarmte und sie doch aufnahm. Als die kleine Gruppe zurückkam, wirkten die Mädchen wie richtig gute Freunde. Im Dunkel und in der Leere draussen war auch Platz für Karin, sie hatte die Mädchen positiv beeindruckt.

Erst jetzt begriffen die Erwachsenen die Tragweite von Karins Problem. Ihre Körpersprache stiess vor den Kopf, war unverständlich und stimmte nicht mit dem überein, was sie sagte. Ihr Körper war eher ein Gefäss, dass sie an einen gewünschten Ort brachte, als ein Instrument um sich damit auszudrücken. Ihre Bewegungen waren eckig und nicht im Fluss und wenn sie las, geschah das ohne Rhythmus. Sie nahm die Grenzen ihres Körpers nicht wahr. Ihr fehlte eine körperliche Beweglichkeit, welche ihre Kommunikation unterstützte. Etwas war falsch mit ihrem Körperbewusstsein, was nach Merleau-Ponty darauf zurückzuführen ist, dass man Sinne und Motorik nicht adäquat anwenden kann. ${ }^{483}$ Die Wahrnehmung des eigenen Körpers verlangt auch, dass man die anderen im Raum richtig wahrnimmt. ${ }^{484}$

Die Beschreibung von Karin und Ali haben einige Gemeinsamkeiten, auch wenn Karin noch eine Primarschülerin ist. Beide haben ein Problem, ihre Stimme und ihr Körper in kulturell als richtig wahrgenommener Weise zu beherrschen: Karin liest laut, obwohl das niemand anderes macht, Ali redet immer unerträglich laut. Ali insistierte viel-

483 nach Merleau-Ponty, Maurice: Phänomenologie der Wahrnehmung, Berlin, Walter de Gruyter, 1974. 484 Olsson, Mellan Rum, 2003, 41-43. 
leicht in Bezug auf den Bus, weil er glaubte, er sei nicht verstanden worden, weil seine Gegenüber mit für ihn nicht interpretierbarer Mimik antworteten. Seine Kleidung, Frisur, Körpersprache und seine Sprache über seinen Körper stiess vor den Kopf, signalisierte Agression und ärgerte die anderen, weil er die Regeln nicht einhielt. Er konnte Leute und Gegenstände im Raum nicht in anerkannter Weise wahrnehmen. Seine Sprache wurde als minderwertig angesehen.

Diese Diskrepanz zwischen Körperbewusstsein und angepasster Kommunikation kann Systemintegration verhindern, obwohl Ali sozial integriert zu sein schien. Möglicherweise beruhte seine Stellung in der Klasse auch auf seiner Gewaltbereitschaft.

Es kann aber auch sein, dass durch eine unangepasste Körpersprache Sozialintegration verhindert wird, sozusagen ein passiver Ausschlussprozess stattfindet, jemand aussen vor gehalten wird. Ein Beispiel soll diesen Sachverhalt illustrieren. Immer, wenn ich Loredana direkt auf Ausschlussprozesse ansprach, kam sie mit Alice. Alice falle ihr sofort ein, sobald es um Ausschluss gehe, sagte sie. Bei ihr wende Loredana aktiv Ausschlussprozesse an. Auch hier scheint ein Problem ähnlich wie bei oben erwähnter Karin vorhanden zu sein, dass Alice sich in der körperlichen Kommunikation nicht angepasst verhält.

Folgende Ausschlussprozesse wende Loredana bei Alice an: Sich aufregen; aus dem Wege gehen; Kopfhörer an und weggehen. Besonders aus der Fassung habe Loredana gebracht, dass Alice sogar dann "mitlatsche", wenn sie die Kopfhörer aufsetzt und weggeht. Alle gehen Alice aus dem Weg. Sie hatte nie Kollegen. Sie schaut blöd (Loredana macht es vor: glotzt einem durchdringend an und hebt dann wissend die Augenbrauen). "In unserer Klasse wird sie zwar nicht gemobbt, aber man meidet sie." ${ }^{485}$

$485 \mathrm{FB} 05,5$. 
Loredana hat mir sogar einen Zettel in A4-Format überlassen, der in einer langweiligen Stunde zwischen ihr und einer Freundin hin und her geworfen wurde. Der Zettel ist über und über vollgeschrieben mit Schmähungen über Alice, die sich an ihrer Pferdeliebe entzündeten. Ich versuche die Reihenfolge einzuhalten:

Sie ist ja selber ein Pferd.

Schau ihre "wunderschönen" Pferdehaare ${ }^{486}$ an!

Wunderschön mit Wellchen drin und ab und zu ein Zöpfchen ...

Ich bin lieber blöd als dass ich keine (unklar) habe.

Was macht sie den ganzen Tag: Lesen, Lernen, Zeichnen, Pferdeheftchen lesen ...

Die tollen Pferde, die sie malen kann ...

Wenn sie so super intelligent ist, sollte sie doch ins Gym oder nein, besser an die Uni

Und Alice, was könnte sie?

Sie?

Die Reiterin!

Ist sie wirklich in der richtigen Fachrichtung? Landdienst würde ihr gefallen ...

Du schnallst es: sie sollte in die Fachrichtung Asozial. ${ }^{487}$

Dann hat sie noch tolle Tatoos ...

... die sie schon ein halbes Jahr am Arm hat.

Ich hoffe, wir beiden werden noch zweieinhalb Jahre miteinander verbringen mit viel Spass, : ) WENDY ${ }^{488}$

486 Original: Rosshoor: Stopfmaterial für eine Matratze.

487 Anspielung auf das Bestreben, an die Fachmittelschule für Soziale Arbeit gehen zu wollen.

488 Pferdezeitschrift für Mädchen. 
Wendy

Hippi

Sie striegelt sich selber...

...und putzt ihre Hufe.

Tut ihren eigenen Stall ausmisten.

Dann müssen wir beiden gute Noten machen.

Das schaffen wir schon. : )

Auch hier wird über das Äussere gespottet, über angeblich fehlende schulische Leistungen und über Alices Hang zu Pferden wie bei Karin. Die letzten Zeilen stellen dagegen eine Art Selbstbestätigung dar, dass die Schmähenden es schon schaffen werden.

Nach diesem Einblick über die Schwierigkeit, den kulturell anerkannten Umgang im sozialen Bereich zu erlernen, soll eine Beschreibung der Abschlussparty der Schulreisewoche das Übergangsritual zum Schulende näher bringen.

\subsection{Rituelles Ende der Schulzeit auf dem Zeltplatz}

Am letzten Tag, dem Freitag, waren wie jeden Tag die Jugendlichen auch unterwegs am Strand, mit ihren gemieteten Fahrrädern oder auf dem Sportplatz. Beim Mittagessen gab es die ersten Vorboten der abendlichen Abschiedsparty:

Heute soll das Nachtessen schon um 19h stattfinden anstatt um 20h, erfahre ich am Mittag im Küchenzelt. Ich solle auch noch (den Jugendlichen) mitteilen, dass es zum Nachtisch Mousse au Chocolat gebe. Wer möchte, solle einen Becher mitbringen. Ich verkünde also die frohe Botschaft beim Essen. Nach dem Essen gehen die "Kinder" 
(Müller) das Mousse holen. Fiona kriegt zwei Riesenkugeln, die sie unmöglich essen kann. Alle wundern sich, hat sie dem Servierer schöne Augen gemacht? Plötzlich ein Aufquietschen am Mädchentisch: Fiona streckt die Zunge heraus mit Schokomousse drauf. Nanny spricht es aus: "Es sieht aus wie Scheisse!" Ein Klatschen, und Fiona ruft: "Das war Rosa!". Die sieht so aus, als könne sie kein Wässerlein trüben. Jedenfalls eskaliert das Mousse-Gewerfe. Auch im Zelt gibt es Opfer. Das Gekreische wird lauter und die Situation unübersichtlich. Fiona und Rosa sind bekleckert, die an zwischen den Zelten gespannten Leinen trocknenden Badetücher versaut, diverse Hosen und Tops anderer Mädchen ruiniert. Monika und Fiona gehen duschen, um sich die Mousse aus Haaren und Tüchern zu waschen. ${ }^{489}$

Während das Herumwerfen mit Esswaren noch als harmloser Spass interpretierbar war, gab es für diese Woche der Übergangsphase am Ende der Schulzeit die zu erwartenden Drogen- und Alkoholexzesse, obwohl beides für die Jugendlichen verboten war. Diese Diskrepanz zwischen alltagsweltlichem Umgang und Prohibition blieb nicht ohne Folgen:

Janine und Sonia haben sich beim Kiffen erwischen lassen. Gisler: "Die kiffen - ich hatte schon vor einem halben Jahr den Verdacht." Anscheinend roch es aus einem Zelt so, dass man es gemerkt hatte. In diesem Zusammenhang sei auch der Name Fiona und Stefan gefallen. Janine ist jeden-

489 FB04, 80. 
falls sauer auf Fiona, weil die gepetzt hätte (obwohl sie mitgekifft hatte). ${ }^{490}$

Gisler und Sandmann, ein Sozialarbeiter, der zur Betreuung mitgereist war, nehmen Sonia und Janine nach dem Frühstück zwei Tage vor Ende der Schulabschlussreise in die Zange. Nachher berichtet Gisler: "Ich hatte sie vor die Wahl gestellt: Zugeben und alles (Gras) abgeben und Telefon an die Eltern am Freitag-Morgen (dem letzten Tag) oder Beweisverfahren und in zwei Stunden den nächsten Zug nach Hause. Dann ist Janine eingeknickt und hat zugegeben. Sonia sei hart geblieben, aber als Janine zugegeben hatte ..." ${ }^{491}$

Das ist der Stoff, aus dem die Erinnerungen sind. Zwei in einer liminalen Phase stehende Menschen übertreten Regeln und konfligieren mit den Autoritäten. Das schweisst zusammen und bildet Zusammenhalt, der oft auch im späteren Leben weiterträgt.

Wie auch immer: Am Freitag abend sollte der Höhepunkt der Reise, die Abschlussparty, gefeiert werden. Die Klasse und deren Betreuer waren tagsüber in Narbonne zu Stadtbesichtigung gewesen. Dort hatte uns Gisler die schlechte Nachricht ins Café überbracht, wo einige Mädchen der Klasse versammelt waren, um auf die Abfahrt des Busses zu warten, dass die Strandparty ausfallen würde, weil der Reiseveranstalter sich weigere, die versprochenen Esswaren und Utensilien dafür bereitzustellen. Als die erste Enttäuschung überwunden war, waren die anwesenden Mädchen einverstanden, etwas zu improvisieren, mit den Spenden der Betreuer etwas Süssgetränk und Chips zu kaufen und sonst zu feiern. So kamen aus Narbonne jede Menge Nüsse, Süssgetränke, Schokoladen, Chips und andere Essenwaren in einen Vorratssack für abends nach dem Abendessen. Dann stieg also die Schulabschluss-Party, die

490 FB04, 79.

491 Ebd., 79. 
trotz ihrer Improvisiertheit und Einfachheit den Schluss der obligatorischen Schulzeit rituell markierte.

Tatsächlich tauchten alle Schülerinnen und Schüler nach dem Abendessen und Abwasch von irgendwoher auf und setzten sich brav an die Tische. Das Partyzeug kommt auf den Tisch, aber die Stimmung bleibt gespannt. Bald setzten sich die ersten ab: die Basketball-Jungs zum Sportplatz und Tobias, der irgendwas mit einem Mädchen hat, wie gemunkelt wird. Bald sind die meisten weg, es blieben noch Janine, Sonia, Rasiah und Müller, Gisler und ich. Janine und Sonia machten Show: Einen Höhlenbewohner-Auftritt zum Nintendo-Soundtrack von Yoshi ${ }^{492}$ - "Uuugh, Hopp, aaah ...!" Das war im Gemeinschaftszelt der 2D. Bald wechseln wir aber ins andere Gemeinschaftszelt, dort war es wärmer.

Janine und Sonia machten weiter Show. Irgendwann fangen sie an, sich das Gesicht mit Schminke vollzuschmieren ("Oh ja, wir machen einen Punk aus dir!") und nach weiteren Opfern für Schminkaktionen Ausschau zu halten. Müller leiht irgendwo Boulekugeln aus und wir Spielen Boule. So sind Gisler, Müller und ich noch eine Zeit lang aus der Schmink-Gefahrenzone. Zunächst spielte Rasiah auch noch mit. Ich habe den Eindruck, sie sucht Anschluss bei Sonia, Janine und Fiona; bald wird Rasiah, die das Interesse verlor ("So'n scheiss AHV ${ }^{493}$-Spiel, Mann!"), als Spie-

492 Eine Spielfigur aus Super-Mario. Die Mädchen liessen den Game-Soundtrack laufen.

493 Alters- und Hinterbliebenen-Versicherung, ein Teil des Schweizerischen Pensionssystems. Gemeint ist ein Spiel für Rentner. 
lerin aber von einem Busfahrer aus Dresden abgelöst, der sehr nett mitspielt, obwohl er das Spiel nicht kennt.

Nachdem sich Janine, Sonia und Fiona die Gesichter vollgeschmiert haben (und fotografiert), kommt Gisler dran, sie kriegt einen Zigeunerschnauz und ein Peacezeichen aufgemalt. Auch sie wird fotografiert. Janine ist wie in Trance: "Das Peacezeichen, geil, Mann!" es geht weiter und weiter hoch, sie steigert sich immer mehr. Müller muss auch hinhalten fürs Schminken.

Um nicht auch geschminkt zu werden, gehe ich schauen, was die anderen Jugendlichen gerade machen. Doch diese sind wohl in alle Himmelsrichtungen zerstreut. Als ich in das Zelt zurückkomme, treffe ich Müller wieder, der gerade eine ausgedehnte Abschminkkur hinter sich hat. Er wollte sich gerade eine Flasche Roséwein holen, die ich im Kühlschrank gelagert hatte. Ob die mir gehöre? Ich biete ihm sie an. Wir gehen zurück zur Party. ${ }^{494}$

In dieser Episode regiert zunächst die Langeweile. Die organisierte Party ist ein Flop, eigentlich passiert gar nichts. Alle gehen weg, ausser zwei Mädchen, die erkennen, dass jetzt ein besonderer Moment ("unser Abschlussabend") mit besonderen Regeln anbricht, die fordern, dass die Lehrer für Schabernack hinhalten. Zuerst ahmen die Beiden zwei liminoide Figuren der kindlich-jugendlichen Spielewelt nach, dann beginnen sie, sich zu maskieren, sich eine andere Identität zu geben, und ihrer Umwelt andere Identitäten aufzuzwingen.

Gleichzeitig steigern sie sich in das Rauschhafte, in die Exstase des liminalen Zustandes. Auch ist sich Janine ganz klar bewusst, dass dieses rauschhafte zu diesem Zeitpunkt statthaft ist. Dies weist auf eine universale Komponente dieser Situation hin. Danach beginnt die eigent494 FB04, 83-84. 
liche Party, alle sind wieder versammelt, es wird Wein getrunken und zusammen geredet, die Spannung vom Anfang ist weg.

Gleichzeitig scheinen verschiedene Schüler an zarten Banden zu weben.

Tobias verschwindet mit einem (...) Mädchen ins Umgebungsdunkel. Daniel scheint ins Fadenkreuz verschiedener Jungdamen geraten zu sein, anscheinend verschwand er mit jemandem. Wer hat eine/n abgekriegt? Wer nicht? ist die Frage, die beschäftigt.

Gisler fasst das Problem des jugendlichen Zwischen-den-WeltenStehens zusammen: "Saufen, Vögeln, Rauchen, aber du musst ihnen sagen, dass sie die Zähne putzen sollen und ins Bett gehen!' Die Rückfahrt nach Basel am nächsten Tag war im Gegensatz zur Hinfahrt unbemerkenswert. Wir fuhren um etwa zwanzig Uhr los; die jungen Leute waren bedeutend ruhiger und gelassener als auf der Hinfahrt.

Die Nacht verlief ruhiger. Die Lehrer hatten beschlossen, den Fehler der Hinfahrt nicht zu wiederholen und einen Actionfilm einzulegen. Es gab den Langweiler "Woman in Red", was die Jugendlichen sicher niederhielt und dann, ab ein Uhr, "Der Schuh des Manitou", der mit querlaufenden Streifen technisch unperfekt, plötzlich um zwei Uhr abgewürgt wurde. Danach war einigermassen Ruhe, und ich versuchte zu schlafen, mit mässigem Erfolg. (...) Um 7h waren wir endlich in Basel. ${ }^{495}$

Für mich war die Schule vorerst vorbei, aber nicht für die Jugendlichen. Für sie gab es Abends eine offizielle Abschlussfeier in der gros495 Ebd., 85. 
sen Halle der Schule. Das war das Übergangsritual zum Ende der Schule. Dort wurde eine Rede gehalten und die Zeugnisse ausgeteilt. Danach gab es eine Disco. Erst dann war die obligatorische Schulzeit zu Ende. Am nächsten Tag machte die Klasse noch einen Brunch zum endgültigen Abschied. Danach waren Sommerferien, die Klasse ging endgültig auseinander. 


\section{Die Welt der Erwachsenen}

In diesem Abschnitt werden die Schicksale der einzelnen Schülerinnen und Schüler der WBS-2D in Bezug auf ihren beruflichen Werdegang weiter begleitet, um den weiteren Weg in das Erwachsenenleben zu verfolgen. Hier geht es um das einnahmenseitige ökonomische Submoratorium und dessen Weiterentwicklung. Es soll beobachtet werden, welche Schwierigkeiten und Probleme die jungen Menschen nach der Schule antrafen und wie sie mit ihnen umgingen.

\subsection{Direkter Einstieg in die Arbeitswelt}

Nach der Schulabschlussreise und den Sommerferien wurde entgültig klar, wer von den Schülerinnen und Schülern der 2D auf Anhieb den Sprung ins Berufsleben geschafft hatte und eine vollwertige Lehre anfangen konnte und bei wem der weitere Weg noch unklar war. Die mit den unklaren Perspektiven gingen in verschiedene Brückenangebote weiter. Eine Lehre anfangen konnten Daniel, der eine Lehre als Motorradmechaniker antrat, Claude, der als Elektroniker begann und Monika, die als Bäcker/Konditor-Lehrtochter einsteigen konnte.

Im Gegensatz zu Daniel, der den Kontakt mit mir abbrach, und Claude, dessen Ausbildung im ersten Jahr wie eine Schule funktionierte, konnte Monika über ihre erste Zeit in der Lehre bei einem Grossverteiler berichten und darüber erzählen, wie die Integration einer Lehrtochter in den Lehrbetrieb und dessen Struktur erfolgt. Die markant anders gehandhabte Integration von Halil in seinen Lehrbetrieb wird weiter unten zum Thema. Er konnte in einem Malergeschäft eine Vorlehre anfangen. Danach werden exemplarisch einige andere Integrationsverläufe aus der WBS betrachtet. Zum Schluss dieses Kapitels wird be- 
schrieben, wie das Ringen um eine Integrationsperspektive im Brückenangebot Basis gehandhabt wurde, welche Faktoren eine Klärung behinderten oder förderten.

\subsubsection{Berufliche Integration einer Lehrtochter}

Ich treffe Monika in einem Café. Sie macht einen aufgeblühten Eindruck. Ich erlebe sie als locker und unternehmungslustig. Sie plaudert drauflos, wie es ihr gefällt, wohl von der Last befreit, Rücksicht auf irgendwelche Klassenkameraden nehmen zu müssen. Sie habe sich in der Klasse 2D relativ unwohl gefühlt. Zu den alten Klassenkameraden hat sie keinen Kontakt mehr, ausser manchmal flüchtig zu Akdis, den sie ab und zu noch im Tram sieht.

Hier wird deutlich, wie froh diese junge Frau war, aus der ihr nicht entsprechenden ambivalenten Situation der Gleichaltrigengruppe herauszukommen und weiterzukommen. Sie hatte bereits deutliche Perspektiven entwickelt. Dennoch musste sie zunächst um ihren Platz in der Hierarchie der neuen Firma ringen, um sich in eine bereits vorhandene Gruppenstruktur zu integrieren.

Sie komme eigentlich mit allen gut aus, ist mit allen per $\mathrm{Du}$, ausser mit dem Lehrmeister. Mit einem Vorarbeiter, dem Chef der Konditorei, habe sie am Anfang Probleme gehabt: Der wollte "Papi spielen" und hat sie immer in einem herabsetzenden Babyton angesprochen.

Dann sagte sie: "Adrian, mit mir kannst du normal reden." Er: "Was sie sich herausnimmt, sie sei nur Lehrling." Dieser Vorfall war am Donnerstag, und sie hat am Wochenende hin und her überlegt, was sie machen solle, das Problem war sehr schwierig für sie. Darüber hat sie auch 
mit ihren Eltern gesprochen. Jedenfalls hat Monika am nächsten Montag mit dem Lehrmeister geredet, und der hat vorgeschlagen, eine Aussprache zu machen. Das sei erfolgt, und seit dem sei die Sache in Ordnung und es gäbe keine Probleme mehr. ${ }^{496}$

Ich weise Monika darauf hin, dass der Vorgesetzte vielleicht selber früher als Lehrling habe unten durch müssen - das habe man damals so gemacht - und dass er deshalb versucht habe, die neue Lehrtochter an die unterste Stelle in der Hierarchie einzuteilen.

Die Lehrfirma führte zu Beginn der Lehre einen Einführungsanlass durch, der die ganze erste Woche dauerte. Man nahm also die Integration der neuen Mitarbeiterinnen und Mitarbeiter sehr ernst. Dieser Anlass funktionierte, wie es die ethnologische Theorie voraussagt, nach den Regeln des dreiteiligen Übergangs. Es gab eine Arbeitsteilung zwischen Schule und Lehrbetrieb: Da die Schule bereits das Abtrennungsritual durchgeführt hatte, die Abschlussreise und das Schulabschlussfest, musste die Lehrfirma bloss das communitasförmige Schwellenritual und das Angliederungsritual abhalten.
Alle neuen Lehrlinge des Grossverteilers haben sich am Montag der ersten Arbeitswoche im Gundelipark getrof- fen, einer sehr grossen Filiale. Als erstes gab es eine Vor- stellungsrunde. Alle Lehrlinge, die in diesem Jahr anfin- gen, stellten sich vor. Das waren alle möglichen Berufe, man glaubt gar nicht, was es da alles gibt. Dann hat man Gruppenarbeiten gemacht, um sich kennenzulernen.

Danach folgt das Angliederungs- und Integrationsritual, das aus formelhaften Handlungen besteht und eine neue Identität aufbaut. Die in 496 Alle Zitate in diesem Abschnitt: Bauer, Monika, 1-2. 
die Statushierarchie der einzelnen Filialen einzuführenden neuen Lehrlinge bringen ihren Namen der Gruppe zur Kenntnis. Dann erhalten sie die äusseren Insignien der Gruppenangehörigkeit, die Arbeitsuniform, und werden über ihre hierarchische Anbindung belehrt. Zum Schluss werden sie belohnt.
Am Dienstag wurden alle Lehrlinge einer Filiale zusam- mengenommen und in den lokalen Betrieb eingeführt. Sie wurden allen Angestellten ihrer lokalen Filiale vorgestellt. Dann bekamen sie Arbeitskleidung, und der Chef der Filia- le stellte sich allen vor. Am Donnerstag begannen sie mit den ersten kleineren Arbeiten, und danach wurden sie in das erste und aus diesem Grund verlängerte Wochenende entlassen.

Jetzt sind die frisch integrierten Lehrlinge in der Lage, sich zu orientieren und werden, noch im geschützen Rahmen, mit den ersten, leichten Arbeiten betraut. Die durch die Integration hergestellte Arbeitsatmosphäre, die den Integrierten noch neu ist und Schwierigkeiten bereiten kann, wird also das erste Mal durch kleinere Arbeiten getestet und durch immer schwierigere verbessert und ausgebaut.
Am Montag drauf ging es "echt" los. Dann wurden ihr leichtere Arbeiten anvertraut, und dann immer wieder schwerere. Die Arbeitszeit ist 6.45 bis 15.30. Das sei schon eine Umstellung gewesen. (...) Es gefällt ihr sehr gut. Sie findet das total kreativ, selber backen zu können und Patis- serie herzustellen.

Wie gut die Integration gelingt, wenn die zu Integrierende motiviert ist und den ihr zugedachten neuen Satz von kulturellen Eigenschaften 
bereitwillig annimmt, zeigt sich in einem bereits übernommenen Arbeitsethos.

Monika lacht, als ich sage, das (Backwerk) kommt alles aus einer Maschine ... das stimme ja gar nicht. Sie müsse immer noch Äpfel, die aus der Schälmaschine rauskommen, selber schneiden und die Wähenbleche ${ }^{497}$ damit belegen. Jedenfalls werde sie zuerst anderthalb Jahre im Grossmarkt arbeiten, dann ein halbes Jahr im Gundelipark ${ }^{498}$, dann ein Jahr im Messegrossmarkt. Dann sei sie ausgebildete Bäckerin/Konditorin, und sie freue sich darauf, danach die Welt kennen zu lernen. Sie möchte dann mal auf einem Schiff arbeiten gehen, also Bäckerin auf einem Kreuzfahrtschiff, wo man in der weiten Welt rumkommt. Oder nach Davos, in die Berge, das würde sie auch reizen.

Die Phantasie führt bereits über die Perspektive der Lehrzeit hinaus, in die der Lehre folgenden Wanderjahre. Sie sollen den Anfängerstatus überwinden helfen und die Aneignung des Raumes fördern. Beides demonstriert den eigenverantwortlichen Erwachsenenstatus und festigt ihn.

\subsubsection{Integration eines Lehrlings Vorlehre A}

Gegenüber dieser sozusagen vollständigen Integration einer Person in die Struktur einer Firma wie oben beschrieben werden Menschen, die kürzere Ausbildungen wie Vorlehren absolvieren, anders in die Firmenstruktur eingebunden. Als Beispiel für einen Schüler aus der WBS-2D,

497 Wähe: Flache Fruchttorte.

498 Grosses Einkaufszentrum, Grossmarkt. 
der eine Vorlehre anfangen konnte, soll Halil dienen, weil dieser Fall am besten dokumentiert werden konnte. Er fing bei einem Malergeschäft in einem Basler Vorort eine Vorlehre $\mathrm{A}^{499}$ als Maler an. Als ich ihn im Aufenthaltsraum für Lehrlinge interviewe, wirkt er nicht besonders glücklich. Die Strapazen des Arbeitslebens, wie etwa um fünf Uhr aufstehen, scheinen ihm zuzusetzen. In der Schule war er schmal, jetzt wirkt er recht kräftig und älter. Ob das an den zwanzig Kilo schweren Farbdosen liegt, die er im Lager stapelt? Jedenfalls bereut er seinen Wunsch, arbeiten gegangen zu sein und nicht die Schule fortgesetzt zu haben.

H: Ja. Also in der Schule ... also wenn ich jetzt an die alte Zeit denke, würde ich denken, ja, wieder Schule und so aufstehen und so ... ich dachte immer, wenn ich schaffe ${ }^{500}$, dann später aufstehen ...aber jetzt weiss ich, dass man sogar viel früher aufsteht ...

N: Hat dir das Mühe gemacht, am Anfang, diese Umstellung.

H: Am Anfang schon, und jetzt auch noch.

$\mathrm{N}$ : Also einfach weil du müde bist am morgen ... oder so ...

H: Das schon, das Schaffen ist kein Problem, aber das früh Aufstehen am Morgen, das ist das Problem. ${ }^{501}$

Der Übergang von Halil ins Firmenkollektiv, seine Integration als Teammitglied weist im Gegensatz zum Bericht von Monika darauf hin, dass er nicht tief in die Arbeitsgemeinschaft eingebunden wurde.

499 Pro Woche zwei Tage Schule und drei Tage in der Lehrfirma. Eine Vorlehre A dauert meist ein Jahr, im Falle von Halil ein halbes Jahr. Es sollen allgemeine Berufskenntnisse vermittelt werden.

500 arbeiten.

501 Demir, Halil, 1, 1. 
$\mathrm{N}$ : Und was ist denn an diesem Mittwoch passiert, wo du hierher gekommen bist? Wurdest du hier besonders empfangen oder so?

$\mathrm{H}$ : Nein. Ich habe alle ganz normal begrüsst und so, sind alle gekommen, und zum (Abteilungsleiter), wer ist der, was macht der ... am Anfang haben alle gedacht, ich bin hier Lehrling. Aber dann habe ich gesagt, ich mache hier keine Lehre.

$\mathrm{N}$ : Einfach eine Vorlehre. Hast du denn mit dem Chef reden können? Bist du vorgestellt worden?

$\mathrm{H}$ : Dem grossen Chef? Irgendwie ich und mein Lehrer, sind ... nicht mit dem grossen Chef, sondern mit dem Personalchef ... haben geredet, mussten Vertrag unterschreiben und so Sachen.

(...)

$\mathrm{N}$ : Und dann ... hast du noch was bekommen, um zu arbeiten?

H: Wo ich vorgestellt worden bin? Ich hätte schon am Nachmittag anfangen können, aber ich hatte noch kein Überkleid. Und mit dem konnte ich dann am nächsten Tag anfangen.

$\mathrm{N}$ : Und dann haben sie es dir gegeben?

$\mathrm{H}$ : Ja, also ich habe eines bekommen von meinem Chef, ein altes, bis meine eigenen gekommen sind.

(...)

$\mathrm{N}$ : In weiss?

H: Jajoja.

$\mathrm{N}$ : Ja, das ist die traditionelle Malerfarbe, weiss ... Dann ... haben sie es dir angepasst, mit Massband und so, oder hast du einfach eines bekommen, so ein Kleid? 
$\mathrm{H}$ : Nein, er ist ja schon fast so gross wie ich ...

$\mathrm{N}$ : Hat er eines von seinen gegeben?

H: Ja. Ich habe jetzt Zuhause noch zwei bis drei. Damit, wenn eines richtig dreckig ist ...

$\mathrm{N}$ : Was haben sie noch erzählt? Wie die Kaffeemaschine funktioniert und so?

$\mathrm{H}$ : Ja, genau.

$\mathrm{N}$ : (...) Und wo die WCs sind, und so Zeugs.

$\mathrm{H}$ : (Ja), und es ist so ... mein Chef ist auch ... wir haben

hier ja die Bahnmalerei und das Spritzwerk. Und da hat er mich jedem vorgestellt.

N: Per Du oder Sie? Schon Sie, oder?

H: Aja. Aber hier sind wir nicht (per Du) ... mit (dem Kollegen) Rolli zusammen (...) schon Du.

N: Deine Kollegen und so, die Arbeiter und so ... sind das solche die schon lange hier arbeiten?

$\mathrm{H}$ : Ich bin schon der Jüngste hier. ${ }^{502}$

Zunächst gab es eine Unsicherheit über seinen Status. Er wurde nicht dem "grossen" Chef vorgeführt wie Monika und ist auch nicht mit allen per Du, sondern er wurde hierarchisch weiter unten eingebunden und duzt nur seinen engsten Kollegen. Der Anlass war nicht separat, sondern wurde gleichzeitig mit der Vertragsunterzeichnung abgewickelt. Die erste Arbeitskleidung, die er erhält, ist gebraucht. Halil wurde auch sozusagen von seinem Lehrer an die Firma übergeben. Halil idealisiert seine Schulzeit und seine Stellung innerhalb der Klasse, innerhalb der Gruppe der Hip-Hop-Fans.

$\mathrm{H}$ : Ah ja, ich würde schon wieder gerne (in die Schule) gehen ...

502 Demir, Halil, 2, 3. 
$\mathrm{N}$ : Hast du nicht gefunden, dass es eher so ... distanziert gewesen ist?

$\mathrm{H}$ : Nein, es war auch gut, dass wir so wenige waren, die anders gewesen sind wie die ganze Klasse. ${ }^{503}$

Auch erscheint ihm einer der Vorteile des Arbeitslebens, das selbst verdiente Geld, nicht als besonders wichtig. Er scheint auch nicht besonders erpicht auf die erwachsene Konsumkultur zu sein. Immerhin scheinen seine Eltern froh zu sein, dass er sich selber unterhalten kann.

$\mathrm{N}$ : Wie nehmen die Familie und Kollegen und so das auf, dass du hier jetzt schaffst ...?

H: Joh, doch, schon, weil ich auch Geld verdiene, hier und so ... und ich kaufe selber einmal ein ...

$\mathrm{N}$ : Ja, das ist noch gut. Machst du denn auch was besonderes mit dem (Geld) ... gehst du in die Ferien und so?

$\mathrm{H}$ : Nein. Ich kaufe mir mal was und so. Das Essen muss man selber bezahlen.

N: Also, das Mittagessen und so.

$\mathrm{H}: \mathrm{Ja}^{504}$

Das Streben nach finanzieller Unabhängigkeit ist ein bekanntes Hindernis, wenn es um den sozialen Aufstieg geht. Menschen aus sozial niedrigen Schichten ziehen oft frühe finanzielle Unabhängigkeit einer höheren Ausbildung vor, was sie in niedrig entlöhnten Stellen und damit in ihrer Herkunftsschicht hält. Möglicherweise liegt dies auch daran, dass sie zuhause ihren Verdienst abgeben müssen und deshalb eher früh ausziehen wollen. Halil und seiner Familie scheint dieses Problem

503 Demir, Halil, 2, 1.

504 Demir, Halil, 2, 3. 
nicht klar zu sein, er möchte weiter in die Schule gehen, weil das bequemer ist und nicht, weil er bessere Chancen erhalten würde.

\subsubsection{Weitere Beispiele aus der 2D}

Claude hat eine Lehre als Elektroniker in einer international bekannten Firma für Steuerungs- und Messtechnik anfangen können. Im ersten Lehrjahr hat er drei Tage Schule die Woche. Er scheint sich gut integriert zu haben. Es gefalle ihm, er müsse aber viel mehr arbeiten, er habe viel Mathematik, Physik und Elektronik. Er käme gut aus mit den Leuten in der neuen Schule, obwohl es einige merkwürdige Leute dort gäbe. Einer, der kaum etwas sagt, und einer, der einem nachläuft und sich freut, wenn einem etwas misslingt. Claude interpretiert dieses Verhalten als merkwürdige Arten, sich in den neuen Klassenverband integrieren zu wollen. Er selbst habe sich mit einem gewissen Samuel angefreundet, weil sie beide das Interesse für Modelleisenbahnen teilten. Es herrsche auch ein guter Klassengeist, der wesentlich besser sei als früher in der WBS-2D. ${ }^{505}$

Rasiah möchte auch eine Lehre machen, hat aber im Gegensatz zu Halil, der unentschlossen wirkt, konkrete Vorstellungen, was sie werden möchte: Medizinische Praxisassistentin. Sie weiss auch, bei welchem Arzt sie diese Ausbildung machen will. Doch ist die Lehrstelle bis zum folgenden Frühjahr besetzt. Deshalb macht Rasiah zunächst eine Vorlehre $\mathrm{B}^{506}$ bei diesem Arzt. Sie erinnert sich nicht wirklich an irgendwelche integrativen Begebenheiten bei ihrem Stellenantritt, was auch daran liegen mag, dass sie selbst bei jenem Arzt in Behandlung war und eine Bekannte von ihr die Lehrstelle gerade innehat. Möglicherweise

505 Boettcher, Claude, 2, 1.

506 Gleich organisiert wie Vorlehre A, es werden jedoch spezielle Berufskenntnisse vermittelt, in diesem Fall im medizinischen Bereich. 
spielt die Tatsache eine Rolle, dass diese Lehrstelle stets von Menschen aus Rasiahs Kultur angetreten wird, was den Übertritt vielleicht besonderen Regeln unterwirft. Unser Gespräch drehte sich hauptsächlich um die Frage, ob sie die Lehrstelle bekomme oder nicht. Das sei überhaupt noch nicht sicher, und sie macht sich deswegen Sorgen. ${ }^{507}$

Wie bereits im Abschnitt über die Submoratorien berichtet, hat Thomas Häberlin hat grosse Schwierigkeiten mit seiner Berufswahl, obwohl er sehr intelligent ist und die IMS anfangen konnte, was eine der besten Aufstiegsmöglichkeiten für einen Schüler aus der WBS darstellt. Im November 2004 telefoniere ich kurz mit seiner Mutter, die auch ratlos wirkt.

Es sei nicht möglich, mit Thomas zu sprechen, da er nach zwei Tagen in der Informatikmittelschule (IMS) zusammengebrochen sei. Er werde jetzt betreut, von einem Psychologen und einem Berufsberater. Er möchte etwas ganz anderes machen, vielleicht mit Tieren. ${ }^{508}$

Auch Klaus, sein engster Freund in der WBS, ist über diese Entwicklung ratlos. Thomas habe die Aufnahmeprüfung geschafft, aber die Schule abgebrochen, habe er im Chat geschrieben. Ob ich mehr wisse? Er habe ja unbedingt dort hin gewollt. Die Tätigkeiten an dieser Schule hätten aber nicht seinen Vorstellungen entsprochen. Jeden Tag müsse man Vorträge halten, es sei zuviel Arbeit, deshalb habe er die Schule verlassen. Klaus glaubt, dass er jetzt ein Zwischenjahr eingelegt hat, daheim sei und nichts macht. Das sei auch nicht so schlimm; er sei ein Jahr jünger als die anderen und könne sich Zeit lassen. ${ }^{509}$ Thomas kann

507 Sunteralingam, Rasiah, Gesprächsnotiz.

508 Gesprächsnotiz vom 4.11.04.

509 Ganz, Klaus 1, 2-3. 
also Entwicklung im ökonomischen Submoratorium wegen seines geringen Alters weiter aufschieben.

Bei Thomas ist der von Glaser und Strauss beschriebene Notfall eingetreten ${ }^{510}$. Er konnte den Schock des Übergangs an die neue Stelle nicht verkraften, sein Übergang verlor die Form. Notfallmassnahmen mussten ergriffen werden, welche die Form wieder herstellen sollen. Eine davon ist ein verordneter Stillstand im ökonomischen Submoratorium, der wegen des noch geringen Alters des Betroffenen noch möglich war, und der helfen soll, Klarheiten in anderen Submoratorien zu erlangen. Hier ist der Knopf definitiv "noch nicht aufgegangen", die Erkenntnis nicht vorhanden, dass es um einen selber geht. Diese Interpretation wird von mehreren Indizien unterstützt: Thomas war in der WBS ein Minimalist. Im Interview mit ihm war ich oft froh, wenn überhaupt eine Antwort auf meine Fragen kam. Im Unterricht engagierte er sich kaum aktiv, obwohl er alle Anforderungen erfüllte. Dem Transkript seines Interviews stellte ich einen Abschnitt voraus, was bei keinem anderen Schüler der Fall war:

Thomas ist extrem maulfaul und nach eigenen Angaben sonst auch faul, lehnt sich zurück, und lässt sich die Würmer zur Nase rausziehen. Das Ergebnis ist, dass ich viel rede, der bekannteste Fehler eines Interviewers. Ich hoffte, es würde als vertrauensbildenden Massnahme wirken. Ich nahm damals an, er lässt mich deshalb auflaufen, weil ich ihm unsympathisch bin oder so, aber nein, es war - echt einfach Faulheit. ${ }^{511}$

Von der Intelligenz her hätte Thomas ins Gymnasium gehen müssen. Isabelle Gisler auf die Frage, weshalb er nicht dort sei:

510 Glaser und Strauss, Status Passage, 1971, 45.

511 Häberlin, Thomas, Prolog. 
G: Faul. Hat er selber gesagt. Gymnasium ist zu anstrengend. Da müsste er was tun. Und WBS ist (einfacher). (Die Noten:) Fünf, fünfeinhalb ${ }^{512} \ldots$ aber Gymnasium hätte er sich eben auf den Hosenboden setzen müssen, das hätte ihn zuviel gekostet.

N. Du meinst also, das ist ein Fall, wo ... er bräuchte einfach ein bisschen mehr Disziplin ... dann könnte er alles machen.

G: Disziplin oder ... Willen. Entweder ... er hat dann den eigenen Willen nicht. Die Eltern haben den Kopf geschüttelt ... was sollen wir machen? Ich habe dann immer wieder das gleiche reingeschrieben in die Berichte, arbeitet ein Minimum, könnte viel mehr ...

N: Ist er denn irgendwie abgelenkt, also, dass er Problem mit sich selber hat?

G: Nee.

N: Mit seiner - was weiss ich - Pubertät oder ...

G: Nein. Hatte ich nicht den Eindruck. Faul. Müde, lethargisch, Ende. Gute Familie, gute Strukturen, ...

N: Jaja, alles perfekt. Wohnt in (einem reichen Vorort) ...

G: Geld, alles perfekt. (...) Schweizer, ganz normale Familie, keinerlei Konflikte oder irgendwelche Problemchen ... $\mathrm{N}$ : Sind die Eltern besonders dominant?

G: Es geht. Bei den Elterngesprächen waren beide Eltern weder dominant noch sehr defensiv, eher so Mittelfeld, haben einfach den Charakter des Sohnes als lethargisch und faul beschrieben. Also sie haben einfach gesagt, er schläft, spielt Computer und macht nichts, was er nicht tun muss,

512 Auf der Schweizer Notenskala von 1 (sehr schlecht) bis 6 (sehr gut) bedeutet 5 gut, in Deutschland wäre das eine 2 . 
und das auch nur in minimalster Ausführung. (Auch ist er desinteressiert an sozialen Kontakten). ${ }^{513}$

Es fragt sich, ob es neben der These, dass noch kein "Knopf-Aufmach"-Moment eingetreten ist, noch weitere Erklärungen für den öfter anzutreffenden jugendlichen Minimalismus? Zunächst ist es eine ausgezeichnete Methode, Autoritäten aller Couleur auflaufen zu lassen, wie in dem Prolog zum Interviewtranskript beschrieben. Doch Thomas' Haltung ging weiter. Gisler führt sie darauf zurück, dass er nie in seinem Leben mit Problemen konfrontiert worden sei. Ihm sei "im Leben alles hinterhergetragen worden ... er braucht im Leben nichts anderes zu tun als Schlafen. Er hat alles zuhause. Er hat auch Geld, (...) er hat auch Intelligenz, er kommt durch, (...) er braucht (in der Schule) nicht zu arbeiten (...) der Rest ist da. Wozu sich bemühen? Vollkommene Energieverschwendung. ${ }^{\not 14}$ Eine reiche Grundausstattung mag erklären, weshalb einer nichts für seine berufliche Zukunft macht, aber Thomas war auch auf sozialem Gebiet minimal, zeigt das Interview mit Gisler.
$\mathrm{N}$ : Hatte er Beziehungen zu anderen Leuten?
G: Er hatte Beziehungen zu wenigen Leuten, und die nur oberflächlich auf bestimmte Themen spezialisiert. Also Klaus, Computerebene, bisschen Claude, da hatte ich so den Eindruck, der kam auch aus (dem Vorort), ...
$\mathrm{N}$ : Zu Thomas Oppliger?
G: Ein bisschen Oppliger, ja, aber wenig, hatte ich den Eindruck. Wobei was die so in der Gruppe privat so getrie- ben haben, das weiss ich natürlich nicht, aber ich habe den Eindruck gehabt, dass er für sich alleine war (...) am An-

513 Gisler, Isabelle, 6-7.

514 Gisler, Isabelle, 8. 
fang noch so ein bisschen Tobias, aber das ist dann auch zerbrochen ... also, da war nicht so sehr viel zu holen.

$\mathrm{N}$ : Also, dann eher so ein Einzelgängertyp.

G: Ja. Auch die kommunikative, soziale Beziehung wäre $\mathrm{zu}$ anstrengend gewesen. ${ }^{515}$

Betrachtet man Thomas Fall aus der Sicht der nicht ökonomischen Submoratorien, gibt es mögliche Interpretationen, obwohl viele Fragen offen bleiben müssen: Thomas scheint im sozialen Bereich nicht in eine Schwellensitutation zu geraten. Wegen seines Desinteresses drängt es sich nicht auf, einen Aufwand zur Integration zu betreiben. Wir wissen nicht, wie er zu der Entscheidung gekommen ist, die IMS zu machen, anscheinend freute er sich auf sie. Die schwierige Prüfung dazu hat er jedenfalls bestanden, möglicherweise glaubte er, der Aufwand sei gering. Seine Krise zu Schulbeginn zu interpretieren, masse ich mir nicht an, jedenfalls sagte er zum Thema IMS im Interview: "Ich freue mich schon (darauf), aber es ist auch ... ich habe auch Schiss davor. Es haben mir viele gesagt, es sei ziemlich streng."

Durch seine zur Schau getragene Faulheit konnte er sehr lange die Ambivalenzen der Moratorien aufrecht erhalten und ebenso die damit verbundenen Freiräume. Möglicherweise war er durch seine relative Jugend dem Zeitplan des Übergangs voraus und konnte sich sozusagen zurücklehnen. Nun kommt das Schulende, da es gilt, sich für seine $\mathrm{Zu}$ kunft zu engagieren, eine Perspektive zu entwickeln. Jetzt funktioniert das Zurücklehnen nicht mehr. Ein Notfall tritt ein, der eine Auseinandersetzung über seine Zukunft in Gang bringt.

Fiona dagegen scheint es gut getroffen zu haben. Zunächst ging sie von der WBS in das "Log in", ein kantonales Brückenangebot in den Bereichen Gestaltung, Technik, Computer und Naturwissenschaften,

515 Gisler, Isabelle, 9.

516 Häberlin, Thomas, 1-2. 
das ausschliesslich für junge Frauen mit Migrationshintergrund geschaffen wurde. Danach konnte Fiona das $\operatorname{Login}^{517}$ anfangen, ein Ausbildungsverbund des öffentlichen Verkehrs, der jährlich 1200 Lehrstellen anbietet. Als ich sie zufällig in einem Supermarkt treffe, erzählt sie mir, dass sie nächstens eine Lehre als Informatikerin bei den Schweizerischen Bundesbahnen anfangen könne.

In die Fachmittelschule (FMS, früher: Diplommittelschule DMS) gingen Devi Mahic, Thomas Oppliger und Sonia Plüss. Sonia hat als einige der wenigen WBS-Schülerinnen die Aufnahmeprüfung für die FMS bestanden, und macht, als ich sie in einem Café treffe, einen selbstsicheren und aufgeblühten Eindruck. Es gefällt ihr in der FMS gut, obwohl sie das hohe Niveau anstrengend findet. Sie treffe niemanden mehr von früher, aus der WBS. Ob in der FMS anders gearbeitet werde? Ja, man sei allgemein viel zielstrebiger. Die Leute arbeiteten konzentrierter. In der FMS gäbe es ziemlich viele Strebertypen.

$\mathrm{N}$ : Wow, was hast du für eine grosse Mappe!

S: Da habe ich meine Entwürfe drin!

Sie zeigt sie mir: eine 5-teilige Arbeit zum Thema Essen in verschiedenen Ländern und in verschiedenen Techniken. Eine Collage (China), eine Bleistiftzeichnung (Schweizer Fondue), ein Foto (Südamerika), eine Guache (ein Kebabsandwich) und noch etwas. Ich bin beeindruckt. Die Arbeit sei wichtig, damit man in den zweiten Jahreskurs kommt, und dann nachher in die FMS-Kunstfachklasse wechseln könne. ${ }^{518}$

517 Die Namensähnlichkeit ist verwirrend. Für das erste Angebot vgl. Schule für Brückenangebote Basel: Log in, http://www.sbabasel.ch/Log_in.53.0.html, 15.11.2010, für das Zweite vgl. login macht Erfolgsstories möglich: Home, http://www.login.org/de/, 15.11.2010. 518 Plüss, Sonia, 2, 1. 
Sonia scheint also einen Fahrplan für ihre weitere Zukunft im Kopf zu haben. Devi hat das auch, doch wirkt sein Entwurf etwas überspannt. Er möchte Architekt werden, nach der FMS an die ETH ${ }^{519}$ Von den früheren Klassenkameraden sieht er niemanden mehr, er habe auch mit dem Basketball aufgehört, die Schule sei zu anstrengend. Er müsse sich jetzt mehr auf seine Zukunft ausrichten. Allerdings sind seine Vorstellungen von der Zukunft etwas zu rosig. Aber anscheinend hat er eine klare Vorstellung von der Wichtigkeit des Timings im Übergang zum Beruf. Es gäbe jedoch auch in der FMS solche, die unentschlossen seien, und ihre Zeit vertrödelten, mit denen wolle er jedoch nichts zu tun haben. Er sieht sich selbst also hinter dem "Knopf-Aufmach-Moment". In der neuen Klasse sei er nicht so gut integriert, was im Licht der unterschiedlichen Stände im ökonomischen Submoratorium auch nicht verwundert. Er habe zwar einige Freunde gewonnen, aber mit denen mache er ausserhalb der Schule nichts. Er begreift also seine Klassenintegration als Arbeitsbündnis.

N: Wie bist du denn auf Architekt gekommen ...?

D: Die Sachen gefallen mir halt so ... verschiedene Häuser bauen und so. Und am PC 3D-Darstellungen, Konstruieren, Mathematik, das ... ist eigentlich alles das was mir gefällt ...

$\mathrm{N}$ : Es hatte eigentlich niemand ... von deinen Schulkumpels (...) eine klare Vorstellung, was sie eigentlich wollen ... die meisten wissen nicht ... sogar der Halil, so recht was sie wollen ... ob sie eine Lehrstelle kriegen oder so ... die haben keine Vorstellung irgendwie ...

519 Die Eidgenössische Technische Hochschule (ETH) in Zürich ist eine technische Universität von Weltklasse. 
D: Ja - das haben die meisten nicht. Es gibt wenig Berufe. Das ist nicht gut. Es werden langsam alle 17, 18, ausser ich, ich bin noch jung ...

$\mathrm{N}$ : Wie alt bist denn du? Noch 16 ?

D: Ja.

(...)

$\mathrm{N}$ : Wenn man auf die WBS zurückschaut, hatte die Klasse wahnsinnig wenig Zusammenhalt ...

D: Das ist auch jetzt so, bei meiner Klasse. Ich habe zwei, drei ... drei gute Kollegen, mit denen komme ich auch jetzt zusammen in die neue Klasse, mit dem Thomas ... komme ich auch in eine ganz neue Klasse, jetzt im zweiten Jahr ist das super - besser wie die jetzt. Weil hier sind jetzt die Hälfte, also mehr wie die Hälfte, so Raucher und so.

N: Kiffer, so (Leute ohne Zukunftsperspektive). Sicher?

D: Solche, die keine Ahnung haben, was sie machen wollen. In der Schule sind sie auch nicht besonders ... Potential hätten sie schon ... aber was machen sie ... lieber rausgehen und vollsaufen oder so ... ist besser.

$\mathrm{N}$ : Jaja, das gibts halt.

D: Die haben, wenn sie 20 sind, 25, nichts. ... Es gibt auch ein paar in der Klasse, drei, vier, die 18, 19 sind. 18 Jahre, und du hast nichts. Das ist nicht gut. Mit 18 bin ich fertig. Es ist halt bisschen hart, sagen wir es so, aber dann, wenn alles gut läuft, dann bin ich mit 22, 23 fertig.

(...)

$\mathrm{N}$ : Wie bist du auf Architekt gekommen?

D: Ich habe Sendungen gesehen, so ... vom Bauen ...

$\mathrm{N}$ : Jaja, ist sicher ein sehr schöner Beruf ... also wenn man Aufträge hat (lacht). 
D: Als Angestellter, also, normal schaffen in einer Firma, sitzt vor dem PC, also, den ganzen Tag, gehst ab und zu schauen und so ... und wenn du denn soweit bist, kannst du selber eine Firma machen ... und wenn du ganz gut bist, wirst Boss. Hast du eine Firma und andere arbeiten für dich. ${ }^{520}$

Diese Aussagen zeigen eine weitere Eigenschaft von Jugend auf: Das Gefühl, dass die ganze Welt nur auf einen gewartet hat. Das Gefühl, alles unter Kontrolle zu haben. Dieses Phänomen wird als Flow bezeichnet. ${ }^{521}$ Flow entsteht zwar in liminalen Situationen, doch habe ich dieses Phänomen nur bei Devi angetroffen. Es könnte auf Faktoren zurückzuführen sein, die ich nicht kenne.

Devi redet über seine jetzigen Kollegen wie über seine WBS-Kameraden abschätzig und negativ. Er selbst aber sieht wegen seines Fleisses seine Zukunft als gesichert an, ja er wird sogar Untergebene haben, die ihm zudienen, glaubt er. Vielleicht reflektiert dieser Optimismus die Migrationserfahrung seiner Familie. Sein Vater ist in den 1980er Jahren in die Schweiz gekommen und hat in der Zwischenzeit eine mittlere Firma in der Baubranche aufbauen können. Gerade kürzlich ist die Familie in einen schönen Neubau umgezogen, allerdings nicht in einem sozial als hoch eingestuften Quartier. Sein Sohn möchte möglicherweise diesen Aufstieg fortsetzen, indem er einen akademischen Beruf wählt, der die Firma seines Vaters weiter voran bringen könnte.

520 Mahic, Devi, 1, 6-8.

521 Flow: Vgl. Csikszentmihalyi, Flow: Das Geheimnis des Glücks, 1998. 


\section{$\underline{8.2 \text { Noch keine Perspektive im Brückenangebot }}$}

Günter Robert, Tobias Holdener, Akdis Saliba, Stefan Staub, Jürg Beller, Janine Maggiorini, Klaus Ganz und Rosa Di Lorenzo gingen in die Brückenangebote Basis oder Basis Plus. Basis Plus ist von den Ansprüchen her schwieriger als Basis und soll dazu befähigen, die Lehre für einen anspruchsvollen Beruf oder die Aufnahmeprüfung in eine weiterführende Schule zu schaffen. Da so viele ehemaligen Schülerinnen und Schüler der WBS-2D in diese Brückenangeboten weitergingen, entschloss ich mich nach Konsultation der Kolleginnen, dort weiter Feldforschung zu betreiben. Drei der sieben oben genannten Jugendlichen fanden sich in der gleichen Klasse wieder, in der 10B. Dort gingen Akdis, Janine und Stefan nun zur Schule. Lehrerin war Paula Lang.

Leider konnte ich den Beginn der Schule nicht miterleben. Erst Anfang November erteilte die Schule die Bewilligungen zur Feldforschung im Brückenangebot Basis. Ich ging daraufhin an einen der Standorte des Brückenangebots, ins Amerbachschulhaus, hinauf zum Zimmer 213, wo Janine, Stefan und Akdis jetzt die Schule besuchten.

Es klingelte zur Pause und die Tür zum Klassenzimmer öffnete sich, Akdis erblickte mich und kam lächelnd auf mich zu. Wir schüttelten die Hände, und auch Stefan und Janine begrüssen mich mit Händedruck. Ich war gerührt, dass sie mich noch kennen wollten, und beruhigt, dass das ethnologische Arbeitsbündnis noch bestand und damit der weitere Fortgang meiner Forschungen sinnvoll war.

Dann kam Paula Lang in den Gang heraus, die Klassenlehrerin. Ich stellte mich vor und wir gingen gemeinsam in das Klassenzimmer und redeten. Die Pause ging zu Ende. Frau Lang stellte mich vor und ich erzählte, was ich machte und was ein Europäischer Ethnologe ist. Janine streckte die Hand hoch und tuschelte aufgeregt mit den Mädchen um sie herum, errötete leicht. Ich war über ihre Empathie so gerührt, dass 
ich mein Ceterum Censeo vergass: dass die Sache völlig anonym ist und niemand Angst haben muss vor Blossstellung.

Es fällt ins Auge, wie homogen die Klasse im Vergleich zur 2D der WBS ist. Die 15 Schülerinnen und Schüler sind nicht durch zwei Leistungszüge getrennt, die auch weitgehend eine ethnische Separierung abbilden. Sie besteht mit zwei Ausnahmen aus Jugendlichen, die einen mindestens teilweisen ausländischen Hintergrund haben. Es ist auch gemütlicher in diesem alten Schulhaus direkt am Rhein. Ein langes, niedriges Bücherregal mit Schriften zur Berufsbildung und Schulmaterial, Computertischchen mit Internetanschluss, Lavabo, Plakate und Tische in Hufeisenform aufgestellt. "Die Kommunikation ist so besser," erklärt Lang.

\subsubsection{Ringen um Perspektive}

In diesem Brückenangebot ist alles auf das Weiterkommen im ökonomischen Submoratorium ausgerichtet, darauf, eine Perspektive zu entwickeln. Darin kommt der Unterstützung des Berufswahl-Prozesses und der Suche nach einer Lehrstelle eine zentrale Bedeutung zu. ${ }^{522}$ Auch Probleme, die in einer Lehre auftauchen, und die eine Integration behindern könnten, werden besprochen, wie etwa das Thema sexuelle Belästigung am Arbeitsplatz. Eine Standortbestimmung und Hilfe bei der Auswahl eines Wunschberufes werden angeboten. Die Lehrerin motiviert, drängt zum Handeln und mahnt. Welche Themen in diesem Zwischenjahr von der obligatorischen Schule zum Berufsleben wichtig sind und diskutiert werden, soll der nachfolgende Abschnitt zeigen, weil diese Themen gekennzeichnet sind vom Ringen um Perspektive, vom

522 Schule für Brückenangebote Basel: Start, http://www.sbabasel.ch/Start.33.0.html, 15.11.2010. 
Überwinden von Blockaden des von Formverlusten bedrohten ökonomischen Submoratoriums.

Es gibt eine Kluft zwischen den Wünschen der jungen Leute bezüglich ihrer beruflichen Perspektive und den institutionellen Möglichkeiten, diesen entgegenzukommen. Die Randbedingungen beeinflussen also die Berufsintegration. Diese wurden im Abschnitt über die historischen Bedingungen der lokalen Jugendlichen bereits angesprochen und sollen an dieser Stelle weiter ausgeführt werden.

Hemmend ist etwa die Trägheit des Dualen Ausbildungssystems, Berufsbilder anzubieten, die für die heutige Dienstleistungsgesellschaft relevant sind. Hier spielen antiquierte Vorstellungen bei Eltern, Lehrern und Gewerbe eine Rolle, was als "anständige Lehre" gilt. Leider sind dabei Berufe gemeint, die wenig mit zukünftigen Wachstumsbranchen zu tun haben.

Zudem sind die Ansprüche der Wirtschaft an die Voraussetzungen für Lehrlinge gestiegen. Dies führt dazu, dass die eigentlich für die Duale Ausbildung vorgesehenen Schulabgänger der WBS von Abgängern der Gymnasiumsstufe verdrängt werden. Ein Abgänger eines Gymnasiums in Basel vertraute mir an, dass er jetzt eine Lehre als Forstwart macht, obwohl ein Forstwart keine gymnasiale Ausbildung benötigt.

Begabungen werden ausserdem nicht honoriert. Eine Schülerin im Brückenangebot Login, das Sprachdefizite bei Jugendlichen mit ausländischem Hintergrund ausgleicht, erzählte zum Beispiel, dass einer Chinesin, die sehr intelligent und mathematisch sehr begabt sei, empfohlen worden sei, eine Lehre als Bäckerin zu machen. Falls dies stimmt, passt dieses anekdotische Indiz in ein Bild, das die Studie bestätigen kann: Man fragt sich, weshalb nicht erkannt wird, welche Chancen eine solche Person für die lokale Wirtschaft im Zeitalter der Globalisierung bedeuten könnte. Möglicherweise wäre es aber besser, könnte die junge Frau mit chinesischem Hintergrund ihre mathematischen und kommu- 
nikativen Fähigkeiten weiterfördern und müsste nicht vier Jahre an einer ihr nicht zusagenden Arbeitsstelle verbringen. Die „klassische“ Lehre gilt den Experten für aus der Form geratene Übergänge als wichtigste Stufe der ökonomischen Erwachsenwerdung. Sie öffnet die Türe zu Weiterbildungsmöglichkeiten. Deshalb werde sie den Jugendlichen empfohlen. ${ }^{523}$ Dieser Gedanke wird weiter unten nochmals aufgegriffen.

Zum Zeitpunkt der Niederschrift dieser Studie entfaltet sich gerade die Diskussion um die Pensionierung der sogenannten "Post-War-BabyBoom"-Generation, also der geburtenstarken Jahrgänge zwischen 1955 und 1963. Deren Pensionierung führt zu einem starken Arbeitskräftemangel nach 2010, glaubt man den Extrapolationen der Bevölkerungsstatistiker.

Um dem absehbaren Arbeitskräftemangel abzuhelfen, dreht sich die Diskussion um die Möglichkeiten, ältere Arbeitnehmer länger im Arbeitsprozess zu halten, etwa durch Erhöhung des Rentenantrittsalters. Jugendliche auszubilden und in den Arbeitsmarkt zu integrieren, um den Mangel an technisch-handwerklichen Fachleuten zu lindern, scheint eigenartigerweise im Jahr 2005 trotzdem noch nicht vordringlich zu sein, wenn von einer WBS-Klasse nur zwei von fünfzehn Jugendlichen eine Lehrstelle finden.

\subsubsection{Widerstände, Hemmungen, Zögern}

Unsere Studie ging davon aus, dass die Jugendlichen gute Gründe dafür haben, sich so zu verhalten, wie sie es tun. Oben genannte äussere Bedingungen sind ihnen durchaus bewusst. Es gab aber auch bei den Jugendlichen selbst gewisse Hemmnisse, Ängste und Ambivalenzen ge-

523 Ledergerber, Beatrice, 3. 
genüber der Arbeitswelt. Sie sind über die Verhältnisse an den Arbeitsplätzen aus verschiedenen Quellen, etwa über ihre Eltern und Freunde, informiert. Deshalb fusst ihr Lavieren in der Berufswahl auf völlig rationalen Überlegungen. Wie diese Hemmnisse, Ängste und Ambivalenzen im Schulalltag thematisiert werden, und welche Lösungen dafür angeboten werden, ist Gegenstand des folgenden Abschnitts.

Zunächst ist der Standort jedes Einzelnen und sein weiterer Lebensweg, also das Entstehen von Perspektive aus Ambivalenz, ein wichtiges Thema, dem immer wieder breiter Raum eingeräumt wird. Wie in den Befunden von Johnsson wird das Leben als ein Projekt aufgefasst ${ }^{524}$ :

Es geht um den weiteren Lebensweg, der offensichtlich als Projekt verstanden wird. Jede/r muss angeben, was er/sie im Leben als ein Ziel hat. Das muss auf einem Blatt eingetragen werden. Amela schreibt etwa: Lehre (Tierpflegerin), Sprachaufenthalt, Vorkurs N, HMS (Weitergehende Schule). ${ }^{525}$

Neben der Bestimmung des eigenen Standortes war auch die Motivation im Unterricht wichtig, diesen trotz allen Schwierigkeiten als Ausgangspunkt für Veränderungen zu nehmen:

Thomas: "Ich habe schon über hundert Bewerbungen geschrieben und es scheisst mich langsam an."

Lang motiviert ihn: “... probieren dranzubleiben ...."526

524 Jonsson, Unga vuxna, deras livsprojekt och tankar om framtida yrke, 1999, 77.

525 FB05, 20.

526 Ebd. 
Auch unterbindet die Lehrerin sofort irgendwelche Bestrebungen, die auf das Herauszögern von Entscheidungen und damit auf das Aufhalten in Freiräumen abzielen. Das Submoratorium muss überwunden werden. Patrizia wird etwa von Frau Lang in die Zange genommen, weil sie gesagt hat, sie werde morgen jener Firma anrufen. Frau Lang verlangt, dass sie das nicht morgen macht, sondern "heute Nachmittag noch" und ihr morgen Bericht erstatte. ${ }^{527}$ Auch spürt sie schnell, ob jemand Ausflüchte macht und unterbindet auch diese.

Atif will den Vorkurs B machen, bleibt aber vage. Da gebe es einen Onkel, der habe eine Garage ... Lang: "Eine Autogarage?"

A: "Ja. Da könnte ich als Automechaniker anfangen ..."

Lang pfeift anerkennend. Dann sagt sie, er müsse sich unbedingt offiziell bewerben. Atif ist zurückhaltend.

Lang: " Das ist mir zu lasch!"

Atif, aufbrausend: "Im schlimmsten Fall mache ich in der Türkei die Militärschule ..."

Lang bleibt hart.

Atif: "Shit!",528

Die Bemerkung mit der Militärschule weist darauf hin, dass ausländische Jugendliche oder ihre Eltern oft noch mit dem Gedanken spielen, in das Herkunftsland zurückzukehren. Dies behindert die berufliche Integration. Umgekehrt ist der Antritt einer Lehrstelle für ausländische Jugendliche meist mit einer vertieften Integration der Familie hierzulande verbunden. Dieser Sachverhalt zeigt, dass jugendliche Migranten mit einem doppelten Übergang und damit mit einer doppelten Integrationsforderung konfrontiert sind: den Übergang in die Berufswelt und

527 Ebd.

528 Ebd. 
den Übertritt in das Gastland. Die Vorwärtsorientierung in traditional-vorwärtsgewandten Familien, die sich durch Selbstbestimmung und Handlungsfähigkeit auszeichnet, ist wichtig in dieser Übergangssituation. ${ }^{529}$

Schon der Gedanke daran, dass man dereinst arbeitslos sein könnte, wird von der Lehrerin nicht akzeptiert.

Lang: "Arbeitslos akzeptieren wir hier nicht als Ziel!"

Jemand: "Aber es könnte ja sein, dass ..."

Lang: "Ja, aber hier arbeiten wir zielorientiert!"

Jemand: "Finger aus dem Arsch!" 530

Es werden immer wieder Berufe ins Gespräch gebracht, die von Frau Lang aber nicht als mögliches Ziel akzeptiert werden.

8.2.1.2 „Sinnvoll“ ist die ,anständige“ Lehre

Es gibt eine Vorstellung von der Sinnhaftigkeit eines Berufes als geeigneter Einstieg ins Berufsleben für eine bestimmte Schülerin oder einen bestimmten Schüler. Lang zu einer Schülerin: "Ja, das wäre sinnvoll für Dich." ${ }^{531}$ Dazu kommt, dass die Berufswahl für die Jugendlichen eine schwierige Wahl darstellt. Die Jugendlichen sind sich zum Teil überhaupt nicht sicher, welchen Beruf sie ergreifen sollen. Paula Lang vermittelt die offizielle Haltung zu diesem Thema: Mal erst etwas "Richtiges" machen, dann kann man immer noch "Künstlerin" werden. Dies deckt sich auch mit den Forderungen der Eltern.

529 Ledergerber, Damit mangelnde Integration nicht zur Desintegration führt, 2005, 24.

530 FB05, 3-4.

531 Ebd., 20. 
Amela will den Vorkurs $B^{532}$ machen. Die Mutter ist dagegen. Sie will, dass Amela eine anständige Lehre machen soll. ${ }^{533}$

Alle machen selbständig Mathe. Es ist still in der Klasse, ausser bei Akdis und Atif, die zusammen kichern. Paula Lang möchte wissen, was so witzig ist. Atif will Rausschmeisser in einer Bar werden. Frau Lang ist nicht gerade begeistert, man merkt das, aber sie behält die Fassung. Meint nur: auch das muss man seriös vorbereiten, sich bewerben, usw. ${ }^{534}$

Hier ringt also die Repräsentantin der Autorität darum, mit dem Beruf umzugehen, den man umgangssprachlich Rausschmeisser nennt. Berufe im Sicherheitssektor sind zwar klar etabliert, wie alle wissen, gehören aber nicht zum als seriös geltenden Kanon, sind nicht "anständig", lässt sie die Klasse merken. Es gibt keine "Rausschmeisser"-Lehre. Deshalb auch die Belustigung.

Erster Schritt zur Beantwortung der Frage, was man werden möchte, ist die Bewusstwerdung der eigenen Fähigkeiten. In einem Brainstorming wurden zunächst Talente gesucht, die in der Klasse vorhanden waren, danach schrieb man eine Liste der daraus folgenden Tätigkeiten auf ein Plakat, das mit dem Titel „Was ist der Sinn des Lebens“ versehen im Schulzimmer aufgehängt wurde.

- Stilberatung (Styling, Haare, Kleider)

- Bad Cops (Rollen für Actionfilm)

532 Berechtigt zum Eintritt in eine künstlerisch orientierte Schule.

533 FB05, 20.

534 Ebd., 10. 
- Popstars (Casting in der Schule)

- Songs/Texte machen (eigene CD's brennen)

- Texte einstudieren

- Broschüre Wortwendungen

- Theater/Tanzproben

- Abschlussfest organisieren

- Werbung machen

- Analyse Fussball

- Lehrstelle finden WIE?

- Filmen

- Forschen (z.B. Wie können Ausländer Deutsch?)

- Multimedia-Arbeit

- Entwicklung von PC-Games

- Snowboard bauen

- Schulhaus verschönern

Es fällt auf, dass viele dieser Fähigkeiten im Bereich Medien, Showgeschäft oder Ästhetik anzusiedeln sind. Doch diese Fähigkeiten werden eher an Universitäten oder Fachhochschulen erworben und nicht in handwerklich-produzierenden Berufslehren, die als Karriere für WBSund Brückenangebotsschüler vorgesehen sind.

Weshalb aber die Brückenangebote stark darauf drängen, eine "anständige" Lehre anzufangen und nicht andere Ziele anzustreben, erklärt Beatrice Ledergerber vom Berufsbildungsamt Basel-Stadt.

L: Zwei Drittel der Jugendlichen machen nach wie vor eine Lehre. Das ist der Königsweg für eine Ausbildung. (...) Wir sagen, wenn jemand mal einen Lehrabschluss hat, dann ist erst mal etwas ganz Wichtiges passiert, Richtung Autonomie. Das ist auch eine der normativen Vorstellungen von uns, dass wenn wir das Gefühl haben, dass sie 
jetzt den Lehrabschluss haben, dann haben sie ein Stück Autonomie gewonnen.

$\mathrm{N}$ : Das ist dann der erste Schritt der wirtschaftlichen Unabhängigkeit ...

L: Ja, zur Weiterbildung, sie können dann weitermachen, oder. Und sie sind in einer Gesellschaft, die sich laufend verändert, können sie mithalten, wenn sie mal mit einer Grundausbildung kommen. ${ }^{535}$

Von Seiten der Autorität wird also die Ansicht vertreten, dass es eine normative Vorstellung gibt, die besagt, dass wenn jemand eine Lehre durchgestanden hat, sie in der Gesellschaft mithalten kann. Die Lehre ist also sozusagen die Feuertaufe oder das Initiationsritual für das Berufsleben. Mit einer Lehre wird aber auch eine entscheidende Weiche gestellt, die es später schwierig macht, etwas anderes zu machen. Es gibt in der Schweiz zu jenem Zeitpunkt praktisch keine Förderung für Berufsumsteiger.

Um an eine Lehrstelle zu kommen, was Ziel des Brückenangebotes Basis ist, gibt es vielfältige Widerstände, die im Umgang mit der Berufswelt abzubauen sind. Die Berufswelt ist das ,richtige“ Leben im Gegensatz zur geschützten Schule und hält einige Härten bereit, soviel haben die Jugendlichen von ihren Geschwistern und Eltern bereits mitbekommen. Ausserdem spiegeln die Bedingungen einer Lehrstelle nicht unbedingt die Wünsche der Jugendlichen, sondern hauptsächlich die Bedürfnisse der Wirtschaft, die sich in der momentanen Situation ihre Arbeitskräfte aussuchen kann. Dieser Sachverhalt wurde bereits als Niedergang des jugendlichen Gesellschaftsvertrags angesprochen. Thomas Oppliger formulierte das am klarsten:

535 Ledergerber, Beatrice, 2 und 4. 
T: Es machen ja ein paar (ehemalige Kollegen) Lehren jetzt, aber die meisten haben Lust dazu, weiter in die Schule gehen, dünkt es mich ...

N: Das Gefühl habe ich auch.

T: Ich finde eigentlich auch, also jetzt eine Lehre anfangen, das finde ich ein bisschen früh.

$\mathrm{N}$ : Früh im Leben.

T: Ja.

$\mathrm{N}$ : Warum findest du das früh?

T: Ich weiss es noch nicht, ich kann es mir aber noch nicht so richtig vorstellen, arbeiten, richtig, Schule liegt mir wie näher.

N: Und das Geld, du würdest ja Geld verdienen, ist dir das gleich?

T: Das ist nicht so wichtig. ${ }^{536}$

Thomas Oppliger befürchtet den den Verlust an Lebensqualität, den er erleiden würde, würde er jetzt mit sechzehn bereits anfangen zu arbeiten. Dieser ist nicht mit dem bisschen Lohn aufzuwiegen, den er als Lehrling erhalten würde. Vielleicht spiegelt diese Aussage auch eine speziell schweizerische Arbeitsmarktsituation, die im Gegensatz zu Deutschland deutlich besser ist. Die Schule hier bietet also den besseren Gesellschaftsvertrag, trotz verschlechterter Bildungsrendite. Erik, ein sechzehnjähriger Schüler der WBS, der ab Herbst 2004 die Fachhochschule für das Transportwesen machte, meinte:

Ich finde mich zu jung für eine Lehre. Ich möchte zuerst noch ein bisschen in die Schule. Dann kann man (nach der

536 Oppliger, Thomas, 1, 7. 
Fachhochschule) noch ein Jahr machen und ist dann KV-ler. ${ }^{537}$

Das Zögern vor der Entscheidung, die Ambivalenz und den Freiraum der Schule zugunsten der viel stärker strukturierten Lehre aufzugeben, ist in diesem Licht ein verständliches Verhalten. Es wird aber von den Autoritäten nicht gerne gesehen. Das Zögern erhöht die Gefahr, dass der Statusübergang aus der Form gerät, weil das Timing sich verschlechtert. Die Erwachsenen haben auch einmal die Strapazen einer Berufslehre, dieser Initiation und Feuertaufe auf sich nehmen müssen, um Eingang in die Berufswelt zu finden.

Manni findet, eine Informatiker-Lehre bei Bell ${ }^{538}$ sei uncool und er habe sie deshalb abgelehnt. Er wolle nicht in einer Wurstfabrik arbeiten. Lang ist entsetzt: "Ja, was glaubst du, was du hier sagst. Glaubst Du, Du kannst hier aussuchen?! Ich habe meine Lehre in Bubendorf gemacht. Jeden Tag mit dem Töffli von (...) Bottmingen nach Bubendorf ${ }^{539}$ - glauben Sie, das sei lustig, im Winter, bei Kälte und Schnee?"

Lang regt sich ob den Widerständen auf und meint:

"Hauptproblem sind nicht die Lehrstellen - ihr ziert euch!

Was soll denn das: Böh, das tönt doof, Gipser, was ist denn

das? Dabei könnte das ein toller Beruf sein!!"540

537 KV-ler: Kaufmännische Angestellte werden durch den Kaufmännischen Verein (KV) ausgebildet und gewerkschaftlich vertreten. Vgl. FB04, 66. 538 Grosser Fleisch verarbeitender Betrieb im Besitz von Coop, einer grossen Detailshandelskette.

539 Töffli: Moped. Von Bottmingen nach Bubendorf sind es rund $25 \mathrm{~km}$ durch städtisch geprägtes Gebiet.

540 FB05, 21. 
Später meint Paula Lang im Gespräch mit mir, dass sie sich Sorgen mache, dass die Jugendlichen keine Lehrstelle finden würden und sie deshalb so empört reagiere. ${ }^{541}$

Weshalb machen sich die Jugendlichen so wenig Sorgen im ihre Zukunft, dass sie das Risiko des Zögerns eingehen? Drei Erklärungen bieten sich an. Erstens mag der Zeitbegriff der Jugendlichen anders sein. Vielleicht denken sie nicht so weit in die Zukunft wie die Älteren oder können die Zukunft weniger gut einschätzen. Dann könnte auch ein falsches Bild der Arbeitswelt eine Rolle spielen: Sie können sich nicht vorstellen, wie es im Berufsleben zugeht und haben deshalb auch von der Schwierigkeit ihrer Situation und der Wichtigkeit der anstehenden Entscheidungen keinen Begriff. Drittens kann es sein, dass die Jugendlichen viel mehr Vertrauen in die Zukunft haben wie Erwachsene und denken, sie seien noch jung und würden es schon (irgendwie) schaffen.

Nicht alle Autoritäten scheinen den Einstieg in die Berufswelt zu forcieren. Akdis meint, dass er jetzt mit sechzehn noch zu jung ist zum Arbeiten, das sei noch zu früh, und sein Vater findet das auch. ${ }^{542}$

\subsubsection{Gute und schlechte Berufe}

Wie das oben vorgestellte Beispiel zeigt, haben die Jugendlichen eine Vorstellung davon, was als schlechter oder guter Beruf sowie was als erstrebenswert und weniger prestigeträchtig gilt. Diese Vorstellungen spielen bei der Berufswahl eine grosse Rolle. Akdis formuliert den Unterschied zwischen guten und schlechten Lehrstellen aus seiner Sicht:

541 Ebd., 23.

542 Saliba, Akdis, 1, 4. 
A: Eine schlechte Lehre ist für mich, wenn man viel stehen muss, viel arbeiten muss, viel körperlich tun muss. Eine gute Lehre ist mehr, wenn man mit dem Kopf arbeiten muss. Weil wenn man zum Beispiel im Rollstuhl landet, kann man immer noch das machen. Wenn man aber im Rollstuhl landet, und hat Schreiner (gelernt) und so, kann man das grad vergessen. (...) Ich finde, es muss mit der Bezahlung stimmen, ist mir egal, was alle sagen, weil wenn man später Familie hat, muss man für die sorgen. Mir ist scheissegal, ob der Job Spass macht und so, Hauptsache die Bezahlung stimmt. Und es ist nichts Illegales. Und man sollte nicht seinen Körper kaputt machen.

$\mathrm{N}$ : Ja, das sieht man bei den Bauarbeitern ... nach 20 Jahren ... Rücken kaputt, Beine kaputt und so ...

A: ... eben, und wenn es regnet und so, muss man trotzdem arbeiten gehen..${ }^{543}$

Akdis möchte also eine Arbeit verrichten, die Kopfeinsatz erfordert und auch bei körperlicher Versehrtheit erledigt werden kann, Spass muss sie nicht in erster Linie machen. Sie sollte gut bezahlt sein und weder illegal noch gesundheitsschädlich sein. Man sollte dem Wetter nicht ausgesetzt sein. Er sieht aber auch ein, dass die Lehre eine Übergangszeit ist, wo man als zu Integrierender unten durch muss.

N: (Die Lehre ist mit) drei Jahren lang ...

A: Aber nach den drei Jahren gehts ab. Lieber drei Jahre so was machen und dann gut arbeiten, wenn es mir auch nicht so gut gefallen würde, würde ich das durchziehen, weil dann würde ich später (besser dran wäre). ${ }^{544}$

543 Saliba, Akdis, 1, 6. 544 Saliba, Akdis, $2,5$. 
Es gibt aber bei den Jugendlichen auch ein Bewusstsein einer ethnischen Komponente bei der Berufswahl. Klaus etwa, der zwei Freunde aus der Basis Plus zum Interview mitgebracht hat, spricht dies an. Er und seine Kollegen finden Detailhandelsfachmann bei Coop oder Migros uncool. Wir sprechen über einen gewissen Darko, der "komisch" sein soll, aber trotzdem eine Lehrstelle bekommen hat, angeblich wegen seinen Eltern.
$\mathrm{K}: \mathrm{Ah!}$.. Dass der die Lehre bekommen hat ...
L: Ja, der macht Einzelhandelsfachmann.
K (abschätzig): Migros!
(...)

Alle (entsetzt): Nein ...

R: Detailhandel im Coop.

N: Detailhandelsfachmann, ach so.

R: Der Darko macht doch im Coop. Seine Eltern schaffen (arbeiten) auch da.

N: Ah, so, da ist der Faktor, der dann spielt. Es spielt eine grosse Rolle, ob du den Schuh wo drin hast, und so.

R: Wenn der keine Beziehungen gehabt hätte, wäre er nicht da rein gekommen. Und zwar nirgends. Und er wäre auch nicht auf eine weitere Schule gekommen.

$\mathrm{N}$ : Was ist er? Ex-Jugoslave irgendwie so?

K: Darko? Ja.

R: Er ist Serbe. ${ }^{545}$

Bei den Mädchen ist das Problem der schlechten Lehre noch akuter, da die ihnen angebotenen Berufe mehr körperliche Strapazen bereithal-

545 Ganz, Klaus, 2, 9. 
ten als die Lehren für Jungen. Folgendes Gespräch zwischen Janine, Sabina und mir soll das verdeutlichen:

J: Ich muss 42 einhalb Stunden (arbeiten) ...(...)

N: 42 einhalb Stunden? Das ist aber noch viel. (...)

S: Aber die Sabrina muss 45 Std. arbeiten. Dann fallen die Sonntage weg, die Feiertage ... da muss sie bis um $12 \mathrm{~h}$ arbeiten.

$\mathrm{N}:$... soviel? Ist das legal?

$\mathrm{J}$ : Ja.

N: Wow.

S. Dort wo die arbeitet haben sie $24 \mathrm{~h}$ am Tag auf.

J: Die haben immer offen. Auch Sonntag.

$\mathrm{N}$ : Im Verkauf ...

S: Die wird Bäckerin.

$\mathrm{J}$ : Ich wollte auch mal Bäckerin werden, das hat mich voll angeschissen.

S: Ich wollte mal Coiffeuse werden. Dann habe ich die Schnupperlehre gemacht und dann ...

$\mathrm{J}$ : ... das wäre noch gut als Coiffeuse ... das würde mich auch interessieren. (...) (Aber) zuwenig Lohn.

(...)

S (zu Janine): Wir zwei im Altersheim waren sowieso die Besten, gell?

J: Sags nicht. Wir waren im Altersheim ...

$\mathrm{S}:$... und erst noch im gleichen.

(...)

S: Das könnte ich mir nicht vorstellen. Das Essen ist wirklich ....

$\mathrm{J}:$... wäääääh. ${ }^{546}$

546 Maggiorini, Janine, 2, 18. 
Obwohl die Beiden die Arbeit im Altersheim als Bestätigung erlebten, wollten sie dort nicht arbeiten, weil bei dieser Institution eine Äusserlichkeit für sie nicht stimmte. Strapaziös in einer Lehre kann auch eine gewisse Eintönigkeit im Arbeitsprozess sein.

Peter lässt den Kopf hängen, weil er eine Schnupperlehre macht als Chemikant ${ }^{547}$. Da muss er am morgen um halb sieben kommen, dann eine Stunde lang irgendwas mischen, dann läuft das zwei Stunden, dann warten sie, dann wird nochmals etwas gemacht, ein paar Minuten, und dann warten sie wieder, und dann ist Mittagspause, und Nachmittags gibts eine Sitzung und nachher um vier geht er wieder. Er macht keinen zufriedenen Eindruck, dort zu sein. ${ }^{548}$

Es gibt natürlich auch Berufe, die aufregend sind, wo man den HipHop-Helden nacheifern kann, viel verdient, tolle Autos fahren kann, viele Mädchen kennen lernt, und die körperlich nicht anstrengend sind: Im Herbst 2005 erreicht mich der Klatsch, dass Peter und Rafael ihre Lehren abgebrochen haben und Dealer und Hehler geworden sind. Man muss sich im klaren sein, dass die Option Illegalität im Hintergrund vorhanden ist: Auch die Drogenbranche mit fünf bis acht Prozent ${ }^{549}$ des Welthandels braucht Nachwuchs und fragt nicht nach Zeugnissen. Diebesgut weiterzuverkaufen bringt manchmal auch einen hübschen Gewinn ein.

547 Fachperson im Produktionsprozess der Chemie- oder Pharmaindustrie, Arbeitsort ist die Fabrik.

548 FB05, 38.

549 Schätzung der Vereinten Nationen für Ende der 1990er Jahre, vgl. World Drug Report - archive, http://www.unodc.org/unodc/en/data-andanalysis/WDR.html, 15.11.2010. 
Dieser Sachverhalt wäre weitere Untersuchungen wert. Es müsste auch abgeklärt werden, wovon es abhängt, dass jemand die Option Kriminalität wählt. Auch müsste untersucht werden, wie der Übergang zur kriminellen Karriere gestaltet ist und wie er rituell markiert wird. Der Untergrund hat sicher seine eigenen Gesetze, die den Neuzutretenden Barrieren in den Weg legen, wie andere Berufe auch.

\subsubsection{Werte und Zukunftsvorstellungen}

Obiger Abschnitt zeigt, dass gewisse Wertevorstellungen bei den Jugendlichen ihren Berufswunsch und ihren weiteren Werdegang mitbestimmen. Dies war auch zu erwarten, doch wollte ich etwas mehr über die Wertestruktur der Jugendlichen der ehemaligen WBS 2D erfahren. Dazu habe ich sie einen Aufsatz schreiben lassen. Unter dem Titel „Montag, den 4. Mai 2024: Ein Tag in meinem Leben“ mussten die Jugendlichen eine A4-Seite mit Text verfassen. Diese sollte "key scenarios" enthalten. Laut Sherry Ortner sollte so ein Bild des Erfolgs oder des guten Lebens entstehen. Jede Kultur hat eine Vorstellung davon, wie Erfolg definiert wird, und welche Mittel am Besten sind, dieses Ideal zu erreichen. "Schlüsselszenarien" ist ihre Bezeichnung für diese Kategorie von Symbolen. Die abgegebenen Aufsätze waren voll von Einsichten auf die Werte der Jugendlichen, was mit dem alltagskulturellen Thema beabsichtigt war. Das Geschlecht der Schreibenden war trotz der Anonymität einfach zu erraten, wenn typische Geschlechterrollen wie der Manns als Verdiener und die Frau als Hausfrau als Grundlage gelten.

Beruflich beschrieben die meisten jungen Männer einen Arbeitsalltag voll Zufriedenheit und ohne finanzielle Sorgen. Die Figuren in den Texten hatten alle gute Jobs, viel Geld und eine Familie oder eine Freundin, wobei jemand eine regelrechte Karriere beschrieb: Automon- 
teur, Automechaniker, Chefmechaniker und ,in ein paar Jahren bin ich reich." Andere Wege zum Reichtum waren Schauspielerei, Rap-Musik oder Sport. Aber viel Geld musste sein: Einer beschrieb seine Utopie, ein Motorradgeschäft zu haben, und umschiffte das Problem, wie man mit dieser Tätigkeit zu viel Geld kommt, indem er sich den nötigen Reichtum durch einen imaginären Sechser im Lotto zuschanzte. In diesen Utopien spielten auch Autos, ein wichtiges Statussymbol von Migranten, eine wichtige Rolle. Alle hatten einen, wenn nicht fünf bis zehn Wagen, Marken wie Ferrari, BMW, Porsche wurden erwähnt. Nur einer nahm in seiner Geschichte die U-Bahn zur Arbeit, weil er nicht als Umweltsünder beschimpft werden wollte. Dieser Aufsatz hatte auch eine konkrete Vorstellung von Arbeit: Computerviren entdecken und neutralisieren. Hier spielte Geld keine Rolle, wohl weil es als selbstverständlich vorausgesetzt wurde.

Bei anderen spielte Geld eine untergeordnete Rolle, das waren wohl die Mädchen. Hier gab es wirkliche Utopien: Zwei mal wurde ein Leben auf einer paradiesischen Südseeinsel oder im Ausland mit einer besten Freundin beschrieben, „ohne Arbeit und Geld“. Hier wuchs das Essen an Bäumen und man lebte am Strand. In einem Fall hatte der Ehemann am Strand ein kleines Bistro.

Die weniger eskapistisch veranlagten Mädchen beschrieben einen vollständigen Alltag, in einem Fall mit zwei Kindern und einem Mann. Dies wirkte derart lebensecht, dass die Verfasserin wissen musste, wovon sie sprach. Dieser Aufsatz beschrieb ein konventionelles Leben mit einer Teilzeitarbeit von 14 bis 17 Uhr und traditioneller Rollenteilung, bis auf das Detail, dass der Mann abends noch das Abendessen zubereitet. Ein weiterer Aufsatz schildert ebenso einen vollständigen traditionell geteilten Alltag mit zwei Kindern, aber hier wird noch eine $60 \%$ Stelle für die Frau ins Bild eingefügt.

Erstaunlicherweise gehen nur drei Aufsätze auf die in zwanzig Jahren zu erwartenden technischen Entwicklungen ein. Diese Aufsätze 
stammen wohl von drei jungen Männern. In ihren Geschichten gibt es keine Hausarbeit mehr, da diese von Robotern übernommen wird. Die bei ihnen beschriebene traditionelle Rollenteilung zwischen Mann und Frau wirkt deshalb etwas luftleer. Es gibt Post, die innert fünf Minuten in alle Winkel der Erde transportiert werden kann, über zweihundert Meter hohe Gebäude, da Autos fliegen können und interstellare Reisen. Diese Reisen haben zur Folge, dass auf der Erde jetzt auch Ausserirdische wohnen, die habe man im Jahre 2019 entdeckt. ,Sie sind genau so nett, gefühlvoll und liebenswürdig“ wie die Menschen. „Deshalb ist es auch keine Seltenheit mehr, das(s) man auf der Erde einen Ausserirdischen als Nachbarn hat", schliesst diese positive, multikulturelle Utopie.

Fasst man die Inhalte dieser kurzen Aufsätze zusammen, stellt man fest, dass die Jugendlichen eine ziemlich konventionelle Vorstellung von Familie haben. Wenn ein Ehepartner vorkommt, dann immer im Zusammenhang mit mindestens zwei Kindern. Auch scheint völlig klar zu sein, dass der Mann arbeitet und die Frau auf die Kinder schaut. Meistens haben die Frauen aber noch Teilzeitstellen. Bei den jungen Männern gibt es Machtfantasien: viel Geld, Autos, Reichtum, Berühmtheit und Frauen. Die Arbeit wirkt bei ihnen im allgemeinen nicht wirklich konkret, aber sie führt auf alle Fälle über verschiedene Karrierestufen zur finanziellen Zufriedenheit oder Reichtum.

Bei den Mädchen steht die Familie im Zentrum. Kinder und der manchmal als einfühlsam beschriebene Mann werden häufig erwähnt. Nebenbei machen die Mädchen einen Teilzeitjob, manchmal einen Beruf, aber oft wird die Erwerbsarbeit gar nicht erwähnt.

Diese konventionellen Schlüsselszenarien lassen darauf schliessen, dass es den Jugendlichen nicht darum geht, die Welt zu verändern. Zum Glück strebt man durch Arbeit, die zwar meistens diffus bleibt, aber viel Geld einbringt. Die Notwendigkeit, in die Berufswelt einzusteigen, ist also mindestens für die männlichen Jugendlichen unbestritten. Die 
Mädchen verbinden sich mit einem Mann, nicht notwendigerweise durch Heirat, und bilden eine glückliche Familie mit mindestens zwei Kindern. Der Beruf spielt hier eine weniger zentrale Rolle. Bei beiden Geschlechtern werden Familienidyllen beschrieben, bei denen man gemeinsam kocht, fern sieht oder liest. Ein traditionelles glückliches Familienleben ist also auch sehr wichtig für die Jugendlichen. Mehr ist aber nicht aus den abgelieferten Arbeiten herauszulesen. Weitergehende Interpretationen würden das Quellenmaterial überstrapazieren.

Dennoch könnte das Streben nach utopischen Zielen im Leben das Ringen um Perspektiven beeinflussen, etwa indem möglichst lange im einnahmenseitigen ökonomischen Submoratorium gezögert wird, um ja nicht den besten Einstieg in die berufliche Laufbahn zu verpassen. In diesem Licht ist auch das dauernde Abwägen, Zögern und Zieren zu sehen. Vielleicht klammern sich die Jugendlichen auch an einen Wunderglauben: Der tolle Mann für die Mädchen, ein Hit als Sänger für die Jungen.

Die Autoritäten hängen das Ziel jedoch deutlich tiefer; für den Staat ist es bereits genügend, wenn eine "anständige" Lehre gemacht wurde. Damit ist die Chance am grössten, dass die jungen Menschen immerhin schon mal aus den Listen der Sozialhilfebezüger raus gehalten werden können, eine Gefahr, welche für die männlichen Jugendlichen mit Migrationshintergrund am grössten ist. So kann das Ziel der staatlichen Integration, das Fehlen von sozialen Konflikten, am besten erreicht werden. 


\section{Freizeit, Öffentlichkeit und Privates}

Im vorhergehenden Abschnitt wurde hauptsächlich die ökonomische Integration der Jugendlichen der WBS-Klasse 2D nachgezeichnet, ausgehend von der Situation der Schulklasse. Diese Analysen betrafen die Einnahmenseite des ökonomischen Submoratoriums, was nicht verwundert, ist doch der Aufbau einer wirtschaftlichen Lebensgrundlage erstes Ziel der Volksschule, der Weiterbildungsschule und der Brückenangebote. Sie war auch der Fokus des ursprünglichen Forschungskonzepts.

Doch gibt es beim Erwachsenwerden auch den Bereich der erwachsenen Konsumkultur, was die Ausgabenseite des ökonomischen Submoratoriums darstellt, das Geschlechtssubmoratorium und das vertikale und horizontale Sozialsubmoratorium. Diese Bereiche werden in der Freizeit und im Privaten weiterentwickelt. Sie sollen in den folgenden Kapiteln besprochen werden, soweit die Quellenlage dies erlaubt. Diese war unerwartet gut: Meine Feldbeziehung zu Janine hat sich im Brückenangebot weiter vertieft. Ich kannte sie bereits über ein Jahr und habe den Wechsel ihrer besten Freundinnen miterlebt. Loredana war jetzt ihre engste Freundin, nicht mehr Sonia. Ich traf Loredana und Janine zu jener Zeit regelmässig, da mich Janine immer wieder in der Schule zu irgendwelchen Unternehmungen einlud. So konnte ich Informationen über die beiden Mädchen und ihre Freunde sammeln. Bald stellte ich fest, dass Martina sehr oft dabei war, dass die Mädchen praktisch immer zu Dritt unterwegs waren. Dieses Material soll im Folgenden auf die anderen Aspekte des Erwachsenwerdens neben dem Berufseintritt analysiert werden. Zunächst wird berichtet, wie ich Martina kennenlernte.

Nach den Sommerferien konnte ich wegen der ausstehenden Bewilligung des Rektorats noch nicht mit meiner Feldforschung in den

Brückenangeboten fortfahren. In der Zwischenzeit werde ich jedoch im 
September 2004 in die „Wohnung“ von Loredana eingeladen. Loredanas Mutter hatte ihr Geschäft aufgegeben, doch die Miete des Raumes lief noch bis in den Mai 2005. Dort im 5. Stock, mit Blick auf das Rangiergelände des Bahnhofs, hatten sich Loredana, Janine und Martina eine Rückzugsmöglichkeit eingerichtet. Sie verbrachten viel Zeit dort, übernachteten und wohnten dort während den Ferien. Immer wieder kamen junge Leute vorbei und besuchten die drei in der Wohnung.

Wie bereits in der Einleitung angetönt, spielt das abgeschiedene Zimmer, gemäss Helena Wulff eine typische Situation, für junge Mädchen eine wichtige Rolle. An dieser Stelle sollen diese Ausführungen kurz rekapituliert werden: In der bequemen Abgeschiedenheit meist eines Schlafzimmers wird die Mädchenfreundschafts-Dyade gepflegt und aufgebaut. Das Zimmer stellt eine private Sphäre dar, in der Musik gehört und mit Make Up und Frisuren experimentiert werden kann oder die Mädchen probieren verschiedene Kleider aus. Dabei werden die Körperhaltungen, Gesten und Sprechweisen der älteren, in geschlechtlichen Dingen erfahreneren Schwestern durchprobiert, während die Mädchen heimlich Zigaretten oder Hanf rauchen. Beste Freundinnen teilen Geheimnisse, reden lange über Jungs, teilen sich über Probleme mit und geben sich Trost. Beste Freundinnen können lange Abende, ganze Wochenenden und Schulferien zusammen verbringen. ${ }^{550}$

Wie Eva Breitenbach in ihrer Fallstudie über Gleichaltrigengruppen feststellt, haben Mädchenfreundschaften in der Adoleszenz einen ausserordentlich hohen Stellenwert für die beteiligten Mädchen. ${ }^{551}$ Diese Freundschaften überdauerten die Sozialsubmoratorien und helfen gleichzeitig, diese zu überstehen. Obwohl diese Freundschaften "eine eigene, lebendige, hinreissende Qualität" besitzten, eine besondere Stimmung, die auf eine tiefe Ernsthaftigkeit, optimistische Leichtigkeit und intensive Gefühle hinwiese, wirkten sie auch normativ: Sie unter-

550 Wulff, Bästa vänner, 1992, 23-28.

551 Breitenbach, Mädchenfreundschaften in der Adoleszenz, 2000. 
stützten gesellschaftliche Zumutungen gegenüber Mädchen und Frauen und setzten sie auch gegenüber den eigenen Gruppenmitgliedern durch. Die Freundinnengruppe sei für ihre Mitglieder sowohl eine liebevolle, unterstützende und kurzweilige Gemeinschaft, aber auch eine kontrollierende und möglicherweise einschränkende. Beide Aspekte kommen in den folgenden Beispielen zum Tragen. In der Literatur gibt es Beschreibungen von Mädchengruppen als eine bestimmte Beziehungsund Kommunikationsstruktur als Instrument der Geschlechtsdarstellung. In funktionalistischer Sichtweise ist die Mädchengruppe ein Arbeitsraum zur Reflexion und Bewältigung von Erfahrungen, wo spezifische Praktiken und Kompetenzen entwickelt werden. ${ }^{552}$

In einer zweiten Phase der Adoleszenz finden sich in einer Gegenbewegung zum Gruppenleben verstärkt Individualisierungsprozesse statt, stellt Breitenbach fest. Diese gehen parallel mit Auslandsaufenthalten, längeren Liebesbeziehungen oder Absetzbewegungen von dominierenden Gruppenkulturen vor sich. Dies führt zu neuen Konflikten; die Mädchen verlieren auf dem Weg zu einer eigenständigen erwachsenen Identität ihre Freundinnen und gewinnen vielleicht andere. Laut Breitenstein bleibt es offen, inwiefern diese Strukturen der Mädchengruppen in das Erwachsenenleben hineinreichen. ${ }^{53}$

Doch es ist nicht nur für Mädchen typisch, dass Jugendliche freistehende Räume vorübergehend besetzen und provisorisch herrichten. Dies sind Räume, die essentiell für ihre Integration sind. Hier werden Verhaltensweisen auf verschiedenen Ebenen eingeübt, Umgang mit Freundinnen und Freunden gepflegt, Zeit zusammen verbracht. Dies ist für die Jugendlichen eine sehr wichtige Zeit. Deshalb möchte ich einige Begebenheiten darstellen, die in diesen acht Monaten dort geschehen sind.

552 Ebd., 303-311.

553 Ebd., 327-328. 
Wir sitzen eine Zeit lang herum und plaudern. Joints werden herumgereicht, Janine bietet mir ein Bier an. Loredana liest ein Brief von ihrer Oma vor, in dem ihr die Idee, während den Herbstferien mit einer Freundin vorbeizukommen, ausgeredet wird. Lustigerweise wird im Brief das Wort Freundin mit der Bedeutung Fremde benutzt. Sie allein sei schon willkommen, aber mit Freundin (lies zwischen den Zeilen: einer Fremden) ... ausserdem gehe es Opa nicht gut und er müsse operiert werden. Janine und Loredana diskutieren, was sie während der Herbstferien machen sollen. Janine geht nach Spanien mit einer Freundin. Loredana bleibt zuhause. ${ }^{55}$

\subsection{Action am Barfüsserplatz}

Von hier aus werden auch Ausflüge unternommen. Zum Beispiel in die Stadt, zu Freundinnen.

"Wir müssen bald weg, einer Freundin Weed ${ }^{555}$ bringen," kündigt Loredana an. Nur mal kurz zum Barfi ${ }^{556}$ und zurück. Wir gehen bald los zur Tramhaltestelle. Janine hat einen Riesenjoint gedreht, den sie raucht, und dann Loredana weiterreicht. Deshalb verpassen wir den Sechser, der Depot angeschrieben hatte. Was jetzt? Loredana checkt den Fahrplan. Die beiden beschliessen, die 300m zum Bahnhof, der nächst grösseren Haltestelle, zu gehen. Ich

554 FB04, 88.

555 Cannabis, Hanf.

556 Barfüsserplatz, zentraler Platz der Stadt Basel, Kreuzungspunkt vieler Strassenbahnlinien (schweizerisch: Trams, von Tramways). 
gehe mit, und unterwegs fragt mich Loredana, wie alt ich bin.

Ich: "Wart mal ... 44."

Sie: "Wow, ich gehe hier mit einem Erwachsenen daher ..."

Ich: "Mein Gott, du bist doch auch praktisch erwachsen ... "

Sie: "Nein, das bin ich nicht!"

Janine stimmt zu und erzählt, dass Loredana letzthin einfach auf ein schreiendes Kind gespuckt habe. Loredana glaubt, sie sei deshalb kindisch. Ich: "Glaubst Du, das wird nachher anders? Ich kenne einen Haufen Erwachsene, die ziemlich kindisch sein können." Beim Rotlicht stellt sich Loredana auf den Fahrradstreifen, wie wenn sie auf Grün warten würde. Einfach ohne Fahrrad. Die Leute glotzen, nehme ich mal an, was mir unangenehm ist. (...)

Am Bahnhof kommt der nächste Sechser nach vier Minuten, nachdem wir gemeinsam auf einer Bank gewartet haben. Die längsten vier Minuten meines Lebens: Die beiden zappelten ständig rum und kicherten und ich hatte den Eindruck, dass sie ständig einen Streich ausheckten. Ich fühlte mich von allen Erwachsenen im Umkreis von 100m beobachtet. Im Sechser legen Loredana wie selbstverständlich die Füsse auf die gegenüberliegenden Bank. Janine hält sich noch zurück, bald macht sie es aber auch. Loredana kann das ja noch mit medizinischen Erfordernissen begründen, da sie wegen einer Bänderzerrung einen eingegipsten Fuss hat, aber Janine? Ich schmunzle, und denke für mich, aha, das ist jetzt eben dieses antisoziale Verhalten, das in den Medien vorkommt. 
Im Tram reden wir über dies und das, während wir in Richtung Stadt gondeln. Loredana habe letzthin eine Halbschwester bekommen. Julia, frisch geboren. Sie täte ihr jetzt schon leid. Ich: "Vielleicht ist dein Vater ihr gegenüber anders als zu Dir?" Sie: "Nein, er bleibt ein Arschloch."

Ich frage Janine, was sie denn geritten habe, dass die eine Schnupperwoche ausgerechnet in Altersheim gemacht habe, wo die Arbeit bekanntermassen hart sei. Sie macht ihren "Alten" nach: Zeigefinger sticht im Worttakt nach unten: "Du machst jetzt die Schnupperlehre!!!" Ein mal muss ich Musik hören von Janine, die die beiden cool finden: Ein Intro, wo ein Rapper was sagt.

Am Barfüsserplatz steigen wir aus und die beiden entfernen sich sehr schnell vom Tram. Ich komme nicht nach und muss rennen. Die beiden gehen schnell zum McDonalds-Ecken, wo die Jugendlichen sich aufhalten, exaltiert, hüpfend, schubsend, kichernd. Janine regt sich über etwas auf. Loredana tauscht etwas aus mit zwei Mädchen an der Treppe zum Leonhardsgymnasium.

Der Polizeiwagen steht am Anfang der Steinenvorstadt, 20 Meter weiter. Ich schwitze: Hoffentlich schnappt mich hier keiner, während ich in Gesellschaft von zwei minderjährigen Mädchen bin, die wohl illegale Substanzen verkaufen. Ich werde ziemlich nervös, wenn ich mir vorstelle, einem Polizisten erklären zu müssen, dass ich ein Ethnologe bin, der sich in die Gepflogenheiten eines fremden Stammes einzuleben versucht, und wenn die kiffen, dann ist mein Job, das aufzuschreiben. Natürlich zappeln Loredana und Janine rum und verhalten sich auch sonst ziemlich auffällig. Ich schwitze weiter. Es passiert aber nichts. Anschei- 
nend fällt das den Autoritäten überhaupt nicht auf, wenn Jugendliche zappeln, hin und herrennen und rumhüpfen auf einer Treppe in der Öffentlichkeit.

Ich schaue unschuldig drein und tue, als ob ich rein zufällig hier stehen würde. Es ist ein Mädchen zu uns gestossen: Martina. Ich werde formell vorgestellt: Obi, das ist Martina. Martina, das ist Obi. Wir lächeln uns an, geben uns aber nicht die Hand. Martina ist 15. Dann machen wir rechtsumkehrt und gehen zur Strassenbahnhaltestelle zurück. Ich will am Automaten ein Ticket lösen, habe aber kein Kleingeld, ausserdem steht Julia mit einem Typen in einer Fünfergruppe Jugendlicher beim Automaten. Loredana und Janine echauffieren sich: Die wollen sie jetzt sicher nicht treffen, die blöde Tussi. Ich, versuche Öl ins Feuer zu giessen, um Begründungen zu erhalten: Vielleicht schon blöde Tussi, aber einen Typen am Arm. Das Argument hat irgendwie keine Wirkung. Martina versteht die ganze Aufregung nicht.

Ich: "Lore und Jani regen sich über die Tussi da auf."

Sie: "Aha? Wer ist denn das?"

Ich: "Ein Mädchen aus der Parallelklasse, die hübscheste vom Schulhaus." Die 15 kommt. Julia und ihr Typ gehen an uns vorbei, grüssen freundlich und Loredana und Janine grüssen zurück, es ist ihnen ausgesprochen peinlich. Als die beiden weg sind, überschlagen sich Loredana und Janine vor "Ooch, ist die blöd ...!"

Der Sechser kommt. Janine rennt zur vordersten Türe und steigt dort ein, es folgen Loredana, Martina und ich. Wir gehen durch das ganze Tram - ein siebenteiliger CombinoTramzug ist rund 40 Meter lang - nach hinten und setzen uns auf die hintersten Sitze. Am Markt steigen drei Män- 
ner ein, Jeans mit schwarzen Turnschuhen, Lumberjackets und Schreibblöcken, die ihnen aus der Jackentasche schauen. Sie benutzen die letzte Türe, als alle anderen Türen bereits blockiert sind. Die schliesst sofort hinter ihnen. Klarer Fall: Fahrausweis-Kontrolle!

Ich überlege blitzschnell: soll ich raushüpfen und meine Forschung versauen? Ich beschliesse, es sein zu lassen, als die Männer zwischen Markt und Schifflände nicht kontrollieren, und versuche mich zu entspannen. Leider erwischt es uns zwischen Schifflände und Rheinbrücke. Ich zücke einen Hunderter und hoffe vergebens, ihn als Spesen dem Nationalfonds anhängen zu können. Loredana und Janine haben auch kein Billet und müssen das Prozedere über sich ergehen lassen, ihre Namen mühsam buchstabieren. Endlich ist es vollbracht. Als die Kontrolleure abgezogen sind, sagt Loredana: "Schau mal, was er geschrieben hat: Di Cotzanzo !!" Wir gehen wieder in die Wohnung. Ich fühle mich recht wohl, nach überstandener Gefahr.

Jedenfalls setzen wir uns alle und entspannen uns. Thomas ist noch zu uns gestossen. Was läuft jetzt, geht die Frage. Super Mario! Ein mir damals noch unbekanntes Spiel. Ein Fernseher wird ausgeborgt, ein Gerät angeschlosen, ich bekomme einen Kontrolgerät in die Hand gedrückt und finde: Ihr müsst mir helfen, was soll ich tun? Ich bekomme genaueste Instruktionen von Thomas. Janine ist auserkoren, mir die einzelnen Games zu erklären. Z.B. auf Plattformen hüpfen, die aus dem Wasser tauchen: Ich: Muss ich jetzt hier drücken? Janine weist mich zurecht: "Sei ruhig und höre zu!" Dann eklärt sie das Spiel in 20 Sekunden. Ich bin beeindruckt von der Effizienz ihrer Erklärung, deren Konzentration und Janines Autorität. 
(...) Ich spiele ungefähr 10 Games (die alle sehr gut sind) und verliere immer als erster, obwohl ich angeblich ziemlich lernfähig bin. Martina: Du hast eine schnelle Auffassungsgabe. Thomas sitzt nebendran und hält sich zurück. Irgendwann verschwinden Janine, Loredana und Thomas, schmieren sich Nutellabrote und machen sich grosse Ovomaltine-Drinks. Martina isst Fastenwähen und Schokolade. Wenn das nicht eine waschechte Hochkaloriendiät ist! Ungefähr um $22 \mathrm{~h}$ gehe ich. ${ }^{557}$

Erving Goffman hat Handlungen, die folgenreich, ungewiss und um ihrer selbst willen unternommen werden, wie im Abschnitt über die Abschlussreise bereits erwähnt, als "action" bezeichnet. Im Augenblick der Action wird ein besonderer, affektiver Zustand hergestellt, der sich in Form von Erregung darstellt. ${ }^{558}$ Bei Jugendlichen hiessen diese Aktionen Nervenkitzel (im Original: kicks). Das Gemeinwesen selbst wird dabei in ein Action-Feld verwandelt, wobei man sich insbesondere der Gleichaltrigen, wehrloser Erwachsener und solcher Personen bedient, die als Symbole der Polizeigewalt wahrgenommen werden. ${ }^{559}$

Das ist genau das, was ablief bei den Jugendlichen, die ich unterwegs beobachtet habe. Da passiert viel mehr als man gewohnt ist innerhalb von einigen Stunden. Es findet eine Beschleunigung statt. Als Feldforscher hatte ich damit umzugehen. "Das ganze Leben geht an einem vorbei und es ist kaum halb elf. Ich war um elf schon zuhause und müde im Bett. Ich hatte deutlich das Gefühl, dass was abgeht, meiner Kontrolle entgleitet, ${ }^{, 560}$ notiere ich.

$557 \mathrm{FB} 05,6$.

558 Goffman, Interaktionsrituale, 1991, 203.

559 Ebd., 231.

560 FB05, 6 . 
Da mitzukommen ist nicht nur eine methodische Herausforderung, sondern auch eine soziale Kompetenz. Goffman bezeichnet das als "handling the action", als eine Fähigkeit, mit den Ereignissen mitzuhalten. Der Begriff Action entstammt der Slangsprache der Welt des Glückspiels, Glücksspiel ist die Reinform von Action. In den Spielsälen von Nevada werden Neulinge unter den Croupiers "von Action im Stich" gelassen, während kaltblütige Habitués, die sich von erfahrenen Spielern nicht verwirren lassen, die "Action zu handhaben" verstehen. ${ }^{561}$

Goffman sieht Action zudem als typisch männliche Tätigkeit. Für Frauen, beobachtet Goffman in den 1960er Jahren in den USA, gibt es nur eine Form von Action, die in Frage kommt: Als Objekt der männlichen Hofierbemühungen. Spielerische Versuche sind im Bereich der Anbahnung von Beziehungen zwischen den Geschlechtern für das Erwachsenwerden nötig, deshalb ist diese Art von Action auch wichtig.

In der Jugendphase herrscht auch eine gewisse Schicksalshaftigkeit. Es entscheidet sich sehr viel für das spätere Leben. Menschen in schicksalshaften Situationen, berichtet Goffman, können die Risiken der Schicksalshaftigkeit sozusagen umwenden, wenn sie aus der Not eine Tugend machen und als Spielende in der Herausforderung leben, Action daraus machen.

Dies kann ein Weg zur Mannwerdung sein, da er mit Männlichkeit assoziert wird. Die Jugendlichen müssen die Neuordnung der Dinge und die Erfahrungen der Grenzen und Eroberungen körperlich machen. ${ }^{562}$ Goffman zitiert Ernest Hemmingway, Experte für Männlichkeit:

Männer, die nicht bereit sind, scharf zu kalkulieren, haben ihre eigene Krankheit, die Syphilis. Sie war im Mittelalter die Krankheit der Seefahrer. (...) Es ist die Krankheit von

561 Ebd., 205.

562 Klosinski, Pubertätsriten, 1991, 17. 
allen Leuten, die ein Leben führen, in dem ein Ausserachtlassen der Folgen ganz gang und gäbe ist. (...) Penicillin hat diesem Weg zur Männlichkeit ein Ende gesetzt. ${ }^{563}$

Die Action in dieser Untersuchung wurde von Mädchen unternommen. Sie nehmen ganz selbstverständlich die früher als typisch männlich wahrgenommenen Tätigkeiten und öffentlichen Bereiche in Anspruch für ihre eigene Action. Hofier-Action ist ebenfalls ein Thema: Julia steht für die erwachsene Frau, die scheinbar kein Problem mit ihrem Körper und die Männer um sich herum hat, und mit ihnen umzugehen weiss. Mit diesem Vollbild von Frau wird gerungen: sie wollen sie zwar nicht treffen, lassen das Treffen aber zu, das ihnen dann peinlich ist und lehnen dann Julia als "blöde Tussi", als nichtvorbildhaft ab. Martina ist wohl im Geschlechtssubmoratorium weiter, deshalb verstand sie die ganze Aufregung nicht. Sie weiss, wie mit Männern umgehen. Sie begrüsste mich formal korrekt und unterhielt sich auf eine erwachsene Weise mit mir.

Zinnecker meint, dass die Jugendlichen Meister der von ihm so genannten kleinen Alltagsflips sind, die "kicks" von Goffman. Alltagsflips sind Handlungen, die aus der erwachsenen Alltagsordnung hinausführen, für Abwechslung sorgen und Action versprechen. Diese sind gemäss der Shell-Jugendstudie 81

- Die Nacht durchmachen $(1,2)$

- richtig rumalbern (2)

- Musik irrsinnig laut hören (1)

- Action suchen (1)

- mit einem Fahrzeug durch die Gegen kurven (1)

- sich anders bewegen

- blau machen (2)

563 Zitiert in Goffman, Interaktionsrituale, 1991, 200. 
- verrückte Sachen anziehen

- schwarzfahren

- ältere Leute provozieren $(1)^{564}$

wobei die mit 1 gekennzeichneten Alltagsflips eher arbeiterschichtliche Tätigkeiten darstellen. Sie lösen den Routinealltag in Spannung und Bewegung auf, während die anderen Flips eher bürgerliche Tätigkeiten sind, die darauf hinauslaufen, dass man sich Zwängen von Institutionen entzieht und ganz allgemein den Ernst der Dinge ins Lächerliche zieht. Die Flips mit der 2 kennzeichnen zudem solche, die erst ungefähr mit 18 Jahren wichtig werden, einem Alter, in dem der Nachtraum erforscht und besetzt wird.

\subsection{Auf der Suche nach Normalität}

Das Konzept der Normalität war für diese Studie richtungsweisend; sie ging davon aus, dass Bedeutungskonstruktionen von Normalität einer Gruppe Integrations- und Ausschlussprozesse bestimmten. Ob diese Annahme richtig war, konnte nicht befriedigend beantwortet werden. Dagegen sprach, dass Leute in der Gruppe waren und daher integriert, die eher als unnormal galten. Fabrizio zum Beispiel hat sogar damit geprahlt, unnormal zu sein. Er erzählte, dass er einmal Wasser aus der Wiese $^{565}$ getrunken habe, da er wahnsinnig durstig gewesen sei. Er habe dann erbrechen müssen. Ich bin halt eben etwas widerlich, rechtfertigt

564 Jugendwerk der Deutschen Shell (Hg.): Jugend '81. Lebensentwürfe, Alltagskulturen, Zukunftsbilder, Band 1, Opladen, Leske + Budrich, 1982, 578.

565 Der Fluss Wiese kommt vom Feldberg und fliesst durch den Landkreis Lörrach (Baden-Württemberg) und durch das schweizerische Kleinhüningen in den Rhein. 
er dieses abnormale Verhalten. ${ }^{566}$ Möglicherweise muss davon ausgegangen werden, dass Einzelne innerhalb von Gruppen sich einen Platz darin suchen müssen, wie etwa der Clown, die Anführerin, der Schlaue, die Sportliche, was bereits eine gewisse Abweichung von der Norm bedingt und deshalb Akzeptanz geniesst. Dies wird vor allem in Gruppen der Fall sein, deren konstituierendes Prinzip nicht freiwillig ist, sondern irgendeine Verwaltungsbestimmung konstitutiv wirkt, wie etwa bei Schulklassen oder Rekrutenjahrgängen, oder geografische Faktoren eine Rolle spielen, wie etwa bei Gruppen von Nachbarschafts-, Quartier- oder Dorfjugendlichen.

Für die Annahme sprach, dass Normalität als Mittel eines Ausschlussprozesses eingesetzt werden konnte. Janine und Sonia wurden beispielsweise in ihrer Gruftiphase vor allem von den männlichen Jugendlichen in ihrer Klasse als nicht normal angesehen. Dies führte zu einem Ausschlussprozess: Die Mädchen wurden von den Jungen nicht akzeptiert. Die beiden Mädchen haben sich bewusst in der Sichtweise der Jungen unnormal aufgeführt, um die Jungen zu reizen. Sie wollten gar nicht akzeptiert werden. Der Ausschlussprozess war von ihnen gewollt, stärkte die Abgrenzung innerhalb der Klasse und die Integration zwischen den Beiden. Beide Seiten waren sich darüber einig, dass sich die Beiden unnormal aufführten. Welche Motivation oder Strategie hinter diesen Prozessen stand, lässt sich nur vermuten. Vielleicht müsste man zur Beantwortung der Frage unterscheiden zwischen den inneren Dynamiken von Freiwilligengruppen und denjenigen, die in Nichtfreiwilligengruppen zu beobachten sind.

Jedenfalls konnte festgestellt werden, dass Normalität nicht einfach vorhanden ist, sondern erlernt werden muss.

Empirisch liess sich feststellen, dass die Normalität von Bedeutungskonstruktionen auf drei Ebenen als Thema auftauchte. Zunächst Normalität in der Schule, also die Stellung des Individuums innerhalb 566 FB05, 83. 
der Schulklasse, dann die Frage nach der Normalität eines Individuums und den Wegen dorthin und drittens die für die Jugendlichen wichtige Frage der Normalität von ihnen im Verhältnis zur Gesellschaft. Diese Themenbereiche weisen darauf hin, dass ein Lernprozess stattfindet.

Die Normen, welche bestimmen, wie man als Person sein soll, werden mehrheitlich im alltäglichen Umgang an der Schule erlernt, schreibt Gunnel Olsson. Sie machte diese Aussage zur Grundlage ihrer Doktorarbeit über die physische und mentale Entwicklung von Kommunikation in einer schwedischen Primarschulklasse. ${ }^{567}$ Methodisch bezog sie sich dabei auf die Phänomenologie von Maurice Merleau-Ponty, dessen Denken um den Zusammenhang zwischen Körper und Bewusstsein kreist. Es ist so, dass das Normalsein unter anderem an der Schule gelernt wird. Doch ist es wahrscheinlich so, dass die Schülerinnen und Schüler kurz vor Ende der obligatorischen Schulzeit nicht wie Primarschüler Normalität lernen müssen, sondern dass ihre Normalitätssschulung stark fortgeschritten ist.

Es kann sogar sein, dass ein Jugendlicher, der sich für die Erwachsenen normal verhält, dessen Normalität sich also mit denen der Erwachsenen weitgehend deckt, nicht als normal angesehen wird. Die Klassenlehrerin Isabelle Gisler wundert sich etwa über Tobias, der mit seinen vielen Interessen erwachsen wirke und gesellschaftlich völlig integriert sei, aber in der Klasse trotzdem anstrengen musste, akzeptiert und integriert zu sein. Seine Tätigkeiten galten als zu aktivistisch und deshalb als nichtjugendlich und im Umfeld der WBS-Normalität als unnormal.

IG: Er war kein Aussenseiter, das hat er gut geschafft (...) es war ja eher so, dass er derjenige wer, der die meisten Interessen hatte, was er erzählte, Chor und Freizeit, Pfadfinder, Theater, und nebenher noch dies und noch ein Praktikum gemacht, und die anderen (geben) schlafen (an) oder

567 Olsson, Mellan Rum, 2003. 
machen Schwimmen ... von daher war er schon von seinen Interessen ganz wo anders.

$\mathrm{N}$ : Aber er war ja normaler, also (...) er war ja irgendwie nicht schwarz wie ... oder erkennbarer Ausländer wie Akdis, sondern er war ja ein Basler.

IG: Genau.

$\mathrm{N}$ : Also völlig integriert. Und trotzdem hat er eine Aussenseiterfunktion (...).

IG: Die Frage ist, ob die deswegen da ist, weil so viele auf unserer Stufe, sozusagen nicht normal sind? Weil die auch dadurch entstehen, weil eben Ausländer da sind, abgestossen, ausgestossen, wirklich durchgeknallte, dass dann das Niveau der Normalität wieder ganz wo anders ist, oder ob's eben auch an ihm liegt, also wenn ich jetzt zum Beispiel meine Klasse jetzt betrachte, die ich habe, da sind auch viele Schweizer Kinder, da wär er trotzdem vom Typ in eine gewisse Aussenseiterposition gerutscht, weil er einfach so dieses - sag ich mal - typisch normale, orientierungslose, pubertierende revolutionäre, freche, was man so kennt einfach, (...) nicht so hat. Er war immer der höfliche, zurückgezogene, trotzdem liebenswerte Schüler von nebenan, ohne Ecken und Kanten. (...) ... und normal in der Phase wäre eben auch mal anzuecken (...). ${ }^{568}$

Tobias Aussenseiterfunktion setzte ihn unter Anpassungsdruck. An dieser Aussage von Isabelle Gisler zeigt sich auch eine Ungleichzeitigkeit: Sie ist durch die von der Achtundsechziger-Pädagogik geforderte jugendliche Rebellion gegen irgendwelche gesellschaftlichen Strukturen geprägt, die das Anecken als normal bezeichnen, und Höflichkeit, Liebenswertigkeit und Zurückgezogenheit als nicht normal ansieht.

568 Gisler, Isabelle, 3. 
Doch möglicherweise sind Jugendliche gar nicht per se rebellisch, sondern nett und höflich. Man darf nicht vergessen, dass auch zur Zeit der Jugendunruhen von 1968 die Mehrheit der Jugendlichen nicht am Demonstrieren auf der Strasse war.

Doch Tobias mit seiner Theatererfahrung ist kreativ mit dem Normalitätsdruck seiner Aussenseiterstellung umgegangen. Wir waren zu viert im Gespräch: Klaus, zwei seiner Freunde, die nicht aus der Klasse stammten, und ich.

Nicholas: Lustig - beim Tobias ist ja irgendwie das Gerücht rumgegangen dass er eine Lehrstelle hat beim Standesamt. (...) Es gibt einen Haufen Leute, die das felsenfest glauben, dass der beim Standesamt ist.

Klaus: Wir haben jetzt auch einen in der Klasse, da haben wir gerade vorher darüber geredet: Der hat gesagt der hat eine (Lehrstelle), aber ob wir es ihm glauben, sei nicht so wichtig.

N: Das ist wie mit der Freundin: Behaupten, man hat eine ...

K: Auch der Tobias ...

$\mathrm{N}$ : Ja.

$\mathrm{K}$ : Der hat das ganz am Anfang ... wir sind ja beide aus dem Gym(nasium) gekommen, ich und Tobias, aber nicht aus dem gleichen ... und sind dann halt in die Klasse gekommen ... und dann sind irgendwie Gerüchte entstanden, Tobias hatte noch nie eine Freundin und so, und dann hat er irgendwie noch eine erfunden. Hat gesagt, jo, sie wohne am Münster und so, aber er sei noch nie bei Ihr daheim gewesen ...

$\mathrm{N}$ : Das Standesamt ist auch am Münster ...

$\mathrm{K}$ : Das war komisch, irgendwie ... 
$\mathrm{N}$ : Was hat der bloss mit dem Münster?

$\mathrm{K}$ : Er hat gesagt, sie gehe dort ins Gymnasium. Hat er gesagt. Sie wohne dort und ... (...). Keiner hat es ihm geglaubt.

N: Jaja. Auch mit der Lehrstelle war das ja so merkwürdig. Ich habe ja mit dem Chef vom Standesamt telefoniert und gefragt, ob es den Tobias gäbe (lacht) ... er hat gesagt: nein. ${ }^{569}$

Um sich als normal darzustellen, und nicht ausgeschlossen zu werden, spielte Tobias schlicht und einfach seine Reife vor. Er behauptete, eine Freundin und eine Lehrstelle zu haben, und veredelte beide, indem er sie mit dem Basler Heinrichsmünster, ehemalige Kathedrale des Bistums und Zentrum des vornehmsten Quartiers in Basel, in Verbindung brachte. Dies bringt auch eine soziale Sehnsucht zum Ausdruck.

Die Frage, wie sie gegenüber der Erwachsenenwelt wirkten, war für die Jugendlichen sehr wichtig, man könnte die Auseinandersetzung fast als Sehnsucht nach dem Normalen bezeichnen. Einmal fragt mich Loredana: Was hältst du von uns? Ich bin baff und weiss keine Antwort. Ich denke drüber nach, versichere ich. ${ }^{570}$ Ich komme nicht zu einer Lösung. Dennoch taucht das Thema immer wieder auf. Später fragt mich Loredana: "Wie kommt dir das so rein, wie wir hier hausen, in dieser Wohnung?" Ich bin von der Frage überfordert. Erwachsene würden nie die Einrichtung einer Wohnung eines anderen Erwachsenen kommentieren, das wäre hochgradig unhöflich. Es sei sehr gemütlich und angenehm in der Wohnung, erwidere ich. Loredana räumt immer wieder auf, hält die Leute dazu an, die Aschenbecher zu leeren, Flaschen wegzubringen und Ordnung zu halten, deshalb habe ich noch nie über das Hausen nachge-

569 Ganz, Klaus, 2, 6.

570 FB05, 5. 
dacht. Aber Loredana ist jugendlich und erwartet deshalb anscheinend Richtlinien aus der Sicht der erwachsenen Normalität.

Einmal treffen wir uns im Eingang, und beim Hochfahren im Lift fragt mich Loredana: "Weshalb gibst du dich mit uns ab?" Ich spreche es aus: "Mit Abschaum und so?" Sie schmunzelt. ${ }^{571}$ Anscheinend erwartet sie, dass Erwachsene Jugendliche irgendwie als abartig empfinden. Ich sage wahrheitsgemäss, weil ich sie und die anderen mag. Doch die Frage scheint sie stark zu beschäftigen. Später greift Loredana sie nochmals auf.

Loredana fragt mich: Wie komme ich dir rein?

Wieder bin ich überfordert. Ich frage: Inwiefern?

L: Ja, bin ich denn normal?

Ich: Normal wie?

L: Naja, normal halt.

Ich: Normal wie wer?

L: Was ich mache und so.

Ich: Ja, machst du was Schlechtes?

L: --?

Ich: Eben. ${ }^{572}$

Die Auseinandersetzung mit der Normalität geht also ziemlich tief. Loredana lässt sich auch nicht durch Rückfragen abspeisen, insistiert und kommt schliesslich zu der Einsicht, dass es keine Normalität gibt ausser der ausgehandelten, da die Frage, ob man etwas Schlechtes mache, auf die Konvention des Schlechten zielt.

Schmerzhafter war die Auseinandersetzung über das Normale bei der Schülerin Fiona, die grosse Probleme mit ihrer Familie hatte. Jeden-

571 Ebd., 30.

572 Ebd., 44. 
falls musste Isabelle Gisler bei ihr feststellen, dass sie Stimmen hört. Fiona selbst empfand das nicht als abnormal, eher als belästigend.

IG: Die ganze Zeit war das dann eher so, naja, ich hör die halt, und jetzt kam ich, und sagte, du das ist nicht in Ordnung, das hindert dich auch am Lernen, das hindert dich auch an deinem Leben, (...)

NS: Kurz: das ist nicht normal.

IG: Ja. Natürlich nicht.

NS: Und wie stellt sie jetzt die Normalität wieder her, ist jetzt die Frage. ${ }^{573}$

Erst das Gespräch mit der Klassenlehrerin hat Fiona davon überzeugen können, in Bezug auf das Stimmenhören professionelle Hilfe in Anspruch zu nehmen. In diesem Fall hat also eine normativ arbeitende Autoritätsperson die Bedeutungskonstruktion von Fiona, dass die Stimmen nicht abnormal seien, in Frage gestellt. Das Stimmenhören wurde als nicht normal definiert. So entstand Handlungsbedarf.

IG: Wir haben dann über (...) sechs Wochen (...) hin und her haben wir dann entschieden (...), das Kind dann herauszunehmen aus der Familie, was für Fiona ein enormer Schritt war, eh, weil sie einfach einsehen musste, dass sie einfach diese ganzen Strukturen, mit Stimmen hören und Kopfschmerzen und nicht Schlafen können und so weiter, aufgrund ihrer Vergangenheit und ihrer familiären Struktur, (...) dass sie dem nur beikommen kann, wenn sie sich von ihrer Familie trennt $(. . .)^{574}$

573 Gisler, Isabelle, 16.

574 Gisler, Isabelle, 16. 
Die Methode der Wiederherstellung von Normalität war also, das Mädchen aus den stark belastenden Familienstrukturen herauszunehmen und in ein Umfeld zu setzten, das die gesellschaftliche Normalität repräsentiert. Sie zog in ein Wohnhaus für Mädchen aus Familien mit schwierigen Strukturen ein, wo sich die Situation für Fiona stark verbesserte. Doch auch das war für Isabelle Gisler verdächtig, da sie dahinter eine Verdrängungsstrategie ähnlich wie bei Rosa witterte, weil dann doch manchmal Devianzen stattfanden.

IG: Wobei sie auch schon in diesem ersten Haus schon sehr viele Freiheiten hatte, wobei ich auch da wieder mit den Betreuern sehr stark in Kontakt war, das lief alles gut, der Betreuer hat immer gesagt, es läuft fast zu gut, dafür, dass immer alles Scheisse ist in ihrem Leben, läufts fast zu gut, so bisschen wie bei der Rosa, sie schwimmt so auf diesem Perfektionsding, dann kam aber wieder was. Dass sie dann irgendwie im Kaufhaus die Sachen hat mitgehen lassen, zusammen mit den anderen und so, und kam wieder so diese Vernunftssachen. Auf der einen Seite will sie ja so ne Ausbildung, und sie ist auch so vif, dass sie von der Reife her weiss, was sie dazu braucht, und will das zielstrebig erreichen, und trotzdem kamen dann so kleine Wellen $(\ldots)^{575}$

Fiona war den anderen, wie bereits oben erwähnt, im Geschlechtsmoratorium weit voraus. Im ersten Jahr der WBS wurde sie schwanger. Nach längerem Drängen der Klassenlehrerin gab Fiona ihre Schwangerschaft zu. Gisler interpretierte diese Schwangerschaft als Insel des Normalen in einer chaotischen Familie, als Sehnsucht nach Normalität.

575 Ebd., 17. 
NS: Was ist dann passiert?

IG: Hatte einen Abgang. (...) Gott sei Dank. Wobei sie auch da ambivalent war, ich hab dann natürlich damals mit ihr auch so ein bisschen ein Gespräch geführt, (sozusagen von Frau zu Frau) Und mir hat sie gesagt, irgendwie hätte sie das Kind schon gern. Und da war auch klar, das wäre auch mal was Gutes gewesen, so was Normales, ohne dieses ganze, chaotische Drumrum (...) Ist natürlich sehr (schwierig) ... ach, diese Entscheidung, ich kenn das ja auch mit 16 will jeder mal schwanger sein. ${ }^{576}$

Zum normalen Erwachsenwerdensprozess der jungen Mädchen gehört also laut Gisler auch die Auseinandersetzung mit der eigenen Fruchtbarkeit, mit der Möglichkeit, schwanger zu werden. Bemerkenswert ist aber, dass all diese Auseinandersetzungen über die Normalität von Fiona überhaupt nichts mit ihrer Integration zu tun hatten.

Eine weitere Dimension der Normalität ist die Sichtweise der Gesellschaft, was als normal gilt. Hier gibt es durchaus Platz für abweichendes Verhalten. Jugendliche dürfen sich abweichend verhalten, der sie begleitende Ethnologe unterliegt aber normalisierten Urteilskriterien und darf das nicht.

Dass der Ethnologe als Vertreter der Normalität agiert, wissen die Jugendlichen ganz genau. Ich hatte etwa die Interviewfrage, ob die Eltern der Jugendlichen geschieden sind oder nicht und stellte sie in einem Gruppengespräch mit Daniel, Klaus, Jürg, Akdis und Devi. Sie spürten sofort, dass die Frage etwas plump darauf zielte, ob die Jugendlichen ein nach herkömmlichen gesellschaftlichen Normen normales Elternhaus hatten oder nicht.

576 Ebd., 18. 
N. Und die Eltern ... ja jetzt kommt die Frage ... ob die geschieden sind oder nicht ... eben, keiner muss beantworten. Daniel: Es sind beide da (alle lachen ...)

Klaus: Alles normal bei mir (wieder lachen alle) ...

$\mathrm{N}$ : (zu Jürg): und bei dir ....

J: (brummelt): ... normal ...

Akdis: Nein! Vater und Mutter noch da.

Devi: (in der allgemeinen Belustigung nicht verständlich). 577

Die Jugendlichen haben durch ihr Amüsement die Frage als ausgehend von veralteten gesellschaftlichen Normen entlarvt, weil sie völlig normale Elternhäuser haben, ausser Jürg, der bloss mit seinem Vater zusammen lebte. Der sagte seine Antwort eher leise, da die Frage nach der Mutter auftauchen könnte. Alle durchschauen aber den Versuch des Forschers und amüsieren sich darüber, irgendwelche Abweichungen festzustellen, die prickelnd sein könnten.

Die Ansichten in der Gesellschaft, was als Normal erachtet wird, entwickeln sich in einer Zeit der schnellen gesellschaftlichen Veränderungen rasant, was zu einem Auseinanderklaffen von gesellschaftlichen Erwartungen und Möglichkeiten zur Erreichung von Normalität führt. Die gesellschaftliche Sicht auf die Normalität ist auch ein wichtiges Herrschaftsinstrument. Deshalb ist die Auseinandersetzung um die Wege der Erwachsenwerdung auch politisch wichtig.

Stefan Blülle, Leiter der Abteilung Jugendschutz des Justizdepartementes schlägt vor, dass die Gesellschaft die Probleme der Jugendlichen beim Übergang in die Erwachsenenwelt als normal ansehen solle.

B: Wenn sie jetzt mit dem Herrn (...) reden, von der Sozialhilfe, dann wird er ihnen sagen, es ist hochdramatisch, (mit

577 Gruppengespräch 1, 3. 
dem Austritt aus der obligatorischen Schule) haben 50\% der Lehrlinge noch keine Lehrstelle.

$\mathrm{N}$ : Mehr.

B: Also: Wenn man natürlich das Modell hat, dass die Wirtschaft sechzehnjährige Lehrlinge braucht, in einer hochtechnisierten Arbeitswelt, dann ist man unter Umständen mit dem Modell falsch, oder? (...) Orientieren wir uns an veralteten Modellen .... ?

$\mathrm{N}$ : ... ganz genau, so würde ich das auch einschätzen ...

B: ... also das wäre dort, was, das man untersuchen müsste. Aber jetzt haben in der Zwischenzeit haben sich neue Formen von Ausbildung entwickelt, die diese Zeit überbrücken, und das müsste man jetzt eben beforschen, und zwar nach dem Konzept Normal. Das müssen wir machen: wir müssen den Weg hier (...) normalisieren, dass wir uns entscheiden, welche Teilgruppe muss man noch am Schluss oder wenn man jetzt von hier her zurückschaut, problematisieren.

$\mathrm{N}$ : Mmmh. Genau.

B: Und das sind die mit em ... em ... vermutlich, das ist meine Hypothese, dass es die sind, mit mangelnden Schlüsselkompetenzen. ${ }^{578}$

Personale Schlüsselkompetenzen sind gemäss Blülle: Konstruktiver Umgang mit Agression, Bewältigung von Depression, konstruktiver Umgang mit Frustrationen, Umgang mit Zeit, Umgang mit Geld, Körperpflege, Schreiben, Rechnen, Umgang mit Freizeit, Ernährung, Haushaltsführung und aktives Verstehen von Handlungsabläufen. Die aus der Sicht der Erwachsenengesellschaft mangelnde Normalität oder

578 Blülle, Stefan, 9. 
mangelndes erwachsenes Verhalten auf diesen Feldern führt also, vermutet Blülle, zu Schwierigkeiten der beruflichen Integration.

Hier besteht eine Catch-22-Situation ${ }^{579}$ : mangelndes Erwachsensein führt zu Ausschluss, doch sind die Jugendlichen eben nicht erwachsen, sondern jugendlich, können also diese Forderungen gar noch nicht erfüllen. Hier wird also normativ auf die Jugendlichen eingewirkt und gleichzeitig wird ihre Integration verhindert. Um diesem Problem abzuhelfen, fordert Blülle zurecht die Entstigmatisierung und Normalisierung der ausgedehnten Brückenzeit zwischen Schule und Lehre.

\subsection{Die Wohnung: Spielplatz der Normalität}

Die "Wohnung" ist bereits eingeführt worden. Dieser Ort ist meistens Schauplatz der Gespräche und Begebenheiten, die im Folgenden reproduziert und analysiert werden. In der Zurückgezogenheit der Wohnung konnten sich die Jugendlichen im Winter 2004/2005 treffen. Hier kamen sie täglich nach der Schule und der Arbeit zusammen. In den Schulferien konnte hier tagelang abgehangen werden. Janine, Loredana

579 Catch-22 ist der Titel eines Romans von Joseph Heller aus dem Jahre 1961. In Catch-22 versucht US-Flieger-Captain John Yossarian im Italienfeldzug, sein eigenes Leben zu schützen, indem er sich geisteskrank schreiben lässt, um nach Hause geschickt zu werden. Die Krankschreibung macht aber die Regel Catch-22 mit paradoxen Begründungen unmöglich: Wer im Wahnsinn des Krieges verlangt, wegen Geisteskrankheit nach Hause geschickt zu werden, hat einen perfekt funktionierenden Verstand, kann also nicht geisteskrank sein. Deshalb wird er nicht nach Hause geschickt. Solche Paradoxa sind Teil des Machtgefälles zwischen Individuum und Staat und halten durch ihre logische Unangreifbarkeit Menschen an dem vom System gewünschten Platz, weil ihre Zirkelschlüssigkeit nicht zu durchbrechen ist. (Heller, Josef: Catch-22, 7. Auflage, London, Random House, 1994). 
und Martina waren sozusagen die Stammbesatzung, während Loredana deutlich als Chefin über das ganze Geschehen amtete.

Loredanas Höhle war nach den Prinzipien von Gemütlichkeit und Geborgenheit eingerichtet. In der Wohnung gab es links neben dem Eingang ein Kajütenbett, darunter und längs der Wand links lagen Matrazen, die mit Decken und Kissen zum bequemen Liegen einluden. Räucherstäbchen, Fotos, CD’s und Selbstgebasteltes sorgten für Atmosphäre und Dekoration. Über den Matrazen hingen Plakate des Hanf-Helden Bob Marley und von gerade aktuellen Filmen wie „Finding Nemo“. Gegenüber dem Eingang war eine Wand mit Fenster, ein CDStänder und Reste aus der Einrichtung des Geschäfts der Mutter wie etwa einen höhenverstellbaren Stuhl. Diese Gegenstände verschwanden aber im Laufe der nächsten Monate. In der Mitte des Raums stand ein niederer Tisch, auf dem sich ein grosser, bunt bemalter Papp-Alien ausbreitete, eine Bastelarbeit aus der Schule.

Rechts vom Eingang stand das Schlagzeug von Loredana, an der Wand rechts war ein kleiner Tisch mit den persönlichen Sachen von Loredana, unter anderem Fotos ihrer Familie. Erstaunlicherweise wurde zu Beginn praktisch kaum Alkohol getrunken, erst als die Jungen 16 wurden, fingen sie an, Alkohol hierher zu bringen.

Hier trafen sich die jungen Leute aus der Umgebung. Häufig klingelte es und jemand kam herein und mache es sich bequem. Insgesamt kristallisierte sich ein harter Kern von rund einem halben Dutzend jungen Menschen heraus, die sich immer wieder hier trafen. Sie sassen da, hörten Musik und plauderten, durchaus auch in kleineren Gruppen. Manchmal drehte sich die Diskussion um Themen, die mit den Submoratorien des Erwachsenwerdens zu tun hatten.

Diese Gespräche waren die wenigen Gelegenheiten, den Anspruch dieser Arbeit umzusetzen, in einer emischen Sichtweise zu beobachten, wie Normvorstellungen ausgehandelt wurden. Allerdings hatte ich nicht den Eindruck, dass diese Auseinandersetzungen die Integration von 
Gruppenmitgliedern gefährdeten. Sie fanden gemäss meinen Beobachtungen erst statt, wenn jemand in der Gruppe bereits integriert war. Solche Normen bespricht man anscheinend mit Vertrauensleuten. Ausserdem wurde ich in die Diskussionen mit einbezogen, wirkte also auch gestaltend auf die Normendiskussionen der Jugendlichen ein. Meine Rolle war dabei manchmal die des Vertreters der Erwachsenenwelt, der die Normen erläutern sollte. Diese Stellung fand ich aus methodischen und sozialen Gründen schlecht. Methodisch, weil ich zu stark in die Feldsituation eingriff und sozial, weil ich mich nicht in die Rolle eines Statuspassagen-Experten drängen lassen wollte, der irgendwie über den Jugendlichen stand.

Zum Verständnis dieser Gruppengeschehnisse tragen auch die Überlegungen von Hans Oswald bei. Oswald hat sich mit dem Phänomen der Peer-Group auseinandergesetzt. ${ }^{580}$ Diese Beziehungen zu den Gleichaltrigen sind sehr wichtig für die Entwicklung von soziokognitiven Fähigkeiten, stellt er fest. Das englische Wort Peer-Group heisst soviel wie eine Gruppe von Menschen, die den gleichen Status haben. Ursprünglich bezeichnete man alle Ränge des englischen Adels, die gleichberechtigt waren, als Peers. Im Rückgriff auf Gedanken aus Jean Piagets Buch "Das moralische Urteil beim Kinde" aus dem Jahre 1932 glaubt Oswald, dass ein positiver sozialisatorischer Einfluss der sogenannten Peer-Gruppe auf das Individuum ausgeübt wird. Besonders der beste Freund oder die beste Freundin sind für diesen Einfluss sehr wichtig. ${ }^{581}$ Oswald weist darauf hin, dass $77 \%$ der Jugendlichen angeben, sich regelmässig oder öfter mit einer Clique zu treffen und dass der Höhepunkt der Wichtigkeit der Clique für seine Untersuchten bei 18

580 Oswald, Hans: Die Beziehung zu Gleichaltrigen, in: Jugendwerk der Deutschen Shell (Hg.); Jugend '92, Lebenslagen, Orientierungen und Entwicklungsperspektiven im vereinigten Deutschland, Band 2, Opladen, Leske + Budrich, 1992, 319-332.

581 Ebd., 319. 
Jahren liegt. ${ }^{582}$ Für den Integrationsverlauf des horizontalen Sozialsubmoratoriums, also die Herausbildung von gleichberechtigten Sozialbeziehungen ist die Gleichaltrigengruppe eine Institution mit sehr hoher Bedeutung. In der Wohnung konnte in Ruhe über die Grenzverläufe des Normalen in den verschiedenen Submoratorien diskutiert werden.

\subsubsection{Geschlecht, Körper, Sexualität}

Sexualität war ein Thema, das immer wieder besprochen wurde. Dies weist darauf hin, dass das Geschlechtssubmoratorium mit fünfzehn für die meisten bereits durchlaufen war, dass sich die Jugendlichen aber noch im Status der Anfänger im Geschlechtsbereich empfanden, was Diskussionsbedarf auslöste. Ich war überrascht über die sexuelle Reife der Jugendlichen und über das hohe Niveau ihrer Auseinandersetzung mit dem Thema. Die Mädchen besprachen diese Fragen eher unter sich, junge Männer waren dabei geduldet. Doch diese wurden manchmal auch unfreiwillig in die Diskussion einbezogen. Etwa als Melanie, Loredanas neue Freundin, im Juni 05 in der Wohnung vorbeikam. Melanie war 19, also rund drei Jahre älter als die anderen und hatte gerade einen neuen Dildo gekauft.

Sie zeigte ihn herum. Den habe sie gerade bei Beate Uhse gekauft. Das ist ein ganz moderner, gutes Design und alles. Man kann die Geschwindigkeit des Vibrators einstellen und er ist aus einem speziellen Material, damit die Gleitcreme gut flutscht, und sei spritzwasserfest, kann also auch unter der Dusche gebraucht werden. Ausserdem sei er deshalb so eigenwillig geformt, damit man auch an den GPunkt gut herankomme, den mann ja beim normalen Ge-

582 Ebd., 322-323. 
schlechtsverkehr normalerweise nicht erreichen könne. Die anwesenden Jungs schwiegen. Das Thema G-Punkt wurde noch weiter besprochen, und wir haben uns noch lang und breit über das Gerät unterhalten, Vor- und Nachteile, Design, Anwendung, usw. Jede/r durfte mal anfassen, vibrieren lassen und die Geschwindigkeit einstellen. ${ }^{583}$

Melanie tritt hier als Wissenschaftlerin auf, die sachlich das Thema erörtert. Der Dildo ist nicht anstössiger als ein Küchen-Mixer. Nichts wird ins Lächerliche gezogen, schlüpfrige Witze blieben aus, die jungen Männer schweigen und hören zu, welche designmässigen Vorschläge zur Lösung eines technischen Problems beim Geschlechtsverkehr umgesetzt wurden. Die Ernsthaftigkeit hat mich verblüfft.

Ein anderes Mal wurde die Ernsthaftigkeit des Themas noch deutlicher. Pascal hatte einen ganzen Bund Jugendhefte des Magazins "4Teens", die Loredana gehörten, neben sich liegen und ging die Ratgeberseiten durch. Pascal und Ich amüsierten uns prächtig:

Pascal liest aus 4-Teens vor: "Hör mal, was diese arme Sau hier schreibt: Beim Onanieren denke ich immer an Mario. Mario ist so schön! Er ist viel lieber und interessanter als alle Mädchen! (...) Der Name ist geändert. Und jetzt der Hammer: Die Red schreibt: Um Deine Anonymität wirklich zu wahren, haben wir den Namen nochmals geändert." Wir Jungs lachen, nicht aber die Mädchen.

Der nächste Brief an die Ratgeberseite: "Meine Brüste sind ungleich gross, was soll ich tun?" Pascal: "O Gott!!" Die Mädchen lachen nicht. Dann lässt Pascal das Vorlesen

583 Ebd., 8. 
sein. Alle fesselnden, also schrägen Artikel sind bereits durch. $^{584}$

Dass die anwesenden Mädchen Janine und Loredana nicht lachen und eher pikiert sind, liegt vielleicht daran, dass die beiden Probleme für sie nicht Stoff für Witze sind. Pascal spürte das und liess das Vorlesen sein. Loredana ist lesbisch und hat vielleicht die Schwierigkeit des verzweifelten jungen Mannes mit seiner homosexuellen Identität nachempfinden können. Auch das Problem der ungleich schnell wachsenden Brüste ist gerade für Mädchen relevant.

\subsubsection{Treue und Beziehungen}

$\mathrm{Zu}$ einer regelrechten Diskussion über Normalität kam es, als die Themen Treue und Promiskuität besprochen wurden. Für Martina gilt Untreue als Beschiss am Partner. Untreue ist nicht per se schlecht, sondern im Prinzip ein Vertrauensbruch neben anderen möglichen, der die Beziehung gefährden könnte und deshalb für sie nicht in Frage käme. Jedenfalls wird das Thema weiter besprochen. Rafael bringt ein für ihn besonders krasses Beispiel:

Fabia, eigentlich die Freundin von Peter, hat anscheinend mit einem Typen Fellatio gemacht. Rafael sagt "gelullt", mit der entsprechenden Gestik, um die Sache für alle klar zu machen. Danach sei sie wieder mit Peter schmusen gegangen. Als der später vom Betreffenden von der Fellatio erfuhr, ist er extrem ärgerlich geworden. ${ }^{585}$

584 Ebd., 20.

585 Ebd., 40. 
Dass Rafael dieses Verhalten krass findet, zeigt, dass es sich an den Grenzen der Normalität abspielt und deshalb für ihn faszinierend ist. Diesen Bericht ergänzt er mit der Behauptung, dass alle Mädchen dieser Familie so seien, was der Normalitätsfrage eine überindividuelle Wendung gibt:
Ausserdem sei Fabia eine Schlampe, erzählt Rafael. Die macht es mit jedem und allem. Mit dem da, jenem, mit Pe- ter und anderen. Und ihre Schwester auch und die dritte Schwester auch noch gerade. ${ }^{586}$

Dieser Klatsch dient dazu, den Ruf dieser Mädchen in der Gegend zu beeinflussen. Einerseits schwingt in Rafaels Erzählung Bewunderung über die professionelle Qualität von Fabias Schlampenhaftigkeit mit, andererseits wird ihr Verhalten vor allem von den beteiligten Männern als nicht akzeptabel verurteilt. Martina dagegen hebt sich von solchen Praktiken ab, indem sie betont, dass solches Verhalten für sie Verrat wäre, den sie vor sich selber nicht billigen könnte. Im Gegensatz zur Feststellung von McRobbie und Garber, dass eine jungen Frau mit schlechtem Ruf sozial erledigt sei ${ }^{587}$, scheint Rafaels Schauerbericht keine weiteren sozialen Konsequenzen zu haben. Das sexuelle Verhalten scheint als Privatsache angesehen zu werden. Man könnte sogar von einer Art Bewunderung bei Pascal sprechen. Diese Überlegungen deuten darauf hin, dass Fabias angebliches Verhalten durchaus in der Reichweite des Normalen anzusiedeln ist, ja sogar auf eine hohe Kompetenz hinweist, die bewundert wird. Nicht Fabia ist in der Erzählung von Rafael die Gelackmeierte, sondern die beteiligten Männer sind es, die entsprechend wütend werden.

586 Ebd., 39.

587 McRobbie und Garber, Girls and Subcultures, (1975) 2002, 213. 
$\mathrm{Zu}$ einer anderen Gelegenheit werden auch die Erfahrungen der Älteren in die sexuelle und allgemeine Recherche über das Leben aufgenommen.

Während wir über alles mögliche reden, trifft Janine ein. Sie setzt sich und nestet sich in den Kissen ein. Dann fragt sie mich: Wie ist das so mit der Liebe in deinem Alter? Ich denke: Bitte? Liebe in deinem Alter? Janine: Und wie ist das mit dem Halt? Kriegt man später im Leben doch auch nochmals Halt? Nach längerem Nachdenken antworte ich: Die Erotik bleibt sich eigentlich gleich. Halt hatte ich noch nie welchen, obgleich jetzt, wo ich Kinder habe, hat sich das insofern geändert, dass ich so tun muss, als ob ich Halt habe. Janine ist interessiert und fragt mehrmals nach Details. ${ }^{588}$

In dieser Passage kommt die Sehnsucht nach dem Ende der Submoratorien zum Ausdruck. Was ist eigentlich das Ziel des Erwachsenwerdens? Gibt es irgendwann einen Zeitpunkt, wo das Geschlechtssubmoratorium und das ökonomische Submoratorium überwunden sind? Leider muss ich Janine enttäuschen: Grundsätzlich sind die Submoratorien nie überwunden. Stabile Situationen sind immer nur provisorisch. Für eine geordnete Welt, in der beispielsweise Kinder behütet heranwachsen können, reicht es, stabile Situationen vorzuspiegeln. Aber jederzeit kann die Arbeitsstelle wegfallen, gerade wenn jemand, wie häufig in jungen Jahren, in Projekten und Praktika eingebunden ist. Immer kann ein Beziehungspartner aufgeben oder aufgrund verschiedener Ursachen wegfallen. Es ist klug, sich dessen bewusst zu sein.

$588 \mathrm{FB} 05,43$. 


\subsubsection{Gender: Sexualität und Männlichkeit}

Ganz nebenbei erfahre ich, dass Loredana lesbisch ist. Weil sie gerade Liebeskummer mit einer Frau gehabt hat. Sie wisse nicht, ob sie Melanie kriege. Eines Abends scheint sie Erfolg gehabt zu haben, denn Loredana taucht trotz mehrmaligen Anschreibens per SMS nicht auf. Erst um 21 Uhr kommt sie vom Stelldichein in die Wohnung und ist exaltiert und glücklich. Wir freuen uns mit ihr. ${ }^{589}$

Die sexuelle Ausrichtung ist anscheinend ein völlig uninteressantes Thema. Nur nebenbei wird sie überhaupt erwähnt, und es spielt keine Rolle, dies in einer Umgebung von jungen Männern und gleichaltrigen Mädchen zu tun. Dies zeigt, dass die sexuelle Ausrichtung vollständig als Privatsache angesehen wird, über die man keine Meinung zu haben hat. Freude macht sich breit, als bekannt wird, dass eine neue Beziehung entstanden ist, welche Loredana glücklich macht. Diese Episode zeigt, dass das sexuelle Submoratorium zu diesem Zeitpunkt im Leben bereits durchlaufen ist und als selbstverständlicher Bestandteil der Identität angesehen wird.

Wirklich verblüfft bin ich über die Tatsache, dass ich anscheinend der einzige erwachsene Mann bin, den Loredana kennt. Einmal streicht sie mir über die Backe, um meinen Bart zu spüren. Ich frage sie, weshalb sie das macht.

Loredana: Du bist der einzige Mann, den ich kenne ...

Ich: Habe ich mir schon gedacht ...

Fabrizio: Ja, und ich?

Loredana: Du bist ein Typ, kein Mann ...

589 Ebd. 
Fabrizio macht deutlich: Ja, ein Milchbubi und so. ${ }^{590}$

Hier kommt der Erfahrungsunterschied zwischen jungendlichen und erwachsenen Männern zum Ausdruck. Jugendliche trifft Loredana in ihrem Umfeld an und weiss deshalb, was sie sind. Erwachsene Männer dagegen scheinen in ihrem Sozialbereich kaum aufzutauchen, und wenn, dann als hierarchisch höherstehende Figuren, denen man sicher nicht über die Backe streichen kann oder Fragen stellen über das Wesen der erwachsenen Männlichkeit.

Auch werden in den Gesprächen in der Wohnung allgemein den Frustrationen des Erwachsenwerdens Luft verschafft, was Solidarität erzeugt. Pascal liest einen Klagebrief aus dem Jugendmagazin “4Teens" vor:

"Meine Eltern wollen immer, dass ich arbeite und meine anderen Geschwister müssen das nicht.” Die arme Sau, meint Pascal, und überhaupt: Eltern!! Kann man denn nicht einfach rein kommen und in sein Zimmer gehen? Nein - sie müssen ständig kommen und fragen: Wie geht es dir? Gut? Hast Du wieder gekifft? Hast du Probleme? Möchtest Du reden? Ohgottohgottohgott!!”, regt er sich auf. . $^{591}$

Ich musste lachen: genau dieses Gefühl hatte ich auch als Jugendlicher. Dass die Eltern viel zu besorgt um einen sind und einen nicht in Ruhe lassen. Die Jugendlichen teilen das Gefühl, dass die enge Überwachung und die ständigen Sorgen der Eltern einem auf die Nerven gehen, und fühlen sich deshalb stärker in der Gruppe integriert.

590 FB05, 54.

591 Ebd., 35. 
Auch wird im Jugendalter mit den Grenzen des Geschmacks und der Tabus experimentiert, was den Betreffenden der Lächerlichkeit preisgeben kann. Deshalb passiert das in der Abgeschiedenheit der Wohnung. Rafael erzählt eine Gewaltgeschichte, die ihn fasziniert hat, und experimentiert mit dem Hakenkreuz-Tabu:

Ein Muskelprotz erschlägt einen Schwarzen. Dann wird er von der Polizei gejagt, und als sie ihn endlich eingekreist haben und verhaften, steht er mit nacktem Oberkörper da und auf seiner Brust ist ein Hakenkreuz. Rafael: So geil, Mann!

(...)

Danach versucht Rafael sich ein Hakenkreuz auf den Unterarm zu malen. Irgendwie klappt es nicht richtig; er muss aufhören und auf einem Blatt ausprobieren, wie man aus zwei Haken ein Hakenkreuz macht. Ein klassisches Jungnazi-Problem, denke ich amüsiert.

(...)

Loredana und ich sagen: Hör doch auf mit dem Scheiss! Er: Ist doch keine Sache.

Nachdem er ein Hakenkreuz in der Grösse eines Bierdeckels auf den Unterarm gemalt hat, probiert er einen grossen Kreis darum herum zu malen und das Kreuz zu schattieren, damit es dreidimensional hervortritt. Mit schmerzverzerrtem Gesicht erkennt er nach einer Weile, dass der Stift doch ungewöhnlich schwer läuft und der Unterarm wund wird. Wir machen uns über ihn lustig. (...)

Kurze Zeit später will er Döner holen. Doch was soll er mit dem Hakenkreuz machen? Wenn die Dönertypen es se- 
hen, gibt es Ärger. Er ängstigt sich und zieht den Ärmel so weit wie möglich herunter. Wir lachen. ${ }^{592}$

Das Hakenkreuz bekommt hier die Rolle des schlimmstmöglichen Schlechtigkeitssymbols und ist deshalb faszinierend. Schlimmer als ein Totschläger mit Hakenkreuz auf der Brust kann man aus der Sicht der Gesellschaft nicht werden. Doch dies ist nicht normal. Das weiss Rafael und versucht die Wirkung des Symbols zu kaschieren, als er bei Ausländern Essen holen will. Nicht normal wäre es, auf die schmackhaften Errungenschaften der fremden Esskulturen zu verzichten.

\subsubsection{Konsumkultur: Hanf und Alkohol}

Wie Philip Lalander und Thomas Johansson ${ }^{593}$ schreiben, hat Drogengebrauch wie Sex die Eigenschaft, von starken Tabus belegt zu sein, weil beide Bereiche den Fortbestand der Gesellschaft in ihren Grundlagen betreffen. Wären die Menschen ständig unter Drogen- oder Alkoholeinfluss, würde die Gesellschaft nicht funktionieren. Würden sie ihr sexuelles Begehren nicht kontrollieren können, würden die Sozialbeziehungen zusammenbrechen. Die Gesellschaft versucht deshalb über die Schule und andere Institutionen, Handlungen mit Drogen, Alkohol und Sex zu disziplinieren, besonders bei Jugendlichen.

Beide Bereiche haben zudem eine symbolische Bedeutung, die zusätzlich durch Mystik aufgeladen ist. Nacktheit ist nur deshalb anziehend, weil im Alltag kaum nackte Haut zu sehen ist, und Drogen werden durch die Prohibition zusätzlich sozusagen mit Widerstandsenergie aufgeladen. Drogen stellen deshalb eine Verbindung zu einer anderen

592 Ebd., 34ff.

593 Lalander, Philip und Thomas Johansson: Ungdomsgrupper i teori och praktik, 2. Auflage, Lund, Studentlitteratur, 2002. 
Welt her, die ausserhalb der Ordnung der modernen Gesellschaft liegt. ${ }^{594}$

Im Gegensatz zum altbekannten Alkohol, der aus historischen Gründen mit (christlicher) Gemeinschaft, Tradition und Kultur verbunden ist und dessen Konsum unter gewissen Bedingungen als relativ unproblematisch angesehen wird, verankerten sich Drogen erst in den 1950er und 1960er Jahren im breiteren Bewusstsein der Gesellschaft. Sogenannte Drögeler wurden stigmatisiert und als Menschen zweiter Klasse angesehen, eine Art Unkraut im modernen Staat, das als gefährlich, verfallen, problematisch und gescheitert angesehen wurde. Drogen wurden zum Staatsfeind, gegenüber dem der Staat versuchte, Handlungsfähigkeit zu demonstrieren. ${ }^{595}$

Es zeigte sich, dass diese Politik zum Scheitern verurteilt war. Trotz grossem Ressourceneinsatz von Seiten des Staates gelang es nicht, das Angebot an Drogen wirksam zu verkleinern, im Gegenteil, die Preise etwa für Opiate sanken im Laufe der Zeit immer mehr, neue Drogen wie Extasy und Kokain kamen in grossen Mengen auf den Markt und erfreuen sich grosser Beliebtheit. Nach den schlimmen Erfahrungen mit offenen verelendeten Drogenszenen am Platzspitz in Zürich und in anderen Städten gab es eine Veränderung in der Drogenpolitik. Basel etwa ging in den letzten fünf Jahren des 20. Jahrhunderts dazu über, die vergleichsweise harmlosen Cannabisprodukte nicht allzu intensiv zu verfolgen und die Ressourcen auf die Bekämpfung der sogenannt harten Drogen zu konzentrieren.

Dies führte zu einem Boom von Hanfläden, die sich aus den sogenannten Headshops ${ }^{596}$ entwickelt hatten und mit allen möglichen Hanf-

594 Lalander und Johansson: Ungdomsgrupper i teori och praktik, 2002, 159. 595 Ebd., 160.

596 Läden, in denen man inmitten von Kunsthandwerk aus Indien, dem Nahen Osten und China Utensilien zum Hanfkonsum kaufen konnte, wie etwa Wasserpfeifen. 
produkten handelten, unter anderem auch Seifen, Badezusätze und Kleider. Begünstigt wurde diese Entwicklung durch eine Besonderheit der schweizerischen Gesetzgebung: Hanfprodukte sind legal, wenn sie nicht als Droge verwendet werden. Als Renner erwiesen sich in der Folge sogenannte Duftsäckchen, die als Mottenschutz in den Schrank gehängt werden sollten oder Stoffkissen mit Hanfblättern, die als Badezusatz ins Badewasser getaucht werden konnten. $\mathrm{Zu}$ beweisen, dass die Konsumentinnen zuhause mit den Hanfblättern nicht etwas anderes als die vorgesehenen Verwendungen anstellten, war für die Behörden zunächst schwierig, bis sich die Toleranzgrenze von $0,3 \% \mathrm{THC}^{597}$ als Beurteilungskriterium zur Unterscheidung von Drogen- und Nichtdrogenhanf durchsetzte und die Polizei mit den entsprechenden Analysemitteln ausgestattet wurde.

Doch waren in der Zwischenzeit in den ersten Jahren des neuen Jahrtausends in vielen Schweizer Kantonen hunderte von neuen Hanfläden aus dem Boden geschossen, die je nach kantonal unterschiedlicher Drogentoleranz, mehr oder weniger offen Hanfprodukte verkauften. Diese Situation und die Ablehnung einer Liberalisierung der Drogenpolitik durch das Eidgenössische Parlament nach einem politischen Rechtsrutsch ${ }^{598}$ sowie der Druck von Frankreich und Deutschland, die ihre restriktive Drogenpolitik durch den massenhaften Schmuggel aus der Schweiz unterwandert sahen, führte dazu, dass in einer konzertierten Aktion im 2003 sämtliche Hanfläden von der Polizei durchsucht,

597 Tetrahydrocannabinol, psychoaktiver Wirkstoff im Hanf.

598 Die Schweiz wurde von 1959 bis 2003 von einer Koalition aus SP (Sozialdemokraten, Mitte Links), FDP (Freisinnig Demokratische Partei, Mitte Rechts), SVP (Schweizerische Volkspartei, national-konservativ) und CVP (Christlichdemokratische Volkspartei, christlichdemokratisch) regiert, wobei die SVP einen, die anderen Parteien je zwei Bundesräte stellten. Ab 2003 waren es zwei SVP- und bloss ein CVP-Bundesrat, weil die nationalkonservative SVP in den 1990er Jahren zur stärksten Partei aufgestiegen war. 
verdächtige Vorräte und Gelder beschlagnahmt und die Lieferanten und Ladenbesitzer des Drogenhandels angeklagt wurden. Der Hanfhandel war wieder in den Untergrund gezwungen worden, behielt aber die in der Boomzeit neu erworbenen meist jugendlichen Konsumentinnen und Konsumenten bei.

Leider hatte sich der Hanf in den Jahren der Quasilegalisierung als Droge der Jugendlichen etabliert, was wohl an seinem Potential als symbolische Verweigerung der Fleissigkeitsforderung der protestantischen Arbeitswelt liegt. ${ }^{599}$ Der Paradigmenwechsel ging gar so weit, dass Hanfkonsum praktisch normalisiert wurde. In den Klassen, die ich beobachtete, vermute ich, dass bis zu einem Drittel der Schülerinnen und Schüler regelmässig Hanf konsumierten, zum Teil sogar am Morgen vor Schulbeginn oder über Mittag. Diese Entwicklung führte in den Schulen zu enormen Problemen und verstärkten Präventionsanstrengungen.

Doch sollen hier die kulturell relevanten Seiten des Hanfkonsums beleuchtet werden, die Symbolik der Droge, und nicht die zweifellos facettenreiche gesellschaftliche Diskussion um Drogen. Generell hilft Konsum von illegalen weichen Drogen, Gefühle der Einsamkeit aufzuheben und ein starkes Gefühl der Gemeinschaft zu erzeugen. Das Verbotene und Heimliche schweisst Individuen zusammen, wirkt also integrativ. Dass die Gruppe es geschafft hat, eine illegale Substanz zu beschaffen, macht sie stärker, hebt deren Selbstvertrauen und Gefühl für Exklusivität. Jugendliche machen etwas, wovon die Erwachsenen abraten, demonstrieren also Selbstbestimmung über sich und ihren Körper.

Während der Schulzeit zusammen Cannabisprodukte zu rauchen ist eine sehr starke Demonstration von Gemeinschaft. Die Konsumenten wissen, dass sie entgegen allen Empfehlungen etablierter Autoritäten handeln, was Gefühle der Freiheit aufkommen lässt und die Erfahrung eines "heimlichen Raumes, wie eine Höhle, die den Tentakeln der Ge-

599 Lalander und Johansson, Ungdomsgrupper i teori och praktik, 2002, 160. 
sellschaft und deren Kontrollmechanismen entweicht." ${ }^{600}$ Die Heimlichheit stärkt ein soziales Band zwischen den Eingeweihten und konstituiert einen Rückzugsraum.

Drogengebrauch hat auch Auswirkungen auf die Konstruktion von Identität. Legt man sich gewisse Drogen als Symbole zu, versucht man, sich eine Identität zu schaffen, wie sie etwa ein Fussballfan mit einer Bierflasche zeigt. Ausserdem demonstriert ein Mädchen, das kifft, Erwachsensein in dem Sinne, als dass sie mit Stoffen hantiert, die nichts für Kinder oder Jugendliche sind. Ein junger Lateinamerikaner erzählte, dass er und seine übrigen Gangmitglieder sich als stark und cool ansahen, weil sie verschiedene Drogen getestet hatten. Sie konnten sich so als Aussenseiter definieren und gleichzeitig Unterschiede zu anderen Jugendlichen markieren, die als jung, unerfahren und uncool angesehen wurden. ${ }^{601}$

Erfahrung zu demonstrieren ist wichtig. Drogengebrauch ist ein wichtiges Ritual des Erwachsenwerdens, wo die Jugendlichen zeigen, dass sie die Kindheit verlassen. Man ist nicht länger ein Kind, das elterlicher Obhut bedarf, sondern eine erwachsene Person, die in die gefährliche Welt hinausgeht. Ein symbolisches Kapital wird geschaffen, also eine Ressource, die in gewissen sozialen Zusammenhängen als wertvoll angesehen wird, um eine bestimmte Position in der Gruppe zu erlangen. ${ }^{602}$ Dieses Kapital kann aber nur in der Subkultur verwendet werden, nicht als gesamtgesellschaftliche Ressource. Wer will schon einem zukünftigen Arbeitgeber bereitwillig erzählen, dass er grosse Drogenerfahrung hat?

Eine weitere symbolische Bedeutung von Drogen ist die Markierung von Time-Out-Zonen. Drogen gehören in die liminale Phase im Sinne Victor Turners, eine Phase, in der die Mitglieder einer Gruppe symboli-

600 Ebd., 162.

601 Ebd., 164.

602 Ebd., 165. 
sieren, dass die in einem anderen kulturellen Modus sind wie während der gewöhnlichen Alltagsordnung. In einer liminalen Phase ist das Leben abenteuerlich, Flow ${ }^{603}$ entsteht, Menschen kommen einander näher und etwas tun, das sonst abgelehnt wird. Dieses Aufatmen im Verhältnis zum Stress des Alltagslebens, das "chillen" ${ }^{604}$, das bloss Da-Sein erlaubt es den Jugendlichen, Ansprüche der Erwachsenen und der Schule eine Zeit lang zu vergessen und in ein Jetzt-Erlebnis einzutauchen. Die Tyrannei der Zeit ist im Rausch aufgehoben, die Erziehung zur Pünktlichkeit und zum industriezeitlichen Wesen ist momentan vergessen. Dieses Time-Out kann auch die Bedeutung einer wohlverdienten Belohnung oder eines Höhepunktes im Dasein annehmen, welche die Alltagsroutine unterbricht.

Drogenkonsum ist auch eine Möglichkeit, etwas Abenteuerliches ins Leben zu bringen, die Sehnsucht nach starken Erlebnissen zu befriedigen. Im Rausch wird die nahe Zukunft unsicher, im Vergleich mit der Ordnung und Schematik der Schule wird das Dasein intensiv. Man kann neue Freunde treffen, sexuelle Beziehungen einleiten, etwas aufregendes und cooles erleben, die hektische Leere überwinden aber auch bloss sein. ${ }^{605}$ Auf die Nähe zum Konzept der Action ist hier aufmerksam zu machen.

Die Droge Hanf war wesentlich früher in das Leben der Jugendlichen gekommen als Alkohol. Deshalb war die Auseinandersetzung um den normalen Umgang auch weiter fortgeschritten. Manchmal konnte diese Auseinandersetzung die Form einer Komödie annehmen:

Peter und Pascal haben aus Alufolie einen riesigen Konus gebastelt. Mit diesem absurden Riesenjoint veranstalten sie

603 Vgl. Csikszentmihalyi, Mihaly: Flow: Das Geheimnis des Glücks, 1998. 604 Janine und ihre Freundinnen verwenden das Wort als Bezeichung für Entspannen, sich zurücklehnen, Musik geniessen. 605 Lalander und Johansson, Ungdomsgrupper i teori och praktik, 2002, 169. 
eine Parodie auf zwei total verkiffte Typen. Sie saugen übertrieben daran, blasen imaginären Rauch aus, hängen schlaff da, stöhnen und rollen die Augen. Dabei waren sie total sauber. Alle lachen: Es war wirklich lustig. ${ }^{606}$

In diesem kleinen Sketch demonstrieren Peter und Pascal nicht normalen Umgang mit Cannabis. Riesige Mengen werden eingenommen, körperliche Apathie stellt sich ein, Augenrollen weist auf Wahnsinn hin, Gestöhne auf die fehlende Kontrolle körperlicher Funktionen. Das gespielte Verhalten ist ausserhalb der Normalität und wirkt deshalb ulkig. Es reflektiert auch die Ängste, welche die Gesellschaft mit dem Konsum von Cannabis verbindet und ist deshalb auch eine Kritik.

Doch sorgte das Thema Hanf auch für ernsthafte Streitereien. Ein Grund dafür ist dessen Illegalität, was ihn ausserhalb der Normalität setzt. Gleichzeitig ist er für die Schülerinnen und Schüler der WBS völlig alltäglich und deshalb normal. Innerhalb dieses Widerspruchs galt es, eine eigene Position zu finden.

Loredana hat ein schlechtes Gewissen, dass sie Hanf raucht. Sie macht sich auch über das Suchtpotential Sorgen. Deshalb hat sie mit Janine einen - wie sie es nennt - Nichtkiffversuch angefangen, der beweisen soll, dass sie nicht süchtig sind und deshalb jederzeit in die Grenzen der Normalität und Legalität zurückkehren können. Der Versuch läuft zum Zeitpunkt folgender Beobachtung anscheinend schon zwei Tage.

Janine scheint nicht so begeistert zu sein vom Nichtkiffversuch, obwohl Loredana ihr ständig in den Ohren liegt damit, dass sie ein Suchtmolch sei, der nicht von der Droge wegkommen könne. Janine: Das ist kein Problem für mich.

606 FB05, 38. 
Die beiden dürfen pro Tag nur drei Züge eines Joints reinziehen. Natürlich stellt sich heraus, dass Janine vor ihres Auftauchens in der Wohnung am Rhein unten gewesen ist und dass sie dort vom coolen Toni einen Zug angeboten bekommen hätte und natürlich nicht ablehnen konnte. Loredana ist sauer. "Wenn ich nicht gefragt hätte, hättest du es mir nicht erzählt, oder?"

Janine schweigt und windet sich nachher irgendwie raus. Es ist schwer festzustellen, ob sie wirklich ein schlechtes Gewissen hat. Ich glaube nicht.

Die neu hinzugekommene Martina macht sich lustig über die beiden, die sich in den Haaren liegen. Trotz ihrem Versuch macht Loredana dann später beim Kiffen mit und zieht nochmals an einem Joint, der ihr hingehalten wird.

(...) Zunächst nicht, dann aber doch. Loredana nimmt einen Zug und ist somit als Heuchlerin entlarvt. ${ }^{607}$

Hier handelt es sich wohl um einen Machtkampf: Janine und Loredana tragen ihre Differenzen über den richtigen Umgang mit Drogen aus. Janine macht auf schlechtes Gewissen, das sie eigentlich nicht hat und Loredana ist sauer, weil sie ihren Vorsatz hat brechen müssen. Beide wahren so ihr Gesicht gegenüber dem von der Gesellschaft angepriesenen Kodex, was als richtigen Umgang mit Hanf gilt. Es gilt das zwar das Verbot, das sich in Janines schlechtem Gewissen und Loredanas Druckversuchen äussert, doch das fehlende körperliche Suchtpotential des Hanfs macht es zu einem relativ harmlosen Genussmittel, was beide auch wissen, weil sie es kennen. Dies äussert sich in den erlaubten zwei Zügen. Deshalb ist der Nichtkiffversuch von vorne herein zum Scheitern verurteilt, weil er gar keiner ist - es wird trotzdem gekifft - und weil er von der falschen Voraussetzung ausgeht, dass es eine Sucht zu 607 Ebd., 34. 
überwinden gäbe. Und Sucht sei schädlich. Die Grenze der Normalität wird genau festgelegt und liegt bei zwei Zügen. Zwei Züge gelten noch nicht als süchtig und daher als normal.

Dieser Zugang deckt sich eigentlich sehr gut mit dem allgemein verbreiteten Umgang mit Cannabisprodukten: illegal, aber relativ harmlos, höchstens gesundheitsschädlich. ${ }^{608}$ Die beiden Mädchen adaptieren also auf der einen Seite sehr weit die doppelbödige Haltung der Erwachsenen gegenüber weichen Drogen und gehen ihren eigenen Weg, legen ihre Norm fest, erhalten sich also im Bereich der des eigenen Körpers Selbstbestimmung.

Nachdem Loredana und Melanie ein Paar geworden sind, und Melanie Loredana das Kiffen austreiben wollte und es daher verbot, bedauerte es Loredana, nicht kiffen zu können.
Als wir in der Küche eine Zigarette rauchen, fragt sie mich, weshalb ich nicht wissen will, weshalb sie nicht mehr kifft. Ich meine, dass es mir nicht wichtig ist, zu wis- sen, warum sie nicht kifft, sondern es ist einfach wichtig, dass sie nicht mehr kifft. Sie insistiert, und als ich nachge- be und frage, weshalb denn, dann meint sie: wegen Mela- nie. Melanie mag das nicht. Ob sie trotzdem rauchen solle? Ich: Nein. Wenn ihr Melanie wichtig ist. ${ }^{609}$

Hier wird der Forscher auf eine Entwicklung aufmerksam gemacht, die ihm nach Meinung der Beforschten nicht entgehen darf. Doch der möchte sich nicht in eine Richtung drängen lassen. Melanie war deshalb so streng mit dem Kiffen, weil ihr Vater ein Alkoholiker ist. Sie übertrug die Gefährlichkeit des Alkohols auf den Hanf und wollte ihn Loredana austreiben, was aber nie ganz gelang. Auch dieser Konflikt

608 FB05, 34.

609 Ebd., 53. 
weist auf Allgemeines hin: Die Gesellschaft wertet im Umgang mit Drogen, also Tabak, Alkohol, Cannabisprodukten und harten Drogen, die Gefährdungspotentiale dieser Substanzen verschieden. Doch diese Kriterien sind nicht objektiv, sondern als historisch gewachsen und kulturell determiniert einfach zu durchschauen. Sie sind einfach zu relativieren. Deshalb die Wirkungslosigkeit von Melanies Bemühungen. Loredana sucht Rat bei mir. Ich kann aber nicht auf etwas Allgemeingültiges hinweisen, sondern nur auf die Beziehungsebene, darauf, dass Melanie wahrscheinlich geht, wenn Loredana ihre Bedingungen nicht honoriert. Diese sind arbiträr: Tabak ja, Cannabis und Alkohol nein. Melanie übt so Macht aus; es ist die Frage, ob Loredana sich unterwirft oder nicht.

Mit der Zeit wurden einige Jugendliche, welche die Wohnung aufsuchten, 16 und fingen an, Alkohol zu trinken. Ich war erstaunt, dass das nicht früher eintrat. Damit tauchte das Thema des kulturell akzeptierten Umgangs mit diesem Rauschmittel auf. Die Gefährlichkeit des Alkohols war für die Jugendlichen nicht auf Anhieb einsichtig, da er ja überall zu kaufen ist. Nachdem Janine im März 2005 sechzehn geworden war, begann sie billige Alkoholika mitzubringen. Bald einmal wurden die konsumierten Mengen so gross, dass Janine die negativen Aspekte des Alkoholrauschs kennenlernte. Damit lernte sie auch den für die hiesige Trinkkultur wichtigen Aspekt der richtigen Dosis und wie man sozial und konsumierend mit Betrunkenheit umging. Dies produzierte weitere Verwerfungen:

Als ich reinkomme, ist Pascal da, und Janine liegt rum. Janine richtet sich auf und beginnt mit mir zu reden. Sie muss getrunken haben und ich verstehe sie kaum. Loredana ist auch da. Plötzlich geht Janine aufs WC und beginnt zu erbrechen. Zunächst machen wir Witze drüber und rufen ab und zu was rein ... Wie gehts und so ... aber das Rö- 
cheln hört nicht auf. Ich bringe mal Wasser, das wird genommen, dann Eistee, den sie ablehnt.

Pascal eilt und besorgt eine Banane, aber offensichlich hält die sich auch nicht lange im Magen. Loredana erklärt: Die hat den ganzen Tag nichts gegessen und dann einen halben (Liter) weissen (Wein) und einen halben (Pfirsichwein) reingeleert. Und verträgt nix. Wir anderen lassen uns von Janines Zustand nicht beirren und plaudern. Die Stimmung ist gut.

Es wird bald zehn Uhr und Loredana meint, dass jetzt Janis Eltern anrufen werden und fragen, wo sie bleibt. Oh, nein! Was sollen wir sagen? Janine ist nicht transportfähig, sagt sie, die würde in ein Auto kotzen.

Loredana: Wir erzählen einfach, was ist - nicht Lügen und dass ihr schlecht ist.

Ich: Wir könnten erzählen, dass sie was schlechtes gegessen hat ... aber bei der Fahne fällt sofort auf, dass das nicht stimmt ...

... und sowieso ist es unglaubwürdig, sagt Loredana, da Janine ziemlich heikel ist und nichts isst, was komisch sein könnte.

Pascal sieht besorgt aus. Wir debattieren, was zu tun ist. Loredanas Mobiltelefon klingelt. Loredana macht eine abschneidende Handbewegung: Absolute Stille! Loredana nimmt an und es sind Janis Eltern. Wir halten den Atem an. Loredana erklärt, dass es Janine schlecht ist und dass sie auf dem WC festsitzt und nicht weg kann. Absolut unmöglich. Diskussion hin und her. Bald meint Loredana entgeistert zu uns, das Mikrofon zuhaltend: Sie kommen sie holen! Oh, Nein! Alles erstarrt im Schreck. Loredana insistiert am Telefon, dass Janine in ein Auto kotzen würde 
in diesem Zustand. Das sei kein Problem, sie kämen trotzdem, wiederholt Loredana laut, um uns ins Bild zu setzen. Oh, Gott! Sie beendet das Gespräch. Was jetzt?

(...) Janine kriecht aus dem Bad und macht auf allen Vieren einen dramatischen Auftritt, bleich, aber eindringlich: Ruf an, Lore, die dürfen nicht hier her kommen! Pascal kaut auf der Unterlippe. Loredana ärgert sich, ruft aber trotzdem an.

Loredana kämpft mit der Technik und endlich Verbindung. Loredana erklärt, dass sie nicht kommen sollen ... aber ergebnislos, sie sind schon unterwegs, echot sie laut. Was sollen wir bloss tun? Abwarten ist nicht gut, und Loredanas Idee, ich soll mich doch in der Küche verstecken, ist auch nicht gerade toll. Also ist bald klar, dass alle hier raus müssen. Je schneller, desto besser. Janine wird unbarmherzig von Loredana angetrieben ... wir schaffen es in den Lift. Janine sieht aus wie eine Leiche. Unten haut sie sofort ab. Loredana warnt: halt, nicht in die Richtung, die sehen dich!

Wir gehen in Richtung Büsche. Ich gehe ganz normal mit dem Velo davon ... die Kids verstecken sich hinter einem Busch: da kommt einer! Ich finde es gescheiter, einfach weiter zu gehen, aber niemand folgt. An der nächsten Ecke schliesse ich das Velo ab und gehe zurück ... was machen die bloss? Plötzlich eilt mir Loredana entgegen: Die Eltern sind da gewesen. Und weil keiner aufgemacht hat, sind sie wieder gegangen. Sie haut ab. Janine, Pascal und ich hintendrein.

Bald kommen wir in die nahe gelegene Neubausiedlung. Keine Gefahr mehr. Janine ist wieder munter geworden an der frischen Luft. Sie kriegt einen Anruf und eilt voraus. 
Ich halte Pascal und Loredana auf und verkünde: Sie telefoniert! Loredana meint wegen meiner Wahnsinnserkenntnis sarkastisch zu mir: Gratuliere! Und dann mit Blick auf Janine besorgt: Wieso geht die so schnell?

An der nächsten Ecke, zur Durchgangsstrasse, bricht Janine fast zusammen. Sie ruft: Die hat mir das Telefon aufgehängt! (...) Wir gehen alle langsam zur Bushaltestelle. Janine sitzt in der Scheisse. Hätte um neun zuhause sein sollen und jetzt ist halb elf. Und besoffen. Tja, immerhin ein Lichtblick: morgen wird sie ein Praktikum als Kinderhortfrau anfangen. ${ }^{610}$

Wichtig ist in dieser Stelle, dass Janine und die Freunde sich in der Situation trotz Berauschtheit geschickt verhalten. Das für den Erwachsenen und der Betrunkenen absolut zu vermeidende Treffen mit den Eltern wird tatsächlich knapp vermieden, mit Hilfe von Leuten, die zielsicher das Kommando übernehmen, einem in der Not beistehen, verhandeln, organisieren, auf der Flucht helfen, kurz: völlig erwachsen reagieren. Ausserdem scheint Janine nicht extrem betrunken gewesen zu sein, sie erholt sich nämlich relativ schnell wieder. So ist sie einen Schritt weiter in der Kontrolle des Alkohols und auf dem Weg zu einem normalen Verhältnis dazu. Das ist keine schmerzfreie Sache: Am Tag darauf Janine ändert ihren Namenskommentar im Chatprogramm Messenger von "Ich bin soooo froh, ein Mädchen zu sein" auf "!Das Leben ist ein Arschfick!". ${ }^{111}$

610 Ebd., 51-52.

611 Ebd., 52. 


\subsubsection{Real Time: Das Leben in Echtzeit}

Wohl hören die Jugendlichen Radio, gehen ins Kino oder schauen fern. Doch wird wesentlich weniger Zeit für diese Zentrum-PeripherieMedien aufgewendet wie in meiner Jugend, was als grosser Fortschritt zu selbstbestimmten, dezentralen und deshalb manipulationsärmeren Kommunikationsformen zu werten ist. Hier haben sie auch eine grosse Kompetenz.

Es wird zu viert "Shooter" auf der "Glotze" gespielt. Eine mir neue Unterhaltungsform. Die Geräuschkulisse macht baller, baller. Ich hoffe bloss, denke ich amüsiert, dass die Kids später nicht glauben, das sterben gehe so von sich, dass ein Blutschleier über den Bildschirm läuft und die Schrift "Game over" erscheint. Jedenfalls sind sie geschickte Spieler und die Stimmung ist sehr entspannt. ${ }^{612}$

Die Subkultur der Warez, das sind Internetnutzer, die illegal beschaffte Software untereinander verbreiten, weist darauf hin: der virtuelle Raum des Internet wird für die Jugendlichen immer wichtiger. Das Internet bietet sich mit seiner Unübersichtlichkeit und der Möglichkeit, Identitäten zu erfinden oder zu verbergen, für die Selbstsuche in der Jugendphase geradezu an.

Verblüfft war ich, nachdem Loredana im Januar 2006 mit Melanie Schluss gemacht hatte: Wir sassen zu zweit in Loredanas Zimmer bei Loredanas Mutter zuhause. Loredana erzählte mir eine komplizierte Geschichte, deren Kernaussage darauf hinauslief, dass sie am Samstagabend Melanie stehen liess und mit Mona losgezogen sei, und sie dann bei ihr übernachtet habe. Sie habe jetzt mit Melanie Schluss gemacht.

612 Ebd., 33. 
Während sie erzählte, startete sie ihr Notebook und loggte sich in einem Chat für "Schwule, Lesben und ihre Freunde" ein und schaute nach neuen Nachrichten, besonders denen ihrer neuen Partnerin. Gleichzeitig checkte sie ihre SMS ab. Woher die beunruhigende Nachricht kam, die sie veranlasste, Melanie anzurufen, weiss ich nicht mehr, auch nicht, ob Melanie wegen einer SMS angerufen hat. Jedenfalls telefonierte Loredana mindestens zwei Stunden lang mit Melanie, entschuldigte sich mehrmals, tröstete und hörte sich die Beschwerden an.

$\mathrm{Zu}$ Beginn dieses Telefonsgesprächs kam Sabine herein, eine mir noch unbekannte Freundin von Loredana, bestaunte mich, den Alten, der hier sass und setzte sich wie selbstverständlich vor Loredanas Computer und chattete während der ganzen Dauer des Telefongesprächs von Loredana mit verschiedenen Leuten. Ab und zu ging ein neues Desktop-Fenster mit einem "Pling"-Geräusch auf, als Leute sich einloggten. Sabine stubste dann jeweils Loredana an und wies auf die Einträge. Loredana tippte dann auch ein paar Wörter oder schrieb mit der freien Hand ein SMS, ja nahm sogar einmal mit dem anderen Ohr ein Gespräch auf dem Mobiltelefon entgegen, ohne das Gespräch mit Melanie zu unterbrechen. Auf diese Weise wurde Loredanas Umgebung in real time über das Ende ihrer Beziehung mit Melanie auf dem Laufenden gehalten.

\subsubsection{Verhältnis zu den Autoritäten}

Auch das Verhältnis zur Schule und damit zu der wichtigsten Autorität im Leben der Jugendlichen ist auch in der Freizeit ein Thema, denn die Schülerinnen und Schüler müssen Hausaufgaben machen, sich für Prüfungen vorbereiten, Projekte realisieren. Pascal hat seine Hausaufgaben wieder einmal nicht gemacht. Er berichtet stolz, dass er $120 \mathrm{Mal}$ schreiben muss: "Ich soll daran denken, dass ich meine Aufgaben 
pünktlich, gewissenhaft und ordentlich ausführen soll (der Satz ist von mir erfunden, jedenfalls ist er ewig lang)". Er berichtet, dass er beim 79 sten Mal angekommen sei. Ich finde das happig. Loredana widerspricht.

Loredana: "Obi, er hat es verdient."

Pascal protestiert.

Loredana: "Weshalb machst du deine Uffzgi ${ }^{613}$ nie?"

Pause.

Pascal, kleinlaut: "Er hätte mir wenigstens 2 Tage Zeit geben können."

Die Seiten sollen morgen ausgestellt werden in der Klasse. ${ }^{614}$

Loredana findet es anscheinend nur blöd, dass Pascal seine Aufgaben nie macht. Für die männlichen Jugendlichen ist es eine Art Ehrensache oder Härtetest, möglichst lange die Aufgaben nicht zu machen und dann stolz lange Strafaufgaben bewältigen zu müssen. Der Lehrer war sich offenbar dessen bewusst und machte Pascal mit seiner kurzen Frist den Stolz sehr schwer. Loredana dagegen lernt unentwegt und lässt sich von fehlenden Grundlagen im Allgemeinwissen nicht entmutigen.

Loredana lernt Erster Weltkrieg. Sarajewo, Schlieffenplan, uneingeschränkter U-Bootkrieg, prägt sie sich murmelnd ein. Zwischendurch helfe ich mit Infos aus. Z.B. "Die Deutschen haben ja das Elsass und Lothringen 1871 erobert, und die Franzosen wollten das gerne wieder haben." Loredana weiss nicht, was das Elsass ist. Janine und ich,

613 Hausaufgaben.

$614 \mathrm{FB} 05,36$. 
entgeistert: "Naja, das Elsass! Mulhouse! St. Louis! Strassbourg! Das da drüben, in Frankreich. Du weisst schon!”'15

Für Martina dagegen ist es glasklar, dass man sich in der Schule anstrengen muss. Sie ist eine der Besten in der Klasse, intelligent und umtriebig. Dass Pascal nur einer und zweier im Zeugnis hat, findet sie entsetzlich. Sie als Gymnasiastin hat nur gute Noten. Sonst würde sie hinausfliegen, und das kommt nicht in Frage, sagt sie. ${ }^{616}$

\subsection{Rumhängen am Rhein}

Ende Mai 2005 wurde die Wohnung aufgelöst. Damit gehörte diese Rückzugsmöglichkeit für die Jugendlichen der Vergangenheit an. Der Raum, den die Jugendlichen für ihre Integration nutzen konnten, verschloss sich. Doch vorläufig machte das nichts: der Sommer stand vor der Tür und die Aktivitäten verlagerten sich an das Rheinbord. Die rechte Rheinseite besteht aus einer breiten Promenade, die immer wieder von tribünenartigen Treppenbuchten hinunter zum Wasser unterbrochen wird. Viele Bäume spenden angenehmen Schatten, der Rhein lädt zum Bade. Mit diesem stimmungsvollen Bericht über eine wichtige Tätigkeit der Jungendlichen, das Rumhängen, einer Diskussion über den Begriff Devianz und einem Ausblick soll der empirische Teil dieser Arbeit abgeschlossen werden.

An einem sehr heissen Augusttag stellte ich fest, dass ich um 15.30h schon mit meinen Pflichten fertig war. Ein Bad wäre jetzt sehr erfrischend, dachte ich. Ich treffe Loredana am vereinbarten Punkt vor der Bank auf dem Marktplatz,

615 Ebd., 36.

616 Ebd., 39. 
wo sie mit zwei Mädchen aus ihrer Klasse abgemacht hat, Caroline und Celine. Die runzeln die Stirn, als ich angeschleppt werde von Loredana, aber ich sage, dass ich die Loredana schon länger kenne. Trotzdem will die Konversation nicht richtig in Gang kommen, doch als wir das Rheinbord gegenüber dem Hotel Dreikönig betreten, ist die erste Verwunderung schon überwunden.

Bald kommt Janine dazu, die begrüsst mich und zieht sich dann um. Loredana und Janine haben Bikinitops und über die Knie reichende Hosen an. Caroline ein Bikinitop und kurze Hosen. Ich ziehe mich auch um. Celine zieht sich nicht um. Vielleicht aus religiösen Gründen?

Danach werde ich bekniet, auch von der Rheinbrücke zu springen. Ich habe Bedenken und lehne ab. (...) Jedenfalls gehen die drei los, steigen auf die Rheinbrücke, gehen vor bis zum Bogen links neben dem Käppelijoch und steigen auf die Brückenbrüstung. Sofort sammeln sich Schaulustige um sie. Dann Gejohle von den Zaungästen: Springt! Springt! Pfiffe und aufmuntendes Geklatsche. Man hört eins, zwei, drei, aber niemand springt. Ein Moment der Unsicherheit: Celine und ich beraten, was los sein könnte. Dann nochmals, herzhafter: Eins, Zwei, Drei! Sie hüpfen. Drei Mädchen mit aufgebauschten Hosen fliegen durch die Luft, mindestens 10 Meter weit. Pluff, ins Wasser. Janine und Caroline tauchen sofort wieder auf, Loredana erst nach einer Weile, in der ich mir schon Sorgen mache. Geklatsche von den Zuschauern. Die drei schwimmen zur Seite und klettern am Ufer angelangt, die Treppen hinauf. Triumphierend lächeln sie, Celine und ich gratulieren. Als Janine und Loredana abziehen, um Hanf einzukaufen, bleibe ich zurück und plaudere etwas mit Caroline. Es gibt 
aber auch lange Schweigeperioden, in denen sie mal ein kurzes Spiel auf dem Handy macht ("Mist, bloss 1240 Punkte, dabei wollte ich 1300 Punkte überschreiten. Der Kollege hatte die ...") Nach dreiviertel Stunden kommen Janine und Loredana zurück, das Geschäft scheint abgewickelt, die Ware wird aufgeteilt unter den Mädchen. Gleichzeitig rollen sie zwei Joints und rauchen sie. Danach wird gebadet. Auch ich steige in den Rhein. Wir lassen uns ungefähr 50 Meter treiben und steigen dann wieder raus. Janine staunt wegen mir. "Meine Alten würden nie im Rhein schwimmen gehen. Wieso machst du das? Du bist doch auch alt, oder? Mein Vater würde sich nie auf den Boden setzen. Schon gar nicht so lange. Macht das nicht weh? Die Alten sagen immer, es täte ihnen weh, so lange auf dem Boden zu sitzen." Ich zucke mit den Schultern. Es ist zwar nicht so bequem wie auf einem Sofa, doch es geht. Ausserdem ist die Lage gut, das Wetter und die Stimmung auch.

Wir sitzen da und plaudern bis ungefähr um 19h. Es ist sehr angenehm, manchmal steige ich und die Mädchen ins Wasser, wir lassen uns treiben. Um sieben kühlt es merklich ab, und ich möchte langsam aus den feuchten Badehosen. Die Mädchen haben Hunger und müssen aufs Klo. Wir beraten, wie das laufen soll, ich kann ja nicht bei Janines Eltern auftauchen, die in der Nähe des Rheins wohnen. Das würde Fragen geben. Sie beschliessen, bloss dort zu kochen und dann wieder an den Rhein zu kommen. Ich soll dort warten.

Ich ziehe mich hinter einem Busch um, und die Mädchen ziehen sich an. Dann steigen wir auf die Fahrräder und fahren in Viererformation los. Bald steigen wir ab, und ich 
bekomme die Instruktion, auf dem Bänkchen am Rhein zu warten. Welches Bänkchen? Da ist nur Böschung. Loredana beschreibt den Zugang. Tatsächlich: Jemand hat ein Metallgitter unterhalb eines Gestrüpps installiert, dass als Fussraste für einen Betonrand dient. Im Kreuz ist ein Balken, so dass Fussraste, Betonrand und Balken eine bequeme Bank für 3-4 Leute bilden. Ein versteckter Ort und nicht einsehbar. Unter den Füssen rauscht der Fluss, und man darf nichts fallen lassen, sonst ist es weg.

Nach einer Stunde oder so tauchen die Mädchen wieder auf. Sie haben panierten Fisch, Pommes Frites, panierte Pouletteile, Sandwiches und vier Liter Eistee dabei. Zudem Mayonnaise und Ketchup. Ein Festessen! Ich haue rein, eingeladen, und das Salami-Ei-Salat-Butter-Brötchen ist ausgezeichnet ("Mit Liebe von Loredana für Dich gemacht!", meint Janine). Nach dem Essen tun sich die Enten und eine benachbarte Gruppe Jugendlicher an den Pommesresten gütlich. Wir plaudern ab und an und schauen dem Mond zu, wie er untergeht. Janine verabschiedet sich um zehn, Loredana will auch gehen. Es ist halb elf geworden. Sie bringen Janines Eltern das Geschirr zurück. Ich verabschiede mich. ${ }^{617}$

Paul Corrigan ${ }^{618}$ hat sich Gedanken darüber gemacht, was Jugendliche mit dem stundenlangen Rumhängen und Nichtstun bezwecken. Dies sei eine Erwachsenensicht: Die Jugendlichen entfliehen der elterlichen Wohnung, wo die Eltern ihrer Meinung nach bloss im Wohnzim-

617 Ebd., 55-57.

618 Corrigan, Paul: Doing Nothing, in: Hall Stuart und Tony Jefferson: Resistance Through Rituals, Youth Subcultures in post-war Britain, London, Routledge, (1975) 2002, 103-105 
mer sässen und langweilig wären. Das Nichtstun sei eine intensive Zeit, die meistens mit Reden und Geschichtenerzählen verbracht werde, manchmal wird eine körperliche Aktivität eingeschoben, bei Corrigans Jugendlichen waren dies Balgereien mit anderen Jugendgruppen aus der Nachbarschaft oder störendes Verhalten wie Flaschenzerschlagen. ${ }^{619}$

Die Aktivitäten des Rumhängen können angeblich leicht deviante Formen annehmen. Dieser Begriff stammt aus dem englischen Sprachraum und bezeichnet zunächst einmal abweichendes Verhalten. Verwendet wird er für unerwünschte Verhaltensweisen Jugendlicher. Tony Blair, zum Zeitpunkt der Niederschrift dieser Zeilen Premierminister von Grossbritannien, legt den Ursprung von deviantem Verhalten in die 1960er Jahre, als Jugendliche angeblich ohne elterliche Disziplin und ordentlichen Rollenvorbildern aufwuchsen, was dazu führe, dass sie keine Verantwortung für sich oder gegenüber andern empfänden.

Doch dies ist eine klassische "o tempora, o mores!"-Erklärung, die wie weiter oben in dieser Studie diskutiert, nicht hält. Früher waren die Jugendlichen auch deviant, geht man von literarischen Zeugnissen aus. In Kiplings Stalky \& Co. aus dem Jahre 1899 beispielsweise mogelt sich eine Jugendbande durch die Schule. Die Bande baute etwa Hütten in einem gerodeten Gelände, dessen Zutritt streng verboten ist, und rauchten dort heimlich Pfeifen. Dieses Verhalten sei eine gute Vorbereitung für das Erwachsenenleben: Nach der Schule geht Stalky nach Indien, wo er mit seiner Rücksichtslosigkeit und seinen devianten Erfahrungen des Jungenlebens genau die richtigen Qualifikationen mitbringt, in der Britischen Kolonialverwaltung zu reüssieren. ${ }^{620}$

Die deviante Zeit in der Jugend kann also als liminale Zeit der teilweise freiwillig gewählten und gemeinsam durchzustehenden Prüfungen aufgefasst werden, wo für die Menschen in der liminalen Situation charakteristischen tiefen Freundschaftsbande geknüpft werden können.

619 Ebd., 103.

620 The Economist, 5.2.2005, 32. 
Diese Freundschaften und der kulturell richtig erlernte Umgang mit dem Regelwerk der Gesellschaft wird also zur wichtigen Voraussetzung, um als Erwachsener sozial aufzusteigen.

Doch Corrigans Beobachtungen stammen aus dem England der 1970er Jahre. Diese Tätigkeiten kommen an oben beschriebenen Nachmittag vor. Aber es sind auch andere, konstruktive, zu verzeichnen: Reden, dasitzen, manchmal ein Bad nehmen, ein Handy-Spiel machen, sinnieren, gut essen. Dazu wird wie bei Kiplings Stalky eine eigene Topografie eingenommen, die anders ist als die der Erwachsenen und doch den zentralen öffentlichen Raum im die Mittlere Rheinbrücke intensiv nutzt, ohne Rücksicht auf Verbote. Janine spricht sogar den Unterschied an zum erwachsenen Raumverhalten, indem sie sich mir gegenüber wundert, dass ich "als Alter" das mitmache. 


\section{Verläufe der Submoratorien}

Im Theoriekapitel wurde das theoretische Konzept der Submoratorien vorgestellt und in der Tabelle "Jugendliche Integration in die Erwachsenenwelt", aufgefächert. Um dieses Konzept in der Praxis zu prüfen, sollen am Schluss des empirischen Teils dieser Arbeit anhand von einigen Beispielen aus der untersuchten Klasse die Verläufe einiger Submoratorien nebeneinander gestellt werden. Diese wurden anhand der Qualität zur Verfügung stehenden Information ausgesucht. Bei vielen Jugendlichen war die Informationslage für eine solche Kategorisierung zu schlecht. Dies beruht darauf, dass im ursprünglichen Forschungsprogramm die Frage nach Integrations- und Ausschlussprozessen im Fokus stand. Erst im Verlauf dieser Forschung hat sich das Konzept der gestaffelt und parallel durchlaufenen Submoratorien herausgeschält, was aber nur noch teilweise in die Feldforschung eingeflossen ist. Zudem erschwerte die Tatsache, dass die Jugendlichen sich nicht gerne erforschen liessen, die Datenerhebung. Wie auch immer: In diesem Abschnitt sind die Fragen erkenntnisleitend, ob es für Mädchen andere Submoratorien gibt als für Jungen, und ob Jugendliche mit ausländischem oder schweizerischem Hintergrund unterschiedliche Verläufe haben.

Bei der Frage nach der Herkunft der Jugendlichen möchte ich nicht näher auf die Schwierigkeit eingehen, was als schweizerisch und ausländisch gilt. Dazu müsste ich eine Trennschärfe zwischen den Kategorien ausländische und schweizerische Jugendliche einführen, die es nur de jure, aber nicht in Wirklichkeit gibt. Die Antwort von Halil auf die Frage, ob er sich eher als Schweizer oder als Türke fühle, weist auf die Komplexität dieser Kategorisierung hin: "Ich fühle mich immer noch wie ein Türke, obwohl ich vor zwei Wochen den Schweizer Pass erhal- 
ten habe." ${ }^{\prime 21}$ Der Punkt ist das "immer noch": Als neuer Schweizer erwartet er also, dass er sich dereinst wie ein Schweizer fühlen werde. Doch dieses Gefühl sei bis jetzt nicht aufgekommen. Die „ausländischen" Jugendlichen sind in dieser Arbeit mit entsprechenden Vornamen versehen. Die meisten Jugendlichen haben ihre ausländische Herkunft betont, Nanny lehnte es sogar ab, den Schweizerpass als Zweitnationalität zu erhalten.

Grund dafür könnte sein, dass die meisten Jugendlichen an der WBS einen ausländischen Hintergrund haben, da könnte es von Vorteil sein, nicht als Reinschweizer zu gelten. Im Zweifelsfall griff ich auf die Selbstdefinition der jungen Leute zurück, um ihre Position zu klären. Im Lichte dieser Überlegungen habe ich vier Jugendliche im Folgenden ausgewählt, die als Schweizer zu bezeichnen sind. Die folgenden Beschreibungen stellen eine Momentaufnahme im Juni 2004 dar, kurz nach Abschluss der WBS und bevor die Jugendlichen sich zerstreuten.

Thomas Häberlin ist Basler und steckte vor einem Jahr mitten in allen Submoratorien. Sein übermächtiger Vater steht einer Festlegung im beruflichen Bereich entgegen, indem er dem Sohn zu hohe Ziele steckt. Beruflich herrscht also eine unklare Situation. Dieses Verhalten verschlechterte die Stimmung in der Familie und verunsicherte den Jungen stark. Im Bereich der Geschlechterbeziehungen ist Thomas Häberlin am arbeiten, er wurde etwa angeblich im Klassenlager gesehen, wie er mit einem Mädchen nachts wegging. Er hat aber keine feste Freundin. Er hat also im Bereich Geschlechterbeziehungen eine klare Perspektive, aber noch keine Beziehung. Gegenüber der Schulautorität passte er sich an und galt als problemlos, obwohl er ein schlechter Schüler war, schuf sich also auch hier einen Freiraum. Leider ist der Kontakt mit ihm abgebrochen, mehr Informationen waren nicht zu erhalten.

Thomas Oppliger wollte die Informatikmittelschule (IMS) machen, die prestigeträchtigste Option für einen WBSler, stieg aber nach weni621 Demir, Halil 1, 4. 
gen Tagen wegen psychischen Problemen wieder aus. Aus zweiter Hand erfuhr ich, dass er mit dem Leistungsdruck in dieser Schule nicht zurechtgekommen sei, aber niemand ausserhalb der Familie wusste etwas genaueres. Man könne ihn nur noch über den Computer kontaktieren, ausgehen würde er nicht mehr. Als ich ihn nach seinen engsten vier Freunden fragte, nannte er mir Email-Adressen im Ausland und der Schweiz, mit denen er regelmässig chatten würde. Er ist jetzt zuhause und denkt in einem Zwischenjahr nach, wie es beruflich weiter gehen soll, hat seine Mutter berichtet. Sie deutete an, dass er vielleicht eine Arbeit mit Tieren aufnehmen wolle. Hier scheint die Situation völlig unklar zu sein. Geschlechtliche Fragen scheinen bei ihm noch keine Rolle zu spielen, jedenfalls habe ich zu jenem Zeitpunkt keine Kontakte mit Mädchen beobachten können. Gegenüber Autoritäten spielte er auf Zeitgewinn und schaffte sich so Freiräume im vertikalen sozialen Submoratorium. Fragen der Lehrerin beantwortete er sehr zögerlich, oft nur mit ja oder nein, und ihn zu einem Gespräch zu bewegen war sehr schwierig.

Für Sonia Plüss scheint die moratoriumshafte Zeit vorbei zu sein. Im Gegensatz zu ihrer Zeit in der WBS. Dort war sie eher verträumt, kiffte viel und probierte laufend neue Styles aus, hat aber die Eintrittsprüfung in die Diplommittelschule (DMS) als eine der wenigen geschafft, was laut verschiedenen Beobachtern an ihrem starken familiären Umfeld liegt. Ich würde diesen Wechsel als typischen "Knopf-Aufmach"-Moment bezeichnen. Sie arbeitete zu jenem Zeitpunkt an Ihrem Eintritt in die Gestaltungs-Linie der DMS. Die Schullaufbahn differenziert sich nach einem Jahr aus. Sonia hat mir Arbeiten gezeigt, die ihr Engagement in diesem Bereich dokumentieren. Über ihr Verhältnis zum anderen Geschlecht habe ich nichts in Erfahrung bringen können. Jedenfalls hat sie die Style-Experimente beendet und entwickelte eine eigene Stilrichtung, wie sie mir beim Interview berichtete. Sie trug Kleider ihres eigenen Geschmacks und wirkte auch viel selbstsicherer und reifer. 
Claude Boettcher ist in Bezug auf das andere Geschlecht ambivalent, was vielleicht eine Folge seines christlichen Elternhauses ist, wo das Verhältnis zum anderen Geschlecht möglicherweise spezifisch geregelt ist. Er hatte als einziger gleichwertigen Zugang zu den Mädchen und zu den Jungen, was darauf zurückzuführen sein mag, dass die Mädchen ihn mit seinem feinen und zierlichen Körperbau nicht bedrohlich fanden. Er hatte noch keinen Stimmbruch und trug auch ein Halskette aus Holzperlen, was sonst kein Junge tat und was im sozialen Zusammenhang Schule eher ein weibliches Attribut sein könnte. Doch hat er die IMS angefangen und fühlt sich dort zuhause, die Berufsperspektive ist also klar. Sein Vater, ein Elektroingenieur, hat stark mitgeholfen, die berufliche Festlegung zu fördern. Soweit ich das beurteilen kann, hat er keine familiären Schwierigkeiten. Seine Strategie gegenüber Autoritäten war Schlitzohrigkeit. Doch damit stösst er an Grenzen. Als er im Schullager gefragt wurde, ob er für einen bestimmten Streich verantwortlich sei, kamen ihm die Lehrer in einem ironischen Chor zuvor und riefen: "Was, ich??” um eine für Claude typische Reaktion zu parodieren. Dieser Vorfall zeigt, dass er sich immer weniger durch vorgespieltes kindliches Verhalten Freiräume in Hierarchien offenhalten kann.

Nach diesen vier als schweizerisch beschriebenen vier Jugendlichen sollen weitere vier Jugendliche vorgestellt werden, die durch ihren nichtschweizerischen kulturellen Hintergrund gekennzeichnet sind. Akdis Saliba ist ein symphatisch wirkender Mensch, hat aber mit seinem arabisch klingenden Namen Schwierigkeiten, seine berufliche Zukunft zu festigen. Er plant mangels Alternativen ein Auslandsjahr in London bei einem Verwandten. Die berufliche Perspektive ist also unklar. Die anderen Submoratorien hat er durchlaufen. Er legt in letzter Zeit viel Wert darauf, mit Mädchen zu verkehren und sich ihnen im besten Licht zu zeigen. Im Moment ist er immer in der Nähe von Mädchengruppen zu beobachten, was ich als Zeichen eines starken Interesses und deshalb klarer Perspektiven deute. Gegenüber vermeintlichen Autoritäten wie 
dem Interviewer verhält er sich fast anbiedernd. Gerne bestätigt er im Gespräch vorauseilend die vermuteten Meinungen des Interviewers, nicht ohne zu fragen, ob ich denn die Macht hätte, die Lehrer zu feuern. Er wisse schon, wen er dafür empfehlen würde, versucht er einen Vorteil herauszuholen. Erbittet man etwas von ihm, macht er das schon, erwartet aber eine Gegenleistung. Er kann sich also in eine Hierarchie eingliedern und gut mit ihr umgehen. Eine Besonderheit ist die Perspektive auf religiöse Autoritäten. Er sagte einmal, Gott sei immer der Chef, und einige Mädchen der Klasse, die um ihn herum sitzen, bezeichneten ihn spöttisch als Priester, weil er oft salbungsvoll moralische Platitüden vortrug.

Devi Mahic ist in der DMS und somit am Anfang eines weiteren Wegabschnitts, an dessen Ende die Perspektive zu klären ist. Dieses Submoratorium ist also noch nicht durchlaufen. Er schmiedet grosse Pläne, will Architekt werden und schnödet über seine Klassenkameraden, die angeblich nicht begreifen, worum es geht im Leben. Die Überheblichkeit, die in diesen Worten aufscheint, hat er auch gegenüber den Mädchen in seiner Klasse an den Tag gelegt, die ihn deshalb nicht mochten. Im Bezug auf das andere Geschlecht findet er also nicht den richtigen Ton. Einerseits möchte er mit Mädchen zu tun haben, andererseits verachtet er sie. Devi scheint im beruflichen Bereich also klare Perspektiven zu haben, doch mit dem Geschlechtssubmoratorium scheint das weit weniger der Fall zu sein. Als ich ihn nach der WBS auf seine ehemaligen Schulkameradinnen anspreche, macht er eine wegwerfende Handbewegung. Auch im Umgang mit den Autoritäten hatte er Schwierigkeiten. Die Lehrer wiesen ihn manchmal für mich unverständlich heftig zurecht, weil sie mit seinem sozialen Verhalten unzufrieden waren. Devi hat also weniger Freiräume zur Verfügung wie andere Jugendliche.

Rosa Di Lorenzo hatte mehrere Todesfälle enger Verwandter zu erleiden. Diese Schicksalsschläge führten dazu, dass sie sich stark abkap- 
selte. Dies ist eine Krisensituation, welche das Leben überflutet und die Submoratorien zum Stillstand bringen kann. Sie ist eine hübsche, zierliche junge Frau, die oft von Jungen angesprochen wurde, die sie dann heftig abwies. Sie spürte also den geschlechtlichen Aufbruch, wollte aber mit Jungen nichts zu tun haben, um ihre Energien auf die Schule zu konzentrieren, was nur dank dem Verständnis der Lehrer auch ging. In der energieraubenden familiären Situation wurde also einzig das Berufssubmoratiorium weitergetrieben, das Geschlechtssubmoratorium stark verlangsamt. Gegenüber der lehrerlichen Autorität verhielt sie sich ausweichend. So hielt sie sich Freiräume auf, was ausnahmsweise von den Lehrern akzeptiert wurde. Über die berufliche Perspektive weiss ich nichts, vermute aber auch hier Unklarheit, da sie sich in der Schnupperlehre passiv verhielt und jetzt das Brückenangebot Basis besucht. Rosas Entwicklung steckt also in den Zweideutigkeiten der Submoratorien, und sie hat meiner Meinung nach die Entwicklung von Perspektiven wegen ihrer tragischen Biografie zu jenem Zeitpunkt eingestellt.

Fiona De La Vega ist in Bezug auf das andere Geschlecht völlig klar und hat feste männliche Freunde. Beruflich schliesst sie das Submoratorium ab: Zunächst hat sie eine Lehrstelle abgelehnt, weil sie noch besser Deutsch lernen wollte. Dazu besucht sie jetzt das Log-In, ein Brückenangebot für junge Frauen mit Migrationshintergrund. Von dort aus konnte sie in das Login (die beiden Angebote heissen wirklich fast gleich) wechseln, einem Ausbildungsverbund verschiedener Firmen des öffentlichen Verkehrs, und eine Lehre als Informatikerin anfangen. Diesen Prozess kann als Übergang von Ambivalenz zu Perspektive aufgefasst werden. Gegenüber Autoritäten hatte Fiona eine klare Haltung und wusste ganz genau, wie weit sie gehen konnte. Beispielsweise spielte sie im Französisch oft den Clown, weil sie wusste, dass der Französischlehrer sie mochte. Ihr Verhalten zog normalerweise keine Konse- 
quenzen nach sich ausser ein gelegentliches Gespräch mit dem Lehrer unter vier Augen, das sie offensichtlich nicht als Strafe auffasste.

Zusammenfassend kann man sagen, dass die schweizerischen Jugendlichen bezüglich des Submoratoriumsbereichs Körper und Geschlechterbeziehungen eher weniger weit gefestigt sind als die ausländischen. Im beruflichen Bereich können sich die schweizerischen Jugendlichen viel mehr erlauben. Sie können etwa das Submoratorium ausdehnen und Freiräume schaffen, da ihre Chancen auf dem Arbeitsmarkt dank Beziehungen und sozial höhergestellten Eltern besser sind. Hier sind die ausländischen Jugendlichen in der Regel energischer in der Ausbildung einer Perspektive. In Bezug auf das Submoratorium Autoritäten und Verhalten in einer Hierarchie lässt sich kaum eine Tendenz herauslesen, die mit der Herkunft zu tun hat. Es gibt sowohl schweizerische wie ausländische Jugendliche, die sich gegenüber Autoritäten Freiräume zu verschaffen wussten, anderen gelang das nicht so gut, waren sie Schweizerinnen oder Ausländer.

Teilen wir die Jugendlichen nach Geschlecht ein, können folgende Tendenzen ausgemacht werden: Bei den weiblichen Jugendlichen scheinen die Submoratorien früher auszulaufen, sie sind meist entwickelter in den Perspektiven als die männlichen Jugendlichen. Sie sind von der Berufsperspektive her eher gefestigt, wobei die Ansprüche tiefer sind. Im Umgang mit der Familie oder Autoritäten scheint bei Mädchen die Ambivalenz jedoch grösser zu sein.

In Bezug auf das Verhältnis zum anderen Geschlecht und den Körper sind bei beiden Geschlechtern mehrheitlich klare Perspektiven auszumachen, was darauf hindeutet, dass das Geschlechtssubmoratorium in den meisten Fällen mit 15 bereits durchlaufen ist. Doch hier gibt es methodische Probleme: Es ist enorm schwierig, in diesem heiklen Bereich überhaupt verwertbare Aussagen zu erhalten und zu beurteilen, weil die Jugendlichen nicht gerne darüber reden. Oft musste ich mich auf Klatsch und Hörensagen abstützen. Nur von einer jungen Frau 
weiss ich aus erster Hand, dass sie gegenwärtig lesbisch ist, da sie geklagt hatte, dass eine Angebetete sie zurückgewiesen habe. Ich war über die Offenheit überrascht. Es gibt auch verschiedene Sprachen: Ein anderes Mädchen habe ein Verhältnis mit einem jungen Mann angefangen, wie eine Zeugin berichtete, wobei ich keine Änderung im Verhalten der beiden gegenüber einander feststellen konnte. Ich konnte diesen Sachverhalt nicht interpretieren: Möglicherweise heisst ein Verhältnis haben für die Jugendlichen etwas anderes als für Erwachsene oder die Beiden verbergen ihr Verhältnis sehr gut.

Es ist schwierig, ein einheitliches Bild auszumachen. In allen genannten Submoratorien gibt es Ausnahmen und Extreme. Auch macht die rasante Entwicklung der Jugendlichen jede Positionierung unsicher, da sie auf Interviews beruht, die eine Momentaufnahme darstellen. Eine Person ist in geschlechtlichen Dingen völlig erwachsen, während sie in beruflicher Hinsicht völlig ambivalent ist, und bei einer anderen Person ist die Entwicklung umgekehrt. Dies weist darauf hin, dass die erste Person an ihrer beruflichen Perspektive arbeitet, während die zweite sich Zeit nimmt, seinen Umgang mit dem anderen Geschlecht zu verfeinern. Diesen Sachverhalt nehme ich als Bestätigung meiner These, dass die Submoratorien zeitlich gestaffelt durchlaufen werden.

Schliesslich möchte ich noch der Frage nachgehen, wie die Jugendlichen selbst die Frage nach den Submoratorien sehen. Ich konnte nicht direkt nach diesem intellektuell abstrakten Konzept fragen, sondern musste der Situation angemessene Fragen entwickeln und mich auf Beobachtungen stützen. Für die jungen Mädchen und Jungen scheint mit 16 oder 17 die Zeit der Aufschübe und Freiräume abgelaufen. Ich hörte zu jenem Zeitpunkt mehrfach Aussagen wie: "Ach, in der Oberstufe ${ }^{622}$ war das Leben noch locker!”. Oder ein Jugendlicher, der eine Lehre machen muss, die ihm eigentlich nicht zusagt, klagt darüber, dass er jetzt einfach wirklich nicht mehr ablehnen könne. Die moratoriumshaf622 Die Schulstufe vor der WBS. 
ten Freiräume gehen also zu. Dass Rafael und Peter ihre Lehren abgebrochen haben, deutet darauf hin, dass sie zwar die Notwendigkeit einsehen, berufliche Ambivalenzen abzubauen und sich Perspektiven zu schaffen, dass dies aber in der alltäglichen Praxis angesichts des sich verschlechternden jugendlichen Gesellschaftsvertrags nicht einfach durchzuhalten ist, wenn man nicht weiss, was man will, und deshalb demotiviert ist. 


\section{Schlussbemerkungen und Ausblick}

\subsection{Schlussbemerkungen}

Noch nie wurde in der Schweiz eine Schulklasse derart lange begleitet. Die mikroskopische Untersuchung der sozialen, ökonomischen und geschlechtlichen Integration rund eines dutzend Schülerinnen und Schülern zeigte, wie die Position eines Erwachsenen in unserer Gesellschaft aufgebaut und wie sie mit verschiedenen Ebenen der Gesellschaft verknüpft wird.

Ausgehend von der Frage nach Integrations- und Ausschlussprozessen innerhalb einer Schulklasse hat sich der Forschungsfokus dieser Arbeit aufgrund der Feldforschungsergebnisse bald weiter entwickelt und sich zum Schluss auf Statusfragen konzentriert. Damit konnte eine für diese kulturwissenschaftliche Forschung wenig fruchtbare Perspektive des innen und aussen sowie ablenkende Konzepte wie Ausländer und Einheimische überwunden werden. Dies war deshalb ein Gewinn, da diese Begriffe entweder innerhalb verschiedener Diskussionszusammenhänge verschiedene Bedeutungen erhalten oder aus politischen Debatten vorbelastet sind.

Das Thema Integration und Ausschluss erwies sich als äusserst komplex: Der oder die Einzelne, die Schulklasse und Gruppen in ihr, sowie die Gesellschaft sind mit einer Vielzahl an Zugehörigkeiten untereinander verknüpft. Diese Vielschichtigkeit macht es schwierig, generell von Ausschluss oder Integration zu sprechen. Deshalb wurde hier ein Integrationsbegriff entwickelt, der nach Bedarf zeitlich und zweckrational eingegrenzt werden kann. Es wurde festgestellt, dass Integration immer einem Zweck dient. Sie dient zur Herstellung einer Arbeitsatmosphäre 
für ein bestimmtes Ziel und stellt damit eine funktionierende Gruppe her.

Über die Diskussion von Begriffen wie Normalität oder Identität vor dem Hintergrund klassischer theoretischer kulturwissenschaftlicher Ansätze wie diejenigen um die Theorien der Rites de Passage gelang es, Fragen nach dem Status der Jugendlichen innerhalb des Übergangs ins Erwachsenenleben und damit auch über den Status der ökonomischen, sozialen und geschlechtlichen Selbständigkeit zu stellen. Eine wichtige Rolle hierbei spielte auch die Theorie der Statuspassagen und die praktische Zugangsweise der teilnehmenden Beobachtung, welche entscheidende Hinweise lieferte.

Dank dieser Vielschichtigkeit konnten die sozialwissenschaftlichen Theorien des Erwachsenwerdens weiterentwickelt und differenziert werden, um den empirischen Erkenntnissen gerecht zu werden und die Jugendlichen als kulturelle Akteure in einem wichtigen Lebensabschnitt zu verstehen. Aufschlussreich war das Ringen der Akteure um Normalität auf unterschiedlichen Ebenen.

Die hier vorgelegte Theorie der Submoratorien besagt, dass es drei Entwicklungsfelder gibt, welche parallel und/oder gestaffelt durchlaufen werden. Die Jugendlichen müssen innerhalb dieser Felder Perspektiven entwickeln, wie diese strukturiert werden können. Diese Felder sind das Geschlechtssubmoratorium, welches alle Fragen um Geschlechtsidentität und Beziehungen umfasst. Daneben gibt es das einnahmenseitige ökonomische Submoratorium, welches sich mit Fragen der ökonomischen Selbstständigkeit und dem Unterhalt befasst. Für die Jugendlichen ist in dieser Hinsicht die Frage nach dem beruflichen Werdegang wichtig. Kehrseite davon ist das ausgabenseitige ökonomische Submoratorium, welches sich mit der kapitalistischen Konsumkultur und dem Umgang mit dessen Kräften befasst. Das dritte Feld kann Sozialsubmoratorium genannt werden und teilt sich in das horizontale Sozialsubmoratorium, welches sich mit Freundschaften und dem sozia- 
len Umfeld befasst und dem vertikalen Sozialsubmoratorium, wo es um die Position in Hierarchien und der Anbindung an verschiedene autoritäre Strukturen geht. Innerhalb aller dieser Submoratorien gibt es Statusübergänge und einen Wendepunkt, der als Moment des "Knopf-Aufmachens" bezeichnet wurde. Dieser Moment bezeichnet die Erkenntnis, dass es um einen selber geht, dass man in unserer Gesellschaft selber aktiv werden muss.

Mit diesem theoretischen Konzept wurde es möglich, für jede Einzelne der Schülerinnen und jeden Einzelnen der Schüler, bei denen die Datenlage es erlaubte, sozusagen eine Momentaufnahme seines oder ihres Status im Übergang von der Kindheit zum Erwachsenenleben mit kulturwissenschaftlichen Begriffen und damit wertungsfrei zu definieren.

Es wird deutlich, dass das im theoretischen Teil beschriebene zentrale Konzept der Submoratorien zunächst eine untergeordnete Rolle spielt, um dann im Verlauf der empirischen Forschung immer wichtiger zu werden. Die Forschungsanlage war darauf angelegt, Integrationsund Ausschlussprozesse zu beobachten. Dies wurde zu Beginn der Feldforschung auch gemacht, deshalb sind die Gruppen in der Klasse beschrieben. Erst allmählich wurde der Zusammenhang der Gruppendynamiken mit den Verläufen der Submoratorien deutlich, die Analysekraft des Konzepts erkannt. Deshalb wird das Konzept der Submoratorien im Verlauf der empirischen Datenerhebung immer wichtiger und kulminiert am Schluss in einer Übersicht zum Stand der Submoratorien bei einzelnen Schülerinnen und Schülern, bei denen die Quellenlage dies erlaubte. Der Fokus wandelte sich also von der Gruppe zum Individuum und den Bedingungen, welche seine Gruppenanbindung mitbestimmten.

In der Betrachtung der durch die Feldforschung gewonnen Daten mithilfe der entwickelten theoretischen Werkzeuge liessen sich einige Erkenntnisse über die Gruppe der Schülerinnen und Schülern der Klas- 
se 2D gewinnen. Zunächst einmal wurde festgestellt, dass es eine breite Spanne von Zuständen gibt, die in den Momentaufnahmen über die jungen Leute aufscheinen. Es sollen einige Beispiele nochmals kurz aufgegriffen werden: Thomas Oppliger war ein Jahr jünger wie die anderen, da er eine Klasse übersprungen hatte. Dieser war noch völlig in Ambivalenzen gefangen und bezahlte dies auch mit psychischen Problemen. Sonia dagegen war aus den Submoratorien heraus. Sie hat zum Zeitpunkt des Interviews einen zielsicheren und aktiven Eindruck hinterlassen, in allen Feldern die Ambivalenzen überwunden und Perspektiven entwickelt. Neben diesen eindeutigen Fällen steckten, wie aufgrund des Alters der Beforschten zu erwarten war, viele Jugendliche noch in ambivalenten Situationen. Thomas Häberlin wirkte verunsichert und gleichzeitig gerissen. Er hatte beruflich noch keine Perspektive, war ebenso in geschlechtlichen Dingen am Suchen. Er arbeitete also am Abbau von Ambivalenzen im geschlechtlichen Bereich. Akdis Saliba versuchte, sich beim anderen Geschlecht im besten Licht zu zeigen, ist hier also nicht mehr ambivalent, vermag aber beruflich aufgrund seiner Herkunft nicht Fuss zu fassen, bleibt hier also in der Schwebe. Rosa Di Lorenzo hatte mehrere Todesfälle enger Verwandter zu erleiden. Diese Schicksalsschläge liessen sie sämtliche Entwicklungen im Sozialsubmoratorium und im Geschlechtssubmoratorium abbrechen. Die junge Frau konzentrierte sich mithilfe der Lehrer ganz auf die wegen ihrer energieraubenden Situation wenigen möglichen Schritte der ökonomischen Perspektivensuche.

Der Stand der jungen Menschen in dieser Klasse war also sehr vielfältig. Es können trotzdem Aussagen über die Gruppe gemacht werden. Doch diese Thesen fussen auf lückenhaftem Datenmaterial und sind sicher nicht pauschal auf andere Gruppen übertragbar. Es kann postuliert werden, dass die Mädchen eher früher wie die Jungen die Ambivalenz der Jugend überwinden. Auch kann behauptet werden, dass die ausländischen Jugendlichen eher früher wie die schweizerischen Jungen die 
Ambivalenzen hinter sich lassen und erwachsen werden. Weshalb dies so ist, kann nur eine weitergehende Studie beantworten. Auch wurde festgestellt, dass die Erwachsenwerdung der Geschlechter nicht mehr streng getrennt in eigenen Formen abläuft. Dies lässt darauf schliessen, dass der kulturelle Unterschied zwischen Mädchen und Jungen kleiner geworden sein muss.

Durch die Zusammenfassung einiger Ansätze in verschiedenen Fächern wurde die Europäische Ethnologie um eine Weiterentwicklung der Rites-de-Passage-Theorie für postmoderne, globalisierte Gesellschaften bereichert. Die Theorie der Submoratorien erlaubt es, wertungsfrei Fragen nach dem Zustand eines Übergangs zu beantworten. Dies zwingt dazu, Jugendliche ernst zu nehmen und einen nüchternen Blick auf das familiäre, soziale und ökonomische Umfeld zu werfen, wo Ambivalenz und Perspektive miteinander ringen. Stigmatisierungen haben in diesem Konzept keinen Platz. Die vorgeschlagenen Submoratorien können die Jugendforschung innerhalb und ausserhalb von Schulen bereichern, weil u.a. die Thesen der Entstandardisierung und Entsynchronisierung berücksichtigt werden. Dieser Beitrag möge auch als Aufforderung verstanden werden, die noch vereinzelten und marginalen Ansätze der Europäischen Ethnologie zum Thema Schule und den darin verbrachten Lebensabschnitten weitere Forschungsarbeiten zu widmen.

Da diese Arbeit zunächst von gruppenbasierten Integrations- und Ausschlussprozessen ausging und das zentrale theoretische Konzept der Submoratorien erst im Verlauf der Forschung entstanden ist, verschiebt sich der Fokus der Feldforschung allmählich von Gruppendynamiken zum Individuum und den Bedingungen, welche seine Gruppenanbindung mitbestimmt. Deshalb ist die Datenlage zum Thema Submoratorien zum Teil unsystematisch. Es wäre wünschenswert, wenn zukünftige Forschungen zum Thema Erwachsenwerden das Konzept der Submoratorien einbeziehen, überprüfen und verfeinern würden. 
Ein weiterer Forschungsansatz könnten es sein, den Einfluss der neuen Medien, Digitalisierung und des Internets auf die Übergangsphase von Kinderheit und Jugend zu untersuchen. Diese neuen Kommunikationsmöglichkeiten beeinflussen ganz sicher die Verhandlungen der jungen Menschen über ihre Identität. Auch könnte es lohnend sein, in nichtoffiziellen Bereichen versuchen herauszufinden, wie Menschen in Übergängen von Ambivalenz zu Struktur gelangen. Wie konstituiert sich etwa eine kriminelle Karriere? Es müsste auch abgeklärt werden, wovon es abhängt, dass jemand die Option Kriminalität wählt. Auch müsste untersucht werden, wie der Übergang zur kriminellen Karriere gestaltet ist und wie er rituell markiert wird. Der Untergrund hat sicher seine eigenen Gesetze, die den Neuzutretenden Barrieren in den Weg legen, wie andere Berufe auch.

Es fragt sich auch, ob die Theorie auf andere Übergangsvorgänge anwendbar gemacht werden kann ausser dem Erwachsenwerden von Jugendlichen in der Schweiz. Da die Theorie unabhängig von Altersangaben zur Definition von Jugend macht, müssen kulturelle Phänomene zur Definition des Verhältnisses von Ambivalenz und Perspektive herangezogen werden. Diese sind in dieser Arbeit überhaupt nicht systematisch erarbeitet worden, sondern wurden aus verschiedenen Literaturen zusammengetragen. Wie auch immer: Die Theorie wird damit auf andere Kulturen und Zusammenhänge übertragbar. Sie könnte auf fremdkulturelle Zusammenhänge angepasst werden, da in anderen Kulturen sicherlich andere Statusübergänge üblich sind.

Die wichtigste Einschränkung dieser Studie war die immer noch recht kurze Beobachtungszeit. Um die Integrationsarbeit von Individuen und Altersgruppen besser zu begreifen und Ansätze zu ihrer Unterstützung entwickeln zu können, wäre eine teilnehmende Beobachtung über längere Zeiträume wünschenswert. Dies wäre eine Anpassung an die sich im Zuge der weiter fortschreitenden gesellschaftlichen Differenzierung, Veränderung und internationalen Verflechtung vermutlich 
weiter verlängernden Integrationsprozesse. Die Berufswelt diffenziert sich weiter aus: Viele Berufe, die es zum jetztigen Zeitpunkt gibt, gab es 2005 noch überhaupt nicht, andere sind vollständig abgegangen. Bei einigen hochspezialisierten Berufsbildern verstehen die Älteren unter uns die damit verbundenen Anforderungsprofile kaum mehr. Es wäre interessant zu wissen, wie sich die Integration in die Berufswelt in Verlauf und in den Anforderungen verändert.

Die Schweiz wird aufgrund der Bevölkerungs- und Wirtschaftsdynamik ein Einwanderungsland mit sich weiter differenzierender Ökonomie bleiben und kommt praktisch nicht umhin, diese Situation wissenschaftlich weiter zu begleiten. Die Institutionen des Bildungswesens, des Jugend- und Familienschutzes, aber auch die Akteure im privaten Sektor stehen vor der enormen Aufgabe, sich laufend an neue Integrationsanforderungen anzupassen. Glücklicherweise wird immer mehr anerkannt, dass der Übergang in die Berufswelt langwierig sein kann und für alle Mitglieder von Alters- und Bevölkerungsgruppen immer wieder akut werden kann. Es ist zu hoffen, dass die Zuständigen von einer eher notfallmässigen Bereitstellung von Möglichkeiten zu einem planmässigeren, wissenschaftlich fundierten Aufbau von Regelstrukturen finden können, und zwar nicht nur für Jugendliche.

Dabei sind die Akteure als Partner zu verstehen. Wer es vermag, diese Perspektive aufzugreifen, wird auch dazu gelangen, Jugendliche und andere Menschen mit Integrationsbedarf als Ressource zu verstehen und eine Defizitperspektive hinter sich zu lassen. Beispielsweise sind Jugendliche aus schwächeren Schulen sozial kompetent, haben viele Fähigkeiten, sind intelligent und freundlich, doch werden diese Ressourcen in der Berufswelt wenig anerkannt. Dies ist schade, denn unsere Gesellschaft ist in Zukunft auf diese Menschen angewiesen. Möglicherweise sollte überlegt werden, ob nicht neben der bewährten dualen Ausbildung ähnlich wie Fachhochschulen im Bereich der höheren Ausbildung Lehrklassen eingerichtet werden können. Diese könnten eine 
vollwertige Ausbildung auch in Berufen, die üblicherweise durch Berufslehren erlernt werden, ermöglichen. Trittbrettfahrende Firmen, welche die Ausbildung von Fachpersonal vernachlässigen, könnten zur Finanzierung dieser Institutionen herangezogen werden. So könnte die ökonomisch oft verständliche Zurückhaltung dieser Firmen in der Bereitstellung von Lehrmöglichkeiten überwunden werden. Auch sind die Berge von Bewerbungsdossiers, welche vor allem fremdkulturelle Jugendliche schreiben müssen, erschreckend hoch. Deshalb ist die traditionelle Ansicht zu hinterfragen, dass Schulabgehende grundsätzlich selber Arbeit suchen müssen. Diese Tradition benachteiligt weniger gut vernetzte Jugendliche, d.h. diejenigen mit weniger tiefen Wurzeln in der lokalen Gesellschaft oder tiefem Status.

Diese Entwicklung betrifft auch die hier untersuchten Jugendlichen. Sie stehen immer noch im Integrationsprozess: Nur zwei aus der Klasse 2D erreichten eine befriedigende Integration in einen Lehrbetrieb. Eine von ihnen will allerdings nach der Lehre nicht in ihrer niedrig qualifizierten Stelle bleiben. Alle anderen müssen sich mit Praktika, Uebergangslösungen und Brückenangeboten weiter um ihre Integration bemühen. Das Phänomen der „lebenslangen Integration“ und das damit verbunde Verbleiben in prekären Verhältnissen bleibt deshalb ein immer wichtigeres wissenschaftliches Forschungsfeld, das auch für andere Gruppen relevant ist. Wie gehen „Expats“, Flüchtlinge, Sans-Papiers, hochqualifizierte Einwanderer und andere Menschen in Dauerübergängen mit den sich ihnen stellenden Problemen um? Die Problematik der Integration in eine sich ständig verändernde Arbeitswelt und an ständig welchselnden Orten bleibt für alle Beschäftigten des privaten Sektors akut und sollte in ihren vielen Aspekten weiter wissenschaftlich erforscht werden. Am liebsten aus einer wissenschaftlichen, möglichst neutralen Sicht: Diese beweglichen Ziele der Integration sind nicht nur negativ zu beurteilen. Neben dem zugegebenermassen enormen Aus- 
schlusspotential dieser Entwicklungen müssten auch die Vorteile dieser Gesellschaftsdisposition besser verstanden werden.

Aus Sicht der Erkenntnisse des Uebergangs von der Kinder- in die Erwachsenenwelt öffnen sich auch interessante Perspektiven auf die Altersforschung. Der Tod war zwar schon immer ein traditioneller volkskundlicher Forschungsbereich und die Europäische Ethnologie erforscht seit langem das Sterben, beispielsweise Sterbebräuche und -rituale. Doch in wie weit lässt sich das Alter ethnologisch ähnlich wie die Jugend als Uebergangsphase auffassen, wo sich Strukturen nach und nach auflösen und wo es Wendepunkte gibt? Wann beginnen diese Prozesse und wie laufen sie ab? Der ökonomische Wendepunkt wird wohl mit der Pensionierung markiert. Gibt es aber auch soziale und das Geschlechtsleben betreffende Uebergänge, etwa nach dem Tod eines Ehepartners? Wie sind solche Abläufe ähnlich wie in der Adoleszenz mit körperlichen Prozessen gekoppelt? Das Phänomen der Langlebigkeit des Grossteils der westlichen Menschen im 21. Jahrhundert eröffnet hier ein weites ethnologisches Forschungsfeld.

\subsection{Ausblick}

Viele Passagen der vorliegenden Arbeit sind beschreibend. Diese narrativen Stellen sollen den Anspruch erfüllen helfen, ein differenziertes Bild von Basler Jugendlichen zu zeichnen, die erwachsen werden in den Jahren zwischen 2003 und 2005. Ich erzähle von Menschen, ihren Ängsten, Gefühlen, Freuden, Lastern, Handlungsweisen und Motivationen. Diese Stellen sollen die Menschen anschaulich werden lassen und durch ihre Lebensnähe ebenso viel zum Verständnis der Jugendlichen beitragen wie qualitative ethnologische Analysen oder quantitative sozialwissenschaftliche Zahlenreihen. So hoffe ich, dass es gelungen ist, die recht unbekannte gesellschaftliche Gruppe, und in ihr besonders die 
Mädchen mit fremdkulturellem Hintergrund und Mädchen mit sozial nicht ausgeschöpftem Potential, besser zu verstehen. Als Ausklang dieser Arbeit sollen nochmals die Jugendlichen in den Mittelpunkt gestellt werden. Es wird berichtet, was zum Zeitpunkt der Niederschrift im Winter 2006/2007 über ihren weiteren Werdegang in Erfahrung zu bringen war. Dies war erstaunlich wenig, denn die Jugendlichen gingen nach dem Schulabschluss bald getrennte Wege und wollten mit der Vergangenheit nichts mehr zu tun haben. Einzig diejenigen, mit denen ich während der Feldforschungsphase auch ausserhalb der Schule Kontakt hatte, waren zu weiteren Auskünften bereit, die anderen verschlossen sich ausnahmslos.

Im Juni 2005 schloss ich die Felderhebung ab. Thomas Oppliger, Tobias Holdener und Nanny van Eijk haben schon nach Abschluss der WBS den Kontakt mit mir abgebrochen. Sie waren auch nach mehrmaligem Nachhaken nicht mehr bereit, weitere Auskünfte über ihren Werdegang abzugeben und erklärten direkt oder durch ihre Eltern, dass sie zu keiner weiteren Zusammenarbeit bereit wären. Häufig wurde mit Zeitmangel argumentiert. Diese Haltung musste ich akzeptieren. Einige Schüler tauchten vollständig ab. Wohin Akdis Saliba nach dem Jahr im Brückenangebot gekommen ist, blieb mir verborgen. Zwei Möglichkeiten hat er erwähnt: einen Auslandsaufenthalt bei einem Onkel in London oder eine weiterführende Ausbildung. Ich habe ihn nach der WBS nur einmal getroffen und sonst nicht mehr gesehen, konnte also keine Informationen aus erster Hand mehr über ihn erlangen. Auch seine noch zugänglichen ehemaligen Klassenkollegen wussten nichts über ihn. Stefan Staub ist rund einen Monat vor Schluss des Brückenangebots Basis doch noch von der Schule verwiesen worden, weil er zu viele Absenzen hatte. Dies ist meine letzte Information, ein Kontakt war nicht mehr möglich. Mit Jürg Beller konnte ich im Herbst 2004 das letzte Mal direkt sprechen. Er war damals im Brückenangebot Basis und suchte eine Lehrstelle. Rosa Di Lorenzo war schon in der WBS mir gegenüber sehr 
verschlossen und es gelang mir danach erst recht nicht mehr, weitere Informationen über ihren Werdegang zu erlangen oder direkt mit ihr zu sprechen.

Diese völlige Nachrichtensperre von einigen Schülerinnen oder Schülern war für mich überraschend. Die Aufzählung der wenigen indirekten Informationen über die weiteren biografischen Schritte eines Teils der untersuchten jungen Menschen zeigt vor allem eins: Der Klassenverband zerfiel nach Schulende sehr schnell. Der Eindruck drängt sich auf, dass die jungen Menschen es sehr eilig hatten, nichts mehr miteinander zu tun zu haben. Auch wurde der Forscher nicht mehr mit Informationen versorgt, was aktiv angekündigt wurde oder passiv erfolgte. Diese Beobachtung stützt die Annahme, dass Integration, hier in einen Klassenverband, ein Arbeitsbündnis ist. Es wird nur so lange aufrecht erhalten, wie es dem oder der Einzelnen nützt, um bestimmte Ziele zu erreichen, etwa wie hier die obligatorische Schule zu durchlaufen. Fällt dieses zwingende Element weg, zerfällt das Arbeitsbündnis sofort. In diesem Licht ist auch das Ausgrenzen des Forschenden zu sehen: Wozu soll jemand informiert werden, wenn dieser im Sozialgefüge Schule keine Rolle mehr spielt? Für manche wäre das reine Zeitverschwendung gewesen. Möglicherweise möchte man auch nicht mehr mit jemandem zu tun haben, der einem in einer verletzlichen Lebensphase erlebt und beobachtet hat.

Immerhin gelang es mir, über einige Schülerinnen und Schüler doch noch weitere, oft bruchstückhafte Informationen zu erlangen. Meistens konnte ich sie bei zufälligen Treffen in ein kurzes Gespräch über sich und ihre ehemaligen Kollegen zu verwickeln. Ein telefonischer Kontakt, ein zum vorne herein arrangiertes Treffen oder sogar ein Interview in einem ruhigen Rahmen waren überhaupt nicht möglich. Solche Avancen wurden schnell abgeblockt. In den nächsten Abschnitten sollen diese punktuellen Informationen dargestellt werden. 
Sonia Plüss war seit Herbst 2004 in der FMS, wie auch Loredana Di Costanzo und Devi Mahic, und die drei scheinen gute Perspektiven zu haben. Fiona De La Vega ging nach der obligatorischen Schule in das Login, einem Brückenangebot, das sprachliche Lücken bei Jugendlichen schliessen soll. 2005 begann sie eine Lehre bei den Bundesbahnen als Informatikerin. Rasiah Sunteralingam besass angeblich die Möglichkeit, nach einer Vorlehre B eine Lehrstelle als medizinische Praxisassistentin anzufangen. Thomas Häberlin hat zwar die Informatikmittelschule (IMS) anfangen können, fiel aber nach einigen Tagen wegen psychischer Probleme wieder heraus. Danach machte er ein Zwischenjahr bis in den Sommer 2005. Da dieser Fall eher tragisch war, habe ich nach einem schwierigen telefonischen Gespräch mit seiner Mutter nicht mehr nachgefragt, was aus ihm geworden ist. Halil Demir machte eine Vorlehre A als Maler bis in den Frühling 2005. Monika Bauer hat eine Lehre als Bäckerin/Konditorin angefangen und sich anscheinend gut eingearbeitet. Daniel Spreng hat seine Wunschlehre als Motorradmechaniker angefangen und ist zufrieden, wie er am Telefon erklärte. Leider konnte ich nicht mehr in Erfahrung bringen, da er mir gleichzeitig sagte, dass er keinen Kontakt mehr wünsche. Claude Boettcher hat die IMS angefangen und hat sich dort gut eingelebt. Als ich ihn zufällig traf, plauderten wir ein wenig. Es geht ihm gut und ihm gefällt die Lehre, auch wenn er ziemlich viel arbeiten muss, wie er sagt. Klaus Ganz hat das Brückenangebot Basis Plus gemacht. Günter Robert wollte in die Kinderbetreuungsbranche, fand aber zunächst nichts. So ging er in das Brückenangebot Basis.

Am längsten konnte ich den Informationsfluss über die drei Mädchen Janine Maggiorini, Loredana Di Costanzo und Martina aufrecht erhalten. Janine fing, wie bereits erwähnt, im Mai 2005 ein einjähriges Praktikum in einer Kinderkrippe an. Am Ende ihres Praktikums konnte sie keine Lehrstelle in diesem Bereich finden und suchte schliesslich erfolgreich nach einem weiteren Praktikumsplatz. Im Winter 2006 lernte 
sie Christoph kennen und baute mit ihm eine feste Beziehung auf, die auch bei letzter Nachfrage 2007 noch bestand. Zu jenem Zeitpunkt rauchte Janine überhaupt nicht mehr und arbeitete weiterhin in der Krippe.

Loredana hat die FMS, Richtung Soziale Arbeit, angefangen und schien diese Schule gut zu durchlaufen. Sie traf sich weiter mit Martina und Janine. Im Herbst 2006 arbeitete sie an einer Arbeit für die Schule über die rechtliche Stellung von gleichgeschlechtlichen Paaren. Für die Abfassung dieser Arbeit bat sie mich um Hilfe. So stellte ich Ihr einen Computerarbeitsplatz zur Verfügung, an dem wir ein Mal gemeinsam etwa eine Stunde lang redaktionelle Arbeiten verrichteten. Dabei kreiste das Gespräch auch um ihre ehemaligen Schulfreunde. Nach Abschuss dieser Arbeit ging sie nicht mehr auf meine Kontaktversuche ein. Sie war zu jenem Zeitpunkt mit Mona zusammen, allerdings gab es in der Beziehung anscheinend Unterbrüche.

Von Martina weiss ich noch weniger, da sie als Gymnasiastin von den drei Mädchen diejenige war, die während der Feldforschungsphase schon am distanziertesten war. Einen grossen Auftritt hatte sie im Theater ihres Gymnasiums als Tod in Camus' „Pest“, wo sie als Schauspielerin eine gute Bühnenpräsenz zeigte. Vermutlich ist sie jetzt bald mit dem Gymnasium fertig. Die Frage hat sie immer wieder beschäftigt, welche Richtung sie nach der Schule einschlagen solle.

Bereits 2006 sind die Jugendlichen allesamt aus meinem Blickfeld verschwunden, die Informationen versiegten vollständig. Nur gelegentlich sah ich Janine, die offenbar in der Strasse arbeitete, in der ich damals wohnte. Wir winkten uns manchmal über die Fahrbahn zu oder wechselten ein paar Worte miteinander. 


\section{Literatur, Quellen, Anhang}

\section{Literaturnachweise}

Andersson, B.-E.: "Does School Stimulate Young People's Development?", in: Johnsson, B. (Hg.): Studies on Youth and Schooling in Sweden. Stockholm, HLS Förlag, 1995.

Baacke, D.: Jugend und Jugendkulturen, 3. Auflage, Weinheim und München, Juventa, 1999.

Bachmann-Geiser, B.: Der Zürcher Schulsylvester, Zürich, Kommissionsverlag Hug \& Co., 1984.

Bauman, Z.: Flaneure, Spieler und Touristen. Essays zu postmodernen Lebensformen, Hamburg, Hamburger Edition, 1997.

Baudrillard, J.: Selected Writings, Oxford, Oxford University Press, 1988

Baumann, J.: Von Schulhäusern, Schulmeistern und der Schule in Oberwil, Separatum aus Baselbieter Heimatblätter 1986, Liestal, 1986.

Behnken, I. und J. Zinnecker: Lebenslaufereignisse, Statuspassagen und biografische Muster in Kindheit und Jugend, in: Jugendwerk der Deutschen Shell (Hg.): Jugend '92. Lebenslagen, Orientierungen und Entwicklungsperspektiven im vereinigten Deutschland. Band 2: Im Spiegel der Wissenschaften, Opladen, Leske \& Budrich, 1992, 127-143. 
Bendix, R.: Amerikanische Folkloristik. Eine Einführung, Berlin, Dietrich Reimer Verlag, 1995.

dies.: Was über das Auge hinausgeht: Zur Rolle der Sinne in der ethnografischen Forschung, SAVk 102 (2006), 71-84.

Bendix, R., M. Kraul, C. Kessler, M. Nietert und K. Koch: Fenster in die Schulkultur: Organisation und Gestaltung ritueller Übergänge, Zs. f. Vk I/106, Münster, u.a., Waxmann, 2010, 1-22.

Bergmann, M.: Vortrag "Wer ist drinnen und wer ist draussen? Dynamiken der soziokulturellen und politischen Integration und des Ausschlusses in der Schweiz", Hochschule für Pädagogik und Soziale Arbeit, Basel, 2005.

Binder, S.: Schule und Migration, Einblicke in die Praxis des interkulturellen Lernens, in: SWS-Rundschau, Schwerpunkt „ZuwandererInnen-Integration mit Formen interkulturellen Lernens in Wien“, 4/42, Wien, 2002, 422-440.

Bourdieu, P.: Die feinen Unterschiede: Kritik der gesellschaftlichen Urteilskraft, Frankfurt a. M., Suhrkamp, 2010.

Breidenstein, G.: Teilnahme am Unterricht, Ethnografische Studien zum Schülerjob, Wiesbaden, VS Verlag für Sozialwissenschaften, 2006.

Breidenstein, G. und H. Kelle: Geschlechteralltag in der Schulklasse, Ethnografische Studien zur Gleichaltrigenkultur, Weinheim und München, Juventa, 1998. 
Breitenbach, E.: Mädchenfreundschaften in der Adoleszenz. Eine fallrekonstruktive Untersuchung von Gleichaltrigengruppen, Opladen, Leske + Budrich, 2000.

Bürgin, D.: Autoinitiationsversuche - Mangelgeburten aus der Not, in: Kosinski, Günter (Hg): Pubertätsriten, Bern Stuttgart Toronto, Hans Huber, 1991, 165-175.

Bunar, N. und M. Dahlstedt: Segregationen, 'community action' och skolan som arena, Umeå, Norrköping, Växjö, PfMI, 2000.

Bundesverfassung der Schweizerischen Eidgenossenschaft vom 18.4.1999: Präambel.

Burckhardt-Sebass, C. und E. Liebl: Maturvergnügungen der Basler Schülerinnen, Basel, 1964.

Cicero, M. Tullius: Erste Rede gegen Catilina, Abschnitt 2.

Corrigan, P.: Doing Nothing, in: Hall, Stuart und Tony Jefferson (Hg.): Resistance Through Rituals. Youth Subcultures in post-war Britain. London, Routledge, (1975) 2002, 103-105.

Csikszentmihalyi, M.: Flow: Das Geheimnis des Glücks, 6. Auflage, Stuttgart, Klett Cotta, 1998.

Dencik, L.: Framtidens skola. K-U-L, Stockholm 1997.

Denzin, N. K.: Sociological Methods. A Sourcebook, Chicago, Aldine, 1970. 
Eberhard, H.: Schulbräuche: Eine volkskundliche Untersuchung der traditionellen Elemente an den Volksschulen der Stadt Graz, Graz 1976, unpubliziert.

Eggmann, S. und C. Renggli: Schlussbericht zum NFP51-Workshop "Begriffe und Theorien zu Integration und Ausschluss", Basel 2005.

Eickelpasch, R. und C. Rademacher: Identität, Bielefeld, transcript, 2004.

Ein Aufklärer des Alltags. Hermann Bausinger im Gespräch mit Wolfgang Kaschuba, Gudrun M. König, Dieter Langwiesche, Bernhard Tschofen, Wien, Köln, Weimar, Böhlau, 2006

Eisner, M.: Die Zunahme von Jugendgewalt - Fakt oder Artefakt? in: Eisner, Manuel und Patrik Manzoni (Hg.): Gewalt in der Schweiz. Studien zu Entwicklung, Wahrnehmung und staatlicher Reaktion, Chur 1998.

Ely, M.: Qualititiv forskningsmetodik i praktiken - circlar inom circlar, Lund, 1994.

Enzensberger, H. M.: Ach, Europa!: Wahrnehmungen aus 7 Ländern, Frankfurt a. M., Suhrkamp, 1987

Erdheim, M.: Zur Entritualisierung der Adoleszenz bei beschleunigtem Kulturwandel, in: Kosinski, Günther (Hg.): Pubertätsriten, Bern Stuttgart Toronto, Hans Huber, 1991, 79-88.

Erikson, E. H.: Jugend und Krise. Die Psychodynamik im sozialen Wandel, Stuttgart, Klett-Cotta, 1974. 
Fend, H.: Frühentwicklung in der Adoleszenz und ihre Risiken, in: Kosinski, Günter (Hg.): Pubertätsriten, Bern Stuttgart Toronto, Hans Huber, 1991, 102-128.

Fillitz, T. (Hg.): Interkulturelles Lernen. Zwischen institutionellem Rahmen, schulischer Praxis und gesellschaftlichem Kommunikationsprinzip. In: Bildungsforschung 18, Insbruck, Wien Bozen, Studien Verlag, 2003.

Fischer, A. und J. Zinnecker: Einleitendes zur Jugendstudie 1992, in: Jugend '92. Lebenslagen, Orientierungen und Entwicklungsperspektiven im vereinigten Deutschland. Band 1: Gesamtdarstellungen und biografische Portraits, Opladen, Leske und Budrich, 1992, 9-22.

Folkuniversitetet, Stockholm: Ausgeteilte Fotokopien im Unterricht in den Sprachintegrationskursen III - V, 1999.

Frebel, L. und S. Helms: Wunderbare Jahre? Magisterfilm, Institut für Kulturanthropologie/Europäische Ethnologie, Göttingen, 2009.

Furlong, A. und F. Cartmel: Young People and Social Change. Individualization and Risk in Late Modernity. Buckingham, Open University Press, 1997.

Geertz, C.: Dichte Beschreibung, Beiträge zum Verstehen kultureller Systeme, 4. Auflage, Frankfurt a. Main, Suhrkamp, 1995.

Giddens, A., The Consequences of Modernity, Oxford 1990: Polity und Modernity an Self Identity. Self and Society in the Late Modern Age, Oxford, Polity, 1991. 
Glaser, B. und A. Strauss: The Discovery of Grounded Theory, Chicago, 1967.

dies.: Status Passage, London, Routledge \& Kegan Paul, 1971.

Göttsch, S. und A. Lehmann (Hg.): Methoden der Volkskunde, Positionen, Quellen, Arbeitsweisen der Europäischen Ethnologie, 2. Auflage, Berlin, Reimer, 2007

Götz, Irene: Empirische Erhebungen in Industriebetrieben und bürokratischen Organisationen, in: Göttsch, Silke und Albrecht Lehmann (Hg.): Methoden der Volkskunde. Positionen, Quellen, Arbeitsweisen der Europäischen Ethnologie, 2. Auflage, Berlin, Reimer 2007, 249-270.

Goffman, E.: Interaktionsrituale, Über Verhalten in direkter Kommunikation, Frankfurt a. M., Suhrkamp stw 594, 1991.

Gross, P.: Bastelmentalität: ein 'postmoderner' Schwebezustand?, in: Thomas Schmid (Hg.): Das pfeifende Schwein, Berlin, Wagenbach, 1985, 63-84.

Gyr, U.: Welschlandaufenthalte als Übergangs- und Kontaktmuster, in: Hugger, Paul (Hg.): Handbuch der Schweizerischen Volkskultur, Leben zwischen Tradition und Moderne, Ein Panorama des schweizerischen Alltags, Band 1, Zürich 1992, 119-128.

Hansson Scherman, M.: Att vägra vara sjuk. Göteborg, Acta Universitatus Gothoburgensis, 1994. 
Hauser-Schäublin, B.: Teilnehmende Beobachtung, in: Beer, B.: Methoden und Techniken der Feldforschung, Berlin 2003, 33-54.

Havighurst, R.J.: Developmental Tasks and Education, New York, 1992.

Hebdige, D.: Subculture. The Meaning of Style, 14. Auflage, London und New York, Routledge. 1979.

Heiz, W.: Die "Unerreichbaren". Probleme der Jugendberatung, Dissertation, Zürich 1983.

Heller, J.: Catch-22, 7. Auflage, London, Random House, 1994.

Herlyn, Gerrit: Ritual und Übergangsritual in komplexen Gesellschaften, Sinn und Bedeutungszuschreibungen zu Begriff und Theorie, Hamburg, Lit Verlag, 2002.

Herrmann, E.: Methoden und Möglichkeiten der Soziografie in der Schule (anhand oberfränkischen Materials), Heimatbeilage zum Amtlichen Schulanzeiger des Regierungsbezirks Oberfranken, Bayreuth, 1985.

Hobsbawm, E.: Age of Extremes. The Short Twentieth Century 19141991, London, Abacus, 1994.

ders.: Mass-Producing Traditions: Europe, 1870-1914, in: Hobsbawm, Eric und Terence Ranger (Hgs.): The Invention of Tradition, 8. Ausgabe, Cambridge, Canto, 1992, 263-308. 
ders.: Interesting Times: A Twentieth-century life, London, Abacus, 2003.

Honer, A.: Einige Probleme lebensweltlicher Ethnografie. Zur Methodologie und Methodik einer interpretativen Sozialforschung, in: Zeitschrift für Soziologie 4, 1989, Jahrgang 18, 297-312.

Hugger, P.: Pubertätsriten - einst und jetzt - aus der Sicht des Volkskundlers, in: Kosinski, Günter (Hg): Pubertätsriten, Bern Stuttgart Toronto, Hans Huber, 1991, 25-39.

Jacobsson, A.: En plats i klassen, en plats i världen, en studie om eleverna i en klass i norra Botkyrka, Botkyrka, Mångkulturellt Centrum, 1995.

Jonsson, B.: Unga vuxna, deras livsprojekt och tankar om framtida yrke, in: Hagström (red.): Ungdomar i övergångsåldern - handlingsutrymme och rationalitet på väg in i arbetslivet, Lund, Studentlitteratur, $1999,58-89$.

Jugendstudie der Deutschen Shell, Frühjahr 2002.

Jugendwerk der Deutschen Shell (Hg.): Jugend ' 81 . Lebensentwürfe, Alltagskulturen, Zukunftsbilder, Band 1, Opladen, Leske + Budrich, 1982.

dass.: Jugend '92. Lebenslagen, Orientierungen und Entwicklungsperspektiven im vereinigten Deutschland, Opladen, Leske + Budrich, 1992. 
Kaschuba, W.: Kulturalismus: Kultur statt Gesellschaft? in: Geschichte und Gesellschaft 21, Heft 1, Göttingen, Vandenhoeck \& Ruprecht, 1995,83 .

Kaufmann, V., M. M. Bergman und D. Joye, Motility: Mobility as Capital, in: International Journal of Urban and Regional Research, Volume 28.4, 745-756.

Kelle, H.: Mädchen und Jungen in Aktion, Ethnografische Ansätze in der schulischen peer culture Forschung, in: Horstkemper, Marianne und Margret Kraul (Hg.): Koedukation - Erbe und Chancen, Weinheim, Deutscher Studien Verlag, 1999, 157-183.

dies.: Ethnografische Methodologie und Probleme der Triangulation, am Beispiel der Peer Culture Forschung bei Kindern, in: Zeitschrift für Soziologie der Erziehung und Sozialisation (ZSE) 21 (2001) 2, 192208.

Keupp, H.: Auf der Suche nach der verlorenen Identität, in: Keupp, Heiner und Helga Bilden (Hg.): Verunsicherungen, Göttingen, Hogrefe, 1989, 47-69.

Kipar, M.: Penne ade! Festaktivitäten zum Abitur zwischen Tradition und Suche nach neuen Formen, Münster, Waxmann, 1994.

Klosinski, G.: Pubertätsriten - Äquivalente und Defizite in unserer Gesellschaft. Einführende Anmerkungen des Jugendpsychiaters, in: Kosinski, Günter (Hg): Pubertätsriten, Bern Stuttgart Toronto, Hans Huber, 1991, 11-20. 
Krahn, H.: The School to Work Transition in Canada: New Risks and Uncertainties, in: Heinz, Walter R. (Hg.): The Life Course and Social Change: Camparative Perspectives, Weinheim, 1991, 43-69.

Kramer, J.: Sonderbare Europäer. Gesichter und Geschichten, Frankfurt a. M., Eichborn, 1993.

Küffer, G.: Volkskundliches im Deutschunterricht, in: Schweizerische Lehrerzeitung 22, 1917, 199-201.

Kullberg, B.: Etnografi i klassrummet, Lund, Studentlitteratur, 1996.

Lalander, P. und T. Johansson: Ungdomsgrupper i teori och praktik, 2. Auflage, Lund, Studentlitteratur, 2002.

Ledergerber, B.: Damit mangelnde Integration nicht zur Desintegration führt, SuchtMagazin 1, Basel, 2005, 22-24.

Lévi-Strauss, C.: Rasse und Geschichte, in: Lévi-Strauss, C.: Strukturale Anthropologie II, Frankfurt a.M., 1975, 365-407.

Lindner, R.: Die Angst des Forschers vor dem Feld. Überlegungen zur teilnehmenden Beobachtung als Interaktionsprozess, in: Zeitschrift für Volkskunde 77, 1981, 51-66.

Link, J.: Versuch über den Normalismus: wie Normalität produziert wird, 2. Auflage, Opladen, Westdeutscher Verlag, 1999.

Lockwood, D.: Soziale Integration und Systemintegration, in: Zapf, Wolfgang (Hg.): Theorien des sozialen Wandels, Köln und Berlin, Kiepenheuer \& Witsch, 1970, 124-137. 


\section{Ludwig-Uhland-Institut für Empirische Kulturwissenschaft: Von}

der Volkskunde zur empirischen Kulturwissenschaft, mit Beiträgen von Hermann Bausinger, Utz Jeggle, Gottfried Korff, Martin Scharfe, u.a., Tübingen 1974.

Lyotard, J.-F.: The Postmodern Condition. A Report on Knowledge, Minneapolis, 1984, University of Minneapolis Press und Baudrillard, J.: Selected Writings, Oxford, Oxford University Press, 1988.

McRobbie, A. und J. Garber: Girls and Subcultures, in: Hall, Stuart und Tony Jefferson: Resistance Trough Rituals, Youth subcultures in post-war Britain, London, Routledge, (1975) 2002, 177-188.

Meier, J. (Hg.): Lehrproben zur deutschen Volkskunde, Berlin und Leipzig, Walter de Gruyter \& Co., 1928.

Mercier, H.: Les Promotions du Collège de Genève de 1798 jusqu'a nos jours, Genève, Villard \& Rabot, 1934.

Merleau-Ponty, M.: Phänomenologie der Wahrnehmung, Berlin, Walter de Gruyter, 1974.

Metje, U. M.: "Hier haben ja alle Löffel Löcher" Forschung im eigenen Land: Eine ethnologische Untersuchung über Strassenkids, in: Ethnopsychoanalyse 6 . Forschen, erzählen und reflektieren, Frankfurt a. Main, Brandes \& Apsel, 2001, 42-69.

Mezger, W.: Die Bräuche der Abiturienten: Vom Kartengruss zum Supergag, ein Beitrag zur Schülervolkskunde, Konstanz, Universitätsverlag Konstanz GmbH, 1993. 
Muri, G.: Pause! Zeitordnung und Auszeiten aus allitagskultureller Sicht, Frankfurt a. M., Campus, 2004.

Myers, M.: The teacher-researcher. Urbana, Illinois, ERIC Clearinghouse on Reading and Communication Skills and the National Council of Teachers of English, 1985, 2-5.

Neidhardt, F.: Die junge Generation: Jugend und Gesellschaft in der Bundesrepublik, Opladen 1967.

Newell, W. W.: Games and Songs of American Children, New York, Harper and Brothers, 1883.

Ochsner, P., U. Kenny und P. Sieber: Vom Störfall zum Normalfall. Kulturelle Vielfalt in der Schule, Chur und Zürich, Rüegger, 2000.

Oelkers, J.: Jugendbewegung als Erziehungsform: Historische Anmerkungen, in: Kosinski, Günther (Hg.): Pubertätsriten, Bern Stuttgart Toronto, Hans Huber, 1991, 64-75.

Olk, T.: Jugend und Gesellschaft, in: Heitmeyer, W. (Hg.): Interdisziplinäre Jugendforschung, Weinheim, Juventa, 1986.

Olofsson, G.: Om begreppen segregation och integration, in: Olofsson, Gunnar et al.: Integration och segregation - i teori och praktik, Växjö 2001, 10-12.

Olsson, G.: Mellan Rum. En studie i fysisk och mental utveckling av kommunikation med utgångspunkt i en mellanstadieklass, Stockholm, Brutus Östlings Bokförlag Symposion, 2003. 
Oring, E.: Dyadic Traditions. Journal of Folklore Research 21: 19-28.

Ortner, S.: On key symbols, American Anthropologist 75(5), 1973: 1338-1346.

Oswald, H.: Die Beziehung zu Gleichaltrigen, in: Jugendwerk der Deutschen Shell (Hg.); Jugend '92. Lebenslagen, Orientierungen und Entwicklungsperspektiven im vereinigten Deutschland, Band 2, 319332.

Parker, H.: Aus Jungen werden Männer. Kurze Adoleszenz in einem innerstädtischen Wohnbezirk, in: Clarke, John, u.a., Jugendkultur als Widerstand. Milieus, Riutale Provokationen. Honneth, Axel, Rolf Lindner und Rainer Paris (Hg.), Frankfurt a. M., Syndikat, 1979, 181-216.

Reh, S., K. Rabenstein und C. Schelle: Unterricht als Interaktion, Bad Heilbrunn, Klinkhardt Verlag, 2010.

Roberts, K.: Structure and Agency: The New Youth Research Agenda. In: Bynner, J., Chisholm, L., \& Furlong, A.: Youth, Citizenship and Social Change in a European Context. Aldershot, Ashgate, 1997.

Rolshoven, J.: Europäische Ethnologie - Diagnose und Prognose einer kultur- und sozialwissenschaftlichen „Volkskunde“, Österreichische Zeitschrift für Geschichtswissenschaft, Wien, 2004, 73-87.

dies.: Integrations- und Ausschlussprozesse von Jugendlichen in Ausbildungsgruppen. (Schlussbericht Projekt Nr. 4051-40-69227 im Rahmen des NFP 51 "Integration und Ausschluss"), 2006. 
Rousseau, J.-J.: Emile oder Über die Erziehung, 1762, herausgegeben von M. Rang, Stuttgart, Reclam, 1968.

Runfors, A.: Mångfald, motsägelser och marginalisieringar. En studie av hur invandrarskap formas i skolan, Stockholm, Prisma, 2003.

Sauter, S.: Wir sind Frankfurter Türken. Adoleszente Ablösungsprozesse in der deutschen Einwanderungsgesellschaft, Frankfurt a. Main, 2000.

ders.: Der Forscher als Mentor, in: Ethnopsychoanalyse 6. Forschen, erzählen und reflektieren, Frankfurt a. M., 2001, 70-93.

Schenda, R.: Lüthi, Max, in: Enzyklopädie des Märchens, 8, 1996, 1307-1313.

Schmidt-Lauber, B.: Das qualitative Interview oder: Die Kunst des Reden-Lassens, in: Göttsch, Silke und Albrecht Lehmann (Hg.): Methoden der Volkskunde. Positionen, Quellen, Arbeitsweisen der Europäischen Ethnologie, 2. Auflage, Berlin, Reimer, 2007, 169-188.

dies.: Feldforschung. Kulturanalyse durch teilnehmende Beobachtung, in: Göttsch, Silke \& Albrecht Lehmann (Hg.): Methoden der Volkskunde. Positionen, Quellen, Arbeitsweisen der Europäischen Ethnonogie, 2. Auflage, Berlin, Reimer, 2007, 219-248.

Schmidt, L.: Brauch ohne Glaube, in: Scharfe, Martin (Hg.): Brauchforschung, Darmstadt, Wissenschaftliche Buchgesellschaft, 1991, 105135. 
Schumann, M. und B. Fuhs (Fotos): Übergangsverläufe: Ende der Jugendzeit? in: Jugendwerk der Deutschen Shell (Hg.): Jugend '92. Lebenslagen, Orientierungen und Entwicklungsperspektiven im vereinigten Deutschland. Band 1: Gesamtdarstellungen und biografische Portraits, Opladen, Leske + Budrich, 1992, 173-183.

Schwab, G.: Sagen des klassischen Altertums, Wien Heidelberg, Überreuther, 1974.

Schweizerischer Nationalfonds, Division Targeted Research, National Research Programmes (NRP): NRP 51 "Social Integration and Social Exclusion", Final scientific report.

Sennett, R.: Der flexible Mensch. Die Kultur des neuen Kapitalismus, Berlin, Berlin Verlag, 1998.

Spranger, E.: Psychologie des Jugendalters, 28. Auflage, Heidelberg, 1966.

Stone, D. A.: Gatekeeping Experts and the Control of Status Passages, in: Heinz, Walter R. (Hg.): The Life Course and Social Change: Comparative Perspectives, Weinheim, 1991, 203-220.

Strauss, A.L.: Qualitative Analysis for Social Scientists, Cambridge, Cambridge University Press, 1987.

Strübin, E.: Baselbieter Volksleben, Sitte und Brauch im Wandel der Gegenwart, 2. Auflage, Basel, 1967.

ders.: Jahresbrauch im Zeitenlauf, Kulturbilder aus der Landschaft Basel, Liestal, Verlag des Kantons Basel-Landschaft, 1991. 
Svensson, S.: Folkkulturkunskap som undervisningsämne i seminarierna, Rig 1941, Separatdruck, 75-78.

Trümpy, H.: Schule, Volksbrauch und Volksglaube, Separatum aus der Basellandschaftlichen Schulnachrichten, 1964, 72-78.

Turner, T. S.: Transformation, Hierarchy and Transcendence: A Reformulation of Van Gennep's Model of the Structure of Rites de Passage, in: Moore, Sally F. und Barbara G. Myerhoff: Secular Ritual, Assen/Amsterdam, Van Gorcum, 1977, 53-56.

Turner, V.: Das Ritual, Struktur und Anti-Struktur, Frankfurt/NewYork, Campus 95, 1989.

Unterweger, G.: Klasse und Kultur. Verhandelte Identitäten in der Schule, Zürich, 2002 (Zürcher Beiträge zur Alltagskultur, Bd. 12).

Van Gennep, A.: Übergangsriten (Les rites de passage), Frankfurt/New York, Campus, 1986.

Von der Mühll, J.: Basler Sitten, Basel, 1944.

Von Trotha, T.: Zur Entstehung der Jugend. Kölner Zeitschrift für Soziologie und Sozialpsychologie 34, 1982, 254-277.

Walcott, H.: Criteria for an Ethnografic Approach to Research in Schools. Human Organisation 34 (2), 111-127. 
Wallace, C. und S. Kovacheva: Youth in Society. The construction and Deconstruction of Youth in East and West Europe, Basingstoke, MacMillan Press Ltd., 1998.

Webb, P.: Unobstuctive Measures. Nonreactive Research in the Social Sciences, Chicago, Rand McNally \& Co., 1966.

Whitman, W.: Grasblätter. Übersetzt von Jürgen Brocan, München, C. Hanser, 2009.

Willis, P. E.: Learning to Labour. How working class kids get working class jobs, 7. Auflage, Aldershot, Gower, 1983.

Wirsing, M.: Oberfränkisches Schulleben in älterer Zeit am Beispiel des früheren Schulortes Pilgramsreuth, Ldkr. Hof, Heimatbeilage zum Amtlichen Schulanzeiger des Regierungsbezirks Oberfranken 138, Bayreuth, 1987.

Wulf, C. (Hg.): Einführung in die Anthropologie der Erziehung, Weinheim und Basel, Beltz Verlag, 2001.

ders. (mit Birgit Althans, Kathrin Audehm, Gerald Blaschke, Nino Ferrin, Ingrid Kellermann, Ruprecht Mattig, Sebastian Schinkel): Die Geste in Erziehung, Bildung und Sozialisation: Ethnografische Feldstudien, Wiesbaden, VS Verlag, 2011.

Wulff, H.: Bästa vänner. Förtrogna flickor på upptäcksfärd i livet, in: Kulturella perspektiv. Svensk etnologisk tidskrift 3-4, 1992, 23-31.

Zeugin, P. und D. Gros: Jugendkultur in der Schweiz, Kurzfassungen der Projekte des NFP 21, Basel, 1991. 
Zinnecker, J.: Sozialgeschichte der Mädchenbildung. Zur Kritik der Schulerziehung von Mädchen im bürgerlichen Patriarchalismus, Weinheim, Juventa, 1973.

ders.: Metamorphosen im Zeitraffer: Jungsein in der zweiten Hälfte des 20. Jahrhunderts, in: Geschichte der Jugend, Band II. Von der Aufklärung bis zur Gegenwart, Levi, Giovanni und Jean-Claude Schmitt (Hg.), Frankfurt a. M., S. Fischer, 1997, 460-505.

ders.: Jugend als Moratorium. Essay zur Geschichte und Bedeutung eines Forschungskonzepts, in: Reinders, Heinz und Elke Wild (Hg.): Jugendzeit - Time Out? Zur Ausgestaltung des Jugendalters als Moratorium, Opladen, Leske + Budrich, 2003, 37-64.

ders: Alles ist möglich und nichts ist gewiss. Deutschlands erste Jugendgeneration im 21. Jahrhundert, in: Coolhunters. Jugendkulturen zwischen Medien und Markt, Frankfurt a. Main, Suhrkamp, 2005, 175190.

\section{Quellen}

\section{II.1 Dokumente amtlicher Stellen}

Abteilung Jugend, Familie und Prävention des Justizdepartementes Basel-Stadt: Die Situation sozial schwacher Jugendlicher im Übergang zwischen Schule und Beruf, Übersicht über Programme, Akteure, Schnittstellen in Basel-Stadt (und Basel-Land), 24. Oktober 2003. 
Erziehungsdepartement, Ressort Schulen: Medienmitteilung "Leitbild für die Schulen des Kantons Basel-Stadt", 1.12.2005, Kanton BaselStadt, Medienmitteilungen, Leitbild für die Schulen des Kantons BaselStadt, http://www.bs.ch/mm/ed/2005/12/ed-20051201-001.html, 4.3.2009.

Kantonale Integrationsstelle Basel-Stadt "Integration Basel": Integration Basel-Stadt, Basel 2004.

Weiterbildungsschule Basel-Stadt: Jahresbericht 2000/2001.

Weiterbildungsschule Basel: Bericht der Arbeitsgruppe "Allgemeine Integrationsarbeit” zur Vorbereitung der Strukturänderung, 2003.

Weiterbildungsschule Basel: Bericht der Arbeitsgruppe "Leitbild" zur Vorbereitung der Strukturänderung, 2003.

\section{II.2 Originaldokumente}

15 anonymisierte Aufsätze der WBS 2D: Montag, der 4. Mai 2024, ein Tag in meinem Leben, Spickzettel, Wurfzettel, Schreibzeug, Plakate, Compact Discs, Hefte, Chat- und SMS-Protokolle. 


\section{II.3 Presseartikel}

Basler Zeitung, Basel: 25.9.2004 Interview mit Niklaus Gruntz, Leiter des Amtes für Berufsbildung und Berufsberatung Baselland, Beilage Stellefant, 1; 25.11.2004, 20; 10.3.2005, 1; 17.3.2005, 1; 23.3.2005, 40; 28.5.2005 Interview mit Reto Baumgartner, Leiter Berufsbildung beim Gewerbeverband Basel-Stadt, Beilage Stellefant, 1.

The Economist, London: 5.2.2005, 70.

\section{II.4 Websites}

Basel-Stadt Statistik Startseite, http://www.statistik-bs.ch, 4.3.2009. Bling-Bling - Wikipedia, http://de.wikipedia.org/wiki/Bling-Bling, 28.5.2006.

Kobe Bryant - Wikipedia, http://de.wikipedia.org/wiki/Kobe_Bryant, 28.2.2009.

Daniel Küblböck - Wikipedia, http://de.wikipedia.org/wiki/Daniel_K $\% \mathrm{C} 3 \%$ BCblb\%C3B6ck, 2.3.2009

Destiny's Child - Wikipedia, http://de.wikipedia.org/wiki/Destiny's_Child, 15.6.2006.

Gangsta-Rap - Wikipedia, http://de.wikipedia.org/wiki/Gangsta-Rap, 30.7.2012

Log in, http://www.sba-basel.ch/Log_in.53.0.html, 15.11.2010 login macht Erfolgsstories möglich: Home, http://www.login.org/de/, 15.11.2010

No Angels - Wikipedia, http://de.wikipedia.org/wiki/No_Angels, 29.2.2012 
Online-Liste der freien Lehrstellen der Ämter für Berufsbildung und Berufsberatung BS/BL, Lehrstellennachweis beider Basel, http://lenabb.ch/, 31.1.2006.

Outsourcing - Wikipedia, http://de.wikipedia.org/wiki/Outsourcing, 22.2.2012

Schule für Brückenangebote Basel: Start, http://www.sba-basel.ch, 4.3.2009.

Szenenforschung, basierend auf Material von Ronald Hitzler, Universität Dortmund und anderen Quellen, http://www.jugendszenen.com, 15.1.2006.

Tages-Anzeiger Online, http://www.tagesanzeiger.ch, 26.12.2005 Website Deutsche Gesellschaft für Erziehungswissenschaften, Kommission Qualitative Bildungs- und Biographieforschung, http://www.dgfe.$\mathrm{de} / \mathrm{sektionen/sektion-2-allgemeine-erziehungswissenschaft/kommis-}$ sion-qualitative-bildungs-und-biographieforschung.html, 11.2.2012. Weiterbildungsschule Startseite, http://wbs-basel.ch, 4.3.2009

Wiki - Wikipedia, http://de.wikipedia.org/wiki/Wiki\#Vorl.C3.A4ufer, 4.3.2009.

World Drug Report - archive, http://www.unodc.org/unodc/en/data-andanalysis/WDR.html, 15.11.2010.

\section{II.5 Interviews}

\section{Einzelgespräche}

Bauer, Monika 2.3.05, unstrukturiertes, längeres Gespräch, etwa eine Stunde

Beller, Jürg: Mai/Juni 04: strukturiertes Einzelgespräch, etwa eine Stunde 
Blülle, Stefan, Abteilung Jugendschutz des Justizdepartementes BaselStadt

Boettcher, Claude: Mai/Juni 04: 1. strukturiertes Einzelgespräch, etwa eine Stunde; 1.12.04, 2. unstrukturiertes Kurzgespräch, eine halbe Stunde

De La Vega, Fiona: Mai/Juni 04: 1. strukturiertes Einzelgespräch, etwa eine Stunde; 13.1.05, 2. unstrukturiertes, längeres Gespräch, etwa eine Stunde

Demir, Halil:Mai/Juni 04: 1. strukturiertes Einzelgespräch, etwa eine Stunde; 13.4.05, 2. unstrukturiertes, längeres Gespräch, etwa eine Stunde

Di Costanzo, Loredana, Mai/Juni 04: strukturiertes Einzelgespräch, etwa eine Stunde

Ganz, Klaus (und zwei Kollegen, die spontan mitkamen) 14.4.05, 1. unstrukturiertes, längeres Gespräch, etwa eine Stunde; Mai/Juni 04: 2. strukturiertes Einzelgespräch, etwa eine Stunde, Gisler, Isabelle, Klassenlehrerin WBS 2D, strukturiertes Einzelgespräch, etwa zwei Stunden

Häberlin, Thomas, Mai/Juni 04: strukturiertes Einzelgespräch, etwa eine Stunde

Holdener, Tobias, Mai/Juni 04: strukturiertes Einzelgespräch, etwa eine Stunde,

Ledergerber, Beatrice, Amt für Berufsbildung Basel-Stadt, strukturiertes Einzelgespräch, etwa zwei Stunden

Maggiorini, Janine: Mai/Juni 04: 1. strukturiertes Einzelgespräch, etwa eine Stunde; 8.6.05, 2. unstrukturiertes, längeres Gespräch, etwa eine Stunde

Mahic, Devi: Mai/Juni 04: 1. strukturiertes Einzelgespräch, etwa eine Stunde; 13.4.05, 2. unstrukturiertes, längeres Gespräch, etwa eine Stunde 
Müller, Sandra, Vorstandsmitglied und Nachwuchsbetreuerin des RTV Handballklub Basel

Oppliger, Thomas, Mai/Juni 04: strukturiertes Einzelgespräch, etwa eine Stunde

Plüss, Sonia: Mai/Juni 04: 1. strukturiertes Einzelgespräch, etwa eine Stunde; 2.3.05, 2. unstrukturiertes, längeres Gespräch, etwa eine Stunde Robert, Günter: Mai/Juni 04: 1. strukturiertes Einzelgespräch, etwa eine Stunde; 16.3.05, 2. unstrukturiertes, längeres Gespräch, etwa 1,5

Stunden

Saliba, Akdis: Mai/Juni 04: 1. strukturiertes Einzelgespräch, etwa eine Stunde; Mai 05, 2. unstrukturiertes, längeres Gespräch, etwa eine Stunde

Schaffner, Werner, Pensionierter, Riehen

Spreng, Daniel, Mai/Juni 04: strukturiertes Einzelgespräch, etwa eine Stunde

Staub, Stefan, Mai/Juni 04: strukturiertes Einzelgespräch, etwa eine Stunde

Sunteralingam, Rasiah, Mai/Juni 04: strukturiertes Einzelgespräch, etwa eine Stunde

Van Eijk, Nanny, Mai/Juni 04: strukturiertes Einzelgespräch, etwa eine Stunde

\section{Gruppengespräche}

Beller, Jürg, Saliba, Akdis, Mahic, Devi, Ganz, Klaus, Spreng, Daniel, 22.1.04,

Boettcher, Claude und Oppliger, Thomas, 29.1.04,

Van Eijk, Nanny, Sunteralingam, Rasiah, Di Lorenzo, Rosa, Bauer, Monika im Januar 04

Robert, Günter, Demir, Halil, Holdener, Tobias, Häberlin, Thomas im Januar 04 


\section{II.6 Feldberichtstage}

2003: 12. September, 14. und 24. November, 1., 2., 5., 10., 12., 15. und 19. Dezember

2004: 7., 9., 14., 17., 21., 23. und 28. Januar, 4., 11. und 13. Februar, 16., 17., 22., 25., 26., 27. und 31. März, 3. und 4. April, 5., 11.,18., 25. und 26. Mai, 3., 8., 11. und 17. Juni, eine Woche ab dem 18. Juni, 20. September, 6. Oktober, 24. November, 3. und undatiert im Dezember 2005: 6., 13., 14., 25. und 29. Januar, 9., 10. und 24. Februar, 2., 16. 22. und 30. März, 6. und 20. April, 3., 4., 7., 18., 24. und 25. Mai, 8. und 14. Juni, 11. August

\section{Anhang}

III.1 Leitfaden strukturiertes Interview vom Juni 2004

Prolog

Erklärung, was ich mache, dass die Informationen geheim bleiben und dass keine echten Namen in der Auswertung erscheinen werden.

Sprichst Du noch andere Sprachen ausser Deutsch?

Was wird zuhause geredet?

Freizeitverhalten

1. Ein typischer Samstagabend 
2. Ein typischer Mittwochabend

3. Die vier besten Schulkumpels

4. Die vier, die Du am wenigsten magst

5. War das vor 3 Monaten auch so?

6 . Bist du in einem Verein?

7. Musik

7.1 Ist sie für dich wichtig?

7.2 Hören Deine Kolleginnen die gleiche Musik?

8. Machst du Games/Spiele?

9. Gehst Du zur Kirche?

10. Wie informierst du Dich? (Zeitungen, TV, Internet)

11. Was machst du in den (bevorstehenden) Frühlingsferien?

Schule

12. Wieso sitzen alle Mädchen am Fenster?

13. Haben die E-ler einen anderen Umgang als die G-ler?

14. Wer bestimmt, wie das Klassenzimmer eingerichtet wird?

15. Gibt es einen Klassengeist?

16. Gehst du gerne zur Schule?

17. Beliebteste Lehrer/Unbeliebsteste Lehrer

18. Verhältst Du Dich in der Schule anders als ausserhalb?

19. Machst Du Dir Sorgen um Deine Zukunft?

20. Was hast du für Zukunftspläne?

Erziehung

21. Was machen Deine Eltern?

22. Legen sie Wert auf ein bestimmtes Verhalten von Dir?

23. Darfst Du rauchen? 
24. Wirst Du streng erzogen?

25. Wirst Du anders erzogen als ein CH-Kind (bei Ausländern)?

Werte

26. Was ist das wichtigste im Leben?

27. Gibt es andere Aufgaben im Leben für Männer und Frauen?

28. Was ist Integration?

Schweiz

29. Lebst Du gerne in Basel?

30. Kommt Dir das Leben hier typisch Schweizerisch vor?

31. Werden Deiner Meinung nach die Ausländer anders behandelt als die Schweizer?

32. Gibt es Unterschiede in der Bahandlung von Ausländern?

33. Hat man als Schweizer bessere Chancen?

Herkunft (nur bei AusländerInnen)

34. Welche Erinnerungen hast Du an Dein Heimatland?

35. Wollen Deine Eltern wieder zurück?

36. Bist Du eher Schweizer/in oder ...?

Die Frage 26 wurde nachträglich eingefügt. Folgende Fragen fielen aus dem Interviewleitfaden heraus, da sie keine klaren Antworten ergaben:

1. Wozu ist die Schule gut?

2. Gibt es Deiner Meinung nach einen Unterschied im Verhalten der Mädchen und der Jungen in der Schule?

3. Als was siehst Du Dich mit 30? 
III.2 Fragen für das unstrukturierte Interview, Herbst 2005

1. Wie sieht er/sie die Klasse als Ganzes im Nachhinein?

2. Mit wem besteht noch Kontakt heute?

3. Sieht er/sie noch jemanden von früher?

4. Einzelne Personen aus ihrem/seinem Bekanntenkreis in der Klasse

5. Fühlt er/sie sich benachteiligt?

6. Erzählen, was in der Zeit vom Ende der WBS bis jetzt passiert ist

7. Bewerbungen

8. Markante Leute aus der Klasse (z.B. Stefan, Tobias, Thomas H.)

III.3 Liste von Denkeinheiten

Nach Kullberg, Etnografi i klassrummet, 1996, 124ff wurden diese Denkeinheiten ausgeschieden und per Datenauswertung mithilfe eines Wikis miteinander verbunden.

Stichwort

Action!

Angst

Arbeitswelt

\section{Erläuterung}

Definition. Rolle. Bedeutung. Beispiel. Beobachtungen, welche die Scheu/Angst vor der Verantwortung der Erwachsenenwelt dokumentieren oder interpretieren.

Ansichten über die Arbeitswelt 
Auffallen

Ausflug

Aus- oder Inländer

Ausschlussprozesse

Berufe

Bilder der Alten

Bild der Jungen

Bild in Medien

Biografie

Computer

Cool

Dissen

Drogen: Alkohol

Drogen: Cannabis

Drogen: Sonstige

Druck

Ehre

Eltern

Erwachsen
Bloss nicht auffallen. Bedingung des Erwachsenseins.

Reisen in die Umgebung.

Staatsbürgerliche und ethnische Unterschiede und ihre Bedeutung im Alltag. Theorie. Praxis: Dissen. Beobachtungen und Überlegungen

Beobachtungen über die Bewertung ei-n zelner Berufe.

Was die Jungen glauben, denken die Alten über sie

Was die Jungen über die Alten denken Der Mediendiskurs über die Jugendl-i chen. Nicht was berichtet wird, sondern welches Bild der Jugendkultur, der Ju gendlichen wird vermittelt.

Ansichten über das Leben. Als was wird die Biografie aufgefasst?

Umgang, Nutzung, "digital divide" Theorie. Praxis.

Begriff, Beispiele

Gebrauch, Bedeutung

Gebrauch, Bedeutung

Tabak, Harte Drogen, Kaffee, Zucker, Tee, u.a. (Gebrauch, Bedeutung)

Du musst! Zwänge für die Jugendli chen. Trimmen für den Arbeitsmarkt. Ehrbegriff der jungen Männer. Väter. Mütter. Rolle. Verhalten während der Pubertät. Strategien.

Was heisst erwachsen sein? 
Essen

Fähigkeiten

Fasnacht

Formelles

Forscher/Beforschte

Freiheit

Frust und Euphorie

Freundschaft (Mädchen)

Freundschaft (Jungen)

Geheimsprache

Geld, Schulden

GeschichteJugend

Gesellschaftl. Debatten

Geschlechterbeziehungen

Gewalt

Girl-Kultur

Gruppen

GruppenFallbeispiele

Gruppenarbeit

Handgemenge

Heiterkeit, Humor

Hilfsethnologen
Klassische Volkskunde

Was können Jugendliche?

Ansichten, Mitmachen, Meinungen

Schulen, Lehren, Gesetze und sonstige äussere Rahmenbedingungen.

Wie entwicktelt sich diese Beziehung? Historische Beispiele für Sonderstellung von gewissen Jugendgruppen (Studenten oder Gesellen, z.b).

Demotivation und Motivation im Erwachsenwerdensprozess

Allgemeine Gedanken, Werte

Allgemeine Gedanken, Werte

Gruppenkultur Loredana, Janine

Bedeutung von Geld

Die Geschichte der Jugend im Lichte des gesellschaftlichen Wandels Theorie Bilder junger Frauen: Teenage mütter, TV-Blondinen, "Dorfmatratze" Alles, was zwischen Mädchen und Jungen abläuft in Bezug auf Liebe, Sexualität, usw.

Quellen-Stellen. Vgl. auch Delinquenz Alles, was zum Girl-Sein gehört. Aussenbeobachtungen, Dynamiken, Theorien. Siehe auch Klassengeist Beschreibungen einzelner Gruppen In der Schule (Kochen, Projekte, usw) Physische Auseinandersetzungen. Als ethnologisches Analyseinstrument. Gewährsleute 
Höhle

Informelles

Integration

Janine und Sonia

Jobben

Jugendkultur

Julia

Kindisch

Klassengeist

Kleidung

Kommunikation

Kontakte

Konflikte

Kulturtechniken

Lehrer

Mathe

Medienkonsum

Musik

Mobiltelefon

Mutprobe
Ort, an dem am Übergang in die Erwachsenenwelt gearbeitet wird Rhizome, Beziehungen, Freunde Mikroprozesse, Begriff für die Jugendli chen, Feldbegrifflichkeit Eine Dyade. Vgl. auch Freundschaft (Mädchen)

Nebenjobs, Bedeutung, Beispiele Gibt es die? Merkmale, Diskussion. Eine Schülerin der Parallelklasse. An geblich die Schönste, ist immer von Jungen umgeben.

Was ist für die Jugendlichen kindisch? Emische Definition, Thema allgemein Gibt es den? Ansichten, Meinungen Bedeutung, Styles, usw. Unterschiede zu Erwachsenen Wer hat sie mit wem? Offizielle Konfliktlösung in der Schule Mangel als Ausschlussprozess, Grund für Absagen

Charaktisierung einzelner, Beobachtungen

Bedeutung eines Faches für die Schulkultur (Mädchen)

Allgemeine Beobachtungen

Wie wird Musik in den Alltag integriert?

Nutzung, Bedeutung

Siehe auch: Action 
Nazi

Normen

Orte

Peinlich

Rassismus

Recht

Rumhängen

Position

Schattenwirtschaft

Scheissdreck

Schlimmste, der

Schule Lehre

Schulstunden, gestört

Schwärmen

Spätzünder

Sport

Sozialbeziehungen
Umgang mit derjenigen Ideologie, die das absolut Böse darstellt.

Wie wird Normalität ermittelt und um gesetzt?

Welche Punkte in der Topografie sind interessant?

Peinliche Situationen und deren Deutung.

Quellenstellen, Reaktionen

Juristische Fragen um die Jugend. Status Jugendlicher.

Einfach irgendwo sitzen.

Das Aushandeln der sozialen Stellung innerhalb der Klasse, des Schulhauses und in der Gesellschaft

Aktivitäten, für die man keine Lehre braucht

Scheissdreck machen: Aktivitäten, die einem die Zukunft versauen, Leute ver ärgern

Warum ist er der Schlimmste? Die Stel lung des Schlimmsten.

Unterschiede Schule und Lehre.

Ereignisse, die den normalen Lauf einer Schulstunde stören.

Verhalten junger Mädchen

Begriff. Vgl. auch Moment des Übergangs

Wer macht was? Bedeutung

Vitamin B, vgl. auch Informelles 
Staat

Staatsbürgerschaft

Streiche

Style

Südfrankreich

Symbolik

Tabu

Tanzen

Töffli

Tradition

Unsicherheit

JungenMädchen

Jungen sind in der

Wohnung

Vorbilder

Zeitbegriff

Zickeln
Rolle, Eingriffsmöglichkeiten, Institutionen, Polizei (ohne Schule)

Nationaliät, Ethnie, Schweizertum

Leute ärgern. Ist Kinderkram.

Style-Typen. Definiert ein Style eine

Gruppe?

Dichte Beschreibung der Abschluss

fahrt. Er lebnisse, Deutungen.

Symbole und ihre Bedeutungen im

Wandel, Symbole des Erwachsenseins

Wie verändern sich die Tabus beim

Übergang zum Erwachsenenleben?

Let's go - Disco!

Niedergang und Comeback des typi-

schen Jugendlichenfahrzeugs. Mobilität

Traditionen, Bräuche, Rituale aus der

Sicht der Jugendlichen

Zaudern vor Berufswahl.

Welche Bereiche sind betroffen?

... Schule schlecht, dann haben sie mehr

Freunde: Das Phänomen der faulen

Jungs und fleissigen Mädchen. Erklä rungsversuche.

Bemühungen um eine Rückzugsmöglichkeit, eigene Wohnung Aus den Medien, sonstige Unterschiede in der Zeitauffassung von Jugendlichen und Erwachsenen. Auch: $\mathrm{Zu}$ früh, zu spät Eine Form des Neckens, Anmachens, Aufziehens 
Zielstrebig

Zukunft
Jugendliche, die einen klaren Weg vor sich sehen (oder den Weg vor sich klar sehen)

Wie sehen die Jugendlichen ihre Zukunft?

Gruppierte Themen:

Delinquenz, Devianz, Antisoziales Verhalten

Antisoziales Verhalten

Gesetzesbruch

Lügen

Randale

Stehlen

Der Übergang

Übergänge

Übergangsangebote

Abscheidungsrituale

Moment, der
Definition. Allgemeine Diskussion. Be obachtungen im Feld. Brechen von Regeln. Legalität, Polizei, usw.

Die Unwahrheit sagen.

Ist Fun. Oder nicht?

Klauen.
Allgemeines, Theorie

Professionelle Initiativen, den Übergang ins Berufsleben zu erleichtern.

Vgl. Südfrankreich

Zeitpunkt des Übergangs individuell:

Den Knopf aufmachen. Sozial: Das ei gentliche Übergangsritual 
Angliederungsrituale

Integration in einen neuen Bereich:

Lehrfirma, neue Schule, usw.

Hinweis: Eine Fülle von Quellen, Materialien, Literaturnachweisen und weiterer, in dieser Arbeit nicht verarbeiteter Inhalte können eingesehen werden auf http://www.lampenwerk.ch/disswiki, 5.5.2012. Hier finden sich eine Suchfunktion und nach Wiki-Manier vernetzte thematische Seiten. Einsicht nach Anfrage auf admin@lampenwerk.ch. 